\title{
WestVirginiaUniversity
}

THE RESEARCH REPOSITORY @ WVU

Graduate Theses, Dissertations, and Problem Reports

2021

\section{Majorana Nanostructures and Their Electrostatic Environment}

\author{
Benjamin David Woods \\ West Virginia University, bdw0010@mix.wvu.edu
}

Follow this and additional works at: https://researchrepository.wvu.edu/etd

Part of the Condensed Matter Physics Commons

\section{Recommended Citation}

Woods, Benjamin David, "Majorana Nanostructures and Their Electrostatic Environment" (2021). Graduate Theses, Dissertations, and Problem Reports. 8241.

https://researchrepository.wvu.edu/etd/8241

This Dissertation is protected by copyright and/or related rights. It has been brought to you by the The Research Repository @ WVU with permission from the rights-holder(s). You are free to use this Dissertation in any way that is permitted by the copyright and related rights legislation that applies to your use. For other uses you must obtain permission from the rights-holder(s) directly, unless additional rights are indicated by a Creative Commons license in the record and/ or on the work itself. This Dissertation has been accepted for inclusion in WVU Graduate Theses, Dissertations, and Problem Reports collection by an authorized administrator of The Research Repository @ WVU.

For more information, please contact researchrepository@mail.wvu.edu. 


\title{
Majorana Nanostructures and Their Electrostatic Environment
}

\section{Benjamin Woods}

\author{
Dissertation submitted \\ to the Eberly College of Arts and Sciences \\ at West Virginia University \\ in partial fulfillment of the requirements for the degree of \\ Doctor of Philosophy in \\ Physics

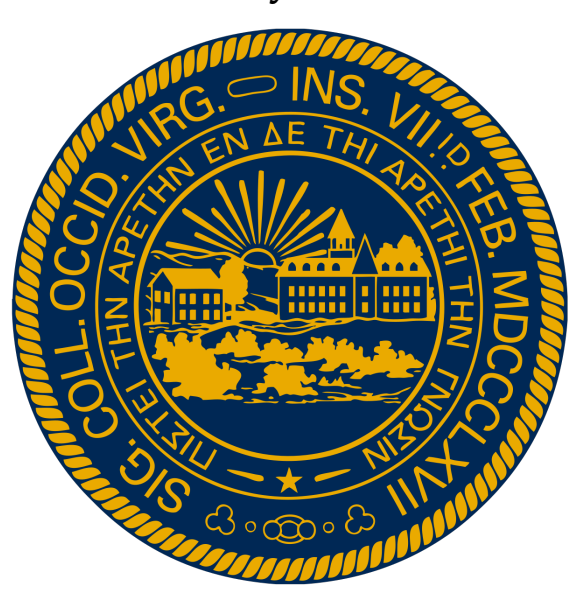 \\ Tudor Stanescu, Ph.D., Chair \\ Mikel Holcomb, Ph.D. \\ Matthew Johnson, Ph.D. \\ Aldo Romero, Ph.D. \\ Adrian Tudorascu, Ph.D.

\section{Department of Physics and Astronomy \\ Morgantown, West Virginia 2021}

Keywords:

Majorana zero modes, Topological superconductivity, Electrostatics,

Schrödinger-Poisson, Charge impurities 


\section{Abstract \\ Majorana Nanostructures and Their Electrostatic Environment \\ Benjamin Woods}

Majorana zero modes (MZMs) are zero-energy excitations emerging in one- and two-dimensional topological superconductors. These exotic modes have attracted much attention in the last decade due to their topological protection and non-Abelian statistics, which make them possible building blocks for topological quantum computation. In particular, semiconductorsuperconductor (SM-SC) nanostructures have attracted the most attention with several measurements being consistent with the presence of MZMs. Debate continues, however, whether MZMs or topologically-trivial Andreev bound states are responsible for such measurements.

In order to interpret experimental results distinguishing MZMs from Andreev bound states and gain a better understanding of what conditions need to be met in order for MZMs to be achieved in semiconductor-superconductor nanostructures, detailed modeling is required. In this thesis, work is presented that addresses this need with particular focus placed on understanding the electrostatic environment. A formalism is developed to solve the Schrödinger-Poisson equations for large systems. Additionally, effective models are constructed that accurately capture the low-energy physics governing Majorana devices while significantly reducing the computational complexity.

Various problems currently of importance to the community are addressed. As a first example, we study subband occupation in Majorana nanowires as a function of device parameters. We find that moderate values of surface charge density dramatically limits the parameter space lying in the optimal regime in which only a few well-separated subbands are occupied. As a second example, we study how the electrostatics affects the magnetic proximity effect in SM-SC-magnetic insulator nanostructures. The geometric layout of the three material components is shown to play a key role in determining the magnitude of the magnetic proximity effect and whether topological superconductivity is achievable. A detailed study of charge impurity disorder within Majorana nanowires is presented. We show that rather low charge impurity densities $\left(\sim 10^{15} \mathrm{~cm}^{-3}\right)$ destroy the topological superconductivity and resulting MZMs, indicating that significant improvements in material purity should be a top community priority. We also show that intersubband coupling arising from disorder or other non-uniformities, can pin trivial Andreev bound states near zero-energy, mimicking the phenomenology of MZMs. Lastly, original device designs in planar SM-SC nanostructures are presented. Periodic modulations of the superconductor are shown to significantly increase the topological gap and improve the robustness of MZMs against disorder and other non-uniformities. 


\section{Acknowledgements}

I would first like to thank my advisor, Dr. Tudor Stanescu, for his guidance, patience, and encouragement throughout my graduate years. It has been a true pleasure learning from you. I also want to thank Dr. Sankar Das Sarma from the University of Maryland for his various contributions to several of the projects presented in this thesis. I am also grateful to Trey Cole and Purna Paudel for collaborating with me over the last few years.

My years in Morgantown have been memorable in large part because of my wonderful friends. I am especially thankful to Nikki, Tristan, and Hasan. Many thanks to my parents for their love, support, and encouragement. I also want to thank Emily for being a sister to look up to.

Finally, thank you to my wife, Kaitlin, for your unending love, support, and sacrifices. I would not have been able to get through this without you. 


\section{Contents}

$\begin{array}{ll}\text { Abstract } & \text { ii }\end{array}$

Acknowledgements

1 Introduction 1

1.1 Topological quantum matter . . . . . . . . . . . . . . 2

1.2 Quantum computation ................. 10

1.2.1 Classical computing . . . . . . . . . . . . . 10

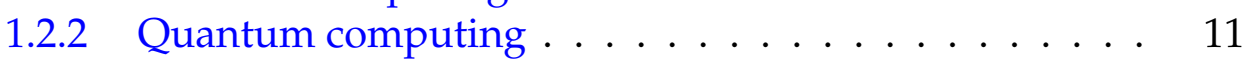

1.2.3 Quantum supremacy ...................... 12

1.2.4 Topological quantum computation . . . . . . . . . . . . 14

1.3 Majorana zero modes in semiconductor-superconductor nanostructures . . . . . . . . 17

1.4 Outline of thesis . . . . . . . . . . . . . . . . . . . 19

2 Theoretical Background 21

2.1 Mean field theory of superconductivity . . . . . . . . . . . 21

2.1.1 Bogoliubov-de Gennes transformation . . . . . . . . . . 23

2.1 .2 Majorana basis ............... 27

2.1 .3 Topological superconductivity . . . . . . . . . . 28

2.2 Kitaev chain model . . . . . . . . . . . . . . . . . . . 30

2.3 Rashba Majorana nanowires . . . . . . . . . . . . . . . . . . 35

2.4 Non-Abelian Statistics of Majorana Zero Modes . . . . . . . . 40

3 Motivations 43

4 Toward realistic modeling of Majorana nanowires 46

4.1 Formalism . . . . . . . . . . . . . . . . 48

4.2 Numerical method for solving the Poisson equation . . . . . . 56

4.2.1 Statement of the problem ................ 56

4.2.2 Weak form of the Poisson equation . . . . . . . . . 57

4.2 .3 Projection method . . . . . . . . . . . 58

4.2.4 Finite element mesh and basis functions . . . . . . . . 59

4.2.5 Calculation of matrix elements . . . . . . . . . . . 61

4.3 Numerical solution of the effective mass Hamiltonian . . . . . 63

4.3.1 Statement of the problem .................. 63

4.3.2 Reduction to spinless problem .......... 64

4.3.3 Weak form, basis functions, and matrix assembly . . . 64

4.4 Calculation of charge density from wavefunctions . . . . . . . 65

4.5 Model of the superconductor . . . . . . . . . . . . . 66 
4.6 Subband occupation in

semiconductor-superconductor nanowires . . . . . . . . 67

4.6.1 Device and modeling . . . . . . . . . . . . . . 69

4.6 .2 Results ................... 72

InSb ....................... 83

Estimation of spin-orbit coupling . . . . . . . . . 84

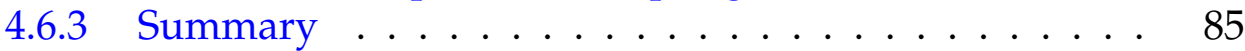

4.7 Electrostatic effects and topological superconductivity in semiconductorsuperconductor-magnetic insulator hybrid wires . . . . . . . 87

4.7.1 Mediated Proximity Scenario . . . . . . . . . . . . . 89

4.7 .2 Model ...................... 90

4.7 .3 Results . . . . . . . . . . . . . . . . 94

4.7 .4 Conclusion . . . . . . . . . . . . . . 97

5 Effects of Charge Impurity Disorder and Other Non-Uniformities in Majorana Nanowires

5.1 Charge impurity effects in hybrid Majorana

nanowires .................... 100

5.1.1 Single charge impurity . . . . . . . . . . . . . . 102

Model .................... 102

Self-consistent Schrödinger-Poisson scheme . . . . . 104

Results . . . . . . . . . . . . . . . 109

5.1 .2 Multiple charge impurities . . . . . . . . . . . . . 118

Model .................... 119

Results .................... 121

Low impurity density regime . . . . . . . . . . . . . . . . . 129

Intermediate impurity density regime . . . . . . . . . 135

Charge impurity statistics . . . . . . . . . . . . . . 141

5.1 .3 Conclusions . . . . . . . . . . . . . . . . 145

5.2 Zero-energy pinning of topologically trivial states in multi-

band semiconductor-superconductor nanowires . . . . . . . 147

5.2.1 Toy model . . . . . . . . . . . . . . . . . . . 149

Homogeneous wires . . . . . . . . . . . . 151

Inhomogeneous wires . . . . . . . . . . . 156

5.2 .2 3D model . . . . . . . . . . . . . . . . 162

Theoretical Model . . . . . . . . . . . . . . 162

Results . . . . . . . . . . . . . . . 167

5.2.3 Summary and Conclusions . . . . . . . . . . . 172

6 Topological superconductivity in a two-dimensional electron gas proximity coupled to periodically patterned superconductors $\quad 175$

6.1 Enhanced topological protection in planar quasi-one-dimensional channels with periodically-modulated width . . . . . . . . 176

6.1.1 One-dimensional model of periodic Majorana nanowires 177

Renormalization of miniband parameters . . . . . . 178

Majorana localization length . . . . . . . . . . . . 184

Topological phase diagram in systems with rectangular periodic potential . . . . . . . . . 185 
Soft confinement and partially-separated Andreev bound states in periodic systems . . . . . . . . . . . 188

Robustness against disorder . . . . . . . . . . . . . . . 190

6.1 .2 Majorana Waveguides . . . . . . . . . . . . . . . . . . . 195

6.1 .3 Summary and conclusions . . . . . . . . . . . . . . . 201

6.1 .4 Acknowledgments . . . . . . . . . . . . . . . . . 203

6.2 Enhanced topological superconductivity in spatially modulated

planar Josephson junctions . . . . . . . . . . . . . . . . . . . 203

6.2 .1 Modeling . . . . . . . . . . . . . . . . . . . . 205

Effective low-energy Hamiltonian . . . . . . . . . . 206

Green's function formalism . . . . . . . . . . . . . . 208

Qualitative effects of modulating the junction width . . 209

6.2.2 Uniform Majorana Josephson Junction Results . . . . . 212

6.2.3 Modulated Majorana Josephson Junction Results . . 216

Modulated Josephson junctions with no phase difference $(\phi=0) \ldots \ldots \ldots \ldots . \ldots . \ldots 217$

Modulated Josephson junctions with phase difference

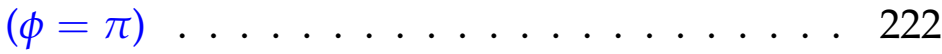

6.2.4 Summary and conclusions . . . . . . . . . . . . . . 223

6.2 .5 Acknowledgments . . . . . . . . . . . . . 226

7 Summary and Outlook 227

A Integration over elements in finite element method 232

A.1 Integrals over 1-dimensional elements . . . . . . . . . . 232

A.2 Integrals over 2-dimensional elements . . . . . . . . . . 233

B Effective mass Hamiltonian with position dependent spin-orbit coupling 236

B.0.1 Statement of the problem . . . . . . . . . . . . 236

B.0.2 Weak form, basis functions, and matrix assembly . . . 237

C Localization length of Majorana zero modes 239

D Lattice Green's functions and self-energy formalism 243

D.1 Green's function and the density of states . . . . . . . . . 243

D.2 Self-energy formalism . . . . . . . . . . . . . . . . 245

D.3 Green's function representation with a non-orthonormal basis set . . . . . . . . . . . . . . . . . . . 246

$\begin{array}{ll}\text { Bibliography } & 248\end{array}$ 


\section{Introduction}

As we shrink down from the macroscopic objects of our everyday lives to mesoscopic and nanoscopic length scales, we leave the realm of classical physics and enter the strange world of quantum mechanics. Fortunately for us, this new physical realm enables new technologies with vast utility. Indeed, humanity's understanding of quantum mechanics has already played a huge role in shaping modern society. The transistor, which is ultimately responsible for all of society's vast computational power, owes its development after all to the understanding of quantum mechanics. Now, with humanity's continuing progress in controlling nature at these small length scales and ultra-low temperatures, another major technological revolution at the hands of quantum mechanics may be on the horizon: quantum computation. While the development of the components of current computational machines is intimately linked to our understanding of quantum mechanics, current information processing occurring in all of our laptops, cellphones, etc. relies solely on classical notions of information processing. Quantum computational algorithms, in contrast, run on machines where the laws of quantum mechanics are directly connected to the processing of information. Remarkably, there are good reasons to believe that quantum computation is more powerful than classical computation for certain types of problems, possibly even providing exponential speedup in some cases. Indeed, there may exist problems that can be solved on a quantum computer that would require a classical computer to crunch numbers for longer than the current age of the universe. In this sense, the quantum computer is superior. ${ }^{1}$

Many platforms for creating qubits, the fundamental building block of quantum computers, have been proposed and are currently being pursued by researchers across the globe [1]. Several difficulties must be overcome, however, to make any physical system a suitable platform for large-scale quantum computing. The greatest of these challenges is limiting quantum decoherence, which is a process in which the information contained in the qubits is lost due to interaction with the surrounding environment [2]. One

\footnotetext{
${ }^{1}$ Since classical physics is just a certain limiting case of quantum mechanics, it follows that quantum computation is at least as powerful as classical computation. Note, however, that any computation that can be performed on a quantum computer can in principle be performed on a classical computer, according to the Church-Turing thesis. The quantum computation may require a smaller number of elementary steps to solve the problem, however, which leads to a speedup compared to the corresponding classical algorithm.
} 
possible solution to this issue is an approach known as topological quantum computation in which information is stored non-locally in quasiparticle excitations known as non-Abelian anyons [3]. It turns out that the nonlocal nature of the quasiparticles makes the system immune to errors arising from local perturbations. A promising platform for the creation of the necessary anyons involves engineering topological edge modes known as Majorana zero modes (MZMs). ${ }^{2}$ In short, MZMs are zero-energy modes localized near topological defects in topological superconductors, which include vortex cores in 2D systems [4] or topological domain edges in 1D systems [5]. Interesting in their own right from the perspective of topological quantum matter [6, 7], MZMs have been among the most studied object in physics in the last decade ever since the discover that they can be engineered in semiconductor-superconductor (SM-SC) nanostructures [8,9]. This thesis studies issues relating to the realization of MZMs in SM-SC nanostructures, with a particular focus placed on understanding and engineering the electrostatic environment of Majorana devices.

Before diving into my research on semiconductor-superconductor Majorana nanostructures, context is first provided on how MZMs fit into the bigger picture of topological condensed matter physics and quantum computation in Secs. 1.1 and 1.2, respectively, along with a brief history and current status of MZMs in SM-SC nanostructures in Sec. 1.3. Finally, an outline for the rest of thesis is given in Sec. 1.4.

\subsection{Topological quantum matter}

As stated above, MZMs are zero-energy modes occurring near topological defects in topological superconductors. What is meant by topological defects and topological superconductors? More generally, what is the meaning of the word "topological" in the context of many-body quantum systems? To give an answer to these questions, we take a step back from MZMs and Majorana nanostructures and look at the broader picture of condensed matter physics.

One key job of a condensed matter physicist is the classification of matter into different distinct equivalence classes known as phases. A particular phase of matter, solid, liquid, gas, superfluid, etc., refers to the collective organization of the elementary constituents of condensed matter, namely how electrons, atoms, and molecules arrange themselves and interact. We do not care about the specific location and velocity of every particle making up a phase of matter, but rather we wish to capture qualitative and quantitative properties that describe some global behavior of the system.

The dominant classification scheme of the $20^{\text {th }}$ century is based on the Landau theory [10]. In this theory, phases of matter are classified according to what symmetries respected by the interactions among the underlying atomic building blocks are spontaneously broken. As a simple example, a

\footnotetext{
${ }^{2}$ Throughout much of the literature, Majorana zero modes are misleadingly referred to as Majorana fermions. They are also referred to as Majorana bound states (MBS). The use of MZM and MBS will be used interchangeably in this thesis.
} 
crystalline solid breaks continuous translation symmetry, while the interactions among the electrons and atomic nuclei respect this symmetry. Symmetry breaking is described quantitatively by a so-called order parameter. For example, the magnetization acts as the order parameter for a ferromagnetic system. Above the Curie temperature, the order parameter is zero and the system is in the paramagnetic phase. Note that the paramagnetic phase preserves the rotational symmetry of the system's underlying interactions. Below the Curie temperature, however, this rotational symmetry is spontaneously broken, the order parameter is non-zero, and the system is in the ferromagnetic phase.

The first quantum phase that did not fit into the Landau paradigm was the integer quantum Hall phase [11], in which a 2D electron gas subject to a strong magnetic field at low temperatures is insulating within the bulk while carrying electric current along its edges. Remarkably, and to the surprise of its discoverers, this current is quantized in units of $e^{2} / h$ independent of the details of the sample. This behavior cannot be explained by any spontaneous symmetry breaking, since the phases with and without quantized conductance have the exact same symmetries. Rather, the quantized conductance behavior is topological in origin and connected to an integer topological invariant known as the TKNN invariant [12]. ${ }^{3}$

Topological invariants are properties that depend only on the topology of a so-called topological space. A topological space is the pair $(X, \mathcal{T})$, where $X$ is a set and $\mathcal{T}$ is a topology. A topology consists of the collection of all open subsets $U$ of the set $X{ }^{4}$ A topological space provides the weakest structure needed to define notions such as continuity, ${ }^{5}$ limits, and connectedness.

\footnotetext{
${ }^{3}$ The TKNN invariant is named in honor of the four physicists who first explained the topological nature of the integer quantum Hall effect two years after its observation [12]. Essentially they derived the conductance of a non-interacting 2DEG in a perpendicular magnetic field through use of the Kubo formula and ended up with the TKNN invariant in the final result. Note that in the modern terminology of topological physics, the TKNN invariant is understood to be the first Chern number, which is the integral of what is known as the Berry curvature over the entire 2D Brillouin zone [13].

${ }^{4}$ The open subsets $U_{i}$ making up a topology $\mathcal{T}$ of a set $X$ must satisfy three axioms [14]:

1. $\varnothing, X \in \mathcal{T}$,

2. If $U_{1}, U_{2} \in \mathcal{T} \Longrightarrow\left(U_{1} \cup U_{2}\right) \in \mathcal{T}$,

3. The intersection of finitely many open subsets is also an open subset of the topology, i.e. if $U_{1}, U_{2}, \ldots, U_{N} \in \mathcal{T}$ where $N \in \mathbb{N} \Longrightarrow U_{1} \cap U_{2} \cap \cdots \cap U_{N} \in \mathcal{T}$.
}

Note that there are many different topologies that one can choose for a given set $X$.

${ }^{5}$ A function $f: X \rightarrow Y$ from a set $X$ of the topological space $\left(X, \mathcal{T}_{X}\right)$ to a set $Y$ of the topological space $\left(Y, \mathcal{T}_{Y}\right)$ is defined to be continuous iff

$$
\forall V \in \mathcal{T}_{Y}: f^{-1}(V) \in \mathcal{T}_{X}
$$

where $f^{-1}(V)$ is the pre-image of the the open subset $V$, which is defined as

$$
f^{-1}(V)=\{x \in X \mid f(x) \in V\} .
$$

Note that a function $f$ can be continuous for one set of topologies, $\left(\mathcal{T}_{X}, \mathcal{T}_{Y}\right)$, and discontinuous for another set of topologies, $\left(\mathcal{T}_{X}^{\prime}, \mathcal{T}_{Y}^{\prime}\right)$. 


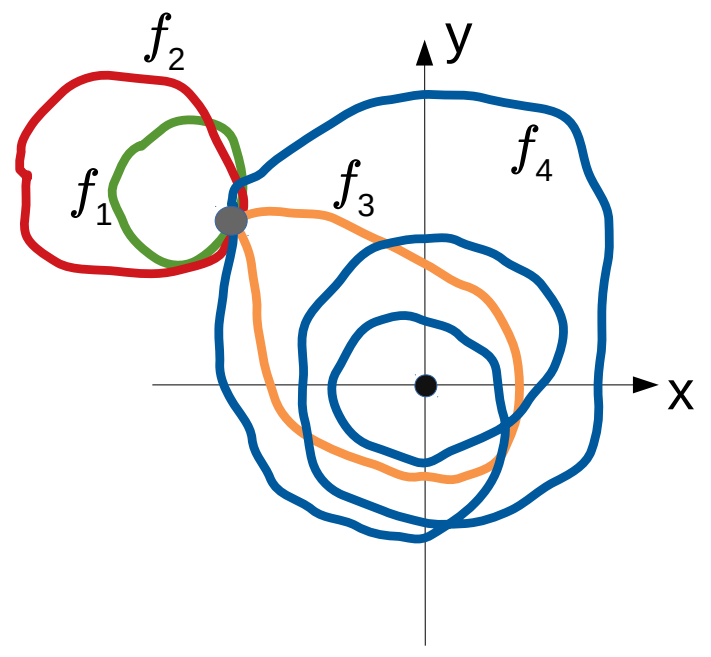

FIGURE 1.1: Schematic of four loops with the same base point (grey point), which are continuous functions $f_{i}:[0,1] \rightarrow X=$ $\mathbb{R}^{2} \backslash(0,0)$, i.e. the $2 \mathrm{D}$ Euclidean plane with the origin excluded. Note that all loops are oriented counterclockwise. Loops $f_{1}$ and $f_{2}$ are topologically equivalence to each other with respect to path homotopy with winding number $v=0$. No other loop pair is path homotopic.

These fundamental notions then allow us to develop more elaborate topological properties of mathematical objects.

As an intuitive example, let us consider the topological property known as path homotopy. First, we define a loop $f:[0,1] \rightarrow X$ with base point $x_{o} \in X$ to be a continuous function such that the two endpoints of the unit interval both map to the base point, i.e. $f(0)=f(1)=x_{0}$. For concreteness, let us take our set $X$ to be the 2-dimensional Euclidean plane with the origin excluded, $X=\mathbb{R}^{2} \backslash(0,0){ }^{6}$ As examples, four loops, $f_{1}, f_{2}, f_{3}$, and $f_{4}$, with the same base point are shown in Fig. 1.1. Two loops are defined to be topologically equivalent ${ }^{7}$ with respect to path homotopy if and only

\footnotetext{
${ }^{6}$ For continuity to be well defined, the topologies of $[0,1]$ and $X$ need to be specified. The topologies of $[0,1]$ and $X$ are chosen to be the subset topologies of the standard topologies of $\mathbb{R}$ and $\mathbb{R}^{2}$, respectively [14]. The generating open sets of the standard topology of $\mathbb{R}^{N}$ are the so-called open balls. The open ball $B_{\mathbf{x}_{o}}(r)$ of radius $r \in \mathbb{R}^{+}$about point $\mathbf{x}_{o}$ is defined as $B_{\mathbf{x}_{o}}(r)=\left\{\mathbf{x} \in \mathbb{R}^{N}|| \mathbf{x}-\mathbf{x}_{o} \mid<r\right\}$. The subset topology is define as follows. Let $\left(Y, \mathcal{T}_{Y}\right)$ be a topological space and $X \subset Y$. The so-called subset topology $\mathcal{T}_{X}$ contains the subsets $U$ defined by

$$
U \in \mathcal{T}_{X} \Longleftrightarrow \exists V \in Y: U=(V \cap X) .
$$

One can then show that $\left(X, \mathcal{T}_{X}\right)$ is a topological space.

${ }^{7}$ Let $M$ be a set. An equivalence relation, $\sim$, is a binary relation on $M$ that satisfies three axioms: For all $a, b, c \in M$,

1. Reflexive: $a \sim a$,

2. Symmetric: $a \sim b$ iff $b \sim a$,

3. Transitive: if $a \sim b$ and $b \sim c$, then $a \sim c$
} 
if it is possible to continuously deform them into one another without going through the origin. ${ }^{8}$ Looking back at the four loops in Fig. 1.1, we can see that $f_{1}$ and $f_{2}$ are topologically equivalent $\left(f_{1} \sim f_{2}\right)$ since loop $f_{2}$ can be deformed into $f_{1}$ by a slight contraction. In contrast, it is impossible to continuously deform loop $f_{3}$ into either $f_{1}$ or $f_{2}$. If we try to do so, clearly the loop must eventually cross over the origin, which is excluded from the topological space we are considering. Loop $f_{3}$ is therefore topologically distinct from $f_{1}$ and $f_{2}$. It is also clear that loop $f_{4}$, which wraps around the origin twice before returning to the base point, is not topologically equivalent to any of the other three loops. Continuing on this way, we see that there exists an infinite number of distinct equivalence classes ${ }^{9}$ of loops with base point $\mathbf{x}_{o}$ that are topologically distinct from one another. Additionally, each equivalence class of loops is completely characterized with respect to path homotopy by a winding number $v \in \mathbb{Z}$ that indicates how many times and in what direction the loop wraps around the origin before returning to the base point. For example, $f_{1}$ and $f_{2}$ both have $v=0$, while $f_{3}$ and $f_{4}$ have $v=1$ and $v=2$, respectively. Note that the winding number does not change if we apply any continuous deformation to a loop, and we therefore call it a topological invariant of the loop.

Having formed some intuitive notion of what is meant by a topological invariant and a continuous deformation, let us turn to quantum mechanics. What continuous deformations and properties potentially invariant under these transformations do we consider in quantum physics for the sake of classifying matter into distinct equivalence classes or topological phases? Let us consider fermionic systems described by non-interacting Hamiltonians ${ }^{10}$ with a bulk spectrum characterized by a finite energy gap between the system's ground state and all of its excited states, i.e. non-interacting fermionic insulators. Two fermionic insulators with non-interacting Hamiltonians are then considered topologically equivalent if there exists a continuous deformation of the Hamiltonian of the first insulator into the Hamiltonian of the

\footnotetext{
${ }^{8}$ More specifically, two loops, $f$ and $g$, with the same base point $x_{0}$ are defined as path homotopic if and only if there exist a continuous function $h:[0,1] \times[0,1] \rightarrow X$ such that $h(r, 0)=f(r)$ and $h(r, 1)=g(r) \forall r \in[0,1]$. In other words, the second argument of the function $h$ continuously interpolates between the two loops.

${ }^{9}$ Let $M$ be an set equipped with an equivalence relation $\sim$. The equivalence class $[a]$ is defined as

$$
[a]=\{b \in M \mid a \sim b\} .
$$

By the symmetric and transitive properties of equivalence relations, two equivalence classes are either equal or share no common elements, i.e. $[a]=[b]$ iff $a \sim b,[a] \cap[b]=\varnothing$ iff $\neg(a \sim b)$. An equivalence relation, therefore, naturally partitions the set $M$ into disjoint subsets. Note that in the example in the main text, the equivalence relation is equivalence with respect to path homotopy.

${ }^{10}$ Non-interacting fermionic Hamiltonians have the generic form,

$$
H=\sum_{i j} \hat{\psi}_{i}^{\dagger} H_{i j} \hat{\psi}_{i}^{\dagger}
$$

where $\hat{\psi}_{i}^{\dagger}$ and $\hat{\psi}_{j}$ are fermion creation and annihilation operators, respectively, that obey the canonical anticommutation relation, $\left\{\hat{\psi}_{i}^{+}, \hat{\psi}_{j}\right\}=\delta_{i j}$.
} 

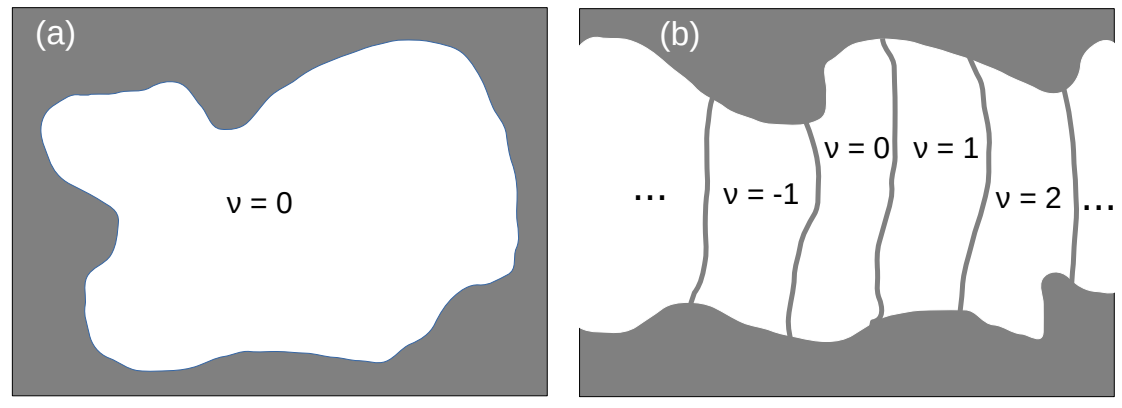

FIGURE 1.2: Schematic topological phase diagram of noninteracting fermionic systems without symmetry constraints in (a) odd spatial dimensions and (b) even spatially dimensions. Moving within the phase diagram alters the parameters of the Hamiltonian. White regions are gapped and are therefore insulator, while grey regions have no energy gap. In odd spatial dimensions (a) all insulators are in the same trivial phase. In contrast, even dimension insulators (b) are characterized by an integer topological invariant $v \in \mathbb{Z}$. It is impossible to transform an insulator with invariant $v$ to an insulator with invariant $v \pm 1$ without closing the gap.

second insulator without closing the bulk energy gap between the ground state and excited states [15]. ${ }^{11}$ If such a continuous deformation does not exist, i.e. one cannot continuously connect the two Hamiltonians without closing the gap, then the two systems belong to two distinct topological phases. ${ }^{12}$ A system undergoes a quantum phase transition from one distinct topological phase to another with the closing and reopening of its bulk energy gap.

How many topological phases exist in the case of non-interacting fermionic insulators? It turns out that the answer depends upon the spatial dimension of the system. Systems with an odd spatial dimension can always be transformed into a trivial insulator without closing the gap. Therefore, these systems all belong to the same topological phase, the trivial topological phase. Systems with an even spatial dimension, on the other hand, are characterized by an integer topological invariant, $v \in \mathbb{Z}$. There are, therefore, an infinite number of distinct topological phases for even dimensional systems. The topologically trivial phase corresponds to $v=0$, while all insulators with $v \neq 0$ are topologically non-trivial. The difference between the classification in odd and even spatial is conceptually illustrated in Fig. 1.2.

\footnotetext{
${ }^{11}$ Note that when deforming the Hamiltonian of the first insulator into the Hamiltonian of the second insulator all intermediate Hamiltonians are required to also be non-interacting fermionic Hamiltonians. Adding many-body interactions is not allowed in this classification scheme. The effects of adding interactions alters the classification [16].

${ }^{12}$ The equivalence relation needs to be slightly altered to deal with insulators with a different number of bands. Note that the core bands of an insulator, i.e. low-energy bands that are also always occupied and thus inert, do not effect its properties. The equivalence relation is then altered such that adding or subtracting a number of trivial core bands to an insulator creates an equivalent insulator in the same topological phase.
} 
We have actually already encountered an example of a topologically nontrivial insulator in the form of the integer quantum Hall phase [11, 12]. Remarkably, the topological invariant $v$ has a direct connection with experiment in the form of the quantized Hall conductance given by $G=v\left(e^{2} / h\right) .{ }^{13}$

As can be seen in Fig. 1.2, it is impossible in even spatial dimensions to transform from an insulator with invariant $v$ to another insulator with invariant $v \pm 1$. How can we visualize such a process? Let us imagine a 2-band model, for simplicity, with a Hamiltonian $H_{\lambda}(\mathbf{k})$ that depends on a control parameter $\lambda$. For $\lambda=0$, suppose the insulator is topologically trivial with a topological invariant $v=0$. The spectrum of $H_{0}(\mathbf{k})$ is schematically shown in Fig. 1.3 (a). For $\lambda=1$, suppose the insulator in topologically nontrivial with an invariant $v=1$. The spectrum of $H_{1}(\mathbf{k})$ is schematically shown in Fig. 1.3 (c). Belonging to two distinct topological phases, at some point in the transformation, $0<\lambda<1$, the gap must have closed. Often times the gap closure occurs at a high symmetry point in the Brillouin zone. For simplicity, assume it occurs at $\mathbf{k}=0$. The evolution of the band energies at $\mathbf{k}=0$ is shown in Fig. 1.3 (b) for $0 \leq \lambda \leq 1$. Near $\lambda \approx 0.5$, the gap closes, signalling a quantum phase transition from the trivial phase with $v=$ 0 to a nontrivial phase with $v=1$. It may be objected that the spectrum of the trivial (a) and nontrivial (c) insulators are not qualitatively different, so what distinguishes between the two insulators? This contains an important lesson; it is impossible to distinguish whether two insulator belong to the same topological phase based on their spectra alone. Rather, the information regarding which topological phase an insulator belongs to is contained in the combination of its spectrum and wavefunctions.

The topological classification of non-interacting fermions can be significantly enriched if further restrictions are placed on the Hamiltonian in the form of symmetries. ${ }^{14}$ Topological phases protected by symmetries are known as symmetry protected phases. According to the Wigner theorem [18], any symmetry transformation of a quantum Hamiltonian is represented by a unitary or antiunitary transformation acting on the Hilbert space. Spatial symmetries are represented by unitary transformations. These types of symmetries allow us to block diagonalize the Hamiltonian according to the irreducible representations of the Hamiltonian symmetry group [19]. Each of the irreducible blocks can be further classified by its non-spatial symmetries, which are represented by an antiunitary transformation. ${ }^{15}$ The most

\footnotetext{
${ }^{13}$ The Hall conductance can also become quantized in fractional units of $\left(e^{2} / h\right)$, which is known as the fractional quantum Hall effect [17]. This effect's origin is also topological, but relies upon electron-electron interactions. It is therefore beyond the scope of the topological classification of non-interaction fermionic insulators.

${ }^{14}$ Transformation between two Hamiltonians, $H_{A}$ and $H_{B}$, that respect the same symmetries are now not allowed to be just any path in the space of all possible non-interacting fermionic Hamiltonian. Rather, all intermediate Hamiltonians between $H_{A}$ and $H_{B}$ must also respect the symmetry constraints.

${ }^{15}$ The operator $\mathcal{O}$ of an antiunitary symmetry can always be decomposed as $\mathcal{O}=U \mathcal{K}$, where $U$ is a unitary matrix and $\mathcal{K}$ is the complex conjugation operator. Note that this implies $\mathcal{O i O}^{-1}=-i$.
} 
(a)

(b)

(c)

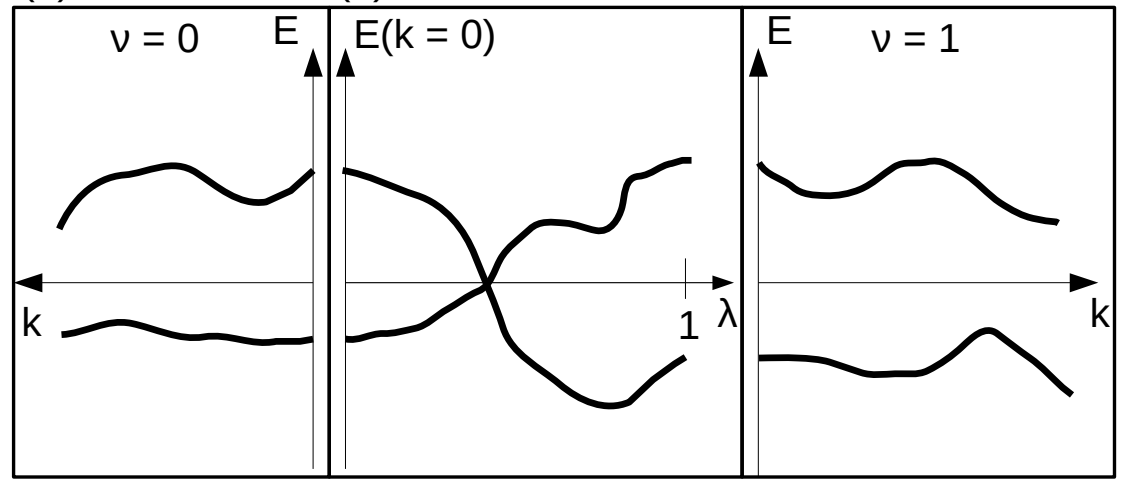

FIGURE 1.3: Illustration of a topological phase transition. The system has a 2-band Hamiltonian $H_{\lambda}$ that depends on a control parameter $\lambda$. The spectrum of a topologically trivial insulator, $v=0$, with $\lambda=0$ is shown in (a), while the spectrum of a nontrivial insulator, $v=1$, with $\lambda=1$ is shown in (c). The evolution of the band energies for $\mathbf{k}=0$ is shown in (b). The system undergoes a topological phase transition with the closing of the gap near $\lambda \approx 0.5$.

generic antiunitary symmetries are time-reversal symmetry $\mathcal{T}$ and chargeconjugation (or particle-hole) symmetry $\mathcal{C} .{ }^{16}$ For each of these antiunitary symmetries, a non-interacting fermionic Hamiltonian (or irreducible block of a non-interacting fermionic Hamiltonian, if the system possesses spatial symmetries) can either possess or not possess the symmetry. If the Hamiltonian does possess a given antiunitary symmetry, squaring that symmetry operator can yield either 1 or -1 . Finally, the Hamiltonian can lack both time-reversal and charge-conjugation symmetry, but the combination of the two is a symmetry of the Hamiltonian. This is known as a chiral symmetry whose operator is denoted by $\mathcal{S}$. With these considerations, there are 10 distinct symmetry classes of non-interacting fermionic insulators. For each of these 10 symmetry classes, it is possible to calculate all of the possible distinct topological phases for a given spatial dimension, which has been coined the ten-fold way [16]. This leads to a periodic table of sorts, often called the periodic table of topological insulators and superconductors, ${ }^{17}$ which is shown in Fig. 1.4. The table indicates how many non-equivalent topological phases each symmetry class has for a given spatial dimension. For example, class $A$ has no symmetry constraints, which is the case we considered before allowing symmetry constraints. The table is consistent will what was previously discussed in that systems in class $A$ with odd spatial dimensions are always

\footnotetext{
${ }^{16} \mathrm{~A}$ time-reversal operator acting on a non-interacting Hamiltonian takes the form, $\mathcal{T}=$ $U_{T} \hat{K}$, where $U_{T}$ is a unitary matrix and $\hat{K}$ is the complex conjugate operator. The Hamiltonian $H$ is said to posses time-reversal symmetry if there exists a $U_{T}$ such that $U_{T}^{\dagger} H^{*} U_{T}^{*}=H$. A charge-conjugation (or particle-hole) operator acting on a non-interacting Hamiltonian takes the similar form, $\mathcal{C}=U_{C} \hat{K}$, where $U_{C}$ is a unitary matrix. The Hamiltonian $H$ is said to posses charge-conjugation (or particle-hole) symmetry if there exists a $U_{C}$ such that $U_{C}^{\dagger} H^{*} U_{C}^{*}=-H$.

${ }^{17}$ The concept of a topological superconductor will be discussed in Sec. 2.1.3.
} 


\begin{tabular}{c|ccc|ccccccccc}
\hline \multicolumn{1}{c}{ Symmetry } & \multicolumn{11}{c}{ Spatial Dimension $d$} \\
Class & $T$ & $C$ & $S$ & 1 & 2 & 3 & 4 & 5 & 6 & 7 & 8 & $\cdots$ \\
\hline A & 0 & 0 & 0 & 0 & $\mathbb{Z}$ & 0 & $\mathbb{Z}$ & 0 & $\mathbb{Z}$ & 0 & $\mathbb{Z}$ & $\cdots$ \\
AllI & 0 & 0 & 1 & $\mathbb{Z}$ & 0 & $\mathbb{Z}$ & 0 & $\mathbb{Z}$ & 0 & $\mathbb{Z}$ & 0 & $\cdots$ \\
\hline Al & 1 & 0 & 0 & 0 & 0 & 0 & $\mathbb{Z}$ & 0 & $\mathbb{Z}_{2}$ & $\mathbb{Z}_{2}$ & $\mathbb{Z}$ & $\cdots$ \\
BDI & 1 & 1 & 1 & $\mathbb{Z}$ & 0 & 0 & 0 & $\mathbb{Z}$ & 0 & $\mathbb{Z}_{2}$ & $\mathbb{Z}_{2}$ & $\cdots$ \\
D & 0 & 1 & 0 & $\mathbb{Z}_{2}$ & $\mathbb{Z}$ & 0 & 0 & 0 & $\mathbb{Z}$ & 0 & $\mathbb{Z}_{2}$ & $\cdots$ \\
DIII & -1 & 1 & 1 & $\mathbb{Z}_{2}$ & $\mathbb{Z}_{2}$ & $\mathbb{Z}$ & 0 & 0 & 0 & $\mathbb{Z}$ & 0 & $\cdots$ \\
All & -1 & 0 & 0 & 0 & $\mathbb{Z}_{2}$ & $\mathbb{Z}_{2}$ & $\mathbb{Z}$ & 0 & 0 & 0 & $\mathbb{Z}$ & $\cdots$ \\
CII & -1 & -1 & 1 & $\mathbb{Z}$ & 0 & $\mathbb{Z}_{2}$ & $\mathbb{Z}_{2}$ & $\mathbb{Z}$ & 0 & 0 & 0 & $\cdots$ \\
C & 0 & -1 & 0 & 0 & $\mathbb{Z}$ & 0 & $\mathbb{Z}_{2}$ & $\mathbb{Z}_{2}$ & $\mathbb{Z}$ & 0 & 0 & $\cdots$ \\
Cl & 1 & -1 & 1 & 0 & 0 & $\mathbb{Z}$ & 0 & $\mathbb{Z}_{2}$ & $\mathbb{Z}_{2}$ & $\mathbb{Z}$ & 0 & $\cdots$ \\
\hline \hline
\end{tabular}

FIGURE 1.4: Periodic table of topological phases characterizing the 10 symmetry classes of non-interacting fermion Hamiltonians. The columns labeled $T C$ and $S$ indicate which symmetry classes have time-reversal, charge-conjugations, and chiral symmetries, respectively. 0 indicates the symmetry is not present, while \pm 1 indicates the symmetry is present and what the symmetry operator squares to. The upper row of numbers indicate the spatial dimension. $\mathbb{Z}$ and $\mathbb{Z}_{2}$ indicates that there are infinite and two distinct topological phases, respectively, while 0 implies that every Hamiltonian in that class is trivial for that spatial dimension.

trivial (indicated by 0 in Fig. 1.4), while those with even dimensions can belong to an infinite number of distinct topological phases (indicated by $\mathbb{Z}$ in Fig. 1.4). Adding time-reversal symmetry that squares to $\mathcal{T}^{2}=-1$ brings us to class $A I I$. Similar to class $A$, class $A I I$ systems in $1 \mathrm{D}$ are always trivial. In contrast, however, class $A I I$ systems in 2D and $3 \mathrm{D}$ can be in one of two topological phases (indicated by $\mathbb{Z}_{2}$ in Fig. 1.4). Class $A I I$ systems in $2 \mathrm{D}$ or $3 \mathrm{D}$ in the topologically nontrivial phase are the so-called topological insulators.

Why should we care about this classification? We saw that the topological properties of the integer quantum Hall phase result in a quantized conductance. This is due to the fact that in this case the conductance is proportional to the integer topological invariant $v \in \mathbb{Z}$ characterizing each phase [12]. In general, the topological invariant may not be directly related to a physical observable. However, there are other physical properties specifically associated with nontrivial topological phases. It turns out that in-gap states always form at topological defects, i.e. boundaries between regions characterized by different topological invariant values. ${ }^{18}$ This is the famous bulk-boundary correspondence of topological quantum matter [21]. For example, in the integer

\footnotetext{
${ }^{18}$ Typically, the in-gap modes coming from the change of the topological phase are bound states localized to one spatial dimension lower than the bulk sample. It is possible, however, that edge modes in topological insulators and superconductor are localized to further lower dimensions in the presence of topological defects in the bulk of the sample [16]. There also exists higher-order topological insulators and superconductors with topologically trivial bulk regions but with surfaces that are topologically nontrivial, which results in protected edge modes bound to smaller dimensions [20].
} 
quantum Hall effect discussed above, the 2D sample in the topological phase is characterized by a non-zero integer topological invariant $v \neq 0$, while the surrounding vacuum is topologically trivial and characterized by $v=0$. This topological phase transition across the sample-vacuum interface must be accompanied by a closure of the bulk gap, which in turn causes in-gap modes that live along the $1 D$ interface. These edge modes are then responsible for the quantized edge conductance. Similarly, in-gap modes occur at the edges and surfaces of 2D and 3D topological insulators, respectively. These modes were first observed experimentally in 2007 [22] and 2008 [23], respectively. Additionally, because the topological phase of the sample is robust against perturbations that neither close the bulk gap nor break the symmetries characteristic of the symmetry class, the in-gap edge modes are also immune to such perturbations. In this sense, the edge modes are topologically protected.

MZMs represent another specific example of topological in-gap edge modes. More specifically, they are 0-dimensional in-gap bound states that occur at the edges of $1 \mathrm{D}$ topological superconductors of classes $B D I, D$, and DIII (see the table in Fig. 1.4) or in the vortex cores of a $2 \mathrm{D}$ class $D$ superconductor. Note that propagating Majorana edge modes can also occur at the 1D edges of 2D topological superconductors of classes $D$ and DIII. These are not MZMs, but rather form in-gap surface bands that cross the Fermi level and connect the highest energy occupied bulk bands to the lowest energy unoccupied bulk bands, similar to the in-gap modes of topological insulators. As argued below, the robustness of MZMs coming from the topological protection against perturbations makes them excellent candidates as the building blocks of a topological quantum computer.

\subsection{Quantum computation}

As stated above, MZMs are a promising platform on which a topological quantum computer may be built. More specifically, the topological protection and non-Abelian exchange statistics of MZMs make them promising candidates for implementing a particular type of quantum computing known as topological quantum computing. What is meant by quantum computation? How does it relate to classical computing? And what makes topological quantum computing appealing when compared to other quantum computing approaches?

\subsubsection{Classical computing}

As a reference to compare quantum computation to, let us begin with a brief description of classical computation. The basic unit of classical information is a bit, which can take two possible values. These two values can be denoted in whatever convention one wishes, but let us stick with the most popular convention with values 0 and 1 . The basic task of a classical computer is to evaluate functions of the form [24],

$$
f:\{0,1\}^{n} \rightarrow\{0,1\}^{m}
$$


where $\{0,1\}^{n}=x_{1} x_{2} \ldots x_{n}$ is the $\mathrm{n}$-bit input string with $x_{i}$ being the value of the $\mathrm{i}^{\text {th }}$ bit and $\{0,1\}^{m}$ is the $\mathrm{m}$-bit output string. Therefore, if we're interested in using a classical algorithm to solve a problem of interest, its inputs needs to be encoded into an n-bit input string, its solution needs to be deciphered from an m-bit output string, and $f$ must be chosen appropriately to obtain the correct solution. ${ }^{19}$ The fundamental components of a classical algorithm are elementary operations, such as NOT, AND, OR, and COPY, acting on one or two bits. For example, the AND operator $(\wedge)$ acting on the bits $x$ and $y$ is given by

$$
x \wedge y=x \cdot y,
$$

which yields 1 if $x=y=1$ and 0 otherwise. A classical circuit can then be built consisting of a sequence of the elementary 1-bit and 2-bit operators (or gates). Remarkably, there exist many small sets of elementary operators, such as the set $\{\mathrm{NOT}, \mathrm{AND}, \mathrm{OR}, \mathrm{COPY}\}$, that are universal, i.e. for any given function with a finite input, there exists at least one classical circuit composed of just those elementary operators that implements that function. Note that when a 1-bit or 2-bit elementary operator transforms an n-bit string $X=$ $x_{1} x_{2} \ldots x_{n}$ into an $m$-bit string $Y=y_{1} y_{2} \ldots y_{m}$, the two bit strings, $X$ and $Y$, need to be "close" in the space of all possible bit strings. By "close", two things are meant: (1) The length of $Y$ can at most differ from the length of $X$ by 1, i.e. $|m-n| \leq 1$. (2) Nearly all of the bits of $X$ and $Y$ must be the same since at least $n-2$ of the bits in $X$ are not involved in the elementary operation. A classical algorithm can then be viewed as a sequence of discrete jumps between "close" bit strings with the $\mathrm{i}^{\text {th }}$ jump dictated by the rule of and $\mathrm{i}^{\text {th }}$ gate of the classical circuit.

\subsubsection{Quantum computing}

Quantum computation is not performed with bits, but rather qubits (quantum bits). A qubit is, essentially, a two-state quantum systems and is the basic unit of quantum information. In contrast to a bit, a qubit is typically in a superposition of 0 and 1, i.e. the quantum state of an isolated qubit takes the form

$$
|q u b i t\rangle=a|0\rangle+b|1\rangle,
$$

where $a, b \in \mathbb{C}$ and $|a|^{2}+|b|^{2}=1$. Note that this contrast between bits and qubits is stark. Whereas a bit can take only 2 possible values, the state of a qubit is one of a continuum of possible states. In other words, a bit is digital, while a qubit is analog. A generic state of an $n$-qubit system takes the form,

$$
|\psi\rangle=\sum_{i_{1} i_{2} \cdots i_{n}} \psi_{i_{1} i_{2} \cdots i_{n}}\left|i_{1} i_{2} \cdots i_{n}\right\rangle
$$

Note that the number of coefficients $\psi_{i_{1} i_{2} \cdots i_{n}}$ grows exponentially with $n$, specifically as $2^{n}$. Analogous to the elementary gates of a classical computer,

\footnotetext{
${ }^{19}$ Of course when algorithms are actually created in the world, they often times are not represented in this form by their programmers. Rather, the problem is represented using higher-order abstractions and compiled into the form of Eq. (1.6) in the final stages.
} 
the fundamental components of a quantum algorithm are quantum gates, which correspond to unitary operations applied to a few qubits, typically one or two. Note that being a unitary transformation implies that the quantum gate is reversible, in contrast to classical gates which are irreversible, (information is lost upon their application) ${ }^{20}$ A quantum computation starts with the n-qubit state being initialized into a computational basis state, i.e. the initial state is given by $\left|\psi_{i}\right\rangle=\left|i_{1} i_{2} \cdots i_{n}\right\rangle_{\text {input }}$, where $i_{m} \in\{0,1\}$ for all $m$. This initial state encodes the (classical) input. The next step in the computation consists in executing a quantum circuit, i.e. a finite sequence of of quantum gates acting on the system's qubits. This sequence of quantum gates implements a unitary transformation $U$ that takes the initial state to the final state, i.e.

$$
\left|\psi_{f}\right\rangle=U\left|\psi_{i}\right\rangle \text {. }
$$

Finally, a measurement is then performed on each qubit projecting the final state onto a computational basis state, i.e

$$
\left|\psi_{f}\right\rangle \stackrel{\text { measurement }}{\longrightarrow}\left|i_{1} i_{2} \cdots i_{n}\right\rangle_{\text {output }}
$$

which yields the (classical) output from the computation. Remarkably, a quantum circuit composed of a finite sequence of quantum gates chosen from a discrete set of universal quantum gates can come arbitrarily close to implementing any of the uncountably infinite possible unitary transformations [7]. Note, however, that nearly all unitary transformation require a number of quantum gates that grows exponentially with the number of qubits.

\subsubsection{Quantum supremacy}

A key concept in both classical and quantum computation is computational complexity. Studying the complexity of a given problem tells us how the size, i.e. the number of gates, of the smallest circuit capable of solving the problem grows with the size of the input. For example, complexity theorists call a problem "easy" if the size of the required circuit to solve the problem grows polynomially in $n$ for $n$-bit inputs. Such a problem belongs to computational complexity class $P$. Otherwise the problem is "hard." Another example of a computational complexity class is $B Q P$. This class relates to whether or not quantum computing is theoretically superior to classical computing. A problem belongs to $B Q P$ if it can be solved, with high probability, on a quantum computer with a polynomial sized circuit. It has yet to be proven that $P \neq B Q P$, but a majority of quantum information scientists and complexity theorists believe it to be true [24]. ${ }^{21}$ Showing this to be the case would be tantamount to proving the supremacy of quantum computation over classical computation.

\footnotetext{
${ }^{20}$ Classical computation can be made reversible. This, however, requires extending the elementary gate set to include 3-bit gates along with some additional overhead [24].

${ }^{21}$ Analogously, there currently exists no proof that $P \neq N P$, but much of complexity theory relies on this conjecture.
} 
While it has not yet been shown that $P \neq B Q P$, there does exist problems where quantum algorithms that have been proven to be polynomially faster, not exponentially faster, than the best possible classical algorithms, such as the Grover algorithm for searching unsorted lists [25]. There also exists quantum algorithms that are exponentially faster than the best known classical algorithm. Examples include the quantum Fourier transform [26] and the Shor algorithm [27] for prime factorization. While it is not proven that these quantum algorithms are exponentially faster than the corresponding best possible classical algorithms, it certainly provides strong evidence for quantum supremacy.

What explains how it is possible that a quantum algorithm can in some instances be more efficient than its classical counterpart? First note that a bit string of a classical computer, $X=x_{1} x_{2} \ldots x_{n}$, can be viewed as corresponding to the computational basis state, $|X\rangle=\left|x_{1}, x_{2}, \ldots, x_{n}\right\rangle$, of a quantum computer. The succinct summary of a classical algorithm given in Sec. 1.2.1 can then be translated into the language of quantum computation; a classical algorithm is, in essence, a sequence of discrete steps between computational basis states that link the (classical) input to the (classical) output. ${ }^{2}$ By contrast, a quantum algorithm uses the full Hilbert space. In other words, the $\mathrm{n}$-qubit state at any given time is generically a linear superposition of computational basis states. The freedom from being restricted to just the computational basis states may provide a "shortcut" between the input and output. If such a "shortcut" exists, the quantum algorithm is then more efficient than the corresponding classical algorithm.

Quantum computation has many possible applications in the real world. The most exciting possibility from a physics perspective is using quantum computation for simulating quantum systems. The simulation of many-body quantum systems using classical computers is very challenging due to the exponential explosion of the Hilbert space with the number of particles, as mentioned in the discussion of Eq. (1.9). The simulation of quantum systems using quantum computers goes beyond what is practically possible by a classical computer as a result of the quantum computer's exploitation of the quantum features of entanglement and superposition. In the words of Richard Feynman [28], "Let the computer itself be built of quantum mechanical elements which obey quantum mechanical laws." These simulations will benefit both research into fundamental science, such as particle physics, and more practical applications, such as problems in chemistry, material science, and strongly correlated materials for technological purposes.

\footnotetext{
${ }^{22}$ The steps of a classical algorithm can reduce or increase the number of bits, while a quantum algorithm cannot change the number of qubits. In this respect, the correspondence is not perfect. Note, however, that an irreversible classical algorithm can always be implemented using a reversible classical algorithm, where the number of bits is conserved, with modest overhead [24]. If we restrict ourselves to reversible classical algorithms, the correspondence between classical bit strings and quantum computational basis states becomes much stronger.
} 


\subsubsection{Topological quantum computation}

In contrast to their classical counterparts, quantum systems tend to be incredibly fragile, and this includes the qubits making up a quantum computer. In essence, this fragility comes from the constant interaction between the qubits and the surrounding environment, which causes entanglement between the system and environment. This entanglement with the environment causes the qubits to undergo decoherence, a process in which the evolution of the qubit subsystem is non-unitary when viewed in isolation from the environment. The quantum information stored in the qubits is essentially lost to the environment and computational errors are created. Computational errors in quantum systems also accrue due to the small errors of quantum gates. For example, suppose a Pauli-Z gate is acting on a single qubit. The desired outcome is given by

$$
\hat{Z}(a|0\rangle+b|1\rangle)=a|0\rangle-b|1\rangle,
$$

where the phase on the $|1\rangle$ basis state has undergone a $\pi$-shift. Due to the continuous nature of the qubit state, however, the actual outcome will be given by

$$
\hat{Z}(a|0\rangle+b|1\rangle)=a|0\rangle+e^{i(\pi+\epsilon)} b|1\rangle,
$$

where $\epsilon$ is the phase-error. Note that classical computation does not have to deal with such small errors due to the discrete, or digital, nature of classical gates. Remarkably, this constant production of errors in quantum computers can in principle be corrected for through quantum error correction [29]. Indeed, it turns out that it is possible to efficiently simulate with only polynomial overhead an ideal quantum computer with one equipped with noisy (but not too noisy) quantum gates. While this is great news for our confidence in the possibility of large scale quantum computing, the quantum gates still need to be of very high fidelity. Furthermore, the amount of overhead coming from error correction protocols decreases as the gate fidelity increases. There is therefore ample motivation to improve the gate fidelity by hardware improvements, if possible.

It turns out that using exotic quasiparticles known as non-Abelian anyons in a type of quantum computing known as topological quantum computing is intrinsically robust against computational errors, as first pointed out by Kitaev [30]. This intrinsic robustness comes from the topological protection and non-local storage of quantum information in anyonic systems [3]. This will be discussed in more detail below, but first, what are anyons and how do they compare to more mundane particles?

In three dimensions, nature only allows particles to be either bosons or fermions, which simply have their collective wavefunction multiplied by 1 and $(-1)$, respectively, upon the the exchange of two identical particles. Two dimensions, in contrasts, allows for a much richer set of exchange statistics that can give rise to anyons, which are neither bosons nor fermions. Remarkably, anyons can be characterized by exchange statistics of arbitrary phase factors $e^{i \theta}$, rather than \pm 1 , or even non-trivial unitary transformations [31]. 
These exotic quasiparticles can have all sorts of strange properties, such as fractional electron charge in the case of the fractional quantum Hall effect [32].

How is it that such strange particles can occur in 2D but not 3D? The answer is topological in nature and relates to our discussion of path homotopy in Sec. 1.1. Imagine exchanging two identical particles twice such that they arrive back at the same positions. Note that this is equivalent to transporting the first particle around the second particle. Imagine the second particle being fixed at the origin of our coordinate system. In light of Fig. 1.1, transporting the first particle around the second (a path like $f_{3}$ Fig. 1.1) is not continuously deformable to the situation of moving the first particle in a circular loop that does not go around the second particle (a path like $f_{1}$ or $f_{2}$ in Fig. 1.1). The two paths have different winding numbers, which are topological invariants that cannot be modified by continuous deformations. What if we introduce a third dimension? Imagine adding a z-axis to Fig. 1.1 such that the space is $\mathbb{R}^{3} \backslash(0,0,0)$. It now becomes possible to continuously deform the loop $f_{3}$ into either $f_{1}$ or $f_{2}$ while avoiding the origin by moving the path intermittently out of the $x y$-plane. Indeed, in $\mathbb{R}^{3} \backslash(0,0,0)$, all loops are path homotopic to each other, i.e. all loops are topologically equivalent. Clearly, moving the first particle in a small circular loop in which the particles are never even close to each other should leave the wavefunction unaffected. Since all paths are continuously deformable to such a path, this then equally holds for all paths. Therefore, exchanging two particles twice must yield the same wavefunction, which further implies a single exchange in $3 \mathrm{D}$ is restricted to the multiplicative factors \pm 1 .

Anyons come into two broad classes, Abelian anyons and non-Abelian anyons. The state describing a system containing $N$ Abelian anyons is nondegenerate [7, 31]. Being a symmetry operation, an exchange of identical particles cannot transform a system between two states with different energies [19]. This fact restricts the effect of an exchange of Abelian anyons to a phase factor, i.e.

$$
\psi \rightarrow e^{i \theta} \psi
$$

under the exchange of any two Abelian anyons present in the system. Performing $m$ exchanges among any Abelian anyons then yields

$$
\psi \stackrel{m \text { exchanges }}{\longrightarrow} e^{i m \theta} \psi \text {. }
$$

Note that phase in Eq. (1.15) does not depend on the order in which the exchanges occur. Hence, the name Abelian anyons. Abelian anyons are not useful for quantum computing. Non-trivial unitary transformations are impossible to produce through their exchanges, and therefore no quantum gates are possible to implement. In contrast to Abelian anyons, a system containing $\mathrm{N}$ non-Abelian anyons contains a degenerate low-energy subspace separated from other states by a finite gap [31]. It is therefore possible that upon an exchange of two non-Abelian anyons, not only can a phase to the wavefunction 
be acquired, but rotations can also occur within the degenerate subspace, i.e.

$$
\psi_{\alpha} \rightarrow \sum_{\beta=1}^{g} \Gamma_{\alpha, \beta} \psi_{\beta},
$$

where $\psi_{\alpha}$ is one basis state in the $g$-fold degenerate subspace and $\Gamma$ is a unitary matrix describing the action of the current exchange. Being matrices, two different exchange operations generically do not commute. Therefore, the order in which the exchanges in a system with $N$ non-Abelian anyons occur matters. Hence, the name non-Abelian anyons. Importantly, the ability to perform non-trivial unitary transformations within a degenerate subspace by exchanging (or braiding) non-Abelian anyons, allows for the implementation of quantum gates. Non-Abelian anyons can therefore serve as a platform for topological quantum computation.

Besides simply being a possible platform for the implementation of quantum computation, topological quantum computation with non-Abelian anyons is attractive due to its intrinsic robustness against computational errors [30]. This occurs for two main reasons: (1) First of all, note that the quantum information is contained within a state residing in a degenerate subspace, which forms a small part of the system's total Hilbert space, and is separated from other states by a finite gap. The states of this degenerate subspace consist in the presence of multiple non-Abelian anyons that are separated from each other in space by a large distance. In this sense, the quantum information stored in the state of the anyonic system is non-local. Remarkably, all local perturbations have vanishing matrix elements within the degenerate subspace $[7,33]$. As a result, the quantum information stored in this subspace does not undergo decoherence due to local perturbations from interactions with the environment. (2) The quantum gates performed by exchanging (or braiding) the non-Abelian anyons depends only upon the topological properties of the path taken in the exchange $[3,7,31]$. In other words, the exact geometric details of the paths in which the anyons are moved around one another to perform a quantum gate are unimportant. This eliminates the small errors that occur due to imprecise quantum gates such as discussed around Eq. (1.13). Overcoming these two huge obstacles afflicting quantum computers makes topological quantum computation a very promising approach.

MZMs are an example of such non-Abelian anyons occurring at topological defects within symmetry-protected 1D or 2D topological superconductors [33]. Importantly, MZMs always come in pairs and result in a ground-state degeneracy. For example, if we have a single (long) SM-SC nanowire segment in the topological phase, $2 \mathrm{MZMs}$ will emerge, one localized at each edge of the 1D segment, and the ground-state will be 2 -fold degenerate. More generally, if there are $2 n$ zero-energy MZMs present, ${ }^{23}$ then the ground state has a $2^{n}$-fold degenerate ground-state. By exchanging the positions of MZMs

\footnotetext{
${ }^{23}$ Multiple nanowire segments each having a pair of MZMs could be present in the system. Alternatively, multiple vortex cores hosting MZMs could be present in 2D topological superconductors.
} 
in the system [34], ${ }^{24}$ we can then perform topological quantum gates as described above. An example showing how exchanging MZMs implements non-trivial unitary transformations is given in Sec. 2.4 after an introduction to the mathematics of MZMs in Sec. 2.1.

\subsection{Majorana zero modes in semiconductor-superconductor nanostructures}

Having discussed how MZMs are connected to the broader context of topological quantum matter and the promise they hold in topological quantum computing, what is the status of the field of MZMs in SM-SC nanostructures? To answer this question, this section presents a brief history of the field starting from about two decades ago. Please see review articles in Refs. [35-39] for additional information.

There were several foundational theoretical papers about MZMs serving as precursors to MZMs in SM-SC nanostructures. Indeed, in 2000 Read and Green showed that zero-energy MZMs can be localized near a vortex ${ }^{25}$ in a spinless $\left(p_{x}+i p_{y}\right)$ 2D-superconductor [4]. The following year, Kitaev considered a 1D model, now known as the Kitaev chain, of a spinless p-wave superconductor [40]. In the Kitaev chain, zero-energy MZMs occur near the edges of the 1D superconducting system when the chemical potential is properly tuned. Being of fundamental importance, we'll revisit the Kitaev chain in chapter 2. These models have two main issues. 1) They are models of spinless fermions, while electrons of course are not. 2) They rely on p-wave superconductivity, which is very rare in nature. Nonetheless, these simplified model provided hints and guidance as to how MZMs could engineered.

Motivated by the works of Read and Green, Kitaev, and others, ${ }^{26}$ theoretical research revealed in 2010 that MZMs could be engineered in SM-SC nanostructures $[8,9,43-45]$ using the combinations of three rather simple ingredients; 1) s-wave superconductivity, 2) Zeeman splitting to realize an effectively spinless system and 3) spin-orbit coupling, which provides the necessary chirality to produce the effective $\mathrm{p}$-wave superconductive pairing. The SM component of the hybrid nanostructure provides the latter two ingredients. The semiconductor should preferably posses a large g-factor to reduce the necessary magnetic field to enter the topological phase and large spin-orbit coupling such that the topological phase is well protected against disorder or other device non-uniformities, making InAs and InSb excellent

\footnotetext{
${ }^{24}$ During the exchange, MZMs must be kept sufficiently far away from each other such that MZMs do not hybridize and break the ground-state degeneracy. What counts as sufficiently far away is set by the Majorana localization length $\xi$ (see Appendix ?? for estimation of the localization length).

${ }^{25} \mathrm{~A}$ vortex in a superconductor is a point or region where the order parameter $\Delta$ vanishes and a $2 \pi$ phase winding of the order parameter occurs around the vortex core.

${ }^{26} \mathrm{Fu}$ and Kane discovered that MZMs could be produced by coupling an s-wave superconductor to a topological insulator shortly before the SM-SC proposals were put forward $[41,42]$. These schemes rely on the chiral in-gap surface (edge) states of a 3D (2D) topological insulator.
} 
candidate materials [46]. The SC component provides the s-wave superconductivity via the proximity effect with the SM.

Following these concrete theoretical proposals, the first experimental observation of a signature of MZMs was reported by Mourik and coworkers [47] in a conductance measurement of an InAs-NbTiN hybrid nanowire. The experiment observed a key prediction of the theoretical models; upon entering the topological phase, a zero-bias conductance peak from electrons tunneling into the edge of the system from a metallic lead should exist due to the Andreev reflection of the incoming electrons into reflected holes by the zero-energy MZM localized at that edge of the system. Soon there after, further experimental groups found evidence for MZMs in SM-SC nanostructures in the form of zero-bias conductance peaks in many SM-SC material combinations, including in InSb-Nb [48], InAs-Al [49], and InAs- $\mathrm{NbN}$ [50] hybrid nanowires. The predicted fractional a.c. Josephson effect was also observed in an InSb-Nb nanowire junction [51].

These experiments were not without problems, however. For example, while the experiments did observe zero-bias peaks as predicted by theory, the size of the conductance peaks were much smaller than what was predicted. Provided the lead has sufficiently strong coupling to the SM-SC hybrid nanowire and the system temperature is sufficiently low, an MZM localized at the edge of the system is predicted to produce perfect Andreev reflection such that an incoming electron at zero-bias should be perfectly reflected as a hole. This results in a predicted quantized zero-bias conductance of $G=2 e^{2} / h[52,53]$, which was not observed in any of the above mentioned conductance measurements. For example the zero-bias conductance peaks in Ref. [47] were $G \approx 0.4-0.7 e^{2} / h$. This was especially troubling, since sources other than MZMs which are topologically trivial were shown around the same time period to also give rise to (unquantized) zero-bias peaks [5457]. Moreover, additional theoretical sources of trivial zero-bias peaks have multiplied in the last near-decade since those early works [57-79]. These first generation SM-SC Majorana experiments were also plagued by a so called "soft-gap," meaning the conductance measurements did not feature sharp coherence peaks in the absence of magnetic field as expected from an s-wave superconductor. Rather, the conductance was only slowly suppressed as the bias approached zero, likely caused by disorder at the SM-SC interface [80].

Significant materials progress has been made [81-88] since the first generation of Majorana SM-SC hybrid nanowire experiments. Indeed many SM-SC nanowire hybrids have had an observed hard superconducting gap with sub-gap conductance exponentially suppressed. This has led to further improved transport experiments consistent with the presence of MZMs. For example, a "zero-bias peak phase diagram" was mapped out as a function of gate voltage and applied magnetic field in an InSb/NbTiN hybrid nanowire system [89], which meant to serve as a mapping out of the topological phase diagram. Unfortunately, it has been shown that trivial Andreev bounds states may be responsible for the observations of that experiment [65]. Additionally, other experiments observed zero-bias peaks with increased magnitude $[90,91]$ compared to the first generation experiments, 
giving more credence to the MZM interpretation. This culminated in 2018 with great excitement when the first apparent observation of a quantized conductance plateau of $2 e^{2} / h$ in a SM-SC nanostructure, specifically in an $\mathrm{InSb} / \mathrm{Al}$ hybrid nanowire [92]. Unfortunately, the measurement of the quantized conductance plateau was later realized to be an error, with the conductance actually being slightly larger than the quantized value of $2 e^{2} / h$ [93], implying an Andreev bound state was being measured. ${ }^{27}$

So have MZMs been observed in SM-SC nanostructures in the last decade? Currently we do not know. The ubiquity of sources of zero-bias conductance peaks besides MZMs makes it impossible to say for sure. Other signatures of MZMs, in combination with zero-bias conductance peaks, need to be observed before we can definitively confirm their presence in SM-SC nanostructures. For example, the predicted Majorana oscillations about zero-energy with increasing magnetic field [98] have never been observed, and neither have correlated zero-bias conductance peaks at the two edges of the system coming from well-separated MZMs [99]. More importantly, even if MZMs have been present in some of the experiments, the community is still far away from achieving topological quantum computation. For this to become a reality, MZMs must come about consistently in a reliable and controllable manner, which is certainly not the current situation. In my opinion, and the opinion of many leading experts in the field, the main obstacle to overcome is reducing disorder in the system, particularly in the SM. Hope is not lost, however. If the disorder is removed from the system, MZMs will emerge. The theory is just too simply ${ }^{28}$ for it to be impossible.

\subsection{Outline of thesis}

In this thesis, I present my research (done in collaboration with several excellent scientists) regarding semiconductor-superconductor (SM-SC) nanostructures engineered with the purpose of giving rise to MZMs. I pay particular attention to the electrostatic environment of Majorana nanostructures. The theory $[8,9,43,44]$ that initially led to the prediction of MZMs in SMSC nanostructures is actually surprisingly simple. The devil is in the details, however. In order to understand the physics and experimental details of realistic SM-SC Majorana devices, there is no choice but to go beyond the minimal theory. This necessarily involves incorporating the details of the electrostatic environment in which the electrostatic potential landscape is shaped by various factors including gate geometry, band bending effects at the SM$\mathrm{SC}$ interface, the potential arising from free charge in the SM, disorder, and

\footnotetext{
${ }^{27}$ It is important to note, however, that even if a truly quantized zero-bias peak were to be observed, we can not automatically conclude that an MZM is present. So called partiallyseparated Andreev bound states [67], also known as quasi-Majoranas [94], can give to quantized zero-bias peaks, along with other mechanisms [95-97]. In a certain sense, however, these states giving rise to the zero-bias peaks are precursors to MZMs, a residue of Majorana physics.

${ }^{28}$ It is based on non-interacting fermion theory after all.
} 
more. Indeed, the electrostatic environment is found to play a crucial, if not dominant, role in understanding and improving Majorana nanostructures.

The rest of the thesis is organized as follows. Chapter 2 provides the theoretical background needed to understand physical models with MZMs and how they arise in SM-SC nanostructures. In chapter 3, I argue why detailed modeling of the electrostatic environment is required for these structures. Chapter 4 provides the formalism for self-consistently solving the Schrödinger-Poisson equations to calculate the electrostatic potential in SMSC nanowires with translation invariance. Two examples of this formalism are then presented. The first studies subband occupancy as a function of system parameters, such as gate voltages and surface charge density. The second example studies the effects of the electrostatics on the magnetic proximity effect in SM-SC-magnetic insulator hybrid nanowires. Disorder in the form of charge impurities in the SM along with its effect on Majorana physics are studied in chapter 5 . The effects of inter-subband coupling arising from potential non-uniformities in a multi-gate system are also studied in 5 . We show that inter-subband coupling can pin topologically trivial Andreev bound states near zero-energy. Planar SM-SC systems with periodically modulated SCs coupled to a two-dimensional electron gas hosted by the SM are studied in chapter 6 . We show that the periodic modulations of the SC increase the robustness of topologically superconductivity against disorder and other nonuniformities in these planar SM-SC systems. Finally, in chapter 7, a summary of results and a future outlook are provided. 


\section{2}

\section{Theoretical Background}

In this chapter, we provide the theoretical background needed to understand Majorana zero modes (MZMs) and how they emerge within condensed matter systems. These interesting modes emerge as the zero-energy excitations at the boundary of topological superconductors. ${ }^{1}$ We therefore begin with the BCS mean-field theory of superconductivity in section 2.1, where we introduce the Bogoliubov-de Gennes transformation, the Majorana basis, and topological superconductivity. Next we describe in section 2.2 the foundational Kitaev chain model as a simple 1D model in which Majorana zero modes emerge. In section 2.3 we show how the Kitaev chain is effectively realized in semiconductor-superconductor hybrid structures. Finally, Sec. 2.4 illustrates the non-Abelian statistics of MZMs.

\subsection{Mean field theory of superconductivity}

Ever since its discovery in 1911 by the Nobel laureate H. Kamerlingh Onnes, superconductivity has been an intense area of study within condensed matter physics. In the superconducting state, electrons are able to carry current with zero resistance, resulting in many technological applications currently in use. Remarkably, a superconductor also manifests a phenomenon known as the Meissner effect where all magnetic flux is expelled from its interior.

How can such a state come about? Finally, after 50 years since its initial discovery, Bardeen, Cooper, and Schrieffer (BSC) put forth a microscopic theory [100] to explain such a state. The theory relies on the formation of Cooper pairs composed of two electrons of opposite momentum and spin. At sufficiently low temperatures, these Cooper pairs form due to an attractive interaction between electrons near the Fermi surface. In conventional superconductors, this attraction interaction arises from the exchange of lattice vibrations, also known as phonons, between electrons. In essence, an electron causes a distortion in the atomic lattice, which the second electron then feels as an attractive potential. Note that such a process is possible because the relaxation rate of lattice distortion is extremely slow compared to time scales associated with the dynamics of the electrons.

\footnotetext{
${ }^{1}$ They can also occur at other topological defect such as the vortices of the superconducting order parameter $\Delta$. A boundary is just one particular type of topological defect.
} 
The BCS theory begins with the Hamiltonian of interacting electrons

$$
H=\sum_{\mathbf{k} \sigma} \varepsilon_{\mathbf{k}} c_{\mathbf{k} \sigma}^{\dagger} c_{\mathbf{k} \sigma}+\sum_{\mathbf{k p q}} \sum_{\sigma \tau} V(\mathbf{k}-\mathbf{p}) c_{\mathbf{k} \sigma}^{\dagger} c_{\mathbf{q}-\mathbf{k} \tau}^{\dagger} c_{\mathbf{q}-\mathbf{p} \tau} c_{\mathbf{p} \sigma},
$$

where $c_{\mathbf{k} \sigma}$ annihilates an electron with momentum $\mathbf{k}$ and spin $\sigma$. The first term is the non-interacting Hamiltonian component, while the second term in Eq. (2.1) is an interaction between two electrons through a pairwise potential $V$. One can show that the most singular terms in the perturbation series for this Hamiltonian come from $q=0$ contributions [101]. The basic approximation of BCS theory is to only retain these term in the interacting component of Eq. (2.1). For simplicity, it is also assumed that $V(\mathbf{k}-\mathbf{p})=V(\hat{\mathbf{k}}-\hat{\mathbf{p}})$, where $\hat{\mathbf{k}}$ is the unit vector in the same direction as $\mathbf{k}$. Finally, since the theory is meant to be a low-energy theory for the electrons near the Fermi surface, the interaction is restricted to electron states near the Fermi surface. The BCS Hamiltonian is then given by

$$
H_{B C S}=\sum_{\mathbf{k} \sigma} \varepsilon_{\mathbf{k}} c_{\mathbf{k} \sigma}^{\dagger} c_{\mathbf{k} \sigma}+\sum_{\mathbf{k p}} \sum_{\sigma \tau} V(\hat{\mathbf{k}}-\hat{\mathbf{p}}) c_{\mathbf{k} \sigma}^{\dagger} c_{-\mathbf{k} \tau}^{\dagger} c_{-} \mathbf{p} \tau c_{\mathbf{p} \sigma} .
$$

The quartic operator in the interaction now represents an electron pair (a Cooper pair) with momenta $\mathbf{p}$ and $-\mathbf{p}$ scattering into another Cooper pair with momenta $\mathbf{k}$ and $-\mathbf{k}$. Different forms of the potential $V(\mathbf{k})$ give rise to different behaviors of the superconducting state. Assuming the potential is independent of spin, we simplify Eq. 2.2 to

$$
H_{B C S}=\sum_{\mathbf{k} \sigma} \varepsilon_{\mathbf{k}} c_{\mathbf{k} \sigma}^{\dagger} c_{\mathbf{k} \sigma}+2 \sum_{\mathbf{k p}} V(\hat{\mathbf{k}}-\hat{\mathbf{p}}) c_{\mathbf{k} \uparrow}^{\dagger} c_{-\mathbf{k} \downarrow}^{\dagger} c_{-}{ }_{-} \downarrow c_{\mathbf{p} \uparrow},
$$

where a factor of 2 comes from summing over spin species. The BCS Hamiltonian (2.3) is certainly more simply than the general interacting Hamiltonian in Eq. (2.1). However, the BCS Hamiltonian still contains quartic interaction terms. We then use mean-field theory to simplify the Hamiltonian into an quadratic Hamiltonian. To do so, we perform a Hartree-Fock like decomposition of the operators. Unlike conventional mean-field theory, where one would expand operators as $c_{\mathbf{k} \uparrow}^{\dagger} c_{\mathbf{p} \uparrow}=\left(c_{\mathbf{k} \uparrow}^{\dagger} c_{\mathbf{p} \uparrow}-\left\langle c_{\mathbf{k} \uparrow}^{\dagger} c_{\mathbf{p} \uparrow}\right\rangle\right)+\left\langle c_{\mathbf{k} \uparrow}^{\dagger} c_{\mathbf{p} \uparrow}\right\rangle$, where $\langle\mathcal{O}\rangle$ indicates thermal expectation value of an operator $\mathcal{O}$, we instead assume that $\left\langle c_{\mathbf{k} \uparrow}^{\dagger} c_{-\mathbf{k} \uparrow}^{\dagger}\right\rangle \neq 0$ and expand the interaction operators in the "Cooper" channel as

$$
\begin{aligned}
c_{\mathbf{k} \uparrow}^{\dagger} c_{-\mathbf{k} \downarrow}^{\dagger} c_{-\mathbf{p} \downarrow} c_{\mathbf{p} \uparrow}= & {\left[\left(c_{\mathbf{k} \uparrow}^{\dagger} c_{-\mathbf{k} \downarrow}^{\dagger}-\left\langle c_{\mathbf{k} \uparrow}^{\dagger} c_{-\mathbf{k} \downarrow}^{\dagger}\right\rangle\right)+\left\langle c_{\mathbf{k} \uparrow}^{\dagger} c_{-\mathbf{k} \downarrow}^{\dagger}\right\rangle\right] } \\
& \cdot\left[\left(c_{-} c_{-} c_{\mathbf{p} \uparrow}-\left\langle c_{-\mathbf{p} \downarrow} c_{\mathbf{p} \uparrow}\right\rangle\right)+\left\langle c_{-\mathbf{p} \downarrow} c_{\mathbf{p} \uparrow}\right\rangle\right] .
\end{aligned}
$$

The key to mean-field theory is to assume that fluctuations around the average are small. When Eq. 2.4 is multiplied through, we then neglect the term 
second order is fluctuations about the mean. The mean-field BCS Hamiltonian then simplifies to

$$
\begin{aligned}
& H_{M F}= \sum_{\mathbf{k} \sigma} \varepsilon_{\mathbf{k}} c_{\mathbf{k} \sigma}^{\dagger} c_{\mathbf{k} \sigma}+2 \sum_{\mathbf{k p}} V(\hat{\mathbf{k}}-\hat{\mathbf{p}})\left(c_{\mathbf{k} \uparrow}^{\dagger} c_{-\mathbf{k} \downarrow}^{\dagger}\left\langle c_{-\mathbf{p} \downarrow} c_{\mathbf{p} \uparrow}\right\rangle+\left\langle c_{\mathbf{k} \uparrow}^{\dagger} c_{-\mathbf{k} \downarrow}^{\dagger}\right\rangle c_{-\mathbf{p} \downarrow} c_{\mathbf{p} \uparrow}\right. \\
&+\left\langle c_{\mathbf{k} \uparrow}^{\dagger} c_{-\mathbf{k} \downarrow}^{\dagger}\right\rangle\left\langle c_{-} \mathbf{p} \downarrow\right. \\
&\left.\left.c_{\mathbf{p} \uparrow}\right\rangle\right) \\
& H_{M F}=\sum_{\mathbf{k} \sigma} \varepsilon_{\mathbf{k}} c_{\mathbf{k} \sigma}^{\dagger} c_{\mathbf{k} \sigma}+\sum_{\mathbf{k}} \Delta_{\mathbf{k}} c_{\mathbf{k} \uparrow}^{\dagger} c_{-\mathbf{k} \downarrow}^{\dagger}+\Delta_{\mathbf{k}}^{*} c_{-\mathbf{k} \downarrow} c_{\mathbf{k} \uparrow}+\left|\Delta_{\mathbf{k}}\right|^{2}
\end{aligned}
$$

where the mean field $\Delta_{\mathbf{k}}$ is defined as

$$
\Delta_{\mathbf{k}}=2 \sum_{\mathbf{p}} V(\hat{\mathbf{k}}-\hat{\mathbf{p}})\left\langle c_{-\mathbf{p} \downarrow} c_{\mathbf{p} \uparrow}\right\rangle .
$$

Note that since the mean-field $\Delta_{\mathbf{k}}$ is defined in terms of expectation values of operators, $\left\langle c_{-\mathbf{p} \downarrow} c_{\mathbf{p} \uparrow}\right\rangle$, this has to be solved self-consistently. Explicitly, the thermal expectation value of the operator is given by

$$
\left\langle c_{-\mathbf{p} \downarrow} \mathcal{C}_{\mathbf{p} \uparrow}\right\rangle=\frac{\operatorname{Tr}\left[c_{-} \mathbf{p} \downarrow \mathcal{c}_{\mathbf{p} \uparrow} e^{-\beta H_{M F}}\right]}{\operatorname{Tr}\left[e^{-\beta H_{M F}}\right]},
$$

where $\beta=\left(k_{B} T\right)^{-1}$ is the inverse temperature and the trace is taken over all eigenstates of the mean-field Hamiltonian.

\subsubsection{Bogoliubov-de Gennes transformation}

We consider the real-space version of the mean-field BCS Hamiltonian in Eq. (2.6),

$$
H_{M F}=\sum_{i j}^{N} \sum_{\sigma \sigma^{\prime}} h_{i \sigma, j \sigma^{\prime}} c_{i \sigma}^{\dagger} c_{j \sigma^{\prime}}+\sum_{i j}^{N} \Delta_{i j} c_{i \uparrow}^{\dagger} c_{j \downarrow}^{\dagger}+\Delta_{i j}^{*} c_{j \downarrow} c_{i \uparrow},
$$

where $N$ is the total number of sites and $c_{i \sigma}^{\dagger}$ creates an electron at site $i$ with spin $\sigma$. We have added the possibility of couplings between the two spin species within the normal Hamiltonian through the matrix element $h_{i \uparrow, j \downarrow}$. This is important because the necessary ingredients of spin-orbit coupling and Zeeman splitting for MZMs to emerge act non-trivial in spin-space. The mean-field formulation of the BCS theory was able to reduce the original quartic Hamiltonian in Eq. (2.3) to the mean-field Hamiltonian in Eq. (2.9), which only contains quadratic operators. The mean-field Hamiltonian looks different than the quadratic Hamiltonians that physicists are usually looking to solve. Due to the terms involving $c_{i \uparrow}^{\dagger} \uparrow_{j \downarrow}^{\dagger}$ and $c_{j \downarrow} c_{i \uparrow}$, we see that the meanfield Hamiltonian couples states with different numbers of electrons. Note, however, that it only couples states with the same parity.

So what do we do? It may appear that the mean-field approximation has been a futile attempt since we're left with finding many-body eigenstates anyway. Fortunately, it is possible to transform the mean-field Hamiltonian 
into a form with the same structure as non-interacting electrons. Namely, we transform the mean-field Hamiltonian such that it takes the form

$$
H_{M F}=\sum_{n=1}^{2 N} E_{n} d_{n}^{\dagger} d_{n}+E_{g},
$$

where $2 N$ is the total number of modes, $E_{g}$ is the ground state energy, $E_{n} \geq 0$, and $d_{n}^{\dagger}$ and $d_{n}$ are fermion creation and annihilation operators that we force to obey the canonical anti-commutation relations,

$$
\begin{gathered}
\left\{d_{n}, d_{m}\right\}=\left\{d_{n}^{+}, d_{m}^{+}\right\}=0, \\
\left\{d_{n}^{\dagger}, d_{m}\right\}=\delta_{n m}
\end{gathered}
$$

just like electron creation and annihilation operators. What kind of structure could the new fermion operators $d_{n}$ have that give us the Hamiltonian in Eq. (2.10)? We find a hint by looking at the commutation of the Hamiltonian with the elementary electron operators,

$$
\begin{aligned}
& {\left[c_{i \uparrow,}, H_{M F}\right]=\sum_{j \sigma^{\prime}} h_{i \uparrow, j \sigma^{\prime}} c_{j \sigma^{\prime}}+\sum_{j} \Delta_{i j} c_{j, \downarrow}^{\dagger},} \\
& {\left[c_{i \downarrow}, H_{M F}\right]=\sum_{j \sigma^{\prime}} h_{i \downarrow, j \sigma^{\prime}} c_{j \sigma^{\prime}}-\sum_{j} \Delta_{j i} c_{j, \uparrow,}^{\dagger},} \\
& {\left[c_{i \uparrow}^{\dagger}, H_{M F}\right]=-\sum_{j \sigma^{\prime}} h_{i \uparrow, j \sigma^{\prime}}^{*} c_{j \sigma^{\prime}}^{\dagger}-\sum_{j} \Delta_{i j}^{*} c_{j, \downarrow},} \\
& {\left[c_{i \downarrow}^{\dagger}, H_{M F}\right]=-\sum_{j \sigma^{\prime}} h_{i \downarrow, j \sigma^{\prime}}^{*} c_{j \sigma^{\prime}}^{\dagger}+\sum_{j} \Delta_{j i}^{*} c_{j, \uparrow},}
\end{aligned}
$$

where $\sigma= \pm 1$ for the two spin types and $\bar{\sigma}=-\sigma$. Obviously, the new annihilation operators $d_{n}$ can not be simple linear combinations of electron annihilation operators. Eqs. (2.13-2.16) do suggest, however, that the new fermion annihilation operators might be linear combinations of both electron creation and annihilation operators. To explore this, we attempt to write the electron creation and annihilation operators in terms of the new fermion operators,

$$
\begin{aligned}
& c_{i \sigma}=\sum_{n} u_{n i \sigma} d_{n}-v_{n i \sigma}^{*} d_{n}^{\dagger}, \\
& c_{i \sigma}^{\dagger}=\sum_{n} u_{n i \sigma}^{*} d_{n}^{\dagger}-v_{n i \sigma} d_{n},
\end{aligned}
$$

where $u_{\text {ni }}, v_{\text {ni }} \in \mathbb{C}$. This transformation is known as a Bogoliubov-de Gennes transformation. The operators $d_{n}^{\dagger}$ and $d_{n}$ are therefore said to create and annihilate a Bogoliubov quasiparticle in state $n$, respectively. By calculating the anti-commutators $\left\{d_{n}, c_{i \sigma}\right\}$ and $\left\{d_{n}^{\dagger}, c_{i \sigma}\right\}$, it is possible to show 
that

$$
\begin{aligned}
& d_{n}=\sum_{i \sigma} u_{n i \sigma}^{*} c_{i \sigma}-v_{n i \sigma}^{*} c_{i \sigma}^{\dagger} \\
& d_{n}^{\dagger}=\sum_{i \sigma} u_{n i \sigma} c_{i \sigma}^{\dagger}-v_{n i \sigma} c_{i \sigma}
\end{aligned}
$$

Looking at Eq. (2.20), we see that we can interpret the creation of a Bogliubov quasiparticle as a superposition of creating and destroying electrons in the system. For this reason, we call $u_{n i \sigma}$ a particle component and $v_{\text {ni }}$ a hole component of the Bogliubov quasiparticle.

How do we now determine the coefficients? To begin, note that the enforcement of the canonical anti-commutation relations on the Bogliubov operators in Eqs. $(2.11,2.12)$, together with the mean-field Hamiltonian form of Eq. 2.10, imply the commutation relations,

$$
\begin{aligned}
{\left[d_{n}, H_{M F}\right] } & =E_{n} d_{n}, \\
{\left[d_{n}^{\dagger}, H_{M F}\right] } & =-E_{n} d_{n}^{\dagger} .
\end{aligned}
$$

If we now plug the definitions of $c_{i \sigma}$ and $c_{i \sigma}^{\dagger}$ in Eqs. $(2.17,2.18)$ into Eqs. (2.13-2.16) and use the commutators in Eqs. (2.21, 2.22), we find that the coefficients satisfy the matrix equation,

$$
\sum_{j} M_{i j} \psi_{j, n}=E_{n} \psi_{i, n}
$$

where $\psi_{i, n}=\left(u_{n i \uparrow}, u_{n i \downarrow}, v_{n i \uparrow}, v_{n i \downarrow}\right)^{T}$ and the matrix $M_{i j}$ is given by

$$
M_{i j}=\left(\begin{array}{cccc}
h_{i \uparrow, j \uparrow} & h_{i \uparrow, j \downarrow} & 0 & -\Delta_{i j} \\
h_{i \downarrow, j \uparrow} & h_{i \downarrow, j \downarrow} & \Delta_{j i} & 0 \\
0 & \Delta_{i j}^{*} & -h_{i \uparrow, j \uparrow}^{*} & -h_{i \uparrow, j \downarrow}^{*} \\
-\Delta_{j i}^{*} & 0 & -h_{i \downarrow, j \uparrow}^{*} & -h_{i \downarrow, j \downarrow}^{*}
\end{array}\right) .
$$

Collecting all $\psi_{i, n}$ into one large vector, $\Psi_{n}$, and all of the matrix blocks $M_{i j}$ into one large matrix, $H_{B d G}$, we have the Schrödinger-like equation,

$$
H_{B d G} \Psi_{n}=E_{n} \Psi_{n}
$$

Note that $\Psi_{n}$ is referred to as a Nambu spinor in the literature, while $H_{B d G}$ is referred to (with some abuse of language) as the Bogoliubov-de Gennes Hamiltonian, or simply the BdG Hamiltonian. If we order the Nambu spinor in the block form,

$$
\begin{aligned}
& \Psi_{n}=\left(u_{n 1 \uparrow}, \ldots, u_{n N \uparrow}, u_{n 1 \downarrow}, \ldots, u_{n N \downarrow}, v_{n 1 \uparrow}, \ldots, v_{n N \uparrow}, v_{n 1 \downarrow}, \ldots, v_{n N \downarrow}\right)^{T} \text {, } \\
& \Psi_{n}=\left(\begin{array}{c}
\mathbf{u}_{n \uparrow} \\
\mathbf{u}_{n \downarrow} \\
\mathbf{v}_{n \uparrow} \\
\mathbf{v}_{n \downarrow}
\end{array}\right),
\end{aligned}
$$


the BdG Hamiltonian also takes a block form and is given by

$$
H_{B d G}=\left(\begin{array}{cc}
h & \widetilde{\Delta} \\
-\widetilde{\Delta}^{*} & -h^{*}
\end{array}\right) .
$$

Here $h$ is the "normal" state Hamiltonian matrix from Eq. (2.9). Note that the hole-hole block (lower right block) has a negative sign out in front of $h^{*}$, which accounts for the fact that particles and holes have opposite energy. The complex conjugation is accounting for the fact that pairing exists between electrons of opposite momentum and spin (see Eq. 2.7). Additionally, $\widetilde{\Delta}$ is the (superconductive) pairing or "anomolous" matrix and is anti-symmetric $\widetilde{\Delta}=-\widetilde{\Delta}^{T}$. The anti-symmetric nature of $\widetilde{\Delta}$ is a reflection of the electron operator identity, $c_{i \sigma} c_{j \sigma^{\prime}}=-c_{j \sigma^{\prime}} c_{i \sigma}$.

To determine the coefficients in our quasiparticle operators, it appears that we just solve for the eigenvalues and eigenvectors of Eq. (2.25). The careful reader, however, will notice that the dimensions of $H_{B d G}$ are $4 \mathrm{~N} \times$ $4 N$, while the quadratic Hamiltonian should only admit of $2 N$ quasiparticle modes. ${ }^{2}$ How do we reconcile this fact?

At the risk of being uninspiring, we first consider the simplest Hamiltonian imaginable, namely

$$
H_{\text {boring }}=\varepsilon c_{1}^{\dagger} c_{1}
$$

where we have a single orbit with energy $\varepsilon$. The eigenstates of this boring system are the vacuum, $|0\rangle$, and the state with the orbital occupied, $|1\rangle$. Suppose $\varepsilon>0$ such that the ground state is the vacuum $|0\rangle$. There is nothing stopping us from performing a Bogoliubov-de Gennes transformation by defining a Bogliubov operator, $d_{1}=c_{1}^{\dagger} \Leftrightarrow d_{1}^{\dagger}=c_{1}$, in which case the transformed Hamiltonian is

$$
H_{\text {boring }}=-\varepsilon d_{1}^{\dagger} d_{1}+\varepsilon \text {, }
$$

where we see that the Bogoliubov quasiparticle energy is $-\varepsilon$. The ground state now is $\left|1_{d}\right\rangle=d_{1}^{\dagger}\left|0_{d}\right\rangle$, where the subscript indicates we're in the basis of the transformed Bogoliubov quasiparticles. Clearly, the quasiparticle created by $d_{1}^{+}$is simply a hole. The point of this uninspiring example is just that we are always free to redefine our quasiparticle operators such that particles become holes and holes become particles, and if we do, we need to change the sign of the quasiparticle energy.

How does the logic of the simple example apply to the generic BdG problem with a matrix of the form given in Eq. (2.27)? It is easy to show that the BdG Hamiltonian has the symmetry

$$
\tau_{x} H_{B d G}^{*} \tau_{x}=-H_{B d G}
$$

where $\tau_{x}$ is the first Pauli matrix acting in particle-hole space. ${ }^{3}$ This implies that if $\Psi_{n}=\left(\mathbf{u}_{n \uparrow}, \mathbf{u}_{n \downarrow}, \mathbf{v}_{n \uparrow}, \mathbf{v}_{n \downarrow}\right)^{T}$ is an eigenstate with energy $E_{n}$, then it is

\footnotetext{
${ }^{2}$ The 2 accounts for spin.

${ }^{3}$ Explicitly, $\tau_{x} H_{B d G}^{*} \tau_{x}=\left(\begin{array}{ll}0 & \mathbb{1} \\ \mathbb{1} & 0\end{array}\right)\left(\begin{array}{cc}h^{*} & \widetilde{\Delta}^{*} \\ -\widetilde{\Delta} & -h\end{array}\right)\left(\begin{array}{ll}0 & \mathbb{1} \\ \mathbb{1} & 0\end{array}\right)=\left(\begin{array}{cc}-h & -\widetilde{\Delta} \\ \widetilde{\Delta}^{*} & h^{*}\end{array}\right)=-H_{B d G}$.
} 
guaranteed that $\tau_{x} \Psi_{n}^{*}=\left(\mathbf{v}_{n \uparrow}^{*}, \mathbf{v}_{n \downarrow}^{*}, \mathbf{u}_{n \uparrow}^{*}, \mathbf{u}_{n \downarrow}^{*}\right)^{T}$ is also an eigenstate but with energy $-E_{n}$. Just like the simple example above, we are free to choose either one (but not both) as the Bogoliubov quasiparticle created by $d_{n}^{\dagger}$. Switching between the two representations of the quasiparticle amounts to letting $d_{n}^{+} \rightarrow d_{n}$ and $E_{n} \rightarrow-E_{n}$. For simplicity, we always choose the quasiparticle with $E_{n} \geq 0$. The ground state $|G S\rangle$ of the mean-field Hamiltonian (2.10) is then the quasiparticle vacuum, i.e. $d_{n}|G S\rangle=0$ for all $n$. Note that the quasiparticle vacuum is not (generically) a state with no electrons. Rather, because $d_{n}$ is composed of a linear combination of electron creation and annihilation operators, $|G S\rangle$ will typically be a complicated linear combination of many-body states with varying numbers of electronic states occupied. If the actual form of the ground state is of interest, it can be found by applying all Bogoliubov annihilation operators to an arbitrary state $|a r b\rangle$. Provided $\left\langle\operatorname{arb} \mid 1_{1}, 1_{2}, \ldots, 1_{2 N}\right\rangle \neq 0$, where $\left|1_{1}, 1_{2}, \ldots, 1_{2 N}\right\rangle$ is the eigenstate with all quasiparticles occupied, it is clear that $\prod_{n} d_{n}|\operatorname{arb}\rangle \propto|G S\rangle$.

\subsubsection{Majorana basis}

In the Bogoliubov-de Gennes transformation, we wrote the quasiparticle operators $d_{n}^{\dagger}$ as a linear combination of electron creation $c_{i \sigma}^{\dagger}$ and annihilation $c_{i \sigma}$ operators. In other words, the quasiparticles have both particle and hole components. As we have discussed, however, the difference between particles and holes is really a matter of perspective and taste. In fact, there is no reason we cannot represent the Bogoliubov quasiparticles using basis states that are linear combinations of particles and holes. One such basis of particular interest is known as the Majorana basis, where we define the Majorana operators,

$$
\begin{aligned}
& \gamma_{\mathbf{i} 1}=\left(c_{\mathbf{i}}^{\dagger}+c_{\mathbf{i}}\right), \\
& \gamma_{\mathbf{i} 2}=i\left(c_{\mathbf{i}}^{\dagger}-c_{\mathbf{i}}\right),
\end{aligned}
$$

where we have combined the site $i$ and spin $\sigma$ indices into the single index i. The Majorana operators are of two flavors or types as indicated by the second subscripts, 1 and 2, respectively. Note that these operators are equal parts particle and hole. More importantly, the operators satisfy the Majorana condition,

$$
\gamma_{\mathbf{i} \mu}^{\dagger}=\gamma_{\mathbf{i} \mu},
$$

which is where the name Majorana basis is derived. From Eq. (2.33), we see that creating and destroying a Majorana are in fact equivalent operations. Also note that that the Majorana operators satisfy the anticommutation relation,

$$
\left\{\gamma_{i \mu}, \gamma_{j v}\right\}=2 \delta_{i j} \delta_{\mu \nu}
$$

The natural question is why is this basis of any particular interest? From the perspective of the BdG Hamiltonian, this is a simply a unitary transformation with an arbitrary basis change. We do this all the time in quantum 
mechanics, so what is the big deal? Indeed, for a generic BdG Hamiltonian, the Majorana basis is nothing special. Consider, for example, an arbitrary BdG eigenstate $\Psi_{n}$ with energy $E_{n}>0$ and created by the Bogoliobuv operator $d_{n}^{+}$. As discussed in the previous section, we automatically know that $\tau_{x} \Psi_{n}^{*}$ also an eigenstate of the BdG Hamiltonian but with energy $-E_{n}$ and is created by the operator $d_{n}$. We can define Majorana operators,

$$
\begin{aligned}
& \widetilde{\gamma}_{n 1}=\left(d_{n}^{\dagger}+d_{n}\right), \\
& \widetilde{\gamma}_{n 2}=i\left(d_{n}^{\dagger}-d_{n}\right),
\end{aligned}
$$

which manifestly satisfy the Majorana condition, $\widetilde{\gamma}_{n \mu}^{\dagger}=\widetilde{\gamma}_{n \mu}$. Combining Eqs. $(2.35,2.36)$ we find

$$
d_{n}=\frac{1}{2}\left(\widetilde{\gamma}_{n 1}+i \widetilde{\gamma}_{n 2}\right)
$$

In words, every Bogoliubov quasiparticle, even one with finite energy, is composed of two Majoranas. Majoranas always come in pairs. There is the special situation of $E_{n}=0$, however. In such a case $\Psi_{n}$ and $\tau_{x} \Psi_{n}^{*}$ are degenerate and the isolated Majoranas themselves become eigenstates of the BdG Hamiltonian. These types of quasiparticles deserve the special name Majorana zero modes to distinguish them from the Majorana components making up any generic BdG state. If you hear somebody discussing the achievement of Majorana fermions, these Majorana zero modes are actually what they mean. Importantly, the mean-field Hamiltonian then has a degenerate ground state. One where the zero-energy quasiparticle is occupied and another were it is not.

\subsubsection{Topological superconductivity}

Recall from Sec. 1.1 of the introductory chapter that free fermion insulators can be classified into different topological equivalence classes, also known as topological phases. Two insulators are defined as equivalent under the classification, i.e. they belong to the same topological phase, if it possible to continuously deform the Hamiltonian of the first insulator into the Hamiltonian of the second without closing the bulk gap. On the other hand, if it is impossible to continuously transform between the Hamiltonians without closing the bulk gap, the insulators belong to different topological phases. It was also pointed out in Sec. 1.1 that the topological classification of free fermion insulators becomes much richer if anti-unitary symmetry constraints in the form of time-reversal, charge conjugation, and chiral symmetries are allowed to be placed on the Hamiltonian. This led to the periodic table of free fermion insulators, which is reproduced in Fig. 2.1.

Looking back at Eqs. $(2.25,2.27)$, it is clear that the BdG Hamiltonian is in fact a free fermion Hamiltonian. The fermions in this case are not electrons, but rather Bogoliubov quasiparticles. In addition, the pairing $\widetilde{\Delta}$ between the 


\begin{tabular}{c|ccc|ccccccccc}
\hline \hline \multicolumn{1}{c}{ Symmetry } & \multicolumn{11}{c}{ Spatial Dimension $d$} \\
Class & $T$ & $C$ & $S$ & 1 & 2 & 3 & 4 & 5 & 6 & 7 & 8 & $\cdots$ \\
\hline A & 0 & 0 & 0 & 0 & $\mathbb{Z}$ & 0 & $\mathbb{Z}$ & 0 & $\mathbb{Z}$ & 0 & $\mathbb{Z}$ & $\cdots$ \\
AIII & 0 & 0 & 1 & $\mathbb{Z}$ & 0 & $\mathbb{Z}$ & 0 & $\mathbb{Z}$ & 0 & $\mathbb{Z}$ & 0 & $\cdots$ \\
\hline Al & 1 & 0 & 0 & 0 & 0 & 0 & $\mathbb{Z}$ & 0 & $\mathbb{Z}_{2}$ & $\mathbb{Z}_{2}$ & $\mathbb{Z}$ & $\cdots$ \\
BDI & 1 & 1 & 1 & $\mathbb{Z}$ & 0 & 0 & 0 & $\mathbb{Z}$ & 0 & $\mathbb{Z}_{2}$ & $\mathbb{Z}_{2}$ & $\cdots$ \\
D & 0 & 1 & 0 & $\mathbb{Z}_{2}$ & $\mathbb{Z}$ & 0 & 0 & 0 & $\mathbb{Z}$ & 0 & $\mathbb{Z}_{2}$ & $\cdots$ \\
DIII & -1 & 1 & 1 & $\mathbb{Z}_{2}$ & $\mathbb{Z}_{2}$ & $\mathbb{Z}$ & 0 & 0 & 0 & $\mathbb{Z}$ & 0 & $\cdots$ \\
All & -1 & 0 & 0 & 0 & $\mathbb{Z}_{2}$ & $\mathbb{Z}_{2}$ & $\mathbb{Z}^{2}$ & 0 & 0 & 0 & $\mathbb{Z}$ & $\cdots$ \\
CII & -1 & -1 & 1 & $\mathbb{Z}$ & 0 & $\mathbb{Z}_{2}$ & $\mathbb{Z}_{2}$ & $\mathbb{Z}$ & 0 & 0 & 0 & $\cdots$ \\
C & 0 & -1 & 0 & 0 & $\mathbb{Z}$ & 0 & $\mathbb{Z}_{2}$ & $\mathbb{Z}_{2}$ & $\mathbb{Z}$ & 0 & 0 & $\cdots$ \\
Cl & 1 & -1 & 1 & 0 & 0 & $\mathbb{Z}$ & 0 & $\mathbb{Z}_{2}$ & $\mathbb{Z}_{2}$ & $\mathbb{Z}$ & 0 & $\cdots$ \\
\hline \hline
\end{tabular}

FIGURE 2.1: Periodic table of topological phases characterizing the 10 symmetry classes of free fermion Hamiltonians. The columns labeled $T C$ and $S$ indicate when the system has timereversal, charge-conjugations, and chiral symmetries, respectively. 0 indicates the symmetry is not present, while \pm 1 indicates the symmetry is present and what the symmetry operator squares to. The numbers over the columns indicate the spatial dimension. $\mathbb{Z}$ and $\mathbb{Z}_{2}$ indicates that there are infinite and two distinct topological phases, respectively, while 0 implies that every Hamiltonian in that class is trivial for the that spatial dimension.

particle and hole degrees of freedom typically opens a superconducting gap. ${ }^{4}$ Therefore, the classification of free fermion insulators also applies to superconductors treated at the BdG mean-field level. Superconductors belonging to phases that cannot be continuously transformed into a topologically trivial superconductor ${ }^{5}$ are called topological superconductors.

What symmetry classes can a BdG superconductor belong to? From Eq. (2.30), it is clear that every BdG Hamiltonian posses the anti-unitary symmetry,

$$
\hat{C} H_{B d G} \hat{C}^{-1}=H,
$$

where the operator $\hat{C}$ is given by

$$
\hat{C}=\tau_{x} \hat{K},
$$

with $\hat{K}$ being the complex conjugation operator and $\tau_{x}$ is the first Pauli matrix acting in particle-hole space. The symmetry operator $\hat{C}$ is a chargeconjugation symmetry operator. ${ }^{6}$ Therefore, every BdG Hamiltonian has charge-conjugation symmetry. Note that charge-conjugation symmetry is

\footnotetext{
${ }^{4}$ It is possible to have a gapless superconductor which is described by a BdG Hamiltonian. We assume for our purposes, however, that a gap exists.

${ }^{5}$ It is easy to create a model for a topologically trivial superconductor. Just let $\mu \rightarrow-\infty$. The ground state of this trivial superconductor is then the true vacuum, i.e. no electrons are present.

${ }^{6} \mathrm{~A}$ charge conjugation symmetry for a non-interacting Hamiltonian has the form $\hat{C}=$ $U_{C} \hat{K}$, where $U_{C}$ is a unitary matrix. The Hamiltonian is said to have charge-conjugation symmetry if $U_{C}^{+} H^{*} U_{C}=-H$
} 
also called particle-hole symmetry, which especially makes sense in the context of superconductors since $\tau_{x}$ is exchanging the particle and hole degree's of freedom. Additionally, the charge conjugation symmetry operator square to 1 , i.e $\hat{C}^{2}=1$. This implies every BdG superconductor belongs to either symmetry class BDI, D, or DIII. Which particular symmetry class of these three that any given superconductor belongs to depends upon if it has timereversal symmetry, and if so, what value the time-reversal operator squares to.

The symmetry class of most importance for MZMs in SM-SC nanostructures is class D, which does not posses time-reversal symmetry. Note that the time-reversal symmetry is broken by the applied magnetic field in Majorana nanostructures. ${ }^{7}$ Looking at the table in Fig. 2.1, class D has topologically non-trivial phases in 1 and 2 dimensions, but not 3 dimensions. Indeed, class $\mathrm{D}$ in $1 \mathrm{D}$ is characterized by a $\mathbb{Z}_{2}$ topological invariant. In other words, there exists only two phases; the trivial phase, which can be continuously deformed into the vacuum, and the non-trivial phase. This non-trivial topological phase is precisely the phase in which MZMs emerge as zero-energy edge modes. In contrast to $1 \mathrm{D}$, class $\mathrm{D}$ in $2 \mathrm{D}$ has an integer topological invariant $\mathbb{Z}$, i.e. there are an infinite number of possible topological phases. A $2 \mathrm{D}$ topological superconductor in class $\mathrm{D}$ with a non-zero topological invariant, $v \neq 0$, has $v$ many chiral, in-gap Majorana modes that propagate on the $1 \mathrm{D}$ edges of the 2D sample. This thesis focuses on SM-SC nanostructures in class D in 1D, where MZMs arise in the topologically non-trivial phase. Note, however, that class DIII systems, where time-reversal symmetry is preserved, have seen an increase of theoretical attention in the last few years [102].

\subsection{Kitaev chain model}

In 2001, Alexei Kitaev introduced a simple toy model, later referred to as the Kitaev chain by the community, of a "quantum wire" in which unpaired Majorana zero modes exist at the two edges of the system [40]. Strikingly simple, the Kitaev chain represents the simplest example of a topological superconductor. The model describes a 1D tight-binding chain of spinless fermions with p-wave superconductivity and a Hamiltonian given by

$$
\mathcal{H}=-\sum_{i=1}^{N} \mu c_{i}^{\dagger} c_{i}+\sum_{i=1}^{N-1} t c_{i+1}^{\dagger} c_{i}+\text { h.c. }+\sum_{i=1}^{N-1} \Delta_{o} c_{i+1}^{\dagger} c_{i}^{\dagger}+\text { h.c. },
$$

where $\mu$ is the chemical potential, $t$ and $\Delta_{o}$ are the hopping and superconducting pairing between nearest neighbor sites, respectively, $c_{i}^{\dagger}\left(c_{i}\right)$ creates (annihilates) a fermion on on the $i^{\text {th }}$ site, and $N$ is the total number of sites in

\footnotetext{
${ }^{7}$ Some models of Majorana nanowires that contain spin happen to have a different timereversal symmetry $\mathcal{T}^{\prime}$ that squares to 1 even when the magnetic field breaks the canonical time-reversal symmetry $\mathcal{T}$ that squares to -1 . Technically, these models are in symmetry class BDI. Typically, however, this symmetry is broken in realistic devices, so symmetry class D is the primary focus.
} 
the chain. Note that h.c. indicates Hermitean conjugate. Also note that onsite superconducting pairing is impossible in a spinless fermion model since $c_{i}^{\dagger} c_{i}^{\dagger}=0$ by the Pauli exclusion principle. As we have discussed in section 2.1.1, we can use a Bogoliubov-de Gennes transformation to recast Eq. (2.40) into the form,

$$
\mathcal{H}=\frac{1}{2} \sum_{i j}\left(\begin{array}{ll}
c_{i}^{\dagger} & c_{i}
\end{array}\right) H_{B d G}^{i j}\left(\begin{array}{c}
c_{j} \\
c_{j}^{\dagger}
\end{array}\right),
$$

where the "first quantized" BdG Hamiltonian takes the form

$$
H_{B d G}^{i j}=\left(\begin{array}{cc}
h_{i j} & \Delta_{i j} \\
-\Delta_{i j}^{*} & -h_{i j}^{*}
\end{array}\right),
$$

with the normal and anomalous Hamiltonian terms given by

$$
\begin{aligned}
h_{i j} & =-\mu \delta_{i j}+t\left(\delta_{i, j+1}+\delta_{i, j-1}\right), \\
\Delta_{i j} & =\Delta_{o}\left(\delta_{i, j+1}-\delta_{i, j-1}\right),
\end{aligned}
$$

respectively.

It is useful to first study the model using periodic boundary conditions. To do so we couple the first and last sites of the original open chain to form a closed ring. Next we Fourier transform into momentum space using

$$
c_{j}=\frac{1}{\sqrt{N}} \sum_{k} \widetilde{c}_{k} e^{-i k j}
$$

where $\widetilde{c}_{k}$ annihilates a fermion with momentum $k, k=\frac{2 \pi n}{N}$, and $n \in \mathbb{Z}$ such that $-\pi<k \leq \pi$. Plugging Eq. (2.45) into Eq. (2.41) yields

$$
\mathcal{H}=\frac{1}{2} \sum_{k}\left(\begin{array}{ll}
\widetilde{c}_{k}^{\dagger} & \widetilde{c}_{-k}
\end{array}\right) \widetilde{H}_{B d G}(k)\left(\begin{array}{c}
\widetilde{c}_{k} \\
\widetilde{c}_{-k}^{\dagger}
\end{array}\right),
$$

where the BdG-Bloch Hamiltonian is given by

$$
\widetilde{H}_{B d G}(k)=\left(\begin{array}{cc}
\varepsilon_{k} & \widetilde{\Delta}_{k} \\
\widetilde{\Delta}_{k}^{*} & -\varepsilon_{k}
\end{array}\right),
$$

with $\varepsilon_{k}=2 t \cos (k)-\mu$ and $\widetilde{\Delta}_{k}=2 i \Delta_{o} \sin (k)$. Eq. (2.47) is easy to diagonalize and we arrive at eigenvalues,

$$
E_{ \pm}(k)= \pm \sqrt{\varepsilon_{k}^{2}+\left|\widetilde{\Delta}_{k}\right|^{2}}
$$

An example spectrum of a Kitaev chain is shown in Fig. 2.2 with parameters given by $t=-1, \mu=0.3, \Delta_{o}=0$ (black solid lines), and $\Delta_{o}=0.25$ (red dashed lines). In the absence of superconductive pairing $\left(\Delta_{0}=0\right)$, the particle and hole degrees of freedom are decoupled in Eq. (2.47). This results in two bands that never mix and that cross each other at zero energy. Once the the pairing is turned on $\left(\Delta_{o}=0.25\right)$, the particle and hole degrees of freedom 


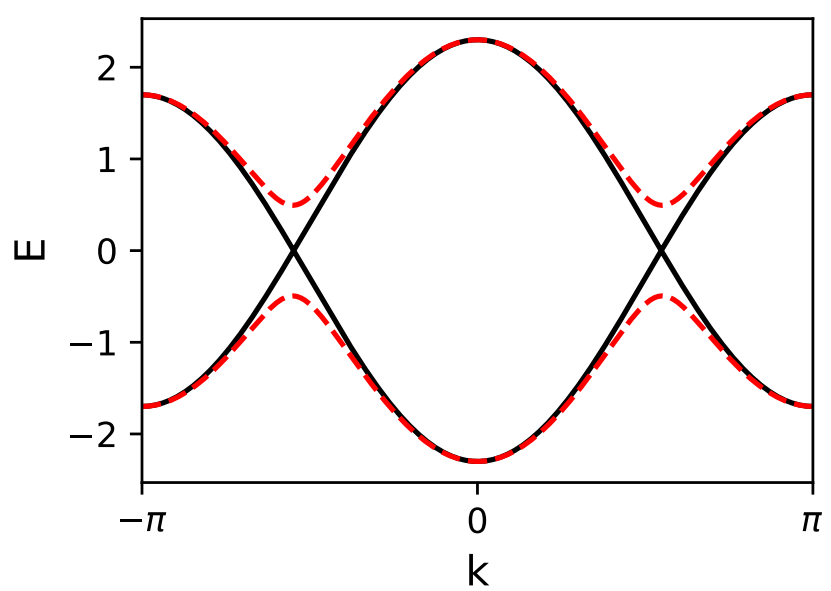

FIGURE 2.2: Bulk spectrum of the Kitaev chain with parameters given by $t=-1, \mu=0.3, \Delta_{o}=0$ (black solid lines), and $\Delta_{o}=0.25$ (red dashed lines). Including pairing, $\Delta_{o} \neq 0$, opens a superconducting gap near the Fermi level.

are coupled, causing the quasiparticle eigenstates to be linear combinations of particles and holes. This results in an avoided crossing of the two bands near zero-energy and we have a gapped system. Are there any parameter choices where the system is not gapped? Looking at Eq. (2.48), we observe that both $\varepsilon_{k}=0$ and $\widetilde{\Delta}_{k}=0$ are needed for the system to become gapless. For $\Delta_{o} \neq 0$, we have $\widetilde{\Delta}_{k}=0$ if and only if $k=0, \pi$. Plugging this into $\varepsilon_{k}$, we find that the system is gapless if $\mu_{ \pm}= \pm 2 t$. Recall from Sec. 1.1 that the onset of a topological phase transition is signalled by the closing of the bulk gap. We therefore can expect $\mu_{ \pm}= \pm 2 t$ to be the boundaries between different topological phases in parameter space.

Now let us return to studying the system for a finite chain with open boundary conditions. Its useful to transform into the Majorana basis, which was introduced in section 2.1.2 and is defined by the Majorana operators,

$$
\begin{aligned}
& \gamma_{i, 1}=\left(c_{i}^{\dagger}+c_{i}\right), \\
& \gamma_{i, 2}=i\left(c_{i}^{\dagger}-c_{i}\right) .
\end{aligned}
$$

Recall that these are operators are named Majorana operators since they are equal to their own Hermitean adjoint, i.e. $\gamma_{i, n}^{\dagger}=\gamma_{i, n}$, with $n=1,2$. We can also write the fermion creation and annihilation operators in terms of the Majorana operators,

$$
\begin{aligned}
c_{i}^{\dagger} & =\frac{1}{2}\left(\gamma_{i, 1}-i \gamma_{i, 2}\right), \\
c_{i} & =\frac{1}{2}\left(\gamma_{i, 1}+i \gamma_{i, 2}\right)
\end{aligned}
$$


Plugging Eqs. $(2.51,2.52)$ into Eq. $(2.40)$ gives the Hamiltonian written in the Majorana basis,

$$
\mathcal{H}=-\frac{\mu}{2} \sum_{j=1}^{N}\left(1+i \gamma_{j, 1} \gamma_{j, 2}\right)+\frac{i}{2} \sum_{j=1}^{N-1}\left(V \gamma_{j, 2} \gamma_{j+1,1}+W \gamma_{j, 1} \gamma_{j+1,2}\right),
$$

where $V=\left(\Delta_{o}+t\right)$ and $W=\left(\Delta_{o}-t\right)$ are the transformed couplings between Majorana basis states on neighboring sites. Generically, the Majorana basis Hamiltonian is just as complicated the Hamiltonian written using the canonical fermion creation and annihilation operators in Eq. (2.41). However, the problem dramatically simplifies in two limits. In the trivial or atomic limit, the parameters are set to $\mu \neq 0$ and $t=\Delta_{o}=0$, and the Hamiltonian is given by

$$
\mathcal{H}=-\frac{\mu}{2} \sum_{j=1}^{N}\left(1+i \gamma_{j, 1} \gamma_{j, 2}\right)=-\mu \sum_{j=1}^{N} c_{j}^{\dagger} c_{j} .
$$

Such a situation is not very interesting since there is no coupling between nearest neighbor sites. Each site can be thought of as atomically isolated in such a case. The ground state of the system has either no occupied states $(\mu<$ 0 ) or every site having an occupied fermionic state $(\mu>0)$. Note that each eigenstate can still be written as a linear combination of Majorana operators, $c_{i}=\frac{1}{2}\left(\gamma_{i, 1}+i \gamma_{i, 2}\right)$, so there is nothing inherently special about the Majorana basis from this perspective. The Kitaev limit [40] is much more interesting. In this limit, the parameters are taken to be $\mu=0$ and $\Delta_{o}=t \neq 0$. In the Majorana basis, this translates into $V=2 t$ and $W=0$, and the Hamiltonian is given by

$$
\mathcal{H}_{\text {Kitaev }}=t \sum_{j=1}^{N-1} \gamma_{j, 2} \gamma_{j+1,1}-\frac{\mu N}{2},
$$

where the unimportant constant comes from summing the terms in Eq. (2.54) without Majorana operators. Notice that because $\mu=0$, Majorana basis states within the same site do not couple. We also only have coupling between Majorana basis states on neighboring sites of differing flavor. Similarly to the the atomic limit, the eigenstates in the bulk of the chain are isolated dimer states. Here, however, the dimers are composed of Majorana states on neighboring lattice sites instead of within one lattice site. In further contrast to the atomic limit, the Kitaev limit is characterized by two Majorana basis states at the two ends of the chain that do not have a partner Majorana basis state to form a dimer state. In fact, the Majorana operators $\gamma_{1,1}$ and $\gamma_{N, 2}$ do not appear in the Hamiltonian (2.55) at all! In such a case, we have two isolated Majorana modes at the edges of the chain with exactly zero energy. Hence they deserve the special name Majorana zero modes. The two MZMs can be combined to form a regular, but highly non-local, Bogoliubov-de Gennes quasiparticle,

$$
\widetilde{d}=\frac{1}{\sqrt{2}}\left(\gamma_{0,1}+i \gamma_{N, 2}\right),
$$


(a)

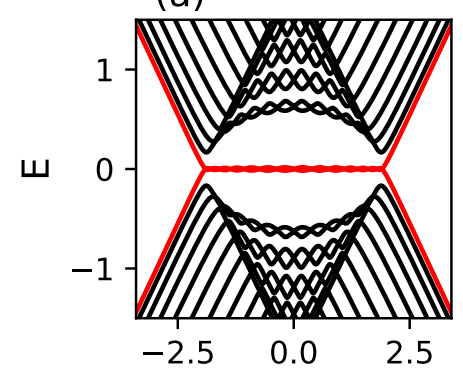

(b)



(c) $\mu$
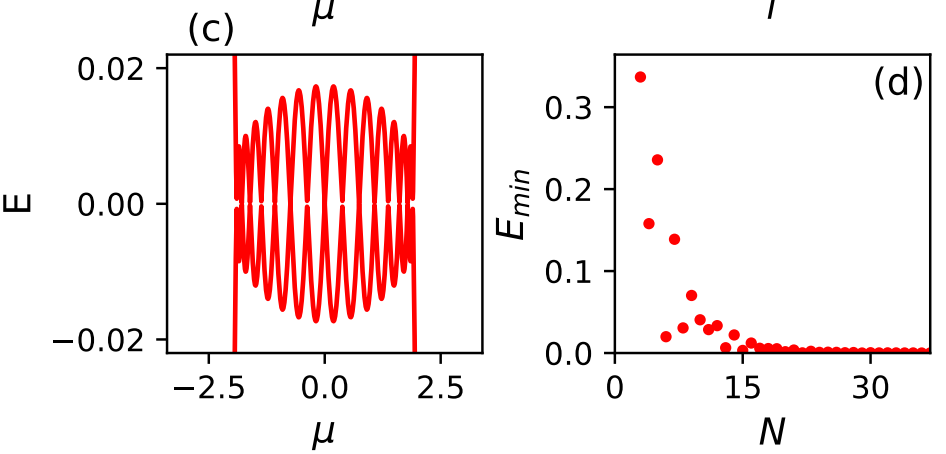

FIGURE 2.3: (a) Spectrum of a finite Kitaev chain with $N=15$ sites, $t=-1$, and $\Delta_{o}=0.25$ as a function of chemical potential $\mu$. Nearly zero-energy Majorana zero modes emerge for $|\mu|<$ $|2 t|$. (b) Modulus square of Majorana zero modes making up a (nearly) zero-energy BdG state for $N=42, t=-1, \Delta_{o}=$ 0.25 , and $\mu=0$. (c) Low-energy spectrum version of (a) which shows Majorana oscillations due to the overlap of the tails of the Majorana zero modes localized on each edge. (d) Lowest energy eigenvalue $E_{\min }$ as a function of chain length $N$ with parameters set to $t=-1, \Delta_{o}=0.25$, and $\mu=0.4$.

with zero energy. Recall from Sec. 2.1.1 that the ground state of a BdG system satisfies the condition,

$$
d_{n}|G S\rangle,
$$

for all quasiparticle operators $d_{n}$. Note, however, that the state

$$
|\widetilde{d}\rangle=\widetilde{d}^{\dagger}|G S\rangle,
$$

has the same energy as $|G S\rangle$ and is therefore also a ground state. The presence of MZMs therefore yields a degenerate ground state subspace.

The Majorana zero modes emerging in the Kitaev limit are quite interesting, but the limit itself requires fine-tuning of the system parameters and Majorana zero modes would only be a purely academic or mathematical interest if their existence required such a tuning. Their existence, however, is not restricted to the parameter choice of the Kitaev limit. Rather their emergence is a a property of the topological nature of the topological phase transition as $\mu$ and $t$ are adjusted. Indeed their existence is a particular instance of the celebrated bulk-boundary correspondence of topological phases of matter. As long as $|\mu|<2|t|$ and $\Delta_{o} \neq 0$, Majorana modes will exist at the edges of 
sufficiently long Kitaev chains. To illustrate this, we show the spectrum of a long Kitaev chain in Fig. 2.3(a) as a function of $\mu$ for fixed $t$ and $\Delta_{o}$. Notice that a pair of (nearly) zero-energy states occur for $|\mu|<2|t|$. Fig. 2.3(b) shows the modulus square of the (nearly) zero-energy states for some particular $\mu$ value. In contrast to the Kitaev limit, these modes are not completely localized at the first and last sites of the system. Rather they exponential decay into the bulk of the chain. Fig. 2.3(c) displays a zoomed in section of the spectrum of Fig. 2.3(a) near zero-energy. Notice that the states are not exactly zero-energy, but rather display small oscillations around zero-energy. These small oscillations occur because of the small overlap between the Majorana zero modes due to the exponential decay of the edge modes into the bulk. As the chain becomes larger, these oscillations become smaller as illustrated in Fig. 2.3(d) and vanish in the thermodynamic limit of $N \rightarrow \infty$.

\subsection{Rashba Majorana nanowires}

The Majorana zero modes emerging in the Kitaev chain are a beautiful result that presents an excellent example of edge modes occurring due to a non-trivial topological phase. However, realizing the Kitaev chain model in a realistic condensed matter system has two main issues. Firstly, the fermions in the Kitaev chain model are spinless, while electrons in nature are of course spin- $\frac{1}{2}$ particles. In principle, one can imagine adding spin to the Kitaev chain model in a trivial fashion. Essentially the model would have one copy of the Kitaev chain for each spin species and have no coupling between the chains. In such a case, there would be two Majorana zero modes at each edge of the system. In the presence of a perturbation, however, that couples the spin species, the two Majorana zero modes at a particular edge of the system would couple and become gapped. We would then be left with no Majorana zero modes. Secondly, the superconductivity in the Kitaev chain has p-wave symmetry (the pairing parameter is an odd function of momentum, $\widetilde{\Delta}_{k}=$ $2 i \Delta_{o} \sin k$ ). P-wave superconductors are extremely rare in nature, so possible materials in which to realized the Kitaev chain directly are few in number. By far the most common superconducting symmetry, especially for elementary superconductors, is s-wave superconductivity, which unfortunately cannot produce Majorana zero modes without further ingredients.

A major breakthrough occurred in 2010 in which it was shown by two groups that a topological p-wave superconductor with Majorana zero modes could be engineered within semiconductor-superconductor hybrid nanowires $[8,9]$. Importantly, the superconducting symmetry of the proposals is swave, meaning that non-exotic superconductors can be employed in the engineering design. The other two amazingly simple ingredients are spin-orbit coupling and Zeeman splitting, both of which are due to properties of the semiconductor in combination with an applied magnetic field or proximity coupled ferromagnet [43].

Consider a nanowire with strong confinement in two directions such that a single spinful subband faithfully captures the physics of the system. It 
is also useful to consider periodic boundary conditions, which makes the momentum $k$ along the length of the wire a good quantum number and allows us to employ Bloch's theorem. The Bogoliubov quasiparticle eigenstates obey the Bogoliubov-de Gennes equation $H_{B d G}(k) \Psi=E \Psi$ with $\Psi(k)=$ $\left(u_{\uparrow}(k) \quad u_{\downarrow}(k) \quad v_{\uparrow}(k) \quad v_{\downarrow}(k)\right)^{T}$ being the Nambu Bloch spinor, and the Bloch BdG Hamiltonian is given by $[8,9]$

$$
\widetilde{H}_{B d G}(k)=\left(\begin{array}{cc}
H_{o}(k) & \widetilde{\Delta} \\
-\widetilde{\Delta}^{T} & -H_{o}^{*}(-k)
\end{array}\right),
$$

where the normal Hamiltonian and pairing matrix are given by

$$
\begin{aligned}
H_{o}(k) & =\left(\frac{\hbar^{2} k^{2}}{2 m^{*}}-\mu\right) \sigma_{o}+\Gamma \sigma_{z}+\alpha k \sigma_{y} \\
\widetilde{\Delta} & =-i \Delta_{o} \sigma_{y}
\end{aligned}
$$

respectively, where $m^{*}$ is the effective mass, $\mu$ is the chemical potential, $\alpha$ is the Rashba spin-orbit coupling coefficient, $\Gamma$ is the Zeeman energy, $\Delta_{o}$ is the superconducting pairing amplitude, $\sigma_{i}$ with $i=0, x, y, z$ are the Pauli matrices in spin space, and $k_{z}$ is the momentum wavenumber along the length of the wire. The first term in the normal Hamiltonian is just the usual kinetic energy of the electrons due to their momentum along the length of the nanowire. Kinetic energy takes the same form independent of spin, so therefore it is multiplied by $\sigma_{o}=I_{2 \times 2}$. The second term is the Zeeman energy due to an applied magnetic field along the length of the nanowire, which we take to be the $z$-direction. This breaks the degeneracy between the spin species as expected due to the $\sigma_{z}$ factor. The last term is the normal Hamiltonian is the Rashba spin-orbit coupling. This is a relativistic effect which couples the motion of the electron to its spin [103]. Microscopically, its origin is an electric field that break the inversion symmetry of the device. Further discussion of Rashba spin-orbit coupling occurs is chapter 4 . Note that the spin-orbit coupling can be viewed as a momentum dependent magnetic field, i.e. an electron with momentum in the $z$-direction "feels" a spin-orbit induced magnetic field pointing in the $y$-direction. Finally, the pairing matrix represent s-wave superconductivity. Pairing can only exist between electrons of opposite spin as indicated by the $\sigma_{y}$ factor in Eq. (2.61). Without loss of generality, the pairing amplitude is assumed to be real, $\Delta_{o} \in \mathbb{R}$. The Bloch BdG Hamiltonian in Eq. (2.59) can also be written more compactly as

$$
\widetilde{H}_{B d G}(k)=\left[\left(\frac{\hbar^{2} k^{2}}{2 m^{*}}-\mu\right) \sigma_{o}+\Gamma \sigma_{z}+\alpha k \sigma_{y}\right] \tau_{z}-\Delta_{o} \sigma_{y} \tau_{y},
$$

where $\tau_{i}$ with $i=o, x, y, z$ are the Pauli matrices in particle-hole space. The form in Eq. (2.59), however, is more physically transparent when first trying to understand the physics.

When beginning to study a BdG system, it is often useful to consider the limit of small pairing $\Delta_{0}$. In other words, we assume the energy scales of the 
normal Hamiltonian components dominate and $\widetilde{\Delta}$ can be treated as a perturbation. Let us then first diagonalize the particle and hole normal Hamiltonian components. The $2 \times 2$ particle Hamiltonian can be diagonalized using the unitary matrix

$$
U_{p}(k)=\left(\begin{array}{cc}
\cos \left(\frac{\theta_{k}}{2}\right) & \sin \left(\frac{\theta_{k}}{2}\right) \\
\sin \left(\frac{\theta_{k}}{2}\right) e^{i \phi_{k}} & -\cos \left(\frac{\theta_{k}}{2}\right) e^{i \phi_{k}}
\end{array}\right)
$$

where $\cos \left(\theta_{k}\right)=\frac{\Gamma}{\sqrt{\Gamma^{2}+\alpha^{2} k^{2}}}$ and $e^{i \phi_{k}}=i k /|k|$ define the auxiliary angles. The unitary matrix $U_{h}(k)$ that diagonalizes the hole normal Hamiltonian component is related the the particle unitary matrix by $U_{h}(k)=U_{p}^{*}(-k)$. We then transform the BdG Hamiltonian in Eq. (2.59) using the block diagonal matrix

$$
U(k)=\left[\begin{array}{cc}
U_{p}(k) & 0 \\
0 & U_{h}(k)
\end{array}\right]=\left[\begin{array}{cc}
U_{p}(k) & 0 \\
0 & U_{p}^{*}(-k)
\end{array}\right] .
$$

This results in the transformed BdG Hamiltonian,

$$
\widetilde{H}_{B d G}^{\prime}(k)=\left[\left(\frac{\hbar^{2} k^{2}}{2 m^{*}}-\mu\right) \sigma_{o}-\sigma_{z} \sqrt{\Gamma^{2}+\alpha^{2} k^{2}}\right] \tau_{z}+\widetilde{\Delta}_{i}^{\prime} \sigma_{z} \tau_{x}+\widetilde{\Delta}_{c}^{\prime} \sigma_{y} \tau_{y},
$$

where the intra-band and inter-band pairing are given by

$$
\begin{aligned}
& \widetilde{\Delta}_{i}^{\prime}=\Delta_{o} \frac{\alpha k}{\sqrt{\Gamma^{2}+\alpha^{2} k^{2}}}, \\
& \widetilde{\Delta}_{c}^{\prime}=\Delta_{o} \frac{\Gamma}{\sqrt{\Gamma^{2}+\alpha^{2} k^{2}}},
\end{aligned}
$$

respectively. Suppose $\Delta_{o} \ll \alpha k_{F} \ll \Gamma$, where $k_{F}$ is the Fermi wavenumber of the lower-energy particle band. The inter-subband pairing term involving $\widetilde{\Delta}_{c}^{\prime}$ can then safely be ignored. Projecting the transformed Hamiltonian (2.65) onto the lower-energy particle and corresponding hole bands we arrive at the effective BdG Hamiltonian,

$$
\widetilde{H}_{B d G}^{\text {eff }}(k)=\left(\frac{\hbar^{2} k^{2}}{2 m^{*}}-\mu^{\prime}\right) \tau_{z}+\left(\frac{\alpha \Delta_{o}}{\Gamma}\right) k \tau_{x}
$$

where $\mu^{\prime}=\mu+\Gamma$. Remarkably, we see that the superconductive pairing of the effective model has p-wave symmetry. In fact, the effective model directly maps onto the Kitaev chain in the continuum limit. Additionally, if $|\Gamma|>|\mu|$, the higher-energy particle band is not occupied, and the system only has one Fermi surface. In essence, the system has become effectively spinless like the Kitaev chain model. We also have $\mu^{\prime}>0$, which implies that the effective system realizes the topological phase of the Kitaev chain and will host MZMs in a system with open boundary conditions.

Understanding how the Rashba-Majorana model is mapped onto the Kitaev model can also be understood graphically. To see this, first note that 
the spectrum of the normal state Hamiltonian, i.e. the spectrum of the two particle-bands in the absence of superconductivity, is given by

$$
E_{ \pm}(k)=\left(\frac{\hbar^{2} k^{2}}{2 m^{*}}-\mu\right) \pm \sqrt{\Gamma^{2}+\alpha^{2} k^{2}}
$$

This spectrum is plotted in Fig. 2.4 with more ingredients being included from left to right. Fig. 2.4(a) shows the spectrum of the system with neither Zeeman splitting nor spin-orbit coupling, i.e. $\Gamma=\alpha=0$. The two bands are degenerate for all values of $k$. Note that the Fermi level is indicated by the dash horizontal line, which is taken to be at zero energy. Also note that $\mu>0$ since the band-edge sinks below the Fermi level. Fig. 2.4(b) shows the spectrum of the system with Zeeman splitting but still without spin-orbit coupling, i.e. $\Gamma \neq 0$ and $\alpha=0$. The Zeeman splitting lifts the degeneracy, and a separation of $2 \Gamma$ develops between the two bands. At this stage the bands are perfectly polarized along the z-axis with the lower and higher energy bands being spin up and down, respectively. Note that the higher energy band rising above the Fermi level indicates that $|\Gamma|>\mu$. Similar to the Kitaev model, the system now is effectively spinless, i.e. the spin degree of freedom has been frozen out, and only one band has a Fermi surface. In contrast to the Kitaev model, however, no superconductive pairing can occur between the states at $k$ and $-k$ of the occupied band since they have the same spin. ${ }^{8}$ Finally, Fig. 2.4(c) shows the spectrum of the system with both Zeeman splitting and spin-orbit coupling, i.e. $\Gamma \neq 0$ and $\alpha \neq 0$. Importantly, the spin-orbit coupling has caused the spin polarization axes at $k$ and $-k$ to slightly cant away from the z-axis and also in opposite directions. This occurs because the spin-orbit coupling is essentially a momentum dependent magnetic field, $H_{S O}=\alpha k \sigma_{y}$. Now superconductive pairing can occur between the states at $k$ and $-k$ of the occupied band (red arrows in Fig. 2.4(c)) since they have a component of their spins that point in opposite directions. Moreover, since the magnitude of the spin-orbit coupling grows as the magnitude of momentum $|k|$ grows, the canting of the spins toward the y-axis also increases with increasing $|k|$. This leads to an increased intra-band, superconductive pairing $\widetilde{\Delta}_{i}^{\prime}$ with increasing $|k|$, just as we find in Eq. (2.66). Adding a small pairing $\Delta_{o}$ to the system then realizes an (effectively) spinless p-wave superconductor that maps onto the Kitaev model as was found in Eq. (2.68).

While the small $\Delta_{o}$ regime maps directly onto the Kitaev chain for large $\Gamma$, the existence of MZMs does not depend upon this limit. Indeed, MZMs exist due to the topological properties of the system, which should not depend upon perturbation arguments. Rather, the MZMs should continue to exist if the bulk gap does not close. Starting from the topological phase of the small $\Delta_{o}$ limit, $|\Gamma|>|\mu|$, we can continuously increase $\Delta_{o}$ until the bulk gap closes

\footnotetext{
${ }^{8}$ Recall from Sec. 2.1 that s-wave superconductive pairing occurs between electrons with opposite momentum and spin, i.e. an electron with momentum $k$ and spin $\uparrow$ can pair with an electron with momentum $-k$ and spin $\downarrow$.
} 

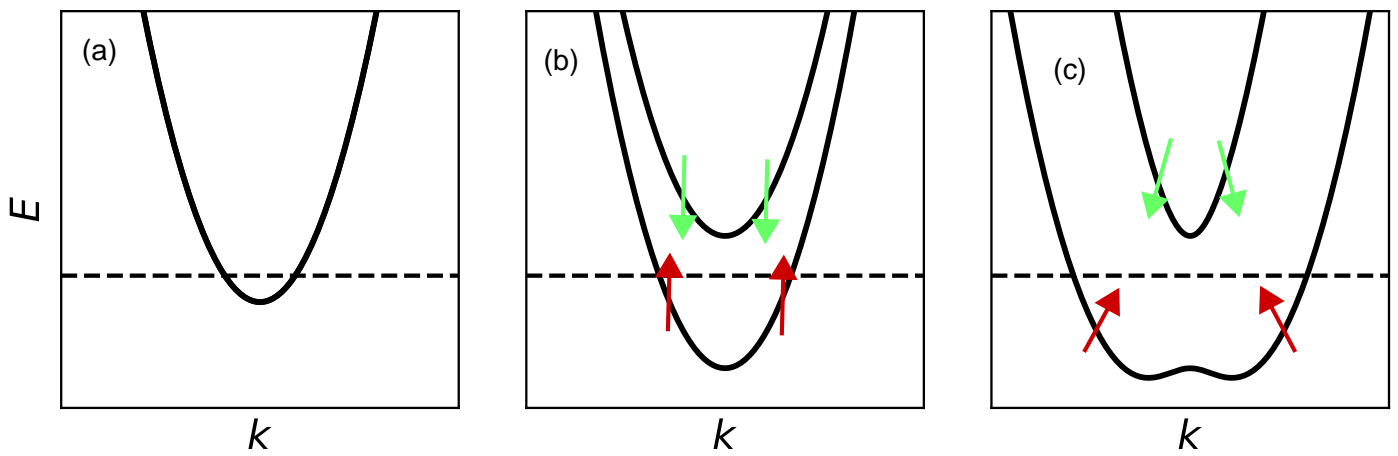

FIGURE 2.4: Normal spectrum of the Rashba Majorana model, i.e. only the particle spectrum before the addition of superconductivity. Horizontal line is the Fermi level, which is assume to be at $\mathrm{E}=0$. (a) Spectrum before either Zeeman splitting and spin-orbit coupling is included. Spectrum is degenerate everywhere. (b) Spectrum with Zeeman splitting, $\Gamma \neq 0$, but still without spin-orbit coupling. The bands are perfectly spin polarized along the z-axis as indicated by red and green arrows. Spectrum with both Zeeman splitting, $\Gamma \neq 0$, and spin-orbit coupling, $\alpha \neq 0$. Spin polarization axis is no long perfectly along the z-axis and is $k$ dependent.

at

$$
\Delta_{o, \text { crit }}=\sqrt{\Gamma^{2}-\mu^{2}} .
$$

Since the topological phase transition only occurs with the closing of the bulk gap, this implies that system is in the topological phase whenever,

$$
|\Gamma|>\sqrt{\mu^{2}+\Delta_{o}^{2}}
$$

This is numerically verified in Fig. 2.5. The spectrum of a $2 \mu \mathrm{m}$ wire is shown in Fig. 2.5 (a) as a function of Zeeman energy $\Gamma$. Other system parameters are given by $m^{*}=0.03 m_{0}$, where $m_{0}$ is the bare electron mass, $\alpha=20 \mathrm{meV} \cdot \mathrm{nm}$, $\Delta_{o}=1 \mathrm{meV}$, and $\mu=1 \mathrm{meV}$. For zero Zeeman energy, $\Gamma=0$, the spectrum is gapped with no states with energies $|E|<\Delta_{o}$. With increasing $\Gamma$, the gap decreases until it finally closes at $\Gamma=\sqrt{\mu^{2}+\Delta_{o}^{2}}=\sqrt{2} \mathrm{meV} \approx 1.4 \mathrm{meV}$ as predicted by Eq. (2.71). The bulk gap then reopens with increasing $\Gamma$ and remains open for the rest of the shown range of $\Gamma$. Additionally, within the gap there are two (nearly) zero-energy BdG states, which are linear combinations of two MZMs. These MZMs are shown in Fig 2.5 (b) for $\Gamma=2 \mathrm{meV}$. The MZMs are heavily localized on the two edges of the system and exponentially decay into the bulk. This is to be expected since topological edge modes occur at the interfaces between regions of differing topological phases [21]. In this case, the two regions are the topological nanowire and the trivial vacuum. For comparison, the Majorana basis states making up the second lowest positive-energy state are shown in Fig. 2.5 (c). In contrast to the localized MZMs in Fig. 2.5 (b), the state in Fig. 2.5 (c) is delocalized across 

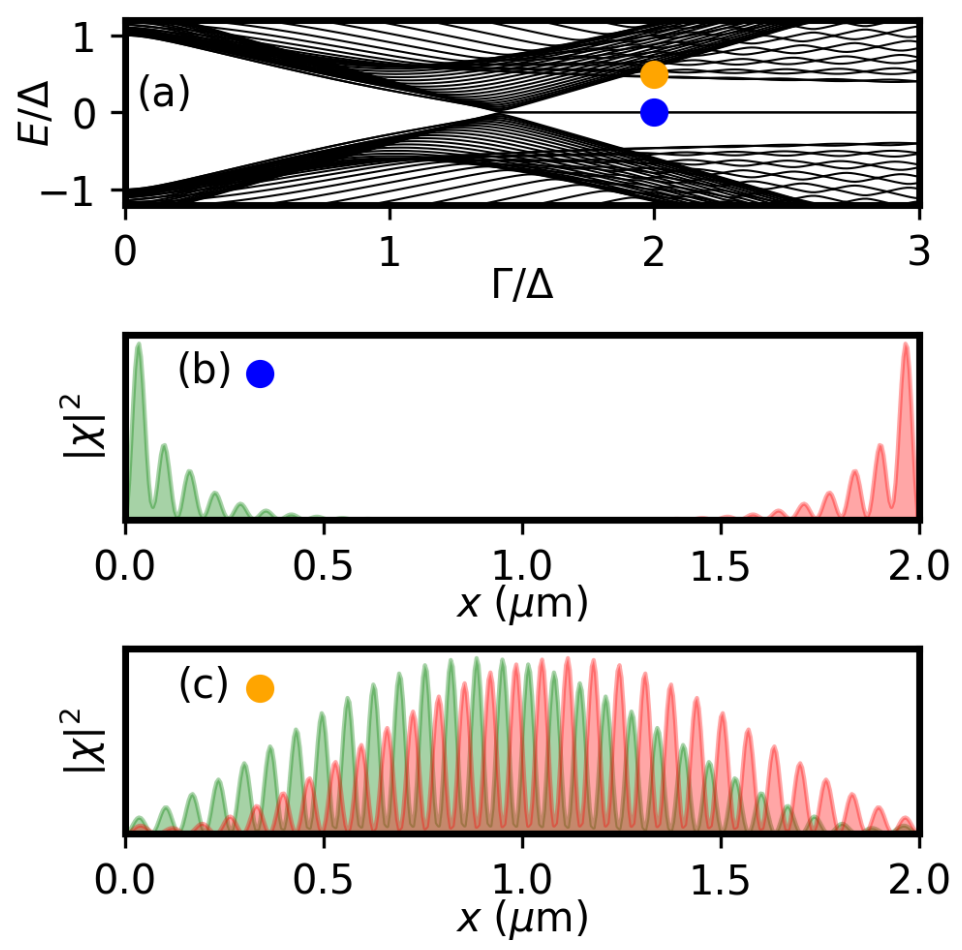

FIGURE 2.5: (a) Spectrum of a Rashba Majorana nanowire as a function of Zeeman energy $\Gamma$. System parameters are $L=2 \mu \mathrm{m}$, $m^{*}=0.03, \alpha=20 \mathrm{meV} \cdot \mathrm{nm}, \Delta=1 \mathrm{meV}$, and $\mu=1 \mathrm{meV}$. The system undergoes a topological phase transition with the closing of the bulk gap at $\Gamma_{\text {crit }}=\sqrt{\mu^{2}+\Delta^{2}}=\sqrt{2} \mathrm{meV} \approx 1.4 \mathrm{meV}$. Majorana zero modes exist at (nearly) zero-energy for $\Gamma>\Gamma_{\text {crit }}$. The Majorana zero modes making up the two nearly zeroenergy BdG states for $\Gamma=2 \mathrm{meV}$ (blue dot) are shown in (b), while the Majorana modes that make up the next lowest positive energy BdG state (orange dot) are shown in (c). Notice that the Majorana modes in (c) are highly overlapping in contrast to the Majorana zero modes in (b).

the entire wire, i.e. it is a bulk state. Additionally, the two Majorana basis states making up the state are highly overlapping in contrast to the topological MZMs in Fig. 2.5 (b).

\subsection{Non-Abelian Statistics of Majorana Zero Modes}

Having discussed the elementary mathematics behind Bogoliubov-de Genne quasiparticles and Majorana operators and shown how MZMs can emerge in the Kitaev and Rashba Majorana models, we now illustrate the non-Abelian statistics of MZMs. Recall from Sec. 1.2.4, that the non-Abelian statistics of MZM allow for the implementation of quantum gates within topological quantum computation. The non-Abelian statistics of MZMs are easiest to illustrate in the context of MZMs localized at the vortex cores of $2 \mathrm{D}$ p-wave 


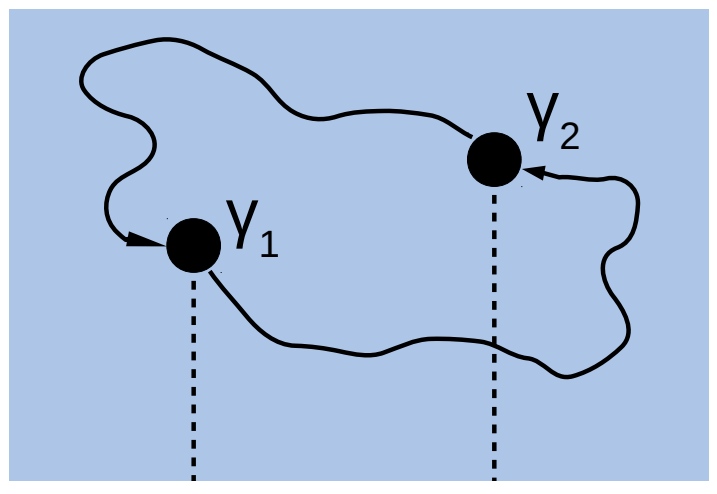

FIGURE 2.6: Two Majorana Zero Modes, $\gamma_{1}$ and $\gamma_{2}$ are exchanged following the paths of the solid black lines. Dotted lines represent the branch cuts where all fermion operators pick up a minus sign when crossed. Upon exchange, the Majorana operations transform according to $\gamma_{1} \rightarrow-\gamma_{2}$ and $\gamma_{2} \rightarrow \gamma_{1}$.

superconductors [104]. To begin, let us consider a system with two MZMs, ${ }^{9}$ which correspond to the Majorana operators $\gamma_{1}$ and $\gamma_{2}$. Recall from Sec. 2.1.2 that a regular BdG fermion can be formed from a linear combination of the Majorana operators, which is given by

$$
d=\frac{1}{2}\left(\gamma_{1}+i \gamma_{2}\right)
$$

Since the Majorana operators, $\gamma_{1}$ and $\gamma_{2}$, are MZMs, the system has 2 -fold degenerate ground state subspace spanned by the states $|0\rangle$ and $|1\rangle=d^{\dagger}|0\rangle$, where $|0\rangle$ is the BdG vacuum containing no BdG quasiparticles. What happens if the two MZMs are now exchanged? First note that the superconductive pairing has a $2 \pi$ phase winding around the center of each vortex, i.e. $\Delta(\mathbf{r})=h(r) e^{i \theta}$ in the vicinity of a vortex core, where $r$ is the radial distance from the vortex, $\theta$ is the polar angle, and $h(0)=0$. This superconducting phase can be gauged away by redefining the electron creation and annihilation operators as

$$
\begin{aligned}
\tilde{c}_{i}^{\dagger} & =c_{i}^{\dagger} e^{-i \theta_{i} / 2}, \\
\tilde{c}_{i} & =c_{i} e^{i \theta_{i} / 2},
\end{aligned}
$$

where $\theta_{i}$ is the polar angle of position $i$. Note that due to the factor of $1 / 2$ in the phase and the ambiguity of $\theta_{i}$, there exists branch cuts emanating from the vortex cores where the electron operators must discontinuously flip sign, as shown in Fig. 2.6. The MZMs are exchanged using the paths shown in solid black lines. At some point in the exchange, the first MZM crosses the branch cut emanating from the the second MZM. It therefore picks up a minus sign. In contrast, the second MZM crosses no such branch cut. Upon

\footnotetext{
${ }^{9}$ Technically, the objects that will be exchanged are vortices carrying Majorana zero modes, but we will simply refer to them as MZMs [7]
} 
exchange, the Majorana operators therefore transform as

$$
\gamma_{1} \rightarrow-\gamma_{2}, \quad \gamma_{2} \rightarrow \gamma_{1} .
$$

The unitary operator that implements such a transformation, i.e. $U \gamma_{1} U^{\dagger}=$ $-\gamma_{2}$ and $U \gamma_{2} U^{+}=\gamma_{1}$, is given by [7]

$$
U=\frac{1}{\sqrt{2}}\left(1+\gamma_{1} \gamma_{2}\right) .
$$

Using the identity, $\gamma_{1} \gamma_{2}=i\left(1-2 d^{\dagger} d\right)=i(1-2 \hat{n})$, the operator $U$ is rewritten as

$$
U=\frac{1}{\sqrt{2}}(1+i-2 i \hat{n})
$$

and therefore acts on the two ground states as

$$
\begin{aligned}
& U|0\rangle=e^{i \frac{\pi}{4}}|0\rangle, \\
& U|1\rangle=e^{-i \frac{\pi}{4}}|1\rangle .
\end{aligned}
$$

From this example, we see that a system composed of only two MZMs is somewhat uninteresting, since only an occupation-dependent phase is acquired through the exchange. This may have been guessed at the outset, since the mean-field BCS Hamiltonian in Eq. (2.9) conserves fermion parity. Indeed, a series of MZM exchanges in a generic system of $2 \mathrm{~N}$ MZMs must always conserve fermion parity.

To illustrate the non-Abelian statistics of MZMs, a system with at least four MZMs is needed. From four MZMs, two regular fermions can be created. Any pairing can be chosen, but let us define the two fermions by

$$
d_{1}=\frac{1}{2}\left(\gamma_{1}+i \gamma_{2}\right), \quad \quad d_{2}=\frac{1}{2}\left(\gamma_{3}+i \gamma_{4}\right) .
$$

The 4-fold ground state subspace is then given by the states $\left|n_{1} n_{2}\right\rangle$ with $n_{i} \in\{0,1\}$. Suppose the second and third MZM are exchanged. Clearly from the previous example, this corresponds to the unitary transformation, $U_{23}=(1 / \sqrt{2})\left(1+\gamma_{2} \gamma_{3}\right)$. This transformation can be rewritten in terms of the regular fermion creation and annihilation operators, yielding

$$
U_{23}=\frac{1}{\sqrt{2}}\left[1+i\left(f_{1}^{\dagger} f_{2}^{\dagger}-f_{1} f_{2}^{\dagger}+f_{1}^{\dagger} f_{2}-f_{1} f_{2}\right)\right] .
$$

Finally, applying this to a ground state basis state yields

$$
U_{23}\left|n_{1} n_{2}\right\rangle=\frac{1}{\sqrt{2}}\left(\left|n_{1} n_{2}\right\rangle+i\left|1-n_{1}, 1-n_{2}\right\rangle\right) .
$$

This clearly accomplishes a rotation within the degenerate ground state subspace, and therefore proves that MZMs posses non-Abelian exchange statistics. In this manner, quantum gates can then be implemented. 


\section{Motivations}

In chapter 2, I discussed how it is possible to effectively realize the 1D Kitaev chain using a SM-SC nanostructure with s-wave superconductivity, spin-orbit coupling, and Zeeman splitting from either an applied magnetic field or coupling the SM-SC system to a magnetic insulator. What then motivates us to go beyond the minimal modelling of the last chapter? More specifically, what need is there to perform simulations of the 3D hybrid nanowire in which the details of the electrostatic environment are included? To provide such motivations, I discuss in this short chapter how the electrostatics affect the various aspects of the physics to provide such motivations.

Let us being with how the electrostatics impacts the superconductivity proximity effect. In the minimal Rashba model of chapter 2 and Refs. [8, 9, 44], it was simply assumed that the system had an induced superconductive pairing $\Delta_{o}$. In order for a subband in the hybrid system to acquire a superconducting gap, however, the wavefunction of the states making up the subband need to extend into the SC, with the size of the induced pairing being proportional to its spectral weight in the SC. The SC, after all, is where superconducting correlations exist, while the SM does not provide such correlations, i.e. $\Delta_{o} \neq 0$ and $\Delta_{o}=0$ in the SC and SM, respectively. What then controls how much of a state resides in the SC? Clearly, a dominate factor will be the electrostatic potential landscape within the SM, where the spectral weight in the SC of states will tend to increase if an electric field "pushes" ${ }^{1}$ the states in the SM toward the SM-SC interface.

Spin-orbit coupling, being a relativistic effect caused by a lack of inversion symmetry of the electrostatic potential, will also be affected by the electrostatic environment. The asymmetry has two sources. The first occurs at the atomic level due to an asymmetry between the anions and cations making up the SM. This is known as Dresselhaus spin-orbit coupling [103] and is intrinsic to the SM material and its lattice structure. Obviously, this spin-orbit coupling is uncontrollable except for the choice of SM and any influence the alterable growth conditions may have on what lattice structure is realized. The second asymmetry is known as structural asymmetry, where an average electric field exists over a length scale (tens to hundreds of $\mathrm{nm}$ 's) much larger than the atomic scale. This gives rise to so-called Rashba spin-orbit coupling [105]. This type of spin-orbit coupling is controllable to some extend because it possible to apply and change electric fields by gating the system. All

\footnotetext{
${ }^{1}$ i.e. the potential energy of the SM basis states residing near the SM-SC interface is lower than those away from the SM-SC interface.
} 
else being equal, it is desirable to maximize the component of the spin-orbit vector perpendicular to the Zeeman field, which increases the topological gap protecting the topological phase and resulting MZMs from disorder and other non-uniformities and decreases the localization length $\xi$ of the MZMs. To a first approximation, this is accomplished in a subband deriving from the conduction band of the SM by simply maximizing the average electric field weighted by the spectral weight of the state's wavefuction. Other effects, such as SM-SC interface effects, can also play a role. For schemes in which holes (absence of an electron) are responsible for the topological, the physics of spin-orbit coupling is more complicated due to a degeneracy of the light and heavy hole bands at the edge of the SM valence band [46]. At the same time, however, the spin-orbit coupling of SM valence band holes is typically larger than SM conduction band electrons.

The final of the three main ingredients, namely Zeeman splitting, will also be affected by the electrostatic environment. In the case where the Zeeman splitting is coming from a magnetic insulator coupled to the SM-SC system, the reasoning is similar to what was discussed for the superconducting proximity effect; The size of the Zeeman splitting will depend crucially on how the electrostatic potential landscape pushes states toward or away from the magnetic insulator. ${ }^{2}$ In the case where an external magnetic field supplies the Zeeman splitting, confinement effects from the electrostatic environment are know to affect the g-factor [46]. Additionally, including orbitals effects of the magnetic field is only possible through the magnetic vector potential in a $2 \mathrm{D}$ or 3D Hamiltonian. These effects can have both negative [106] and positive [107] consequences with respect to achieving the topological phase. For example, states with a non-vanishing expectation value of angular momentum along the length of the nanowire will have enhanced g-factor [107]. An evaluation of such negative and positive consequences, however, requires accurate knowledge of the wavefunction profiles in the nanowire, which can only be found by including the various details of the electrostatic environment.

Besides its impact on the three basis ingredients needed for engineering a topological superconductor and MZMs, the electrostatic environment plays the dominant role in creation of topologically trivial Andreev bound states (ABSs). As discussed in chapter 1, the formation of trivial ABSs through various mechanisms is playing a central theme in current research on MZMs in SM-SC nanostructures. Additionally, all such mechanisms are intimately related to the electrostatic environment of the hybrid device. The electrostatics role in ABS formation is therefore arguably the most important issue that needs to be addressed by more realistic models. As an example, soft confinement at an edge of the proximitized region can lead to near zero-energy partially-separated ABS [54, 67, 108], also known as quasi-Majoranas [94], which can even give rise to quantized conductance [68]. It is impossible to estimate, a priori, the likelihood of the formation of these types of states unless information is known about the degree of softness (the strength of the electric field) of the effective potential near the edge of the proximitized region. To

\footnotetext{
${ }^{2}$ We study this effect in Sec. 4.7.
} 
gain such information, a model of the electrostatic potential is clearly necessary. As another example, it is known that random Hamiltonians in class D, which is the symmetry class to which the SM-SC systems typically belong, give rise to (trivial) zero-energy peaks in the density of states [55]. Effective randomness can arise in the Hamiltonian due to disorder in the system. This disorder not only will give rise to disordered effective potentials for the subband from the MZMs may arise, but also will produce inter-subband coupling (see Sec 5.2 of chapter 5), further increasing the randomness of the Hamiltonian. To quantify the importance of inter-subband coupling it is necessary to have knowledge of the subband structure of the nanowire, which subsequently requires the incorporation of the electrostatic details (see Sec 4.6 in chapter 4 ). In addition, estimation of the disorder strength from various sources also requires detailed models incorporating electrostatic details. For example, to quantify the strength of disorder coming from charge impurities inside the SM it is necessary to solve a self-consistent SchrödingerPoisson problem including the screening from the gates, superconductor, and redistribution of free charge surrounding the charge impurity (see Sec. 5.1 of chapter 5). Note that these issues relating to the formation of trivial ABSs plays a central role in the current debate over whether MZMs have actually been observed in SM-SC nanostructures. Only by the fruitful interplay of experiment, theory, and numerical modeling can we hope to see a resolution of this controversy. Indeed, our detailed study on charge impurity disorder presented in Sec. 5.1 paints a somewhat dire picture regarding the chances that MZMs have been observed. On the hand, it provides material scientists with the clear goal of reducing the impurity density below $\sim 10^{15} \mathrm{~cm}^{-3}$.

Finally, in addition to its use in understanding the negative effects of disorder and other non-uniformities within the device, knowledge of the electrostatic environment can also be leveraged to devise new and improved designs of SM-SC nanostructures for engineering MZMs. For example, introducing periodic potentials for Majorana nanostructures can provide increased spin-orbit coupling and robustness against disorder. We present two such designs in planar SM-SC nanostructures in chapter 6. 


\section{Toward realistic modeling of Majorana nanowires}

In this chapter, the journey toward realistic modeling of Majorana nanostructures begins. In particular, capturing the various aspects of the electrostatics environment is a key goal. To do so, numerical methods to find selfconsistent solutions to the Schrödinger-Poisson equation are devised. The numerical methods are then applied to two Majorana nanowire systems as examples of their utility.

There are many questions to consider when creating a model. What type of models should be used for the semiconductor (SM) and superconductor (SC)? How should the coupling between the SM and SC be incorporated? At what level should the electron-electron interactions be treated at? What imperfections within the device are to be included? The answers to these various questions depends of course upon the specific research question that is being studied. It depends on how realistic of a model is necessary and what aspect of the device needs a detailed description.

It is typically easiest to make progress by first creating simple models and then adding additional details as needed. In this spirit, the problem of simulating a "general" Majorana nanowire is not considered in this chapter. Rather, the systems are limited to wires of infinite length with translation invariance. Within these systems, the Schrödinger-Poisson equations are solved to characterize the electrostatic environment and its effects on the electronic states of the system. Note that this includes the effects of any metallic gates, band-bending at the SM-SC interface, and electron-electron interactions at the mean-field. Note that the constraint restricting us to systems with translation invariance excludes disorder and other non-uniformities along the length of the device. These complications, however, will be addressed in chapter 5. In addition, incorporating the SC explicitly into the Hamiltonian of the device when self-consistently solving for the electrostatic potential turns out to significantly increase the computational complexity of the problem. We therefore assume weak-coupling between the SM and SC when solving for the electrostatic potential self-consistently, which allows us to exclude the SC from the Hamiltonian of the system. ${ }^{1}$ This does not imply, however, that the presence of the superconductor is not important in this limit. It still plays a

\footnotetext{
${ }^{1}$ The SC is explicitly included, however, in the Hamiltonian after the electrostatic potential has been determined in the results presented in Sec. 4.7. Additionally, at the time of this writing, we are currently working on incorporating the SC explicitly in the Hamiltonian
} 
huge role in determining the electrostatic potential within the semiconductor through band-bending at the SM-SC interface.

As discussed in chapters 1 and 3, disorder and device non-uniformities are currently a central obstacle to overcome in order to consistently and controllably produce topological superconductivity and Majorana zero modes (MZMs) in SM-SC nanostructures. Indeed, much of the motivation for performing more realistic modeling of SM-SC Majorana nanostructures is to study disorder and other non-uniformities beyond what is possible in the minimal models of Sec. 2.3. What relevance then does modeling Majorana nanowires in the clean limit with neither disorder nor other non-uniformities to real-life experiments?

There are two main ways in which the clean limit is relevant. Firstly, several aspects of the device can be addressed in the clean that are not possible using the minimal model of Sec. 2.3. For example, theoretical evaluation of the spin-orbit coupling strength is only possible using information about the electrostatic potential [46]. ${ }^{2}$ The subband structure and occupation can also be studied in this limit. Indeed, these type of results are presented in Sec. 4.6. As is shown in chapter 5 , knowledge of the subband occupation and energy separation between subbands is important to understand the robustness of topological superconductivity and MZMs against disorder. As a final example, proximity effects can be studied within this clean limit. In fact, the effects of the electrostatic environment on the magnetic proximity effect in a SM-SC-magnetic insulator Majorana nanostructure is studied in Sec. 4.7. The second way that the clean limit is relevant to realistic systems is that it serves as a starting step in building low-energy effective theories for systems involving further complications. For example, this is the case in the study of charge impurity disorder in Sec. 5.1 of the next chapter. In principle, a brute force solution to the 3D-Schrödinger-Poisson equations could be sought by a straight-forward extension of the model described in Sec. 4.1 to non-translation invariant systems. In practice, however, the brute force approach to the problem quickly becomes numerically intractable due to an explosion of the number of degrees of freedom. The problem becomes numerically tractable, however, by using the solution of the clean system found through the methods of this chapter as an intermediate step to build an effective low-energy theory. A second example of building an effective lowenergy theory by exploiting the methods of this chapter is presented in Sec. 5.2 of the next chapter. There the system has broken translation symmetry due to multiple back gates along the length of the wire.

The rest of this chapter is organized as follows. In section 4.1, we provide the framework of the Schrödinger-Poisson equations for systems with translation invariance and weak SM-SC coupling. The numerical techniques used

when solving for the self-consistent potential. See Sec. 4.5 and chapter 7 for more discussion relating to this work.

${ }^{2}$ While the results are not included in this thesis, our work in Ref. [109] evaluates the spinorbit coupling of a SM-SC Majorana nanostructure in which the SC fully encircles the SM nanowire and the electrostatic potential is self-consistently calculated with a SchrödingerPoisson formalism very similar to Sec. 4.1. 


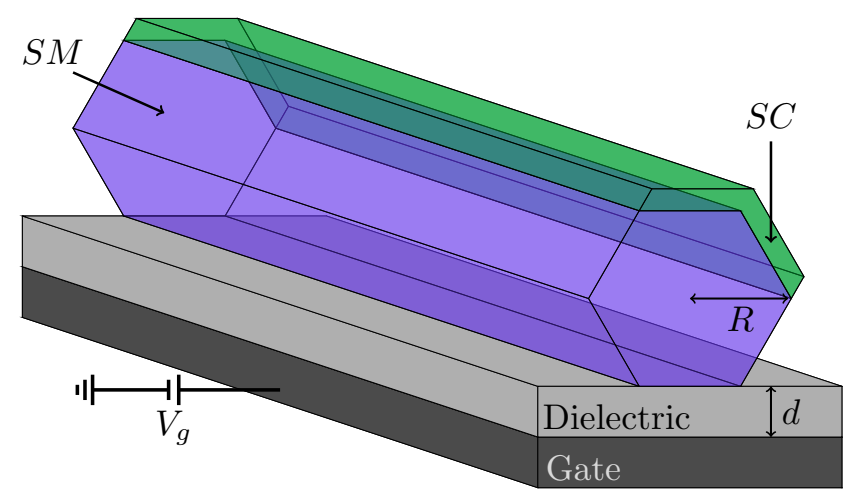

FIGURE 4.1: Schematic representation of the SM-SC hybrid device. A semiconductor nanowire (purple) of radius $R$ is proximity coupled to a thin superconductor (green). The band edges of the low-energy SM subbands can be tuned near the Fermi level using a back gate (black) separated from the wire by a thin dielectric layer (gray).

for the individual steps in solving the Schrödinger-Poisson equations are presented in Secs. 4.2 - 4.4. As discussed above, the solution to the translation invariance problem serves as the starting point in constructing effective theories in the next chapter. Therefore, an effort is made to be rather thorough in presenting the numerical methods. In Sec. 4.5, issues regarding the inclusion of the SC into the device are discussed. An example application of the numerical solution to the Schrödinger-Poisson equations is presented in Sec. 4.6. There the subband structure of a SM-SC device with a prototypical geometry used in current SM-SC Majorana nanowire experiments is studied. The effects of electrostatic control parameters, device geometry, and surface charge density are discussed. Finally, in Sec. 4.7, the Schrödinger-Poisson formalism is apply to study the magnetic proximity effect in a SM-SC-magnetic insulator Majorana nanowire. The electrostatics of the device are shown to play a dominate role in determining the magnitude of the magnetic proximity effect.

\subsection{Formalism}

Consider the system shown in Fig. 4.1, which is meant to represent the paradigmatic example of a SM-SC Majorana nanowire system. For simplicity, the system is taken to be infinite along the length of the wire. The hybrid system is composed of a semiconducting nanowire of radius $\mathrm{R}$ (purple), a thin superconducting layer (green) covering two facets of the nanowire, a metallic bottom gate (dark grey), and a dielectric layer (light grey) separating the bottom gate from the nanowire. Each of these components plays differing roles to bring about topological superconductivity and MZMs. The SM nanowire provides quasi-1D subbands with strong spin-orbit coupling and large Zeeman splitting with the addition of an external magnetic field. The requirements for strong spin-orbit coupling and large Zeeman field make 
semiconductors with small band gaps, such as InAs and InSb, ideal candidate materials. The SC provides the s-wave superconductivity through the superconducting proximity effect. Essentially, the states in the semiconductor hybridize with states in the superconductor. This results in some of the spectral weight of the quasi-1D subbands residing in the superconducting layer, which produces an induced superconducting gap in the subbands of the SM. Together, the SM and SC provide the three necessary ingredients to bring about topological superconductivity. ${ }^{3}$ Recall from Sec. 2.3, however, that one of the band-edges of an SM subbands must be tuned close to the Fermi level. Being a small energy window on the sub-meV scale, it is unlikely that a subband band-edge will just so happen to fall at the Fermi level. Applying voltage on the bottom gate, however, allows the subbands to be moved to some extent up and down in energy. The idea is that for some voltage range(s), a subband band-edge will reside near the Fermi level and topological superconductivity will be achieved for a strong enough Zeeman splitting. Lastly, the dielectric simply insulates the SM nanowire against contact with the backgate. Besides the fundamental roles just laid out, there also exists an interplay between any two components. For example, the gate affects the induced superconductivity in the SM subbands by "pushing" or "pulling" the states towards or away from the SM-SC interface depending upon the applied voltage.

How should such a system be modeled? More specifically, what details need to be included in a model to understand the electrostatic environment of the hybrid device? To begin, it is useful to consider the different energy scales that are involved in the problem. One energy scale is associated with the superconductivity, spin-orbit coupling, and Zeeman splitting. These are all characterized by an energy scale in the $\lesssim 1 \mathrm{meV}$ regime. Note that this energy scale is associated with the Majorana physics of the device, i.e. this energy scales dominates the physics of the Majorana Rashba model in Sec. 2.3. Another energy scale is associated with the electrostatic potential fluctuations across the transverse profiles of the SM nanowire. This energy scale is typically tens to hundreds of $\mathrm{meV}$ and is determined by the back gate and the details of the band bending at the SM-SC interface. Finally, the typical energy separation between the bottom band-edges of the occupied SM subbands and the Fermi level represents another energy scale, which in turn determines the free charge density in the SM nanowire. For all cases except systems with very low subband occupation, this energy scale is tens of meV.

With these energy scales in mind, we can begin to reason about what to include and exclude in the model. Let us start with how to model the SM. Consider the electronic band structure of a typical semiconductor with a direct band gap as shown in Fig. 4.2. The shown band structure consists of valence bands in the heavy and light hole bands along with the split-off band and a conduction band at higher energy. The conduction and valence band edge are separated by an energy gap $E_{g}$, which is typically a few hundred

\footnotetext{
${ }^{3}$ Recall from Sec. 2.3 that the three ingredients needed to produce topological superconductivity and MZMs in the Majorana Rashba model are spin-orbit coupling, Zeeman splitting, and s-wave superconductivity.
} 


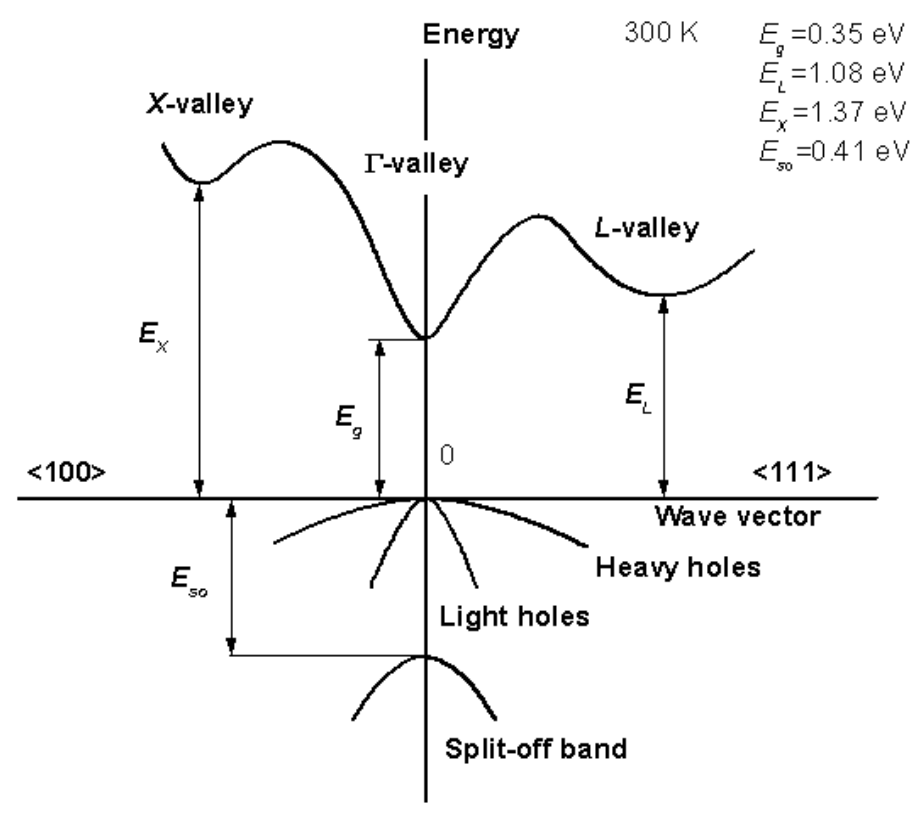

FIGURE 4.2

$\mathrm{meV}$, at the center $(\Gamma)$ point of momentum space. Away from the $\Gamma$ point, the conduction and valence bands are separated by a larger energy. In the bulk crystal, the chemical potential falls somewhere in the band gap. Therefore, the valence band is (nearly) full and the conduction band is (nearly) empty. What then happens when the SM is confined to a nanowire and incorporated into the Majorana nanostructure? Confinement-induced subbands form out of these bands, and the system will have many conduction (dominated) and valence (dominated) subbands. ${ }^{4}$ Additionally, some conduction subbands may become partially occupied or valence subbands may become partially depleted. Whether conduction electrons or valence band holes are the primary charge carrier in a Majorana device depends upon the details of the device, such as the sign of the band-bending at the SM-SC interface and gate voltages. Both cases are interesting, but let us consider the case of conduction subbands becoming occupied. This case is simpler and occurs for both Majorana devices using InAs and InSb as the SM. The low-energy physics of the Majorana device will be determined by the properties of the subbands near the Fermi level. As stated in the paragraph above, the occupied conduction subbands dip only a few tens of meV below the Fermi level. Therefore, these subbands derive primarily from the lowest energy sector of the bulk conduction band, namely the $\Gamma$-valley as shown in Fig. ??. Within this energy range of a few tens of $\mathrm{meV}$, the bulk conduction band is well approximated by a

\footnotetext{
${ }^{4}$ I use the word dominated to describe conduction dominated and valence dominated subbands because the subbands are technically a mixture of both bulk conduction and bulk valence band states due to coupling between the bulk bands upon confinement. It still makes sense to talk of a conduction dominated subband, however, because bulk valence bands contribute only a small amount. Similarly for valence dominated subbands.
} 
simple quadratic dispersion,

$$
E_{c}(\mathbf{k}) \approx \frac{\hbar^{2} \mathbf{k}^{2}}{2 m^{*}}+E_{c, o}
$$

where $m^{*}$ is the effective mass and $E_{c, o}$ is the conduction band energy at $\mathbf{k}=0$. The effective mass is much smaller than the bare electron mass. For example, $m_{\mathrm{InAs}}^{*}=0.026 m_{0}$ and $m_{\mathrm{InSb}}^{*}=0.015 m_{0}$ [46], where $m_{0}$ is the bare electron mass. The simplest model that will reproduce this spectrum in a uniform system is an effective mass Hamiltonian given by

$$
H_{o}=\frac{\hbar^{2}}{2 m^{*}}\left(\hat{k}_{x}^{2}+\hat{k}_{y}^{2}+\hat{k}_{z}^{2}\right)-e \phi(\mathbf{r})
$$

where the momentum has been promoted to an operator $k_{j}=-i \partial_{j}, \mathbf{r} \in \mathbb{R}^{3}$ is a position vector, and $\phi$ is the electrostatic potential. Note that for a uniform system with $\phi=-E_{c, o} / e$, the effective mass Hamiltonian (4.2) reproduces the desired spectrum in Eq. (4.1). Also note that this electrostatic potential does not include the potential fluctuations occurring on the atomic scale due to the crystal being made up of discrete nuclei. The effect of that atomic scale periodic potential is approximated for in the value of the effective mass $m^{*}$. Rather, $\phi$ accounts for potential fluctuations on a large length scale compared to the atomic scale.

What determines the electrostatic potential $\phi$ ? Electrostatic theory teaches us that the electrostatic potential is determined by the Poisson equation,

$$
\nabla \cdot(\varepsilon(\mathbf{r}) \nabla \phi(\mathbf{r}))=-\rho(\mathbf{r}),
$$

where $\varepsilon$ is the dielectric constant and $\rho$ is the charge density. Note that the Poisson equation is altered from its typical form in free space due to the piecewise dielectric function, which takes a different value within each material. The charge density $\rho$ in Eq. (4.3) comes from electrons occupying conduction subband states, which will be discussed further below. In addition to Eq. (4.3), we also need boundary conditions to determine $\phi$. From the perspective of the electrostatics, the SC is a metal. Assuming the SC is grounded, an electric field can be produced by applying a voltage on the bottom gate. This yields one boundary condition, i.e. $\phi(\mathbf{r})=V_{g}$ for $\mathbf{r}$ at the top surface of the bottom gate, where $V_{g}$ is the applied voltage. In principle, the electrostatic potential $\phi$ should then vanish at the surface of the grounded SC. Complex chemistry and physics occur at the SM-SC interface, however, with some charge exchange between the SM and SC. This, in turn, causes bandbending of the SM bands. No simple effective model can incorporate the subtle details of such a process. In substitution of this, the band-bending effect is included in the model by enforcing a boundary condition of $\phi(\mathbf{r})=V_{S C}$ for $\mathbf{r}$ at the SM-SC interface [110]. This value will vary depending upon the SM and SC material combinations. Typical values, however, are in the hundreds of meV [111]. In principle, the other boundary conditions could be chosen as the enforcing the potential to vanish at infinity, i.e. $\phi \rightarrow 0$ as $|\mathbf{r}| \rightarrow \infty$. 
This choice makes numerical simulations difficult, since the simulation region cannot extend to infinity. Instead, we force the electric field to vanish on all boundary of our simulation region. This will be specified in Sec. 4.2 when discussing the numerical solution method to the Poisson equation.

What about the spin-orbit coupling and Zeeman splitting that is suppose to arise from the SM? As mentioned above, the energy scale characterizing these ingredients is $\lesssim 1 \mathrm{meV}$, which is small compared to the energy scales associated with the ingredients in the effective mass Hamiltonian in Eq. (4.2). Therefore, these terms are not crucial when determining the electrostatic potential $\phi$ self-consistently. The opposite is certainly not the case, however, namely the electrostatic potential $\phi$ plays a crucial role in determining the size of the spin-orbit coupling and Zeeman splitting. The spin-orbit coupling and Zeeman splitting are intimately linked to the coupling between the conduction and valence band energy, the energy $E_{s o}$ separating the light and heavy hole band-edges from the split-off band-edge, and the electrostatic environment [46]. To accurately determine the sizes of the spin-orbit coupling and Zeeman energy, an 8-band $\mathbf{k} \cdot \mathbf{p}$ model that includes of the bulk bands in Fig. 4.2 should be used [112]. Expressions can be found, however, using Löwdin perturbation methods $[46,113,114]$ that integrate out the valence bands to approximate the spin-orbit coupling and Zeeman splitting from the effective mass model (4.2). The spin-orbit coupling Hamiltonian component takes the form [114],

$$
H_{S O}^{\prime}=\frac{J}{2}(\mathbf{E} \times \mathbf{k}-\mathbf{k} \times \mathbf{E}) \cdot \boldsymbol{\sigma},
$$

where $\mathbf{k}=-i \nabla$ is the momentum operator, $\mathbf{E}=-\nabla \phi$ is the electric field, $\sigma$ contains the Pauli spin matrices $\sigma_{i}$ as components, where $i=x, y, z$, and $J$ is a constant related to the energy gaps between the bands labeled in Fig. 4.2. Provided the nanowire has a sufficiently small radius, the terms proportional to $\hat{k}_{x}$ and $\hat{k}_{y}$ are negligible and only the $\hat{k}_{z}$ terms are kept. To simplify things further, if the electric field $\mathbf{E}$ is fairly constant and in the $x-y$ plane, the spinorbit can be approximated as

$$
H_{S O}=\alpha_{R} \hat{k}_{z}\left(\boldsymbol{\sigma} \cdot \mathbf{n}_{\perp}\right),
$$

where $\alpha_{R}$ is the Rashba spin-orbit coefficient, and $\mathbf{n}_{\perp}$ is a unit vector in the $x-y$ plane perpendicular to $\mathbf{E}$. It is worth noting that the spin-orbit coupling acts as a momentum-dependent magnetic field. The Zeeman splitting Hamiltonian component takes the simply form [46],

$$
H_{Z}=\Gamma \sigma_{z}
$$

where $\Gamma=(1 / 2) g^{*} \mu_{B} B$ is the Zeeman energy, $g^{*}$ is the effective g-factor of SM conduction band, $\mu_{B}$ is the Bohr magneton, $B$ is the applied magnetic field strength along the z-axis. This clearly breaks the spin-degeneracy between the two spin species for all values of the momentum $k_{z}$. The magnetic field also contributes via the orbital effect, which can have important effects 
$[106,107]$ The orbital effect is partially incorporated through the effective $g^{*}$ factor, but the momentum operator $\mathbf{k}$ should also be appropriately altered in all Hamiltonian terms to $\mathbf{k} \rightarrow-i \nabla+(e / h) \mathbf{A}$, where $\mathbf{A}$ is the magnetic vector potential, and $h$ is Planck's constant. In the discussion below about numerically solving the effective mass Hamiltonian, the simplified spin-orbit and Zeeman terms in Eqs. $(4.5,4.6)$ will be included and the orbital effect will be ignored. The full Hamiltonian for the SM is then given by

$$
H_{S M}=H_{o}+H_{S O}+H_{Z} .
$$

Let us now briefly consider the superconductor. For simplicity, it is assumed in this section that the SM-SC coupling is weak. In other words, the $\mathrm{SM}$ states are assumed to have a small spectral weight in the $\mathrm{SC}$ region. This allows us to not explicitly include the SC in the Hamiltonian when studying the electrostatic environment, which dramatically lowers the computational cost of calculating the eigenstates of the Hamiltonian and ultimately the charge density $\rho$. Note that this does not imply that the presence of the $\mathrm{SC}$ does not play an important role in our model. Indeed, the boundary condition $\phi(\mathbf{r})=V_{S C}$ enforced at the SM-SC interface dramatically effects the electrostatic potential and the electronic states of the system. Additionally, this is not to say that the SC is unimportant for Majorana physics. Clearly how the SM-SC couple is important to the proximity effect that provides the necessary induced superconductivity in the SM subbands to realize MZMs. Note however, that the energy scale associated with the superconductivity is $\lesssim 1 \mathrm{meV}$, which is far smaller than the energy scales associated with $H_{0}$ in Eq. (4.87). Therefore, it is well justified to treat and understand the superconducting proximity effect after the electrostatic potential is determined. Given the importance of understanding the SM-SC coupling beyond the weak-coupling limit, however, Sec. 4.5 is devoted to discussing the issues of incorporating it into the model. For now, however, Eq. (4.7) represents the full Hamiltonian of the system.

The electronic states of the system are determined by the Schrödinger equation. Explicitly, the $n^{\text {th }}$ electronic state $\psi_{n}$ of the system satisfies

$$
H_{S M} \psi_{n}(\mathbf{r})=E_{n} \psi_{n}(\mathbf{r}),
$$

where $E_{n}$ is the energy of the eigenstate. Note that $\psi_{n}$ has two components due to the electron spin, which we can denote as

$$
\psi_{n}(\mathbf{r})=\left(\begin{array}{l}
\psi_{n, \uparrow}(\mathbf{r}) \\
\psi_{n, \downarrow}(\mathbf{r})
\end{array}\right),
$$

where $\psi_{n, \uparrow}\left(\psi_{n, \downarrow}\right)$ is the spin up (down) component..$^{5}$ As mentioned earlier, the eigenstates and their respected energies determine the charge density $\rho$

\footnotetext{
${ }^{5}$ These spin components are what the Pauli spin matrices $\sigma_{j}$ act on. As an example, $\sigma_{x} \psi_{n}=\left(\begin{array}{ll}0 & 1 \\ 1 & 0\end{array}\right)\left(\begin{array}{l}\psi_{n, \uparrow} \\ \psi_{n, \downarrow}\end{array}\right)=\left(\begin{array}{l}\psi_{n, \downarrow} \\ \psi_{n, \uparrow}\end{array}\right)$, which has exchanged the spin components.
} 


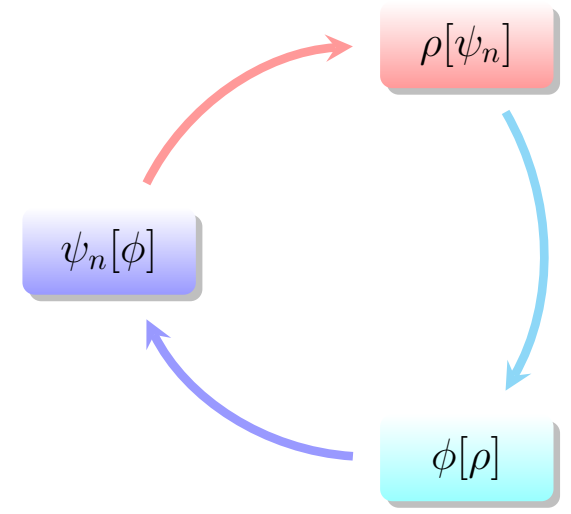

FIGURE 4.3: Functional relations between the charge density $\rho$, electrostatic potential $\phi$, and the eigenstates $\psi_{n}$ of the Hamiltonian. Quantities in brackets indicate functional dependence.

is the nanowire. This relation is given by

$$
\rho(\mathbf{r})=-e \sum_{n}\left|\psi_{n}(\mathbf{r})\right|^{2} F\left(E_{n}, T\right),
$$

where $F(E, T)=\left(1+\exp \left(E /\left(k_{B} T\right)\right)\right)^{-1}$ is the Fermi function, $k_{B}$ is the Boltzmann constant, and $T$ is the temperature. Note that we use the convention that $E=0$ is the Fermi level. Note that each spinor component of $\psi_{n}$ is summed over in Eq. (4.10).

Eqs. $(4.3,4.8,4.10)$ are collectively referred to as the Schrödinger-Poisson equations. As illustrated in Fig. 4.3, the electrostatic potential $\phi$, charge density $\rho$, and eigenstates $\psi_{n}$ have non-trivial, circular relationship among each other. The Schrödinger-Poisson equations therefore require a self-consistent solution where Eqs. $(4.3,4.8,4.10)$ are simultaneously satisfied. Note that with the exception of $\rho=0$ (no free charge in the system), the charge density $\rho$ and eigenstates $\psi_{n}$ have a non-linear relationship. Therefore, no generic analytic solution is to be found to find such a self-consistent solution. Rather, we must resort to iterative numerical methods in which we creep up to the solution starting from an initial guess for the self-consistent charge density, whether it be an educated guess or simply a shot in the dark.

A flowchart of the iterative method is shown in the Fig. 4.4. From the initial input for the charge density $\rho_{i n}=\rho_{0}$, the electrostatic potential $\phi$ is computed from the Poisson equation (4.3). Next the eigenstates of the Schrödinger equation are calculated (4.8) where the Hamiltonian contains the electrostatic potential from the previous step. ${ }^{6}$ Then the output charge density $\rho_{\text {out }}$ is calculated from these eigenstates using Eq. (4.10). The charge density, electrostatic potential, and eigenstates only represent a self-consistent

\footnotetext{
${ }^{6}$ In practice, not all of the eigenstates of the Schrödinger equation need to be computed since only the occupied eigenstates contribute to the charge density (see Eq. (4.10)). Rather it is adequate to find only the eigenstates $\psi_{n}$ with energies $E_{n} \lesssim 10 T$, where $T$ is the temperature used in the Fermi function.
} 


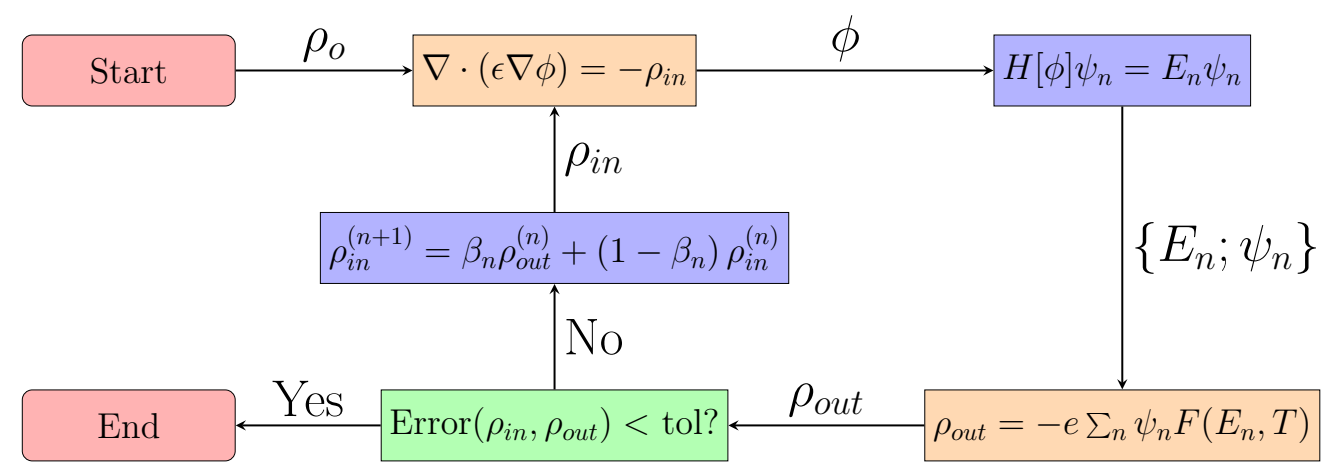

FIGURE 4.4: Flowchart of the iterative algorithm to find a selfconsistent solution to the Schrödinger-Poisson equations.

solution of the Schrödinger-Poisson equations if $\rho_{\text {in }}=\rho_{\text {out }}$. If it is found that $\rho_{\text {in }}=\rho_{\text {out }}$ within some chosen tolerance, the loop is terminated. The selfconsistent solution has been found. Otherwise, a new input charge density is generated and the loop is iterated again. The process continues until a self-consistent solution is found.

With each iteration of the self-consistent solution algorithm needing an input charge density $\rho_{i n}$, a method is needed to generate such functions. We choose to use a simply mixing method, which can be described as follows: Let $\rho_{i n}^{(n)}$ and $\rho_{\text {out }}^{(n)}$ be the input and output charge density of the $n^{\text {th }}$ iteration of the loop in the flowchart in Fig. 4.4. The input charge density of the next iteration is then given by

$$
\rho_{\text {in }}^{(n+1)}=\beta_{n} \rho_{\text {out }}^{(n)}+\left(1-\beta_{n}\right) \rho_{\text {in }}^{(n)},
$$

where $0<\beta_{n}<1$ is the mixing coefficient of the $n^{\text {th }}$ iteration. Different values of the mixing coefficients can be employed. Typically, I have found success with the first few iterations have larger values $\left(\beta_{n} \approx 0.1-0.3\right)$, while the later iterations have smaller mixing coefficients. In my experience, as the charge density comes closer and closer to convergence, usually a smaller mixing coefficient is needed to further reduce the error.

In principle, the algorithm just outlined is enough to solved any 3-dimensional Schrödinger-Poisson problem. We have not yet, however, taken advantage of the homogeneous nature of the system. Due to the translation invariance along the length of the wire, $k_{z}$ is a good quantum number, and the eigenstates can be written as

$$
\psi_{n}(\mathbf{r})=\psi_{\alpha, k_{z}}(\mathbf{r})=\varphi_{\alpha, k_{z}}(x, y) \frac{e^{i k_{z} z}}{\sqrt{L}}
$$

where $L$ is the length of the wire, ${ }^{7} k_{z}=n \frac{2 \pi}{L}$ with $n \in \mathbb{Z}$ such that $-\frac{\pi}{L}<$ $k_{z} \leq \frac{\pi}{L}$, and $\varphi_{\alpha, k_{z}}$ is the $\alpha^{\text {th }}$ transverse mode for momentum $k_{z}$ satisfying the

\footnotetext{
${ }^{7}$ We will take the limit $L \rightarrow \infty$ shortly. Technically, $k_{z}$ can only take on finitely many values when $L$ is finite, $k_{z}=n \frac{2 \pi}{L}$, with $n \in \mathbb{Z}$ such that $-\frac{\pi}{L}<k_{z} \leq \frac{\pi}{L}$.
} 
Bloch-Schrödinger equation,

$$
\mathcal{H}_{N}\left(k_{z}\right) \varphi_{\alpha, k_{z}}(x, y)=E_{\alpha}\left(k_{z}\right) \varphi_{\alpha, k_{z}}(x, y),
$$

where $\mathcal{H}_{N}$ is the Bloch Hamiltonian obtained by letting $\hat{k}_{z} \rightarrow k_{z}$ in Eq. (??). Note that $\varphi_{n, k_{z}}$ are normalized over the $x-y$ plane. Plugging Eq. (4.12) into Eq. (4.10), the charge density can be written in the form,

$$
\rho(x, y)=-\frac{e}{L} \sum_{\alpha} \sum_{k_{z}}\left|\varphi_{\alpha, k_{z}}(x, y)\right|^{2} F\left(E_{\alpha}\left(k_{z}\right), T\right) .
$$

If we now take the limit of $L \rightarrow \infty$, the sum over $k_{z}$ becomes an integral. Eq. (4.14) then becomes

$$
\rho(x, y)=-\frac{e}{2 \pi} \sum_{\alpha} \int_{-\infty}^{\infty}\left|\varphi_{\alpha, k_{z}}(x, y)\right|^{2} F\left(E_{\alpha}\left(k_{z}\right), T\right) d k_{z}
$$

where the integral runs over all possible values of momentum.

Having introduced the Schrödinger-Poisson equations and outlined the algorithm to find a self-consistent solution, how do we perform the individual steps of solving the Poisson equation for the electrostatic potential $\phi$, finding the eigenstates $\psi_{\alpha, k_{z}}$ of the Bloch-Schrödinger equation, and finally calculating the charge density $\rho$ ? In Sec. 4.2, we describe how we solve the Poisson equation (4.3). We'll address the Schrödinger equation (4.8) in Sec. 4.3. Finally, we show how to calculate the charge density is Sec. 4.4.

\subsection{Numerical method for solving the Poisson equa- tion}

Some simple geometries allow for an analytic or semi-analytical solution to the Poisson equation. These problems are rare, however, and typically we have to fall back onto numerical methods. A powerful numerical method to find approximate solutions to the Poisson problem, and partial differential equations in general, is the finite element method (FEM) [115], which we provide an elementary introduction to by way of a case study of the Poisson equation. We refer the reader to Ref. [115] for a more complete and rigorous study of FEM methods.

\subsubsection{Statement of the problem}

To keep this section self-contained, we state the generic Poisson problem that can be solved using finite element method. Consider a connected region of d-dimensional space $\Omega \subset \mathbb{R}^{d}$ shown schematically in Fig. (4.5). Find the potential $\phi$ that satisfies the Poisson equation,

$$
\nabla \cdot(\epsilon(\mathbf{r}) \nabla \phi(\mathbf{r}))=-\rho(\mathbf{r}),
$$




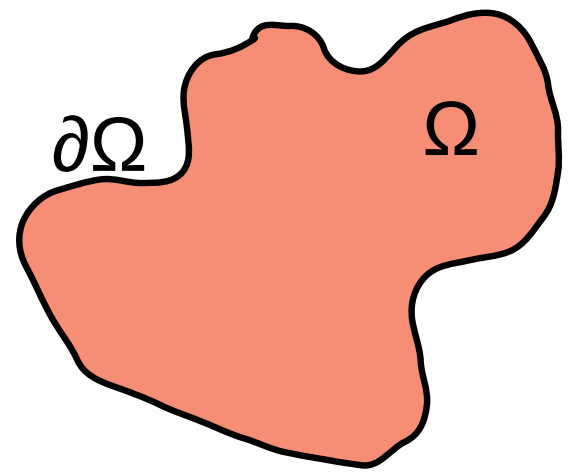

FIGURE 4.5: Schematic depiction of a connected region $\Omega$ of $d-$ dimensional space. The boundary of the space is denoted by $\partial \Omega$.

$\forall \mathbf{r} \in \Omega$. The boundary conditions are given by

$$
\begin{aligned}
\phi(\mathbf{r})=u(\mathbf{r}), & \mathbf{r} \in \partial \Omega_{D}, \\
\frac{\partial \phi(\mathbf{r})}{\partial n}=v(\mathbf{r}), & \mathbf{r} \in \partial \Omega_{N},
\end{aligned}
$$

where $\partial \Omega_{D}$ and $\partial \Omega_{N}$ are the regions of the surface in which the potential satisfies Dirichlet and Nuemann boundary conditions, respectively. The total surface is of course their union, $\partial \Omega=\partial \Omega_{D} \cup \partial \Omega_{N}$.

\subsubsection{Weak form of the Poisson equation}

The first step in finding the solution to the Poisson equation is to transform the differential equation (4.16) into a form more accessible for numerical treatment. We multiply both sides of Eq. (4.16) by a generic function $f$, known as a test function, and integrate over the whole domain,

$$
\int_{\Omega} f[\nabla \cdot(\varepsilon \nabla \phi)+\rho] d \Omega=0,
$$

where we have brought the $\rho$ term onto the left hand side and suppressed the position dependence of all functions. Note that Eq. (4.19) implies Eq. (4.16) due to the arbitrary nature of the function $f$. In other words, Eqs. (4.16, 4.19) are completely equivalent and equally deserve to be named the Poisson equation. We now make use of the vector identity,

$$
f \nabla \cdot(\epsilon \nabla \phi)=\nabla \cdot(f \epsilon \nabla \phi)-\nabla f \cdot(\epsilon \nabla \phi)
$$

which the reader can easily verify by applying the product rule to the first term on the right hand side. Plugging this into Eq. (4.19) yields

$$
-\int_{\Omega} \nabla f \cdot(\epsilon \nabla \phi) d \Omega+\int_{\Omega} \nabla \cdot(f \epsilon \nabla \phi) d \Omega+\int_{\Omega} f \rho d \Omega=0 .
$$


The second term can now be integrated using Stokes' theorem

$$
\int_{\Omega} \nabla \cdot(f \epsilon \nabla \phi) d \Omega=\oint_{\partial \Omega}(f \epsilon \nabla \phi) \cdot \mathbf{n} d S
$$

where the integral runs over the whole surface $\partial \Omega, d S$ is a differential element of the surface, and $\mathbf{n}$ is the unit vector normal to the surface element. We now assume that $f=0$ for any position on the surface where Dirichlet boundary conditions are imposed, i.e. $f(\mathbf{r})=0 \forall \mathbf{r} \in \partial \Omega_{D}$. The remaining region of the surface has Nuemann boundary conditions imposed which we can substitute in Eq. (4.18) for $\nabla \phi \cdot \mathbf{n}$. These two considerations imply that Eq. (4.21) can be written as

$$
-\int_{\Omega} \nabla f \cdot(\epsilon \nabla \phi) d \Omega+\int_{\Omega} f \rho d \Omega+\int_{\partial \Omega_{N}} f \epsilon v d S=0 .
$$

In the finite element literature, this is referred to as the weak form of the Poisson equation. In what sense is it weaker than the strong form of the Poisson equation in Eq. (4.16)? Observe that Eq (4.23) has no second-order partial derivatives in contrast to Eq. (4.16). Eq. (4.23) therefore places weaker constraints on differentiability than Eq. (4.16) in the sense that $\phi$ and $f$ need only to be once weakly differentiable. ${ }^{8}$

\subsubsection{Projection method}

So far, no approximations have been introduced. The differential equation has simply been transformed into an integral equation. The approximation comes in by projecting the potential $\phi$ and test functions $f$ onto a finite basis set $\mathcal{B}$. Namely we assume the potential can be written as the expansion,

$$
\phi(\mathbf{r})=\sum_{m=1}^{M} C_{m} g_{m}(\mathbf{r})
$$

where $g_{m} \in \mathcal{B}$ is the $m^{\text {th }}$ real valued basis function, $C_{m} \in \mathbb{R}$, and $M$ is the total number of basis functions in $\mathcal{B}$. In principle, we generically will only recover the exact solution for the potential by including an infinite number of basis function. The error between the approximate and exact solutions, however, can be reduced by expanding the basis set. In practice, we typically observe that the error becomes negligible for a relatively small number of basis states (on the order of hundreds to thousands) for 1D and 2D problems. Plugging

\footnotetext{
${ }^{8}$ The weak derivative is a generalization of the normal derivative to functions that are not necessarily differentiable. It essentially requires the integration by parts formula to hold. Let $f$ and $g$ be differentiable functions. The integration by parts formula is given by $\int_{a}^{b} f(x) g^{\prime}(x) d x=-\int_{a}^{b} f^{\prime}(x) g(x) d x+\left.f(x) g(x)\right|_{a} ^{b}$. We then generalize this concept as follows. The function $h$ is the weak derivative of the function $k$ if and only if $\int_{a}^{b} k(x) g^{\prime}(x) d x=$ $-\int_{a}^{b} h(x) g(x) d x$ for all infinitely differentiable functions $g$ such that $g(a)=g(b)=0$. Note that $k$ does not need to be differentiable for the integral $\int_{a}^{b} k(x) g^{\prime}(x) d x$, and hence its weak derivative $h$, to be well-defined.
} 


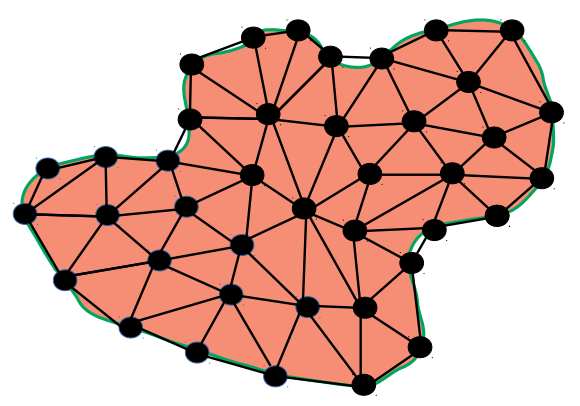

FIGURE 4.6: Example of a 2D mesh (approximately) covering the simulation region (red shading). The mesh is composed of $N_{E}$ triangular elements $\Omega_{E} \subset \Omega$, where $\Omega$ is the entire simulation region. Each element has three vertices, which are also known as nodes, shown as black dots.

in the expansion of Eq. (4.24) into Eq. (4.23) and letting $f=g_{n}$, we find the matrix equation

$$
\sum_{m=1}^{M} L_{n, m} C_{m}=R_{n}, \quad \forall n \in[M],
$$

where $[M]=\{1,2, \ldots, M\}$,

$$
\begin{aligned}
L_{n, m} & =\int_{\Omega} \nabla g_{n}(\mathbf{r}) \cdot\left(\epsilon(\mathbf{r}) \nabla g_{m}(\mathbf{r})\right) d \Omega, \\
R_{n} & =\int_{\Omega} g_{n}(\mathbf{r}) \rho(\mathbf{r}) d \Omega+\int_{\partial \Omega_{N}} g_{n}(\mathbf{r}) \epsilon(\mathbf{r}) v(\mathbf{r}) d S .
\end{aligned}
$$

Apart from enforcing the Dirichlet boundary conditions, Eq. (4.25) determines the coefficients $C_{m}$ for our approximate solution.

\subsubsection{Finite element mesh and basis functions}

One can envision many possible choices for the basis functions. An appropriate choice is problem dependent, but typically it is beneficial from a computational cost perspective to choose a basis set such the resulting matrix $L$ is sparse. Moreover, we need to readily and efficiently be able to compute the integrals in Eq. $(4.26,4.27)$. A smart choice for basis states will therefore be localized (to produce a sparse matrix $L$ ) and have simple analytical forms (to allow for efficient calculation of the necessary integrals). The simplest choice for basis states that satisfy these two properties are linear piecewise functions. For concreteness, let us focus on $d=2$ for the rest of this section. The method applies, however, to problems of other dimensions by appropriate alteration of the basis states.

To introduce these basis functions, we first define the mesh covering the domain of the problem. An example mesh is shown in Fig. 4.6. ${ }^{9}$ The mesh is composed of two types of entities; 1 ) An element $\Omega_{e}$ is a region of space that is a proper subset of the total space, $\Omega_{e} \subset \Omega$, where $e=1,2, \ldots, N_{E}$ is

\footnotetext{
${ }^{9}$ An example of a structured mesh used in our work is shown in Fig. 4.20.
} 


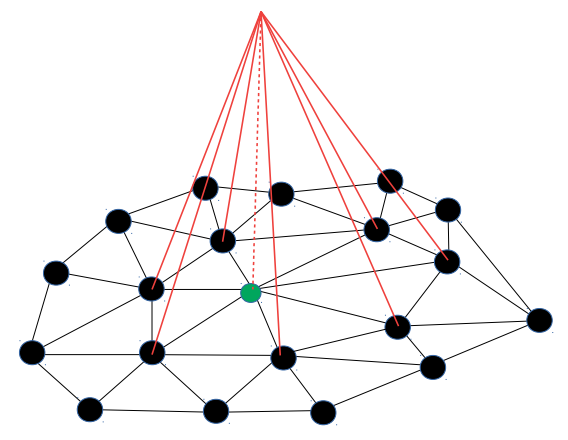

FIGURE 4.7: Example of a 2D "hat" basis function. The nodes of the $2 \mathrm{D}$ mesh are represents as circles. The unique linear piecewise basis function $g$ associated with the green node is shown in red. The value of the basis function is $g=1$ at the green node and $g=0$ at every black node.

the element index, and $N_{E}$ is the total number of elements. Distinct elements have no common interior points, $\left(\Omega_{e} \backslash \delta \Omega_{e}\right) \cap\left(\Omega_{e^{\prime}} \backslash \delta \Omega_{e^{\prime}}\right)=\varnothing$ for $e \neq e^{\prime}$. The total space $\Omega$ is the union of all elements, $\Omega=\cup_{e=1}^{N_{e}} \Omega_{e}$. For $d=2$, we employ triangle meshes in our work where the elements are triangles ${ }^{10}$ as shown in Fig. 4.6. Note that the elements do not need to be of the same size or be geometrically similar to each other. Indeed, elements will typically be made smaller in regions where the solution is required to be of high resolution. 2) A node $\mathbf{r}_{n}$ is a vertex of the triangular elements, where $n=1,2, \ldots, N_{N}$ is the node index, and $N_{N}$ is the total number of nodes in the mesh. Of course, each element has three nodes and a node is shared among several elements. To make this notion explicit, we can define maps from nodes to elements and elements to nodes. Let the map $h$ be defined as

$$
h(n)=\left\{e \in\left[N_{E}\right] \mid \mathbf{r}_{n} \text { is a vertex of } \Omega_{e}\right\},
$$

where $n$ is a node index. In other words, the set $h(n)$ contains all of the element indices of elements that have $\mathbf{r}_{n}$ as a vertex. We can also define a map $q$ from element indices to set of node indices,

$$
q(e)=\left\{n \in\left[N_{N}\right] \mid \mathbf{r}_{n} \text { is a vertex of } \Omega_{e}\right\},
$$

where $e$ is an element index. In other words, the set $q(e)$ contains the three node indices of the vertices of the element $\Omega_{e}$.

With each node $\mathbf{r}_{m}$ we associate a basis function $g_{m}$ that is defined to be the unique linear piecewise function that satisfies

$$
g_{m}\left(\mathbf{r}_{n}\right)=\left\{\begin{array}{ll}
1, & n=m \\
0, & n \neq m
\end{array},\right.
$$

\footnotetext{
${ }^{10}$ An element being a triangle is only once such choice in $2 \mathrm{D}$, but it is by far the most common. Other forms of FEM use rectangles or curvilinear polygons for the elements in 2D. In $1 \mathrm{D}$, an element is a line segment. In 3D, the most common element choice is a tetrahedron.
} 
where $\mathbf{r}_{n}$ is the $n^{\text {th }}$ node of the mesh. As promised, the basis functions have a simple analytical form

$$
g_{m}(\mathbf{r})=\left\{\begin{array}{ll}
a_{m}^{(e)}+b_{m}^{(e)} x+c_{m}^{(e)} y, & \mathbf{r} \in \Omega_{e} \text { where } e \in h(m) \\
0, & \mathbf{r} \in \Omega_{e} \text { where } e \notin h(m)
\end{array},\right.
$$

where $a_{m}^{(e)}, b_{m}^{(e)}, c_{m}^{(e)} \in \mathbb{R}$ are coefficients uniquely defined such that Eq. (B.14) is satisfied. ${ }^{11}$ Fig. 4.7 illustrates an example of such a basis function. The basis functions are sometimes referred to as tent or hat functions for obvious reasons. Observe that $g_{m}$ is nonzero only within the elements to which the $m^{\text {th }}$ node belongs, i.e. $g_{m}(\mathbf{r}) \neq 0$ if and only if $\mathbf{r} \in \Omega_{e}$ where $e \in h(m)$. Hence the basis functions only overlap with basis functions corresponding to nearest neighbor nodes, fulfilling our desired property of basis function locality. It follows from Eqs. (4.24, B.14) that $\phi\left(\mathbf{r}_{m}\right)=C_{m}$. This provides an easy way of implementing the Dirichlet boundary conditions by simply imposing

$$
C_{m}=u\left(\mathbf{r}_{m}\right), \forall \mathbf{r}_{m} \in \partial \Omega_{D}
$$

Note that if $u(\mathbf{r})$ is constant between two boundary nodes, then Eq. (4.32) perfectly matches the boundary condition in that region. If, on the other hand, $u(\mathbf{r})$ is not constant between two boundary nodes, then then Eq. (4.32) imposes a linear interpolation of the boundary condition between those points. This interpolation becomes more and more accurate, however, as the distance between boundary nodes shrinks.

\subsubsection{Calculation of matrix elements}

The last piece of the puzzle in solving the Poisson equation is to calculate the matrix elements in Eqs. $(4.26,4.27)$. Let us first focus on $L$ matrix elements,

$$
L_{n, m}=\int_{\Omega} \nabla g_{n}(\mathbf{r}) \cdot\left(\epsilon(\mathbf{r}) \nabla g_{m}(\mathbf{r})\right) d \Omega,
$$

which we have rewritten here for convenience. The integral in Eq. (4.33) runs over all of space within the simulation region. The basis functions vanish almost everywhere except due to the locality evident in Eq. (4.31). Moreover, the integrand is non-zero at position $\mathbf{r}$ if and only if both $g_{n}(\mathbf{r})$ and $g_{m}(\mathbf{r})$ are non-zero. We can therefore restrict the integration to elements having both nodes $\mathrm{n}$ and $\mathrm{m}$ as vertices. That is we integrate in all regions $\Omega_{e}$ where $e \in h(n) \cap h(m)$. In addition, we will always be taking $\epsilon$ as being constant within a material. Provided there exist no element in the mesh that is partially within multiple materials, which we will impose as a constraint when generating meshes, then $\epsilon$ is constant within each element and is denoted as $\epsilon_{e}$. With these considerations, Eq. (4.33) becomes

$$
L_{n, m}=\sum_{e \in h(n) \cap h(m)} \epsilon_{e} \int_{\Omega_{e}} \nabla g_{n}(\mathbf{r}) \cdot \nabla g_{m}(\mathbf{r}) d \Omega_{e},
$$

\footnotetext{
${ }^{11}$ See appendix A for expressions for $a_{m}^{(e)}, b_{m}^{(e)}$, and $c_{m}^{(e)}$ within each element.
} 
where each integral in the sum is restricted to an element $\Omega_{e}$ as denoted by the subscript of the integral sign. Obviously, if nodes $\mathbf{r}_{n}$ and $\mathbf{r}_{m}$ have no common element(s), $L_{n, m}=0$. Plugging the analytical formula for $g_{n}$ and $g_{m}$ from Eq. (4.31 into Eq. (4.34), $L_{n, m}$ finally becomes

$$
L_{n, m}=\sum_{e \in h(n) \cap h(m)} \epsilon_{e}\left(b_{n}^{(e)} b_{m}^{(e)}+c_{n}^{(e)} c_{m}^{(e)}\right) A_{e}
$$

where $A_{e}$ is the area of element $\Omega_{e}$.

We now turn to the first term in $R_{n}$, which involves the charge density $\rho$ and is given by

$$
R_{n}^{\rho}=\int_{\Omega} g_{n}(\mathbf{r}) \rho(\mathbf{r}) d \Omega .
$$

We once again can restrict the integral to elements in which $\mathbf{r}_{n}$ is vertex and plug in the analytical formula for $g_{n}$, which yields

$$
R_{n}^{\rho}=\sum_{e \in h(n)} \int_{\Omega_{e}}\left(a_{n}^{(e)}+b_{n}^{(e)} x+c_{n}^{(e)} y\right) \rho(\mathbf{r}) d \Omega_{e} .
$$

At first glance it appears that we've simplified $R_{n}^{\rho}$ as much as possible since $\rho$ could be any function. It must be remembered, however, that $\rho$ comes from summing over occupied wavefunctions of the Hamiltonian as we can see in Eq. (4.14). The wavefunctions, as will be discussed in the next section, are also projected onto a finite element basis. It therefore follows that $\rho$ is not such generic function, but rather can only contain a finite number of terms that we know (up to multiplicative factors) once the basis is chosen for the wavefunctions. Indeed, the charge is shown in Sec. 4.4 to take the form,

$$
\rho(\mathbf{r})=\sum_{m, p} A_{m p} g_{m}(\mathbf{r}) g_{p}(\mathbf{r}),
$$

where $g_{m}$ and $g_{p}$ are the same basis function used in the expansion of the electrostatic potential. Plugging Eq. (4.38) into Eq. (4.37) yields

$$
R_{n}^{\rho}=\sum_{e \in h(n)} \sum_{m, p \in q(e)} A_{m p} I_{m n p}^{(e)}
$$

where $m, p \in q(e)$ in the inner summation means that $m$ and $p$ are restricted to the three vertices of the element $e$ and $I_{m n p}^{(e)}$ is given by

$$
I_{m n p}^{(e)}=\int_{\Omega_{e}} g_{m}(\mathbf{r}) g_{n}(\mathbf{r}) g_{p}(\mathbf{r}) d \Omega_{e},
$$

which is a third order polynomial integral over the element $e$. The above integrals are tedious, but it is possible to work out analytical expressions for their evaluations given in terms of only the coordinates of the vertices of the triangular element over which the integral is being performed. Appendix A provides the necessary analytic expressions for calculating integrals, such as 
the integral in Eq. (4.40), encountered in finite element problems in 1D and 2D.

\subsection{Numerical solution of the effective mass Hamil- tonian}

Having described our numerical method for solving the Poisson equation in the previous section, we move on to finding the numerical solution for the eigenstates of the Hamiltonian. The method is similar to the method employed to solve the Poisson equation, so we will only briefly mention aspects already covered. While the eigenstates are technically two-components spinor functions, the problem of finding the eigenstates can be reduced in this simple case to finding the eigenstates of a spinless Hamiltonian. This is not the case for more detailed models that we will encounter later, however. Therefore, we show as an example how to solve for the two-components eigenstates of an effective mass Hamiltonian with position dependent spinorbit coupling in Appendix B.

\subsubsection{Statement of the problem}

Consider a connected space $\Omega^{\prime} \subseteq \Omega \subset \mathbb{R}^{2}$, where $\Omega$ is the domain of the Poisson problem in the previous section. Find Bloch eigenstates $\varphi_{\alpha}$ that satisfy the Bloch-Schrödinger equation

$$
\mathcal{H}_{N}\left(k_{z}\right) \varphi_{\alpha}\left(\mathbf{r}, k_{z}\right)=E_{\alpha}\left(k_{z}\right) \varphi_{\alpha}\left(\mathbf{r}, k_{z}\right),
$$

where $\mathcal{H}_{N}$ is the Bloch Hamiltonian, $E_{n}$ is the eigenenergy, and $\varphi_{\alpha}$ is a twocomponent spinor,

$$
\varphi_{\alpha}\left(\mathbf{r}, k_{z}\right)=\left(\begin{array}{c}
\varphi_{\alpha, \uparrow}\left(\mathbf{r}, k_{z}\right) \\
\varphi_{\alpha, \downarrow}\left(\mathbf{r}, k_{z}\right)
\end{array}\right) .
$$

The normal Hamiltonian is given by

$$
\begin{aligned}
\mathcal{H}_{N}\left(k_{z}\right) & =\mathcal{H}_{o}\left(k_{z}\right)+\mathcal{H}_{\Gamma}+\mathcal{H}_{S O}\left(k_{z}\right), \\
\mathcal{H}_{o}\left(k_{z}\right) & =\left[\frac{\hbar^{2}}{2 m^{*}}\left(-\nabla^{2}+k_{z}^{2}\right)-e \phi(\vec{r})\right] \sigma_{o}, \\
\mathcal{H}_{\Gamma} & =\Gamma \sigma_{z} \\
\mathcal{H}_{S O}\left(k_{z}\right) & =\alpha_{R} k_{z} \sigma_{x},
\end{aligned}
$$

where $\nabla^{2}=\partial_{x}^{2}+\partial_{y}^{2}$, and $\sigma_{j}$ with $j=\{x, y, z\}$ are the Pauli matrices acting in spin-space. Note that $k_{z} \in \mathbb{R}$ is the z-component of the momentum and is a good quantum number. The boundary conditions are given by

$$
\varphi_{\alpha}\left(\mathbf{r}, k_{z}\right)=0, \quad \mathbf{r} \in \partial \Omega^{\prime},
$$


i.e. we assume that the wavefunction vanishes everywhere on the boundary of $\Omega^{\prime}$.

\subsubsection{Reduction to spinless problem}

Suppose $\widetilde{\varphi}_{\alpha \sigma}$ satisfy

$$
\mathcal{H}_{o}\left(k_{z}=0\right) \widetilde{\varphi}_{\alpha \sigma}(\mathbf{r})=\varepsilon_{\alpha} \widetilde{\varphi}_{\alpha \sigma}(\mathbf{r}),
$$

where $\widetilde{\varphi}_{\alpha \sigma}(\mathbf{r})=\left(\widetilde{\varphi}_{\alpha}(\mathbf{r}) \delta_{\sigma, \uparrow}, \widetilde{\varphi}_{\alpha}(\mathbf{r}) \delta_{\sigma, \downarrow}\right)^{T}$ has only one non-zero spinor component. In other words, $\widetilde{\varphi}_{\alpha \sigma}$ is an eigenstate with energy $\varepsilon_{\alpha}$ of the $k_{z}=0$ Hamiltonian in the absence of Zeeman splitting and spin-orbit coupling. We're free to use $\widetilde{\varphi}_{\alpha, \sigma}$ as a basis for the full Hamiltonian for arbitrary $k_{z}$ and $\Gamma$. Calculation of the matrix elements yields,

$$
\int_{\Omega^{\prime}} \widetilde{\varphi}_{\alpha, \sigma}^{\dagger} \mathcal{H}_{N}\left(k_{z}\right) \widetilde{\varphi}_{\beta, \sigma^{\prime}} d \Omega^{\prime}=\delta_{\alpha, \beta}\left[\left(\varepsilon_{\alpha}+\frac{\hbar^{2} k_{z}^{2}}{2 m^{*}}\right) \delta_{\sigma, \sigma^{\prime}}+\Gamma\left(\sigma_{z}\right)_{\sigma, \sigma^{\prime}}+\alpha_{R} k_{z}\left(\sigma_{x}\right)_{\sigma, \sigma^{\prime}}\right],
$$

where we see that all matrix elements vanish between basis state with different orbits indices. The full states therefore have energies given by

$$
E_{\alpha, \pm}\left(k_{z}\right)=\left(\varepsilon_{\alpha}+\frac{\hbar^{2} k_{z}^{2}}{2 m^{*}}\right) \pm \sqrt{\Gamma^{2}+\alpha_{R}^{2} k_{z}^{2}}
$$

We therefore only need to numerically calculate the states $\widetilde{\varphi}_{\alpha}$ which are eigenstates of the spinless Hamiltonian,

$$
h_{o}=-\frac{\hbar^{2}}{2 m^{*}} \nabla^{2}-e \phi(\vec{r}) \text {, }
$$

satisfying

$$
h_{o} \widetilde{\varphi}_{\alpha}(\mathbf{r})=\varepsilon_{\alpha} \widetilde{\varphi}_{\alpha}(\mathbf{r}) .
$$

\subsubsection{Weak form, basis functions, and matrix assembly} form,

Just as we did for the Poisson problem, we convert Eq. (4.52) to the weak

$$
\begin{gathered}
\int_{\Omega^{\prime}} f_{1}\left(-\frac{\hbar^{2}}{2 m^{*}} \nabla^{2}-e \phi-\varepsilon_{\alpha}\right) \widetilde{\varphi}_{\alpha} d \Omega^{\prime}=0 \\
\int_{\Omega^{\prime}} \nabla f_{1} \cdot\left(\frac{\hbar^{2}}{2 m^{*}}\right) \nabla \varphi_{\alpha}-f_{1}(e \phi) \widetilde{\varphi}_{\alpha} d \Omega^{\prime}=\varepsilon_{\alpha} \int_{\Omega^{\prime}} f_{1} \widetilde{\varphi}_{\alpha} d \Omega^{\prime},
\end{gathered}
$$

where $f_{1}$ is the test function and we have transformed the second-order derivative to a dot product between two first-order derivatives through the use of integration by parts. Note that Eq. (4.54) contains no boundary terms due the condition of a vanishing of the wavefunction at the boundary (see Eq. 
(B.9)). Next, we project Eq. (4.54) onto a finite basis set. We choose the same piecewise-linear functions defined in Eq. (B.14) as we did for the Poisson problem, except we restrict them to vertices of elements in the domain $\Omega^{\prime}$, i.e.

$$
\widetilde{\varphi}_{\alpha}(\mathbf{r})=\sum_{m} g_{m}(\mathbf{r}) D_{m, \alpha}
$$

This leads to the generalized eigenvalue problem,

$$
\sum_{m} h_{n m} D_{m \alpha}=\varepsilon_{\alpha} \sum_{m} S_{n m} D_{m \alpha},
$$

where the matrix element $h_{n m}$ is found by substituting $g_{n}$ and $g_{m}$ into the left hand side of Eq. (4.54) for $f_{1}$ and $\widetilde{\varphi}_{\alpha}$, respectively. Similarly, the overlap matrix element $S_{n m}$ is found by substituting $g_{n}$ and $g_{m}$ into the right hand side of Eq. (4.54) for $f_{1}$ and $\widetilde{\varphi}_{\alpha}$, respectively. Note that these matrix elements are calculated in the same manner as was done for the Poisson problem in section 4.2.5. The only difficulty at first sight appears to be calculating integrals involving the potential,

$$
\phi_{n m}=\int_{\Omega^{\prime}} g_{n}(\mathbf{r}) \phi(\mathbf{r}) g_{m}(\mathbf{r}) d \Omega^{\prime},
$$

since the potential can in principle be any continuous function. Note, however, that the potential in our problem comes from solving the Poisson equation using the numerical scheme in Sec. 4.2, where the potential is expanded in the form given in Eq. (4.24). The matrix element $\phi_{n m}$ is then given by

$$
\phi_{n m}=\sum_{l=1}^{M} C_{l} \int_{\Omega^{\prime}} g_{n}(\mathbf{r}) g_{l}(\mathbf{r}) g_{m}(\mathbf{r}) d \Omega^{\prime},
$$

which has an expression given by a linear sum of analytical integrals provided in Appendix A. Once all of the matrix elements are computed, sparse matrix methods are used to find the low-energy eigenstates satisfying Eq. (4.56).

\subsection{Calculation of charge density from wavefunc- tions}

In Eq. (4.59), we found the charge density $\rho$ to be expressed as an integral over $k_{z}$ of the modulus square of the wavefunction weighted by the Fermi function F. As we showed in Sec. 4.3.2 the transverse profile $\varphi_{\alpha}$ is independent of $k_{z}$, which implies that $\left|\varphi_{\alpha, k_{z}}\right|^{2}$ can be factored out of the integral in Eq. (4.59). The charge density is then given by

$$
\rho(x, y)=-\frac{e}{2 \pi} \sum_{\alpha}\left|\varphi_{\alpha}(x, y)\right|^{2} \int_{-\infty}^{\infty} F\left(E_{\alpha+}\left(k_{z}\right), T\right)+F\left(E_{\alpha-}\left(k_{z}\right), T\right) d k_{z \prime}
$$


where the $E_{\alpha \pm}$ are given in Eq. (6.14) and account for the two spin subbands for each value of $\alpha$. The integrals are then easily calculated using Simpson's method. Plugging in the projection expansion given in Eq. (4.55) for $\varphi_{\alpha}$ into Eq. (4.59) yields

$$
\rho(x, y)=-\frac{e}{2 \pi} \sum_{\alpha} \mathcal{F}_{\alpha} \sum_{n, m} D_{n \alpha} D_{m \alpha} g_{n}(x, y) g_{m}(x, y),
$$

where $\mathcal{F}_{\alpha}$ denotes the integral of Fermi functions in Eq. (4.59). Reversing the order of summation, the charge density is given by

$$
\rho(\mathbf{r})=\sum_{n, m} A_{n m} g_{n}(\mathbf{r}) g_{m}(\mathbf{r}),
$$

with

$$
A_{n m}=-\frac{e}{2 \pi} \sum_{\alpha} \mathcal{F}_{\alpha} D_{n \alpha} D_{m \alpha} .
$$

Note that Eq. (4.61) has the same form as Eq. (4.38), as promised in Sec. 4.2.5.

\subsection{Model of the superconductor}

In Secs. 4.1 and 4.3 the SM-SC coupling was assumed to be weak. The SC was therefore excluded from the Hamiltonian. Again, it is stressed that the SC still played an important through the electrostatic boundary condition at the SM-SC interface, which accounted for band-bending. If it is desired to explicitly incorporate the SC into the Hamiltonian, how might that be? First of all, energy scale considerations indicate that the energy scale associated with the superconductivity is $\lesssim 1 \mathrm{meV}$, which is far smaller than the energy scales associated with $H_{o}$ in Eq. (4.2). Therefore, the case in which the properties of the electrostatic environment in infinite nanowires systems are of primary concern, treating the SC as a normal metal $\left(\Delta_{o} \rightarrow 0\right)$ is well justified. A straight-forward idea is then to continue the effective mass Hamiltonian (4.2) used for the SM into the SC (which is currently being treated as a metal), i.e.

$$
H_{S C}=\frac{\hbar^{2}}{2 m_{S C}^{*}}\left(\hat{k}_{x}^{2}+\hat{k}_{y}^{2}+\hat{k}_{z}^{2}\right)-\mu_{S C}
$$

where $m_{S C}^{*}$ and $\mu_{S C}$ are the effective mass and chemical potential of the superconductor, respectively. Superconductor materials, such as $\mathrm{Al}$, that are used in SM-SC Majorana devices are well approximated using a nearly free electron model with $m_{S C}^{*} \approx m_{0}$ and a large chemical potential. For example, the chemical potential of aluminium is $\mu_{A l} \approx 11 \mathrm{eV}$.

This model runs into a problem, however. For a translation invariant nanowire, the momentum along the length of the wire, $k_{z}$, is a good quantum number. This implies that any states of the SM and SC that couple need to have the same $k_{z}$. As noted in the discussion of the SM model in Sec. 4.1, the subbands only fall up few tens of meV below the Fermi level. Combined with the small effective mass $m^{*}$ of the SM, the typical Fermi-momentum $k_{z, F}$ 
of the SM subbands is very small. In contrast, the lowest-energy subbands of the SC are many eVs below the Fermi level, and therefore have a huge Fermimomentum compared to the SM subbands. This result in negligible coupling between the SM subbands and the lowest-energy SC subbands. The only SC subbands that will couple effectively with SM subbands therefore need to have a large confinement energy very close to $\mu_{S C}$. Recall from elementary quantum mechanics, that the energy separation between confinement induced states increases for the higher energy bands. Additionally, the energy separation grows as the confinement region shrinks. ${ }^{12}$ Due to the combination of the large SC chemical potential $\mu_{S C}$ and the small thickness of the SC layer, the energy separation between SC subbands with band-edges near the Fermi level is typically on the meV energy scale. This fact typically leads to small coupling between the SM and SC. This contradicts the experimental observation of an induced superconducting gap $\Delta_{i n d}$ comparable to the parent gap $\Delta_{o}$ of the SC layer.

To fix this issue of weak coupling, disorder in the SC can be included. Some studies have explicitly incorporated disorder into the Hamiltonian [116118], but the included disorder is translation invariant, which is unphysical. Another strategy is treat the SC as a bulk SC, which is equivalent to averaging over disorder realizations [119]. Unfortunately, this makes the problem challenging from a modeling standpoint due to the continuum of states in the bulk superconducting metal. While understanding how to solve such a problem is important, we choose to not to deal with such issues in this thesis $^{13}$ when solving for the electrostatic potential self-consistently. For one thing, the computational expense of including the SC explicitly, as done in Refs. [116-118], is numerically expensive. Secondly, it is not clear that much insight is gained from including it from the perspective of understanding the electrostatic ${ }^{14}$ unless the disorder fluctuations along the length of the wire are included.

\subsection{Subband occupation in semiconductor-superconductor nanowires}

As a concrete example, let us now apply our formalism to a Majorana hybrid nanowire. Specifically, we consider quasi one-dimensional (1D) SM-SC devices having a transverse cross-section as shown schematically in Fig. 4.8. Note that the system is infinite in the $z$-direction. We focus largely on the determination of subband occupation as a function on system parameters, by

\footnotetext{
${ }^{12}$ For example, the energy separation between particle in a box states in $1 \mathrm{D}$ is given by $E_{n+1}-E_{n}=\frac{\hbar^{2} \pi^{2}}{2 m^{*} W^{2}}(2 n-1)$, where $W$ is the width of the box.

${ }^{13}$ We are currently working on such a problem. See chapter 7 for ideas relating to this problem.

${ }^{14}$ One insight from explicitly including the SC is that the SM subbands are shifted down in energy [120].
} 


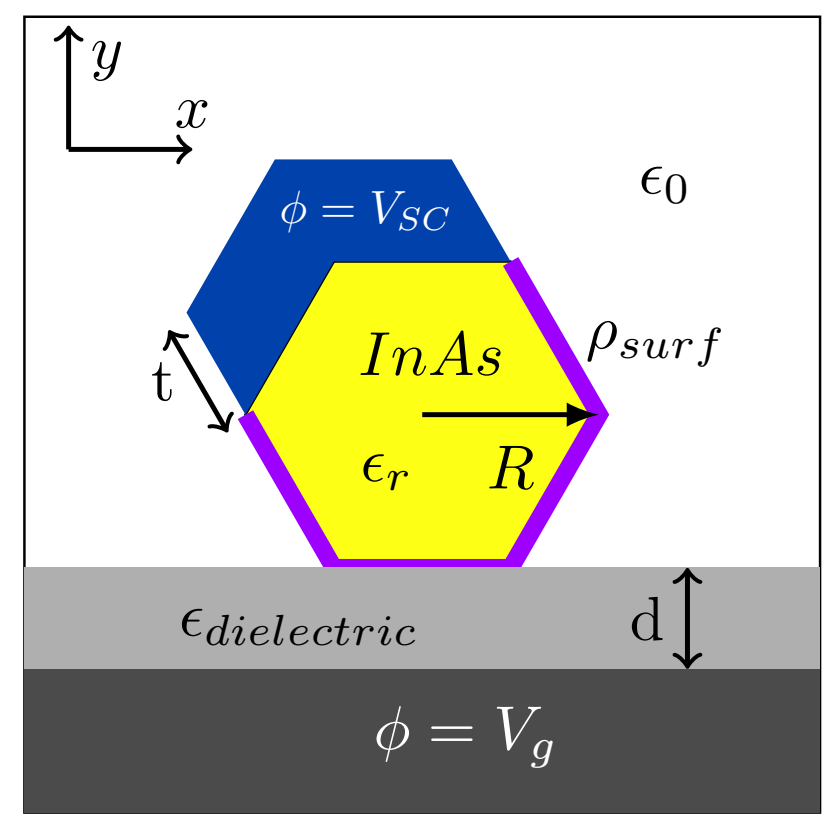

FIGURE 4.8: Transverse profile of the hybrid device studied in this section. The wire is infinite in the $z$-direction. An InAs nanowire (yellow) of radius $\mathrm{R}$ is partially covered by a superconductor (blue) and placed on an insulating substrate (light gray). The device is gated from below (dark gray) to tune the electrostatic potential. A uniform surface charge (purple) is placed on the InAs surfaces other than the SM-SC interface.

solving self-consistently the Schrödinger-Poisson equations for the conduction electrons of the semiconductor nanowire. The results of this section are adopted from our work in Ref. [121]

As discussed in chapters 1 and 3, a key concern in the field of MZM in SM-SC nanostructures is the possibility of topologically trivial Andreev Bound states (ABSs) mimicking the MZM phenomenology. While some of the ABS-producing mechanisms such as, for example, long-range inhomogeneous potentials (e.g., soft confinement) and inhomogeneous pairing, can be understood within a strictly one dimensional model, other mechanisms rely on or are strongly enhanced by the presence of multiple occupied subbands in wires with finite thickness. For example, the inter-band coupling mechanism produces low-energy ABSs though mixing multiple subbands in the presence of, e.g., an inhomogeneous electrostatic potential [72, 122]. ${ }^{15}$ Note that this is a specific example of trivial low-energy states that emerge generically in systems with only particle-hole symmetry [74] (i.e. systems in the D symmetry class). Also note that, at high occupancy, the presence of an inhomogeneous effective potential (generated by electrostatic gates, strain, etc.) is similar to the presence of disorder.

\footnotetext{
${ }^{15}$ Our work showing how inter-band coupling can lead to low-energy ABSs will be presented in chapter 5 .
} 
There are thus many reasons to avoid high occupancy in semiconductor nanowires while searching for topological Majorana zero modes. It is therefore important to theoretically establish the criteria for subband occupancy in the experimentally relevant nanowires. To establish whether or not a hybrid device is in the many-subband regime, it is essential to determine the expected number of occupied subbands and the corresponding characteristic subband spacing.

A critical feature that we include in our model is a finite surface charge density on the InAs nanowire, which is known to have an accumulation of surface charge, apparently due to surface point defects [123] or surface hydrogen impurities [124, 125]. While several studies have investigated devices using a Schrödinger-Poisson framework [110, 116, 117, 126-128], only some have included the effects of surface charge [117, 128]. Here we focus on understanding the possible effect of the surface charge on the subband occupation and the inter-subband energy spacing. We find that regimes characterized by many occupied subbands are possible and even likely within realistic windows of system parameters. On the other hand, gating the system to reach the few-subbands regime is usually not possible (except for rather narrow parameter windows) due to the onset of holes. Furthermore, the many-subbands regime is typically associated with small values of the inter-subband spacing near the Fermi level, which has highly detrimental consequences for the realization of robust MZMs. Finally, to assess whether a device is in the optimal few-subbands or the undesirable many-subband regimes, we propose a measurement of the LDOS on the exposed facets of the nanowire, e.g., using an STM. We calculate the expected characteristic zero-energy LDOS features obtained by varying the applied gate potential within different regimes of device parameters and asses the capabilities and limitations of such a measurement.

The remainder of this section is organized as follows. In Sec. 4.6.1 we discuss the hybrid semiconductor-superconductor device, the model used to described its electronic properties, and the methods used in the calculations. The results of our numerical analysis are presented in Sec. 4.6.2. These results include the dependence of the subband occupation, average subband separation near the Fermi level, and local density of states on relevant system parameters. Our conclusions are presented in Sec. 4.6.3.

\subsubsection{Device and modeling}

We consider quasi one-dimensional (1D) semiconductor-superconductor (SM-SC) devices having a transverse cross-section as shown schematically in Fig. 4.8. For concreteness, we consider zinc-blende-structured InAs semiconductor wires partially covered by a superconductor, e.g., $\mathrm{Al}$, grown epitaxially on two faces of the nanowire. The proximitized wire is gated from below, to be able to tune the electrostatic potential within the system, and considered to be infinitely long. To estimate the dependence of the occupancy of confinement-induced conduction subbands on the applied gate potential, we model the nanowire using the simple effective mass model described in 
Sec. 4.1. Similarly, we use the iterative procedure described in Sec. 4.1 to solve the Schrödinger-Poisson Eqs. $(4.8,4.3,4.10)$ self-consistently. The only additional ingredient that we add on to the formalism of Sec. 4.1 is the fixed surface charge [123]. The charge density takes the form,

$$
\rho(\vec{r})=\rho_{f}(\vec{r})+\rho_{\text {surf }}(\vec{r}),
$$

where $\rho_{f}$, which is given by the occupied eigenstates of the Hamiltonian and given by Eq. (4.10), has to be calculated self-consistently, while $\rho_{\text {surf }}$ represents an immobile surface charge density. Following Ref. [117], we model this surface charge as a uniform layer of (positive) charge of thickness $\ell$ on the nanowire surfaces other than the semiconductor-superconductor interface (see Fig. 4.8).

We note that, for a given surface charge density $\sigma=\rho_{\text {surf }} \ell$, the potential $\phi(\vec{r})$ depends weakly on the value of $\ell$, as long as $\ell \ll R$. The superconductor is not explicitly included in the Hamiltonian, but it plays a key role in the Poisson problem by setting the Dirichlet boundary condition $\phi(\vec{r})=V_{S C}$ at the SM-SC interface, where $V_{S C}$ represents the work function difference between the semiconductor and the superconductor [110]. The exact value of the work function difference is not known, hence $V_{S C}$ will be treated as a phenomenological model parameter. In this work we consider $V_{S C}>0$ on the order of $10^{2} \mathrm{mV}$. Note that a non-zero work function difference results in the bending of the InAs conduction bands near the semiconductorsuperconductor interface. We also impose Dirichlet boundary conditions, $\phi(\vec{r})=V_{g}$, on the top surface of the back gate. In addition, we impose von Neumann-type boundary conditions on the top, left, and right surfaces of a box of side length $b$ surrounding the wire. We emphasize that the potential within the nanowire is negligibly affected by these boundary conditions e.g., the exact value of $b$ or whether we choose von Neumann or Dirichlet boundary conditions - provided $b \gg R$. Finally, to reduce the number of parameters in our analysis, we notice that the eigenstates of Eq. (4.52) are unaffected by the transformation $\mu \rightarrow \widetilde{\mu}=0, V_{S C} \rightarrow \widetilde{V}_{S C}=V_{S C}+\mu / e$, $V_{g} \rightarrow \widetilde{V}_{g}=V_{g}+\mu / e$, where $\mu$ is the chemical potential defined with respect to the bottom of the conduction band in a system with $\phi(\vec{r})=0$. Our results are given in terms of $\widetilde{V}_{S C}$ and $\widetilde{V}_{g}$ (with $\widetilde{\mu}=0$ ); we stress that, in systems with nonzero chemical potential, the values of these parameters are shifted (by $\mu / e$ ) with respect to the 'bare' work function difference and gate potential, respectively. Thus, all one has to do is to redefine $V_{S C}$ and $V_{g}$ by including the chemical potential.

To account for the possible emergence of holes, we estimate the corresponding $\widetilde{V}_{g}$ values using a Luttinger model $[46,129]$ for the InAs light and 
heavy hole bands, considering them as decoupled from the conduction electrons. We consider the wire to be grown along the [111] direction. The Hamiltonian is given by

$$
H_{\text {Lutt }}=\left[\begin{array}{cccc}
-P-Q & S & R & 0 \\
S^{+} & -P+Q & 0 & R \\
R^{+} & 0 & -P+Q & -S \\
0 & R^{\dagger} & -S^{\dagger} & -P-Q
\end{array}\right],
$$

where

$$
\begin{aligned}
& P=\gamma_{1} \frac{\hbar^{2} k^{2}}{2 m}+E_{\text {gap }}+\mu+e \phi(\vec{r}), \\
& Q=\gamma_{3} \frac{\hbar^{2}}{2 m}\left(k_{x}^{2}+k_{y}^{2}-2 k_{z}^{2}\right), \\
& S=\frac{2}{\sqrt{3}}\left(2 \gamma_{2}+\gamma_{3}\right) \frac{\hbar^{2}}{2 m}\left(k_{x}-i k_{y}\right) k_{z}, \\
& R=\frac{1}{\sqrt{3}}\left(\gamma_{2}+2 \gamma_{3}\right) \frac{\hbar^{2}}{2 m}\left(k_{x}-i k_{y}\right)^{2},
\end{aligned}
$$

and we have employed the axial approximation, for simplicity [46]. The $\gamma_{i}$ coefficients, known as the Luttinger parameters, take the values $\gamma_{1}=20.4$, $\gamma_{2}=8.3$, and $\gamma_{3}=9.1$ for InAs. The valence band is separated from the conduction band by a bandgap, given by $E_{g a p}=0.418 \mathrm{eV}$ for InAs, and we have incorporated the chemical potential $\mu$ and electrostatic potential $\phi$ into the diagonal term $P$. When the model is implemented on the nanowire, we take $k_{x} \rightarrow-i \partial_{x}, k_{y} \rightarrow-i \partial_{y}$, and use the finite element method as we have done for the conduction electrons to solve for the eigenstates near the valence band edge. Note that incorporating the four bands of the Luttinger model into the finite element method is a straightforward extension of the numerical methods employed in the two band model of Appendix B. We stress that the charge density from populated hole states is not included in the electrostatics calculations. Rather, we are interested in the $\widetilde{V}_{g}$ values for which the hole states begin to populate.

While the main focus of this section is understanding subband occupancy, our calculations can also provide an estimate of the expected Rashba spinorbit coupling, which is crucial for the realization of Majorana bound states. Starting from an $8 \times 8$ Kane model that incorporates the coupling between conduction and valence bands, quasi-degenerate perturbation theory [113] can be used to arrive at an effective $2 \times 2$ model for the conduction electrons given by [114]

$$
\begin{aligned}
H_{2 x 2}= & {\left[\frac{\hbar^{2}}{2 m^{*}}\left(-\partial_{x}^{2}-\partial_{y}^{2}+k_{z}^{2}\right)-\mu-e \phi(x, y)\right] \sigma_{o} } \\
& +\left(\alpha_{x} \sigma_{x}+\alpha_{y} \sigma_{y}\right) k_{z \prime}
\end{aligned}
$$


where the Rashba coefficients, $\alpha_{x}$ and $\alpha_{y}$, are given by

$$
\begin{aligned}
& \alpha_{x}(x, y)=\frac{e \widetilde{P}^{2}}{3}\left(\frac{1}{E_{\text {gap }}^{2}}-\frac{1}{\left(E_{\text {gap }}+\Delta_{o}\right)^{2}}\right) \partial_{y} \phi(x, y), \\
& \alpha_{y}(x, y)=\frac{e \widetilde{P}^{2}}{3}\left(\frac{1}{E_{\text {gap }}^{2}}-\frac{1}{\left(E_{\text {gap }}+\Delta_{o}\right)^{2}}\right) \partial_{x} \phi(x, y),
\end{aligned}
$$

with $\widetilde{P}, E_{g a p}$, and $\Delta_{o}$ being parameters within the $8 \times 8$ Kane model (see Ref. [46]). Note that eq. (4.70) is the effective mass Hamiltonian in Eq. (??) of Sec. 4.1, except the Rashba spin-orbit coupling has position dependence as made clear in Eqs. (4.71,4.72). Here, we are interested in the matrix elements $\left(\alpha_{i}\right)_{m n} \equiv\left\langle\psi_{m}\left|\alpha_{i}\right| \psi_{n}\right\rangle$, where $\left|\psi_{n}\right\rangle$ is the $n^{\text {th }}$ eigenstate of our Hamiltonian with no spin-orbit coupling. In particular, the diagonal Rashba terms for the eigenstates near the Fermi level play a central role in determining the topological gap and localization length of Majorana bound states.

In the calculations, the following parameter values are used unless otherwise stated: the effective mass is $m^{*}=0.026 m_{0}$ (as we focus on InAs wires). We consider two different values of the nanowire radius, $R=35 \mathrm{~nm}$ and $R=50 \mathrm{~nm}$; the superconductor and dielectric thicknesses are $t=10 \mathrm{~nm}$ and $d=10 \mathrm{~nm}$, respectively; the dielectric constants of the wire, dielectric, and surrounding air are taken to be $\epsilon_{\text {wire }}=15.15 \epsilon_{0}, \epsilon_{\text {diel }}=24 \epsilon_{0}$, and $\epsilon_{\text {air }}=\epsilon_{0}$, where $\epsilon_{o}$ is the permittivity of free space. The layer of surface charge is taken to be $\ell=1 \mathrm{~nm}$. We use Gmsh [130] to create unstructured triangular meshes to be used in the finite element calculation. The characteristic element mesh sizes within the nanowire, dielectric, and surrounding air are taken to be 2 , 2.5 , and $5 \mathrm{~nm}$, respectively. To accommodate the small value of $\ell$ for the surface charge layer, we use a separate mesh with characteristic element mesh size of $1 \mathrm{~nm}$ inside the nanowire to calculate $\phi$ due to the surface charge.

\subsubsection{Results}

We first investigate the dependence of the subband occupation on the (shifted) gate and superconductor potentials for a wire of radius $R=35 \mathrm{~nm}$. The results are shown in Fig. 4.9. The three panels correspond to three different values for the surface charge density $\sigma=\rho_{\text {surf }} \ell$. Note that spin subbands are counted separately, so each change of color/shade represents the occupation of an additional pair of (degenerate) spin subbands. We focus on negative gate voltages since we are interested in limiting the number of occupied subbands. Note that, in addition to increasing the number of occupied subbands, attractive (i.e. positive) gate potentials will move the conduction electrons away from the SM-SC interface and, consequently, will suppress the superconducting proximity effect.

The subband occupation in the absence of surface charge $(\sigma=0)$ is shown in Fig. 4.9(a). As expected, there are no conduction electrons in the wire for $\widetilde{V}_{S C}=0$ and $\widetilde{V}_{g} \leq 0$, as the chemical potential is below the bottom of 


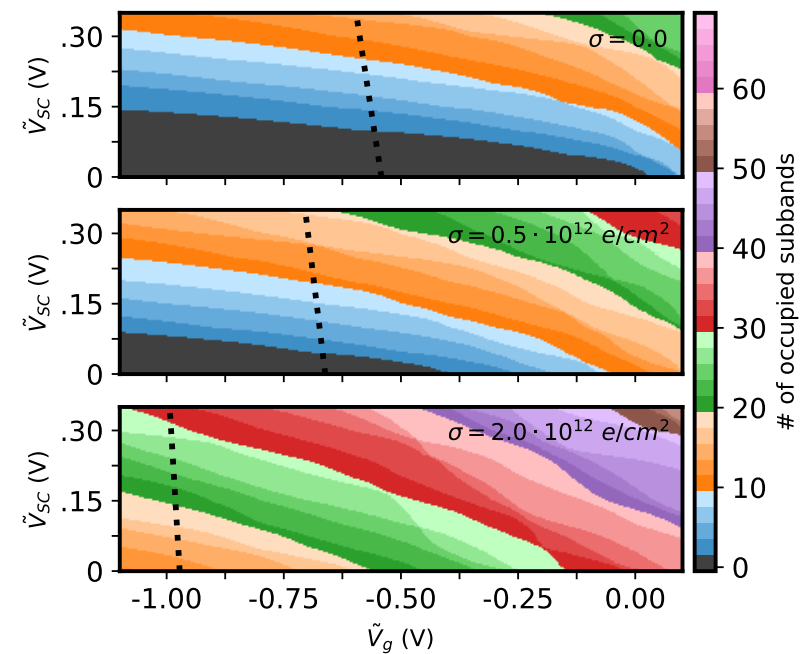

FIGURE 4.9: Subband occupation for a wire of radius $R=$ $35 \mathrm{~nm}$ as function of the (shifted) gate potential $\widetilde{V}_{g}$ and the (shifted) work function difference $\widetilde{V}_{S C}$. The panels correspond to three different values of surface charge $\sigma$. The negative gate voltage regime to the left of the dashed lines is characterized by the emergence of holes at the bottom of the nanowire (i.e. near the interface with the dielectric). Note that each spin subband is counted separately (hence, each color/shade corresponds to one pair of (degenerate) spin subbands).

the conduction band. Increasing $\widetilde{V}_{S C}$ (for a fixed value of the gate potential) bends the conduction band near the SM-SC interface resulting in an increasing number of occupied subbands (up to about 26 for $\widetilde{V}_{g}=0$ and $\widetilde{V}_{S C}=325 \mathrm{mV}$ ). Of course, in the actual system $\widetilde{V}_{S C}$ is fixed and the available tunning parameter is the gate potential $\widetilde{V}_{g}$. An important feature revealed by our results is the possibility of tunning across several subband pairs using moderate gate voltages. Note that this property is independent of the work function difference, provided $\widetilde{V}_{S C}$ is large enough so that the system is not nearly depleted at $\widetilde{V}_{g}=0$ (i.e. $\widetilde{V}_{S C}>100 \mathrm{mV}$ ). The possibility of observing experimentally these subband crossings will be discussed below.

A crucial question is whether single-subband or few-subbands occupancy can be obtained by applying a negative-enough gate potential. The relevance of this question becomes clear in the context of realizing topological superconductivity and Majorana zero modes (MZMs) using hybrid SM-SC structures, as high occupancy is not only detrimental to the stability of MZMs (as a result of being associated with smaller topological gaps), but also generates ubiquitous trivial low-energy states in the presence of disorder or system inhomogeneities $[55,56,59,61,62,72,122,131]$. To answer this question, we first notice that, in principle, the conduction bands will eventually become completely depleted for a large enough value of $\left|\widetilde{V}_{g}\right|$. However, it is possible that the valence bands become partially depleted (i.e., holes emerge) 


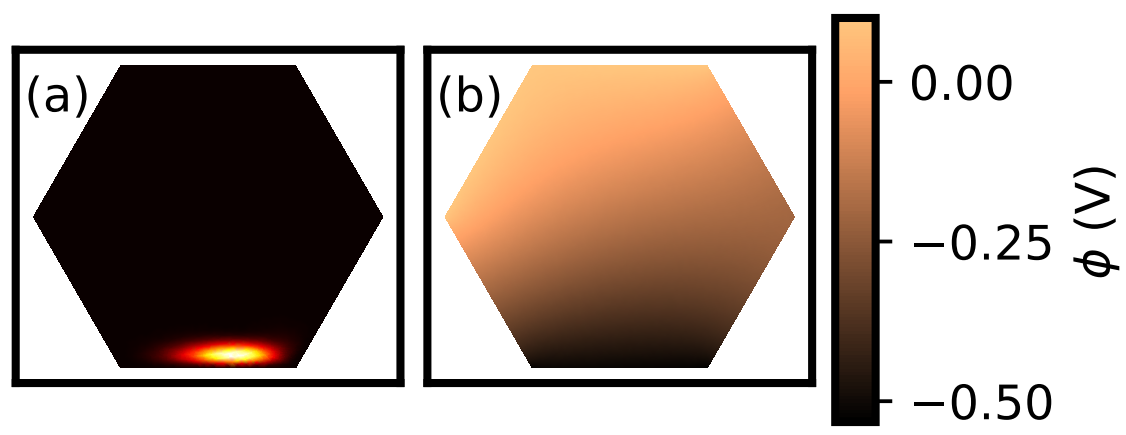

FIGURE 4.10: Highest energy valence band eigenstate, $|\psi|^{2}$ (a) and corresponding electrostatic potential, $\phi(b)$. The system parameters are y $R=35 \mathrm{~nm}, \widetilde{V}_{S C}=0.1 \mathrm{~V}, \widetilde{V}_{g}=-0.6 \mathrm{~V}, \sigma=0$. Note that the emerging holes are strongly localized near the back gate due to the large negative electrostatic potential in that region.

before reaching this regime. The emergent holes are localized near the interface with the dielectric (i.e., as close as possible to the back gate), as shown in Fig. 4.10. Consequently, the holes experience practically no proximity effect from the parent superconductor. As a result, a SM-SC hybrid system in this regime will consist of a quasi-1D topological superconductor (made of proximitized conduction electrons) and a parallel "normal-metal" channel (made of holes). Of course, the hole channel will, in general, completely destroy the topological properties of the MZMs supported by the parallel 1D superconductor; hence, its emergence has to be avoided. To account for the possible emergence of holes, we estimate the corresponding $\widetilde{V}_{g}$ values using the Luttinger model for the InAs light and heavy hole bands, as described in Sec 4.6.1. The dashed black lines in Figs. 4.9-4.13 indicate the critical (shifted) gate voltage associated with the emergence of holes. Note that the electrostatic screening from the holes (which is significant, particularly considering their higher effective mass) has not been explicitly taken into account in our calculations. This implies that the actual lever arm associated with $\widetilde{V}_{g}$ is strongly reduced within the hole-regime left of the dashed lines. Practically, from the perspective of Majorana physics, the dashed lines mark the limit of negative (shifted) potentials consistent with the realization of topologically-protected MZMs. For example, Fig. 4.9(a) shows that holes begin to emerge near $\widetilde{V}_{g} \approx-550 \mathrm{mV}$. This limits drastically the window of $\widetilde{V}_{S C}$ values associated with the few-subband regime, $\widetilde{V}_{S C}<200 \mathrm{mV}$. This window is even smaller in panel (b) and absent in panel (c). Finally, note that, as a result of the holes being localized very close to the back gate (see Fig. 4.10), the critical gate voltage associated with their emergence depends weakly on the (positive) work function difference $V_{S C}$ at the SM-SC interface, as clearly demonstrated by the (almost vertical) dashed lines in Figs. 4.9 and 4.11 .

The impact of surface charge (which is responsible for band-bending near the surface of the SM wire) on the subband occupation is illustrated in Fig. 


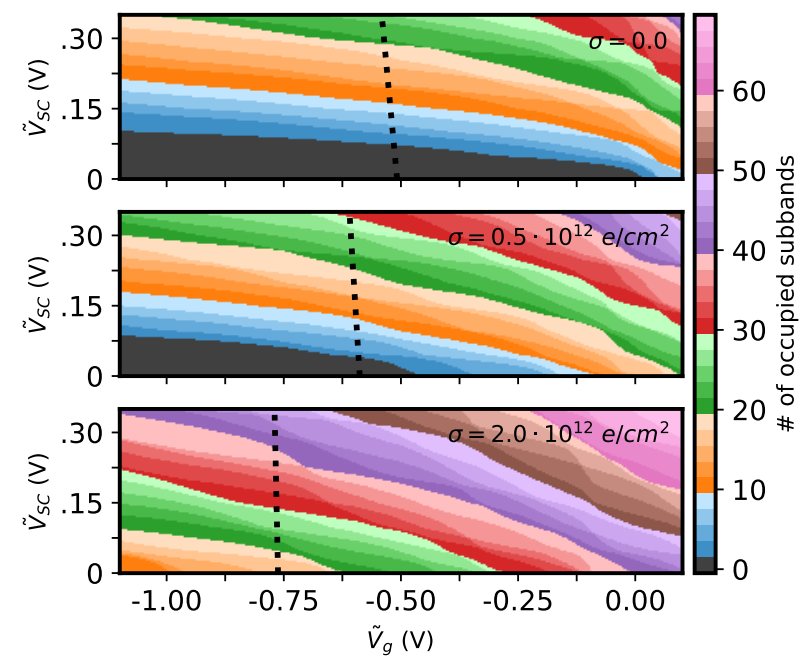

FIGURE 4.11: Same as Fig. 4.9 for a wire of radius $R=50 \mathrm{~nm}$. Note that the number of occupied subbands increases with the radius of the wire.

4.9(b) and (c). The chosen surface charge values are consistent with experimental estimates for InAs [123] and have been used in previous theoretical studies of Majorana devices [117, 128]. As expected, the main effect of the (positive) surface charge is to increase the overall number of occupied subbands. The effect can be clearly seen in panels (b) and (c), as the subband occupations are shifted down and to the left relative to panel (a). Note that, despite the fact that the gate potentials for which holes begin to populate the wire become more negative as $\sigma$ increases, the window of $\widetilde{V}_{S C}$ values associated with the few-subband regime shrinks and eventually vanishes, as illustrated in panel (c). For example, the single-subband regime can be attained in Fig. 4.9(b) only for $\widetilde{V}_{S C} \lesssim 75 \mathrm{mV}$, while in Fig. 4.9(c) the lowest number of occupied subbands (before holes start emerging) is ten, corresponding to $\widetilde{V}_{S C}=0$.

In addition to $\widetilde{V}_{S C}$ and $\widetilde{V}_{g}$, the subband occupation has a significant dependence on the radius of the wire. To illustrate this feature, in Fig. 4.11 we shows the subband occupation for the same $\widetilde{V}_{S C}-\widetilde{V}_{g}$ parameter ranges as Fig. 4.9 , but for a thicker wire with $R=50 \mathrm{~nm}$. On the one hand, wires with larger diameters may be less prone to disorder, as compared to thinner wires, but, on the other hand, the reduced confinement implies a smaller intersubband energy spacing. This leads to a higher subband occupancy for any given set of potential parameters, as manifest from the comparison of Figs. 4.9 and 4.11. Note that the $R=35 \mathrm{~nm}$ and $R=50 \mathrm{~nm}$ wires generate qualitatively similar trends, with the thicker wire being associated with higher occupancies. It is still possible to tune the system through several subbands before the hole states become populated. However, the gate voltages associated with the emergence of holes shift with increasing $R$ toward less negative 
values, due to the combined effects of reduced confinement, reduced electrostatic screening, and larger separation between the back gate and the superconductor. This implies that, if attaining the few-subband regime (ideal for realizing MZMs) is not possible by gating a given system (due to the emergence of holes), reducing the diameter of the wire is a possible solution.

The fundamental reason why high subband occupancy is detrimental to realizing Majorana bound states is that the corresponding (typical) value of the inter-subband energy spacing for subbands near the Fermi energy is significantly reduced as compared with the few-subband regime. On the one hand, this is due to a weaker transverse confinement of the high-energy subbands (as compared to the low-energy subbands) by the effective potential $\phi(\vec{r})$ and, on the other hand, to a larger effective mass associated with highenergy subbands (which is an effect of the non-parabolicity of the conduction band). Note that our effective mass approximation does not capture the second effect, which requires a multi-orbital modeling of the semiconductor nanowire [46, 109]. The reduced inter-subband energy separation associated with high subband occupancy makes the system susceptible to perturbations such as disorder and inhomogeneous effective potentials due to the presence of multiple gates, strain, etc. If the subbands are not well separated, these perturbations lead to inter-subband mixing [72, 132], which, in turn, induces (topologically-trivial) low-energy Andreev bound states (ABSs) [122]. Note that this type of near-zero energy states are generic in class D systems [74, 133] and were explicitly shown to emerge in proximitized nanowires (in the presence of spin-orbit coupling and nonzero Zeeman field) within a large window of local gate potentials [72,122]. We emphasize that the emergence of this type of low-energy states does not require the explicit presence of disorder, as multiple subband occupancy leads to an effective random matrix Hamiltonian in the presence of any perturbation that couples the subbands (e.g., applied gate potentials, strain, etc.). Finally, note that certain types of trivial low-energy states - the so-called quasi-Majorana [94], or partiallyseparated ABS states [67] - mimic very faithfully the local phenomenology of Majorana zero modes [68, 108].

To evaluate the dependence of the inter-subband energy separation on the relevant system parameters, we calculate the average subband energy separation near the Fermi level as functions of the (shifted) gate potential $\widetilde{V}_{g}$ and the (shifted) work function difference $\widetilde{V}_{S C}$ for two wires with radii $R=35 \mathrm{~nm}$ and $R=50 \mathrm{~nm}$. The results are shown in Figs. 4.12 and 4.13, respectively. The average subband separation near the Fermi level is defined as follows. Let the $n^{\text {th }}$ subband be the "Fermi subband", i.e. the subband having its bottom closest to the Fermi level. The energy at the bottom of the Fermi subband is $E_{n}$. We define the average subband separation as the weighted average of the gaps between the Fermi subband and the subbands immetiadely below and above it. Explicitly, we have

$$
(\Delta E)_{\text {ave }}=\lambda\left(E_{n}-E_{n-1}\right)+(1-\lambda)\left(E_{n+1}-E_{n}\right),
$$

where $\lambda=\left(E_{n+1}+E_{n}\right) /\left(E_{n+1}-E_{n-1}\right)$. We first point out that the average 


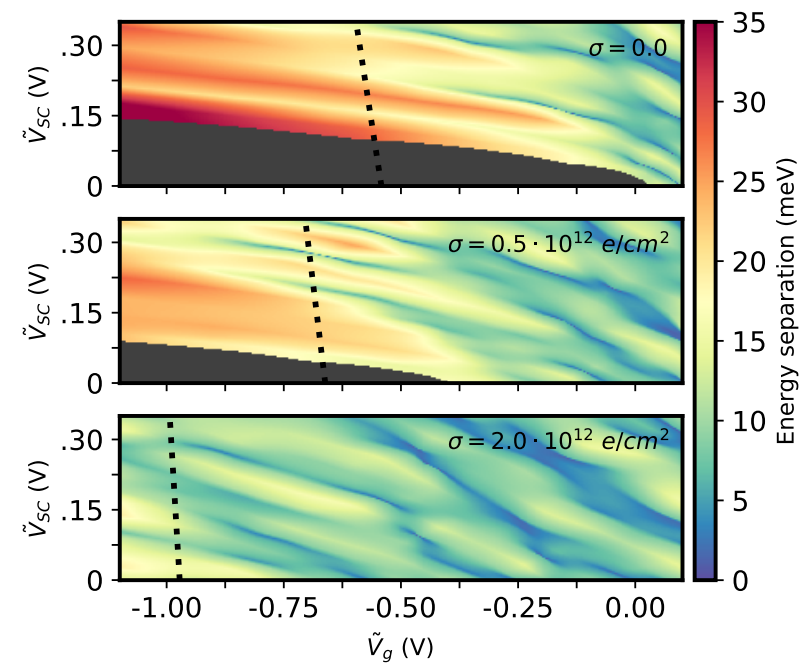

FIGURE 4.12: Average subband separation near the Fermi level, as defined by Eq. (4.73), for a wire of radius $R=35 \mathrm{~nm}$ as function of gate voltage, $\widetilde{V}_{g}$, and SM-SC work function difference, $\widetilde{V}_{S C}$. The panels correspond to three different values of surface charge, $\sigma$, while the dashed lines indicate the emergence of holes.

energy separation tends to increase as $\widetilde{V}_{g}$ becomes more negative. This effect is due to the increased confinement generated by the negative gate potential, which pushes the electron wave function towards the semiconductorsuperconductor interface, combined with the reduction of subband occupation. By contrast, increasing $\widetilde{V}_{S C}$, while also enhancing the effective confining potential, increases the subband occupation as well, the net effect on the subband energy separation being minimal.

The results shown in Figs. 4.12 and 4.13 suggest that the optimal system for realizing robust MZMs would be a thin wire with no surface charge (or a small $\sigma$ ), as the corresponding spectrum is characterized by a large intersubband energy separation for moderate negative gate potentials, before the emergence of holes in the system. For example, the zero surface charge results in Fig. 4.12(a) present an excellent outlook for the realization of MZMs, as the Fermi level subband separation (right before the onset of hole states) is $(\Delta E)_{\text {ave }} \approx 15-30 \mathrm{meV}$, depending upon the exact value of $\widetilde{V}_{S C}$. The situation becomes progressively less promising with the addition of surface charge, as can be seen in Figs 4.12 (b) and (c). This is due to the reduced confinement caused by the positive surface charge pulling the wave functions away from the semiconductor-superconductor interface. Increasing the diameter of the wire further reduces the confinement and, consequently, the subband energy separation, as manifest when comparing the results in Fig. 4.12 and Fig. 4.13. In addition, in thicker wires the hole regime sets in at less negative gate potentials, making the small inter-subband energy separation problem even worse. In Fig. 4.13(c), for example, the subband energy separation is $(\Delta E)_{\text {ave }} \approx 2-7 \mathrm{meV}$ for all acceptable values of the gate voltage (i.e. outside 


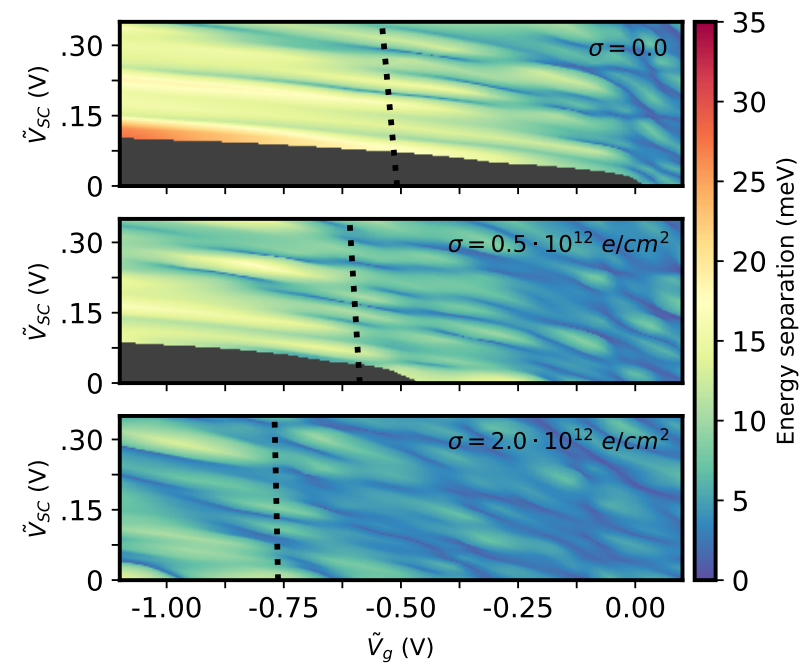

FIGURE 4.13: Same as Fig. 4.12 for a wire of radius $R=50 \mathrm{~nm}$. Note that higher subband occupation (see Figs. 4.9 and 4.11) is consistently associated with smaller inter-band separation.

the hole regime) due to the combined effects of large surface charge and large wire diameter. As an estimate of the relative impact of these factors, we note that the average subband energy separations in Figs. 4.12(c) (large $\sigma$, small $R$ ) and 4.13(a) $(\sigma=0$, large $R)$ are comparable. This points to the importance of using wires with the smallest possible diameter in order to keep the subband occupancy at a minimum. Finally, we note that the effective mass model used here predicts larger subband separation energies than more detailed (multi-orbital) models, which take into account the nonparabolicity of the conduction band [37,46]. Hence, the actual subband separation is expected to be smaller than the predictions based on the results shown in Figs. 4.12 and 4.13. These nonparabolicity effects are larger for high-energy subbands and will generate an additional reduction of the inter-band separation in the high-occupation regime. Thus, our calculations presented here provide the most optimistic estimates for inter-subband energy separations with the corresponding realistic level separations in experimental nanowires likely being even smaller.

Our results show clearly that i) regimes characterized by many occupied subbands are possible and even likely within realistic windows of system parameters, ii) gating the system to reach the few-subbands regime is usually not possible (except narrow parameter windows) due to the onset of holes, and iii) the many-subbands regime is typically associated with small values of the inter-subband energy spacing near the Fermi level, which has highly detrimental consequences for the realization and observation of robust Majorana zero modes. The natural question is how to measure experimentally the subband occupation of a proximitized wire? This would facilitate the optimization of the growth and fabrication procedures in order to reach the 'ideal' few-subbands regime. Our key observation is that the most striking feature associated with a quasi-1D subband becoming occupied is a van 

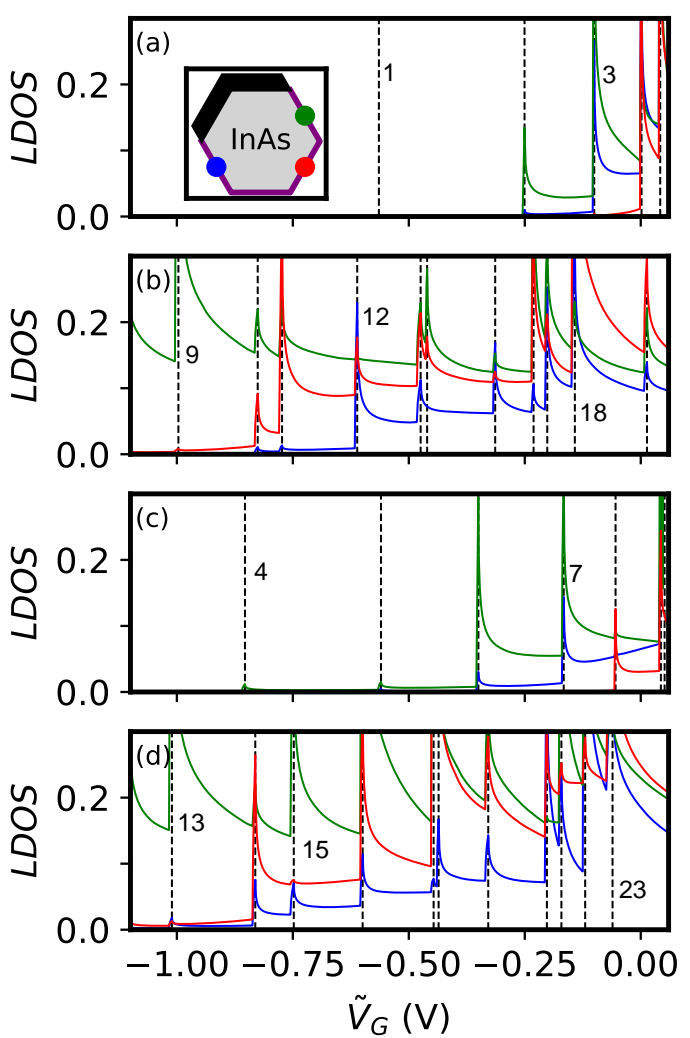

FIGURE 4.14: Local density of states at zero energy versus (shifted) gate voltage $\widetilde{V}_{g}$ for a wire of radius $R=35 \mathrm{~nm}$. The LDOS is normalized with respect to the maximum in each panel. The three sets of lines correspond to the local density of states in the regions marked by the corresponding colored dots in the insert of panel (a). The dashed lines indicate the $\widetilde{V}_{g}$ values where the bottom of a pair of spin subbands cross the Fermi level. Numbers next to dashed lines indicate subband pair index. The model parameters corresponding to different panels are: (a) $\widetilde{V}_{S C}=0.1 V, \sigma=0$, (b) $\widetilde{V}_{S C}=0.1 V, \sigma=2 \cdot 10^{12} \mathrm{e} \cdot \mathrm{cm}^{2}$, (c) $\widetilde{V}_{S C}=0.25 \mathrm{~V}, \sigma=0$, (d) $\widetilde{V}_{S C}=0.25 \mathrm{~V}, \sigma=2 \cdot 10^{12} \mathrm{e} \cdot \mathrm{cm}^{2}$.

Hove singularity in the density of states (DOS), or the local DOS (LDOS), at the Fermi energy. This is the well-known square-root van Hove singularities associated with 1D subbands in semiconductor quatum wires pointed out more than thirty years ago [134]. Probes that are highly sensitive to this van Hove singularity would be good candidates for experimentally estimating the subband occupation.

A straightforward and powerful tool for measuring the local density of states is scanning tunneling spectroscopy [135]. To anticipate the capabilities and limitations of such a tool, we calculate the LDOS on different facets of a proximitized nanowire with a transverse profile as shown in Fig. 4.8. More specifically, we assume that the system is in the normal state (i.e. above the superconducting critical temperature) and that the SM-SC coupling is not too high (i.e. it is not much larger than the gap of the parent superconductor) and focus on the LDOS at zero energy as a function the applied gate potential 
$\widetilde{V}_{g} \cdot{ }^{16}$ As the applied potential becomes more negative and the occupied SM conduction subbands are depleted, the presence of a subband bottom near the Fermi energy is signaled by a peak in the LDOS. The results for a wire of radius $R=35 \mathrm{~nm}$ and different representative combinations of $\widetilde{V}_{S C}$ and $\sigma$ values are shown in Fig. 4.14. A similar plot for $R=50 \mathrm{~nm}$ is shown in Fig. 4.15. The blue, red, and green lines correspond to the LDOS on the three exposed facets of the wire (which are potentially accessible to an STM measurement), as indicated by the corresponding colored dots in the inset of Fig 4.14(a). The dashed lines indicate the $\widetilde{V}_{g}$ values corresponding to the bottom of a pair of (degenerate) spin subbands crossing the Fermi level, while the numbers indicate the index of the corresponding pair.

The first significant feature is the presence of well-defined peaks in the LDOS (due to van Hove singularities) that coincide with subband bottoms crossing the Fermi energy (dashed lines). Note that the peaks measured on different facets that are associated with the same subband pair occur at exactly the same value of $\widetilde{V}_{g}$, but may have significantly different amplitudes. These amplitudes depend on the transverse profile of the subband, and, in general, are different on different facets of the wire. Moreover, the lowest energy subbands are "pushed away" by the negative gate potential and strongly confined near the SM-SC interface. Consequently, they have negligible amplitudes on the exposed facets of the wire and are practically "invisible" in a LDOS measurement. For example, the first three subband pairs in Fig. 4.15(a) have no visible peak in the LDOS, while the fourth pair generates a significant signal only on the "green" facet (i.e. close to the SM-SC interface and as far as possible from the potential gate). This is illustrated in Fig. 4.16 (a) and (b), which shows the profiles of the eigenstates for the first and fourth subband pairs, respectively. The state shown in panel (a) is confined at the SM-SC interface and has negligible weight on any exposed surface, which make it "invisible" to a LDOS probe. By contrast, the state shown in panel (b) has some weight on the "green" facet, making it potentially "visible" in a

\footnotetext{
${ }^{16}$ To calculate the LDOS of states, we first note that the dispersion of the $n^{\text {th }}$ subband is given by

$$
E_{n}(k)=E_{n, o}+\frac{\hbar^{2} k^{2}}{2 m^{*}}
$$

where $E_{n, 0}$ is the energy of the subband bottom. This translates into a density of states

$$
D_{n}(E)=\frac{1}{2 \pi} \sqrt{\frac{m^{*}}{\hbar^{2}}} \frac{\Theta\left(E-E_{n, o}\right)}{\sqrt{E-E_{n, o}}},
$$

where $\Theta(E)$ is the Heaviside step function. We note the strong 1D square-root van Hove singularity in Eq. 4.75. The LDOS is then obtain by multiplying the density of states of each subband by the weight of the wavefunction inside the spatial region interest. Explicitly, we have

$$
\operatorname{LDOS}(E)=\sum_{n} D_{n}(E) \int_{\Omega}\left|\psi_{n}(\vec{r})\right|^{2} d \vec{r},
$$

where the sum runs over all subbands and $\Omega$ is the region of interest. We note that the wavefunction of each subband is independent of $k$ within this effective mass model. To eliminate the singularity in Eq.(4.75) coming from the subband bottoms, we average the density of states over a small energy interval $\delta E=0.2 \mathrm{meV}$.
} 

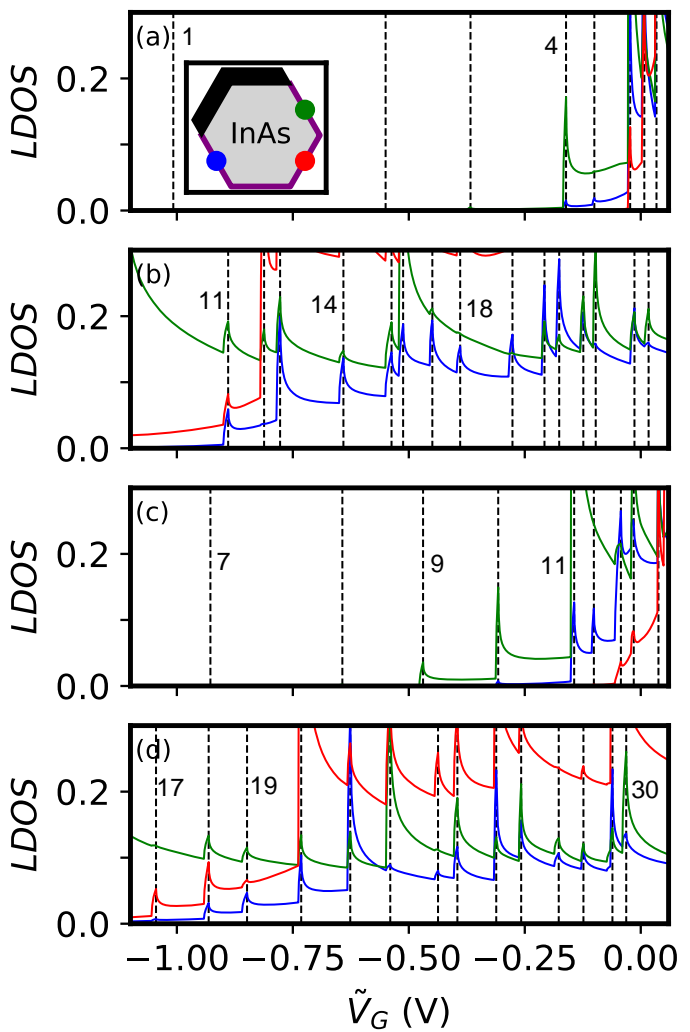

FIGURE 4.15: Same as Fig. 4.14 for a wire of radius $R=50 \mathrm{~nm}$. Note that the ranges of gate potentials over which LDOS peaks are visible are similar to those in Fig. 4.14, but there are more "invisible" subbands.

LDOS measurement on that facet. The existence of "invisible" subbands prohibits a simple counting of the subband occupation number. In other words, the number of observed LDOS peaks as function of the applied gate potential (obtained, for example, in an STM experiment) is typically lower than the number of occupied subbands.

Nonetheless, the very observation of a peaked LDOS structure would have enormous implications and would contain significant information regarding the hybrid system and its suitability for realizing Majorana physics. First, the presence of sharp LDOS peaks signals the existence of well defined subbands within the nanowire. Disorder will generically broaden these peaks, as states become localized within different regions of the wire [134]. In the presence of strong disorder, the peaked structure is washed out, the notion of subband becomes meaningless, and the possibility of realizing Majorana zero modes is practically lost. For the hybrid system to be able to host MZMs, it is therefore essential that the nanowire mobility in the SMSC hybrid be large, so that any disorder-induced collisional broadening is smaller than the inter-subband energy separation[134]. Moreover, even if a peaked structure can be observed, one has to check its independence on the position along the wire. Basically, a structure dependent of the position 

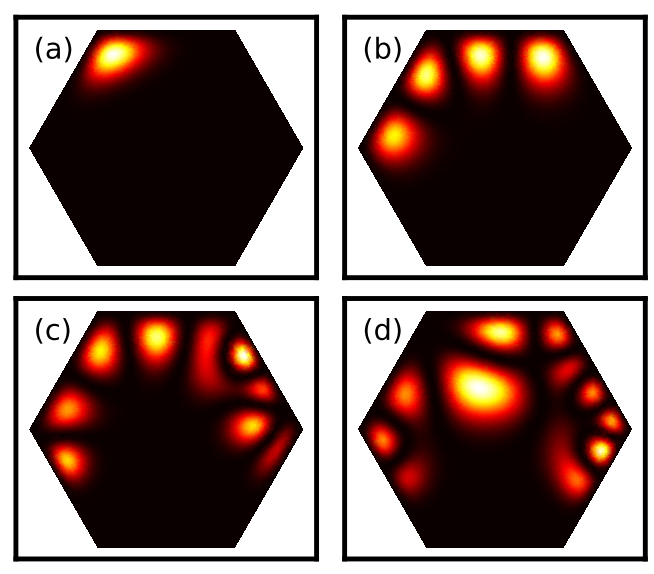

FIGURE 4.16: Wave function profiles, $|\psi|^{2}$, corresponding to different confinement-induced subbands marked by dashed lines in Fig. 4.15. Panels (a) and (b) correspond to the first and fourth subband pairs (dashed lines 1 and 4) in Fig. 4.15(a), respectively, while (c) and (d) correspond to the subbands marked by dashed lines 11 and 14 in Fig. 4.15(b), respectively. Note that the state in (a) has negligible weight near any of the three exposed facets, which explains its "invisibility" in the LDOS.

along the wire is a clear indication of long-range effective potential variations, a scenario likely to lead to the emergence of partially-separated ABSs (i.e. quasi Majoranas), rather than well-separated MZMs. Finally, each identification of a LDOS peak (corresponding to the bottom of a subband being at the Fermi level) provides a value of the gate potential at which Majorana physics should be explored. In other words, if the system can support MZMs (i.e. if it is uniform and clean enough), the optimal gate voltage regimes for the realization of these MZMs will necessarily correspond to the chemical potential being close to the bottom of a confinement-induced subband, which is indicated by the LDOS peak.

The details of the LDOS structures shown in Figs. 4.14 (wire of radius $R=35 \mathrm{~nm}$ ) and 4.15 (wire of radius $R=50 \mathrm{~nm}$ ) reveal additional information about the system. For example, let us compare a wire with no surface charge, $\sigma=0$, panels (a) and (c), and a wire with large surface charge density, $\sigma=2 \cdot 10^{12} \mathrm{e} / \mathrm{cm}^{2}$, panels (b) and (d). In the absence of surface charge the LDOS for all three facets shows a peak structure that diminishes rapidly as $\widetilde{V}_{g}$ become more negative and the wave functions become strongly confined near the SM-SC interface. By contrast, the LDOS peaks for the wire with large surface charge remain moderately large across the whole scan of $\widetilde{V}_{g}$. In particular, the LDOS peaks in the upper right facet region (green curve) are quite large. The qualitative differences between the two cases are determined by the attractive potential associated with the positive surface charge, which keeps a significant fraction of the wave function weight near the exposed edges making them "visible" in the LDOS, while the negative gate potential 
tends to push the states towards the semiconductor-superconductor interface. This is illustrated in Fig. 4.16 (c) and (d), which show the eigenstates of the eleventh and fourteenth subband pairs, respectively, for a wire with large surface charge density. The upper right facet maintains especially prominent peaks because of its larger spatial separation from the repulsive back-gate. Note that the observation of multiple LDOS peaks - as, e.g., in the case of the states shown in Fig. 4.15 panels (b) and (d) - is a clear indication of high subband occupancy, a regime that is not favorable to the realization of stable MZMs, as argued above. Also, the observation of only a few peaks over a moderate range of gate potentials is not necessarily an indication that the system is in the few-subband regime [see, e.g., Fig. 4.15(c)]. However, combined with a reduction of the wire diameter, such an observation indicates a clear path toward the few-subbands regime. Note that the qualitative (and even some of the semi-quantitative) features are very similar in the corresponding panels of Figs. 4.14 and 4.15. Assume, for example, that the system is in a regime corresponding to panels (c), i.e., $\widetilde{V}_{S C}=0.25 \mathrm{~V}, \sigma=0$, and that the lowest observed peaks occur around $\widetilde{V}_{g} \approx-0.3 \mathrm{~V}$. In the thinner wire [Fig. 4.14(c)] the peak corresponds to the $n=6$ subband pair (i.e. 12 occupied spin sub-bads), while in the thicker wire [Fig. 4.15(c)] the peak corresponds to the $n=10$ subband pair (i.e. 20 occupied spin sub-bads). All other conditions being equal, the thinner wire is clearly more suitable for realizing stable MZMs. Finally, we emphasize that i) the LDOS measured in the normal states (i.e. above the critical superconducting temperature) already contains a wealth of information about the feasibility of Majorana physics in a SM-SC hybrid system and, should experimental LDOS data become available (e.g., from future STS measurements on SM-SC structures), ii) it is essential to perform detailed numerical studies (using realistic models) of the dependence of the LDOS features on key factors, such as disorder and long range potential inhomogeneities. We emphasize that experimental studies of nanowire LDOS manifesting clear 1D van Hove singularities at the subband bottoms (which necessitate that the inter-subband energy separations are larger than disorder broadening) along with the occupancy of only a few (ideally just one) orbital subband(s) are essential steps in advancing the search for MZMs in SM-SC hybrid systems.

\section{InSb}

InSb, another III-V semiconductor often used in the fabrication of Majorana devices, is characterized by an effective mass significantly lower than InAs. To asses the consequences of having a lower effective mass, we provide a calculation of the subband occupation and energy separation for InSb similar to the calculation done for InAs in the main text. We use the same device setup and values of the material parameters corresponding to InSb: $m^{*}=0.015 m_{0}$ and $\epsilon_{\text {wire }}=16.8 \epsilon_{0}$. The materials parameters for the corresponding Luttinger model are $\gamma_{1}=34.8, \gamma_{2}=15.5, \gamma_{3}=16.5$, and $E_{\text {gap }}=0.235 \mathrm{eV} \mathrm{[46].} \mathrm{InSb} \mathrm{does} \mathrm{not} \mathrm{have} \mathrm{a} \mathrm{large} \mathrm{surface} \mathrm{charge} \mathrm{density,}$ like InAs, so we set $\sigma=0$. The subband occupation and average separation 


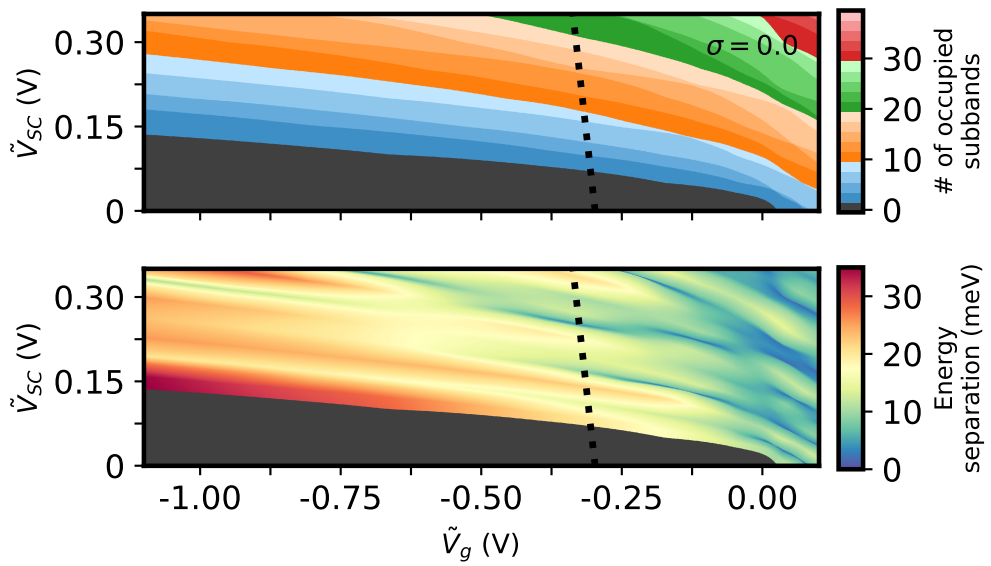

FIGURE 4.17: (a) Subband occupation for an InSb wire of radius $R=50 \mathrm{~nm}$ as function of the (shifted) gate potential $\widetilde{V}_{g}$ and the (shifted) work function difference $\widetilde{V}_{S C}$. The negative gate voltage regime to the left of the dashed lines is characterized by the emergence of holes at the bottom of the nanowire (i.e. near the interface with the dielectric). (b) Average subband separation near the Fermi level, as defined by Eq. (4.73).

near the Fermi level for an InSb nanowire of radius $R=50 \mathrm{~nm}$ are shown in Fig. 4.16 (a) and (b), respectively. Comparing the InSb subband occupation to that of the InAs wire in Fig. 4.11(a), we notice that the InSb wire has slightly fewer occupied subbands for a given set of potentials due to its smaller effective mass, $m_{I n S b}^{*} / m_{I n A s}^{*}=0.57$. This effect is also seen in Fig. 4.17(b), which shows larger subband separations than the InAs results shown in 4.13(a). However, while the reduced subband occupation of InSb may provide some advantage, note that the gate voltage at which holes begin to emerge (dashed lines in Fig. 4.17) is shifted to significantly less negative values compared to InAs due to the reduced bandgap of the InSb. This small bandgap inhibits $\mathrm{InSb}$ nanowires of reaching the few subband occupation regime except for small $\widetilde{V}_{S C}$, just as in the InAs case. Lastly, we note that non-parabolicity effects (not included in our calculations) are stronger in InSb due to the small band gap. Therefore, the subband separation in the large subband occupation regime may be significantly reduced from the values predicted by the effective mass model used here. We conclude that, although the lower effective mass may provide some advantages, using InSb does not automatically solve the high-occupancy problem in Majorana devices. Since this is a potentially serious problem, it should be further investigated, both theoretically and experimentally, with the goal of identifying controlled procedures for driving the system in the few-subbands regime.

\section{Estimation of spin-orbit coupling}

While the main focus of this section is understanding subband occupancy, our calculations also provide an estimate of the expected Rashba spin-orbit 
coupling as explained in Sec. 4.6.1. The spin-orbit coupling strength is crucial for the realization of Majorana bound states. Fig. 4.18 shows the Euclidean sum of the diagonal Rashba coefficients, $\left(\alpha_{n}=\sqrt{\left(\alpha_{x}\right)_{n n}^{2}+\left(\alpha_{y}\right)_{n n}^{2}}\right)$, for the eigenstate closest to the Fermi level as a function of gate voltage, $\widetilde{V}_{g}$. Results for InAs and InSb are shown in Fig. 4.18 (a) and (b), respectively. We consider two values of the work function difference, $\widetilde{V}_{S C}=0.15,0.3 \mathrm{~V}$, along with the effects of zero and nonzero surface charge for InAs. The radius is taken to be $R=50 \mathrm{~nm}$. Beginning with the zero surface charge cases (blue and green dots), we observe that spin-orbit coupling generally increases with increasing the magnitudes of both $\left|\widetilde{V}_{g}\right|$ and $\widetilde{V}_{S C}$. This is due to the increasing of the electric field as the difference between $\widetilde{V}_{S C}$ and $\widetilde{V}_{g}$ grows. Ignoring the jumps (associated with different subbands being near the Fermi level), $\alpha$ is roughly linear in $\widetilde{V}_{g}$, as should be expected from Eqns. (4.71 and 4.72). Also note that $\alpha$ is significantly higher for InSb (as compared to InAs) due to its smaller bandgap. The addition of surface charge on the uncovered InAs facets (red and orange dots) results in a significant reduction of the spin-orbit coupling, as shown in Fig. 4.18(a). This can be understood as the surface charge partially counteracting the structural asymmetry caused by the difference between $\widetilde{V}_{S C}$ and $\widetilde{V}_{g}$. The electrostatic potential becomes more uniform with the addition of the surface charge and therefore suppresses the Rashba spinorbit coupling. This reduction of the spin-orbit coupling points to another concern stemming from the presence of surface charge on InAs nanowires.

\subsubsection{Summary}

We have studied the subband occupation of proximitized InAs nanowires as a function of the applied gate potential and the semiconductor-superconductor work function difference in the presence of surface charges using an effective mass model and solving the corresponding Schrödinger-Poisson equations self-consistently. We find that for realistic values of the surface charge density the system is characterized by many occupied subbands, while reducing the occupation and driving the system into a few subband regime using a (negative) gate potential is precluded by the emergence of holes in the semiconductor valence band, unless the system is characterized by a small work function difference $\widetilde{V}_{S C}$. The many subband regime is characterized by small inter-subband energy separation near the Fermi level. The small subband energy separation, in combination with the sheer number of occupied subbands, makes the system susceptible to subband mixing in the presence of disorder, potential inhomogeneities, strain, and other perturbations, which generically produces low-energy Andreev bound states. We emphasize that, even in the absence of disorder, a many subband system with nonzero Zeeman field, spin-orbit coupling, and induced superconductivity effectively behaves as a class D system due to the practically uncontrollable inter-subband coupling that occurs once translational symmetry is broken. Consequently, topologically-trivial low-energy states will emerge generically 


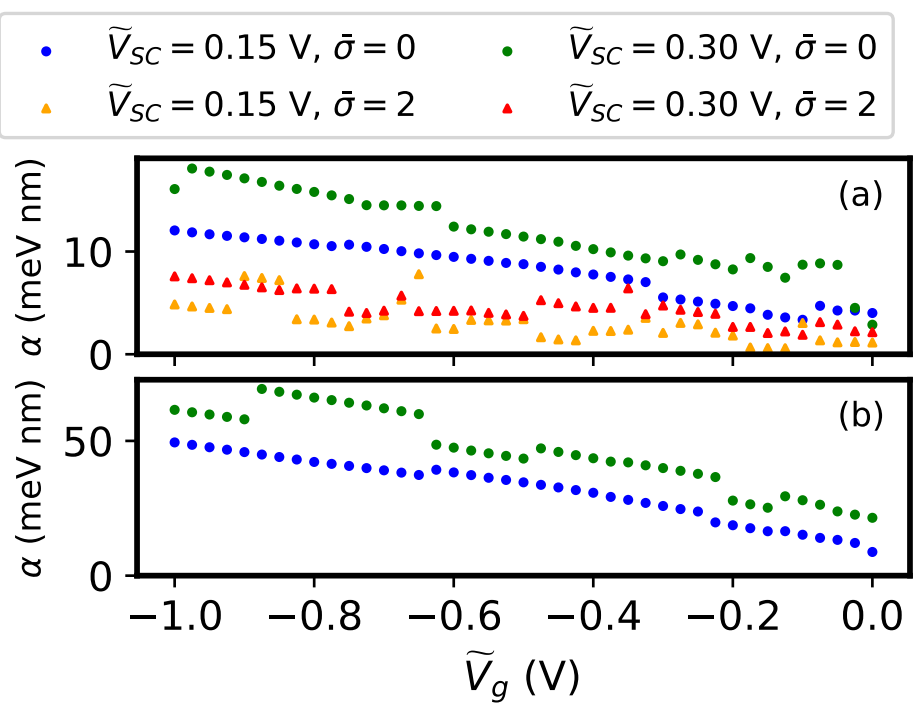

FIGURE 4.18: Diagonal Rashba spin-orbit coefficient for eigenstate closest to the Fermi energy as a function of gate voltage $\widetilde{V}_{g}$ for multiple work function, $V_{S C}$, and surface charge, $\sigma$, values, with $\sigma=\bar{\sigma} \cdot 10^{12} \mathrm{e} / \mathrm{cm}^{2}$. Panels (a) and (b) are for InAs and InSb, respectively. Note in (a) that the presence of surface charge significantly reduces spin-orbit coupling for InAs. Also note that the jumps in $\alpha$ occur due to the eigenstate closest to the Fermi level changing with $\widetilde{V}_{g}$.

in such a system as the control parameters (e.g., gate potentials and Zeeman fields) are tuned. Therefore, the many subband regime should be systematically avoided to ensure the realization of stable topological superconductivity and Majorana zero modes. In other words, in addition to the commonly recognized conditions associated with the realization of topologicallyprotected MZMs - such as having long wires and the absence of disorder having low-occupancy, i.e., chemical potential values consistent with one or a few occupied subbands, represents an essential requirement. We note that using a semiconductor with a lower effective mass, e.g., InSb, does not automatically solve the problem, as illustrated by the results shown near the end of Sec. 4.6.2. A smaller gap leads, on the one hand, to the emergence of holes at less negative values of the gate potential, hindering the depletion of the wire. On the other hand, a smaller gap enhances the non-parabolicity effects (not included in our effective mass model), which results in a further reduction of the inter-subband separation at high occupancy.

All these considerations makes it clear that measuring the subband occupancy and, ultimately, being able to reduce it to the few-subbands regime are critical tasks for building Majorana devices. To identify experimentally systems that may be in the many subband regime and to be able to optimize the growth and fabrication procedures, we propose a measurement of the LDOS on the exposed facets of the nanowire, e.g., using an STM. We note that such a measurement is not capable, in general, to give the exact number of occupied subbands, as states strongly localized near the SM-SC interface 
may remain "invisible". On the other hand, such a measurement can provide critical information regarding the suitability of a SM-SC hybrid structure to host Majorana physics, even when performed in the normal state (i.e. above the critical superconducting temperature). In particular, the observation of peaks in the LDOS as function of the applied gate voltage indicates that the system is clean-enough to have some well-defined subbands. Furthermore, the positions of the peaks provide the values of the gate voltage where Majorana physics should most likely emerge. The presence of disorder is expected to destroy the LDOS peaks, while a LDOS peak structure dependent on the spatial position along the wire may be an indication of long-range effective potential variations, which may generate quasi-Majorana modes. If LDOS peaks are completely absent in the nanowires, the system is simply unsuitable for studying Majorana physics because disorder would then completely suppress the emergent Majorana modes. Even if the LDOS peaks are seen, one must worry about the subband occupancy question, as discussed in depth in our work.

This work is supported by the Laboratory for Physical Sciences and Microsoft. T.D.S. was supported in part by by NSF Grant No. DMR-1414683.

\subsection{Electrostatic effects and topological supercon- ductivity in semiconductor-superconductor-magnetic insulator hybrid wires}

As a second example application of our formalism, we study topological superconductivity in hybrid wires composed of semiconductor, superconductor, and magnetic insulator components. More specifically, we investigate the impact of electrostatics on the proximity effect between the magnetic insulator and the semiconductor of the hybrid structures and how this affects the topological phase diagram. This section is adapted from work original published in Ref. [136].

Recall that a Zeeman splitting of sufficiently high strength is necessary in order to achieve the topological superconducting phase and MZMs in SM-SC nanostructures. Additionally, increasing the Zeeman splitting increases the robustness of the topological phase and resulting MZMs against disorder and generic device non-uniformities. Naively, it then seems that applying an external magnetic field with as large as strength as possible would optimize the chances of creating a robust topological phase. Increasing the field, however, will eventually destroy the superconducting gap of the parent superconductor, causing the loss of any possibility of topological superconductivity. In addition, using an applied magnetic field imposes serious constraints on the possible device layout for Majorana-based topological qubits [137].

A possible solution is to create the required Zeeman field by proximity coupling the semiconductor to a magnetic insulator $[43,44]$. Recently, this possibility has been explored experimentally using InAs nanowires with epitaxial layers of superconducting $\mathrm{Al}$ and ferromagnetic $\operatorname{EuS}[119,138,139]$. A 
key finding was that an effective Zeeman field $\Gamma_{e f f}^{S C}$ of order $1 \mathrm{~T}(\sim 0.05 \mathrm{meV})$ emerges in the superconductor in the absence of an applied magnetic field, but only in nanowires with overlapping shells of superconductor and ferromagnetic insulator [119]. Correlated with the emergence of an effective Zeeman field in the superconductor was the observation of zero-bias conductance peaks for charge tunneling into the end of the semiconductor wire, which is consistent with the presence of topological superconductivity. These features are absent in hybrid structures with non-overlapping $\mathrm{Al}$ and EuS covered facets [119].

The crucial question concerns the physical mechanism responsible for the startling contrast between the phenomenologies observed in the two setups. Furthermore, one may ask if, based on the understanding of this mechanism, one can identify efficient knobs for controlling the magnitude of the effective Zeeman field emerging in the nanowire, to ensure that the topological superconducting phase is accessible and robust.

A natural candidate for explaining the difference between the behaviors associated with the two setups is the ferromagnetic exchange coupling occurring inside the SC in the overlapping geometry due to spin-dependent scattering at the Al-EuS interface [140-144]. In turn, the proximity effect generated by the exchange-coupled superconductor inside the spin-orbit coupled nanowire could lead to the emergence of a topological superconducting state. In this scenario, the effective Zeeman field $\Gamma_{e f f}^{S M}$ required to drive the SM nanowire into the topological regime is induced "indirectly", via the $\mathrm{Al}$ layer. Consequently, it is controlled by the strength $\widetilde{\gamma}$ of the effective coupling between the semiconductor and superconductor, which also determines the size of the induced superconducting gap and the critical Zeeman field associated with the topological quantum phase transition (TQPT) [145]. In particular, the minimum value of the critical field is given by $\widetilde{\gamma}$ and can be significantly larger that the induced gap in the strong coupling limit [145]. This poses a serious problem for the "mediated proximity" scenario. As explicitly shown below, the topological condition $\Gamma_{e f f}^{S M}>\widetilde{\gamma}$ is inconsistent with the experimental parameters reported in Ref. [119] and, more importantly, is generally inconsistent with robust topological superconductivity.

In this section we investigate a different scenario involving the "direct" proximity effect at the semiconductor - magnetic insulator (SM-MI) interface. We show that the strength of the effective Zeeman field $\Gamma_{\text {eff }}^{S M}$ induced in the wire by proximity to the MI is controlled by electrostatic effects, which, in turn, depend on the geometry of the SC layer and on the applied gate potential. In essence, because of the finite work function difference between the SM wire and the SC shell, the wave functions characterizing the low-energy states in the wire are strongly "attracted" toward the superconductor, regardless of whether the SM and SC are in direct contact or separated by a MI layer. This means away from the SM-MI interface in the non-overlapping setup and toward the SM-MI interface in the system with overlapping MI and SC layers (see Fig. 4.19). As a result, the induced $\Gamma_{e f f}^{S M}$ has significantly higher values in the overlapping structure as compared to the non-overlapping setup. 




FIGURE 4.19: Top panels: Schematic representation of the hybrid structure studied in this work corresponding to a semiconductor nanowire (yellow) with (a) non-overlapping (setup 1) and (b) overlapping (setup 2) layers of superconductor (pink) and magnetic insulator (green). An external potential is applied using a back-gate (black) separated from the wire by an insulating dielectric layer (gray). Parameters: $R=50 \mathrm{~nm}, d=10 \mathrm{~nm}$. Bottom panels: Wave function profile of the second lowest transverse mode for parameters corresponding to this mode being near the Fermi level: $V_{g} \approx-1.1 \mathrm{~V}, V_{S C}=0.15 \mathrm{~V}$.

By performing self-consistent Schrödinger-Poisson calculations, we demonstrate that the overlapping setup is consistent with the emergence of topological superconductivity over a large window of system parameters and applied gate potentials, in sharp contrast with the non-overlapping structure. Our findings support the feasibility of topological superconductivity in SMSC-MI hybrid structures and provide guidance for controlling the system and enhancing the robustness of the topological phase.

\subsubsection{Mediated Proximity Scenario}

Before we present our model calculations, let us briefly discuss the "mediated proximity" scenario. In the strong coupling limit, satisfying the topological condition $\Gamma_{\text {eff }}^{S M}>\widetilde{\gamma}$ requires a large effective Zeeman field $\Gamma_{\text {eff }}^{S C}$ inside the SC, possibly exceeding the Chandrasekhar-Clogston (CC) limit [146, 147]. Even assuming that spin-orbit coupling induced by proximity to the SM wire prevents the closing of the SC gap, its value (and, implicitly, the size of the topological gap) will be very small. On the other hand, in the weak/intermediate regime the induced SC gap and effective Zeeman field 
are approximately given by [145]

$$
\Delta_{i n d} \approx \frac{\tilde{\gamma} \Delta}{\widetilde{\gamma}+\Delta^{\prime}}, \quad \Gamma_{e f f}^{S M} \approx \frac{\widetilde{\gamma} \Gamma_{e f f}^{S C}}{\widetilde{\gamma}+\Delta^{\prime}},
$$

where $\Delta$ is the order parameter of the parent SC in the presence of the ferromagnetic exchange coupling generated by the MI. Using these relations, we can rewrite the topological condition $\Gamma_{e f f}^{S M}>\widetilde{\gamma}$ in terms of the effective Zeeman field inside the SC and the induced gap as

$$
\Gamma_{e f f}^{S C}>\frac{\Delta^{2}}{\Delta-\Delta_{\text {ind }}} \geq \Delta
$$

First, we note that the parameters characterizing the recent experiment [119], i.e., $\Gamma_{\text {eff }}^{S C} \sim \Delta_{\text {ind }} \sim 0.05 \mathrm{meV}$, do not satisfy Eq. (4.78). Second, note that the CC limit restricts the Zeeman field to [146, 147]

$$
\Gamma_{e f f}^{S C} \leq \frac{\Delta}{\sqrt{2}}
$$

in order for the superconducting state to survive. Clearly, Eq (4.78) and Eq. (4.79) can not be satisfied simultaneously. This leads to the conclusion that the "mediated proximity" mechanism does not enable the realization of robust topological superconductivity in SM-SC-MI hybrid structures and suggests that the investigation of the "direct" proximity effect at the SM-MI interface is critical for understanding the low-energy physics in these systems.

\subsubsection{Model}

The SM-SC-MI hybrid system studied in this section is represented schematically in the top panels of Fig. 4.19. We focus on two setups corresponding to the non-overlapping (setup 1 in Fig. 4.19) and overlapping (setup 2) configurations investigated in the recent experiment [119]. We do not address explicitly the proximity effect between the MI and the SC (in setup 2), but focus instead on the impact of electrostatics on the proximity-induced Zeeman field and pairing potential at the SM-MI and SM-SC interfaces, respectively.

The SM-SC-MI hybrid system, assumed to be infinitely long, is described by the Hamiltonian

$$
H\left(k_{z}\right)=H_{o}\left(k_{z}\right)+H_{b} .
$$

The first term, which includes the SM wire and the SC and MI layers, is given by

$$
\begin{aligned}
H_{o}\left(k_{z}\right)= & {\left[-\nabla_{\perp} \cdot \frac{\hbar^{2}}{2 m^{*}\left(\mathbf{r}_{\perp}\right)} \nabla_{\perp}+\frac{\hbar^{2} k_{z}^{2}}{2 m^{*}\left(\mathbf{r}_{\perp}\right)}-\mu\left(\mathbf{r}_{\perp}\right)-e \phi\left(\mathbf{r}_{\perp}\right)\right] \tau_{z}, } \\
& +\left[\Gamma\left(\mathbf{r}_{\perp}\right) \sigma_{z}+\alpha\left(\mathbf{r}_{\perp}\right) k_{z} \sigma_{y}\right] \tau_{z}+\Delta\left(\mathbf{r}_{\perp}\right) \sigma_{y} \tau_{y}
\end{aligned}
$$




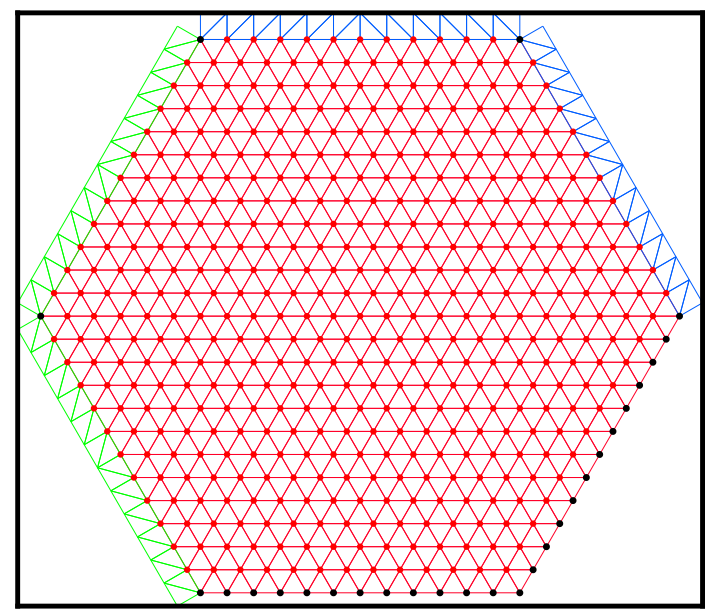

FIGURE 4.20: Finite element mesh used for simulation of setup 1 of Fig. 4.19. A basis function is associated with each vertex in the mesh. The SM region is shown in red. A single layer of the MI and SC meshes are shown in green and blue, respectively. The rest of the MI and SC regions extend to infinity by repeating the shown single layers, but the degrees of freedom from these regions are integrated out. The spacing between vertices is exaggerated in this figure for clarity. In the actual simulation mesh, the vertex spacing in the SM is $2 \mathrm{~nm}$, while in the MI and $\mathrm{SC}$ regions the vertex spacing is $0.01 \mathrm{~nm}$.

where $m^{*}$ is the subsystem-dependent effective mass, $\nabla_{\perp}$ is the nabla operator in the $x y$-plane (i.e., transverse to the wire axis), $\mu$ is the chemical potential, $\phi$ is the electrostatic potential, $\Gamma$ is the Zeeman energy, $\alpha$ is the Rashba spin-orbit coefficient, $\Delta$ is the superconducting pairing, $k_{z}$ is the Bloch wavenumber along the length of the wire, and $\sigma_{i}$ and $\tau_{i}$ are Pauli matrices acting in spin and particle-hole space, respectively. Note that these parameters are piece-wise functions with respect to the SM, SC, and MI regions. In particular, $\alpha, \Delta$, and $\Gamma$ are uniform and non-zero only within the SM, SC, and $\mathrm{MI}$ regions, respectively. Each region, therefore, provides a necessary ingredient for topological superconductivity, as captured by, e.g., the minimal 1D models $[8,9]$. For simplicity, we have neglected transverse spin-orbit coupling. The three regions are characterized by different effective masses and chemical potential values (relative to the bottom of the corresponding bands). Specifically, we have $m_{S M}^{*}=0.023 m_{0}, m_{S C}^{*}=m_{0}, m_{M I}^{*}=m_{0}$ [148] (where $m_{0}$ is the free electron mass), $\mu_{S M}=0, \mu_{S C}=10 \mathrm{eV}$, and $\mu_{M I}=-1 \mathrm{eV}$ [138].

We model the SC region of the device as a semi-infinite bulk superconductor, which avoids including disorder as an ingredient needed to reproduce the experimentally observed induced gaps [116] for systems with thin superconductor layers. As shown in Fig. 4.20, a bulk superconducting region is attached to each facet of the SM which is in direct contact with the SC. Note that we do not attach a bulk superconducting region to the top of the MI region in setup 2 (see Fig. 4.19(b)), since we are not considering the direct 
proximity effects between the MI and SC regions. To control the coupling between the SM and bulk SC(s), we include a barrier potential at the SM-SC interface,

$$
H_{b}=V_{b} \sum_{n} \delta\left(u_{n}-\sqrt{3} R / 2\right) \tau_{z}
$$

where $V_{b}$ is the barrier strength, $\delta$ is the Dirac delta function, the sum runs over all SM-SC interfaces, and $u_{n}$ is the coordinate normal to the $n^{\text {th }}$ SM-SC interface.

Similarly, we also model the MI as a bulk magnetic insulator by attaching a bulk MI region to each facet of the SM which is in direct contact with the MI (see Fig. 4.20). Note that the wavefunction exponentially decays into the MI due to the large negative chemical potential $\mu_{M I}$ of the MI. The decay length is extremely small $(<1 \mathrm{~nm})$, so even a MI layer of a few nm's behaves essentially identical to an semi-infinite MI region.

Inclusion of semi-infinite bulk SC and MI regions inhibits us from performing a straightforward, brute-force numerical calculation in which the SC and MI degrees of freedom are included in a finite dimensional Hamiltonian matrix. To overcome this issue, we employ a Green's function approach in which the degrees of freedom of the bulk SC and MI regions are integrated out and included as a self-energy. The Green's function restricted to the SM is given by

$$
G_{S M}\left(k_{z}, \omega, \Gamma\right)=\left(\omega S_{S M}-H_{S M}\left(k_{z}\right)-\Sigma_{M I}\left(k_{z}, \omega, \Gamma\right)-\Sigma_{S C}\left(k_{z}, \omega\right)\right)^{-1},
$$

where $H_{S M}$ is the matrix representation of the Hamiltonian (4.80) restricted to the SM region (red region in Fig. 4.20), $\Sigma_{M I}$ and $\Sigma_{S C}$ are the momentum and energy dependent self-energies due to the MI and SC semi-infinite regions, respectively, and $S_{S M}$ is the overlap matrix whose elements are the overlap of the SM basis functions. The self-energies are computed using the surface Green's functions of the semi-infinite regions, which are calculated using an accelerated iterative algorithm $[149,150]$ as described in Appendix D. The MI self-energy is only weakly dependent on $k_{z}$ and $\omega$ over the relevant inverse length and energy scales of the problem due to the large effective mass $m_{M I}^{*}$ and large negative chemical potential $\mu_{M I}$ of the MI. It can therefore be well approximated by simply ignoring such dependencies. We approximate the MI self-energy as

$$
\Sigma_{M I}\left(k_{z}, \omega, \Gamma\right) \rightarrow \Sigma_{M I}(0,0, \Gamma)=\Sigma_{M I}^{\text {static }}(\Gamma) .
$$

The electrostatic potential $\phi$ is governed by the Poisson equation,

$$
-\nabla_{\perp} \cdot \varepsilon\left(\mathbf{r}_{\perp}\right) \nabla_{\perp} \phi\left(\mathbf{r}_{\perp}\right)=\rho\left(\mathbf{r}_{\perp}\right),
$$

where $\varepsilon$ is the region-dependent permittivity and $\rho$ is the charge density. The potential is subject to Dirichlet boundary conditions on the back-gate, $V_{g}$, and superconductor, $V_{S C}$, e.g. the superconductor is treated as a perfect metal with regards to the electrostatics. Solving the Schrödinger-Poisson equations 
in the presence of the bulk superconductor is a non-trivial task due to the continuum of states with energies outside of the superconducting gap. This requires an extension of the formalism presented in Sec. 4.1, and is left for future work. We therefore work in the limit of $V_{b} \rightarrow \infty$ (e.g. the SC is uncoupled from the SM and MI regions within the Hamiltonian) when solving for the electrostatic potential, $\phi$, self-consistently. The resulting potential is then used in the Hamiltonian for non-infinite $V_{b}$. Note that the presence of the superconductor still plays a key role in determining the potential since it provides a boundary condition for the Poisson equation. Note that approximating the MI self-energy in Eq. (4.84) in the static limit causes the effective Hamiltonian in the absence of the SC self-energy to be energy independent, i.e. we define the effective Hamiltonian,

$$
H_{\text {eff }}^{\phi}\left(k_{z}, \Gamma\right)=H_{S M}\left(k_{z}\right)+\Sigma_{M I}^{\text {static }}(\Gamma) .
$$

We therefore can directly apply the formalism from Sec. 4.1 when solving for the electrostatic potential $\phi$ self-consistently. Note that we have used the superscript $\phi$ to indicate that this effective Hamiltonian is used for solving for $\phi$ self-consistently. Also note that we set $\Gamma=0$ in Eq. (4.86) when solving for $\phi$.

Eigenstates of the full Hamiltonian (including the bulk SC) are indicated by the poles of the Green's function (4.83). We are particularly interested in finding zero-energy states, since they indicate topological phase transitions. This is equivalent to finding the zero-energy states of another effective, energy-independent Hamiltonian,

$$
H_{\text {eff }}^{\text {top }}\left(k_{z}, \Gamma\right)=H_{S M}\left(k_{z}\right)+\Sigma_{M I}^{\text {static }}(\Gamma)+\Sigma_{S C}\left(k_{z}, 0\right),
$$

where the zero-energy SC self-energy is used. This effective Hamiltonian completely describes the system with respect to calculating the topological phase diagram. Note that we have used the superscript "top" to indicate that this effective Hamiltonian is used solely to calculate the topological phase diagram. Importantly, the effective Hamiltonian (4.87) lends itself to numerical calculations since it only contains the finite number of SM degrees of freedom. We are also interested in calculating the induced superconducting gap with $\Gamma=0$ to assess the coupling strength between the SM and SC. To do so, we need to calculate subgap states, i.e. states below the superconducting gap of the parent superconductor. Both of the self-energies in (4.83) are purely real within the superconducting gap of the parent superconductor, implying that any subgap states must satisfy the generalized eigenvalue equation,

$$
H_{\text {eff }}^{\text {ind }}\left(k_{z}, \omega\right) \psi\left(k_{z}\right)=\omega S_{S M} \psi\left(k_{z}\right),
$$

where $|\omega|<|\Delta|$ and $H_{\text {eff }}^{\text {ind }}$ is defined as

$$
H_{\text {eff }}^{\text {ind }}\left(k_{z}, \omega\right)=H_{S M}\left(k_{z}\right)+\Sigma_{M I}^{\text {static }}(\Gamma=0)+\Sigma_{S C}\left(k_{z}, \omega\right) .
$$




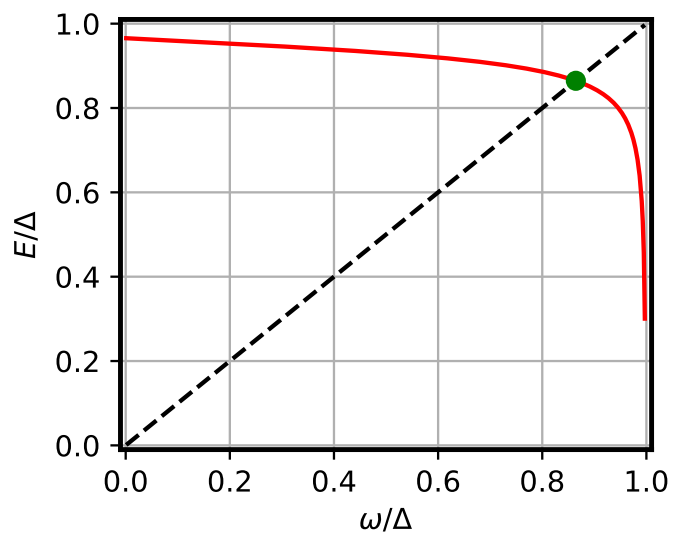

FIGURE 4.21: The lowest postive eigenvaule (red solid line) of $H_{\text {eff }}^{\text {ind }}\left(k_{z}, \omega\right)$ is plotted as a function of input $\omega$ for some example system parameters with $\Gamma=0$. The energy of a subgap state is found where the eigenvalue curve intersects (indicated by green dot) the line $E=\omega$ (black dashed line).

Note that we have used the superscript "ind" to indicate that this energydependent, effective Hamiltonian is used for calculating the induced gap at $\Gamma=0$. Also note that in Eq. (4.88) $\omega$ appears both within the effective Hamiltonian and as the eigenvalue. Therefore, we must solve the eigenvalue equation self-consistently, e.g. the input $\omega$ needs to be equal to one of the eigenvalues of the effective Hamiltonian. To understand how we can find such as $\omega$, we plot in Fig. 4.21 the lowest positive eigenvalue (red solid line) of $H_{\text {eff }}^{\text {ind }}\left(k_{z}, \omega\right)$ as a function of $\omega$ for some example system parameters with $\Gamma=0$. Equation (4.88) is satisfied when the eigenvalue curve interests the line $E=\omega$ (black dashed line). We notice that the lowest positive eigenvalue of $H_{\text {eff }}^{\text {ind }}\left(k_{z}, \omega\right)$ is monotonic over the range $-|\Delta|<\omega<|\Delta|$. Therefore, a simply bisection algorithm allows us to find $\omega$ satisfying (4.88), provided it exists, by iteratively reducing the sub-interval in which $\omega=E_{1}(\omega)$ is possible, where $E_{1}$ is the lowest positive eigenvalue of the effective Hamiltonian (4.89).

\subsubsection{Results}

First, we determine the dependence of the number of occupied subbands on the applied gate potential $\left(V_{g}\right)$ and identify the values of $V_{g}$ corresponding to the bottom of a certain subband $n$ being at the chemical potential, which provides the optimal condition for the emergence of topological superconductivity. The results are shown in Fig. 4.22 (blue disks). Note that the differences in subband occupancy between setups 1 and 2 are very small, which demonstrates that electrostatic effects depend weakly on the location of the magnetic insulator layer. Next, we solve the generalized eigenvalue equation $H_{\mathrm{eff}}^{\phi}\left(k_{z}, \Gamma\right) \psi=E S_{S M} \psi$ corresponding to $V_{b} \rightarrow \infty$ and $\Gamma \rightarrow 0$ and calculate the (linear) susceptibility $\chi_{\Gamma, n}=\Gamma_{e f f, n}^{S M} / \Gamma$ characterizing the direct proximity effect at the SM-MI interface. Here, $\Gamma_{e f f, n}^{S M}=\frac{1}{2}\left(E_{n, \uparrow}-E_{n, \downarrow}\right)$, where 


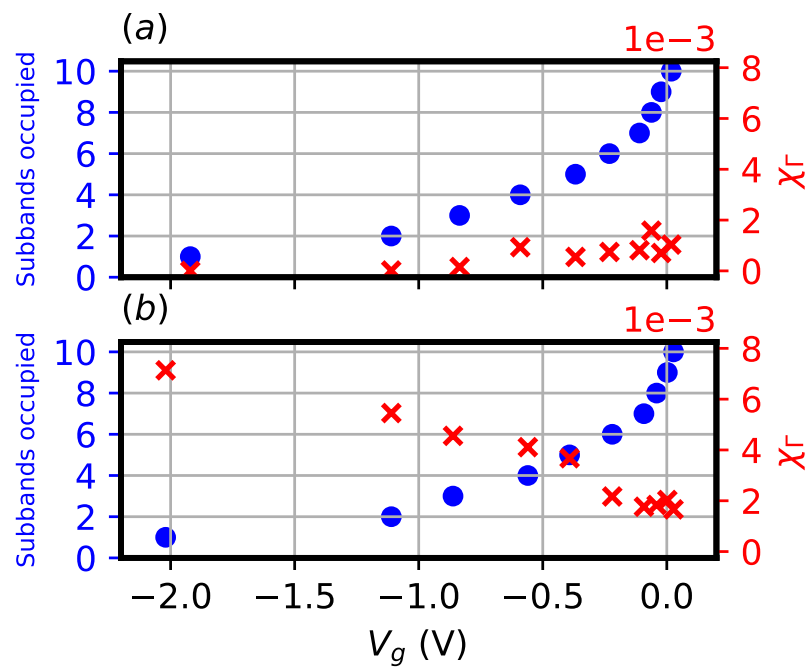

FIGURE 4.22: Blue disks: Subband occupancy as a function of the applied gate potential $V_{g}$ for (a) setup 1 and (b) setup 2. The values of $V_{g}$ correspond to the bottom of a certain subband $n$ being at the chemical potential. Note the negligible difference between the two setups. Red crosses: Linear susceptibility $\chi_{\Gamma, n}=\Gamma_{e f f, n}^{S M} / \Gamma$ characterizing the direct proximity effect at the SM-MI interface. Note the significant difference between (a) setup 1 and (b) setup 2, indicative of a much stronger direct SM-MI proximity effect in the overlapping configuration as compared to the non-overlapping setup. The system parameters are: $V_{b} \rightarrow \infty, \Gamma \rightarrow 0$, and $V_{S C}=0.15 \mathrm{~V}$.

$E_{n, \uparrow}$ and $E_{n, \downarrow}$ are the energies of the spin up and spin down eigenstates of the $n^{\text {th }} \mathrm{SM}$ subband for $k_{z}=0$ and $V_{b} \rightarrow \infty$. The results are shown in Fig. 4.22 (red crosses). Note the striking difference between the two setups. Particularly significant is that in the low-occupancy regime $n \lesssim 5$, which is expected to be most favorable for realizing robust topological superconductivity [121], the susceptibility for setup 2 (overlapping layers) is 5-50 times higher than the corresponding susceptibility for setup 1 . This behavior is determined by electrostatic effects, which result in the wave function of the lowest energy mode (relative to the Fermi level) being localized in the vicinity of the superconductor, as shown, for example, in Fig. 4.19 (c) and (d). For setup 1, this implies a wave function localized away from the SM-MI interface (hence, weak SM-MI proximity effect), while for setup 2 the wave function has a significant amplitude at the interface with the magnetic insulator, leading to large values of $\chi_{\Gamma}$. We note that the wave functions associated with higher energy transverse modes are more delocalized, reducing the difference between the two setups. However, the high-occupancy regime is characterized by a small inter-subband spacing, which makes the topological phase susceptible to disorder and other types of system inhomogeneity [121].

Having elucidated the key role played by electrostatics in determining the strength of the SM-MI proximity effect, we calculate the topological phase diagram as a function of the bare Zeeman field $\Gamma$ and the applied gate voltage 


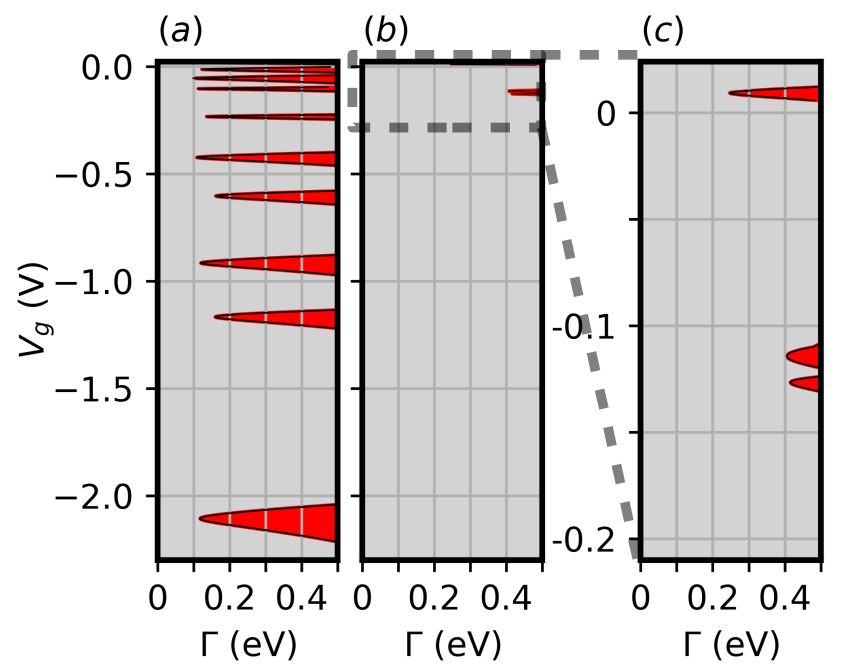

FIGURE 4.23: Topological phase diagram as function of the (phenomenological) Zeeman field $\Gamma$ characterizing the MI and the applied gate potential $V_{g}$ for (a) the overlapping structure (setup 2) and (b) the non-overlapping structure (setup 1). Panel (c) is an inset corresponding to the high occupancy regime in panel (b). Note that setup 2 is consistent with the emergence of topological superconductivity within a significant parameter window, in sharp contrast with setup 1 . The system parameters are: $V_{S C}=0.15 \mathrm{~V}$ and $V_{b}=2.75 \mathrm{eV} \cdot \mathrm{nm}$

$V_{g}$ for a hybrid system with $\Delta_{o}=0.3 \mathrm{meV}$ [151] and a SM-SC interface barrier $V_{b}=2.75 \mathrm{eV} \cdot \mathrm{nm}$. The phase boundary separating the trivial and topological superconducting phases are obtained by finding $\Gamma$ such that the equation $H_{\text {eff }}^{\text {top }}\left(k_{z}=0, \Gamma\right) \psi=0$ is satisfied. The results are shown in Fig. 4.23. Note that the overlapping configuration (setup 2) is consistent with the emergence of a topological phase for $\Gamma \sim 100-200 \mathrm{meV}$ and $V_{g}$ near the optimal values corresponding to the bottom of a certain subband being at the Fermi level [see Fig. 4.23(a)]. By contrast, the non-overlapping structure (setup 1) cannot support topological superconductivity for $\Gamma<500 \mathrm{meV}$, except in the highoccupancy regime [see Fig. 4.23(b) and (c)]. We emphasize that including the "indirect" proximity effect for setup 2, reduces the parent SC gap $\Delta$ and generates an effective Zeeman field $\Gamma_{e f f}^{S C}$ inside the parent superconductor, which favors the emergence of topological superconductivity and further enhances the already substantial difference between the two setups.

To demonstrate the robustness of our results, we investigate the dependence of the minimum critical field $\Gamma_{c, \min }^{n}$ characterizing the topological phase transition associated with subband $n$ on the strength of the effective SM-SC coupling $\widetilde{\gamma}$ for two values of the SM-SC work function difference $V_{S C}$. The effective coupling is calculated from Eq. (4.77) as $\tilde{\gamma}=\Delta_{\text {ind }} \sqrt{\Delta_{o}+\Delta_{\text {ind }}} / \sqrt{\Delta_{o}-\Delta_{\text {ind }}}$ [145], where $\Delta_{\text {ind }}$ is the induced gap for $\Gamma_{\text {eff }}^{S M}=0$. The results shown in Fig. 4.24 confirm the striking difference between the overlapping (dashed lines) and the non-overlapping (solid lines) setups. More specifically, the (bare) 
(a)

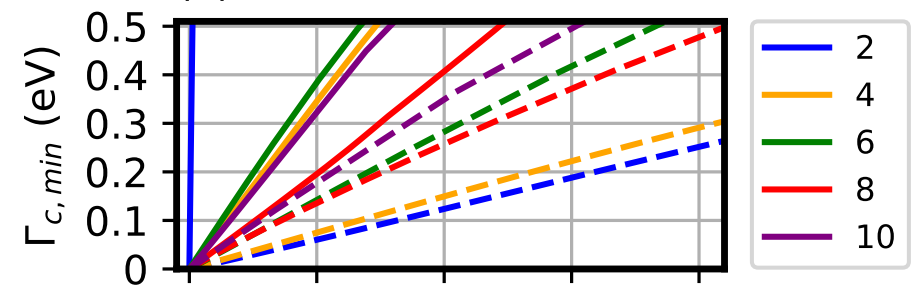

(b)

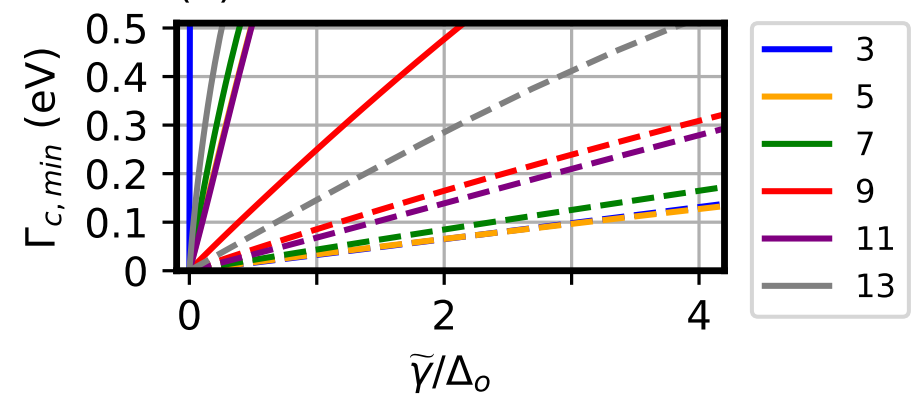

FIGURE 4.24: Dependence of the minimum critical Zeeman field $\Gamma_{c, \text { min }}^{n}$ on the effective SM-SC coupling $\widetilde{\gamma}$ for two values of the SM-SC work function difference: (a) $V_{S C}=0.15 \mathrm{~V}$ and (b) $V_{S C}=0.3 \mathrm{~V}$. The full lines correspond to setup 1, while the dashed lines are for the overlapping structure (setup 2). Note that the minimum critical Zeeman fields required for the emergence of a topological SC phase are systematically larger (by up to three orders of magnitude) in the non-overlapping configuration as compared to the overlapping setup.

minimum critical Zeeman fields required for the emergence of a topological SC phase are systematically larger (by up to three orders of magnitude) in the non-overlapping configurations as compared to the overlapping setup. A comparison between panels (a) and (b) in Fig. 4.24 shows that this trend increases with $V_{S C}$. Including the "indirect" proximity effect can only enhance the difference between the two configurations. Note that the spin splitting in EuS, $\Gamma \approx 0.14-0.19 \mathrm{eV}$ near the zone center [152], is consistent with the emergence (in overlapping structures, within a significant $\widetilde{\gamma}$ range) of topological SC induced by "direct" proximity effect (alone).

\subsubsection{Conclusion}

In conclusion, we have demonstrated that electrostatic effects play a critical role in determining the strength of the (direct) proximity effect between a magnetic insulator and a semiconductor wire in semiconductor-superconductormagnetic insulator (SM-SC-MI) hybrid structures. This decisive impact of electrostatics is rather generic in proximity-coupled hybrid nanostructures, beyond the SM-SC-MI system discussed as a specific example. Here, the electrostatic effects are controlled by the applied gate potential and by the geometry of the superconducting layer, regardless of whether the SM and SC are in direct contact or separated by a MI layer. We have argued that the "indirect" 
proximity effect emerging in structures with overlapping SC and MI layers is generally insufficient for the realization of a topological superconducting phase in the hybrid system. However, in these overlapping structures electrostatics favors the realization of low-energy transverse modes with large amplitudes near the SM-MI interface, which, in turn, results in a strong proximity effect between the MI and the SM wire and the emergence of a large effective Zeeman field consistent with the presence topological superconductivity. By contrast, such large proximity-induced Zeeman fields do not occur in non-overlapping structures within similar parameter windows. On the one hand, our results suggest that the recently reported experimental findings [119] are consistent with the presence of small proximity-induced Zeeman fields and topologically-trivial superconductivity in non-overlapping structures and significant effective Zeeman fields in the overlapping setup, large-enough to generate topological superconductivity in a homogeneous system. On the other hand, our findings suggest possible strategies for enhancing the robustness of the topological superconducting phase realized in a SM-SC-MI hybrid system. For example, using a lateral gate (instead of or in addition to a back gate) may enable a better control of the amplitudes of the relevant wave functions at the interfaces between the SM wire and the magnetic insulator or the parent superconductor. In addition, changing the areas of the SM-SC and SC-MI interfaces (e.g., having three facets covered by superconductor) can significantly affect the strength of the induced SC pairing potential and effective Zeeman field. Finally, since the "indirect" proximity effect alone cannot generate topological superconductivity and is not required to generate it, as shown in this study, but has the rather undesired effect of reducing the superconducting order parameter of the parent $\mathrm{SC}$, it may be convenient to reduce the effective coupling at the SC-MI interface, e.g., by adding a thin nonmagnetic insulating layer. This would have a minimal impact on the electrostatics, while enhancing the induced SC gap. Of course, quantitative estimates of the topological gap within these scenarios require a more detailed modeling of the hybrid structure that explicitly includes the proximity effect at the SC-MI interface. 


\section{Effects of Charge Impurity Disorder and Other Non-Uniformities in Majorana Nanowires}

"To understand the actual world as
it is, not as we should wish it to be,
is the beginning of wisdom."

Bertrand Russell

We come now to a key, if not the key, obstacle currently facing the realization of Majorana qubits, namely disorder and other general non-uniformities within the hybrid nanowire device.

From the outset, it is worth discussing a question that routinely comes up at non-specialized talks about Majorana physics and sets the stage for our motivation behind studying disorder and other non-uniformities; Do Majorana zero modes (MZMs) actually exist in semiconductor-superconductor (SM-SC) devices? The current answer is that we do not know if MZMs have been achieved in the laboratory. There certainly have been many experimental observations of zero-bias conductance peaks in tunneling spectroscopy that at the very least have been argued to be consistent with MZMs [47$50,87,89,153-158]$, if not making the stronger claim of finding signatures of their presence. Several mechanisms, however, have been theoretically shown over the last decade to produce nearly zero-energy topologically trivial states known as Andreev bound states (ABSs) (see Ref. [75] for a review), including soft confinement [54, 99, 108], inhomogeneous superconductive pairing [58, 69] and spin-orbit coupling [159], SNS junctions [63, 160, 161], coupling to quantum dots $[65,66,73,162]$ and leads probing the bulk of the nanowire $[70$, 71], disorder [55, 56, 59-62, 64, 74, 77], and inter-subband coupling [72]. Especially concerning are trivial ABS states known as partially separated ABS states [67] or quasi-Majorana [94] which mimic the local phenomenolology of MZMs nearly perfectly [97]. Most, if not all, of the experimental observations reporting zero-bias conductance peaks as evidence for MZMs are quite likely actually observing trivial ABS. Among the evidence for this interpretation is a lack of observations of other signatures of MZMs apart from a 
zero-bias conductance peak such as Majorana energy oscillations with increasing magnetic field and zero-bias conductance correlations between the two edges of the nanowire. Even if we grant that true MZMs have been observed in some experiment in SM-SC nanowires, however, the community would still be very far from quantum computing since we need to be able to reliably and consistently produce nanowires with robust and controllable MZMs.

All hope is not lost however. Consider the related question; Suppose the issues of disorder and device non-uniformities are solved or brought beneath a tolerable level. Will MZMs emerge? The resounding answer is yes! We can be confident in this answer because of the simplicity of the theory underlying the emergence of MZMs in SM-SC heterostructures. After all, the theory is built on free fermion theory with the three rather simple ingredients of s-wave superconductivity, Zeeman splitting, and spin-orbit coupling. ${ }^{1}$ On another positive note, the presence of trivial ABSs is not completely a bad signal with respect to the prospects of eventually achieving MZMs. Indeed, ABS physics can be viewed as residual Majorana physics, where ABSs are a precursor to MZMs in the sense that MZMs will necessarily emerge if either the disorder and other non-uniformities are brought beneath a certain tolerance level or the Zeeman energy is sufficiently increased without destroying superconductivity. Ultimately, trivial ABSs occur when the topological Majorana condition is locally satisfied within a small region of the device. MZMs will then emerge when this region is enlarged to (nearly) the entire proximitized region of the device.

In this context, we present two studies centered on the topics of disorder and device non-uniformity. In Sec. 5.1, we present the first study, which investigates the effects of charge impurities on Majorana physics in SM-SC hybrid nanowires. The second study is presented in Sec. 5.2 which shows that level-repulsion generated by inter-subband coupling can lead to the pinning of topologically trivial low-energy modes near zero energy. Other types of disorder are also possible. These deserve to be studied and I briefly discuss this in the outlook of these thesis in chapter 7.

\subsection{Charge impurity effects in hybrid Majorana nanowires}

In this section we ask a fundamental questions about the physics of SMSC Majorana nanowires: What happens if the nanowire, instead of being pristine, has disorder arising from unintentional charge impurities residing in it? The scenario considered in this question is not hypothetical, since unintentional charge impurities ("low doping") constitute the commonest type of disorder in high-quality semiconductor materials [163]. There is evidence that the experimental nanowires do, in fact, have substantial disorder. Given the considerable confusion about the situation surrounding the Majorana

\footnotetext{
${ }^{1}$ If we are going to start questioning free fermion theory at this point, I am content to quit and work on something else.
} 
nanowire experiments and the intrinsic difficulty of directly measuring disorder in hybrid nanostructures, we think it is appropriate to take a quantitative, microscopic approach to the problem. We do this in two main steps. First, we provide a quantitative characterization of the effective potential generated by a charge impurity embedded inside a semiconductor wire proximity-coupled to a superconductor layer by solving self-consistently the associated threedimensional Schrödinger-Poisson problem. Next, using the single impurity effective potential obtained self-consistently, we construct disorder potentials associated with the presence of multiple charge impurities and solve numerically the Bogoliubov-de Gennes (BdG) equations that describe the hybrid system in the presence of s-wave superconductivity, spin-orbit coupling, Zeeman splitting, and disorder arising from charge impurities. We also carry out first principles charge transport calculations and determine the tunneling differential conductance as a function of various systems parameters (e.g., disorder strength, chemical potential, and Zeeman splitting). Along the way, we introduce a number of quantities that facilitate the characterization of the low-energy physics in the presence of disorder (e.g., the Majorana separation length and the edge-to-edge correlation) and describe several protocols that enable a more efficient extraction and use of experimentally accessible information (e.g., construction of zero-bias conductance correlation maps).

We emphasize that our work is of considerable importance to the development of a Majorana-based topological quantum computer (TQC), as it addresses a critical outstanding problem facing the realization of topological qubits using hybrid nanostructures, which is the platform Microsoft Corporation is working on. In particular, our finding that charge impurities in the environment lead to serious complications regarding the realization and observation of Majorana zero modes has obvious direct implications for the development of Majorana-based qubits and TQC. Our work provides a full microscopic-based description of how experimentally available Majorana nanowire behave in the presence of charge impurity disorder of varying strength. More importantly, our work provides a clear future direction regarding what needs to be done for progress in the field, as well as quantitative measures of the maximum allowed impurity concentrations consistent with the full manifestation of topological MZMs in hybrid nanostructures. In particular, based on our extensive realistic calculations, we provide specific materials quality and semiconductor purity targets which must be achieved to create a topological qubit, providing a clear blueprint for future progress towards building a TQC. Our work establishes a clear goal of using nanowires with impurity concentrations around $10^{15}$ per $\mathrm{cm}^{3}$ or lower for TQC hardware to be feasible using Majorana qubits. This is a challenging target, but by no means an impossible one.

The remainder of this section is organized as follows. The case of a single charge impurity embedded within a proximity-coupled nanowire is investigated in Sec. 5.1.1. Section 5.1.2 is dedicated to the multi-impurity case. Our concluding remarks are presented in Sec. 5.1.3. Much of this section is adapted from our paper found in Ref. [76]. 


\subsubsection{Single charge impurity}

In this section, we investigate a single charge impurity embedded within a semiconductor (SM) nanowire proximity-coupled to a superconductor (SC). In particular, we address the key question regarding the magnitude and characteristic length scale of the potential inhomogeneity induced by the charge impurity. The screening due to the presence of the superconductor and of a nearby metallic gate, as well as the effects due to the redistribution of free charge within the SM wire are incorporated using a position-dependent selfconsistent Schrödinger-Poisson scheme. We first introduce our model for describing the SM-SC hybrid structure with an embedded charge impurity and describe the self-consistent Schrödinger-Poisson calculation method. We then turn to the results of our analysis.

\section{Model}

We consider the hybrid device represented schematically in Fig. 5.1, which consists of a hexagonal semiconductor nanowire of radius $R$ (purple in Fig. 5.1) having a thin superconducting layer (green) deposited on two of its facets. A metallic back gate (black) separated from the hybrid nanowire by a thin dielectric layer of thickness $d$ (gray) is used to tune the band edges of the low-energy SM subbands near the Fermi level. Up to minor modifications of the device geometry, e.g., having additional side gates, or depositing the SC on more than two facets, this setup corresponds to the most prevalent type of SM-SC hybrid device used experimentally for exploring Majorana physics [47-49, 82, 89, 97, 154, 164-167]. The key additional ingredient, which represents the focus of this study, is a charge impurity $Q$ embedded inside the SM wire, as is indicated in Fig. 5.1 by a yellow sphere. In our theory, the effects induced by the presence of the charge impurity are calculated exactly within a position-dependent self-consistent Schrödinger-Poisson formalism.

At this stage, the SM nanowire is modeled using a simple effective mass Hamiltonian given by

$$
H=-\frac{\hbar^{2}}{2 m^{*}} \nabla^{2}-e \phi(\vec{r}),
$$

where $m^{*}$ is the effective mass, $\nabla^{2}$ is the Laplacian operator in 3-dimensional space, and $\phi$ is the electrostatic potential inside the wire. We assume that the wire is infinitely long. The potential $\phi$ must satisfy the Poisson equation,

$$
\nabla \cdot[\epsilon(\vec{r}) \nabla \phi(\vec{r})]=-\rho(\vec{r}),
$$

where $\epsilon(\vec{r})$ is a material dependent dielectric constant taking different values inside the dielectric, the SM wire, and the surrounding vacuum and $\rho$ is the charge density within the wire. We impose Dirichlet boundary conditions on the bottom gate and the surface of the superconductor with potential values $V_{g}$ and $V_{S C}$, respectively. Note that the boundary condition on the SC surface accounts for the band-bending of the SM conduction band near the SM-SC interface $[110,111]$. In addition, we impose Neumann boundary conditions 


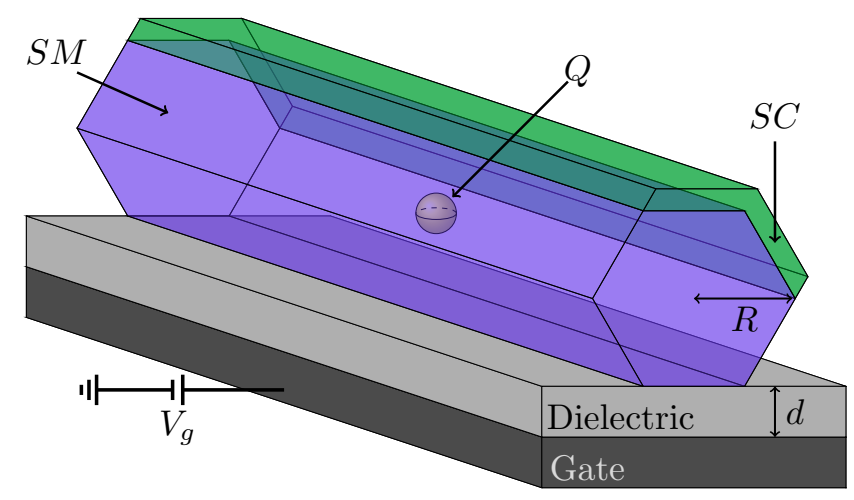

FIGURE 5.1: Schematic representation of the SM-SC hybrid device with an embedded charge impurity. A semiconductor nanowire (purple) of radius $R$ is proximity coupled to a thin superconductor (green). An impurity (yellow sphere) of charge $Q$ embedded within the semiconductor nanowire will create a potential inhomogeneity. The band edges of the low-energy SM subbands can be tuned near the Fermi level using a back gate (black) separated from the wire by a thin dielectric layer (gray).

on the sides and top of the full simulation region for Eq. (5.2), which are a distance $b \gg R$ away from the nanowire. Note that this choice of boundary conditions on the outer boundaries has negligible impact on the potential within the nanowire [121]. It is convenient (and physically appealing) to break the total charge density into three components,

$$
\rho(\vec{r})=\rho_{o}(x, y)+\rho_{\text {imp }}(\vec{r})+\rho_{\text {red }}(\vec{r}),
$$

where $\rho_{0}$ is the free charge density inside the SM wire in the absence of a charge impurity, $\rho_{i m p}$ is the charge density associated with the impurity, and $\rho_{\text {red }}$ accounts for the redistribution of free charge due to the presence of the impurity, i.e. describes the screening cloud. Note that $\rho_{o}$ is translation invariant along the direction parallel to the wire, which we take as the $z$ direction. The charge impurity is modeled as a small sphere of radius $R_{i m p}$ and uniform charge density given by

$$
\rho_{i m p}(\vec{r})=\left\{\begin{array}{ll}
\frac{3 Q}{4 \pi R_{i m p}^{3}}, & \left|\vec{r}-\vec{r}_{i m p}\right| \leq R_{i m p} \\
0, & \left|\vec{r}-\vec{r}_{i m p}\right|>R_{i m p}
\end{array},\right.
$$

where $\vec{r}_{i m p}=x_{i m p} \hat{e}_{x}+y_{i m p} \hat{e}_{y}$ is the position vector of the impurity. Note that, without loss of generality, we assume $z_{i m p}=0$. Finally, the free charge density is related to the occupied electronic states,

$$
\rho_{f}(\vec{r})=\rho_{o}(x, y)+\rho_{r e d}(\vec{r})=-2 e \sum_{n}\left|\psi_{n}(\vec{r})\right|^{2} f\left(E_{n}, T\right)
$$

where $\rho_{f}$ is the total free charge density, $E_{n}$ and $\psi_{n}$ are the $n^{\text {th }}$ eigenenergy of the Hamiltonian (5.1) and the corresponding eigenstate, respectively, $f$ is 
the Fermi function, $T$ the temperature, and the factor of 2 accounts for spin degeneracy. Note that Eq. (5.5) couples Eqs. (5.1) and (5.2), known as the Schrödinger-Poisson equations. The free charge density and the electrostatic potential are given by the self-consistent solution of these equations.

Before presenting our method for solving the Schrödinger-Poisson problem, a few comments about the model are warranted. First, note that we have neglected the key ingredients responsible for the emergence of Majorana physics in a SM-SC hybrid structure, namely proximity-induced superconductivity, spin-orbit coupling, and Zeeman splitting. These additional contributions to the effective Hamiltonian, which will be included in the finite wire model discussed in Sec. 5.1.2, are characterized by energy scales much smaller than the typical inter-band spacing associated with the Hamiltonian in Eq. (5.1), the potentials $V_{g}$ and $V_{S C}$, and the bare potential of the charge impurity. In other words, the spatial profile of eigenstates $\psi_{n}$ and, implicitly, the charge density $\rho_{f}(\vec{r})$ and the potential $\phi(\vec{r})$ are mainly determined by the terms already included in Eq. (5.1) and by the boundary conditions, while the additional terms are expected to generate small perturbations. Also note that we do not explicitly include the SC subsystem in the Hamiltonian, but consider it in the boundary conditions. Of course, the coupling between the SM and SC is crucial for inducing superconductivity within the SM wire through proximity effect. Moreover, it is known that the proximity coupling to the superconductor renormalizes the low-energy spectrum of the hybrid system [7] and generates a shift of the SM subbands [168]. However, these effects can be accounted for in our model by modifying the effective mass and appropriately shifting $V_{g}$ and $V_{S C}$. Consequently, to avoid the dramatic increase of the computational cost associated with including the SC in the Hamiltonian, we do not explicitly consider the SC degrees of freedom. We stress, however, that the SC still plays an important role in our model due to the band-bending generated by the Dirichlet boundary condition imposed on $\phi$ at the SC surface.

\section{Self-consistent Schrödinger-Poisson scheme}

We start by decomposing the electrostatic potential into three components, similar to Eq. (5.3). Explicitly, we have

$$
\phi(\vec{r})=\phi_{o}(x, y)+\phi_{\text {imp }}(\vec{r})+\phi_{\text {red }}(\vec{r}),
$$

where $\phi_{o}$ is the electrostatic potential in the absence of a charge impurity, and $\phi_{i m p}$ and $\phi_{\text {red }}$ are solutions of the Poisson equation with $\rho_{i m p}$ and $\rho_{\text {red }}$ as source terms, respectively. The Dirichlet boundary conditions for non-zero values of $V_{g}$ and $V_{S C}$ are imposed on $\phi_{0}$, while $\phi_{i m p}$ and $\phi_{\text {red }}$ are subject to trivial boundary conditions. Next, we rewrite the Hamiltonian as

$$
H=H_{0}+H^{\prime},
$$


with

$$
\begin{aligned}
& H_{o}=-\frac{\hbar^{2}}{2 m^{*}} \nabla^{2}-e \phi_{o}(x, y), \\
& H^{\prime}=-e \phi_{\text {imp }}(\vec{r})-e \phi_{\text {red }}(\vec{r}) .
\end{aligned}
$$

Here, $H_{0}$ is the Hamiltonian of the clean system (i.e., the wire without a charge impurity) and $H^{\prime}$ represents the perturbation due to the presence of the impurity. We first solve the Schrödinger-Poisson equations with $H=H_{0}$. Details regarding the self-consistent numerical procedure can be found in Chapter 4. The key output of this initial calculation is a set $\left\{\left(\varepsilon_{\alpha, 0}, \varphi_{\alpha}\right) \mid \alpha \in\right.$ $\mathbb{N}\}$ of transverse eigenenergies and corresponding eigenmodes. Note that the transverse wavefunction $\varphi_{\alpha}$ satisfies the eigenvalue equation

$$
H_{o}\left[\varphi_{\alpha}(x, y) e^{i k z}\right]=\left(\varepsilon_{\alpha, o}+\frac{\hbar^{2} k^{2}}{2 m^{*}}\right)\left[\varphi_{\alpha}(x, y) e^{i k z}\right],
$$

for arbitrary values of $k$. In other words, $\varphi_{\alpha}$ represents the $k$-independent transverse profile of the $\alpha$ subband for a clean system, while $\varepsilon_{\alpha, o}$ is the energy of the corresponding band edge (i.e., bottom of the band). Since $\left\{\varphi_{\alpha}\right\}$ is a complete, orthonormal set of transverse functions, we use it as a basis to expand the states of the full Hamiltonian (5.7). Explicitly, we have

$$
\psi_{n}(\vec{r})=\sum_{\alpha} \varphi_{\alpha}(x, y) g_{n, \alpha}(z)
$$

where $\psi_{n}$ is the $n^{\text {th }}$ eigenstate of Eq. (5.7) and $g_{n, \alpha}(z)$ is a yet-undetermined function of $z$. In principle, all subbands may contribute to each eigenstate. In practice, however, only a limited number of low-energy subbands contribute significantly to the low-energy eigenstates of the Hamiltonian. We therefore project the eigenstate (5.11) of the full Hamiltonian onto a low-energy subspace defined by subbands with $\varepsilon_{\alpha, o}<\varepsilon_{c u t}$, where $\varepsilon_{c u t}$ is a finite cutoff energy larger than any other relevant energy scale in the problem. Note that the accuracy of this low-energy projection can be tested by increasing $\varepsilon_{c u t}$, i.e., including additional transverse modes into the low-energy basis. The basis is large-enough if further increasing it generates a negligible change of the final results.

Next, we point out that introducing a charge impurity breaks the continuous translation invariance along the $z$ axis, making the assumption of an infinite system rather inconvenient. To address this issue, we impose periodic boundary conditions with a supercell of length $\ell$ sufficiently large so that charge impurities in neighboring supercells have a negligible effect on one another. In these conditions, the electrostatic potential within the large supercell will be practically identical to the potential of an infinitely long system within a region of length $\ell$ containing the impurity. We introduce the 
following Fourier transforms of the potential and charge density

$$
\begin{aligned}
& \phi_{i}(\vec{r})=\sum_{m} \widetilde{\phi}_{i, m}(x, y) e^{i G_{m} z}, \\
& \rho_{i}(\vec{r})=\sum_{m} \widetilde{\rho}_{i, m}(x, y) e^{i G_{m} z},
\end{aligned}
$$

where $G_{m}=2 \pi m / \ell$ is a reciprocal lattice vector, $m \in \mathbb{Z}$, and $i \in\{$ imp, red $\}$ designates different components defined in Eqs. (5.3) and (5.6). Plugging Eqs. (5.12) and (5.13) into the Poisson equation (5.2) yields

$$
\left[\nabla_{\perp} \cdot\left(\epsilon \nabla_{\perp}\right)-\epsilon G_{m}^{2}\right] \widetilde{\phi}_{i, m}(x, y)=-\widetilde{\rho}_{i, m}(x, y),
$$

for all possible values of $m$, where $\nabla_{\perp}$ is the del operator in the $x$-y plane. This reduces the original 3-dimensional Poisson equation to a set of independent 2-dimensional inhomogeneous screened Poisson equation equations with screening factor, $G_{m}$. Note that the screening factor in Eq. (5.14) suppresses $\widetilde{\phi}_{i, m}$ away from the source, $\widetilde{\rho}_{i, m}$. To aid the reader's intuition, note that the solution of the screened Poisson equation in $3 \mathrm{D}$ free space due to a unit charge at the origin is given by

$$
\phi_{3 D}(\mathbf{r})=\frac{e^{-G r}}{4 \pi \epsilon_{0} r}
$$

where $r$ is the radial distance from the unit charge and origin and $G$ is the screening factor. Notice that $G \rightarrow 0$ (no screening) yields the well know $1 / r$ potential of a unit charge from elementary electrostatics. We of course are solving $2 D$ screened Poisson equations, but the intuition is not as clear since the $2 D$ solution analogous to Eq. (5.15) involves Bessel functions. Nonetheless, the $3 D$ solution grants some intuition in understanding the behavior of the $2 D$ solution. These $2 D$ screened Poisson equations equations are significantly less costly numerically, as compared to the original $3 D$ Poisson equation. As a result, we are able to efficiently perform high resolution calculations of the self-consistent potential near the impurity. By contrast, achieving similar results using a brute force approach to the $3 D$ Poisson equation would require a dense discretization around the impurity, which would lead to significant costs in terms of both memory and computational time.

With periodic boundary conditions, the low-energy expansion of the eigenstates of the full Hamiltonian becomes

$$
\left|n, q_{z}\right\rangle=\sum_{\alpha=1}^{\mathcal{E}_{\alpha, 0}<\varepsilon_{\text {cut }}} \sum_{m}\left|\alpha, m, q_{z}\right\rangle A_{\alpha, m}^{n, q_{z}}
$$

where $q_{z} \in(-\pi / \ell, \pi / \ell]$ is the crystal momentum in the $z$-direction, $A_{\alpha, m}^{n, q_{z}}$ $=\left\langle\alpha, m, q_{z} \mid n, q_{z}\right\rangle \in \mathbb{C}$, and the basis state $\left|\alpha, m, k_{z}\right\rangle$ is given by

$$
\left\langle\vec{r} \mid \alpha, m, k_{z}\right\rangle=\varphi_{\alpha}(x, y) \frac{e^{i\left(G_{m}+q_{z}\right) z}}{\sqrt{L}},
$$


where $L$ is the total length of the system and the bra-ket notation is introduced for convenience. Note that $q_{z}$ is a good quantum number due to the discrete translation symmetry with period $\ell$. Calculation of the Hamiltonian matrix elements yields

$$
\begin{aligned}
& \left\langle\alpha, m, q_{z}\left|H_{o}\right| \beta, m^{\prime}, q_{z}\right\rangle=\varepsilon_{\alpha, m}\left(q_{z}\right) \delta_{\alpha, \beta} \delta_{m, m^{\prime}}, \\
& \left\langle\alpha, m, q_{z}\left|H^{\prime}\right| \beta, m^{\prime}, q_{z}\right\rangle=\widetilde{V}_{i m p, m-m^{\prime}}^{\alpha, \beta}+\widetilde{V}_{r e d, m-m^{\prime}}^{\alpha, \beta}
\end{aligned}
$$

where

$$
\begin{aligned}
\varepsilon_{\alpha, m}\left(q_{z}\right) & =\varepsilon_{\alpha, o}+\frac{\hbar^{2}}{2 m^{*}}\left(G_{m}+q_{z}\right)^{2}, \\
\widetilde{V}_{i, m}^{\alpha, \beta} & =-e \int \varphi_{\alpha}^{*} \widetilde{\phi}_{i, m} \varphi_{\beta} d x d y,
\end{aligned}
$$

with $i \in\{i m p, r e d\}$. Using this representation, the charge density can be expressed in the following compact form

$$
\begin{aligned}
\widetilde{\rho}_{f, m}(x, y)= & \frac{-2 e}{L} \sum_{n, q_{z}} \sum_{\alpha, \beta} \sum_{m^{\prime}} A_{\alpha, m^{\prime}}^{n, q_{z} *} A_{\beta, m^{\prime}+m}^{n, q_{z}} \\
& \times f\left[E_{n}\left(q_{z}\right), T\right] \varphi_{\alpha}^{*}(x, y) \varphi_{\beta}(x, y),
\end{aligned}
$$

where $E_{n}\left(q_{z}\right)$ is the eigenenergy of the $n^{\text {th }}$ eigenstate with crystal momentum $q_{z}$ and the factor of 2 again accounts for spin-degeneracy. Eq. (5.21) shows that both $\phi_{\text {imp }}$ and $\phi_{\text {red }}$ generically have diagonal and off-diagonal matrix elements corresponding to intra- and inter-subband couplings. Consequently, the eigenstates of the full Hamiltonian will be linear combinations of basis states involving several transverse modes. However, if the energy spacing between subbands is significantly larger than the perturbation terms, $\widetilde{V}_{i m p, m}^{\alpha, \beta}$ and $\widetilde{V}_{r e d, m}^{\alpha, \beta}$, with $\alpha \neq \beta$, the inter-subband mixing is small and the subband index $\alpha$ becomes an "almost good" quantum number. This motivates us to consider the independent subband approximation, in which we neglect any Hamiltonian matrix element between different subbands, i.e. $\left\langle\alpha, m, q_{z}|H| \beta, m^{\prime}, q_{z}\right\rangle=0$ for $\alpha \neq \beta$, when calculating the self-consistent potential. Within this approximation, the subband index becomes a good quantum number, and we can write the eigenstates as

$$
\left|\alpha, n, q_{z}\right\rangle=\sum_{m}\left|\alpha, m, q_{z}\right\rangle \mathbb{A}_{\alpha, m}^{n, q_{z}}
$$

where $\mathbb{A}_{\alpha, m}^{n, q_{z}}=\left\langle\alpha, m, q_{z} \mid \alpha, n, q_{z}\right\rangle$. The free charge density reduces to

$$
\begin{aligned}
\widetilde{\rho}_{f, m}(x, y)= & \frac{-2 e}{L} \sum_{n, q_{z}} \sum_{\alpha} \sum_{m^{\prime}} \mathbb{A}_{\alpha, m^{\prime}}^{n, q_{z} *} \mathbb{A}_{\alpha, m^{\prime}+m}^{n, q_{z}} \\
& \times f\left[E_{\alpha, n}\left(q_{z}\right), T\right]\left|\varphi_{\alpha}(x, y)\right|^{2} .
\end{aligned}
$$


Finally, we can write the matrix elements of $\phi_{\text {red }}$ in a compact form by introducing the subband Green's function, $\widetilde{g}_{m, \alpha}$, defined as the solution of the Poisson equation,

$$
\left[\nabla_{\perp} \cdot\left(\epsilon \nabla_{\perp}\right)-\epsilon G_{m}^{2}\right] \widetilde{g}_{m, \alpha}(x, y)=-e\left|\varphi_{\alpha}(x, y)\right|^{2},
$$

with trivial boundary conditions, and the Green's function tensor,

$$
\widetilde{g}_{m, \alpha}^{\beta, \gamma}=\int \varphi_{\beta}^{*} \widetilde{g}_{m, \alpha} \varphi_{\gamma} d x d y
$$

With these notations, the relevant matrix elements become

$$
\widetilde{V}_{r e d, m}^{\beta, \gamma}=\sum_{\alpha} \widetilde{g}_{m, \alpha}^{\beta, \gamma} n_{\alpha, m}
$$

with

$$
n_{\alpha, m}=\frac{2}{L} \sum_{n, q_{z}} \sum_{m^{\prime}} \mathbb{A}_{\alpha, m^{\prime}}^{\left(n, q_{z}\right)^{*}} \mathbb{A}_{\alpha, m^{\prime}+m}^{\left(n, q_{z}\right)} f\left[E_{\alpha, n}\left(q_{z}\right), T\right],
$$

for $m \neq 0$. If $m=0$, the structure of Eq. (5.28) remains the same, but the quantity $n_{\alpha, 0}$ associated with the clean system must be subtracted, as it is already incorporated into $\phi_{0}$. Note that, while Eq. (5.27) gives both diagonal and off-diagonal matrix elements, within the independent subband approximation only the diagonal contributions containing tensor elements of the form $\widetilde{g}_{m, \alpha}^{\beta, \beta}$ are relevant for the self-consistent calculation of the potential. Also, we point out that, once $\widetilde{g}_{m, \alpha}^{\beta, \gamma}$ and $\widetilde{V}_{i m p, m}^{\alpha, \beta}$ have been calculated using the self-consistent wavefunctions of the clean system, the problem reduces to finding $n_{\alpha, m}$ self-consistently. We perform this task using a simple iterative mixing scheme. An iteration is numerically inexpensive, since each subband corresponds to an independent 1D Schrödinger equation that determines the eigenstates $\left|\alpha, n, q_{z}\right\rangle$. In fact, the largest computation cost corresponds to calculating the elements $\widetilde{g}_{m, \alpha}^{\beta, \gamma}$ of the Green function tensor. Also note that, while the subbands are independent as far as solving the Schrödinger equation is concerned, they still affect each other through Eq. (5.27), since $n_{\alpha, m}$ enters the expression of $\widetilde{V}_{r e d, m}^{\beta, \beta}$ for all $\alpha$ and $\beta$. Therefore our independent subband approximation still captures the main contribution due to inter-subband electrostatic screening. In addition, we have explicitly checked that neglecting inter-subband coupling has a negligible effect on the spectrum of the full Hamiltonian.

Once the self-consistent solution is found, we Fourier transform the matrix elements of the potential back to real space and define the effective impurity potential matrix elements,

$$
V_{\alpha, \beta}(z)=\sum_{m}\left(\widetilde{V}_{i m p, m}^{\alpha, \beta}+\widetilde{V}_{r e d, m}^{\alpha, \beta}\right) e^{i G_{m} z}
$$

These quantities provide information regarding the amplitude and characteristic length scale of the potential inhomogeneity induced by the charge 
impurity. Note that the diagonal element $V_{\alpha, \alpha}(z)$ can be interpreted as an effective 1D potential for the $\alpha$ subband. On the other hand, the off diagonal element $V_{\alpha, \beta}(z)$ couples the subbands $\alpha$ and $\beta$ in a position dependent manner.

For the numerical calculations we choose parameter values that roughly correspond to the currently existing InAs-Al and InSb-Al nanowire-superconductor platforms, while being somewhat on the reasonably optimistic side. We emphasize that our qualitative and semi-quantitative conclusions do not depend on the details of our parameter choice. Specifically, we have used the following parameter values: radius of the SM nanowire $R=35 \mathrm{~nm}$, dielectric thickness $d=10 \mathrm{~nm}$, superconductor thickness $W_{S C}=10 \mathrm{~nm}$, SM permittivity $\epsilon_{S M}=15.15$, dielectric permittivity $\epsilon_{d}=24$, effective mass $m_{\text {eff }}=0.023$, work function difference $V_{S C}=110 \mathrm{meV}$, radius of the charge impurity $R_{i m p}=2.5 \mathrm{~nm}$, supercell size $\ell=500 \mathrm{~nm}$, energy cutoff for the transverse modes $\epsilon_{c u t}=20 \mathrm{meV}$, kinetic energy cutoff of plane waves along the $z$ direction $\epsilon_{c u t}^{k i n}=3 \mathrm{eV}$, Fourier coefficients satisfying $|m| \leq 200$ are used for the electrostatic potential and charge density expansions, and transverse mesh spacing within the semiconductor for the Poisson, screened Poisson, and Schrödinger equations $a_{S M}=1 \mathrm{~nm}$.

\section{Results}

To understand the qualitative and quantitative characteristics of the effective potential generated by a charge impurity embedded inside the semiconductor wire, we start with a calculation of the impurity potential $\phi_{i m p}$, which corresponds to the second term in the decomposition given by Eq. (5.6). We note that $\phi_{\text {imp }}$ is the solution of the Poisson equation (5.2) with a source term given by $\rho_{i m p}$ from Eq. (5.4) and homogeneous Dirichlet boundary conditions on the surface of the superconductor and the metallic gate. Consequently, in addition to the bare $1 / r$ potential of the charge impurity, $\phi_{i m p}$ includes the screening effect due to the presence of the SC layer and metallic back gate. However, it does not include the screening effect due to the redistribution of the free charge within the wire, which corresponds to $\phi_{\text {red }}$ in Eq. (5.6). Note that if we instead used $Q=-e$ in Fig. 5.2, $\phi_{i m p}$ would simply pick up an overall minus sign due to the linearity of Eq. (5.14). This will not be the case, however, for the redistribution potential $\phi_{f}$, as we show below.

Maps of the screened potential amplitudes at $z=0$ (i.e., in the plane containing the impurity) and $z=10 \mathrm{~nm}$ for two different impurity locations are shown in Fig. 5.2. The left panels correspond to an impurity located in the middle of the wire, while the right panels show the potential of an impurity located near the SM-SC interface. While at $z=0$ the potentials generated by the two impurities are comparable (see top panels in Fig. 5.2), further away the potential of the central impurity is much stronger that the potential generated by the other impurity (lower panels). This indicates that the potential of the impurity located near the SM-SC interface has a significantly shorter decay length than the central impurity, which is the result of a stronger screening by the superconductor. We conclude that, while the characteristic length 


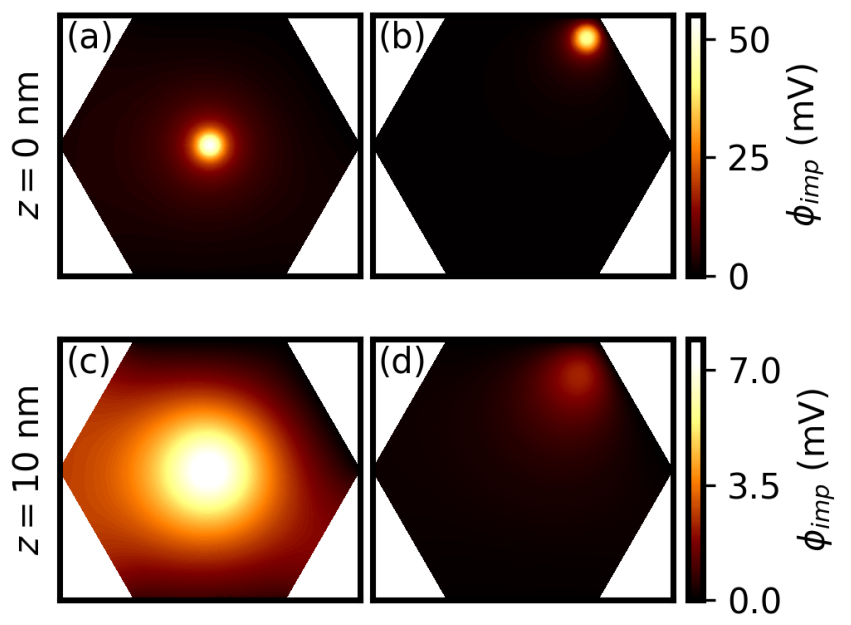

FIGURE 5.2: Impurity potential maps, $\phi_{\text {imp }}(x, y)$, within the semiconductor region for two impurity locations: middle of the wire, $\left(x_{\text {imp }}, y_{\text {imp }}\right)=(0,0)$ [panels (a) and (c)] and close to the SM-SC interface (top and upper right facets), $\left(x_{\text {imp }}, y_{\text {imp }}\right)=$ $(15,25) \mathrm{nm}$, [panels (b) and (d)]. The potential amplitudes at $z=0$, i.e., in plane containing the impurities, are comparable (top panels), while at $z=10 \mathrm{~nm}$ the potential of the central impurity is much stronger than the potential generated by the other impurity (lower panels) as a result of weaker screening by the superconductor. Note the different energy scales for the upper and lower panels. The impurity charge $Q=e$ is used for both impurity locations.

scale of the screened potential depends strongly on the location of the impurity relative to the SM-SC interface and the back gate, the maximum amplitude of $\phi_{i m p}$ is on the order of tens of $\mathrm{meV}$ regardless of the location of the charge impurity. This is at least one order of magnitude larger than the characteristic energy scale associated with Majorana physics. Without additional screening, the presence of charge impurities inside the hybrid device would have catastrophic effects on the stability of topological superconductivity and Majorana zero modes. This is a quantitative finding of extreme importance in the search for Majorana zero modes, as it clearly reveals the fragility of the quantum energy scale associated with Majorana physics (e.g., the topological gap $\sim 0.1 \mathrm{meV}$ or less), which can be easily overwhelmed by the huge (essentially classical) impurity energy scale $(\sim 10 \mathrm{meV})$. This further emphasizes the critical need for clean samples and the role of screening in limiting the impurity-induced potential.

Next, we perform the full, self-consistent Schrödinger-Poisson calculation and determine the effective impurity potential matrix elements defined by Eq. (5.29). For concreteness, we focus on a system that, in the absence of the impurity, has the bottom of the fourth subband at the chemical potential, which is realized by properly tuning the gate potential $V_{g}$. Since Majorana 


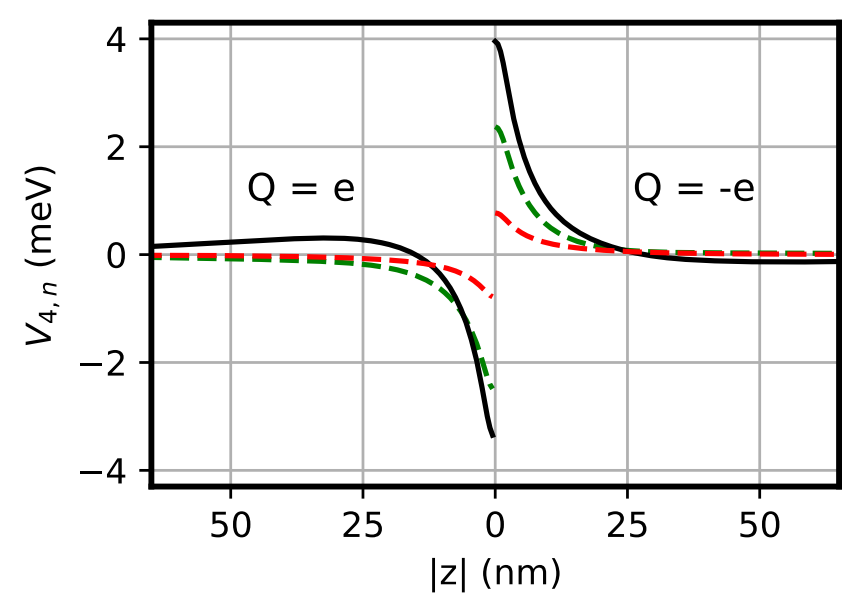

FIGURE 5.3: The dependence of the effective potential matrix elements on the distance $|z|$ from the plane containing the impurity for a system having the chemical potential near the bottom of the fourth subband. The left side corresponds to a positively charged impurity with $Q=+e$, while the right side corresponds to a negative charge, $Q=-e$. Both impurities are located at $\left(x_{i m p}, y_{\text {imp }}\right)=(23,0) \mathrm{nm}$. The black solid lines correspond to the relevant intra-subband effective potential, $V_{4,4}$, while the green and red dashed lines represent the intersubband matrix elements, $V_{4,3}$ and $V_{4,5}$, respectively.

physics is controlled by the top occupied subband, the relevant effective potential matrix elements $V_{\alpha, \beta}$ correspond, in this case, to $\alpha=4$ and $\beta=3,4,5$, with the diagonal element $V_{4,4}(z)$ representing the intra-subband effective impurity potential and the off-diagonal elements $V_{4,3}$ and $V_{4,5}$ providing a measure of the impurity-induced inter-subband coupling. The dependence of the effective potential matrix elements on the distance $|z|$ from the plane containing the impurity is shown in Fig. 5.3. We consider two cases: positive charge impurity, $Q=+e$ (left side of Fig. 5.3), and negative charge impurity, $Q=-e$ (right side). In both cases the location of the impurity in the transverse plane is given by $\left(x_{i m p}, y_{\text {imp }}\right)=(23,0) \mathrm{nm}$. First, we note that the off-diagonal contributions are smaller than, but comparable to the diagonal term. If the inter-subband spacing is much larger than $\Delta E \sim 1 \mathrm{meV}$, the impurity-induced inter-subband coupling is negligible and one can accurately describe the system within the independent subband approximation. If, on the other hand, the inter-subband spacing is comparable to (or lower than) $\Delta E$, inter-subband coupling becomes important and the system has to be treated explicitly as a multi-subband system. In this scenario, the system is expected to be prone to the formation of topologically-trivial low-energy states due to impurity-induced inter-subband coupling [72]. On the other hand, in the independent-subband regime the system is expected to be less sensitive to impurity-induced disorder. This study focuses on the more favorable scenario involving well separated subbands. We note that accessing this regime depends critically on ensuring low subband occupancy [121]. We 
emphasize that in systems characterized by small inter-subband energy separation, which is generically the case at high occupancy (e.g., for $\alpha>10$ ), inter-subband coupling may prevent the realization of a robust topological phase even in the absence of disorder [72]. Here, we focus on the situation corresponding to large inter-subband energy splittings and low subband occupancies, where the inter-subband coupling (induced by, e.g., charge impurities) can be safely neglected. Note that, in principle, the subband occupancy can be kept low by properly tuning the gate voltage, $V_{g}$.

The diagonal matrix elements (full black lines in Fig. 5.3) are characterized by amplitudes of a few meV and decay lengths on the order of $10 \mathrm{~nm}$. In general, the amplitude of the potential generated by a negative charge is slightly larger that the amplitude of a positive charge potential corresponding to the same subband and impurity location. This is a screening effect arising from the free charge being made of electrons, which are more effective in screening a positively charged impurity. Note that the dependence of $V_{4,4}$ on $z$ is not monotonic, being characterized by a fast decay at short distances followed by a change of sign and a slow decay at long distances. Remarkably, this behavior, which turns out to be quite generic, is well captured by the following empirical function

$$
V_{\alpha, \alpha}(z)=B_{i m p}^{\alpha} e^{-|z| / \lambda_{\text {imp }}^{\alpha}}-B_{r e d}^{\alpha} e^{-|z| / \lambda_{r e d}^{\alpha},}
$$

where the four fitting parameters, $B_{i m p}^{\alpha}, B_{r e d}^{\alpha}, \lambda_{i m p}^{\alpha}$, and $\lambda_{r e d}^{\alpha}$, depend on the band index, $\alpha$, and also on the specific location of the impurity, $\left(x_{i m p}, y_{\text {imp }}\right)$. Note that the first and second terms in Eq. (5.30) account for the effective impurity and redistribution potentials, respectively. Moreover, while there are four fitting parameters in the Eq. (5.30), which, in principle, are independent, we find that correlations between the fitting parameters imply that one only needs to input $B_{i m p}^{\alpha}$ or $B_{i m p}^{\alpha}$ and $\lambda_{i m p}^{\alpha}$, i.e., two independent parameters, to obtain a realistic disorder potential (see the Appendix of the published version of this work [76] for details and statistical results regarding the fitting). This has two major implications. First, to understand the dependence of the effective impurity potential on the band index and the position of the impurity, it is enough to study the dependence of the amplitude and decay length on these parameters, which substantially simplifies our analysis. Second, the simple form of Eq. (5.30) provides an extremely useful phenomenological model for describing charge impurities embedded within SM-SC hybrid devices. Combined with our quantitative results described below, this enables the study of disorder generated by charge impurities without actually performing a full, numerically-intensive Schrödinger-Poisson calculation. The validation of the relatively simple empirical fitting of the impurity potential defined by Eq. (5.30) is an important result of our work.

Our next task is to determine the dependence of the amplitude and decay length characterizing the effective disorder potential $V_{\alpha, \alpha}(z)$ on the position of the impurity and the subband index. Here, we define the amplitude as $V_{\alpha, \alpha}(z=0)$, while the decay length $\xi_{\alpha}$ is obtained by finding $z$ such that 


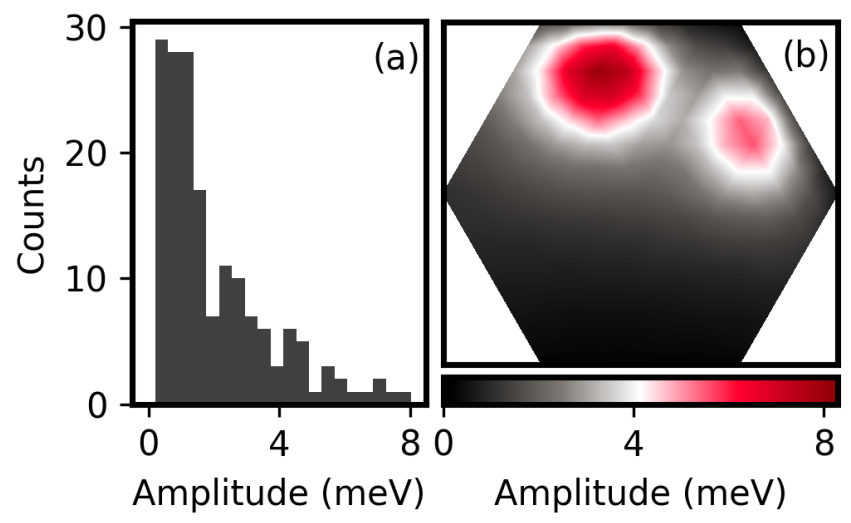

FIGURE 5.4: (a) Histogram of the effective potential amplitude $V_{2,2}(0)$. Data taken from 169 impurity locations sampled evenly over the hexagonal cross-section. (b) Effective potential amplitude $V_{2,2}(0)$ as a function of the impurity position, $\left(x_{i m p}, y_{i m p}\right)$. Note that the largest amplitude corresponds to locations where the second transverse mode has high spectral weight.

$V_{\alpha, \alpha}(z)=V_{\alpha, \alpha}(0) \exp (-1)$. We emphasize that, within the independent subband regime, the only relevant matrix element is the diagonal element corresponding to the top occupied subband. In turn, the occupancy of the SM subbands is controlled by the applied gate potential $V_{g}$.

To acquire some intuition, we first consider a specific example involving a system having the bottom of the second subband near the chemical potential. A map showing the dependence of the amplitude $V_{2,2}(0)$ on the position of the impurity is provided in Fig. 5.4(b). We note that the amplitude of the effective impurity potential depends strongly on the position of the impurity. The largest amplitude corresponds to locations where the second transverse mode has high spectral weight. This is not surprising, considering that $V_{2,2}(0)$ is a matrix element of a short range quantity over the second subband. Also note that, as a result of having a finite work function difference, $V_{S C}$, the lowest energy modes tend to be localized in the vicinity of the SM-SC interface. Higher energy modes, on the other hand, are more evenly spread over the cross section of the wire. The subband-dependent amplitude of the effective impurity potential $V_{\alpha, \alpha}(0)$ exhibit a similar dependence on the position of the charge impurity. To describe quantitatively the distribution of potential amplitudes, we generate a histogram of the amplitude corresponding to 169 impurity locations sampled evenly over the hexagonal cross-section of the wire. The results are shown in Fig. 5.4(a). Note that, as a result of the second subband being localized near the SM-SC interface, the distribution is skewed toward lower amplitudes. For higher energy modes, the amplitude distributions are more uniform, as a consequence of the wider distribution of spectral weight associated with those modes.

Our analysis of the position dependence of $V_{2,2}(0)$ suggests that, in general, a compact characterization of the potential amplitude $V_{\alpha, \alpha}(0)$ can be obtained by simply focusing on the distribution obtained by sampling the 




FIGURE 5.5: Distributions of the intra-subband effective potential amplitude, $V_{\alpha, \alpha}(0)$, (a) and decay length, $\xi_{\alpha},(b)$ for the subbands $\alpha=2-9$ with $Q=-e$. Note that the distributions corresponding to $\alpha=1$ are not shown. The bottom of each subband is tuned to the Fermi level by adjusting the gate potential $V_{g}$. The orange lines indicate the median $(50 \%)$ of the distribution, the boxes correspond to the $25-75 \%$ range, and the whiskers mark the upper and lower boundaries of the distribution. Each subband distribution is sampled over 169 impurity locations evenly distributed over the hexagonal cross-section of the semiconductor wire.

hexagonal cross-section of the wire. Note that the effective potential $V_{\alpha, \alpha}$ is relevant when the bottom of the corresponding subband is in the vicinity of the Fermi level. We characterize the distributions by specifying the minimum and maximum values of the potential amplitude, as well as the values corresponding to the median (50\%), 25\%, and 75\%. A similar procedure can be used to compactly characterize the distribution of decay lengths. The results for subbands $2-9$ are shown in Fig. 5.5. The orange lines indicate the median (50\%), the boxes correspond to the $25-75 \%$ range, and the whiskers mark the upper and lower boundaries of the distribution. We note that the distributions corresponding to a given subband $\alpha$ were obtained for a value of the applied gate potential $V_{g}$ that tunes the bottom of the subband near the chemical potential. As indicated in Fig. 5.5, for $V_{g}=0$ the system has the fourth subband near the chemical potential. Accessing lower energy subbands requires depleting the wire, i.e., applying a negative gate potential. Higher energy bands, on the other hand, become relevant at positive $V_{g}$ values. We note that the typical values of the effective potential amplitude are 


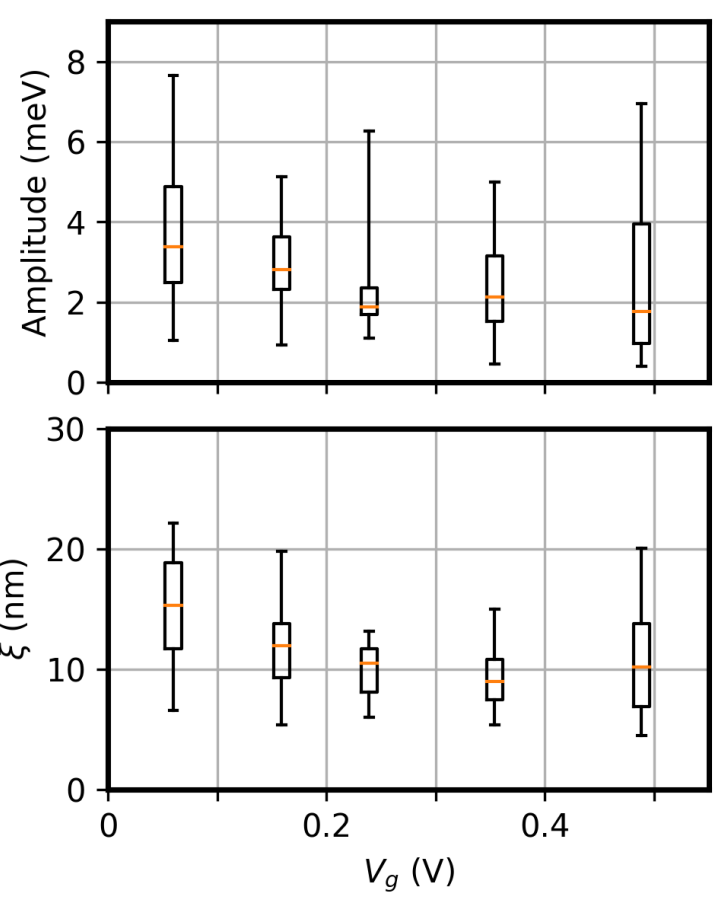

FIGURE 5.6: Same as Fig. 5.5, but for a system without a superconductor layer. The distributions correspond to even subbands with index (from left to right) $2 \leq \alpha \leq 10$. Note that, as compared to the results shown in Fig. 5.5, the typical values of the effective impurity potential amplitude are larger by at most a factor of two, while the typical decay lengths are only slightly larger, which indicates that screening by the superconductor has a rather limited effect.

on the order of $2 \mathrm{meV}$, significantly larger than the typical superconducting energy scales associated with Majorana physics. The typical decay lengths are in the range $8-12 \mathrm{~nm}$ for $\alpha \geq 3$, while the lowest energy subbands are characterized by longer (typical) decay lengths and wider distributions due to the localization of the corresponding transverse modes near the SM-SC interface.

An important question that can be raised at this point concerns the role of the superconductor in screening the impurity potential. To address it, we consider a charge impurity embedded inside a semiconductor wire in the absence of the superconductor layer. Note that the only change with respect to the calculations described above is the elimination of the Dirichlet boundary condition $\phi=V_{S C}$ at the SC surface. The distributions of the intra-subband effective potential amplitude, $V_{\alpha, \alpha}(0)$, and decay length, $\xi_{\alpha}$, for the even subbands with $2 \leq \alpha \leq 10$ are shown in Fig. 5.6. Note that in the absence of superconductor-induced band bending the values of $V_{g}$ associated with different subbands are different from the corresponding values in Fig. 5.5. The key result of this calculation, which is revealed by the comparison of Figs. 5.5 and 5.6 , is that screening by the superconductor does not generate a dramatic 

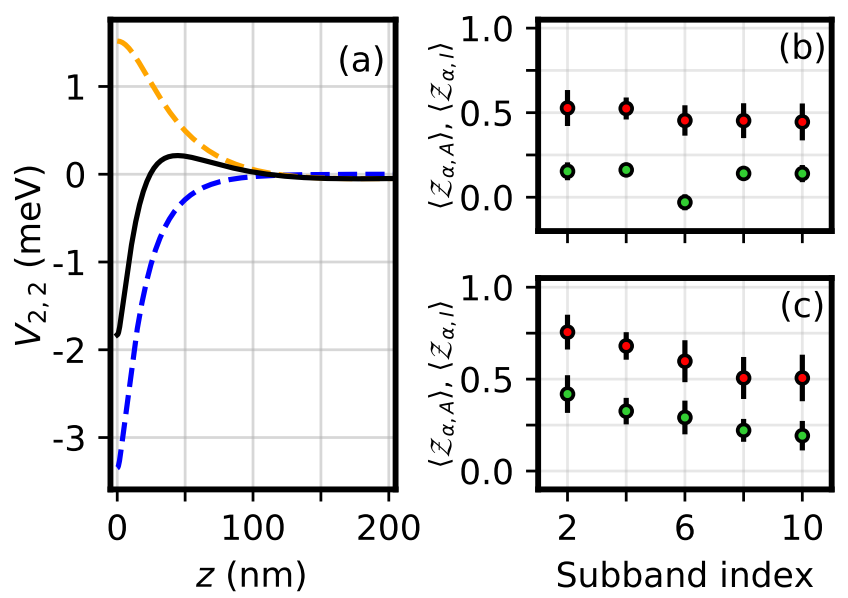

FIGURE 5.7: (a) Position dependence of the effective impurity potential $V_{2,2}$ (black line) and its impurity component, $V_{2,2}^{i m p}$ (blue dashed line), and free charge redistribution component, $V_{2,2}^{r e d}$ (orange dashed line), for a positively charged impurity placed at $x_{i m p}=-18 \mathrm{~nm}, y_{i m p}=10 \mathrm{~nm}$ inside a wire having the second subband tuned to the Fermi level. (b) Average amplitude screening, $\mathcal{Z}_{\alpha}^{(A)}$ (red circles), and average integrated screening $\mathcal{Z}_{\alpha}^{(I)}$ (green circles), for a positively charged impurity, $Q=+e$, embedded in a wire having the chemical potential tuned near the bottom of different subbands. The bars correspond to one standard deviation, which ranges from 0.03 to 0.13. Each subband distribution is sampled over 169 impurity locations evenly distributed over the hexagonal cross-section of the semiconductor wire. (c) Same as (b) for a negatively charge impurity, $Q=-e$. Note that the screening by the free charge of negative impurities is significantly less effective than the screening of positive impurities.

effect, as it reduces the typical amplitude of the effective impurity potential by at most a factor of two and slightly shortens the typical decay length. This behavior is mainly due to the fact that the impurities inside the SM wire are typically located too far from the SM-SC interface for the superconductor to drastically screen out the impurity potential.

Another important question regards the screening of the impurity potential due to the free charge redistribution in the wire. To characterize the renormalization of the band-dependent effective potential due to free charge redistribution, we introduce the amplitude screening factor, $\mathcal{Z}_{\alpha}^{(A)}$, and the integrated screening factor, $\mathcal{Z}_{\alpha}^{(I)}$, defined as follows

$$
\begin{aligned}
\mathcal{Z}_{\alpha}^{(A)} & =\frac{V_{\alpha, \alpha}(z=0)}{V_{\alpha, \alpha}^{i m p}(z=0)}, \\
\mathcal{Z}_{\alpha}^{(I)} & =\frac{\int V_{\alpha, \alpha}(z) d z}{\int V_{\alpha, \alpha}^{i m p}(z) d z},
\end{aligned}
$$


where $V_{\alpha, \alpha}^{i m p}(z)$ is the real-space diagonal matrix element of the impurity potential,

$$
V_{\alpha, \alpha}^{i m p}(z)=\sum_{m} \widetilde{V}_{i m p, m}^{\alpha, \alpha} e^{i G_{m} z}
$$

with $\widetilde{V}_{i m p, m}^{\alpha, \alpha}$ given by Eq. (5.21). Note that, using Eq. (5.29), the effective potential can be written as $V_{\alpha, \alpha}(z)=V_{\alpha, \alpha}^{i m p}(z)+V_{\alpha, \alpha}^{r e d}(z)$, where $V_{\alpha, \alpha}^{i m p}$ includes the bare impurity potential contribution and the screening by the superconductor and the metallic gate, while $V_{\alpha, \alpha}^{r e d}$ is the contribution due to free charge redistribution. A specific example corresponding to a positive charge impurity embedded inside a system having the chemical potential near the bottom of the second subband is given in Fig. 5.7 (a). Note that $V_{\alpha, \alpha}^{r e d}$ has a larger decay length and a smaller amplitude than the impurity potential. This is a general property responsible for the sign change of the effective potential and the "hump" ("dip" for negative impurities) feature starting near $z \approx 30 \mathrm{~nm}$.

The average screening factors averaged over different transverse impurity positions for a system with different occupancy levels are shown in Fig. 5.7 (b) and (c) for positively and negatively charged impurities, respectively. First, note that $\mathcal{Z}_{\alpha}^{(A)}$ is a measure of short-range screening, while $\mathcal{Z}_{\alpha}^{(I)}$ takes into account long-range contributions. Since in general $V_{\alpha, \alpha}^{\text {red }}$ has a longer decay length than $V_{\alpha, \alpha}^{i m p}$, we have $\mathcal{Z}_{\alpha}^{(I)}<\mathcal{Z}_{\alpha}^{(A)}$. Second, the screening by the free charge of positive impurities is significantly more effective that the screening of negative impurities. In particular the integrated screening factor, $\mathcal{Z}_{\alpha}^{(I)}$, has values smaller than 0.2 for all subbands, indicating that the contribution from the "hump" feature almost cancels the contribution from the central dip. In fact, in the case of the sixth subband the average integrated screening factor for $Q=e$ is actually negative, indicating over-screening by the free charge. In addition, we note that the screening of negative impurities is more effective when the subband occupancy increases, while in the case of positive impurities the dependence on the subband index is weak. Our analysis demonstrates that screening due to free charge redistribution in the wire is a significant effect that has to be taken into account to obtain a quantitative description of the low-energy physics in the presence of charge impurities. This is physically reasonable, since the free charge inside the SM wire resides within the same spatial region as the impurity, making its screening effect quantitatively dominant.

We conclude this section with a comment on the relevance of the results obtained here to understanding Majorana physics in semiconductorsuperconductor structures. On the one hand, the matrix elements of the effective impurity potential obtained numerically from the self-consistent solution of the Schrödinger-Poisson problem can be used to investigate hybrid devices containing a finite number of randomly distributed charge impurities. The single impurity matrix elements should represent an excellent approximation, as long as the typical distance between neighboring impurities is much larger than the characteristic decay length $\xi$, so that each impurity 
can be considered as independent. In addition to the "high energy" ingredients described in the model section, the model used in this type of investigation should include the key ingredients necessary for the emergence topological superconductivity, i.e., proximity-induced superconductivity, spin-orbit coupling, and Zeeman splitting. We pursue this path in the next section. On the other hand, the single impurity results described above can be used to construct phenomenological models with an effective impurity potential given by in Eq. (5.30) and relevant parameters, i.e., amplitude $A_{\alpha}$ and decay length $\xi_{\alpha}$, having distributions similar to those shown in Fig. 5.5 (see the Appendix of the published version of this work [76] for more details regarding the construction of phenomenological models). This type of approach enables the efficient investigation of the disordered system over a large parameter space without the need to address a numerically demanding threedimensional Schrödinger-Poisson problem. Hence, in addition to the results discussed below, Majorana device modeling should indirectly benefit from our phenomenological characterization of the impurity potential given by Eq. (5.30).

\subsubsection{Multiple charge impurities}

In this section we consider a hybrid nanowire with multiple embedded charge impurities and investigate the effect of the impurity-induced potential on the low energy physics, focusing on the fate of the Majorana zero energy modes that emerge in the clean system. Our analysis is based on two working assumptions. i) We consider systems with low/intermediate impurity concentrations, which are characterized by average distances between neighboring impurities that are much larger than the characteristic length of the effective (single) impurity potential. This allows us to work within the independent impurity approximation, in which each charge impurity generates an effective potential that is independent of the presence of other impurities and can be described using the approach discussed in the previous section. ii) We assume that the inter-subband spacing is much larger than all other relevant energy scales. This allows us to work within the independent band approximation, which neglects the effects of inter-subband coupling. Within this approximation, the low-energy physics can be accurately captured using an effective single band model. We note that the independent band approximation is expected to break down in systems with high subband occupancy [121]. Also note that in systems with low inter-subband spacing the effects of impurity-induced disorder are expected to be significantly stronger than the effects described below, due to additional contributions from impurityinduced inter-subband couplings [72]. So, the situation discussed here is, in some sense, the most optimistic scenario conducive to the emergence of topological Majorana modes; strong disorder, high subband occupancy, and, implicitly, small inter-subband spacing will simply make the situation worse, with topological physics being practically impossible to achieve in SM-SC hybrid platforms. The effective single band model for a hybrid wire with 
multiple charge impurities is introduced in Sec. 5.1.2. The results of our numerical analysis are discussed in Sec. 5.1.2.

\section{Model}

Within the independent subband approximation, the system can be described using an effective one-dimensional single-band model $[8,9]$ defined by the Bogliubov-de Gennes (BdG) Hamiltonian,

$$
\begin{aligned}
H= & \left(-\frac{\hbar^{2}}{2 m^{*}} \partial_{z}^{2}-\mu-i \alpha_{R} \partial_{z} \sigma_{y}+\Gamma \sigma_{z}\right) \tau_{z} \\
& -\Delta \sigma_{y} \tau_{y}+V_{i m p}(z) \tau_{z}
\end{aligned}
$$

where $m^{*}$ is the effective mass, $\mu$ is the chemical potential, $\alpha_{R}$ is the Rashba spin-orbit coupling coefficient, $\Gamma$ is the Zeeman energy, $\Delta$ is the induced superconducting pairing, $V_{\text {imp }}$ is the effective potential generated by the presence of charge impurities, and $\sigma_{i}$ and $\tau_{i}$, with $i=x, y, z$, are Pauli matrices in spin and particle-hole spaces, respectively. Note that all parameters in Eq. (5.34) are assumed to be position independent, and we use the values $m^{*}=0.023, \alpha_{R}=20 \mathrm{meV} \cdot \mathrm{nm}$, and $\Delta=0.3 \mathrm{meV}$ unless stated otherwise.

On the other hand, the impurity potential has the form

$$
V_{i m p}(z)=\sum_{m=1}^{N_{i m p}} V_{\alpha, \alpha}\left(z-z_{m} ; Q_{m}, x_{m}, y_{m}\right) \text {, }
$$

where $N_{i m p}$ is the total number of impurities embedded within the wire, $V_{\alpha, \alpha}$ is the effective potential generated by a single impurity, i.e., the intrasubband matrix element given by Eq. (5.29), $r_{m}=\left(x_{m}, y_{m}, z_{m}\right)$ describes the position of impurity $m$, and $Q_{m}$ indicates its charge. We assume charge neutrality and consider an equal number of positive $(Q=+e)$ and negative $(Q=-e)$ elementary charges distributed randomly throughout the wire. Each disorder realization corresponds to a specific set of $N_{i m p}$ impurity positions $\left\{r_{m}\right\}$ and a set of $N_{i m p}$ charges $\left\{Q_{m}\right\}$. Note that $\left(x_{m}, y_{m}\right)$ can take 169 different values sampled evenly over the hexagonal cross-section of the nanowire, while $z_{m}$ can take any value corresponding to a lattice site of the discretized version of Eq. (5.34) with $a_{z}=4 \mathrm{~nm}$ being the lattice spacing. For concreteness, we assume that chemical potential is tuned near the bottom of the second subband, so that the relevant matrix elements $V_{\alpha, \alpha}$ entering Eq. (5.35) correspond to $\alpha=2$. These matrix elements are calculated selfconsistently following the procedure described in Sec. 5.1.1. The low-energy eigenvalues and the corresponding eigenstates of the Hamiltonian (5.34) are then obtained using the Lanczos method [169].

To facilitate the connection with experimental tunneling spectroscopy, we also calculate the differential conductance for charge tunneling into the left or the right end of the wire. This is realized by connecting the proximitized wire to semi-infinite leads at both ends and using the Blonder-Tinkham-Klapwijk 
(BTK) formalism [170]. The normal leads are modeled by the Hamiltonians,

$$
H_{L(R)}=\left(-\frac{\hbar^{2}}{2 m^{*}} \partial_{z}^{2}-\mu_{l}+V_{L(R)}(z)\right) \tau_{z}
$$

where the labels $L$ and $R$ designate the left and right leads, respectively, $\mu_{l}$ is the chemical potential of the leads, and $V_{L}$ and $V_{R}$ are tunnel barrier potentials at the left and right ends of the system, respectively. The tunnel barriers are square potential barriers of amplitude $V_{B}$ and length $L_{B}=20 \mathrm{~nm}$ located at the ends of the corresponding leads directly adjacent to the proximitized wire. To evaluate the scattering matrix $S$, we consider the retarded Green's function,

$$
\mathcal{G}(\omega)=\left[\omega-\bar{H}-\Sigma_{L}(\omega)-\Sigma_{R}(\omega)+i \eta\right]^{-1},
$$

where $\bar{H}$ is the (discretized) Hamiltonian containing the sites within the proximitized region, as well as the barrier sites, plus one additional site on each side of the system, immediately outside the corresponding barrier region, $\Sigma_{L}$ and $\Sigma_{R}$ are the self-energies obtained by integrating out the degrees of freedom associated with the left and right leads [149], respectively, and $\eta \in \mathbb{R}^{+}$ accounts for dissipative broadening [171, 172]. Details about the Green's function and self-energy formalism can be found in Appendix D. The boundary elements of the Green's function (D.1) are calculated using the recursive Green's function algorithm [173]. In turn, these elements can be related to the scattering matrix, $S$, using the Fisher-Lee relations [174]. Finally, the scattering matrix elements are used to calculate the local conductance [170],

$$
G_{i}=\frac{e^{2}}{h}\left(2-\operatorname{Tr}\left(S_{i i}^{e e}\right)+\operatorname{Tr}\left(S_{i i}^{e h}\right)\right)
$$

where $S_{i i}^{e e}$ and $S_{i i}^{e h}$ describe the reflection of incoming electrons with energy $\omega$ into electrons and holes, respectively, and $i=\mathrm{L}, \mathrm{R}$. The numerical values of the parameters used in the diferential conductance calculations are $\mu_{l}=$ $20 \mathrm{meV}, V_{B}=40 \mathrm{meV}, L_{B}=20 \mathrm{~nm}$, and $\eta=20 \mu \mathrm{eV}$.

Before discussing the results, a few comments are warranted. By taking the effective impurity potential, $V_{i m p}$, in Eq. (5.35) to be a sum of single impurity potentials, we are neglecting any change of the potential due to inter-impurity coupling. This is expected to be a good approximation, provided the typical spacing between charge impurities is larger than the single impurity potential decay length, i.e., in the low/intermediate impurity density regime. The results shown in Fig. ?? indicate that the decay length is in the range $\xi \approx 5-25 \mathrm{~nm}$, which is significantly less than the typical impurity separation length for low/intermediate impurity densities. Note that for higher impurity densities we find that Majorana physics is completely destroyed by disorder, a conclusion that is unlikely to be modified by including inter-impurity coupling effects. The fact that strong disorder destroys the Majorana physics in nanowires and other superconducting systems is now well-accepted. 
Finally, we note that the generalization of the single-band formalism discussed here to a multi-subband approach is straightforward. The generalized effective model is a one-dimensional multi-subband model with intersubband coupling induced by the off-diagonal matrix elements of the effective potential, $V_{\alpha, \beta}$, with $\alpha \neq \beta$. As shown in Sec. 5.1.1, these elements are typically smaller than, but comparable to the corresponding diagonal elements (see Fig. 5.3). The inter-subband coupling terms are expected to become relevant when the inter-subband spacing $\Delta E$ between subbands close to the Fermi level is comparable to the magnitude of $V_{\alpha, \beta}$, which implies $\Delta E \lesssim 1 \mathrm{meV}$. For the case investigated here, which corresponds to the second subband being tuned near the chemical potential, the inter-subband spacing is $\Delta E \sim 10 \mathrm{meV}$, significantly larger than the amplitude of the effective potential matrix elements. Consequently, we can safely ignore the disorderinduced inter-subband coupling. High occupancy, on the other hand, is associated with a reduction of the inter-subband spacing [121] and a multisubband approach becomes necessary. We emphasize that in the multi-subband regime the system is less robust against disorder [55, 72]. Therefore, our independent-subband treatment provides upper bounds for impurity concentrations consistent with various aspects of Majorana physics. In other words, we are considering the most favorable scenario in order to predict the upper bound on the allowed disorder that would still enable topological Majorana physics to emerge in realistic SM-SC structures.

\section{Results}

The numerical results discussed in this section correspond to a charge neutral system containing an equal number of positively and negatively charged impurities with charges $Q=+e$ and $Q=-e$, respectively. Positive charges create local potential wells, while negatively charged impurities generate effective potential barriers. To gain some intuition regarding the effects induced by the two types of potential perturbations (i.e., "well" and "barrier"), we first consider a wire of length $L=4.2 \mu \mathrm{m}$ having an "artificial" potential perturbation localized near the middle of the wire and consisting of a square potential well (barrier) of width $L_{b}=50 \mathrm{~nm}$ and height $V_{b}=-10 \Delta$ $\left(V_{b}=+10 \Delta\right)$, where $\Delta=0.3 \mathrm{meV}$ is the induced pair potential. The dependence of the corresponding low-energy spectra on the applied Zeeman field is shown in Fig. 5.8, panels (a) and (b). Note that the short-range potential perturbation induces sub-gap states (green lines in Fig. 5.8) when the system is in the topological regime $[67,175]$, which can act as a source of quasiparticle poisoning in Majorana qubits [176]. Also note that the characteristic energy of the in-gap mode generated by the potential barrier is much lower than the energy of the in-gap mode generated by the potential well, except for an isolated zero energy crossing at Zeeman field $\Gamma \approx 5.4 \Delta$.

The difference between the in-gap mode induced by the potential well and that generated by the potential barrier is further illustrated by the dependence of these modes on the amplitude of the square potential. This dependence is shown in fig. 5.8(c) for a fixed value of the Zeeman field, 


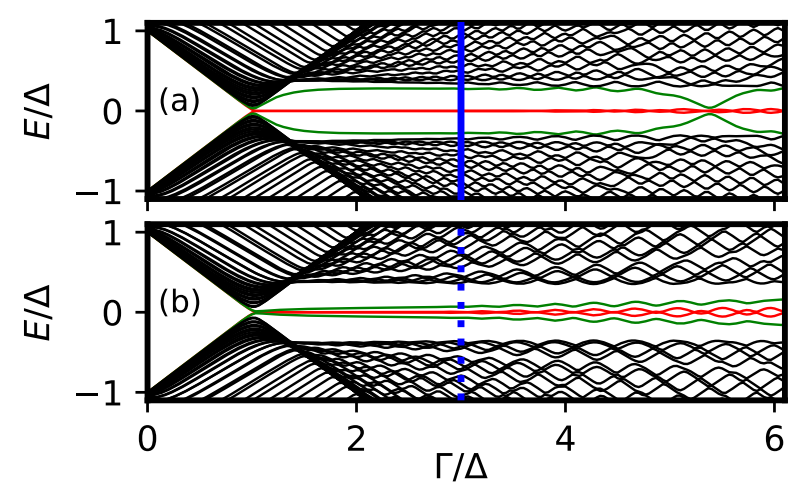

(c)

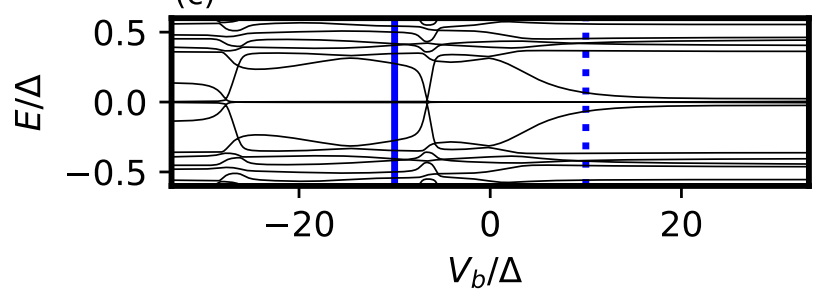

FIGURE 5.8: Low-energy spectrum as a function of Zeeman splitting for a wire of length $L=4.2 \mu \mathrm{m}$ having a square potential well (a) or barrier (b) localized near its center. The width of the square potential is $L_{b}=50 \mathrm{~nm}$ and its height is (a) $V_{b}=-10 \Delta$ and (b) $V_{b}=10 \Delta$. Red and green lines correspond to the first and second lowest energy modes, respectively. (c) Spectrum as a function of $V_{b} / \Delta$ for a Zeeman field $\Gamma=3 \Delta$. Blue solid and dashed lines indicate matching parameters in panel (c) and panels (a) and (b), respectively.

$\Gamma=3 \Delta$. Note that the potential well generates an in-gap mode with energy comparable to the topological gap, except a few isolated Andreev crossings. By contrast, the mode generated by the potential barrier collapses toward zero energy with increasing $V_{b}$. This is a specific example of a near-zero energy subgap mode induced by an inhomogeneous potential, a scenario extensively discussed in the literature.

To identify the nature of the in-gap modes, we calculate the corresponding wave functions in the Majorana representation. More specifically, let $\psi_{ \pm E_{n}}(z)$, with $0 \leq E_{1} \leq E_{2}$, be the lowest energy eigenstates of the BdG Hamiltonian. We define the following Majorana components associated with the low-energy BdG states [16]

$$
\begin{aligned}
\chi_{n}^{A}(z) & =\frac{1}{\sqrt{2}}\left[\psi_{E_{n}}(z)+\psi_{-E_{n}}(z)\right], \\
\chi_{n}^{B}(z) & =\frac{i}{\sqrt{2}}\left[\psi_{E_{n}}(z)-\psi_{-E_{n}}(z)\right] .
\end{aligned}
$$

Note that $\chi_{n}^{A}$ and $\chi_{n}^{B}$ are not eigenstates of the BdG Hamiltonian, except for $E_{n}=0$, and we have $\left\langle\chi_{n}^{A}|H| \chi_{n}^{A}\right\rangle=\left\langle\chi_{n}^{B}|H| \chi_{n}^{B}\right\rangle=0$ and $\left\langle\chi_{n}^{A}|H| \chi_{n}^{B}\right\rangle=i E_{n}$. 


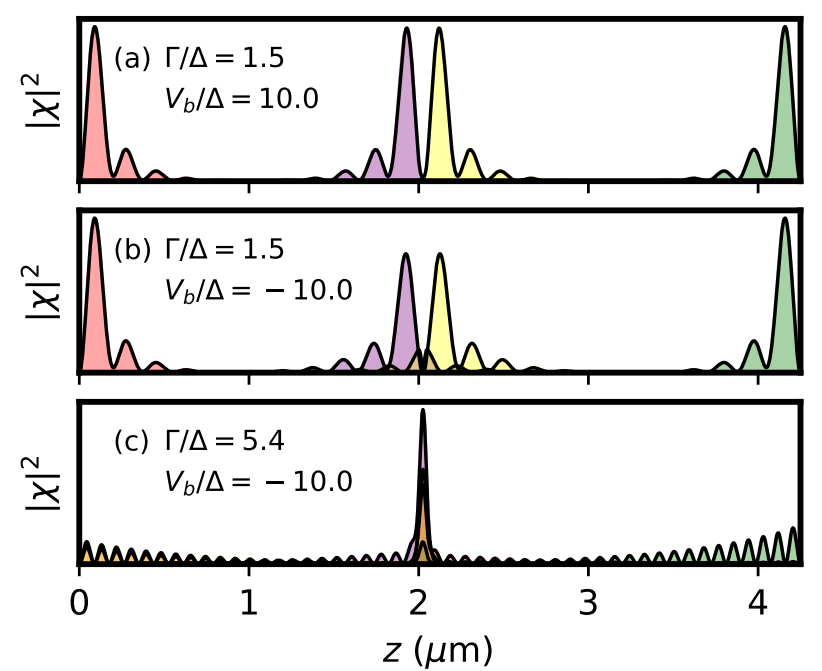

FIGURE 5.9: Position dependence of the amplitude of the Majorana wave functions, $\left|\chi_{n}^{A}\right|^{2}$ and $\left|\chi_{n}^{B}\right|^{2}$, corresponding to the lowest energy states $(n=1,2)$ in Fig. 5.8. The values of the potential height $V_{b}$ and Zeeman field $\Gamma$ are indicated inside each subplot. Note that the lowest energy states $(n=1$, red lines in Fig. 5.8) correspond to a pair of Majorana modes localized near the two ends of the nanowire (red and green modes), while the potential-induced in-gap states $(n=2$, green lines in Fig. 5.8) correspond to a pair of (partially) overlapping Majorana modes localized near the middle of the wire (purple and yellow modes). At the Andreev crossing corresponding to $\Gamma \approx 5.4 \Delta$ in Fig. 5.8(a) the two Majorana modes completely overlap [panel (c)].

The position dependence of the amplitude of the Majorana wave functions corresponding to the in-gap states from Fig. 5.8 are shown in Fig. 5.9. The lowest energy states, $n=1$ (red lines in Fig. 5.8), correspond to a pair of Majorana modes localized near the two ends of the nanowire (red and green modes in Fig. 5.9). On the other hand, the in-gap states induced by the square potential perturbation, $\mathrm{n}=2$ (green lines in Fig. 5.8), correspond to a pair of (partially) overlapping Majorana modes localized near the middle of the wire (purple and yellow modes in Fig. 5.9). Note that the Majorana modes generated by the potential well [Fig. 5.9(b)] have a significantly stronger overlap than the Majorana modes generated by the potential barrier [Fig. 5.9(a)]. Furthermore, at the Andreev crossings, the two Majorana modes $\chi_{2}^{A}$ and $\chi_{2}^{B}$ completely overlap, generating a "regular" Andreev bound state localized in the potential well. In general, however, the in-gap modes generated by the local potential perturbation can be viewed as a pair of partially overlapping quasi-Majorana modes [94] or, alternatively, as a partially separated Andreev bound state (ps-ABS) [108]. As shown below, partially overlapping/separated Majorana modes emerge generically in proximitized wires in the presence of positively/negatively charged impurities. 


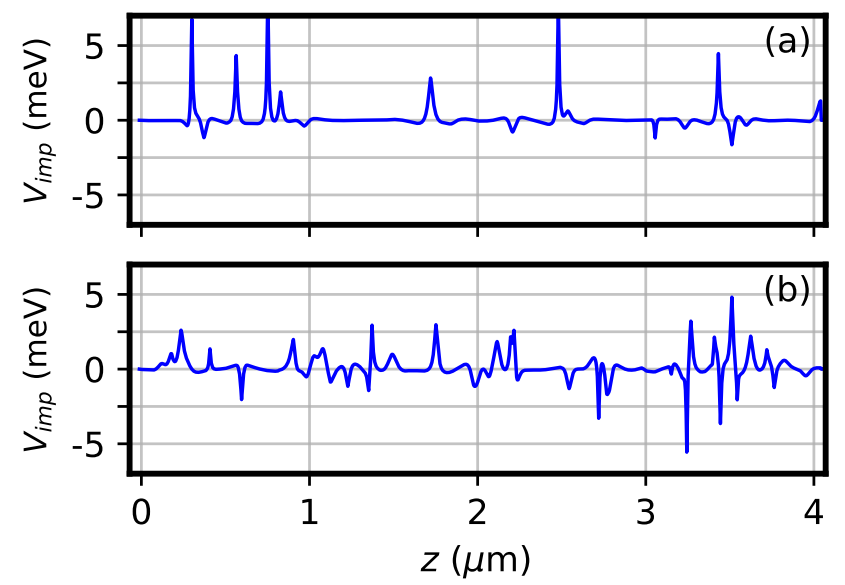

FIGURE 5.10: Position dependence of the effective impurity potential for two specific disorder realizations corresponding to impurity densities (a) $n_{\text {imp }}=1.6 \cdot 10^{15} \mathrm{~cm}^{-3}$ (linear density $\lambda_{\text {imp }}=5 \mu \mathrm{m}^{-1}$ ) and (b) $n_{\text {imp }}=4.7 \cdot 10^{15} \mathrm{~cm}^{-3}$ (linear density $\lambda_{\text {imp }}=15 \mu \mathrm{m}^{-1}$ ). The chemical potential of the wire is tuned near the bottom of the second subband. These impurity potentials are used in the calculations discussed in subsections 5.1.2 and 5.1.2.

Next, we characterize the effective potential generated by charge impurities embedded within the wire by providing some specific examples and calculating the correlation function $\left\langle V_{i m p}(z) V_{i m p}\left(z^{\prime}\right)\right\rangle$. The position dependence of the effective impurity potential $V_{i m p}(z)$ given by Eq. (5.35) for two disorder realizations with impurity densities $n_{i m p}=1.6 \cdot 10^{15} \mathrm{~cm}^{-3}$ and $n_{\text {imp }}=4.7 \cdot 10^{15} \mathrm{~cm}^{-3}$, respectively, are shown in Fig. 5.10. The first example corresponds to a low impurity density of about 5 impurities per micron, while the second example corresponds to an intermediate regime with 15 impurities per micron. These are relatively low impurity concentrations for semiconductor materials, but within the current technological capability. Note that the amplitude of the strongest potential peaks exceeds $5 \mathrm{meV}$, which corresponds to about $17 \Delta$, a significant perturbation (more than an order of magnitude larger than the SC gap) even taking into account its relatively short range. The properties of the system in the presence of the effective potential shown in Fig. 5.10 (a) are discussed in Sec. 5.1.2, while the intermediate impurity density regime corresponding to $V_{i m p}$ given in Fig. 5.10 (b) is investigated in Sec. 5.1.2.

To obtain a more generic characterization of the effective impurity potential, we consider many disorder realizations consistent with given values of the impurity density and calculate the correlation function $\left\langle V_{\operatorname{imp}}(z) V_{\text {imp }}\left(z^{\prime}\right)\right\rangle$. The results for a system with impurity densities $n_{\text {imp }}=0.25 \cdot 10^{16}, 0.5 \cdot 10^{16}, 1$. $10^{16} \mathrm{~cm}^{-3}$, which correspond to linear densities $\lambda_{\text {imp }}=7.9,15.9,31.8 \mu \mathrm{m}^{-1}$, respectively, are shown in Fig. 5.11. Each curve was obtained by averaging 


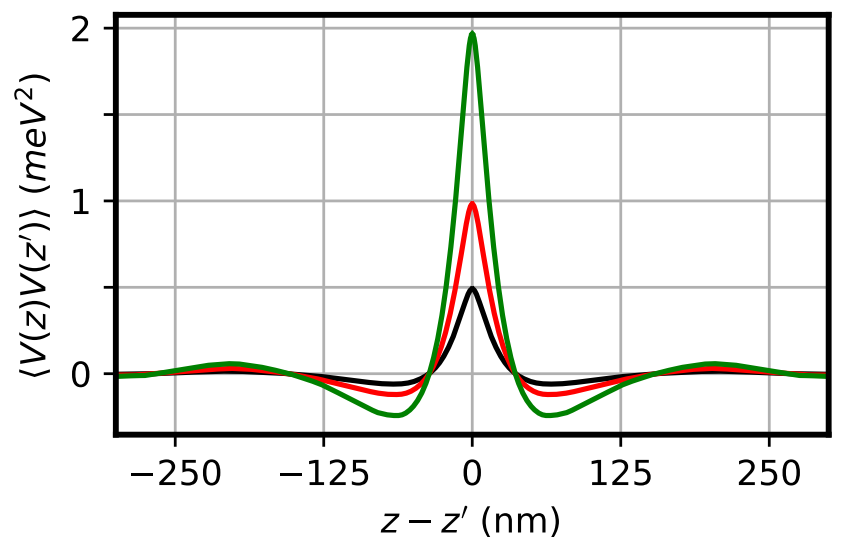

FIGURE 5.11: Correlation of the impurity potential for a system with impurity densities $n_{\text {imp }}=0.25 \cdot 10^{16} \mathrm{~cm}^{-3}$ (black), $n_{\text {imp }}=$ $0.5 \cdot 10^{16} \mathrm{~cm}^{-3}$ (red), $n_{\text {imp }}=1 \cdot 10^{16} \mathrm{~cm}^{-3}$ (green), which correspond to linear densities $\lambda_{\text {imp }}=7.9 \mu \mathrm{m}^{-1}, \lambda_{\text {imp }}=15.9 \mu \mathrm{m}^{-1}$, and $\lambda_{i m p}=31.8 \mu \mathrm{m}^{-1}$, respectively. The system is charge neutral (i.e., contains an equal number of $Q=+e$ and $Q=-e$ impurities, and has the chemical potential near the bottom of the second subband. Each correlation function was obtained by averaging over $5 \cdot 10^{5}$ disorder realizations. Note that the potential correlation scales with the impurity density.

over $5 \cdot 10^{5}$ disorder realizations. Note that the potential correlation function scales with the impurity density. For the intermediate density, $n_{\text {imp }}=$ $0.5 \cdot 10^{16} \mathrm{~cm}^{-3}$, the correlation function is characterized by a central peak of height $\propto 1 \mathrm{meV}^{2}$ and width at half maximum of about $40 \mathrm{~nm}$.

Based on previous studies of disorder effects in Majorana nanowires [54, $56,57,60,64,65,74,77,108]$, we know that the presence of disorder generally induces low-energy sub-gap states. Also, the simple example illustrated in Figs. 5.8 and 5.9 suggests that, at least under certain conditions, these sub-gap states consist of partially overlapping Majorana modes (or ps-ABSs) localized throughout the wire, in general away from the ends of the system. Note, however, that the presence of such non-topological (often called "trivial") ABSs does not necessarily affect the "genuine" topological Majorana zero modes (MZMs) that emerge in the topological regime at the ends of the system, as shown in Fig. 5.9. Therefore, it is of crucial importance to characterize quantitatively the spatial separation between Majorana modes and the edge-to-edge correlation associated with the presence of MZMs at the ends of the wire and investigate the effect of charge impurity-induced disorder on these quantities. To this end, we introduce the Majorana separation length, $\ell_{\text {sep }}$, defined as follows. Let $\psi_{E_{n}}$, with $E_{n} \geq 0$, be a positive energy eigenstate of the BdG Hamiltonian and $\chi_{n}^{(L / R)}$ be its left/right Majorana components. The corresponding Majorana separation length is defined as

$$
\ell_{\text {sep }}^{(n)}=\left\langle z_{n, R}\right\rangle-\left\langle z_{n, L}\right\rangle
$$




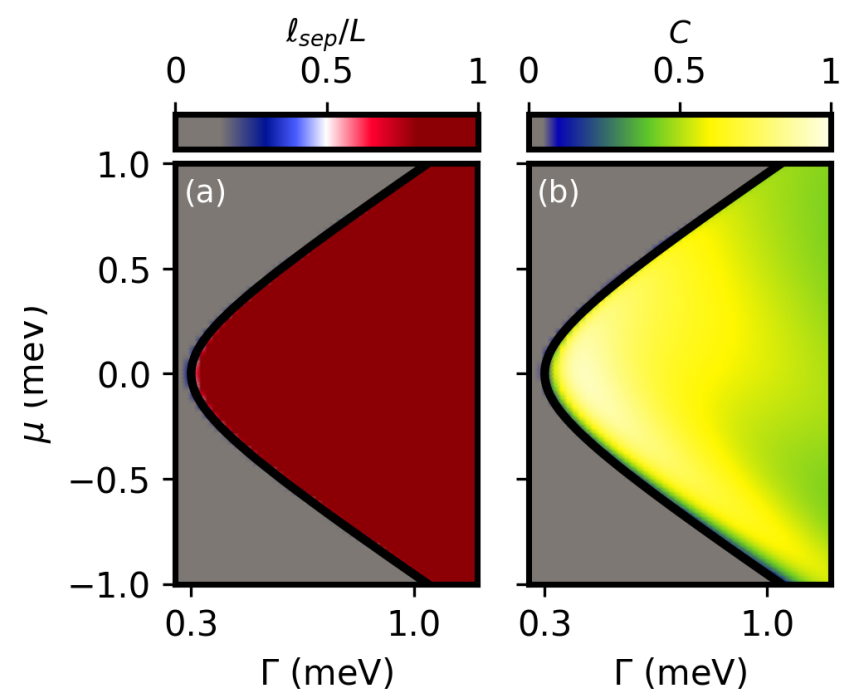

FIGURE 5.12: (a) Majorana separation, $\ell_{\text {sep }}$, and (b) edge-toedge correlation, $C$, maps for a clean system of length $L=4 \mu \mathrm{m}$. The black lines indicate the (bulk) topological quantum phase transition corresponding to $\Gamma=\sqrt{\mu^{2}+|\Delta|^{2}}$. The edge length used in the definition of $C$ [see Eq. (5.45)] is $\ell_{e}=200 \mathrm{~nm}$.

where $\left\langle z_{n, L(R)}\right\rangle$ is the expectation value of the position along the wire corresponding to the left (right) Majorana component. Explicitly, we have

$$
\left\langle z_{n, J}\right\rangle=\sum_{v} \sum_{i=1}^{N_{z}}\left|\chi_{n}^{(J)}\left(z_{i}, v\right)\right|^{2} z_{i},
$$

where $J \in\{L, R\}, N_{z}$ is total number of sites, $z_{i}$ is the (discretized) $z$-coordinate corresponding to site $i$, and we sum over the spin and particle-hole degrees of freedom indexed by $v$. Finally, we have

$$
\ell_{\text {sep }}=\operatorname{Max}_{n}\left[\ell_{\text {sep }}^{(n)} \mathcal{F}\left(E_{n}, \mathcal{U}, \Omega\right)\right]
$$

where $\mathcal{F}$ is a function that filters out the states outside a small energy window centered at $E=0$. The details of the filtering are not important, as this simply corresponds to the energy resolution defining "zero energy" or "zero bias" in the experiment. We choose the filter function to have the form

$$
\mathcal{F}(E, \mathcal{U}, \Omega)=\frac{1}{2}\left[\tanh \left(\frac{E-\mathcal{U}}{\Omega}\right)-\tanh \left(\frac{E+\mathcal{U}}{\Omega}\right)\right]
$$

Note that $\mathcal{F} \approx 0$ for $|E| \gg \mathcal{U}$ and $\mathcal{F} \approx 1$ for $E=0$, while it smoothly interpolates between these values near $|E| \approx \mathcal{U}$ over an energy scale $\Omega$. Throughout the rest of this section we set $\mathcal{U}=0.2 \Delta$ and $\Omega=0.1 \Delta$. These are, most likely, fairly generous estimates for defining the zero-energy modes. Hence, according to Eq. (5.42), $\ell_{\text {sep }}$ measures the largest separation length between the left and right Majorana components of BdG states having a sufficiently 


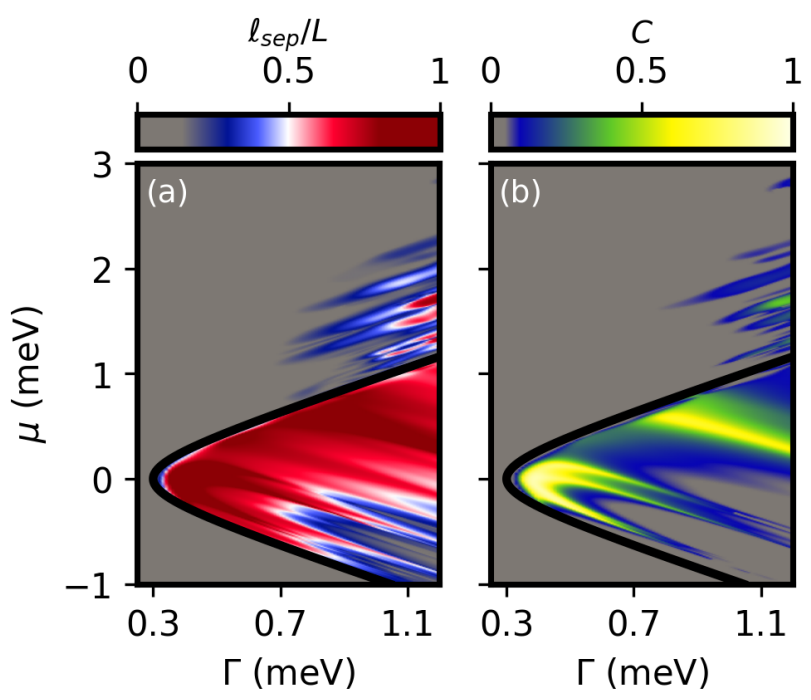

FIGURE 5.13: (a) Majorana separation, $\ell_{\text {sep }}$, and (b) edge-toedge correlation, $C$, maps for a disordered system of length $L=4 \mu \mathrm{m}$ with impurity density $n_{i m p}=1.6 \cdot 10^{15} \mathrm{~cm}^{-3}$ $\left(\lambda_{i m p}=5 \mu \mathrm{m}^{-1}\right)$. The black lines indicate the topological quantum phase transition for a clean system. The edge length used in the definition of $C$ [see Eq. (5.45)] is $\ell_{e}=200 \mathrm{~nm}$. Note that non-negligible values of $\ell_{\text {sep }}$ and $C$ occur outside the nominally topological region, while these quantities are significantly suppressed in some areas within this region.

low energy, so as to be operationally considered a zero-energy state. Next, we define the edge-to-edge correlation associated with the BdG eigenstate $\psi_{E_{n}}$ as

$$
C_{n}=\sqrt{W_{n}^{(L)} W_{n}^{(R)}} \mathcal{F}\left(E_{n}, \mathcal{U}, \Omega\right),
$$

with $W_{n}^{(L / R)}$ being the spectral weight at the left/right end of the system. Explicitly, we have

$$
W_{n}^{(J)}=\sum_{v} \sum_{i}^{\left(\ell_{e}\right)_{J}}\left|\chi_{n}^{(J)}\left(z_{i}, v\right)\right|^{2},
$$

where $J \in\{L, R\}$ and the summation over $i$ is restricted to sites that are within a distance $l_{e}$ of the corresponding edge. Let $n_{0}$ be the state characterized by the largest Majorana separation, i.e., $\ell_{\text {sep }}^{\left(n_{0}\right)}=\ell_{\text {sep }}$. Typically, $n_{0}=1$, i.e., the largest Majorana separation corresponds to the lowest energy mode, unless there is a "regular" (i.e., non-separated) Andreev bound state. We define the edge-to-edge correlation as $C=C_{n_{0}}$. Note that $0 \leq C \leq 1$, with $C \approx 1$ corresponding to a low energy BdG state having its Majorana components localized at the ends of the system, each within a distance $\ell_{e}$ of the corresponding edge.

To benchmark these quantities, we start with a clean system of length $L=4 \mu \mathrm{m}$ and calculate the dependence of the Majorana separation, $\ell_{\text {sep }}$, 

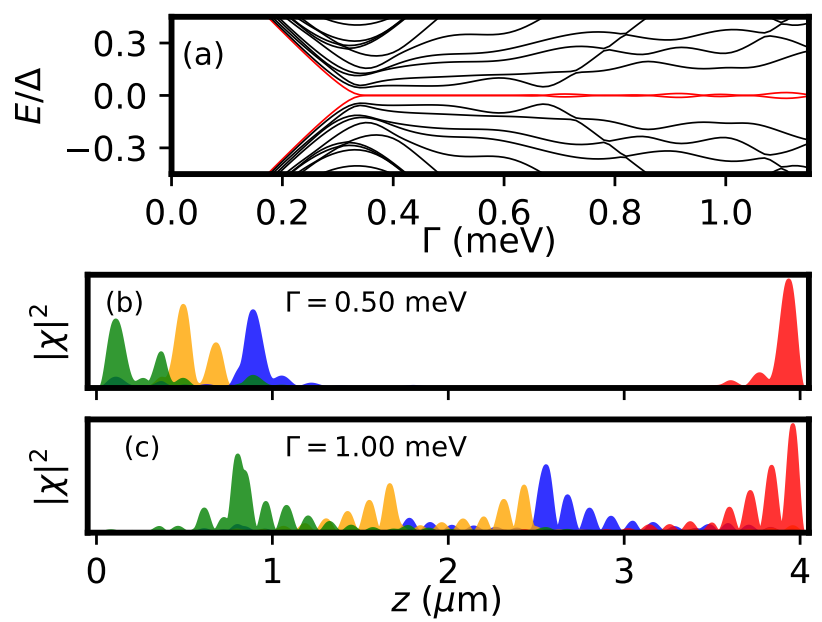

FIGURE 5.14: (a) Low-energy spectrum as a function of the Zeeman field for a system with the same parameters as in Fig. 5.13 and $\mu=0$. Red lines denote the lowest energy mode. (b) and (c) Spatial profiles of the Majorana components corresponding to the lowest BdG eigenstate (red and green) and second lowest energy eigenstate (blue and yellow) for $\Gamma=0.5 \mathrm{meV}$ and $\Gamma=1 \mathrm{meV}$, respectively. Note that in (c) the left Majorana component of the lowest energy state (green) is localized away from the corresponding edge, which causes the collapse of the edge-to-edge correlation $C$ in Fig. 5.13 in the area around $\mu=0, \Gamma=1 \mathrm{meV}$.

and edge-to-edge correlation, $C$, on the Zeeman field and chemical potential. The corresponding "phase diagrams" are shown in Fig. 5.12. The black lines mark the theoretically known phase boundary [177] associated with the topological quantum phase transition. Remarkably, the area characterized by large values of the Majorana separation, $\ell_{\text {sep }} \lesssim L$, and large edge-to-edge correlations, $C>0.5$, practically coincides with the topological phase. This indicates that the two quantities capture meaningful information about the Majorana zero modes and the topological quantum phase transition. Note, for example, that $C$ decreases with increasing Zeeman field as result of increasing the Majorana localization length, $\xi$, which transfers some of the spectral weight outside the edge regions defined by the length scale $\ell_{e}$ in Eq. (5.45). We emphasize that generating two dimensional maps of the relevant quantities as functions of various control parameters, such as the Zeeman splitting and the chemical potential (or applied back gate potential), provides significantly more information than focusing on specific parameter values. As shown below, such maps are mandatory for properly understanding the effects of disorder and should represent the standard in both theoretical and experimental investigations of hybrid systems. We urge experimentalists to always characterize the presence of (near) zero-energy modes by providing two-dimensional "phase diagram" maps in the magnetic field-gate voltage parameter space. 


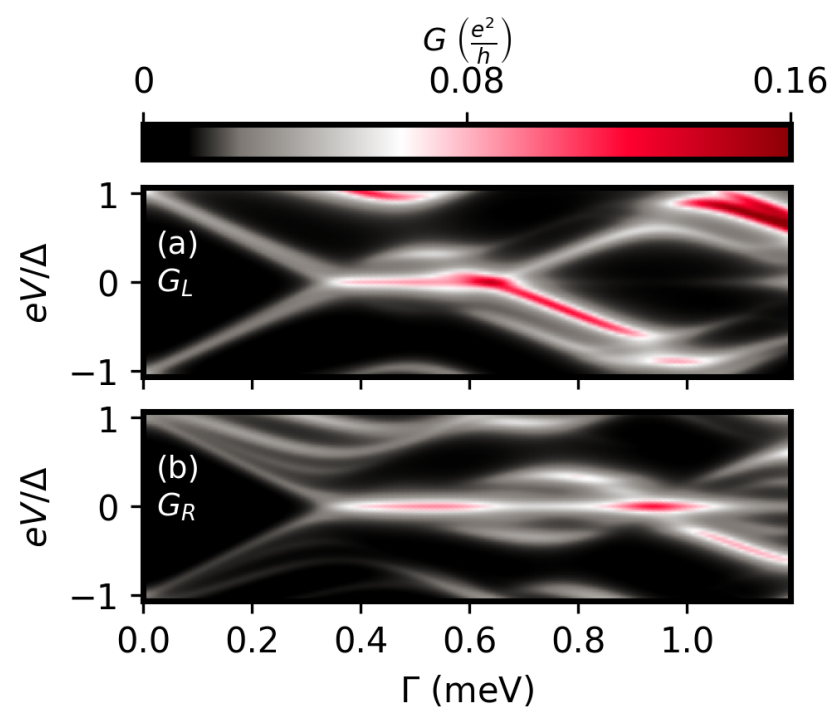

FIGURE 5.15: Local differential conductance at the left (a) and right (b) ends of the wire for a system with the same parameters as in Fig. 5.14. Note that the zero-bias conductance peak characterizing $G_{L}$ is suppressed between $0.7 \lesssim \Gamma \lesssim 1.1 \mathrm{meV}$ as a result of the left Majorana mode being pushed away from the edge, as shown in Fig. 5.14 (c).

\section{Low impurity density regime}

We are now ready to consider a system with randomly distributed charge impurities and investigate the effects of this type of disorder using the quantities introduced above. We start with a specific disorder realization corresponding to a relatively low impurity density, $n_{\text {imp }}=1.6 \cdot 10^{15} \mathrm{~cm}^{-3}$, which means $\lambda_{i m p}=5$ impurities per micron. The position dependence of the impurity potential $V_{i m p}(z)$ for this disorder realization is shown in Fig. 5.10 (a). The maps of the Majorana separation and edge-to-edge correlation as functions of Zeeman field and chemical potential are shown in Fig. 5.13. A comparison of these maps with the corresponding "phase diagrams" in Fig. 5.12 reveals two distinctive features: the emergence of areas with significant values of $\ell_{\text {sep }}$ and $C$ outside the nominally topological region and the substantial suppression of these quantities in certain areas within the topological region. We emphasize that, although the quantitative details of the phase diagram in Fig. 5.13 depend on the specific disorder realization and on the corresponding impurity potential (see Fig. 5.10) used in the calculation, these two distinctive qualitative features are generic.

To better understand the significance of these features, we calculate the low-energy spectrum as a function of the Zeeman field for a fixed value of the chemical potential, as well as the spatial profile of the Majorana components corresponding to certain representative low-energy modes. The results for $\mu=0$ are shown in Fig. 5.14. The low-energy spectrum in Fig. 5.14(a) shows the emergence of a near-zero energy mode for Zeeman fields 
$\Gamma \gtrsim 0.3 \mathrm{meV}$ (red lines). The lowest energy mode is separated from other finite energy states by a small gap that increases significantly for $\Gamma \gtrsim 0.75 \mathrm{meV}$. This behavior may be surprising if judged based on the information in Fig. 5.13 , which, for $\mu=0$, shows a strong suppression of $C$ at higher values of the Zeeman field. However, the spatial profiles of the Majorana components shown in Fig. 5.14(b) and (c) clarify the physics. Indeed, for $\Gamma=0.5 \mathrm{meV}$ the lowest energy state consists of two well separated Majorana modes localized near the ends of the system (green and red). The left (green) Majorana has some overlap with a ps-ABS localized nearby (yellow and blue), which represents the second lowest BdG state, but is weakly affected by the presence of this bound state. Consequently, $\ell_{\text {sep }}$ is comparable to the length $L$ of the wire and the edge-to-edge correlation $C$ is large. By contrast, at $\Gamma=1 \mathrm{meV}$ the left (green) Majorana mode is "pushed" away from the end of the system, which results in a reduction of the Majorana separation length and the collapse of the edge-to-edge correlation.

The example discussed above shows that a hybrid system with a low concentration of charged impurities is consistent with the emergence of well separated, near-zero energy Majorana modes. However, the presence of disorder may "push" these modes away from the ends of the system, which results in low values of the edge-to-edge correlation. In other words, the system can host "genuine" MZMs, but they may be "invisible" to local probes coupled to the ends of the wire. This severely limits the relevance of tunnel spectroscopy as a tool for detecting the emergence of Majorana zero modes in the presence of disorder, even in the weakly disordered situation. To make further connection with experiment, we calculate the local differential conductance for charge tunneling into the left and right end of the system. The results corresponding to a system with the same parameters as in Fig. 5.14 are shown in Fig. 5.15. One can clearly notice two low-energy modes coalescing toward zero energy and generating robust zero-bias conductance peaks (ZBCPs) at both ends of the system. At the left end, the ZBCP persists from $\Gamma=0.3 \mathrm{meV}$ to $\Gamma \approx 0.7 \mathrm{meV}$, then it appears to split. However, as revealed by the data in Fig. 5.14, the apparent splitting is due to a ps-ABS localized near the left end, while the "actual" Majorana mode (i.e., the "green" Majorana) does not become gapped, becoming instead "invisible" to local measurements at the edge, as it gets pushed away from the end of the wire. Within the range $0.7 \lesssim \Gamma \lesssim 1.1 \mathrm{meV}$ there is a robust $\mathrm{ZBCP}$ at the right end of the wire, but no ZBCP at the left end. This example clearly illustrates the difficulty of correctly interpreting tunneling conductance results in the presence of disorder. First, apparent splittings of the ZBCP can be misleading, as they are not necessarily associated with the mode that generates the $\mathrm{ZBCP}$. Second, the absence of edge-to-edge correlation does not necessarily imply the absence of robust, well-separated Majorana modes; it may simply mean that (at least) one of these modes is localized away from the end of the wire. We note that the conductance calculations shown in Fig. 5.15 were done in the tunneling limit, i.e., for high values of the potential barrier amplitude. In addition, we considered some finite dissipation, $\eta=20 \mu \mathrm{eV}$. As a result, the height of the 

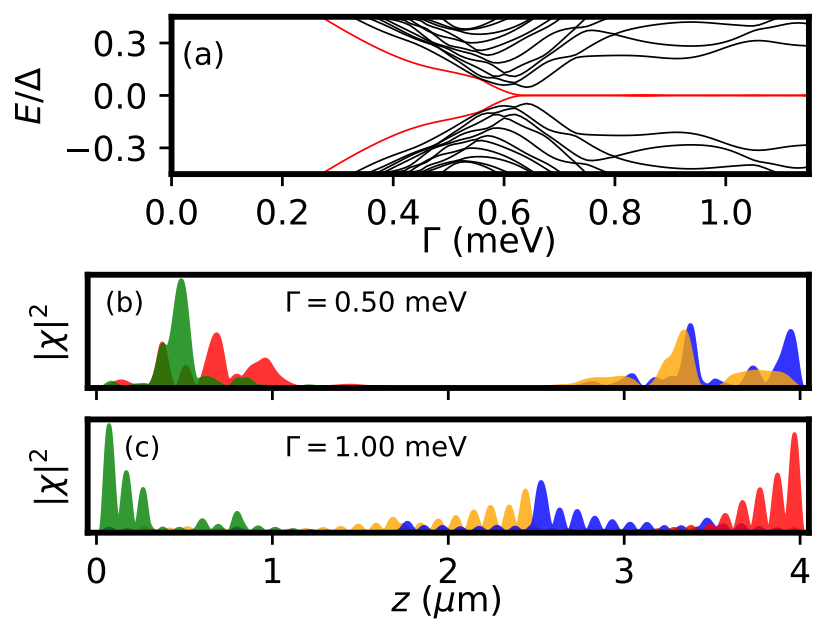

FIGURE 5.16: (a) Low-energy spectrum as a function of the Zeeman field for a system with the same parameters as in Fig. 5.13 and $\mu=0.5 \mathrm{meV}$. Red lines denote the lowest energy mode. (b) and (c) Spatial profiles of the Majorana components corresponding to the lowest BdG eigenstate (red and green) and second lowest energy eigenstate (blue and yellow) for $\Gamma=0.5 \mathrm{meV}$ and $\Gamma=1 \mathrm{meV}$, respectively. Note that in (b), which corresponds to the trivial regime, the Majorana modes strongly overlap, generating two ABSs localized near the ends of the system.

$\mathrm{ZBCP}$ is much smaller than the quantized value and there is some particlehole asymmetry [171, 172, 178]. These issues are well-understood and do not in any way affect our key qualitative conclusion of disorder possibly pushing the zero mode away from the end and making it invisible in standard tunneling spectroscopy. In some sense, this invisibility of the topological Majorana in the tunneling measurement (a false negative) is the ironic counterpart of the ps-ABS misleadingly producing non-topological zero bias conductance peaks mimicking Majorana zero modes (a false positive)!

Next, we consider another horizontal cut through the phase diagram in Fig. 5.13 corresponding to $\mu=0.5 \mathrm{meV}$. For this value of the chemical potential, the system is characterized by large Majorana separations and edgeto-edge correlations in the topological regime, i.e., for $\Gamma \gtrsim 0.6 \mathrm{meV}$. Indeed, the spectrum shown in Fig. 5.16 (a) is characterized by a robust zero energy mode (red line for $\Gamma \gtrsim 0.6 \mathrm{meV}$ ) and a sizable topological gap. Note the presence of finite energy in-gap states in the topologically trivial regime (e.g., red lines for $\Gamma \lesssim 0.6 \mathrm{meV}$ ). These topologically trivial in-gap modes consist of Andreev bound states with strongly overlapping Majorana components localized near the ends of the wire, as shown in Fig. 5.16 (b). In the topological regime, on the other hand, the system is characterized by well separated Majorana modes localized at the ends of the system, as shown in Fig. 5.16 (c) (the green and red Majoranas) and is consistent with the large values of $\ell_{\text {sep }}$ and $C$ in Fig. 5.13.

The conductance traces corresponding to the $\mu=0.5 \mathrm{meV}$ cut are shown 


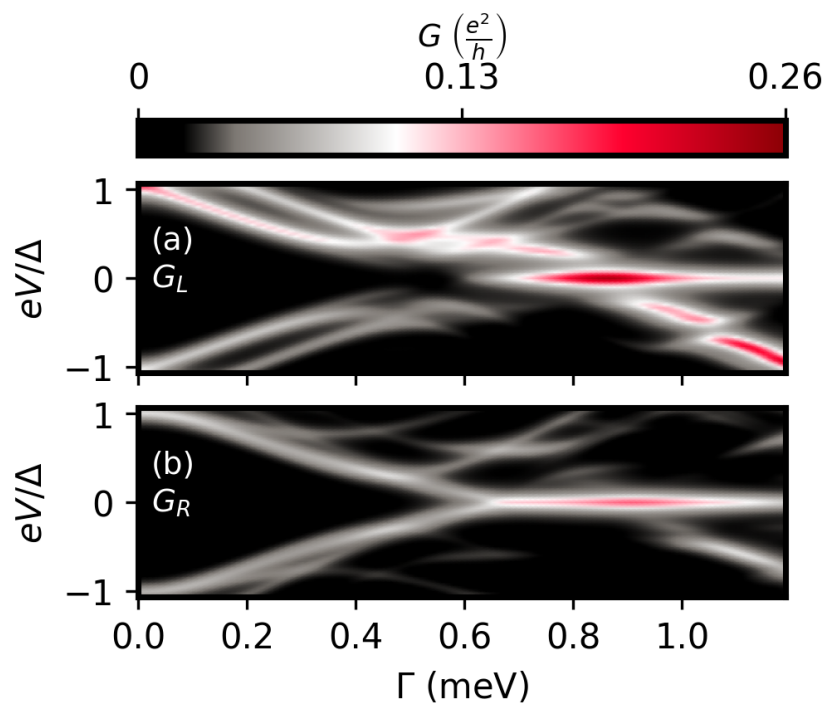

FIGURE 5.17: Local differential conductance at the left (a) and right (b) ends of the wire for a system with the same parameters as in Fig. 5.16. Correlated zero-bias conductance peaks occur at the two end of the system for $\Gamma \gtrsim 0.65$, consistent with the large $C$ values for $\mu=0.5 \mathrm{meV}$ and $\Gamma \gtrsim 0.65 \mathrm{meV}$ in Fig. 5.13. Note the significant enhancement of the ZBCP in (a) due to the Majorana mode hybridizing with a bound state localized within the barrier region, which crosses zero energy at $\Gamma \approx 0.8 \mathrm{meV}$.

in Fig. 5.17. The presence of the MZMs is revealed by the emergence of robust ZBCPs at both ends of the system. Note, however, that the emergence of the $\mathrm{ZBCP}$ looks rather different at the two ends, with two low-energy modes coalescing toward zero energy clearly visible at the right end and no apparent gap closing at the left end. This behavior is due to the fact that the right Majorana mode is adiabatically connected to the ABS localized at the right end of the system, while the left Majorana is connected to a trivial mode that has low spectral weight at the left end of the system and couples weakly to the corresponding probe, thus remaining "invisible." Another significant feature that is clearly manifested in Fig. 5.17 (a) is the enhancement of the $\mathrm{ZBCP}$ weight/height due to the Majorana mode hybridizing with a bound state localized in the barrier region. Indeed, in Fig. 5.17 (a) one can clearly notice an ABS crossing zero energy at $\Gamma \approx 0.8 \mathrm{meV}$. This mode is absent from the low-energy spectrum shown in Fig. 5.16 (a), a clear indication that it is generated by the very presence of the barrier region that couples the system to the normal lead, as this is not included in the calculation of the spectrum. This type of enhancement of the ZBCP due to coupling to an ABS localized at the end of the system is also visible in Fig. 5.15. The results presented in Fig. 5.17 and discussed above indicate a serious problem regarding tunnel conductance measurements: the end-to-end conductance correlations, which are often thought to be the decisive signature for the existence of topological MZMs, may very well be quite imprecise (or even absent) in the presence of (even weak) disorder. The absence of such correlations can be quite generic 

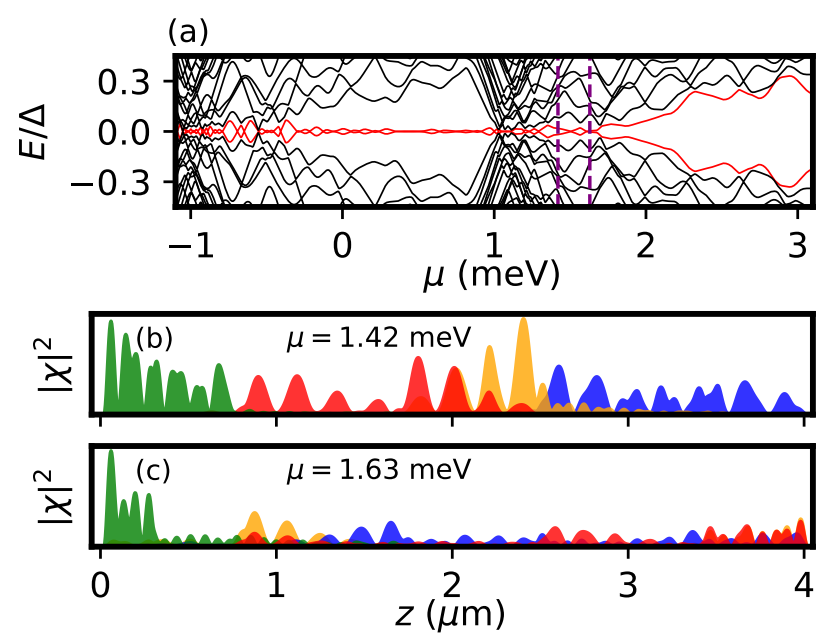

FIGURE 5.18: (a) Low-energy spectrum as a function of the chemical potential for a system with the same parameters as in Fig. 5.13 and $\Gamma=1.1 \mathrm{meV}$. Red lines denote the lowest energy mode. (b) and (c) Spatial profiles of the Majorana components corresponding to the lowest BdG eigenstate (red and green) and second lowest energy eigenstate (blue and yellow) for two values of the chemical potential marked by dashed purple lines in (a). In (c) the Majorana components of the lowest energy mode (green and red) have nonzero spectral weights at the ends of the system, which results in a finite edge-to-edge correlation $C$.

in disordered systems and may imply either that one of the MZMs cannot be accessed through tunnel spectroscopy at the wire end (because it was pushed away), or that the observed zero mode is simply trivial. Comparing the conductance traces at the two ends of the system cannot discriminate between these possibilities. However, generating two-dimensional conductance maps over large parameter regions may provide additional information, as discussed below.

Having clarified the features that characterize the nominally topological region of the phase diagrams in Fig. 5.13, the natural question concerns the nature of the low-energy states responsible for the emergence of high Majorana separations and significant edge-to-edge correlations in the trivial region (of the pristine system) with $\mu>1 \mathrm{meV}$. To address this question, we consider a vertical cut at fixed Zeeman field $\Gamma=1.1 \mathrm{meV}$. The dependence of the low-energy spectrum on the chemical potential along this cut is shown in Fig. 5.18 (a). For $-1 \lesssim \mu \lesssim 1 \mathrm{meV}$ the system is in the nominally topological regime and one can clearly notice the a near-zero energy mode (red lines) protected by a finite gap over most of this interval. The gap collapses for $\mu \lesssim-0.25 \mathrm{meV}$. Most interestingly, low-energy modes are also present for $1 \lesssim \mu \lesssim 1.75 \mathrm{meV}$, i.e., in the nominally trivial regime. To clarify the nature of these states, we calculate their Majorana components for two values of the chemical potential.The results are shown in Fig. 5.18 (b) and (c). The lowenergy states can be viewed as superposition of several partially-overlapping 


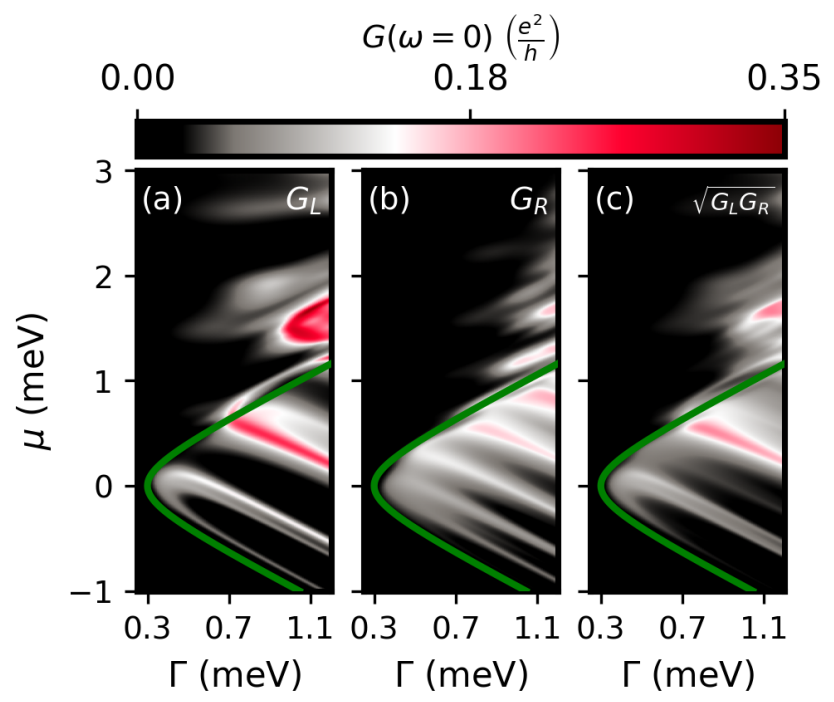

FIGURE 5.19: Zero-bias differential conductance maps for a system with the same parameters as in Fig. 5.13, but having normal leads and tunnel barriers attached at both ends. The conductance at the left $\left(G_{L}\right)$ and right $\left(G_{R}\right)$ ends of a system and the geometric average $\left(C_{G}=\sqrt{G_{L} G_{R}}\right)$ are shown in (a), (b), and (c), respectively. Note that the $C_{G}$ map closely resembles the edgeto-edge correlation map, $C$, in Fig. 5.13.

Majorana modes. Accidentally, Majorana components associated with the lowest energy state can have significant weights at the ends of the system, which generates a finite edge-to-edge correlation, as shown in Fig. 5.13 (b). Such zero modes accidentally arising from the disorder-induced overlap of several Majorana modes cannot be construed as being topological.

We have already pointed out the importance of generating two dimensional maps of the relevant quantities as functions of various control parameters. To further emphasize this point, we calculate the zero-bias differential conductance maps corresponding to charge tunneling into the left $\left(G_{L}\right)$ and right $\left(G_{R}\right)$ ends of a system having the same parameters as in Fig. 5.13. In addition, we define the geometric average of the left and right conductivities as a practical measure of the edge-to-edge correlation. Specifically, we define

$$
C_{G}=\sqrt{G_{L} G_{R}}
$$

The results are shown in Fig. 5.19. We note that the $C_{G}$ map in Fig. 5.19 (c) closely resembles the edge-to-edge correlation map, $C$, in Fig. 5.13. This observation has two important implications. First, $C_{G}$ provides a good measure of the edge-to-edge correlation that can be easily determined experimentally. Second, for large scale calculations (e.g., when doing statistics involving many disorder realizations - see below), one can focus on the numericallyless-expensive quantity $C$, instead of the more experimentally-relevant quantity $C_{G}$, since we find the two to be representing equivalent physics, even in 
the presence of disorder. In addition, we note that for low-impurity concentrations, the (zero energy) conductance maps provide a reasonably good correspondence with the phase diagram of the clean system, particularly in the low-field regime. However, as shown below, this correspondence fades away upon increasing the impurity concentration. This suggests that the systematic mapping of the zero-bias conductance at both ends of the system and of the corresponding correlation $C_{G}$ can provide a powerful experimental tool for assessing the strength of the effective disorder potential. Finally, we note that $C_{G}$ has the highly desirable practical property that it does not require identical tunnel barriers at the two ends. As long as a differences between the two barriers amounts to an overall enhancement/suppression of $G_{L}$ relative to $G_{R}$, the corresponding factor is irrelevant when calculating the correlation $C_{G}$. We note that our calculated conductance shown in Figs. 5.17 and 5.19 is characterized by zero bias values $\left(G_{L}, G_{R}\right.$, and $\left.C_{G}\right)$ smaller than the so-called Majorana quantization value of $2 e^{2} / h$, as we consider relatively high tunnel barriers and include a dissipation term. We emphasize that, in the presence of disorder, fine-tuning the parameters to obtain quantized values of the zero-bias conductance does not provide additional information regarding the nature of the underlying low-energy mode. Instead, producing detailed conductance maps over extended ranges of tuning parameters, similar to those in Fig. 5.19, can provide additional information, including estimates of the disorder strength. We think that generating such comprehensive maps is what experiments should focus on, rather than fine-tuning parameters to achieve Majorana quantization.

\section{Intermediate impurity density regime}

How does the phenomenology discussed above depend on the concentration of charge impurities, i.e., on the disorder strength? To address this question, we consider another specific disorder realization corresponding to an intermediate impurity density, $n_{\text {imp }}=4.7 \cdot 10^{15} \mathrm{~cm}^{-3}$, which means $\lambda_{i m p}=15$ impurities per micron. This is still relatively low disorder in terms of the bulk doping magnitude, but it is three times larger than the low-disorder case $\left(\lambda_{i m p}=5 \mu \mathrm{m}^{-1}\right)$ considered above. The position dependence of the impurity potential $V_{\text {imp }}(z)$ for this disorder realization is shown in Fig. 5.10 (b). We carry out the same calculations as above and construct the maps corresponding to the Majorana separation, $\ell_{\text {sep }}$, and edge-to-edge correlation, $C$, as functions of Zeeman field and chemical potential. The results are shown in Fig. 5.20.

In addition, we introduce a "projection map" based on the following quantity:

$$
P\left(\ell_{\text {sep }}, C\right)=\left\{\begin{aligned}
0, & \ell_{\text {sep }} \leq \ell_{\text {min }} \\
-1, & \ell_{\text {sep }}>\ell_{\text {min }} \text { and } C<C_{\text {min }} . \\
1, & \ell_{\text {sep }}>\ell_{\text {min }} \text { and } C>C_{\text {min }}
\end{aligned}\right.
$$

In essence, $P=0$ corresponds to low Majorana separation lengths (according to a criterion determined by $\left.\ell_{\min }\right), P=-1$ signals well separated Majoranas 


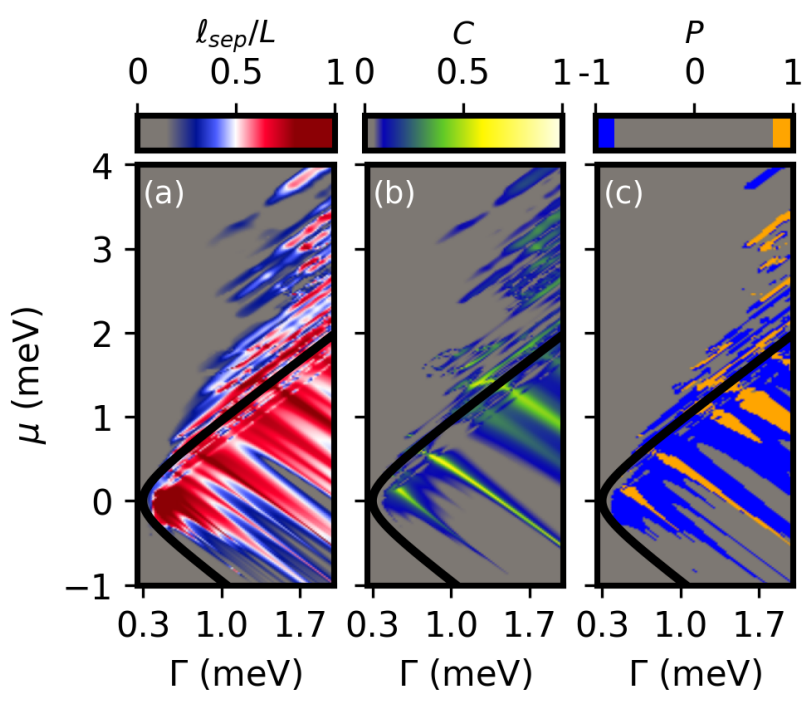

FIGURE 5.20: (a) Majorana separation, $\ell_{\text {sep }}$, (b) edge-to-edge correlation, $C$, and (c) projection, $P$, maps for a disordered system of length $L=4 \mu \mathrm{m}$ with impurity density $n_{\text {imp }}=$ $4.7 \cdot 10^{15} \mathrm{~cm}^{-3}\left(\lambda_{i m p}=15 \mu \mathrm{m}^{-1}\right)$. The impurity potential $V_{i m p}(z)$ for this disorder realization is shown in Fig. $5.10(b)$. The black lines indicate the topological quantum phase transition for a clean system. The projection map in (c) corresponds to $\ell_{\min }=0.5 \mathrm{~L}$ and $C_{\text {min }}=0.25$.

that do not generate a substantial edge-to-edge correlation (e.g., because one of the Majorana modes is pushed away from the end of the system by the disorder potential), while $P=1$ corresponds to the desired scenario involving well separated Majoranas and substantial edge-to-edge correlation. The projection map corresponding to $\ell_{\min }=0.5 \mathrm{~L}$ and $C_{\min }=0.25$ is shown in Fig. 5.20 (c). As compared to the corresponding maps in Fig. 5.13, the suppression of the Majorana separation and edge-to-edge correlation inside the nominally topological region is significantly stronger. When comparing the two figures, note that $\Gamma$ extends to higher values in Fig. 5.20 than Fig. 5.13. Nonetheless, there is a substantial area - blue region in panel (c) - corresponding to large values of the Majorana separation $\left(\ell_{\text {sep }}>2 \mu \mathrm{m}\right)$, but weak edge-to-edge correlation. This suggests that, even at this level of impurity concentration, there are segments of the wire that can be viewed as effectively topological, but their presence cannot be revealed by local measurements at the ends of the wire. By contrast, the areas corresponding to large values of $C$ are reduced to a few small islands. The underlying disorder-induced nonperturbative rearrangement of the Majorana spatial locations and the corresponding signatures revealed by the Majorana phase diagrams are important findings of our work.

To help connect these features to experimentally measurable quantities, we generate the corresponding zero bias conductance maps, as well as the geometric correlation $C_{G}$, for the intermediate disorder case. The results are shown in Fig. 5.21. First, we note the close resemblance between the $C$ map 


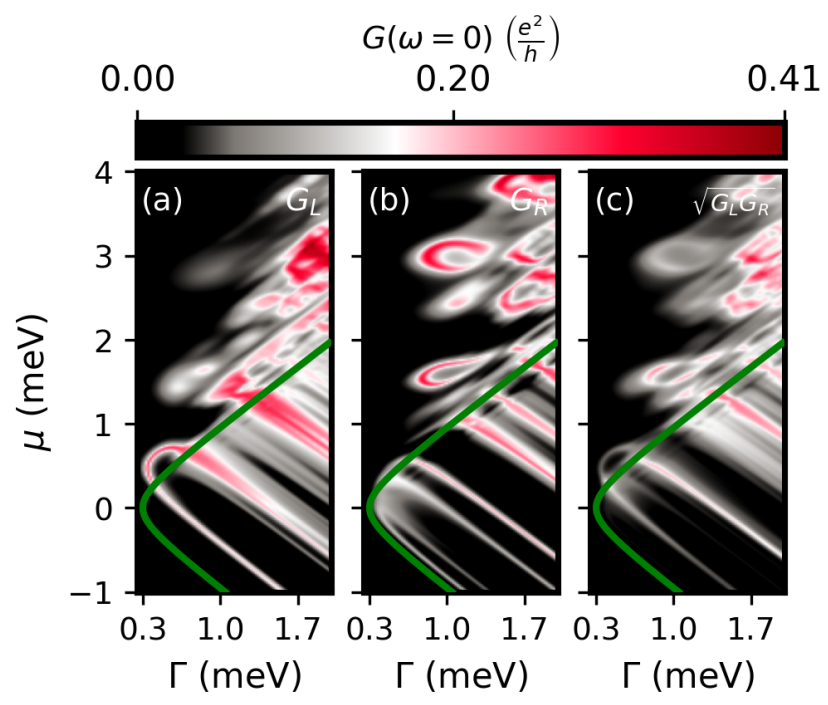

FIGURE 5.21: Zero-bias differential conductance maps for a system with the same parameters as in Fig. 5.20, but having normal leads and tunnel barriers attached at both ends. The conductance at the left $\left(G_{L}\right)$ and right $\left(G_{R}\right)$ ends of a system and the geometric average $\left(C_{G}=\sqrt{G_{L} G_{R}}\right)$ are shown in (a), (b), and (c), respectively. Note that the correspondence between the conductance maps and the topological phase boundary for the clean system (green line) is weak.

in Fig. 5.20 (b) and the $C_{G}$ map in Fig. 5.21 (c), with the exception of a few additional, loop-like features present in the $C_{G}$ map that will be discussed below. Second, we point out that, unlike the low impurity density case shown in Fig. 5.19, the areas of high zero-bias conductance are almost equally distributed inside and outside the nominally topological region. This suggest a shift of the chemical potential associated with the emergence of low-energy modes toward higher values as the impurity density increases, which is consistent with previous studies $[62,179]$. Note that this is not due to an actual shift of the impurity-induced effective potential, as the average value of $V_{i m p}$ is close to zero regardless of the impurity concentration (see Fig. 5.10).

To shed further light on the nature of various streaky and loopy highconductance features in Fig. 5.21, we consider the differential conductance as function of the applied Zeeman field and potential bias for two specific values of the chemical potential, $\mu=0$ and $\mu=3 \mathrm{meV}$, respectively. The first trace cuts through several narrow, uniformly dispersing high-conductance features that are characteristic to the nominally topological region (see Fig. 5.21). As revealed by the results shown in Fig. 5.22, these features are associated with Andreev bound states crossing zero energy at different values of the Zeeman field. Note that robust ZBCPs signaling the presence of well separated Majorana modes are clearly visible at both ends of the system, but within different intervals of Zeeman fields. The presence of these ZBCPs at $\mu=0$ is consistent with the large values of the Majorana separation in Fig. 5.20 (a), while their emergence within different $\Gamma$ intervals is consistent with 


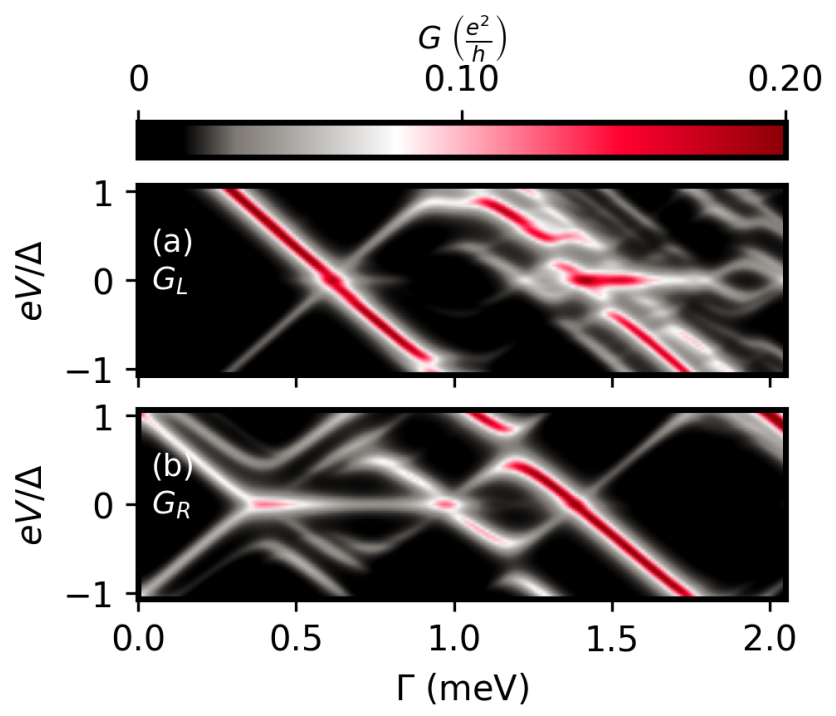

FIGURE 5.22: Local differential conductance at the left (a) and right (b) ends of the wire for a system with the same parameters as in Fig. 5.20 and chemical potential $\mu=0$. Note the strong features associated with Andreev bound states that cross zero energy at different values of the Zeeman field. In (a) the hybridization of these states with the Majorana mode leads to an enhancement of the ZBCP (extremely faint near $\Gamma \approx 0.6 \mathrm{meV}$ and clearly visible above $\Gamma \approx 1.4 \mathrm{meV}$ ).

the low values of $C$ in Fig. 5.20 (b). Also note that, as mentioned before, the $\mathrm{ZBCP}$ is strongly enhanced as a result of the Majorana modes hybridizing with the ABSs localized near the ends of the wire. Particularly interesting is the faint $\mathrm{ZBCP}$ near $\Gamma \approx 0.6 \mathrm{meV}$, which is "revealed" by the strong ABS mode that crosses zero energy at that value of the Zeeman field.

Next, we focus on the $\mu=3 \mathrm{meV}$ trace, which cuts through a loop-like feature in Fig. 5.21 (b) that has no equivalent in Fig. 5.20. The corresponding low-energy spectrum is shown in Fig. 5.23 (a). Note that, with increasing Zeeman field, several low-energy modes accumulate near zero energy, with the first one crossing zero at $\Gamma \approx 0.8 \mathrm{meV}$ (red lines). To understand the nature of the low-energy states, we calculate their component Majorana modes. As shown in Fig. 5.23 (b), for $\Gamma=0.86 \mathrm{meV}$ the lowest energy BdG state consists of a partially separated ABS (ps-ABS) localized near the right end of the wire (red and green Majorana components in Fig. 5.23 (b)). On the other hand, the second lowest energy state is a "regular" ABS consisting of two nearly overlapping Majorana components (orange and blue) localized at the left end of the system. As a consequence, both the Majorana separation and the edge-to-edge correlation have small values in the area around $\mu=3 \mathrm{meV}$, $\Gamma=0.86 \mathrm{meV}$ (see Fig. 5.20). At a higher Zeeman field, $\Gamma=1.73 \mathrm{meV}$, the Majorana components of the lowest energy mode - green and red in Fig. 5.23 (c) - are well separated and localized near the ends of the wire. This explains the large Majorana separation and the finite edge-to-edge correlation characterizing the corresponding region of the "phase diagrams" in Fig. 5.20. Note, 

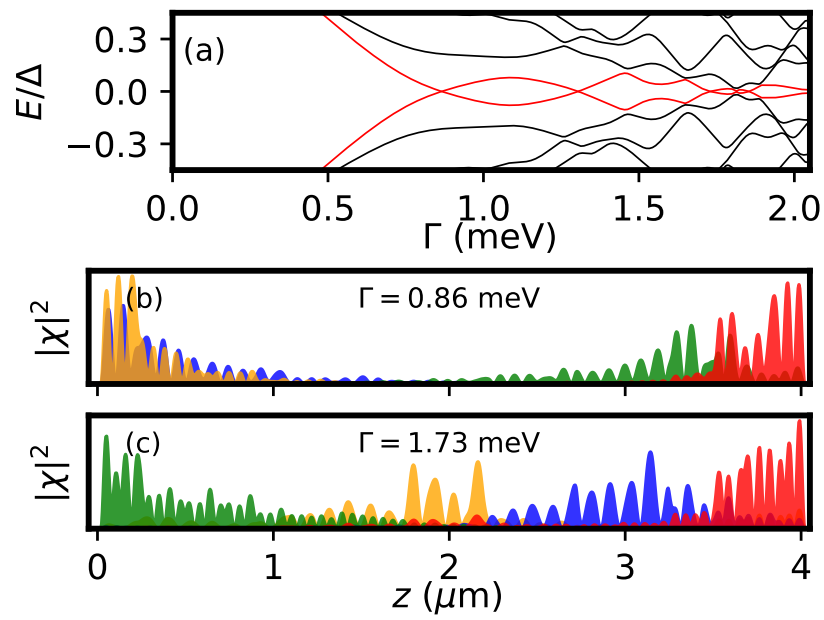

FIGURE 5.23: (a) Low-energy spectrum as a function of the Zeeman field for a system with the same parameters as in Fig. 5.20 and $\mu=3 \mathrm{meV}$. Red lines denote the lowest energy mode. (b) and (c) Spatial profiles of the Majorana components corresponding to the lowest BdG eigenstate (red and green) and second lowest energy eigenstate (blue and yellow) for two values of the Zeeman field, $\Gamma=0.86$ and $1.73 \mathrm{meV}$, respectively. Note that the lowest energy state in (b) has partially separated Majorana components (i.e., quasi-Majorana modes) localized near the right edge, while the lowest energy state in (c) has wellseparated Majorana components.

however, that these well separated Majorana modes have a significant overlap with the Majorana components of higher energy states, with which they can easily hybridize in the absence of an energy gap that would protect them. Consequently, $\ell_{\text {sep }}$ and $C$ are highly sensitive to variations of the control parameters, which explains the "small islands" structure of the corresponding region of the phase diagram in Fig. 5.20.

Our analysis of the low energy spectrum corresponding to $\mu=3 \mathrm{meV}$ suggests that the loop-like feature visible in Fig. 5.21 around that value of the chemical potential is associated with the quasi-Majorana mode (or ps-ABS) emerging at the right edge of the system [see Fig. 5.23 (b)]. To confirm this finding, we calculate the differential conductance at the left and right ends of the system along the same constant $\mu$ cut as the spectrum in Fig. 5.23 (b). The result in Fig. 5.24 (b) clearly shows the emergence of a nearly-zero bias conductance peak at the right edge of the system that practically traces the lowest energy mode [red lines in Fig. 5.23 (a)] for $\Gamma \lesssim 1.4$ meV. A maximum of the zero-bias conductance occurs at $\Gamma \approx 0.8 \mathrm{meV}$, where the quasi-Majorana mode crosses zero energy and the $\mu=3 \mathrm{meV}$ cut intersects the loop-like feature [see Figs. 5.21 (b) and 5.23 (a)]. We conclude that the loop-like features that characterize the zero-bias conductance maps in Fig. 5.21 outside the nominally topological region are generated by quasi-Majorana modes (or ps-ABSs) localized near the ends of the system. 


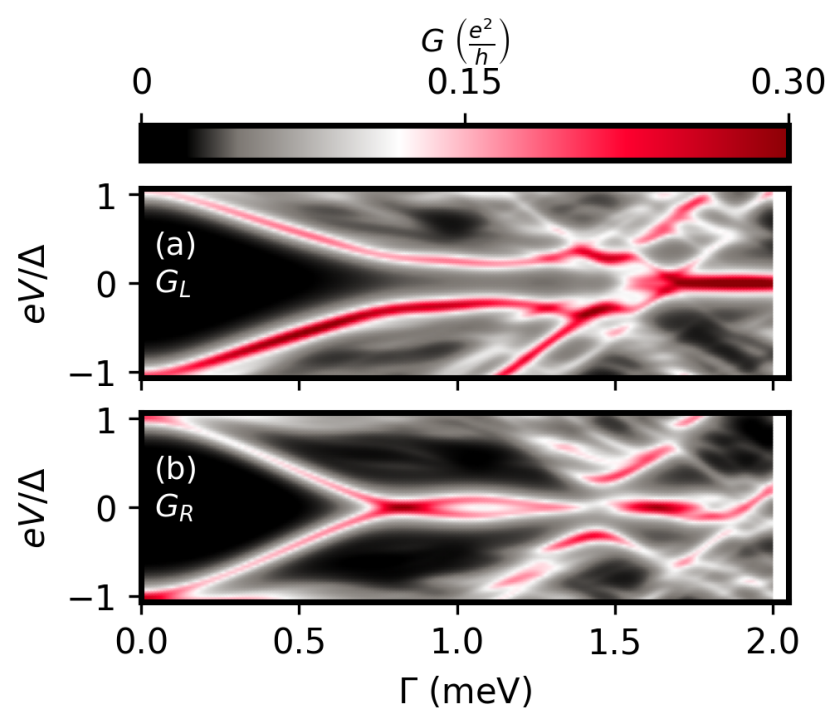

FIGURE 5.24: Local differential conductance at the left (a) and right (b) ends of the wire for a system with the same parameters as in Fig. 5.23, but having normal leads and tunnel barriers attached at both ends. The left conductance has no ZBCP for $\Gamma \lesssim 1.5 \mathrm{meV}$, while the right conductance is characterized by a strong nearly-zero energy feature associated with the loop-like feature in Fig. 5.21 (b) and generated by the quasi-Majorana mode shown in Fig. 5.23 (b). At larger Zeeman fields, the differential conductance is characterized by $\mathrm{ZBCP}$ at both ends of the system, which is consistent with a finite edge-to-edge correlation.

Turning now our attention to the left end of the system, we notice [see Fig. 5.24 (a)] the presence of strong finite bias conductance peaks for $\Gamma \lesssim 1.4 \mathrm{meV}$. These peaks are generated by the ABS localized at the left end of the system and representing the second-lowest BdG state (see Fig. 5.23). We note that, as a result of finite broadening, the contribution of this state to the zero-bias conductance $G_{L}$ is finite, although small. However, when combined with the large quasi-Majorana contribution to $G_{R}$, it generates a non-zero contribution to the correlation $C_{G}$, which can be clearly seen as "shadow" loop-like feature in Fig. 5.21 (c). This spurious correlation feature can be eliminated by considering the finite bias conductance and suppressing $C_{G}$ if the left and right contribution are not associated with conductance peaks located within the same energy window $(E-\delta E, E+\delta E)$, where $\delta E$ is determined by the energy resolution. Nonetheless, the zero-bias conductance maps, including the $C_{G}$ map, can play a crucial role as a first step in characterizing the system and evaluating the effects of disorder. We suggest that this type of comprehensive maps, rather than fine-tuned and post-selected "good looking" traces, including traces with conductance $\sim O\left(2 e^{2} / h\right)$, should be the standard protocol for the experimental characterization of hybrid semiconductor-superconductor devices. Finally, we note that for $\Gamma \gtrsim 1.5 \mathrm{meV}$ the conductance is characterized by ZBCPs at both the left and right ends, as shown in Fig. 5.24. This 
is consistent with the finite edge-to-edge correlation expected in this regime based on the "phase diagrams" shown in Fig. 5.20.

We conclude this section with a few additional remarks on the "phase diagrams" shown in Figs. 5.20 and 5.21. First, we note that within the nominally topological regime all "phase diagrams" are characterized by stripy features that disperse downwards in $\mu$ with increasing Zeeman field. We have shown that in the case of the conductance maps these features are associated with Andreev bound states localized near the ends of the system that cross zero energy. In certain cases the presence of these ABSs may enhance an otherwise "invisible" ZBCP generated by well separated Majorana modes, which results in a finite edge-to-edge correlation. Second, we note that the features located outside the nominally topological region have qualitatively different characteristics. The conductance maps show several rounded, loop-like features that we identified as being associated with partially separated Majorana modes (or quasi-Majoranas). As discussed above, these features can be eliminated from the correlation map using additional finite bias information. The remaining features have a stripy character and are present in all "phase diagrams." However, unlike the stripy features emerging in the topological region, these "trivial stripes" disperse upward in $\mu$ with increasing Zeeman field. Note that a qualitatively similar behavior can be observed even at lower impurity concentrations, as revealed by the "phase diagrams" in Figs. 5.13 and 5.19. These observations suggest that detailed zero-bias conductance maps could help identify nominally topological regions even when the presence of disorder suppresses the "standard" Majorana phenomenology expected in a clean system. Note however, that these results are not expected to hold if the system is characterized by a small inter-subband spacing (i.e., it is not in the in the independent subband regime) or if the disorder strength exceeds a certain threshold (i.e., the system is in the strong disorder regime). For small inter-subband spacings, even weak disorder will make the system behave as a random disordered class $D$ system because of the essentially random nature of the resultant inter-subband couplings that become comparable to the intra-subband terms.

\section{Charge impurity statistics}

We have investigated the effects of impurity-induced disorder for two specific disorder realizations corresponding to two different impurity concentrations. The natural questions are: i) What is the generic behavior of the system for arbitrary disorder realizations corresponding to a given impurity concentration? ii) What is the dependence of the results on the impurity concentration? To effectively address these questions, we need to define some quantities that provide a "global" description of the two-dimensional maps discussed in the previous section. To this end, we first define the "filter function" $\chi\left(\mu, \Gamma ; \ell_{\text {min }}, C_{\text {min }}, E_{\text {min }}\right)$ that selects control parameter values consistent with certain minimum requirements associated with the presence of 

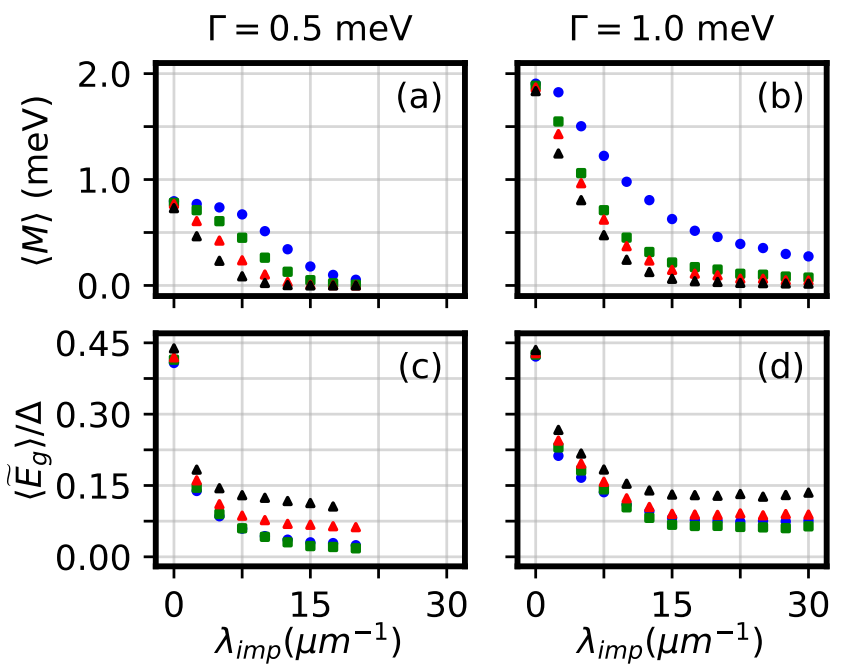

FIGURE 5.25: Disorder-averaged chemical potential range, $\langle M\rangle$, and quasiparticle gap, $\left\langle\widetilde{E}_{g}\right\rangle$, as functions of the impurity density for a system of length $L=4 \mu \mathrm{m}$. The first $(\mathrm{a}, \mathrm{c})$ and second $(\mathrm{b}, \mathrm{d})$ columns correspond to $\Gamma=0.5 \mathrm{meV}$ and $\Gamma=1 \mathrm{meV}$, respectively. The results corresponding to different sets of filter function parameters, $\left(\ell_{\min } / L, C_{\min }, E_{\min } / \Delta\right)$, are color coded: $(0.5,0,0)$ - blue, $(0.5,0.2,0)$ - green, $(0.5,0.2,0.05)$ - red, $(0.5,0.2,0.1)$ - black. Note that the maximum impurity density, $\lambda_{i m p}=30 \mu \mathrm{m}^{-1}$, corresponds to $n_{i m p}=9.4 \cdot 10^{15} \mathrm{~cm}^{-3}$.

well separated Majorana modes capable of generating edge-to-edge correlations. Specifically, we have

$$
\chi(\mu, \Gamma)=\Theta\left(\ell_{\text {sep }}-\ell_{\text {min }}\right) \Theta\left(C-C_{\text {min }}\right) \Theta\left(E_{g}-E_{\text {min }}\right),
$$

where $\Theta(x)$ is the step function, $\Theta(x>0)=1, \Theta(x<0)=0$, and $E_{g}=$ $E_{2}-E_{1}$, with $E_{1}$ and $E_{2}$ being lowest and second lowest positive eigenenergies, respectively, is the quasiparticle gap separating the lowest energy state from the rest of the spectrum. Note that $\chi=1$ if the Majorana separation length is larger than $\ell_{\text {min }}$, the edge-to-edge correlation larger than $C_{\text {min }}$ and the quasiparticle gap larger than $E_{\text {min }}$, while $\chi=0$ otherwise. Next, we introduce the quantity $M(\Gamma)$ defined as the total chemical potential range that satisfies the "good Majorana" criterion, $\chi(\mu, \Gamma)=1$, for a given value of the Zeeman field. Specifically, we have

$$
M(\Gamma)=\int \chi(\mu, \Gamma) d \mu
$$

Note that for a clean system and "reasonable" values of $\ell_{\min }, C_{\min }$, and $E_{\min }$ we have $M(\Gamma)=0$ for $\Gamma<\Delta$, i.e., in the topologically trivial regime, and $M(\Gamma)=2 \sqrt{\Gamma^{2}-\Delta^{2}}$ for $\Gamma>\Delta$. In other words, for a clean system $M(\Gamma)$ is a measure of the "thickness" of the topological region along the $\mu$ direction at a given value of the Zeeman field. For example, $\Gamma \rightarrow \Delta$ (from above) implies $M \rightarrow 0$, precisely giving the lowest Zemman field associated with the 

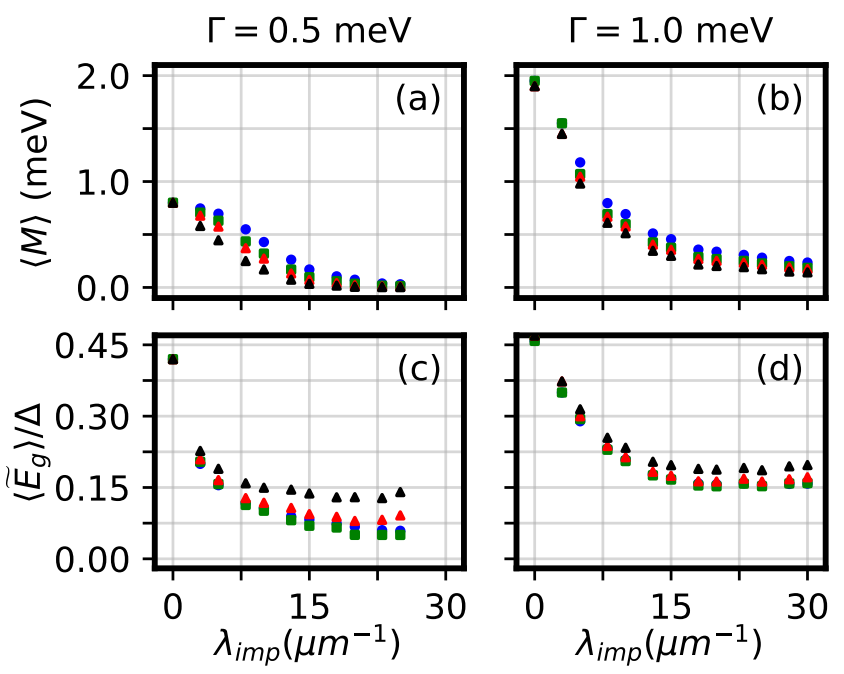

FIGURE 5.26: Same as Fig. 5.25 for a wire of length $L=2 \mu \mathrm{m}$.

pristine TQPT. In addition, we define the average quasiparticle gap within the region satisfying the "good Majorana" condition as

$$
\widetilde{E}_{g}(\Gamma)=\frac{1}{M(\Gamma)} \int E_{g}(\mu, \Gamma) \chi(\mu, \Gamma) d \mu .
$$

To test the relevance of these quantities, we calculate the disorder averages $\langle M\rangle$ and $\left\langle\widetilde{E}_{g}\right\rangle$ as functions of the impurity concentration for two values of the Zeeman field and different sets of filter function parameters, $\left(\ell_{\min } / L, C_{\text {min }}, E_{\text {min }} / \Delta\right)$. The results for a wire of length $L=4 \mu \mathrm{m}$ are shown in Fig. 5.25, while the results corresponding to a shorter wire with $L=2 \mu \mathrm{m}$ are presented in Fig. 5.26 . The averages corresponding to each value of the impurity density, $\lambda_{\text {imp }}$, were calculated using 500 different disorder realizations. Note that if a given impurity realization is characterized by $M=0, \widetilde{E}_{g}$ is undefined, and we do not include it in the calculation of $\left\langle\widetilde{E}_{g}\right\rangle$. First, we observe that $\langle M\rangle$ collapses with increasing impurity density reaching negligible values for impurity densities of the order $10-20$ impurities per micron. This means that for higher impurity concentrations there are practically no "good Majoranas" in the system. We point out that for the Majorana separation criterion we used a rather generous value, $\ell_{\text {min }}=0.5 \mathrm{~L}$, which does not guarantee the localization of the well-separated Majorana modes near the ends of the wire. This is particularly significant in Fig. 5.25 (b), where introducing the edge-to-edge correlation requirement $C_{\text {min }}=0.2$ strongly reduces $\langle M\rangle$ as compared to the case $C_{\min }=0$ (blue dots). On the other hand, the fact that the blue dots in Fig. 5.25 (b) correspond to finite values of $\langle M\rangle$ over the entire range of impurity densities reveals that, even in the presence of relatively strong disorder, the system contains well-separated Majoranas. However, these Majoranas do not generate edge-to-edge correlations. In other words, some segments of a long wire are likely to be in the topological superconducting phase, but 


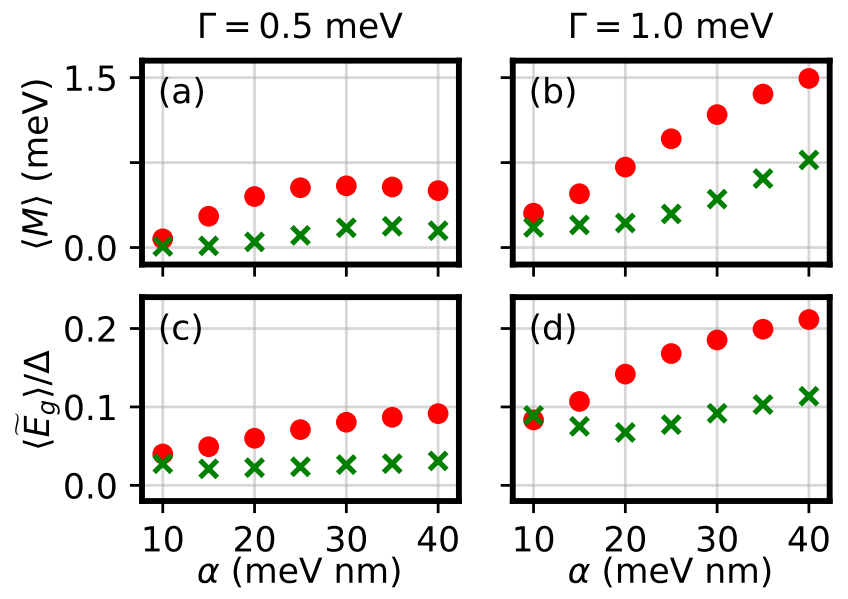

FIGURE 5.27: Disorder-averaged chemical potential range, $\langle M\rangle$, and quasiparticle gap, $\left\langle\widetilde{E}_{g}\right\rangle$, as functions of the spin-orbit coupling strength, $\alpha$, for a wire of length $L=4 \mu \mathrm{m}$. The red circles and green crosses correspond to $\lambda_{i m p}=7.5 \mu \mathrm{m}$, and $\lambda_{\text {imp }}=15 \mu \mathrm{m}$, respectively. The filter function parameters are $\ell_{\text {min }} / L=0.5, C_{\min }=0.2$, and $E_{\text {min }}=0$.

these segments have a concentration-dependent typical length (which is unknown experimentally) that is less than the length $L$ of the wire. Therefore, their presence cannot be established based on the edge-to-edge correlation, which is negligible. This observation is consistent with the specific examples discussed in sections 5.1.2 and 5.1.2. Note that for the shorter system (see Fig. 5.26) imposing the additional filter $C>0.2$ does not reduce $\langle M\rangle$ drastically. This is due to the fact that Majorana modes with $\ell_{\text {sep }}>L / 2$ are significantly more likely to generate edge-to-edge correlations in a shorter wire, as compared to a longer wire. Finally, regarding the average quasiparticle gap, $\left\langle\widetilde{E}_{g}\right\rangle$, we notice a sharp drop at low impurity density, followed by a slower decline toward a density-independent plateau, which starts at $\lambda_{\text {imp }} \approx 15 \mu \mathrm{m}^{-1}$. The height of the plateau is determined by the average inter-state spacing, which depends on the length of the wire being proportional to $1 / \mathrm{L}$.

The "global" quantities introduced above provide useful tools for studying the effects of disorder on the Majorana physics. Most importantly, they reveal the strong dependence of the Majorana physics on the impurity concentration. In particular, observing edge-to-edge correlations at relatively low values of the Zeeman field requires reducing the impurity density below a certain threshold of about $15-20$ impurities per micron. This type of analysis can be also useful for optimizing the system parameters. As an example, we consider the dependence on the spin-orbit coupling strength. Fig. 5.27 shows the dependence of $\langle M\rangle$ and $\left\langle\widetilde{E}_{g}\right\rangle$ on spin-orbit coupling strength $\alpha$ for a wire of length $L=4 \mu \mathrm{m}$ for two impurity densities and two values of the Zeeman field. Typically, increasing the spin-orbit coupling strength enhances both $\langle M\rangle$ and $\left\langle\widetilde{E}_{g}\right\rangle$. However, for $\lambda_{i m p}=15 \mu \mathrm{m}^{-1}$ (green crosses) the dependence of the average energy gap on $\alpha$ is weak, while $\langle M\rangle$ shows a significant enhancement only at larger values of the Zeeman field and for 
$\alpha \gtrsim 25 \mathrm{meV} \cdot \mathrm{nm}$. Finally, we point out that throughout this work the value of the spin-orbit coupling strength was $\alpha=20 \mathrm{meV} \cdot \mathrm{nm}$, which we consider as relatively optimistic. While for large enough Zeeman fields $\langle M\rangle$ and $\left\langle\widetilde{E}_{g}\right\rangle$ can be enhanced by having a stronger spin-orbit coupling, there is not much room for optimizing the low-field regime. Note, however, that at large field values the topological gap itself may be rather small and, again, optimization becomes a challenge even in this regime.

\subsubsection{Conclusions}

We have carried out a comprehensive microscopic theoretical study of disorder effects arising from the inevitable presence of charge impurities in superconductor-semiconductor nanowire hybrid structures, focusing on the fate of the Majorana zero modes expected to emerge in these systems. The work consists of four closely connected, but distinct, theoretical components: (1) developing a fully self-consistent realistic Schrödinger-Poisson scheme to calculate the effective impurity potential arising from the presence of charge impurities, which takes into account electrostatic and screening effects due to the superconductor and potential back gate, as well as the screening by the free charge in the wire; (2) carrying out full solutions of the BdG equations in the presence of disorder by incorporating the effective impurity potential calculated self-consistently for a multi-band system, as well as the superconducting proximity effect, spin-orbit coupling, and applied Zeeman field; (3) obtaining, based on the solutions of the BdG equations, effective "phase diagrams" as functions of the control parameters (i.e., Zeeman field and chemical potential) in the presence of disorder and investigating their dependence on the disorder strength; (4) calculating the tunnel conductance at both ends of the system and generating the corresponding "phase diagrams", which provides insight into the existing tunnel spectroscopy experiments on Majorana nanowires and suggests new directions for enhancing the relevance of such measurements.

Since the work involves multiple aspects, we have specific conclusions regarding each component of the theory already included in the corresponding sections of this article. Instead of repeating what is already described and discussed in depth in sections 5.1.1 and 5.1.2, we summarize our most important conclusions regarding the role of charge impurity-induced disorder from the perspective of the ongoing search for non-Abelian Majorana modes in superconductor-semiconductor nanowire hybrid structures.

We show that the superconductor plays a rather limited role in screening the impurity potential, while substantial screening arises from the free charges in the nanowire. We provide a simple two-parameter empirical fitting formula for the effective screened potential, which should be useful for future simulations of Majorana devices. Quantitatively, we find that the effective impurity potential has typical amplitudes of the order of $1.5-2 \mathrm{meV}$ and typical decay lengths of about $8-12 \mathrm{~nm}$.

We find that disorder produces zero energy states outside the pristine topological phase boundary and we analyze in depth the nature of these 
states and their possible experimental signatures. We also find that, within the nominally topological regime, the system can host well separated Majorana modes even in the presence of significant disorder levels, but typically the presence of these modes is not associated with a significant edge-to-edge correlation. A key finding in this context is that disorder may often push Majorana zero modes away from the wire ends, thus making them invisible to local (end-of-wire) tunnel spectroscopy. Thus, it is entirely possible (and likely) to miss the presence of Majorana zero modes in a disordered nanowire when using tunneling spectroscopy simply because this is a local probe sensitive only to states localized at the wire ends. Hence, in the presence of disorder, long segments within the bulk of the wire may be topologically nontrivial, with Majorana modes emerging at their ends, but the wire ends themselves may contain no Majorana modes, which dramatically reduces the probability of observing edge-to-edge correlations.

We establish that detailed two-dimensional maps of the zero-bias conductance as a function of Zeeman splitting (i.e. magnetic field in the laboratory) and chemical potential (i.e. gate voltage in the laboratory) may be the most effective operational way to search for the "hidden" topological superconductivity and the associated Majorana modes. The current experimental focus on looking for large zero bias peaks with conductance $\sim 2 e^{2} / h$ by fine-tuning the control parameters is unlikely to solve the outstanding questions regarding the nature of the low-energy states responsible for these peaks. First, a large zero bias peak obtained through careful fine-tuning and post selection may have nothing to do with topological Majorana modes, and second, this procedure is likely to lead to strong confirmation bias in the experiment. Instead, creating zero bias conductance maps in the extensive parameter space of gate voltage and magnetic field using the cleanest possible samples and comparing these maps to our theoretical results may be a much more systematic way of searching for Majorana physics, without suffering from any confirmation bias. In addition, this would provide much needed estimates of the disorder strength characterizing actual superconductor-semiconductor hybrid devices and an effective way of testing future materials improvements that aim at reducing disorder.

We find that for reasonably realistic (but still somewhat optimistic) parameter choices, genuine, well-separated topological Majorana modes should exist in nanowires for impurity densities up to $5 \cdot 10^{15} \mathrm{~cm}^{-3}$, which corresponds to around 15 impurities per micron. This would mean that a $2-4 \mathrm{mi}-$ cron long nanowire can contain up to $30-60$ charge impurities, but cleaner samples, with charge impurity density below $10^{15} \mathrm{~cm}^{-3}$, may be necessary in practice, since we ignored any disorder arising from possible interface defects or imperfections. Such a low intrinsic doping of less than $10^{15} \mathrm{~cm}^{-3}$ is a challenge, but is by no means out of reach in semiconductor materials growth, as impurity contents below $10^{13} \mathrm{~cm}^{-3}$ have been achieved in MBEgrown GaAs structures [180]. 


\subsection{Zero-energy pinning of topologically trivial states in multi-band semiconductor-superconductor nanowires}

Understanding the possible mechanisms that cause the pinning of ABSs near zero energy is important for i) distinguishing topological MZMs from non-topological ABSs with similar phenomenology and ii) estimating the relative sizes of the parameter subspaces consistent with the formation of MZMs and low-energy ABSs, respectively, and explaining the apparent ubiquitousness of zero-bias conductance peaks in tunneling experiments. Ultimately, these efforts are meant to guide the engineering of SM-SC hybrid structures toward the reliable, controlled realization of the conditions consistent with the emergence of MZMs. In general, low-energy ABSs can emerge at Zeeman fields below the critical value $\Gamma_{c}$ associated with the TQPT when the system is not homogeneous. The inhomogeneity is generated either by the structure of the hybrid device, e.g., the presence of (multiple) electrostatic gates, the partial covering of the SM wire with superconducting material, and the presence of multiple tunnel contacts [70,71, 157], or by disorder at surfaces and interfaces, e.g., disorder at the surface of the SC film and spatial fluctuations of the work function difference between the SC and the SM wire [126]. The most direct consequence of having such sources of inhomogeneity is an effective potential that varies along the wire, which, in turn, gives rise to low-energy ABSs [67, 108].

One possible explanation for the emergence of ABSs pinned near zero energy over an extended range of parameters [66, 68, 94, 108] (e.g., applied magnetic field) is the partial spatial separation of the two Majorana modes $[68,108]$ (also known as quasi-Majorana states [94]) that constitute the Andreev bound state, which was dubbed a partially-separated ABS (ps-ABS). This partial separation mechanism is quite generic, being responsible for the emergence of low-energy ABSs in various types of non-homogeneous systems, e.g., in the presence of smooth confinement, potential wells/hills, or quantum dots coupled to a proximitized wire. Note, however, that this mechanism was studied theoretically based (almost exclusively) on i) singlesubband ${ }^{2}$ tight-binding models and ii) toy models for the potential profile containing largely arbitrary parameter values. Most importantly, the partialseparation mechanism does not explain the ubiquity of zero-bias features observed experimentally [122]. Therefore, exploring the possibility that other mechanisms may also be responsible for the collapse of ABSs toward zero energy in systems with multi-band occupancy represents a critical outstanding task. In addition, the potential profiles (and other position-dependent system parameters) should be calculated based on the properties of actual hybrid devices, rather than postulated based on arbitrary assumptions. For example, in systems with soft confinement the collapse (and 'sticking') to zero-energy of a ps-ABS localized near the end of the wire is controlled by the slope of the potential [54, 94, 108]. In turn, estimating this slope requires solving a

\footnotetext{
${ }^{2}$ In the remainder of this section, subbands are simply called bands.
} 
challenging 3D Schrödinger-Poisson problem that takes into account the geometry of the heterostructure and the applied gate potentials [126] (and see chapter 4). Without explicitly solving this type of problem, it is difficult (if not impossible) to estimate if the conditions necessary for the emergence of low-energy ABSs (through either the partial separation mechanism, or the newly-proposed inter-band coupling mechanism) are generically satisfied, somewhat likely, or nearly impossible.

While our conclusions are general, we focus on a recent tunneling conductance experiment on InSb/NbTiN hybrid structures, in which low-energy features similar to those predicted theoretically were observed over a considerable parameter range (i.e., tunnel barrier and back gate potentials, magnetic fields, etc.) [122]. Are they generated by MZMs, ps-ABSs (i.e., quasiMajoranas), or some other (topologically-trivial) low-energy ABSs? The experiment provides some useful hints. First, we note that the characteristic length scales associated with the structural inhomogeneity of the device are small: an uncovered region of about $100 \mathrm{~nm}$ (corresponding to the tunneling gate region) and a covered region defined by a bottom gate of about $200 \mathrm{~nm}$ [122]. These small length scales pose serious difficulties to the partialseparation scenario [108]. Second, the single-band model calculations predict that the low-energy ABSs should be fairly well separated in energy from the bulk states (on the scale of the induced SC gap); by contrast, the experiment shows a relatively crowded sub-gap spectrum.

In this section, we show that multi-band occupancy characterizes the heterostructure studied in the recent tunneling conductance experiment [122] over the whole relevant range of control parameters. Furthermore, we demonstrate that, in general, hybrid systems with multi-band occupancy host topologically trivial ABSs that can be pinned near zero energy as a result of a mechanism that involves the coupling of two or more confinement-induced low-energy bands. Band repulsion resulting from this coupling pins the lowest energy state near zero energy over a significant range of control parameters (e.g., Zeeman field). We emphasize that multi-band occupancy (an ingredient that is not included in the vast majority of the theoretical work on Majorana hybrid structures) is crucial for this mechanism to be active. The resulting (topologically trivial) ABSs are characterized by Majorana modes that are not separated spatially. Consequently, the characteristic length scales associated with the collapse and pinning to zero energy of the ABSs generated by this mechanism are significantly smaller (e.g. on the order of the nanowire diameter of $100 \mathrm{~nm}$ ) than those required for the formation of a psABS. In general, in addition to the partial-separation mechanism discussed extensively in the literature, the inter-band coupling mechanism should be viewed as an alternative path for generating low-energy ABSs. This mechanism becomes dominant in systems with multi-band occupancy and shortrange inhomogeneities. We show that this inter-band coupling mechanism is capable of explaining the features observed in the experimental data reported in Ref [122]. In addition, we find that, unlike (partially) separated Majorana modes, the ABS modes generated by the inter-band coupling mechanism retain their particle or hole character down to zero energy (except for a few 
discrete points). Consequently, in the presence of dissipation, the nearly-zero energy conductance features generated by these ABSs can break particle-hole symmetry. We conclude that the observation of nearly-zero differential conductance peaks that break particle-hole symmetry, which is inconsistent with the presence of MZMs, quasi-Majoranas, or any other low-energy modes that involve (partially) separated Majorana bound states, should be attributed to (topologically-trivial) ABSs pinned near zero-energy by level repulsion.

To incorporate the details of the electrostatic environment characterizing the experimental device, we perform 3D Schrödinger-Poisson calculations using an efficient approach developed earlier (see chapter 4 ). We match the geometry of the device (gate sizes, material parameters, superconductor geometry, etc.) in an attempt to be as close as possible to the relevant parameter regime. Within this approach, we demonstrate that inter-band coupling is a direct (and necessary) consequence of the inhomogeneous electrostatic potential along the wire. Moreover, inter-band coupling is expected to be a generic feature at interfaces between regions with different electrostatic environments. Note that the present calculation does not include disorder, which is expected to induce additional interband coupling. Nonetheless, these results emphasize the importance of being able to perform 3D SchrödingerPoisson calculations, rather than assuming translation invariance along the wire. Since our realistic modeling predicts i) multi-band occupancy over the whole range of experimentally-relevant control parameters and ii) strong inter-band coupling, we conclude that the emergence of low-energy ps-ABSs generated by the inter-band coupling mechanism is quite generic, in agreement with the ubiquity of zero-bias conductance peaks observed experimentally.

The remainder of this section is organized as follows. In Sec. 5.2.1, we present a toy model that illustrates the basic principle behind the inter-band coupling mechanism. We introduce the key ideas associated with inter-band coupling in SM-SC hybrid structures and explore multi-band effects in both homogeneous wires and inhomogeneous systems. A detailed 3D model of the device is described in Sec. 5.2.2 along with the corresponding results which show explicitly that inter-band coupling can pin ABSs near zero energy. Finally, in Sec. 5.2.3 we discuss the relevance of our findings and suggest ways to lessen the chance of ABSs emerging as a result of the inter-band coupling mechanism. Much of this section is adapted from our paper found in Ref. [72].

\subsubsection{Toy model}

To illustrate the main ideas underlying the emergence of low-energy ABSs within the inter-band coupling mechanism and to emphasize the main differences between this multi-band scenario and the partial separation mechanism responsible for the formation of ps-ABSs in single-band models, we first consider a multi-band tight-binding toy model that captures the essential aspects of multi-band physics in hybrid structures. We emphasize that the ABSs emerging within the inter-band coupling mechanism consist of two 
overlapping (i.e. non-separated) Majorana modes, yet they still 'stick' near zero energy over a wide range of magnetic field. This is in stark contrast to single band scenarios (or, in general, models that neglect inter-band coupling), which predict either ps-ABSs (consisting of two Majorana modes with significant separation in space $[67,94])$ that stick near zero-energy, or "plain" ABSs (composed of overlapping Majorana modes) that can only cross zeroenergy, without "sticking". Note that the presence of various inter-band coupling terms in the toy model can be fully justified based on the 3D selfconsistent calculations presented in Sec. 5.2.2. However, the toy model has the major advantage that, due to its (relative) simplicity, it makes the physics behind the inter-band coupling mechanism more transparent. Specifically, we consider the following 1D nearest neighbor multi-band Hamiltonian describing a finite SM nanowire weakly coupled to an s-wave superconductor:

$$
\begin{aligned}
H & =\sum_{i}^{N-1} \sum_{\alpha, \beta} \sum_{\sigma} t_{i}^{\alpha \beta} c_{i \alpha \sigma^{+}}^{\dagger} c_{i+1, \beta \sigma}+\text { h.c. } \\
& +\sum_{i \alpha} \sum_{\sigma \sigma^{\prime}}\left[\left(V_{i \alpha}-\mu_{\alpha}-2 t_{i}^{\alpha \alpha}\right) \delta_{\sigma, \sigma^{\prime}}+\Gamma\left(\sigma_{x}\right)_{\sigma \sigma^{\prime}}\right] c_{i \alpha \sigma}^{\dagger} c_{i \alpha \sigma^{\prime}} \\
& +\sum_{i}^{N-1} \sum_{\alpha, \beta} \sum_{\sigma \sigma^{\prime}} \widetilde{\alpha}_{i}^{\alpha \beta} c_{i \alpha \sigma}^{\dagger}\left(i \sigma_{y}\right)_{\sigma \sigma^{\prime}} c_{i+1, \beta \sigma^{\prime}}+\text { h.c. } \\
& +\sum_{i} \sum_{\alpha \beta} \sum_{\sigma \sigma^{\prime}}\left(\alpha_{T}\right)_{i}^{\alpha \beta} c_{i \alpha \sigma}^{\dagger}\left(i \sigma_{x}\right)_{\sigma \sigma^{\prime}} c_{i \beta \sigma^{\prime}}+\text { h.c. } \\
& +\sum_{i \alpha \beta} \Delta_{i}^{\alpha \beta} c_{i \alpha \uparrow}^{\dagger} c_{i \beta \downarrow}^{\dagger}+h . c .,
\end{aligned}
$$

where $c_{i \alpha \sigma}$ annihilates an electron on the $i^{\text {th }}$ site with $\alpha$ and $\sigma$ being band and spin indices, respectively, $t_{i}^{\alpha \beta}$ is a spin-conserving hopping matrix element, $\Gamma$ is the (half) Zeeman splitting due an external magnetic field, $V_{i \alpha}$ is the effective potential of the $\alpha$ band, $\Delta_{i}^{\alpha \beta}$ is a superconducting pairing matrix element, while $\widetilde{\alpha}_{i}^{\alpha \beta}$ and $\left(\alpha_{T}\right)_{i}^{\alpha \beta}$ are longitudinal and transverse spin-orbit matrix elements, respectively. The parameter $\mu_{\alpha}=\mu-\epsilon_{\alpha}$, where $\mu$ is the chemical potential and $\epsilon_{\alpha}$ is the energy of the $\alpha$ band at $k=0$ (in a long, uniform wire), represents the chemical potential relative to the bottom of the band. Note that subtracting the quantity $2 t_{i}^{\alpha \alpha}$ from the on-site energy ensures that the bottom of the $\alpha$ band is at $\epsilon_{\alpha}$. Finally, $\sigma_{i}$, with $i=x, y, z$, are Pauli spin matrices. We note that $\widetilde{\alpha}_{i}^{\alpha \beta}=\alpha_{i}^{\alpha \beta} / 2 a$, where $a$ is the lattice constant of the 1D lattice describing the wire, and $\alpha_{i}^{\alpha \beta}$ has units of energy times length (i.e. the typical units for the spin-orbit coupling constant). By contrast, $\left(\alpha_{T}\right)_{i}^{\alpha \beta}$ does not scale with the lattice spacing, as it models transverse spin orbit coupling between various orbitals delocalized across the transverse section of the wire. The multi-band model (5.51) reduces to the 'standard' single-band model used in the literature if all the matrices are diagonal, $\mathcal{O}^{\alpha \beta}=0$ for $\alpha \neq \beta$ (i.e., there is no inter-band coupling), and one focuses on the top occupied band. We emphasize that the multi-band nature of the model (which involves inter-band 


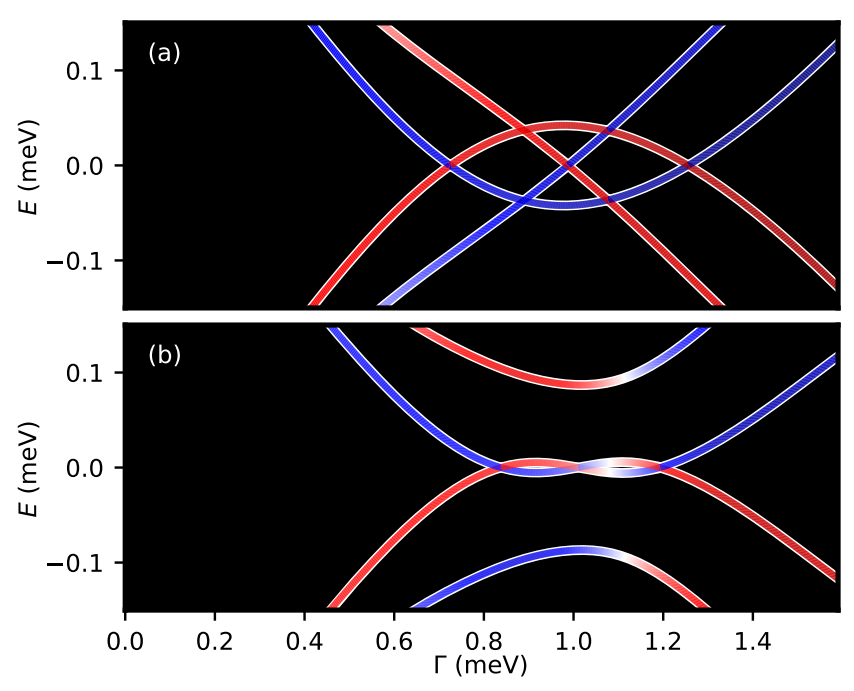

FIGURE 5.28: Spectrum of a two band model with the chemical potential placed between the two bands for a wire of length $L=250 \mathrm{~nm}$. The model parameters are given by $m^{*}=0.03$, $\alpha_{1,1}=\alpha_{2,2}=500 \mathrm{meV} \AA, \Delta_{1,1}=\Delta_{2,2}=0.35 \mathrm{meV}, \mu_{1}=0.28$ $\mathrm{meV}$, and $\mu_{2}=-0.35 \mathrm{meV}$, where $\mu_{\alpha}$ is defined with respect to the bottom of the corresponding band. (a) No inter-band coupling. (b) Inter-band coupling defined by $\Delta_{1,2}=-\Delta_{2,1}=$ $0.21 \mathrm{meV}$ and $\left(\alpha_{T}\right)_{1,2}=0.19 \mathrm{meV}$. Red and blue lines indicate (dominant) particle and hole weights, respectively, while white denotes an equal particle-hole mixture.

coupling as an essential ingredient) introduces new physics that is relevant to understanding many of the features observed in the current experiments on semiconductor-superconductor hybrid structures, as we demonstrate below.

The parameter values used in the numerical calculations are loosely based on the (known) parameters for a typical SM-SC structures (e.g., InAs nanowires proximitized with $\mathrm{Al}$ ) and take into consideration certain symmetry constraints, as discussed below. However, the main point of this section is not to provide quantitative predictions (e.g., to fit specific experimental results), but rather to reveal the role of inter-band coupling in generating low-energy ABSs pinned near zero energy. By contrast, the inter-band couplings obtained within the full 3D calculation of Sec. 5.2.2 are not arbitrary, being determined by the evolution of the transverse profile of the orbitals associated with different confinement-induced bands (determined self-consistently) as one moves along the wire.

\section{Homogeneous wires}

First, let us consider a short homogeneous system with position-independent effective potential $V_{i \alpha}=0$ (for all bands). In addition, we require the Hamiltonian to respect inversion symmetry (see the Appendix of the published 
version of this work [72] for technical details). Note that in the homogeneous case, the near-zero energy states are ABSs consisting of strongly overlapping Majorana modes. We focus on the effects of the inter-band coupling on these low-energy states. More specifically, let us consider a short wire of length $L=250 \mathrm{~nm}$. We describe the wire using a two-band model and assume that the chemical potential lays between the (bottoms of the) two bands, $\epsilon_{1}<\mu<\epsilon_{2}$. The corresponding low-energy spectrum is shown in Fig. 5.28. If the two bands are decoupled, see Fig. 5.28(a), the empty and occupied bands generate particle- (red) and hole-type (blue) states coming toward zero energy from above as the Zeeman field increases from zero. Note that in this discussion we focus on the positive energy sector, but the spectrum is particle-hole symmetric, as clearly shown in Fig. 5.28. The particle mode has one zero energy crossing near $\Gamma \approx 0.95 \mathrm{meV}$ and becomes a hole mode (blue) at larger fields (within the positive energy sector). The hole band exhibits one "oscillation", with two zero-energy crossings (at $\Gamma \approx 0.67 \mathrm{meV}$ and $\Gamma \approx 1.3 \mathrm{meV}$, respectively) within the relevant Zeeman field range. The energy splittings of the low-energy modes (which can be viewed as being induced by the strong overlap of the Majorana components of these modes) are large, i.e. comparable to the induced pairing potential. This is a finite-size effect generated by the short length of the wire, which does not allow the separation of the Majorana components. Note that, formally, the topological condition $\Gamma>\sqrt{\mu_{\alpha}^{2}+\Delta_{\alpha \alpha}^{2}}$ is satisfied for both bands when $\Gamma>0.5 \mathrm{meV}$. Next, upon introducing an inter-band coupling through the off-diagonal pairing and the transverse spin-orbit coupling, the two bands hybridize and generate a mode that sticks to zero energy over a finite range of Zeeman fields, as shown in Fig. 5.28(b) . Note that the lowest-energy mode undergoes two oscillations about zero energy with amplitude significantly lower than the energy splittings characterizing the decoupled system. We conclude that level repulsion induced by inter-band mixing can generate a low-energy mode that sticks to zero-energy over a finite range of Zeeman field.

To strengthen this conclusion, we consider a three-band model of the short wire $(L=250 \mathrm{~nm})$, both with and without inter-band coupling. The corresponding spectra are shown in Fig. 5.29. The parameters of the model corresponding to the first two bands are the same as in Fig. 5.28, while the parameters associated with the third band are provided in the figure caption. Note that, again, in the presence of inter-band coupling, level repulsion pushes one mode toward zero energy over a significant range of Zeeman field (about $0.6 \mathrm{meV}$ ). The near-zero-energy mode is characterized by three low-amplitude oscillations, which may appear in tunneling spectroscopy as a robust $\mathrm{ZBCP}$ (without splitting) due to broadening and finite energy resolution. Furthermore, even the splitting of the low-energy mode away from zero energy for $\Gamma>1.4 \mathrm{meV}$ may not be observable in practice, if the the superconducting gap of the parent superconductor collapses at comparable values of the magnetic field. We conclude that the inter-band coupling mechanism illustrated in these examples can generate low-energy states with local signatures similar to those of topologically-protected MZMs (e.g., a robust ZBCP), 

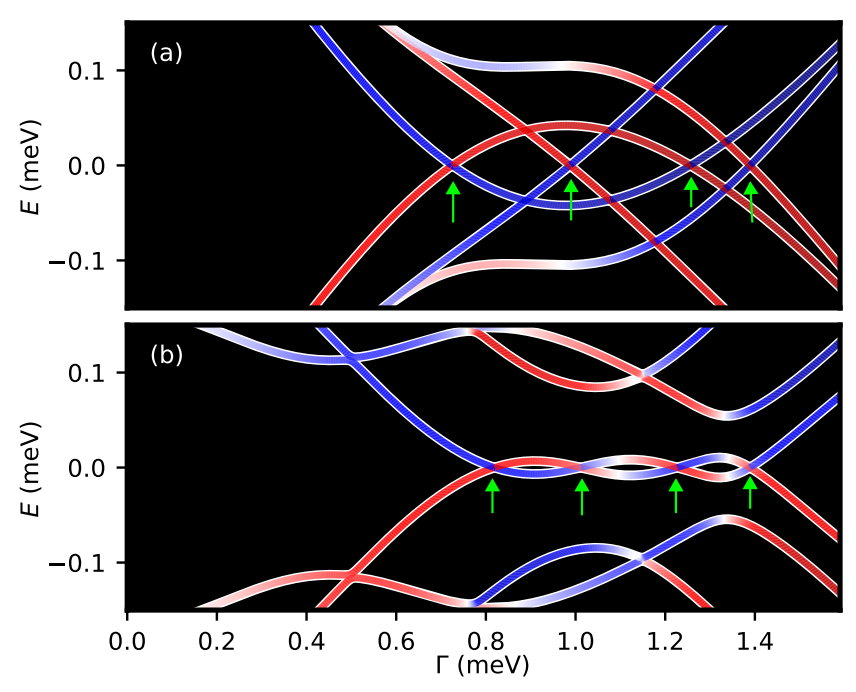

FIGURE 5.29: Spectrum of a three band model with the same parameters for the first two bands as in Fig. 5.28, $\mu_{3}=-0.6$ $\mathrm{meV}$, and $\Delta_{3,3}=0.35 \mathrm{meV}$. (a) No inter-band coupling. (b) Interband coupling defined by $\Delta_{2,3}=\Delta_{3,2}=0.2 \mathrm{meV},\left(\alpha_{T}\right)_{1,3}=$ $0.05 \mathrm{meV}$, in addition to the parameters defined in Fig. 5.28(b).

despite the wire being very short (i.e. being incapable to support two wellseparated Majorana modes localized at the opposite ends of the system).

To gain further insight, we calculate the position-dependence of the wave function amplitude for the states corresponding to the zero energy crossings in Fig. 5.29 (see the green arrows). The results are shown in Fig. 5.30. Note that in the decoupled-band case [panels (a) - (d) in Fig. 5.30], each state is composed of a single band component. By contrast, in the presence of interband coupling, the zero energy states are composed of a mixture of the three bands [panels (e) - (h)]. Note, however, that the separation between the main wave function peaks is similar in the two cases, suggesting that the collapse to zero-energy of the low-energy mode in the band-coupled system is not the result of the component Majorana modes becoming spatially separated. In fact, the explicit calculation of the corresponding Majorana components (see Sec. 2.1.2 for a technical definition) shows that they have nonzero amplitude throughout the entire wire and cannot be identified with the main peaks of the ABS wave function. We emphasize that previous studies using single-band models found the pinning to zero of a low-energy mode to be necessarily associated with the (partial) separation of the Majorana modes $[67,108]$. By contrast, ABSs generated by the inter-band coupling mechanism are not characterized by separated Majorana modes. We note that inter-band level repulsion in homogeneous systems has been previously explored [132], being attributed to spin-orbit coupling. However, it should be pointed out that inter-band coupling can be more general, e.g., it can involve the (induced) superconducting pairing potential, as shown here. This effect can be naturally understood as a proximity-induced coupling of the confinementinduced bands and is expected to be significant in the strong coupling limit 


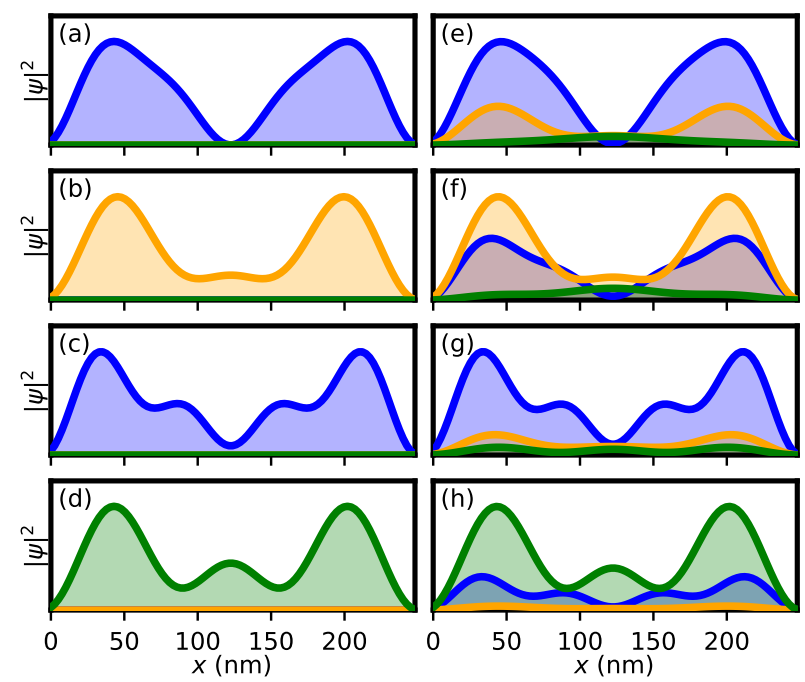

FIGURE 5.30: Wave function amplitudes of the zero energy states indicated by green arrows in Fig. (5.29). Panels (a)(d) and (e)-(h) correspond to Fig. 5.29(a) and Fig. 5.29(b), respectively. The three colors correspond to different band contributions. The spin and particle-hole degrees of freedom are summed over for each lattice site.

[145]. More importantly, homogeneous systems represent a rather ideal limit which may not be easily realizable in practice. A more interesting (and potentially relevant) situation involves inter-band mixing caused by an inhomogeneous electrostatic potential, which we address in the subsection below.

Before closing this subsection, we want to emphasize a fundamental difference between the ABSs generated by the inter-band coupling mechanism described here and the (partially) separated Majorana modes that emerge in a finite wire upon satisfying the 'topological condition' $\Gamma>\Gamma_{c}$. While the Majorana modes represent (almost) equal mixtures of particles and holes (hence, they appear as white lines in our color code representation, see, for example, Fig. 5.32), the ABS modes retain their particle or hole character down to zero energy (except for a few discrete points), as shown in Figs. 5.28 and 5.29. This property can have measurable consequences in a tunneling experiment. It has been shown that, in the presence of dissipation (e.g., from a parent superconductor with sub-gap states) [171, 172, 181-183], the differential conductance is particle-hole asymmetric. Essentially, a state of energy $E_{n}$ with, say, particle character and its hole-type counterpart at energy $-E_{n}$ will generate differential conductance signals of different amplitudes. This asymmetry does not emerge in the case of a split Majorana mode (as long as the splitting is not too large), because both the positive- and negative-energy states are equal mixtures of particle and hole components. By contrast the signature of an ABS generated by the inter-band coupling mechanism can be particle-hole asymmetric (in the presence of dissipation) down to arbitrarily low energy. To illustrate this point, in Fig. 5.31 we compare the differential conductance trace calculated generated (in the presence of finite dissipation) by an ABS 


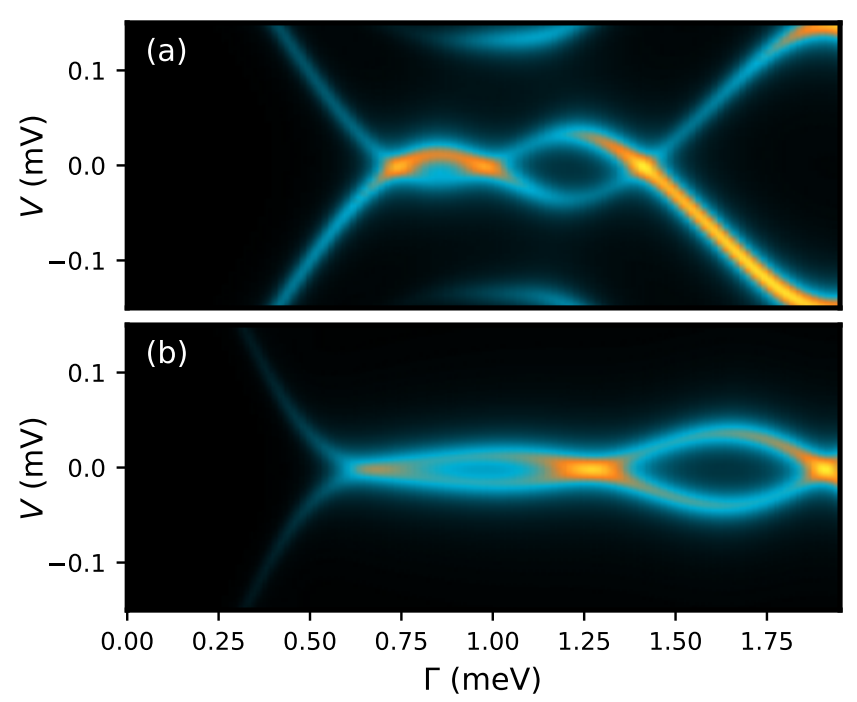

FIGURE 5.31: Differential conductance as function of Zeeman field and bias voltage in the presence of dissipation for a hybrid system that supports (a) an ABS generated by the inter-band coupling mechanism and (b) Majorana bound states in a finite wire. Note that the ABS generates a particle-hole asymmetric zero bias peak, in contrast with the MBS signature, which is particle-hole symmetric. The system parameter for panel (a) are the same as those in Fig. 5.28 except $\mu_{1}=0.2 \mathrm{meV}$ and $\left(\alpha_{T}\right)_{1,2}=$ $0.2 \mathrm{meV}$. In panel (b) we have a wire of length $780 \mathrm{~nm}$ described by a single-band model with $\mu=0.25 \mathrm{meV}, m^{*}=0.03, \alpha_{L}=$ $500 \mathrm{meV} \AA$, and $\Delta=0.35 \mathrm{meV}$. Dissipation was modeled as an imaginary contribution of magnitude $\eta=0.015 \mathrm{meV}$.

pinned near zero energy by level repulsion [panel (a)] and the trace associated with an oscillating Majorana mode [panel (b)]. The differential conductance was calculated using Kwant [184], with dissipation being modeled $[171,172]$ as a diagonal imaginary contribution to the effective Hamiltonian, $i \eta$, where $\eta=0.015 \mathrm{meV}$. Note the manifest low-energy particle-hole asymmetry in panel (a). More specifically, the asymmetric zero bias peak extending from $\Gamma \approx 0.7 \mathrm{meV}$ to $\Gamma \approx 1.0 \mathrm{meV}$ in panel (a) is a clear sign that the underlying BdG state has asymmetric particle-hole character down to very small energies. We emphasize that the asymmetric features discussed here are characterized by an energy scale on the order of the ZBCP width. In particular, the energy scale of the state responsible for the asymmetric peak in panel (a) is on the order of $10 \mu \mathrm{eV}$. Asymmetric features occurring at higher energies (inside or outside the induced gap), e.g., the feature emerging above $\Gamma \approx 1.25 \mathrm{meV}$ in panel (a), do not provide any information regarding the Majorana (or non-Majorana) nature of the lowest energy mode. Based on the fundamental property discussed here, we conclude that any nearly-zero-bias differential conductance feature that does not exhibit particle-hole symmetry should not be attributed to MZMs, quasi-Majoranas, or any other low-energy mode that involves (partially) separated Majorana bound states, but rather to 
the presence of (topologically-trivial) ABSs pinned near zero-energy by level repulsion.

\section{Inhomogeneous wires}

A position-dependent effective potential causes variations (along the wire) of the transverse profiles of the wave functions associated with different confinement-induced bands, which, in turn, induces inter-band coupling [126]. Inhomogeneous potentials can arise in Majorana devices for reasons such as the termination of the $\mathrm{SC}$ covering, the use of multiple electrostatic gates along the wire, an inhomogeneous SC-SM work function difference, the presence of multiple leads used in tunneling spectroscopy, and disorder. We note that the presence of an inhomogeneous potential can induce trivial lowenergy ABSs that stick to zero energy even within single-band models (i.e. ps-ABSs generated via the partial-separation mechanism), as discussed extensively in the literature $[66,68,108]$. Here, we show that the inter-bandcoupling induced by an inhomogeneous potential can also lead to the emergence of ABSs pinned near zero energy, but these ABSs are composed of nonseparated Majorana components. Note that the inhomogeneous potential also breaks the inversion symmetry of the system, which allows the presence of an anti-symmetric component of the longitudinal spin orbit coupling in the toy model, i.e. $\widetilde{\alpha}_{i}^{\alpha \beta}=-\widetilde{\alpha}_{i}^{\beta \alpha}$ for $\alpha \neq \beta$. As a consequence, transverse spin-orbit coupling is not needed to induce inter-band level repulsion within inhomogeneous systems, in contrast to the homogeneous case [132].

Consider a wire of total length $L=1.5 \mu \mathrm{m}$ described by a three band model. We assume all three bands to be empty in the bulk of the wire at zero magnetic field. We also assume a potential well localized near the left end of the wire, within a $100 \mathrm{~nm}$ long region, where the three bands become occupied (i.e. $V_{\alpha}<\mu_{\alpha}$ ) as the result of applying a gate potential, as shown in Fig. 5.32(a). We include a key insight from the 3D model by making the effective potential band-dependent within the gated region (see Sec. 5.2.2). This is due to the fact that that the three bands have, in general, different transverse profiles. For example, the band corresponding to the orange curve in Fig. 5.32(a) may have more weight near the gate, as compared to the other two bands, and, therefore, its effective potential is more affected by the applied gate potential. The inter-band coupling includes terms associated with the (induced) superconducting pairing and the longitudinal spin-orbit coupling, as predicted by the 3D model.

The dependence of the low-energy spectrum on the applied Zeeman field is shown in Fig. 5.32(b). The first notable feature is represented by the subgap states generated by the inhomogeneous potential at low fields, i.e. in the topologically trivial phase characterized by $\Gamma<\Gamma_{c} \approx 2.3 \mathrm{meV}$. The bulk quasiparticle gap vanishes at the TQPT corresponding to $\Gamma=\Gamma_{c}$, then it reopens, simultaneously with the emergence of MZMs. While the existence of ABSs induced by short-range inhomogeneous potentials (which can cross zero energy in the topologically-trivial regime [67]) was discussed in the literature, a remarkable feature of this ABS mode is the pinning near 

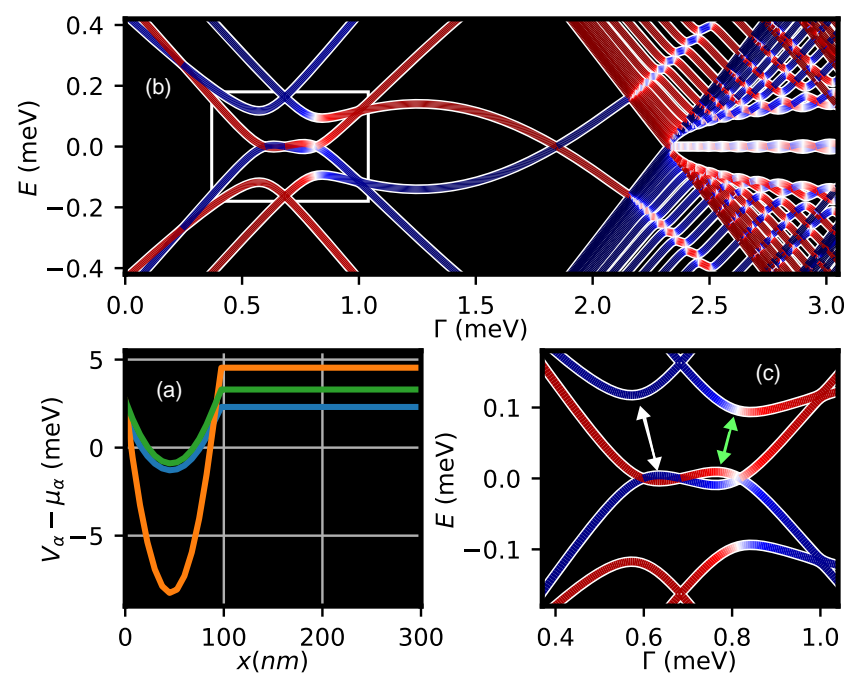

FIGURE 5.32: (a) Band-dependent effective potential (shifted by $\mu_{\alpha}$ ) as a function of position for a wire of length $L=1.5 \mu \mathrm{m}$ in the presence of a gate potential at the left end of the system. We have $\mu_{1}=-2.31 \mathrm{meV}$ (blue), $\mu_{2}=-3.3 \mathrm{meV}$ (green), and $\mu_{3}=-4.54 \mathrm{meV}$ (orange). Within the gate region $(0 \leq x \leq 100 \mathrm{~nm}), V_{i \alpha}$ is assumed to be harmonic with maximum depths of $3.6 \mathrm{meV}, 4.2 \mathrm{meV}$, and $12.8 \mathrm{meV}$, respectively, while the effective potential is zero outside of the gated region. Note that effective potential is shown only for the leftmost $300 \mathrm{~nm}$. (b) Dependence of the low-energy spectrum on the applied Zeeman field. Panel (c) represents a zoomed in look of the region outlined by a white box in panel (b). The model parameters are: $\alpha_{1,1}, \alpha_{2,2}, \alpha_{3,3}=500,333$, and $250 \mathrm{meV}$ $\AA$, respectively, $\alpha_{1,2}=-\alpha_{2,1}=5 \mathrm{meV} \AA, \Delta_{i, i}=0.35 \mathrm{meV}$, and $\Delta_{1,3}=\Delta_{3,1}=0.175 \mathrm{meV}$.

zero energy over a considerable Zeeman field range $[\sim 0.3 \mathrm{meV}$; see Fig. 5.32(c)], despite the very short length scale associated with the inhomogeneity (about $100 \mathrm{~nm}$ ). We emphasize that a single band model with similar parameters predicts zero-energy ABS crossings, but no pinning over a finite Zeeman field range [67]. In fact, the low-energy ABS illustrated in Fig. 5.32, which sticks near zero energy over a substantial range of Zeeman field, is generated by the inter-band coupling mechanism discussed above, hence it requires multi-band occupancy. Reproducing this behavior within a single band model would require an inhomogeneity with characteristic length scale on the order of a micron [67].

Analyzing the structure of the low-energy states associated with the spectrum shown in Fig. 5.32 provides us with a physical picture of the inter-band coupling mechanism responsible for the pinning of the low-energy mode. For example, in the vicinity of the first zero energy crossing near $\Gamma=0.6 \mathrm{meV}$, the lowest energy state has most of its weight coming from the band shown 

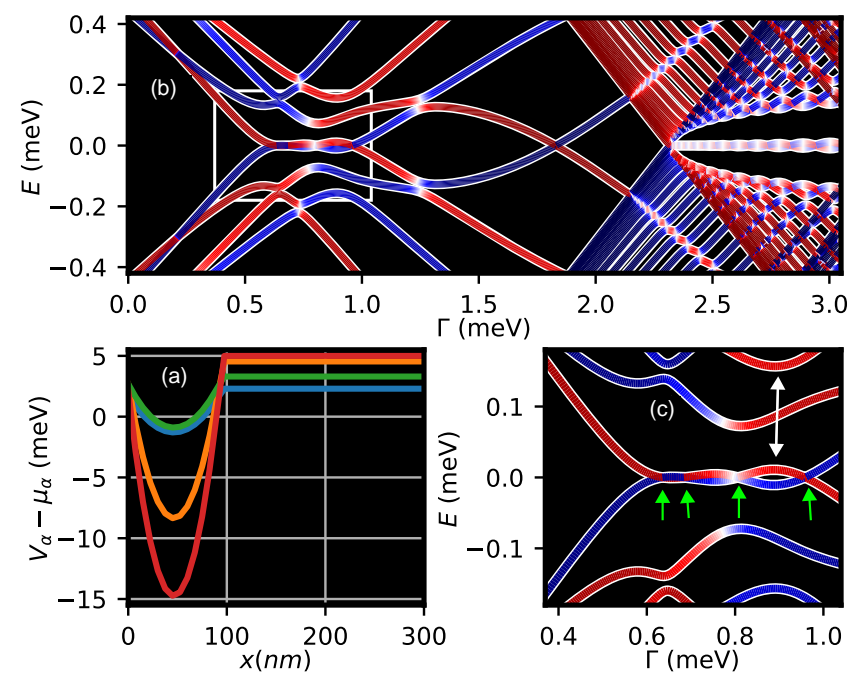

FIGURE 5.33: (a) Band-dependent effective potential (shifted by $\mu_{\alpha}$ ) as a function of position for a wire of length $L=1.5 \mu \mathrm{m}$ in the presence of a gate potential at the left end of the system. The parameters corresponding to the first three bands are the same as in Fig. 5.32. The forth band (red) is characterized by $\mu_{4}=-5 \mathrm{meV}$, while $V_{i, 4}$ is a harmonic well inside the gate region with a maximum depth of $19.75 \mathrm{meV}$ and is zero outside. The other parameters associated with the fourth band are: $\alpha_{4,4}=333 \mathrm{meV} \AA, \alpha_{1,4}=-\alpha_{4,1}=5 \mathrm{meV} \AA, \alpha_{3,4}=-\alpha_{4,3}=72.5$ $\mathrm{meV} \AA, \alpha_{2,4}=-\alpha_{4,2}=10 \mathrm{meV} \AA$, and $\Delta_{3,4}=\Delta_{4,3}=0.1 \mathrm{meV}$. (b) Dependence of the low-energy spectrum on the applied Zeeman field. Panel (c) represents a zoomed in look of the region outlined by a white box in panel (b).

in green in Fig. 5.32(a). In the absence of inter-band coupling, this state simply crosses zero energy and leaves the energy window represented in Fig. 5.32(b) near $\Gamma=1 \mathrm{meV}$. However, in the presence of inter-band coupling, the state hybridizes with another low energy state associated (primarily) with the band corresponding to the 'blue' effective potential in panel (a), which results in the anti-crossing indicated in Fig. 5.32(c) by the white arrow. Note that both of these states are hole-like (blue filling) - here, as before, we focus on the positive energy states - and maintain their hole character throughout the anti-crossing. The primary mechanism responsible for this anti-crossing is the inter-band spin-orbit coupling $\alpha_{1,2}$ between the 'blue' and 'green' bands, which are the main components of the two states. By contrast, the second anti-crossing indicated by the green arrow in Fig. 5.32(c) corresponds to a particle-like and a hole-like state (associated, primarily, with the 'green' and 'orange' bands, respectively) approaching each other. The particle-hole characters are exchanged between the two states through the anti-crossing. Since particle-hole coupling occurs due to SC pairing, we conclude that the primary mixing mechanism responsible for this anti-crossing is the inter-band superconducting pairing $\Delta_{13}$ between the 'green' and the 'orange' bands of 


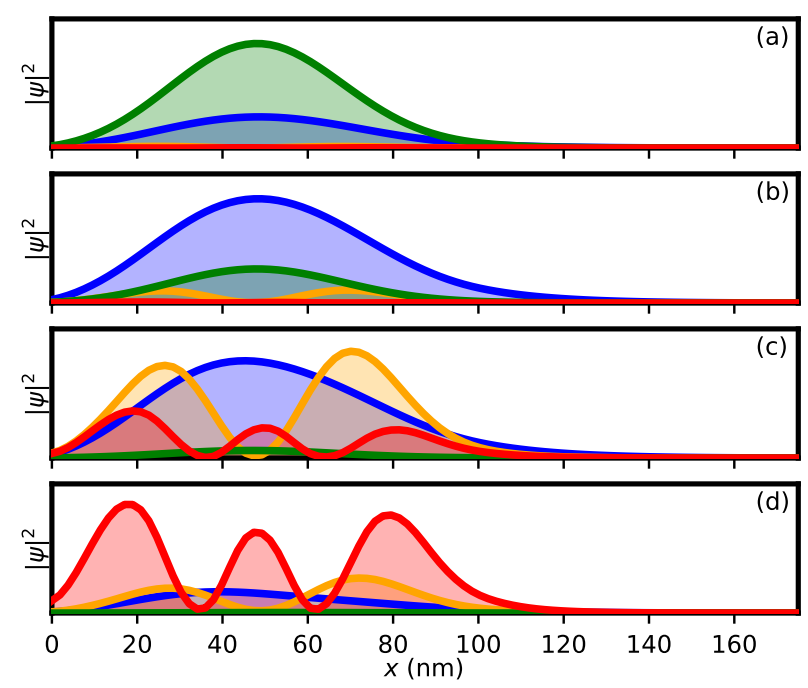

FIGURE 5.34: Wave function profiles corresponding to the zeroenergy states marked by green arrows in Fig. 5.33(c). The calculated amplitudes $|\psi|^{2}$ involve summations over the spin and particle-hole degrees of freedom. Different colors represent contributions from the corresponding bands, using the color code from Fig. 5.33(b). Note that the low-energy ABS mode is localized within the gate region $(0 \leq x \leq 100 \mathrm{~nm})$ and that its band composition changes dramatically with the Zeeman field as a result of strong inter-band coupling.

Fig. 5.32(a).

A more robust (nearly) zero-energy state can be obtained by adding a fourth band to the model described above, more specifically the 'red' band in Fig. 5.33(a), which is characterized by a deep potential well in the barrier region. The corresponding low-energy spectrum is shown in Fig. 5.33(b), with panel (c) representing a zoomed in view of the low-field near-zero ABS. Again, the lowest energy mode is pinned near zero energy over a significant range of Zeeman field $(\sim 0.4 \mathrm{meV})$ in the topologically trivial regime. Note that experimentally the high-field regime (e.g., $\Gamma>1.5 \mathrm{meV}$ ) may be inaccessible due to the collapse of the SC gap of the parent superconductor, so that the most prominent low-energy feature would be the ZBCP generated by the topologically-trivial ABS mode pinned near zero energy. The expanded pinning range (as compared with the three-band model shown in Fig. 5.32) is due to the additional anti-crossing marked by the white arrow in Fig. 5.33(c), which is primarily due to inter-band spin-orbit coupling (described primarily by $\alpha_{3,4}$ ), since the relevant states have particle-like character through the entire anti-crossing (i.e. red filling at positive energies - see Fig. 5.33).

To demonstrate that the anti-crossings are indeed due to inter-band coupling, we calculate explicitly the wave function amplitudes corresponding to the four zero energy crossings marked by the green arrows in Fig. 5.33(c). 

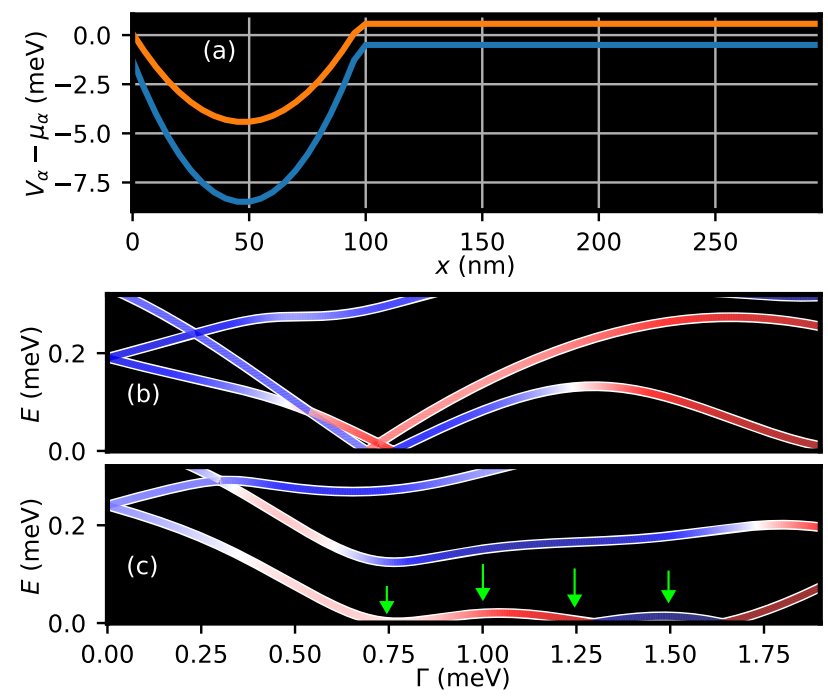

FIGURE 5.35: The system is described by a two-band model with a position- and band-dependent effective potential (shifted by $\mu_{\alpha}$ ) shown in panel (a). The chemical potential is place between the two bands, such that $\mu_{1}=0.5 \mathrm{meV}$ and $\mu_{2}=-0.57 \mathrm{meV}$. The potential well depths are $8 \mathrm{meV}$ and $5 \mathrm{meV}$, respectively. Dependence of the low-energy spectrum on the applied Zeeman field for a wire of length $L=0.3 \mu \mathrm{m}$ in the presence of a gate potential at the left end of the wire for (b) a system without inter-band coupling and (c) a system with inter-band spin-orbit coupling. The other model parameters are: $\Delta_{11}=\Delta_{22}=0.35 \mathrm{meV}$ (only within the proximitized, homogeneous potential region) and, for panel (c), the inter-band coupling is given by $\left(\alpha_{T}\right)_{12}=1.2 \mathrm{meV}$ (only within the inhomogeneous, gate region).

The results are shown in Fig. 5.34. Note that the contributions to the lowestenergy mode from various confinement-induced bands change quite dramatically as the Zeeman field increases, which is a clear indication of inter-band mixing. In addition, the explicit calculation of the corresponding Majorana components reveals the absence of any significant Majorana separation in real-space, which confirms that the partial separation mechanism is not responsible for the pinning of this ABS mode near zero energy.

As a final example, we consider a two-band model representing a short wire of length $L=300 \mathrm{~nm}$ with an inhomogeneous potential as shown in Fig. 5.35(a). This model differs from the previous two examples in two respects: (1) the inter-band coupling is active only within the inhomogeneous potential (gate) region $0 \leq x \leq 100 \mathrm{~nm}$ and (2) the induced superconductivity is nonzero only outside of the gate region. The model corresponds to a setup consisting of a short proximitized wire coupled to a quantum dot representing the uncovered gate region. As the gate-induced potential in the dot region is highly inhomogeneous, we expect the emergence of strong inter-band mixing, as confirmed by the 3D calculation (see Sec. 5.2.2). The dependence 

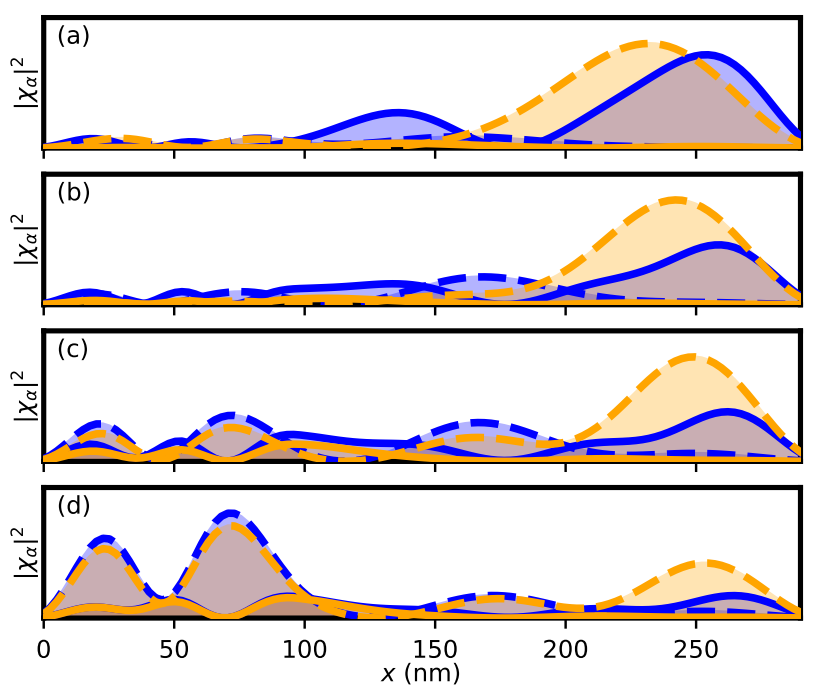

FIGURE 5.36: Majorana wave function profiles corresponding to the low-energy states marked by green arrows in Fig. 5.35(c). The two Majorana wave functions are shown in blue and orange, respectively, while solid and dashed lines indicate the contributions from the first and second bands, respectively.

of the low-energy spectrum on the Zeeman field is shown in Fig. 5.35 for a system with decoupled bands, i.e., without inter-band coupling [panel (b)], and a system with inter-band spin-orbit coupling [panel (c)]. Note that in the proximitized region both band minima are relatively close to the chemical potential, $\left|\mu_{i}\right| \approx 0.5 \mathrm{meV}$, so that in the absence of inter-band coupling they satisfy the topological condition at about the same critical field. However, due to the very short length of the wire, the two pairs of MBSs (one for each band) overlap strongly and the resulting energy splittings have amplitudes comparable to the induced gap, as shown in Fig. 5.35(b). By contrast, interband spin-orbit coupling within the uncovered (gate) region pins the lowest energy mode near zero energy over a very wide range of Zeeman energy from $\Gamma \approx 0.75 \mathrm{meV}$ to $\Gamma \approx 1.6 \mathrm{meV}$. The nature of this low energy mode is revealed by calculating the wave functions of its Majorana components. The amplitudes of the Majorana wave functions corresponding to the low-energy states marked by green arrows in Fig. 5.35(c) are shown in Fig. 5.36. In panel (a), which corresponds to $\Gamma=0.75 \mathrm{meV}$, we notice two Majorana modes (orange and blue, respectively) localized near the right end of the wire. While they overlap strongly, these modes belong to different bands, as indicated by the solid (first band) and dashed (second band) lines. This result can be understood as follows: in the absence of inter-band coupling, the system supports two pairs of (strongly overlapping) MBSs associated with the two bands. When the inter-band coupling is turned on in the inhomogeneous region, the two Majoranas localized near the left end of the system get coupled and morph into a finite energy ABS. This leaves two unpaired Majoranas at the right end of wire that are spatially overlapping, but are (partially) separated in band space. 
Note that, in the short wire studied here, the two Majoranas still have tails leaking into the region where the bands couple. Naively, one would expect significant energy splitting oscillations as a result of this coupling. However, upon increasing $\Gamma$, the lowest energy state is transforming as a result of interband coupling from a pair of band-separated Majoranas localized near the right end of the wire [panel (a)] into a low-energy ABS (predominantly) localized within the uncovered region and characterized by strongly overlapping Majorana modes associated with the second band [panel (d)]. Hence, the robust pinning near zero energy shown in Fig. 5.35(c) is due to a combination of two distinct mechanisms: the band separation of the MBSs localized in the homogeneous (uncoupled) region and the level repulsion (induced by interband coupling) affecting the ABS localized within the uncovered region. We will discuss a similar example within the 3D model, at the end of Sec. 5.2.2. The important message here is that, in general, the inter-band coupling mechanism acts in combination with the partial separation (in real space or band space) mechanism. A combination of these mechanisms in very short systems can result in a rather spectacular pinning of the lowest-energy mode near zero energy. This example further demonstrates that the observation of low-amplitude energy splitting oscillations is not necessarily an indication of topological protection.

\subsubsection{D model}

The 3D model calculations incorporate electrostatic effects due to gateinduced external potentials and the presence of a parent superconductor. Their purpose is twofold. First, we want to understand if the basic assumptions underlying the simplified models used so far in the literature for discussing topologically-trivial ABSs emerging in hybrid systems, or those underlying the inter-band coupling mechanism discussed above are realistic enough. For example, does a specific experimental setup generate an electrostatic confinement that is smooth enough to induce robust ps-ABSs? More importantly for this work, are the actual inter-band couplings strong enough to trigger the level repulsion mechanism discussed in the previous section? Our second purpose is to estimate whether the emergence of low-energy ABSs due to level repulsion is a rather generic occurrence, or rather one that requires a lot of fine tuning.

\section{Theoretical Model}

We now describe the 3D model used to study the effects of an inhomogeneous electrostatic potential and the multi-band physics in Majorana nanowires. A schematic representation of a setup that matches the devices used in a recent tunneling experiment [122] is shown in Fig. 5.37. The basic ingredients include a semiconductor wire (SM) in proximity to an s-wave superconductor (SC), a normal lead (used for tunneling spectroscopy), and various gates to control the electrostatic potential, as shown in panel (a). The transverse profile of the system in the SC covered region in shown in panel (b). Note 

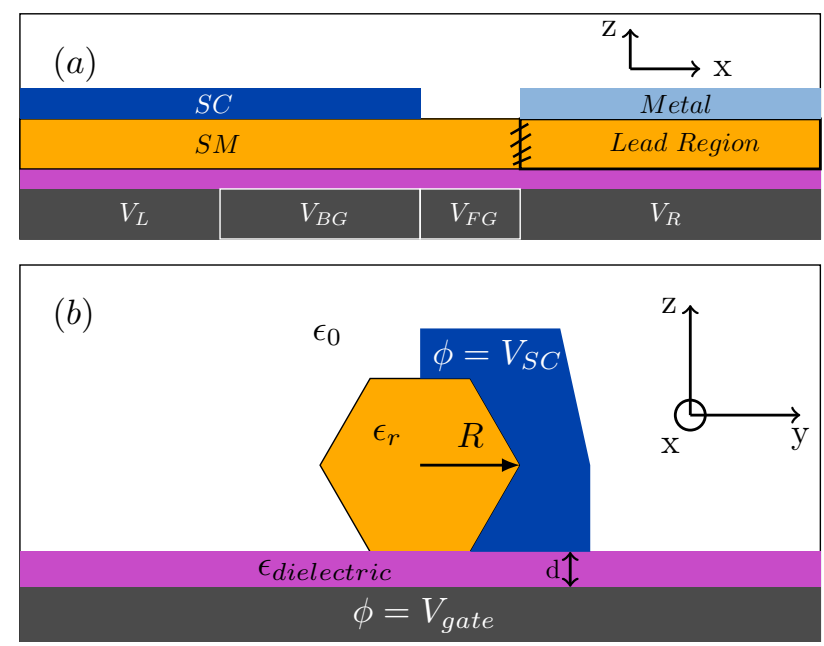

FIGURE 5.37: (a) Schematic representation of the device along the SM wire. The semiconductor nanowire (orange) is proximity coupled to a superconductor (blue) and a metal (light blue) is the lead region. A dielectric layer (purple) separates the nanowire from potential gates (dark grey). There are four regions defined by the external gates and the materials deposited on the SM wire: the left bulk region (gate potential $V_{L}$ ), the biggate region $\left(V_{B G}\right)$, the fine-gate (uncovered) region $\left(V_{F G}\right)$, and the lead region $\left(V_{R}\right)$. The lead region is a continuation of the SM nanowire but in proximity to a metal, which strongly renormalizes its properties. We focus on the physics of the isolated hybrid nanowire, which is disconnected from the lead (as indicated by black lines between the SM and the lead region). (b) Schematic representation of the cross section of the nanowire device in the SC-covered region.

that the SC is treated as a boundary condition, as far as the electrostatic effects are concerned, with a potential $V_{S C}$ set by the work function difference between the SC and SM. The details of the lead region (which consists of a SM wire segment covered by normal metal) are quite difficult to model due to the unknown parameters characterizing this region. The metallic material alters the electrostatic conditions (due to the work function difference between the metal and the SM) and heavily renormalizes the effective parameters of the wire due to strong hybridization between SM and metallic states. Accurately capturing these effects would require to explicitly incorporate the normal metal into the model. Since we are not concerned here with tunneling features, we focus on the physics of an isolated nanowire (i.e. a nanowire that is not coupled to a tunneling probe). However, we still take into account electrostatic effects due to the presence of the lead. The lead region is disconnected from the SM region (as indicated by black lines), but the metal covering (light blue region above lead region) is still incorporated when calculating the external electrostatic potential. This allows us to include the electrostatic screening effect of the lead, without explicitly incorporating the 
normal metal into the Hamiltonian.

There are four different gates with potentials denoted by $V_{L}, V_{B G}, V_{F G}$, and $V_{R}$ respectively. These gates break up the device into four regions: the left bulk region (gate potential $V_{L}$ ), the big-gate region $\left(V_{B G}\right)$, the fine-gate (uncovered) region $\left(V_{F G}\right)$, and the lead region $\left(V_{R}\right)$. Our focus is on the lowenergy physics of the BG and FG regions, which can be probed using tunneling spectroscopy (from the right lead). Consequently, the gate potential $V_{L}$ is set such that the low energy states do not leak significantly into the left bulk region. The low-energy states of interest are therefore confined to the BG and FG regions with the corresponding gates being used as control knobs for the electrostatic potential within these active regions.

The tight-binding model used in the 3D model calculations is constructed [126] by dividing the semiconductor into $N_{x}$ layers along the length of the wire, each containing $N_{\perp}$ sites. The corresponding Hamiltonian, ${ }^{3}$ which does not include superconductivity, is given by

$$
\begin{aligned}
H_{S M}= & \sum_{i, j, m, \sigma} t_{i j}^{\perp} c_{i m \sigma}^{\dagger} c_{j m \sigma}+\sum_{i, m, n, \sigma} t_{m n}^{\|} c_{i m \sigma}^{\dagger} c_{i n \sigma} \\
& +\sum_{i, m, \sigma}\left(V_{i m}+U_{i m}\right) n_{i m \sigma} \\
& +\sum_{i, m, \sigma, \sigma^{\prime}} \widetilde{\alpha}_{R}\left[c_{i(m+1) \sigma}^{\dagger}\left(-i \sigma_{y}\right)_{\sigma \sigma^{\prime}} c_{i m \sigma^{\prime}}+\text { h.c. }\right] \\
& +\sum_{i, m, \sigma, \sigma^{\prime}} \Gamma c_{i m \sigma}^{\dagger}\left(\sigma_{x}\right)_{\sigma \sigma^{\prime}} c_{i m \sigma^{\prime}},
\end{aligned}
$$

where $c_{i m \sigma}^{\dagger}$ creates an electron with spin $\sigma$ localized near the site $i$ of layer $m$, $n_{i m \sigma}=c_{i m \sigma}^{\dagger} c_{i m \sigma}$ is the number operator, $t_{i j}^{\perp}$ and $t_{m n}^{\|}$are intra- and inter-layer nearest neighbor hopping matrix elements, respectively, $\Gamma$ is the (half) Zeeman splitting, and $\widetilde{\alpha}_{R}=\alpha_{R} /\left(2 a_{x}\right)$, where $\alpha_{R}$ is the Rashba spin-orbit coefficient and $a_{x}$ is the distance between layers. Note that we neglect transverse spin-orbit coupling, for simplicity.

The electrostatic effects due to the presence of the potential gates and the superconductor are described by the external potential $V_{i m}$. Explicitly, the potential matrix elements are $V_{i m}=-e\langle i, m|V(\mathbf{r})| i, m\rangle$, where $V(\mathbf{r})$ is the solution of the Laplace equation $\nabla^{2} V(\mathbf{r})=0$ with boundary conditions set by the potential on the superconductor $\left(V_{S C}\right)$ and the external gates $\left(V_{B G}\right.$, $V_{F G}$, etc.). Electron-electron interactions are included at the mean field level through the term $U_{i m}$. Explicitly, the potential matrix elements are $U_{i m}=$ $-e\langle i, m|U(\mathbf{r})| i, m\rangle$, where $U(\mathbf{r})$ is the solution of the Poisson equation with homogeneous boundary conditions. We solve the Laplace and Poisson equation using the methods described in chapter 4.

\footnotetext{
${ }^{3}$ Note that the Hamiltonian in this section is modeled using a tight-binding (finite difference method) approach, while finite element methods were used in chapter 4 when solving the Schrödinger equation. The Poisson equation was solved still with finite element methods.
} 
The Schrödinger-Poisson problem defined by the 3D Hamiltonian in Eq. (5.52), which necessarily involves a large number of degrees of freedom, can be efficiently solved by reducing it to an effective $1 \mathrm{D}$ problem though a projection onto an appropriate low-energy sub-space. Here, we give a brief outline of the projection technique; the details of this procedure can be found in our work in Ref. [126]. The essential observation behind this low-energy projection approach is that the transverse profiles of the (low-energy) states of a finite wire are quite similar to those of an infinite homogeneous wire with electrostatic environment similar to the local environment of the finite system. To incorporate this observation, we define an auxiliary Hamiltonian for each layer:

$$
\begin{aligned}
& H_{a u x}^{(m)}=\sum_{i, j, k, \sigma}[\left.t_{i j}^{\perp}+\left(\frac{\hbar^{2} k^{2}}{2 m^{*}}+V_{i}^{(m)}+U_{i}^{(m)}\right) \delta_{i j}\right] c_{i k \sigma}^{\dagger} c_{j k \sigma} \\
&+\sum_{i k \sigma \sigma^{\prime}} \alpha_{R} k c_{i k \sigma}^{\dagger}\left(\sigma_{y}\right)_{\sigma \sigma^{\prime}} c_{i k \sigma^{\prime},}
\end{aligned}
$$

where $V_{i}^{(m)}=V_{i m}$. The auxiliary Hamiltonian of the $m^{\text {th }}$ layers describes an infinite wire with a translation-invariant external potential that matches the local external potential of the finite wire and whose transverse profile also matches the local transverse profile of the $m^{\text {th }}$ layer. The low-energy $k=0$ eigenstates of the effective Hamiltonian can be viewed as a set of 'molecular orbitals' and provide us with a position-dependent (i.e. layer-dependent) basis for the low-energy sub-space. Finally, the low-energy effective 1D Hamiltonian is obtained by projecting the full 3D Hamiltonian, Eq. (5.52), onto the sub-space spanned by the $n_{o}$ lowest molecular orbitals [i.e. $k=0$ eigenstates of Eq. (5.53)]. Explicitly, we have

$$
\begin{gathered}
H_{S M}^{\mathrm{eff}}=\sum_{m, n, \sigma} \sum_{\alpha, \beta} \tilde{t}_{m \alpha, n \beta}^{\|} c_{m \alpha \sigma}^{\dagger} c_{n \beta \sigma}+\sum_{m, \sigma} \sum_{\alpha} \epsilon_{\alpha}^{m} n_{m \alpha \sigma} \\
+\sum_{m, \sigma \sigma^{\prime}} \sum_{\alpha, \beta}\left[\widetilde{\Delta U}_{\alpha \beta}^{m} \delta_{\sigma \sigma^{\prime}}+\Gamma\left(\sigma_{x}\right)_{\sigma \sigma^{\prime}} \delta_{\alpha \beta}\right] c_{m \alpha \sigma}^{\dagger} c_{m \beta \sigma^{\prime}} \\
\quad+\sum_{m, n, \sigma \sigma^{\prime}} \sum_{\alpha, \beta} i \alpha_{\alpha \beta}^{m n}\left(\sigma_{y}\right)_{\sigma \sigma^{\prime}} c_{m \alpha \sigma}^{\dagger} c_{n \beta \sigma^{\prime}}
\end{gathered}
$$

where $\mathrm{m}$ and $\mathrm{n}$ label the sites of a 1D lattice, $\alpha$ and $\beta$ label the molecular orbitals corresponding to the eigenstates of $H_{a u x}^{(m)}, \epsilon_{\alpha}^{m}$ are the energies of the molecular orbitals for layer $\mathrm{m}$, and the summations marked by a $\bullet$ are restricted to the low energy subspace. The hopping matrix elements $\widetilde{t}_{m \alpha, n \beta}$ are given by

$$
\tilde{t}_{m \alpha, n \beta}^{\|}=\left\langle\varphi_{\alpha}^{m}\left|T^{\|}\right| \varphi_{\beta}^{n}\right\rangle,
$$

where $\left[T^{\|}\right]_{i m, i n}=t_{m n}^{\|} \delta_{i j}$ and $\left|\varphi_{\alpha}^{m}\right\rangle,\left|\varphi_{\beta}^{n}\right\rangle$ are eigenstate of $H_{a u x}^{(m)}$ and $H_{a u x}^{(n)}$, respectively. The spin-orbit matrix elements $\alpha_{m n}^{\alpha \beta}$ are calculated in a similar 
manner. Notice that the inter-band hopping and the inter-band spin-orbit coupling are nonzero if (and only if) the eigenstates corresponding to neighboring layers are different, i.e. if the transverse profile of the states associated with a given 'molecular orbital' change as a function of position along the wire. This occurs when the effective potential is position-dependent. Consequently, we expect inter-band coupling to occur, for example, near the interface of the BG and FG regions [see of Fig. 5.37(b) and (c)] due to the termination of the SC covering and having (in general) different values for $V_{B G}$ and $V_{F G}$. The quantity $\widetilde{\Delta U}_{\alpha \beta}^{m}$ describes the difference between the mean field potential of the auxiliary Hamiltonian $H_{a u x}^{(m)}$ and the actual mean field potential of the 3D Hamiltonian given by Eq. (5.52). This term arises primarily due to charge redistribution along the length of the wire and can lead to barrierlike features between the SC-covered and the uncovered regions [126]. For simplicity, we neglect this term in the current analysis. In other words, we incorporate the mean field self-consistency when calculating the eigenstates of Eq. (5.53), but neglect any fluctuation of the mean field value due to broken translation invariance or nonzero applied magnetic field. These fluctuations are important for quantitative considerations, however, the focus of this work is to illustrate the main qualitative features of multi-band physics in devices with inhomogeneous potentials.

Lastly, we incorporate superconductivity at the mean field level through the pairing term

$$
H_{\Delta}=\sum_{n, m, \alpha, \beta}\left[\left\langle\varphi_{\alpha}^{n}|\Delta| \varphi_{\beta}^{m}\right\rangle c_{n \alpha \uparrow}^{\dagger} c_{m \beta \downarrow}^{\dagger}+\text { h.c. }\right]
$$

with $\Delta_{i j}^{m n}=\Delta_{i}^{m} \delta_{m, n} \delta_{i, j}$, where $\Delta_{i}^{m}$ is zero everywhere except at the SM-SC interface. Note that the FG region does not contribute to pairing due to the termination of the superconductor. Also note that, in general, the inter-band pairing $\Delta^{\alpha \beta}$ can become significant when the gate voltage is comparable to or larger than the superconductor-semiconductor work function difference. The total effective BdG Hamiltonian becomes

$$
H_{B d G}=H_{S M}^{\mathrm{eff}}+H_{\Delta} .
$$

Note that the structure of the effective Hamiltonian is similar to the structure of the toy model in Eq. (5.51). The major difference is that the parameters of the toy model (including the number of occupied bands, the profile of the effective potential, the inter-band coupling parameters, etc.) are largely arbitrary, while the parameters of the effective Hamiltonian are calculated based on the geometric and electrostatic properties of the device. The results presented below show that the conditions required by the mechanism described Sec. 5.2.1 can be realized and are even likely to occur in experimental device. 


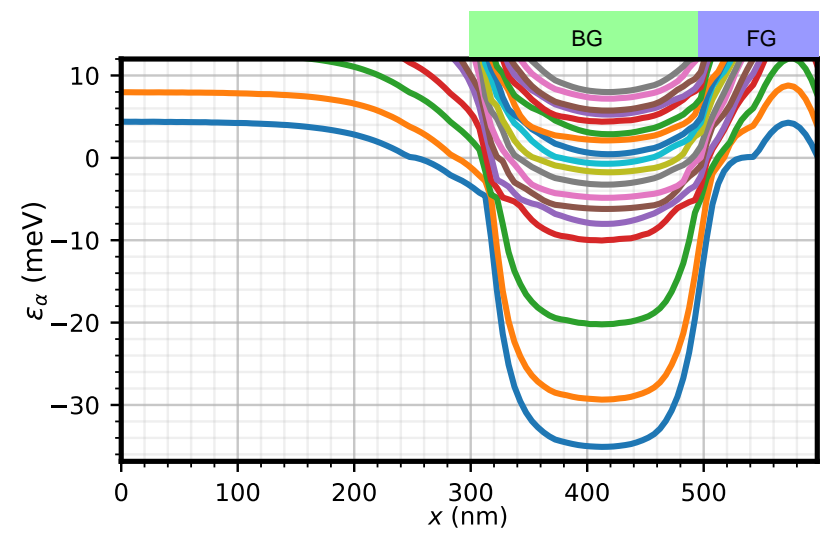

FIGURE 5.38: Effective potential profiles corresponding to different bands for the device represented schematically in Fig. 5.37. The BG region stretches from 300 to $500 \mathrm{~nm}$, while the FG region stretches from 500 to $600 \mathrm{~nm}$. The external potentials are: $V_{S C}=230 \mathrm{mV}, V_{L}=-250 \mathrm{mV}, V_{B G}=364 \mathrm{mV}, V_{F G}=175 \mathrm{mV}$, and $V_{R}=-125 \mathrm{mV}$.

\section{Results}

The main findings discussed in this subsection are: i) for a wide range of experimentally-relevant gate potentials the system is characterized by multiband occupancy (i.e. 5-20 occupied bands), ii) within the 3D model, interband coupling arises naturally in the presence of inhomogeneities, and iii) inter-band coupling produces low-energy states that remain near zero energy over a wide range of Zeeman field due to inter-band level repulsion. Moreover, these "sticky" states occur quite frequently for systems with band occupancy of the order ten (and larger).

Throughout this section, we use the following values for the system parameters: radius of the circle that circumscribes the $S M$ wire $R=70 \mathrm{~nm}$, thickness of the dielectric layer $d=10 \mathrm{~nm}$, permittivity $\epsilon_{r}=17.7$ (wire) and $\epsilon_{\text {dielectric }}=24$ (dielectric), effective mass $m^{*}=0.025 m_{0}$, and Rashba coefficient $\alpha=250 \mathrm{meV} \AA$. The in-plane and out-of-plane lattice spacings are taken to be $a_{\perp}=7 \mathrm{~nm}$ and $a_{\|}=5 \mathrm{~nm}$, respectively. The FG and BG regions are $100 \mathrm{~nm}$ and $200 \mathrm{~nm}$ long, respectively. The SC-SM work function difference is chosen to be $V_{S C}=230 \mathrm{mV}$ and the gap between the bottom of the lowest-energy confinement-induced band and the Fermi level (before the external gates are applied) is $E_{o}=210 \mathrm{meV}$. The geometric and dielectric parameters are chosen to match devices used in Ref. [122]. We note that the pinning mechanism described in Ref. $[185,186]$ cannot be at work in the system studied here, as it requires the presence of same-sign image charges in the dielectric, which is only possible if $\epsilon_{\text {dielectric }}<\epsilon_{r}$.

The effective potential profile along the device corresponding to a given set of gate voltages is shown in Fig. 5.38. Note that the BG and FG regions span from 300 to $500 \mathrm{~nm}$ and from 500 to $600 \mathrm{~nm}$, respectively. As mentioned in the previous section, we are mainly interested in the low-energy physics of states localized (primarily) within the BG and FG regions. For this 


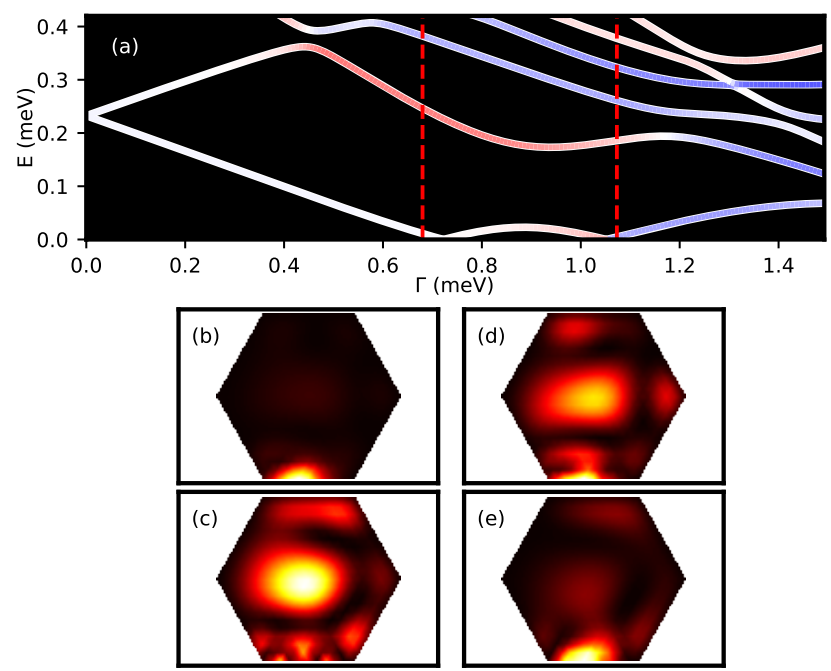

FIGURE 5.39: (a) Low-energy spectrum as a function of Zeeman field for a system with the same parameters as in Fig. 5.38 (only the positive energy sector is shown). The transverse profiles of the first- and second-lowest energy states at $x=400 \mathrm{~nm}$ (i.e. near the middle of the BG region, see Fig.5.38) and Zeeman fields indicated by the red dashed lines in panel (a) are shown in panels (b)-(e). Panels (b) and (c) correspond to the states indicated by the first red dashed line, while (d) and (e) correspond to the second. Note that the states swap characters as the Zeeman field increases from $\Gamma \approx 0.67 \mathrm{meV}$ to $\Gamma \approx 1.13 \mathrm{meV}$, indicating anti-crossing behavior.

reason, $V_{L}$ has been set to a negative value, to suppress the leakage of low energy states in the left bulk region. There are several characteristics of the effective potential that deserve attention. Firstly, the spacing between successive bands is highly band-dependent within the BG region. In particular, the three lowest-energy bands are widely separated (with inter-band gaps on the order of $10 \mathrm{meV}$ ). These three bands have transverse profiles that are pinned near the BG gate, which explains why they sink dramatically within the $\mathrm{BG}$ region upon applying a relatively strong (positive, i.e. attractive) gate potential. For the higher energy bands (fourth band and above) the interband spacing reduces to around $2 \mathrm{meV}$ or less, which dramatically increases inter-band coupling. These higher-energy bands are less confined near the BG gate, as compared to the lowest three bands. Secondly, one notices the rapid variation of the effective potential near the edges of the BG region. This is caused, on the one hand, by the termination of the superconductor at the right edge of the $B G$ region and, on the other hand, by the sudden change of the gate potential from $V_{B G}=364 \mathrm{mV}$ to $V_{L}=-250 \mathrm{mV}$ (at the left edge) and $V_{F G}=175 \mathrm{mV}$ (at the right edge). This sharp variation of the effective potential causes several bands to cross zero energy and, very importantly, to switch order. This behavior, which is connected to a rapid evolution of the transverse profiles of the bands, is responsible for the large inter-band mixing 


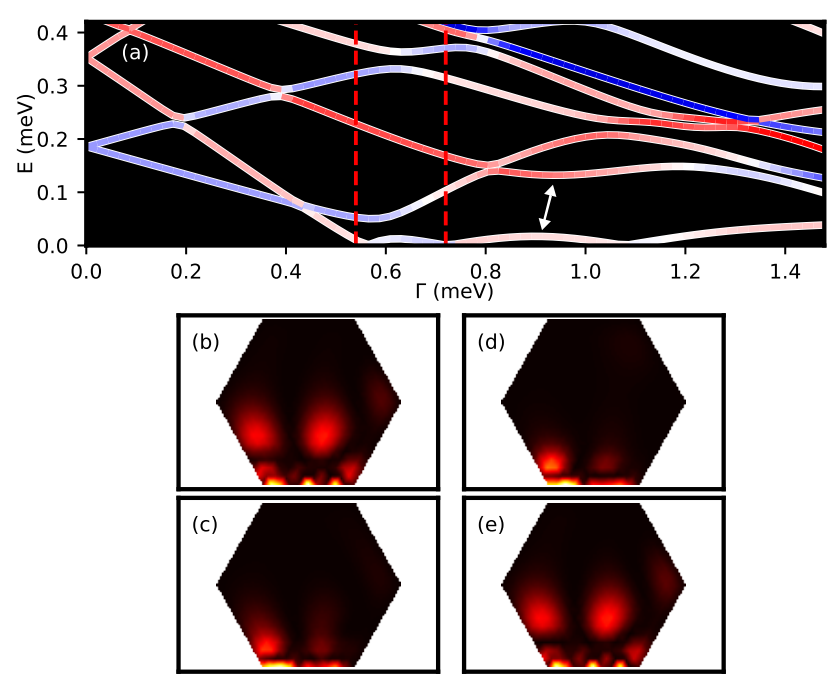

FIGURE 5.40: (a) Low-energy spectrum as a function of Zeeman field for a system with the same parameters as in Fig. 5.38, except $V_{B G}=520 \mathrm{mV}$. Panels (b)-(e) show the transverse profiles of the two lowest-energy states at $x=400 \mathrm{~nm}$ and Zeeman fields indicated by the red dashed lines in panel (a). Again, the wave functions swap character as $\Gamma$ increases, indicating anti-crossing behavior. A second anti-crossing is marked by the white arrow in panel (a).

that occurs within the transition regions.

The dependence of the low-energy spectrum on the Zeeman field for a system with parameters given in Fig. 5.38 is shown in Fig.5.39(a). Note that the spectrum is particle-hole symmetric, but only the positive energy sector is shown. The key feature is the low energy mode that remains near zero energy from about $\Gamma \approx 0.65 \mathrm{meV}$ to $\Gamma \approx 1.15 \mathrm{meV}$. This behavior, which is generated by the inter-band-coupling mechanism, is due to an anti-crossing between the two lowest-energy levels. To demonstrate that this is indeed the case, we calculate the transverse profiles of the two lowest energy states at a position corresponding to the middle of the BG region and Zeeman field values on the two sides of the anti-crossing, $\Gamma \approx 0.67 \mathrm{meV}$ and $\Gamma \approx 1.13 \mathrm{meV}$, respectively. Note that the transverse profile of a given state is determined by the band-components of that state, i.e. the molecular orbitals that provide the dominant contribution to the state. The results are shown in Fig.5.39, panels (b)-(e). The anti-crossing is revealed by the fact that the two levels swap their transverse character, i.e. the lowest energy state at $\Gamma \approx 0.67 \mathrm{meV}$ [panel (c)] becomes the second-lowest state at $\Gamma \approx 1.13 \mathrm{meV}$ [panel (d)] and vice versa [see panels (b) and (e)]. This mechanism is essentially the same as the one discussed in Sec. 5.2.1 in the context of the toy model. We remark again that in experiment, the signature of the low-energy mode will be broadened due to temperature, dissipation, and coupling to the continuum of states in the lead, which may result in the emergence of a relatively robust zero-bias conduction peak mimicking Majorana phenomenology. 
To illustrate the fact that the emergence of low-energy ABSs pinned to zero energy by the inter-band coupling mechanism is quite generic, we provide another example corresponding to a larger BG gate potential, $V_{B G}=$ $520 \mathrm{mV}$. The results are shown in Fig. 5.40. The spectrum shown in panel (a) contains a low-energy mode that remains near zero energy from $\Gamma \approx$ $0.53 \mathrm{meV}$ to $\Gamma \approx 1.2 \mathrm{meV}$ due to two anti-crossings. The first anti-crossing, which takes place from $\Gamma \approx 0.53 \mathrm{meV}$ to $\Gamma \approx 0.72 \mathrm{meV}$, as marked by the red dashed lines in panel (a), is revealed by the swapping of the transverse profiles between the lowest energy levels, as shown explicitly in panels (b)(e). Again, the transverse profiles correspond to the middle of the BG region and the Zeeman fields marked by the red dashed lines in panel (a). The second anti-crossing is indicated by the white arrow in Fig. 5.40(a) is revealed by a similar swapping of the transverse profile character (not shown). This spectrum also mimics the gap opening feature predicted to occur in Majorana hybrid systems at the TQPT, simultaneously with the emergence of the Majorana mode. However, this is not a bulk gap opening, but rather a level repulsion between the lowest energy (localized) modes, which one should generically expect to occur due to inter-band coupling. We also emphasize that the only difference between the system parameters corresponding to Figs. 5.39 and 5.40 is the voltage applied to the BG gate. In fact, varying $V_{B G}-$ which is exactly what is done in experiment - provides many instances of low-energy states pinned near zero energy due to inter-band coupling. None of these states are well-separated, topologically-protected MZMs.

Our final example of low-energy mode pinned near zero-energy by level repulsion is shown in Fig. 5.41(a). The system parameters are the same as in Figs. 5.39 and 5.40, except the BG voltage, which is $V_{B G}=380 \mathrm{mV}$. The lowenergy spectrum [panel (a)] is characterized by three anti-crossings that pin the lowest energy mode near zero energy. The anti-crossings are indicated by red, white, and green arrows, respectively. The most obvious anti-crossing - as revealed by a transverse profile analysis similar to those presented in Figs. 5.39 and 5.40 - is the second one (indicated by the white arrow). By contrast, the first anti-crossing in Fig. 5.41(a) - which involves the first and third energy levels - is not clearly revealed by the transverse wave function profiles. However, it become evident if one analyses the longitudinal profile of the wave functions and their band components. The longitudinal profiles of the first and third lowest-energy modes at the Zeeman fields indicated by the red dashed lines in panel (a) are shown in panels (b)-(e). The lowest energy state shown in panel (b) is mainly composed of a single band and, more importantly, has nearly all of its spectral weight within the BG region (i.e. between $x=300 \mathrm{~nm}$ and $x=500 \mathrm{~nm}$ ). By contrast, the third lowest energy state shown in panel (c) has significant weight in both the BG and left FG regions. Note also that this state has a larger admixture of bands, as compared to the state in panel (b), since it leaks through the BG-FG transition region, where the effective potentials vary rapidly (see Fig. 5.38) and inter-band coupling is large. For $\Gamma \approx 1.15 \mathrm{meV}-$ panels $(\mathrm{d})$ and (e) - the structures of the first and third states have (approximately) reversed, with the lowest energy state having significant weight in both the 


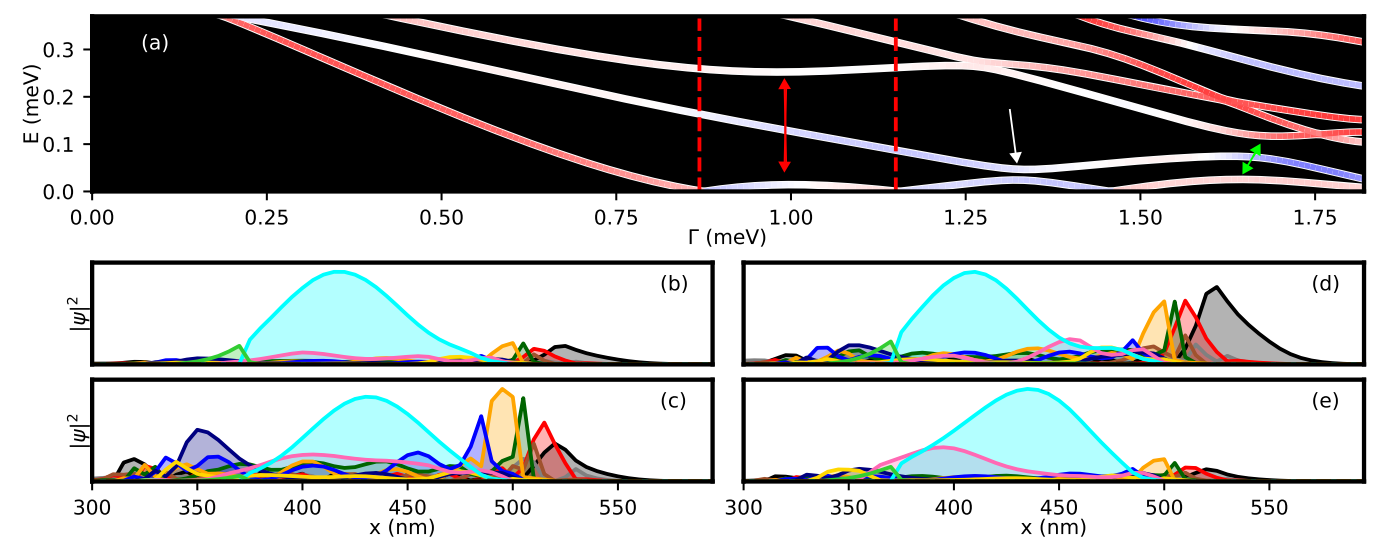

FIGURE 5.41: (a) Low-energy spectrum as a function of Zeeman field for a system with the same parameters as in Figs. 5.38-5.40, except $V_{B G}=380 \mathrm{mV}$. Three anti-crossings indicated by red, white, and green arrows, respectively, pin the lowest energy mode near zero energy over a significant range of Zeeman fields. Panels (b)-(e) show the longitudinal profiles and band composition of the first and third lowest-energy states at Zeeman fields indicated by the red dashed lines in panel (a). The color code for different band contributions is the same as in Fig. 5.38. Note that the state in (b) has little weight in the FG region (500 to $600 \mathrm{~nm}$ ), while the state in (c) has a significant weight in both BG and FG regions. The roles change in (d) and (e), indicating that a resonance between quantum dot states associated with the BG and FG regions is responsible for this anti-crossing.

BG and FG regions, while the third lowest state being localized within the $B G$ region. Consequently, this anti-crossing can be viewed as a resonance between two longitudinally confined, quantum dot-like, states associated with the BG and FG regions, respectively. The third anti-crossing [green arrow in panel (a)] involves a similar mechanism. We note that this coupling between two quantum dot states represent the real space counterpart of the inter-band coupling mechanism discussed in Sec. 5.2.1. In general, the interband coupling (including its real-space version - i.e. the inter-dot coupling) acts in conjunction with the partial separation mechanism for zero-energy pinning discussed extensively in the context of single-band models. This makes the pinning near zero-energy of (topologically-trivial) ABSs a rather generic occurrence in non-homogeneous SM-SC hybrid systems with multiband occupancy. The examples discussed in this section were obtained by changing a single experimentally-controllable parameter: the gate voltage $V_{B G}$. Varying other parameters, e.g., the gate voltage $V_{F G}$, generates similar low-energy states. The ubiquity of ABS modes pinned near zero energy by the inter-band coupling mechanism (possibly in conjunction with the partial separation mechanism) predicted by our 3D model calculations is consistent with the experimental observations on SM-SC devices with a structure similar to the setup considered here [122]. 
We conclude this section with a comment on the role of different interband couplings, as revealed in the 3D model calculations. While normal hopping, longitudinal spin-orbit coupling, and superconducting pairing all contribute to the inter-band coupling within our 3D model, the dominant inter-band contributions are due to longitudinal spin-orbit coupling and normal hopping. Note that the normal hopping and the longitudinal spin-orbit inter-band couplings are generated by a non-uniform electrostatic potential along the wire, while the superconducting inter-band pairing is (largely) independent of this non-uniformity. In the absence of a non-uniform potential we do not see non-Majorana states remaining near zero-energy, indicating that the superconducting inter-band pairing is not sufficient to pin states near zero-energy. We note that we have not assessed the role of transverse spinorbit coupling [132], as we did not include it in our model.

\subsubsection{Summary and Conclusions}

In this work we have studied the emergence of low-energy ABS modes pinned near zero energy in SM-SC hybrid systems with multi-subband occupancy. We have demonstrated that the pinning of these topologically-trivial modes is due to inter-band coupling, which occurs generically in inhomogeneous systems. Impressive zero-energy pinning can be generated by potential inhomogeneities with rather small characteristic lengths scales (of the order of the nanowire diameter, $100 \mathrm{~nm}$ ). We emphasize that this type of behavior cannot be obtained within single-band models with comparable parameters (e.g., effective mass, spin-orbit coupling, induced pairing, etc.). To get a better insight, we first illustrated the effects of the inter-band coupling mechanism using a simple multi-band toy model. We then confirmed this general picture within a realistic 3D model that incorporates the geometric and electrostatic details of actual devices studied in the laboratory [122]. The 3D calculation demonstrates that inter-band mixing occurs naturally in non-homogeneous multi-band systems due to the electrostatic-induced variation along the wire of the transverse profiles associated with different confinement-induced bands. Explicitly solving the 3D Schrödinger-Poisson problem allows us to study realistic device geometries without having to guess the strength of the interband coupling or the spacial profile of the effective electrostatic potential. We stress that within this approach there is no need to fine tune the "intrinsic" model parameters (e.g., effective mass, spin-orbit coupling, chemical potential, etc.) in order to pin ABSs near zero energy. Instead, one can simply tune experimentally-controllable parameters, such as, for example, the gate voltage $V_{B G}$, and identify the regimes consistent with the presence of (relatively robust) low-energy states. We emphasize that this is exactly the same protocol used in the experimental search for Majorana zero modes in SM-SC devices.

The main implications of this study for the ongoing efforts to realize MZMs 
in the laboratory are fourfold. (1) We have shown that the emergence of lowenergy ABSs pinned near zero energy (by the inter-band coupling mechanism) is rather generic in non-homogeneous systems with multi-band occupancy. For example, many low-energy ABSs with properties similar to those illustrated in Figs. 5.39-5.41 can be obtained by sweeping the BG gate voltage within a range on the order of 1V. (2) The level-repulsion generated by inter-band coupling can lead to a rather spectacular pinning of the lowestenergy mode near zero energy in systems (or regions) characterized by veryshort length scales (of the order of $100 \mathrm{~nm}$, the nanowire diameter). This demonstrates that the observation of near-zero-energy features characterized by low-amplitude energy splitting oscillations is not necessarily an indication of topological protection and well-separated MZMs. Moreover, this is not even an indication of partial separation and quasi-Majoranas. (3) We have shown (see Fig. 5.40) that a level repulsion between the lowest energy modes, which is generically induced by the inter-band coupling in the topologically trivial regime, can mimic the gap closing and re-opening feature (simultaneous with the emergence of a near zero energy mode) predicted to occur in Majorana hybrid systems at the TQPT. This possibility has to be taken into account in the interpretation of experiments that study such features in Majorana devices. (4) We identified and illustrated in Fig. 5.31 an experimental signature that could allow one to identify low-energy ABSs generated by the inter-band coupling mechanism. Specifically, any nearly zero-bias differential conductance feature that does not exhibit particle-hole symmetry should be attributed to the presence of (topologically-trivial) ABSs pinned near zeroenergy by level repulsion, rather than MZMs, quasi-Majoranas, or any other low-energy mode that involves (partially) separated Majorana bound states.

Based on the results of this study, it is clear that multi-band physics significantly complicates the interpretation of any experiment involving SM-SC hybrid structures, in particular charge tunneling measurements. An obvious way to reduce the importance of inter-band coupling is to reduce the diameter of the wire as much as possible, without inducing disorder. This will increase the energy spacing between bands and, therefore, reduce the importance of inter-band coupling. On the other hand, large diameter nanowires tend to approach the regime in which many confinement-induced sub-bands cluster near the chemical potential generating large inter-band couplings that control the low energy physics of the system. A second path toward reducing inter-band coupling is to use negative (rather than positive) voltages on the back gates. On the one hand, this reduces the number of occupied bands. On the other hand, it increases the inter-band energy spacing for two reasons; (1) the lowest-energy confinement-induced conduction bands tend to have larger energy spacing due to a lower effective mass (as compared to the high-energy bands) and (2) the negative voltage pushes the wave functions towards the SM-SC interface, increasing the confinement and, consequently, the inter-band spacing. Note that the negative gate voltage can also reduce the inter-band superconducting pairing. Finally, our findings highlight the importance of inhomogeneous effective potentials in generating low-energy 
ABSs. While inhomogeneous potentials have been previously shown to induce low energy topologically-trivial ABSs, this study reveals that in multiband systems the collapse and pinning of ABSs to zero energy can take place even when the characteristic length scale of the potential non-uniformity is on the order of $100 \mathrm{~nm}$. We stress that the electrostatic gradients between different regions of the wire need to be as sharp as possible to reduce inter-band coupling. This problem is the multi-band generalization of the sharp versus smooth confinement discussed extensively within the context of single-band toy models. 


\title{
Topological superconductivity in a two-dimensional electron gas proximity coupled to periodically patterned superconductors
}

\author{
"If you want to improve, be content to be thought foolish and stupid." \\ - Epictetus
}

In previous chapters, we have studied Majorana device physics of semiconductor-superconductor (SM-SC) hybrid nanowires. While SM-SC nanowires have been the primary experimental platform [47-50, 87, 89, 93, 97, 122, 153, 154, 156-158, 187] for studying topological superconductivity and the corresponding Majorana physics in SM-SC nanostructures, planar SM-SC structures have attracted both experimental [90, 155, 188-196] and theoretical attention [179, 197-207] in recent years as an alternative platform. These proposals involve a two-dimensional electron gas (2DEG) with strong spin-orbit coupling and large g-factor (InAs/InSb) in proximity to a SC (often $\mathrm{Al}$ ). A quasi-1D channel is formed either by having a narrow quasi-1D SC strip [90, 155] or a Josephson junction geometry [191, 193, 196, 198, 199] with an unproximitized junction region between two proximitized regions.

In this chapter, we present two designs within the planar SM-SC paradigm for the reliable and consistent achievement of topological superconductivity and Majorana zero modes (MZM). The main feature in both designs is the incorporation of periodic modulations of the SC in proximity to the SM. As we show below, periodic structures provide three main advantages when compared to uniform systems. (1) The total area of the parameter space associated with the topological superconducting phase increases. As pointed out in previous studies $[62,128,208,209]$, this is related to the formation of minibands in the presence of a periodic potential. (2) The topological gap characterizing the topological superconducting phase is typically larger than the corresponding value in a uniform system and, generally, increases with increasing chemical potential. In essence, this occurs as a result of an increased effective spin-orbit coupling within the higher energy minibands. (3) The topological state shows increased robustness against disorder. This is an effect of the increased topological gap combined with the highly oscillatory nature of the states associated with high-energy minibands. The importance of 
this third advantage is obvious in light of our discussion in chapter 5 that disorder is the main obstacle to overcome for the reliable achievement of MZMs in SM-SC nanostructures. The first design [179], which we have named the Majorana waveguide, is alteration of the quasi-1D SC strip systems [90,155] in which the width of the SC strip is periodic modulated and will be studied in Sec. 6.1. The second design [JuncitonPaper] is an alteration of the Majorana Josephson junction design in which the width of the unproximitized junction region is periodically modulated and will be studied in Sec. 6.2.

\subsection{Enhanced topological protection in planar quasi- one-dimensional channels with periodically-modulated width}

In this section, we analytically and numerically study the emergence of MZMs within periodic structures. Much of this section is adapted from Ref. [179], where we first proposed the Majorana waveguide device design.

Before we introduce the actual Majorana waveguide design in the planar 2DEG system, we first consider a purely 1D system based on the minimal model of a Majorana nanowire [8,9], with the addition of a periodic potential in Sec. 6.1.1. Starting from a suitable low-energy basis, we analytically derive effective parameters characterizing each set of periodicity-induced minibands. These parameters include renormalized effective masses, spin-orbit coupling coefficients, Zeeman splittings, and Majorana localization lengths. The derived analytic expressions provide valuable insight into the underlying physics and suggest possible avenues for optimizing the topological properties of the system. Numerical calculations based on an equivalent 1D tight-binding model show excellent agreement with the analytic results and highlight the importance of creating a sufficiently strong periodic potential, without which the periodic structure loses its advantages over the uniform system. The formation of (topologically trivial) partially-separated Andreev bound states (ps-ABSs) $[67,94,108]$ in the presence of a soft confining potential is also explored. We find that the confinement must be softer (i.e. have a smaller slope) within the periodic system, as compared to a uniform one, to ensure the ps-ABS collapse to zero-energy. Thus, the presence of a periodic potential reduces the parameter region associated with the presence of ps-ABSs, which may provide a significant advantage in the search for topologically protected MBSs. In addition, we investigate the effects of potential disorder and find that, typically, the topological phase becomes more robust in the presence of an additional periodic potential. Moreover, in a superlattice the nonlocal (edge-to-edge) correlations indicative of well-separated MBSs localized at the ends of the system are found to become less sensitive to the presence of disorder.

While a periodic potential can, in principle, offer the advantages mentioned above, engineering a strong-enough potential represents a nontrivial task. Naively, one could try to generate such a potential using periodic arrays 
of gates applied to "standard" semiconductor wire-superconductor devices similar to those used in recent Majorana experiments [47, 81, 83, 84, 86, 210]. We find that, unfortunately, the effective periodic potential generated within such a nanowire setup is too weak for the superlattice scheme to provide any notable advantage over the uniform system. For this reason, we introduce and study in Sec. 6.1 .2 a possible alternative which we will refer to as a modulated channel device or a Majorana waveguide. The proposed device (see Fig. 6.10) consists of a two-dimensional electron gas (2DEG) hosted by a semiconductor heterostructure and proximity-coupled to a lithographycally defined superconductor that generates a quasi-1D channel with periodically modulated width. While a periodic potential is not directly applied, the periodic structure of the device results in the formation of minibands similar to those induced by an actual periodic potential, due to scattering at the interfaces between regions of differing width [211]. For not-too-large values of the chemical potential, the topological properties of the system, including the stability of the MBSs, exhibit all the advantageous features identified in the 1D "ideal" periodic model. In addition, we show that the topological phase diagram is not dramatically affected by the details associated with the screening by the superconductor of the confinement potential that defines the modulated channel. Specifically, we consider a confining potential that varies near the edges of the region covered by the superconductor over a finite length scale $\chi$ and show that the phase diagram depends weakly on $\chi$. Furthermore, we argue that $\chi$ can be quite large, which implies that the chemical potential of the electron gas underneath the superconductor is tunable (to a certain degree), providing an important knob for accessing the topological superconducting phase. Taking into account all these findings, as well as the natural ability of the 2DEG system to enable the construction of complex structures, we conclude that the Majorana waveguide and, more generally, patterned 2D structures represent a promising versatile platform for realizing robust Majorana bound states.

\subsubsection{One-dimensional model of periodic Majorana nanowires}

In this subsection, we study analytically and numerically a simple, onedimensional model of a Majorana wire in the presence of a periodic effective potential. We show that topological superconductivity and Majorana zero modes emerge at low values of the applied Zeeman field (on the order of the induced pairing potential) whenever the chemical potential is tuned near the bottom/top of a potential-induced pair of minibands. We determine explicit analytical expressions for the renormalized miniband parameters (e.g., effective mass and spin-orbit coupling) and the Majorana localization length in periodic nanowires. The validity of these analytic expressions is verified numerically. We also investigate the effect of the periodic potential on the topological phase diagram, the emergence of topologically trivial Andreev bound states in systems with soft confinement, and the robustness of Majorana bound states against disorder. 


\section{Renormalization of miniband parameters}

We begin by considering a minimal model of the Majorana nanowire [8, 9] in the presence of a periodic potential. More specifically, we have a onedimensional system (i.e., a Majorana wire) with Rashba spin-orbit coupling, (induced) superconductivity, magnetic field applied parralel to the wire, and a periodic (effective) potential. The system is modeled by the Bogliubov-de Gennes (BdG) Hamiltonian

$$
H_{B d G}=\left(\begin{array}{cc}
H_{o} & -i \Delta \sigma_{y} \\
i \Delta^{*} \sigma_{y} & -H_{o}^{*}
\end{array}\right)
$$

The diagonal (normal wire) component is

$$
H_{o}=\frac{\hbar^{2} \hat{k}^{2}}{2 m^{*}}-\mu+V(x)+\alpha \hat{k} \sigma_{z}+\Gamma \sigma_{x}
$$

where $m^{*}$ is the effective mass, $\mu$ is the chemical potential, $\alpha$ is the Rashba spin-orbit coefficient, $\Gamma$ is the (half) Zeeman splitting, $\Delta$ is the (induced) superconducting pairing, and $\hat{k}=-i \partial_{x}$ is the momentum operator. Note that $\sigma_{i}$ with $i=x, y, z$ are the Pauli matrices acting within the spin space. The potential $V(x)$ is periodic with the period $\ell$, so that $V(x+\ell)=V(x)$. A specific example of a periodic potential used in the calculations is shown in Fig. 6.1(a). We first study the quantum problem described by the BdG Hamiltonian with periodic boundary conditions, so that $k$ represents a good quantum number. In the absence of the magnetic field and periodic potential, the normal Hamiltonian eigenstates are simply given by the spin polarized plane waves

$$
\langle x \mid k, n, \sigma\rangle=\frac{\chi_{\sigma}}{\sqrt{L}} e^{i\left(k+G_{n}\right) x},
$$

where $L$ is the total length of the system, $\chi_{\sigma}=\left(\delta_{\sigma, \uparrow}, \delta_{\sigma, \downarrow}\right)^{T}$ is the spinor corresponding to spin projection $\sigma, G_{n}=2 \pi n / \ell$ is a reciprocal (super)lattice vector, and $\mathrm{k}$ is restricted to the first Brillioun zone, $-\pi / \ell<k \leq \pi / \ell$. The label $n$ is the zone number of the plane wave state corresponding to the (superlattice) Brillioun zone to which the state belongs. The energies of the eigenstates (6.3) are given by

$$
E_{k, n, \sigma}=\frac{\hbar^{2}}{2 m^{*}}\left(k+G_{n}\right)^{2}+\alpha\left(k+G_{n}\right)\left(\sigma_{z}\right)_{\sigma \sigma}-\mu .
$$

To understand the effects of the periodic potential and magnetic field, it is convenient to Fourier transform $V(x)$ and to calculate the matrix elements of the pertubations (i.e., periodic potential and magnetic field) in the plane 

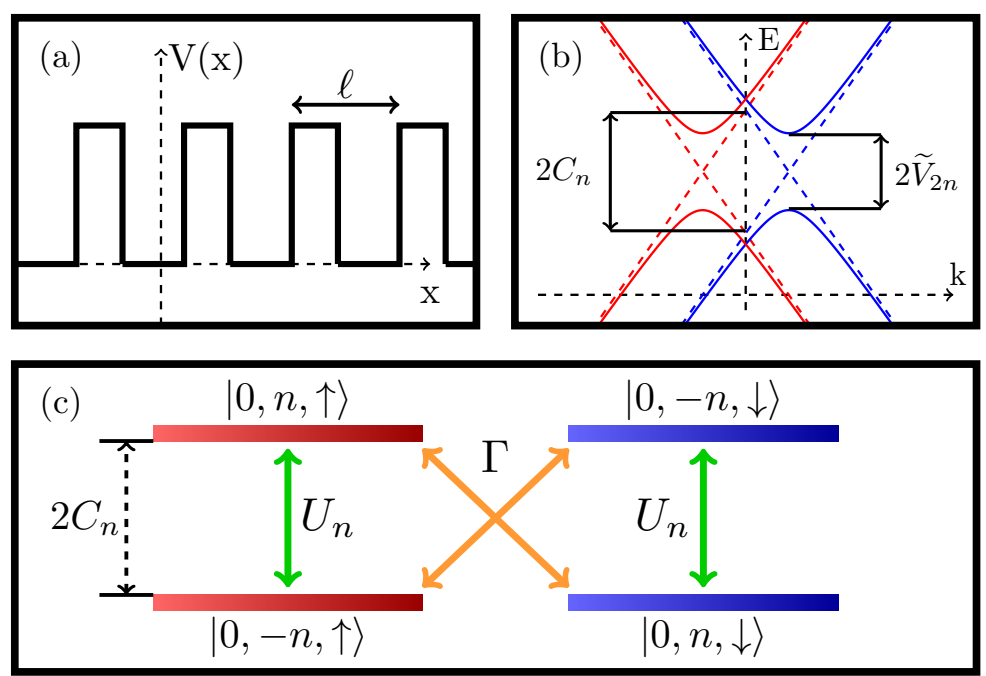

FIGURE 6.1: (a) Rectangular 1D potential profile with period $\ell$. (b) Spectrum of the effective Hamiltonian (6.8) near the zone center for zero magnetic field $(\Gamma=0)$ in the absence (dashed lines) and presence (solid lines) of a periodic potential. The red (blue) lines correspond to spin $\sigma=\uparrow(\downarrow)$. (c) Energy level diagram showing the couplings of the zone $n$ basis states with $k=0$ induced by the periodic potential and the applied magnetic field The color code is the same as in (b).

wave basis given by Eq. (6.3). We have

$$
\begin{aligned}
& V(x)=\sum_{n=-\infty}^{\infty} \widetilde{V}_{n} e^{-i G_{n} x}, \\
& \left\langle k, m, \sigma|V(x)| k, n, \sigma^{\prime}\right\rangle=\widetilde{V}_{n-m} \delta_{\sigma, \sigma^{\prime}} \\
& \left\langle k, m, \sigma\left|\Gamma \sigma_{x}\right| k, n, \sigma^{\prime}\right\rangle=\Gamma \delta_{m, n}\left(\sigma_{x}\right)_{\sigma \sigma^{\prime}}
\end{aligned}
$$

Provided the period $\ell$ is sufficiently small, the energy difference between different minibands, i.e. the difference between $E_{k, m, \sigma}$ and $E_{k, n, \sigma^{\prime}}$ with $|m| \neq$ $|n|$, is much larger than the characteristic energy scales associated with the periodic potential and the magnetic field. Consequently, the basic physics can be understood by treating $V(x)$ and $\Gamma$ as perturbations acting within the subspace of basis states having the same absolute value of the zone number, i.e. $\{|k, n, \uparrow\rangle,|k,-n, \uparrow\rangle,|k, n, \downarrow\rangle,|k,-n, \downarrow\rangle\}$. For $n \neq 0$, the effective normal state Hamiltonian acting within this subspace is

$$
\begin{aligned}
H_{n}(k)= & \left(\begin{array}{cccc}
C_{n}+v_{+} k & U_{n}^{*} & \Gamma & 0 \\
U_{n} & -C_{n}-v_{-} k & 0 & \Gamma \\
\Gamma & 0 & -C_{n}+v_{-} k & U_{n}^{*} \\
0 & \Gamma & U_{n} & C_{n}-v_{+} k
\end{array}\right) \\
& -\mu_{n}+\frac{\hbar^{2} k^{2}}{2 m^{*}},
\end{aligned}
$$


where the effective parameters are

$$
\begin{aligned}
\mu_{n} & =\mu-\frac{2 n^{2} \pi^{2} \hbar^{2}}{m^{*} \ell^{2}}-\widetilde{V}_{0}, \\
C_{n} & =\alpha \frac{2 \pi n}{\ell} \\
v_{n \pm} & =n \frac{2 \pi \hbar^{2}}{m^{*} \ell} \pm \alpha, \\
U_{n} & =V_{2 n} .
\end{aligned}
$$

Notice that for $\Gamma=0$ (i.e., no magnetic field), spin is a good quantum number [i.e., the red and blue bands in Fig. 6.1 (b) and (c) do not couple] and one can exactly diagonalize the Hamiltonian in Eq. (6.8). The energies of the corresponding eigenstates are

$$
\begin{aligned}
\varepsilon_{ \pm, \sigma}^{(n)}(k)= & \pm \sqrt{\left(C_{n}+\left(\sigma_{z}\right)_{\sigma \sigma} \bar{v}_{n} k\right)^{2}+\left|U_{n}\right|^{2}} \\
& +\left(\sigma_{z}\right)_{\sigma \sigma} \alpha k-\mu_{n}+\frac{\hbar^{2} k^{2}}{2 m^{*}}
\end{aligned}
$$

with $\bar{v}_{n}=\frac{1}{2}\left(v_{n+}+v_{n-}\right)$. The spectrum of a typical miniband in the vicinity of $k=0$ is shown in Fig. 6.1(b). When there is no periodic potential $\left(U_{n}=0\right.$, dashed lines), the spectrum consists of two Dirac-like cones with intersections shifted away from $k=0$ due to the spin-orbit coupling. This gives rise to an energy splitting $2 C_{n}$ at $k=0$, i.e. a splitting proportional to the Rashba coefficient $(\alpha)$ and the miniband index $(n)$. Applying a periodic potential opens a gap of size $2\left|U_{n}\right|$ at the nodes of the Dirac cones. Expanding the eigenenergies $\varepsilon_{ \pm, \sigma}^{(n)}$ in the wave vector near $k=0$, we have

$$
\varepsilon_{ \pm, \sigma}^{(n)} \approx \frac{\hbar^{2} k^{2}}{2 m_{n \pm}^{*}}+\left(\sigma_{z}\right)_{\sigma \sigma} \alpha_{n \pm} k-\widetilde{\mu}_{n \pm}
$$

where the effective spin-orbit coupling $\alpha_{n \pm}$, mass $m_{n \pm}^{*}$, and chemical potential $\tilde{\mu}_{n \pm}$ are re-normalized by the periodic potential,

$$
\begin{aligned}
\alpha_{n \pm} & =\left[1 \pm \frac{4 \pi^{2} n^{2}}{\gamma_{n}}\left(\frac{\hbar^{2}}{\left|U_{n}\right| m^{*} \ell^{2}}\right)\right] \alpha, \\
\frac{1}{m_{n \pm}^{*}} & =\left[1 \pm \frac{4 \pi^{2} \hbar^{2} n^{2}}{m^{*} \ell^{2}\left|U_{n}\right| \gamma_{n}}\left(1 \mp\left|\frac{C_{n}}{U_{n} \gamma_{n}}\right|^{2}\right)\right] \frac{1}{m^{*}}, \\
\widetilde{\mu}_{n \pm} & =\mu_{n} \mp \sqrt{C_{n}^{2}+\left|U_{n}\right|^{2}},
\end{aligned}
$$

with $\gamma_{n}=\sqrt{1+\left|C_{n} / U_{n}\right|^{2}}$. Remarkably, both the upper and lower pairs of minibands mimic the spectrum of a uniform Rashba nanowire, but having renormalized effective masses and spin-orbit parameters that depend on the 
characteristics (i.e., amplitude and period) of the periodic potential. This suggests the possibility of optimizing the effective parameters of the nanowire by engineering the periodic potential. For example, Eq. (6.15) shows that, for moderate values of $n$, the renormalized spin-orbit coefficient can be significantly larger than the corresponding bare parameter $\alpha$. Combining Eqs. (6.15) and (6.16), we obtain the effective spin-orbit energy

$$
\widetilde{E}_{S O, n}= \pm \frac{m^{*} \alpha^{2}}{2 \hbar^{2}}+\frac{2 \pi^{2} n^{2}}{\gamma_{n}}\left(\frac{\alpha^{2}}{\left|U_{n}\right| \ell^{2}}\right)
$$

where the first term is the bare spin-orbit energy of the original Hamiltonian (6.2) with no periodic potential, i.e. with $V(x)=0$, and the second term is a potential-induced contribution. Note that this additional contribution increases with the miniband index $(n)$ and can become dominant, as we explicitly show below.

Next, we apply a magnetic field, $\Gamma \neq 0$, which removes the spin-degeneracy at $k=0$. For convenience we incorporate the effects of the magnetic field by writing the Hamiltonian (6.8) in the basis of eigenstates corresponding to $\Gamma=0$. Explicitly, we have

$$
\widetilde{H}_{n}(k)=\left(\begin{array}{cccc}
\varepsilon_{+, \uparrow}^{(n)} & \widetilde{\Gamma} & \Omega & 0 \\
\widetilde{\Gamma} & \varepsilon_{+, \downarrow}^{(n)} & 0 & -\Omega \\
\Omega & 0 & \varepsilon_{-\downarrow \downarrow}^{(n)} & \widetilde{\Gamma} \\
0 & -\Omega & \widetilde{\Gamma} & \varepsilon_{-, \uparrow}^{(n)}
\end{array}\right),
$$

where $\widetilde{\Gamma}(k)$ and $\Omega(k)$ are intra- and inter-miniband pair coupling terms, respectively. The exact expressions for $\widetilde{\Gamma}(k)$ and $\Omega(k)$ are complicated. Focusing on $k=0$ and expanding $\widetilde{\Gamma}$ and $\Omega$ in a power series with respect to the parameter $\left(C_{n} / U_{n}\right)$, we have

$$
\begin{array}{r}
\widetilde{\Gamma}(k=0)=\left(1-\frac{1}{2}\left(\frac{C_{n}}{U_{n}}\right)^{2}+\frac{3}{8}\left(\frac{C_{n}}{U_{n}}\right)^{4}+\mathcal{O}\left(\frac{C_{n}}{U_{n}}\right)^{6}\right) \Gamma, \\
\Omega(k=0)=\left(\left(\frac{C_{n}}{U_{n}}\right)-\frac{1}{2}\left(\frac{C_{n}}{U_{n}}\right)^{3}+\mathcal{O}\left(\frac{C_{n}}{U_{n}}\right)^{5}\right) \Gamma,
\end{array}
$$

where, without loss of generality, we assumed $U_{n} \in \mathbb{R}$. Note that the $( \pm)$ pairs of minibands become decoupled if $\Omega \rightarrow 0$, i.e. in the limit of strong periodic potentials, $\left(C_{n} /\left|U_{n}\right|\right) \rightarrow 0$. As long as the energy separation between the miniband pairs dominates over the Zeeman splitting, i.e. $\sqrt{C_{n}^{2}+\left|U_{n}\right|^{2}} \gg$ $\Gamma / 2$, we can treat $\widetilde{\Gamma}$ as the renormalized Zeeman splitting. From Eq. (6.20) we see that $\widetilde{\Gamma}=\Gamma$ in the limit $\left(C_{n} /\left|U_{n}\right|\right) \rightarrow 0$, but, for finite $\left(C_{n} /\left|U_{n}\right|\right)$, the effective Zeeman splitting is renormalized to smaller values. To understand the physical mechanism responsible for this behavior, we refer to Fig. 6.1(c). Before the application of the periodic potential and magnetic field, the high energy states are $|0, n, \uparrow\rangle$ and $|0,-n, \downarrow\rangle$. These two states cannot couple directly 
because $V(x)$ preserves spin and $\Gamma \sigma_{x}$ preserves the zone number, $n$, therefore the mixing between these states must rely on an indirect path involving both $\Gamma$ and $\left|U_{n}\right|$. The periodic potential, $\left|U_{n}\right|$, couples the (same-spin) upper and lower energy states, but has to overcome an energy gap $2 C_{n}$. Hence, we expect a large effective Zeeman splitting $\widetilde{\Gamma}$ only if $\left(C_{n} /\left|U_{n}\right|\right) \lesssim 1$. Indeed, in the opposite limit, $\left(C_{n} /\left|U_{n}\right|\right) \gg 1$, we find a reduced effective Zeeman splitting, $\widetilde{\Gamma} \approx\left(\left|U_{n}\right| / C_{n}\right) \Gamma$. Since we are interested in realizing Majorana physics, which requires a Zeeman splitting $\widetilde{\Gamma}>\Delta$, we focus of the strong periodic potential regime, $\left(C_{n}\left|U_{n}\right|\right) \lesssim 1$.

To investigate the emergence of topological superconductivity and Majorana bound states, we consider a BdG Hamiltonian with uniform induced pairing potential $\Delta$ and a normal component described by Eq. (6.19). Since a large effective Zeeman splitting is needed for the emergence of Majorana bound states, we focus on the regime $\left(C_{n} /\left|U_{n}\right|\right) \lesssim 1$, which implies $\widetilde{\Gamma} \gtrsim \Omega$. This allows us to use quasi-degenerate perturbation theory [113] to decouple the higher energy miniband pair from the lower energy pair. Note that we implicitly incorporate the effects of the lower energy minibands on the higher energy pair, but a similar analysis can be done by explicitly keeping the lower energy pair in the effective model. To second order in $\Omega$, the effective Hamiltonian describing the higher energy minibands has the form

$$
\begin{aligned}
H_{e f f}^{(n)}= & {\left[\frac{\hbar^{2} k^{2}}{2 m_{n+}^{*}}-\left(\widetilde{\mu}_{n+}-\frac{\Omega^{2}}{2 \sqrt{C_{n}^{2}+\left|U_{n}\right|^{2}}}\right)+\widetilde{\Gamma} \sigma_{x}\right] \tau_{z} } \\
& +\alpha_{n+} k \sigma_{z}+\Delta \sigma_{y} \tau_{y} .
\end{aligned}
$$

This Hamiltonian corresponds to a simple, uniform Majorana nanowire model $[8,9]$ having effective parameters that are renormalized by the periodic potential [see Eqs. (6.15-6.17), (6.20), and (6.21)]. In the regime $\left(C_{n} /\left|U_{n}\right|\right) \ll 1$, the system undergoes a topological phase transition at a (bare) critical Zeeman field

$$
\Gamma_{c}^{2} \approx\left(\tilde{\mu}_{n+}^{2}+|\Delta|^{2}\right)\left[1+\left(1-\frac{\widetilde{\mu}_{n+}}{\left|U_{n}\right|}\right)\left(\frac{C_{n}}{\left|U_{n}\right|}\right)^{2}\right] .
$$

Note that in the limit $\left(C_{n} /\left|U_{n}\right|\right) \rightarrow 0$ we recover the "standard" expression of the critical field for a uniform Majorana nanowire $[8,9]$. When $\Gamma>\Gamma_{c}$ the system is in a topological superconducting phase, with two zero-energy Majorana bound states localized at the edges.

To illustrate the emergence of Majorana bound states in a high energy miniband, we solve numerically the BdG problem described by Eq. (6.1) for a finite system using the finite difference method. For simplicity and clarity we first consider an idealized periodic potential composed of a single harmonic of the form $V(x)=2 U \cos (\pi x / \ell)$, with $\ell=25 \mathrm{~nm}$, so that $\widetilde{V}_{n}=U \delta_{n, \pm 1}$. Other system parameters are $m^{*}=0.026 m_{0}, \alpha=20 \mathrm{meV} \cdot \mathrm{nm}$, $\Delta=0.5 \mathrm{meV}, L=5.1 \mu \mathrm{m}, C_{1} / U_{1}=0.5$. The chemical potential is $\mu=103.5$ meV, which corresponds to $\widetilde{\mu}_{1+}=0$, i.e. the chemical potential is set to the 

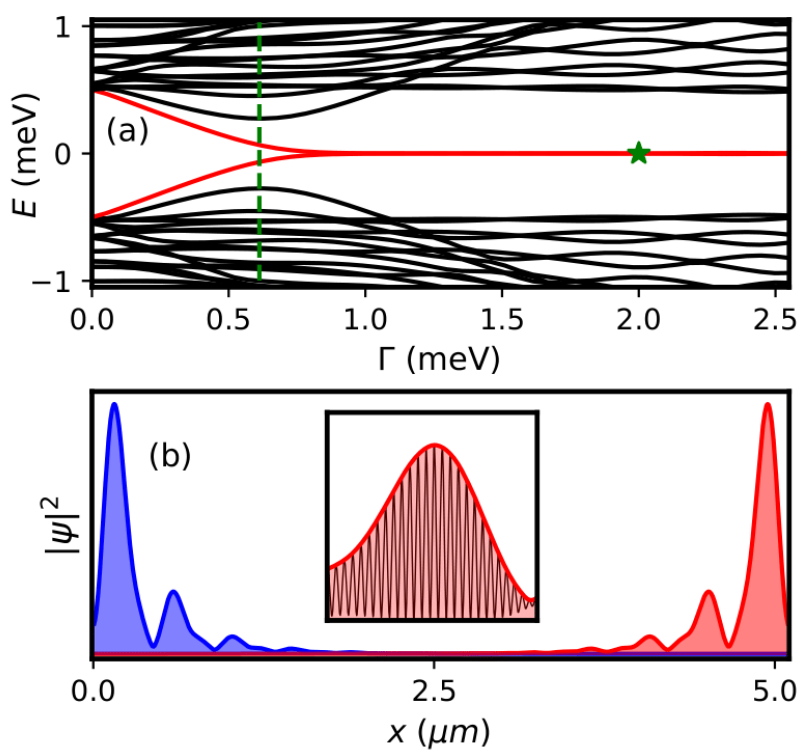

FIGURE 6.2: (a) Low-energy spectrum of a finite system described by Eq. (6.1) as a function of the applied (bare) Zeeman splitting $\Gamma$. The system parameters are $m^{*}=0.026 m_{0}$, $\alpha=20 \mathrm{meV} \cdot \mathrm{nm}, \Delta=0.5 \mathrm{meV}, \ell=25 \mathrm{~nm}, L=5.1 \mu \mathrm{m}$, and $C_{1} / U_{1}=0.5$. The chemical potential is $\mu=103.5 \mathrm{meV}$, corresponding to the bottom of the $|n|=1$ higher energy miniband pair, i.e. $\widetilde{\mu}_{1+}=0$. The green dashed line shows the critical Zeeman splitting predicted by Eq. (6.23), which coincides with the minimum of the bulk gap, as expected. (b) Envelopes of the MBS wave functions corresponding to the green star in (a). The inset shows a zoom-in of the second maximum of the right Majorana. Note the highly oscillatory nature of the Majorana wave function (black lines).

bottom of the $|n|=1$ higher energy miniband pair. The results are shown in Fig. 6.2. Upon applying a sufficiently high Zeeman field, a pair of Majorana modes emerges at zero energy [see Fig. 6.2(a)]. Note that Majorana bound states could not emerge at such a high chemical potential $(\mu=103.5$ $\mathrm{meV}$ ) in a uniform system, as the required Zeeman field would completely destroy superconductivity in the parent superconductor. Rather, the periodic potential has expanded the parameter space consistent with topological superconductivity, as pointed out in Refs. [62, 128, 208]. The green dashed line in Fig. 6.2(a) shows the critical Zeeman splitting predicted by the analytical expression in Eq. (6.23). Note that this value coincides with the minimum of the bulk gap, which occurs at $\Gamma>\Delta$ due the additional $\left(C_{n} /\left|U_{n}\right|\right)$ contribution in Eq. (6.23). The wave functions of the two MZMs corresponding to $\Gamma=2 \mathrm{meV}$, which are obtained using the Majorana representation [67] of the lowest energy states, are shown in Fig. 6.2(b). Note that the broad blue and red curves, which represent the modulus squared of the envelope functions corresponding to the two MZMs localized at the ends of the wire, are very similar to Majorana wave functions emerging in a uniform system. However, 
a zoom-in of the second maximum of the right (red) Majorana mode [see the inset of Fig. 6.2(b)] reveals the highly oscillatory nature of the wave function, which oscillates with a wavelength $\ell$. This rapidly oscillating nature, which is indicative of large $k$ components, represents the source of the enhanced (renormalized) spin-orbit coupling in Eq. (6.15).

\section{Majorana localization length}

A key parameter that characterizes the Majorana bound states is the localization length $\xi$ representing the characteristic length scale of the MBS. This localization length controls, among other things, the amplitude of the energy splitting oscillations due to the partial overlap of the MBSs localized at the opposite ends of a finite wire. A natural question is how does the localization length of a periodic Majorana structure compare with the localization length of the corresponding uniform system? On the one hand, the reduced effective mass [see Eq. (6.16)] favors delocalization, while, on the other hand, the increased spin-orbit coupling [see Eq. (6.15)] enhances the Majorana localization. To determine the relative role of these effects, we study (analytically and numerically) the solutions of the effective Hamiltonian (6.22). Details can be found in Appendix C. We find the the localization length of the MBSs associated with the higher energy miniband pair $(n+)$ has the form

$$
\xi_{n+} \sim \ell_{S O}\left(\frac{\Gamma}{\Delta}\right) \sqrt{1+\frac{4 \widetilde{\mu}_{n+} \widetilde{E}_{S O, n+}}{\Gamma^{2}}+\frac{4 \widetilde{E}_{S O, n+}^{2}}{\Gamma^{2}}},
$$

where $\ell_{S O}=\hbar^{2} /\left(m^{*} \alpha\right)$ is the bare spin-orbit length, while $\widetilde{\mu}_{n}+$ and $\widetilde{E}_{S O, n+}$ are the renormalized chemical potential (6.17) and spin-orbit energy (6.18), respectively. Note that a similar calculation can be done for $\xi_{n-}$. The first two factors in Eq. (6.24) (i.e., those outside the square root sign) are bare parameters entering the original BdG Hamiltonian (6.1). The last factor (i.e., the square root) contains renormalized parameters and leads to a moderate increase of the localization length. We verify Eq. (6.24) numerically by fitting the envelope of the Majorana wave function to an exponential, $|\psi|^{2} \propto e^{-2 x / \xi}$. The results are shown in Fig. 6.3 as a function of $\left(C_{1} / U_{1}\right)$ for fixed Zeeman field, $\Gamma=2 \mathrm{meV}$, and $\widetilde{\mu}_{1+}=0$. Note the excellent agreement between the analytical and numerical results for $\left(C_{1} / U_{1}\right) \lesssim 1.5$, which corresponds to the strong periodic potential regime. Above this threshold, the localization length increases strongly as the critical Zeeman field $\Gamma_{c}$ approaches $\Gamma=2 \mathrm{meV}$. Note that the dashed line shows the localization length of a uniform wire $[V(x)=0, \mu=0]$ with the same bare parameters as the periodic system. In the limit $C_{1} / U_{1} \rightarrow 0$, the periodic and uniform systems have the same localization length, $\xi \approx 600 \mathrm{~nm}$, while the localization length of the periodic system increases with increasing $C_{1} / U_{1}$. At $C_{1} / U_{1}=1, \xi$ is roughly double for the periodic system as compared to the uniform wire. However, this is a rather moderate increase, particularly considering that the renormalized effective mass $m_{1+}^{*}=0.0016 m_{0}$ is significantly smaller than the bare effective mass, $m^{*}=0.026 m_{0}$. Finally, we note that, while the results shown 


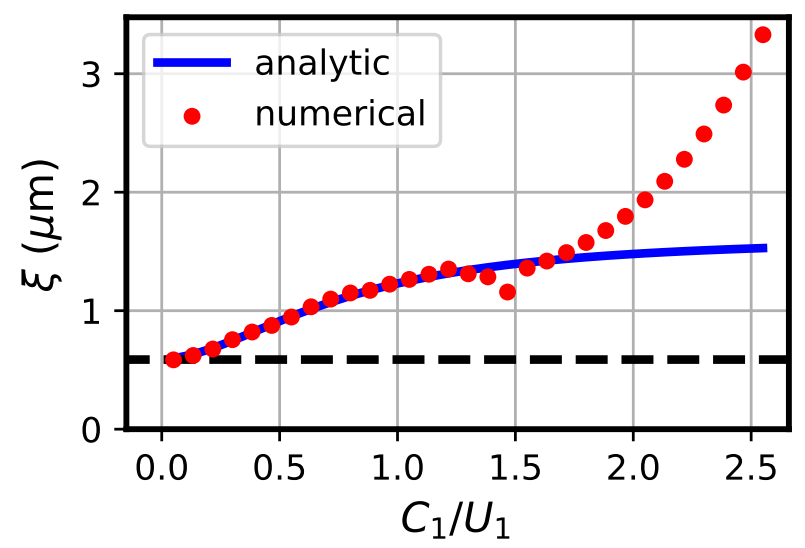

FIGURE 6.3: Localization length $\xi_{1+}$ as a function of $C_{1} / U_{1}$ for a system with $\Gamma=2 \mathrm{meV}$ and $\widetilde{\mu}_{1+}=0$. The blue line shows the analytical result given by Eq. (6.24), while the red dots corresponds to the numerical solution extracted by fitting the envelope of the Majorana wavefuction, $|\psi| \propto e^{-x / \xi_{1+}}$. The system parameters are the same as in Fig. 6.2, except $L=20 \mu \mathrm{m}$ and $C_{1} / U_{1}$ varies. For comparison, the localization length of a uniform wire with the same bare parameters $\left(m^{*}, \alpha\right.$, and $\left.\Delta\right)$ and $\mu=0$ is shown as a black dashed line.

in Figs. 6.2 and 6.3 are based on an idealized periodic potential of the form $V(x)=2 U \cos (\pi x / \ell)$, the basic physics discussed above holds for generic periodic potentials.

\section{Topological phase diagram in systems with rectangular periodic potential}

To investigate the effect of the periodic potential on the topological phase diagram, we consider a system with rectangular periodic potential, as shown in Fig. 6.1(a). Explicitly, we have

$$
V(x)= \begin{cases}V_{o}, & 0 \leq x \leq L_{b a r} \\ 0, & L_{b a r}<x<\ell\end{cases}
$$

where $V_{o}$ and $L_{b a r}$ are the height and length of each potential barrier, respectively, and $V(x+\ell)=V(x)$. The Fourier components of the potential are

$$
\widetilde{V}_{n}=\frac{-i V_{o}}{2 \pi n}\left[\exp \left(\frac{i 2 \pi n L_{b a r}}{\ell}\right)-1\right] .
$$

The energy spectra of the normal system with and without the periodic potential are shown in Fig. 6.4(a) as blue solid lines and black dashed lines, respectively. In the absence of the periodic potential, the dispersion is quadratic and there are no energy gaps. Applying a periodic potential opens energy gaps at the zone center and at the zone boundaries inducing (pairs of) minibands. 


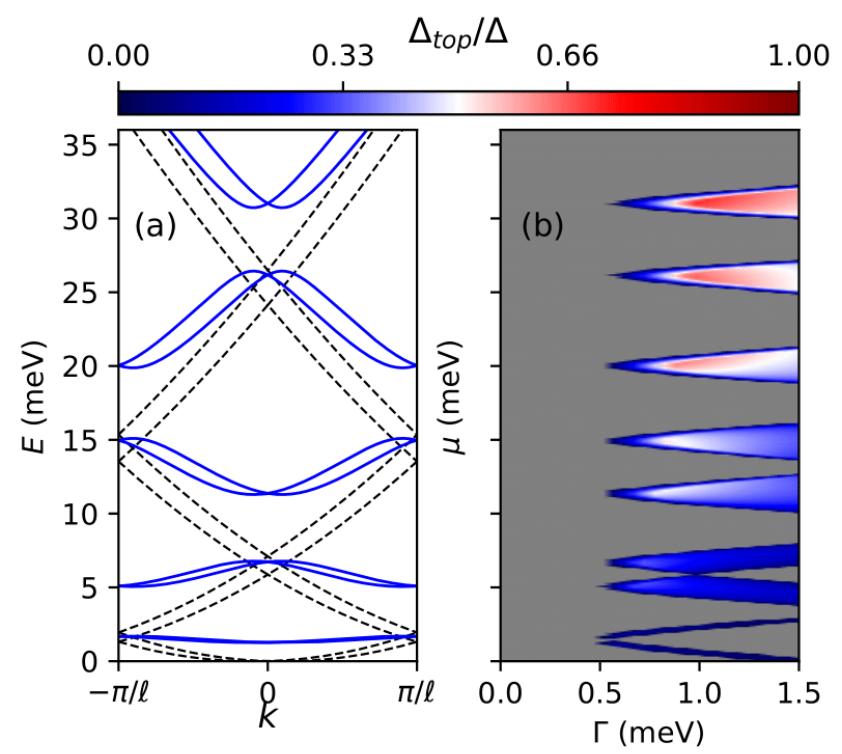

FIGURE 6.4: (a) Low energy spectrum of a normal wire in the absence (black dashed lines) and presence (blue solid lines) of a periodic potential. (b) Topological phase diagram of the periodic system as a function of chemical potential $\mu$ and Zeeman field $\Gamma$. Gray regions are topologically trivial. The color of the topological regions is determined by the size of the topological gap. The system parameters are $m^{*}=0.023 m_{0}$, $\alpha=10 \mathrm{meV} \cdot \mathrm{nm}, \Delta=0.5 \mathrm{meV}, \ell=100 \mathrm{~nm}, L_{b a r}=15 \mathrm{~nm}$, and $V_{o}=20 \mathrm{meV}$.

As noted above, the dispersion of the minibands is similar to that of uniform Rashba nanowires, except that the effective parameters are renormalized and miniband-dependent [see Eqs. (6.14-6.17)]. Each of the minibands can support Majorana bound states, as long as the chemical potential is close to its bottom/top and the Zeeman splitting is strong-enough. For a given set of parameters, we use the Chern-Simon invariant $[16,40], \mathcal{Q}$, to determine whether the system is topologically trivial $(\mathcal{Q}=1)$ or non-trivial $(\mathcal{Q}=-1)$. Fig. 6.4(b) shows the calculated phase diagram as a function of Zeeman splitting, $\Gamma$, and chemical potential, $\mu$. Topologically trivial regions are shown in gray, while the topologically non-trivial regions are colored using a colorscale that indicates the size of the topological gap. Several features are worth pointing out. First, we emphasize that the emergence of a low-field topological phase is associated with the chemical potential being near one of the miniband edges, i.e., either the one at $k=0$ or the $k= \pm \pi / \ell$ band edge. The critical Zeeman field $\Gamma_{c}(\mu)$ has minima near each of these band edges. Note that the corresponding uniform system only supports a low-field topological phase for low values of $\mu$, i.e., for chemical potential values near the bottom of the conduction band. Second, the areas of the parameter space that support topological superconductivity typically increase as one reaches higher energy minibands. This is due to the increasing of the miniband width with energy. For example, the lowest energy miniband has a bandwidth of less 

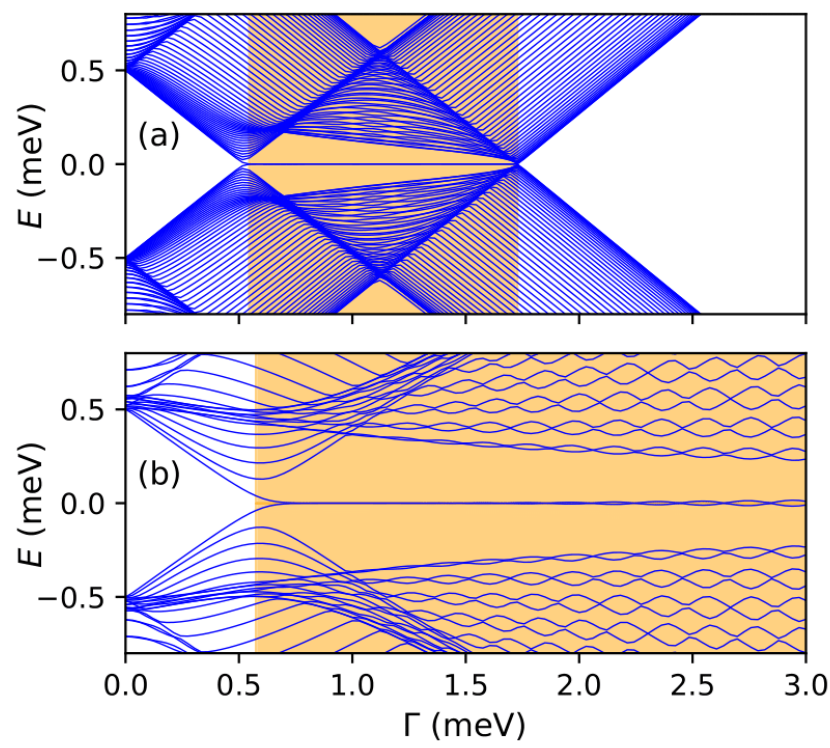

FIGURE 6.5: Energy spectrum of a finite system of length $L=$ $6 \mu \mathrm{m}$ as function of the Zeeman field for a chemical potential (a) $\mu=6.73 \mathrm{meV}$ (corresponding to the top of the second pair of minibands) and (b) $\mu=31.02 \mathrm{meV}$ (bottom of the fifth miniband pair). The topological phase (corresponding to the orange shading) supports a pair of zero-energy Majorana modes. Note that the topological gap is significantly larger in panel (b), i.e., in the topological phase associated with the fifth miniband [see also Fig. 6.4(b)].

than $0.5 \mathrm{meV}$. For any (large-enough) value of the Zeeman field, the single miniband occupancy condition (consistent with the emergence of topological superconductivity) is only satisfied within a narrow chemical potential window comparable to the bandwidth. The increase of the topological phase with the miniband index is further illustrated in Fig. 6.5, which shows the dependence of the low-energy spectrum of a finite wire on the applied Zeeman field for two different values of the chemical potential corresponding to the $k=0$ band edge of the (a) second and (b) fifth pair of minibands, respectively. Both sets of parameters support topological superconductivity and zero-energy MBSs, but in the case of the second miniband [panel (a)] this occurs over a rather narrow range of Zeeman fields. Indeed, the system in panel (a) becomes non-topological for $\Gamma>1.7 \mathrm{meV}$, when the $k= \pm \pi / \ell$ band edge crosses the Fermi level. The third important feature of Fig. 6.4(c) is the increasing of the topological gap as the chemical potential moves into the higher energy minibands. The larger topological gap is a consequence of the larger spin-orbit energy characterizing the higher energy minibands [see Eq. (6.18)]. We conclude that the presence of a periodic potential (i) expands the (low-field) parameter region that supports a topological superconducting phase and (ii) can enhance the size of the (low-field) topological gap. Note that throughout this work we are particularly interested in the low-field 


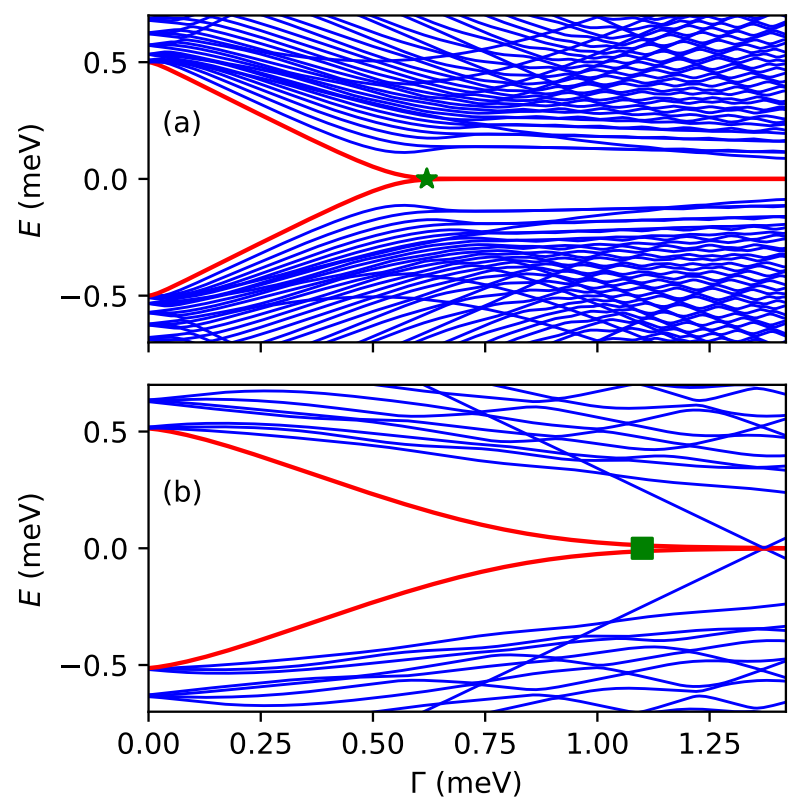

FIGURE 6.6: (a) Low-energy spectrum as a function of the applied Zeeman field for a system with soft confinement (and no periodic potential). (b) Low-energy spectrum of a periodic system with soft confinement. The periodic potential has the form $V(x)=V_{o} \cos (\pi x / \ell)$, with $V_{o}=8 \mathrm{meV}$ and $\ell=20 \mathrm{~nm}$, while the soft confinement has a slope $\kappa=2 \mathrm{meV} / \mu \mathrm{m}$. Both systems support ABS modes that collapse toward (and stick to) zeroenergy as the Zeeman splitting increases. Note, however, that in the periodic system the ABS collapses to zero-energy at a significantly larger Zeeman field.

regime $(\Gamma \lesssim 5 \Delta)$, as strong magnetic fields are detrimental to superconductivity, can lead to the complete collapse of the parent superconducting gap, and, therefore, are less relevant to understanding the physics of experimentally realizable structures.

\section{Soft confinement and partially-separated Andreev bound states in periodic systems}

Systems with inhomogeneous parameters are known to give rise to lowenergy Andreev bound states (ABSs) in the topologically-trivial phase [54$56,58,62,67,69,94,95,108]$. Of particular interest are the trivial near-zero energy modes that mimic the local phenomenology of Majorana bound states, the so-called partially-separated Andreev bound states (ps-ABS) or quasiMajorana modes [94, 108]. In the Majorana representation, these low-energy states are characterized by Majorana components that are partially separated in space, unlike "standard" ABSs, which consist of highly overlapping Majorana components. The energy splitting of the low-energy ABSs (including the ps-ABSs) is sensitive to local perturbations, indicating that these states do not share the topological protection of well separated MBSs. Here, we study 
the emergence of low-energy ps-ABSs in a periodic system due to soft confinement and compare the properties of these states with those of ps-ABSs emerging in "conventional" nanowires with soft confinement and no periodic potential. For clarity, we consider an idealized soft confinement given by the potential

$$
V^{\prime}(x)=-\kappa x,
$$

where $\kappa$ is the slope of the potential. The potential given by Eq. (6.27) has the property that, for sufficiently long wires, induced low-energy states are independent of the chemical potential, up to an overall spatial shift. For small-enough values of $\kappa$ we expect nearly zero energy ps-ABSs emerging for values of the Zeeman splitting slightly above $\Delta$, since there will be a sufficiently-wide region that locally satisfies the topological condition $\Gamma>$ $\sqrt{[\mu-V(x)]^{2}+\Delta^{2}}$. Increasing $\kappa$ shrinks the region of space where the topological condition is met (for a given value of $\Gamma$ ) and the ps-ABS collapses toward zero energy at larger values of the Zeeman field.

The low-energy spectra of a system with soft confinement in the (a) absence and (b) presence of the periodic potential are compared in Fig. 6.6. The periodic potential has the form $V(x)=V_{o} \cos (\pi x / \ell)$, with $V_{o}=8 \mathrm{meV}$ and $\ell=20 \mathrm{~nm}$. We notice that the low-energy spectrum in Fig. 6.6(a), i.e., in the absence of a periodic potential, has a zero energy state emerging at a Zeeman field just above the induced pairing $\Delta=0.5 \mathrm{meV}$. By contrast, the periodic system supports a near-zero energy state only above $\Gamma \approx 1.1 \mathrm{meV}$. This behavior suggests that the presence of a periodic potential reduces the low-field parameter region that supports ps-ABSs (i.e. quasi-Majoranas).

To understand this behavior, we calculate the Majorana components of the ps-ABSs marked by the green star and square in Fig. 6.6 (a) and (b), respectively. The results are shown in Fig. 6.7. Both states are characterized by a partial separation of the Majorana components larger than the widths of the corresponding main peaks of the Majorana wave functions, which is a necessary condition for the collapse of the ps-ABS to zero energy [108]. The key difference between the states in Fig. 6.7(a) and and those in Fig. 6.7(b) is that the "conventional" ps-ABS is characterized by Majorana peak widths that are significantly narrower than the corresponding peaks of the ps-ABS emerging in the periodic system. Consequently, in a periodic wire with soft confinement the partial separation condition [108] is realized at higher values of the Zeeman field, as compared to a "conventional" system, as explicitly shown in Fig. 6.6. Finally, we note that width of the (main) Majorana peak, which controls the Zeeman field associated to the collapse to zero energy of the psABS, is determined by the effective mass. In a periodic system, the effective mass can be significantly reduced, particularly for higher energy minibands [see eq. (6.16)]. Consequently, the presence of a periodic potential reduces the probability of (accidental) ps-ABSs emerging in the (topologically-trivial) low-field regime, and, implicitly, reduces the likelihood of getting false positives when searching for Majorana zero modes. Quantitative estimates of this superlattice-induced reduction require a more realistic modeling of the hybrid system. 

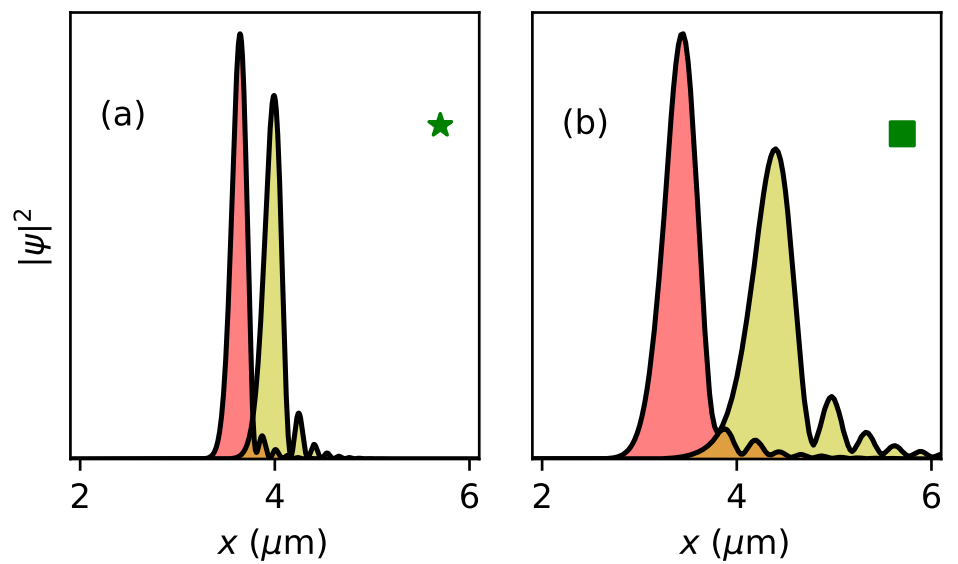

FIGURE 6.7: (a) Majorana wave functions corresponding to the low-energy ps-ABS marked by a green star in Fig. 6.6(a). (b) Envelopes of the Majorana wave functions corresponding to the low-energy ps-ABS marked by a green square in Fig. 6.6(b). Note that the actual wave function oscillates rapidly, as illustrated in Fig. 6.2(b). The main Majorana peaks are wider in the periodic system [panel (b)] as a result of a smaller effective mass. In turn, this requires a higher Zeeman field for satisfying the partial separation condition associated with the collapse of the ps-ABS to zero energy [108] (see Fig. 6.6).

\section{Robustness against disorder}

Our next objective is to investigate the robustness against disorder of the topological superconducting phase realized in a periodic system and compare it with the robustness of the corresponding phase emerging in uniform structures. We note that the presence of disorder, which, to some degree, is inevitable in real systems, can lead to the reduction of the topological gap $[212,213]$ and the emergence of trivial low-energy modes $[55,61,95,214]$. To investigate the effects of disorder, we consider a correlated Gaussian disorder potential, $V^{\prime}(x)$, characterized by

$$
\begin{aligned}
\left\langle V^{\prime}\left(x_{i}\right)\right\rangle & =0, \\
\left\langle V^{\prime}\left(x_{i}\right) V^{\prime}\left(x_{j}\right)\right\rangle & =U^{2} \exp \left(-\frac{\left|x_{i}-x_{j}^{\prime}\right|}{L_{d i s}}\right),
\end{aligned}
$$

where $U$ is the disorder strength, $x_{i}$ is the position along the wire corresponding to lattice site $i$, and $L_{d i s}$ is the disorder correlation length scale. In our numerical calculations we use $L_{d i s}=50 \mathrm{~nm}$, which is a length scale comparable to the typical diameter of a semiconductor nanowire. The correlated disorder is numerically implemented using the scheme described in Ref. [215]. The other system parameters are the same as in Fig. 6.4 and the wire length is $L=5 \mu \mathrm{m}$. For comparison, we also consider a "conventional" disordered 

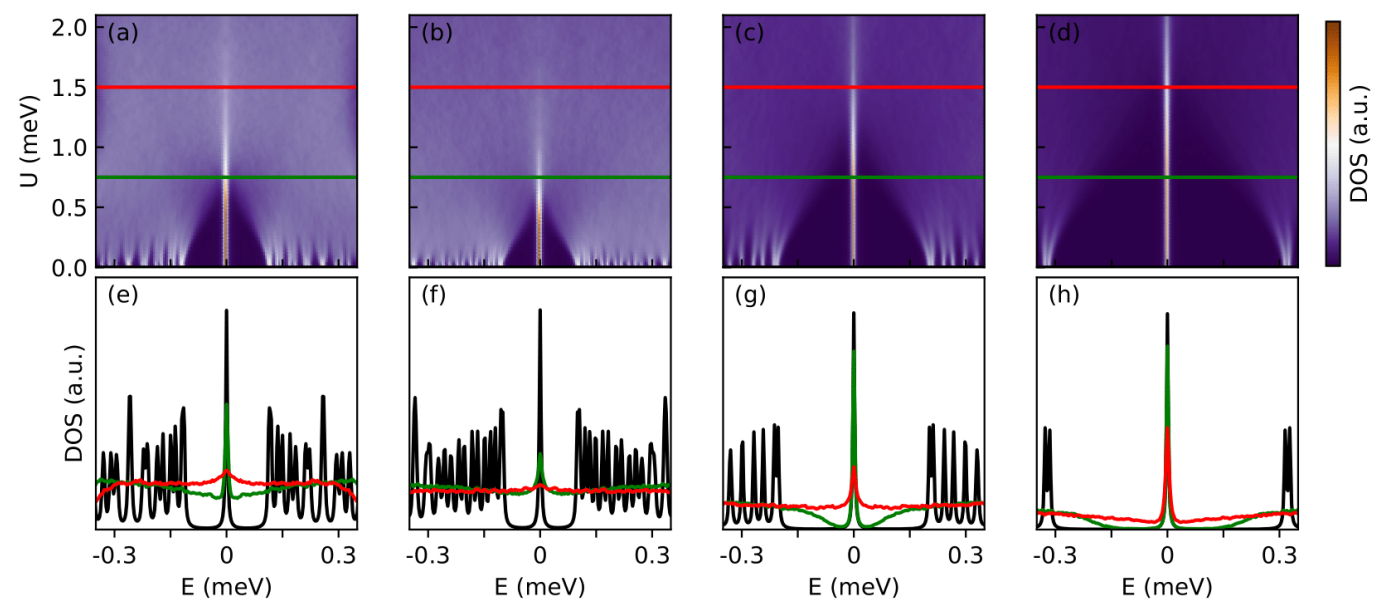

FIGURE 6.8: Density of states averaged over 1000 disorder realizations as function of the disorder strength $U$ for (a) a uniform system with chemical potential $\mu=0$ and (b-d) a periodic structure with chemical potential set to the second (b), third (c), and fourth (d) $k=0$ miniband edges. The Zeeman splitting is fixed at $\Gamma=1.2 \mathrm{meV}$, the length of the wire is $L=5 \mu \mathrm{m}$, the disorder correlation length is $L_{d i s}=50 \mathrm{~nm}$, and other system parameters are the same as Fig. 6.4. The bottom row (e-h) shows line cuts corresponding to the colored lines in the top row: $U=0$ (black lines), $U=0.75 \mathrm{meV}$ (green), and $U=1.5 \mathrm{meV}$ (red). Note that the topological gap and the Majorana zero-energy peak corresponding to higher-energy minibands of the periodic system [panels $(\mathrm{c}, \mathrm{d})$ and $(\mathrm{g}, \mathrm{h})]$ are significantly more robust against disorder than their counterparts emerging in the uniform system [panels (a) and (e)].

wire having the same system parameters and disorder potential, but no periodic potential.

First, we calculate the density of states (DOS) averaged over disorder (using 1000 realizations) as a function of the disorder strength for a fixed value of the Zeeman field, $\Gamma=1.2 \mathrm{meV}$, well inside the topological phase of a clean system. The results are shown in Fig. 6.8 for (a) a uniform system with chemical potential $\mu=0$ and $(\mathrm{b}-\mathrm{d})$ a periodic systems with chemical potential corresponding to the bottom of the second, third, and fourth $k=0$ miniband edges, respectively. In the absence of disorder $(U=0)$, the system is in the topological phase and supports a pair of zero-energy MBSs localized at the edges of the finite wire. The presence of the Majorana modes is signaled by a sharp zero-energy peak in the density of states (see Fig. 6.8). We also note the larger topological gap associated with the higher energy minibands of the periodic system, which is due to a larger effective spin-orbit coupling. Indeed, the zero-disorder topological gap in the third and fourth $k=0$ minibands [panels (c) and (d) in Fig. 6.8] is significantly larger than the corresponding gap in the uniform system [panel (a)]. As the disorder is turned on, the topological gap collapses as low-energy bound states (localized by 
the disorder potential) start to populate the gap. Note that the collapse of the gap to zero energy occurs at significantly higher disorder strengths in the periodic system (with chemical potential inside higher-energy minibands) as compared to the uniform case. This is consistent with the corresponding size of the topological gap at $U=0$. For clarity, in the bottom row of Fig. 6.8 we provide line cuts of the (disorder-averaged) density of states at fixed disorder strengths. One clearly notices the zero energy peak associated with the presence of MBSs and the collapse of the topological gap in the presence of disorder. Remarkably, in the periodic system with a chemical potential inside the fourth miniband [panel (h)], a finite topological gap survives at $U=0.75 \mathrm{meV}>\Delta$ and the zero-energy peak is well defined even at $U=1.5 \mathrm{meV}$. We also note that the (average) density of states corresponding to higher energy minibands is reduced relative to the uniform system DOS due to a smaller (renormalized) effective mass in the periodic system. This results in a large ratio between the zero-energy peak height and the background density of states corresponding to high-energy minibands of the periodic system [see, e.g, Fig. 6.8(h)].

The results discussed above suggest that a periodic system can support (low-field) topological superconductivity and Majorana zero modes that are significantly more robust against disorder than their counterparts realized in a uniform system (having the same parameters), provided the chemical potential is tuned into the higher-energy minibands. An interesting problem that cannot be settled based on our density of states analysis concerns the survival of the zero-energy peaks above the disorder strength corresponding to the collapse of the topological gap (see Fig. 6.8). While it is tempting to attribute the peak to MBSs localized at the ends of the wire, which is definitely the case for weak disorder, one has to keep in mind that generic class $\mathrm{D}$ systems are known to have zero-energy peaks in their density of states even in the absence of topological MBS localized at the boundaries. This phenomenon can be understood in terms of the Griffiths effect [212, 216], i.e. the disorder potential causing fluctuations in the chemical potential that generate short topologically trivial and non-trivial regions throughout the wire. Highly overlapping low-energy MBS emerge at domain walls between these regions. It has been shown that a power-law peak in the density of states at $E=0$ is expected to occur due to such disorder induced states, in contrast with the sharp peak associated with topological MBSs localized at the edges of the wire [216].

To clearly disentangle the contributions generated by topological MZMs from those associated with local MBS pairs (including ps-ABSs, or quasiMajoranas), we introduce the following edge-to-edge correlation function

$$
C=\max (0, \widetilde{C})
$$

with

$$
\begin{aligned}
\widetilde{C} & =\left|\psi_{1, L}\right|^{2}\left|\psi_{1, R}\right|^{2} f\left(E_{1}, \omega_{0}, \Omega\right) \\
& -\sum_{\lambda}^{\lambda \neq 1}\left|\psi_{\lambda, L}\right|^{2}\left|\psi_{\lambda, R}\right|^{2} f\left(E_{\lambda}, \omega_{0}, \Omega\right),
\end{aligned}
$$


where $\psi_{\lambda, L(R)}$ is the left (right) edge component of the positive energy state $\lambda$ and $f$ is a weight function (explicitly defined below) peaked at zero-energy and characterized by an energy window $\omega_{0}$. Note that $C \neq 0$ signals that the lowest energy mode has nonzero weight at both ends of the wire, i.e. it consists of well-separated MBSs. Possible non-local correlations associated with higher energy states result in $\widetilde{C}<0$ [see eq. (6.31)], hence $C=0$. A perfectly symmetric system can support topologically-trivial near-zero energy states that have equal (nonzero) amplitudes at the ends of the system. However, in this situation the $\mathrm{BdG}$ states $\lambda=1$ and $\lambda=2$ are degenerate and have equal $\mathrm{R} / \mathrm{L}$ amplitudes, which leads to the cancelation of the first two terms in Eq. (31). In the presence of disorder, the symmetry is broken and the nearly-degenerate states $\lambda=1$ and $\lambda=2$ are (typically) localized at opposite ends (hence, again, the correlation is zero). There can be disorder configurations that generate accidental nonzero correlations due to nearlydegenerate trivial states, but we found that these situations are rare and the weight of the corresponding contributions is negligible when considering a large-enough number of disorder realizations. Finally, note that the lowest energy state can have a finite energy $\epsilon$ and still generate a well-defined edgeto-edge correlation, as long as $\epsilon<\omega_{0}$. This property is extremely useful for disentangling well-separated MBSs and ps-ABSs in finite (relatively short) wires, as both types of states may be characterized by finite energy splitting oscillations. The weight function is defined as

$$
f\left(E, \omega_{0}, \Omega\right)=H\left(E,-\omega_{0}, \Omega\right)-H\left(E, \omega_{0}, \Omega\right),
$$

with

$$
H\left(E, \omega_{0}, \Omega\right)=\left(1+\exp \left[-2\left(E-\omega_{0}\right) / \Omega\right]\right)^{-1} .
$$

Note that Eq. (6.33) becomes the Heaviside step function in the limit $\Omega \rightarrow 0$. In the numerical calculations we use $\omega_{0}=2 \mathrm{meV}$ to define the range of relevant low-energy states and $\Omega=0.2 \mathrm{meV}$ to define the smooth transition region. Also, we define $\psi_{\lambda, L(R)}$ as the total weight (including summations over the spin and particle-hole degrees of freedom) within the leftmost (rightmost) $200 \mathrm{~nm}$ segment of the wire.

Calculated edge-to-edge correlations averaged over 300 disorder realizations are shown in Fig. 6.9 for both the periodic and uniform systems. The top panels correspond to a clean system $(U=0)$, while the middle and bottom panels correspond to $U=0.5 \mathrm{meV}$ and $U=1 \mathrm{meV}$, respectively. The length of the wire is $L=2 \mu \mathrm{m}$, while other system parameters are the same as in Fig. 6.8. First, we note that for a clean system (see top panels in Fig. 6.9) the areas with $C \neq 0$ correspond to the the topological superconducting phase in Fig. 6.4(b), demonstrating that the edge-to-edge correlations are generated by Majorana modes localized at the ends of the wire. To emphasize this property, we will use the term correlation phase diagram to designate the map $C(\mu, \Gamma)$. Note that the large correlations corresponding to the green regions in panels (a) and (b) are due to strongly localized MBSs characterizing the corresponding clean systems. By comparison, the (clean) MBSs associated with higher energy minibands [panels (c) and (d)] have larger localization 

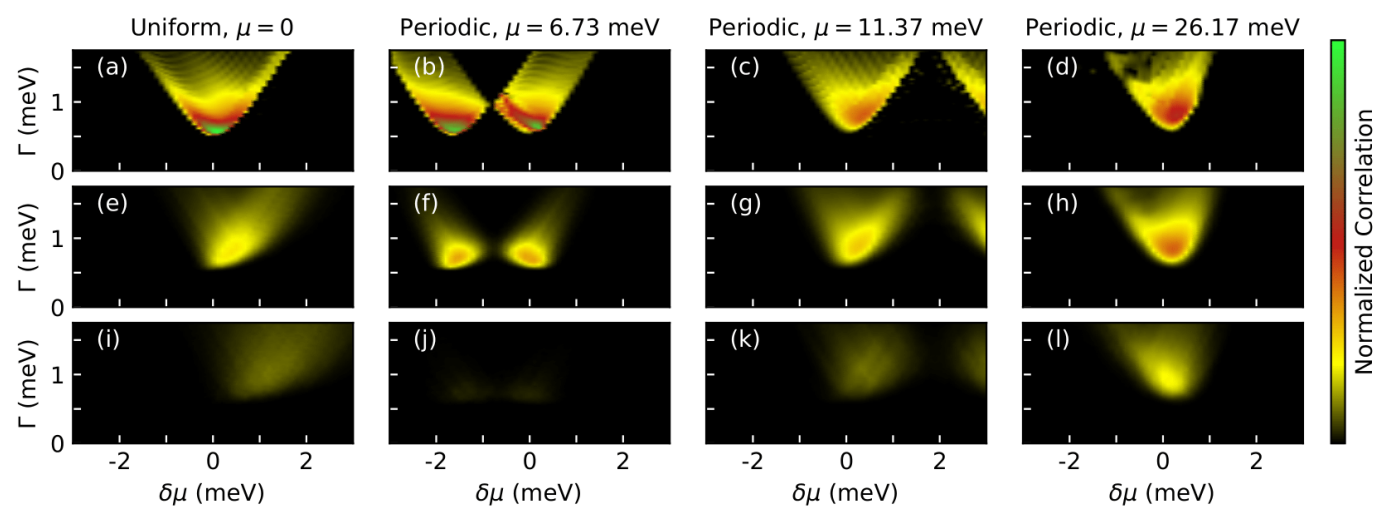

FIGURE 6.9: Correlation phase diagrams for uniform and periodic systems as a function of chemical potential and Zeeman splitting. The correlation strength $C$ is defined by Eq. (6.30) and is averaged over 300 disorder realizations, with each panel being normalized to the maximum value corresponding to a clean, uniform system - panel (a). The disorder strength is $U=0$ for the panels in the top row (a-d), $U=0.5 \mathrm{meV}$ (middle row), and $U=1.0 \mathrm{meV}$ (bottom row). Note that the edge-to-edge correlations are suppressed most rapidly in the periodic system with low chemical potential (second column), followed by the uniform system (first column). In the periodic system with higherenergy minibands (third and fourth columns) the correlations decrease with disorder at a lower rate, indicating an increases robustness of MBSs against disorder.

lengths, hence lower values of $\left|\psi_{1, L(R)}\right|^{2}$ and, implicitly, C. Upon introducing disorder, the edge-to-edge correlation is reduced in all cases, essentially due to the hybridization of end-of-wire MBSs with disorder-induced low-energy states. In addition, one can notice (primarily in the uniform system) a shift of the correlated area toward larger values of $\mu$, which is consistent with the findings of Ref. [62]. However, the most important feature is that the suppression of $C$ does not occur at the same rate for all parameter regimes. While in a periodic system with low chemical potential (second column in Fig. 6.9) the correlations are suppressed even faster than in the uniform system (first column), periodic systems with a chemical potential within higher energy minibands (columns three and four) are characterized by correlations that decrease at a lower rate than the uniform system, which signals the increased robustness of MBSs against disorder. Note that while panels (i) and (k) have similar correlation levels at $U=1 \mathrm{meV}$, the periodic system (third column) is characterized by correlations decreasing with the disorder strength at a significantly slower rate than the correlations in the homogeneous system (first column). We conclude that topological phases and Majorana zero modes realized in periodic systems can be more robust against disorder than their counterparts emerging in uniform system, provided the chemical potential lies within a sufficiently high energy miniband. 


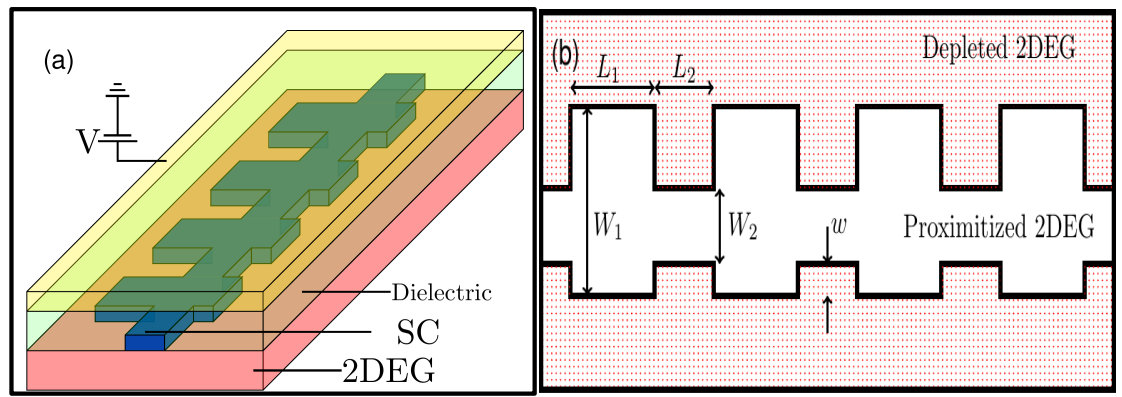

FIGURE 6.10: (a) Schematic representation of the proposed $M a-$ jorana waveguide device. A 2DEG hosted by a semiconductor heterostructure is proximitized by a quasi-1D superconductor with alternating wide and narrow regions. A top gate depletes the 2DEG outside the region covered by the superconductor generating a quasi-1D channel with periodically modulated width. (b) Top view of the periodic quasi-1D channel.

\subsubsection{Majorana Waveguides}

Our detailed analysis of the periodic Majorana system based on the 1D toy model given by Eqs. (6.1) and (6.2) has demonstrated that the presence of a periodic potential could provide significant advantages for realizing Majorana zero modes in semiconductor-superconductor structures, such as increasing the low-field parameter range consistent with topological superconductivity, enhancing the topological gap and the robustness of the Majorana modes against disorder, and reducing the (low-field) parameter space associated with the emergence of topologically trivial low-energy states. However, our analytical expressions for the renormalized effective parameters, corroborated by the numerical results, indicate that these potential benefits can only be obtained if (i) the applied periodic potential is strong-enough and (ii) the chemical potential is tuned within one of the higher energy minibands. In practice, realizing the first condition is highly nontrivial. Our numerical estimates indicate that using, for example, a proximitized semiconductor nanowire $[47,81,83,84,86,210]$ and a periodic arrangement of potential gates is highly unlikely to generate a strong-enough periodic potential.

In this section we approach the critical problem of realizing the periodic potential from a different angle: instead of actually applying an external periodic potential, we propose the realization of an effective periodic potential by modulating the width of the device. More specifically, we propose the realization of periodic Majorana devices based on quasi-1D channels realized in 2D semiconductor heterostructures proximity coupled to superconductor strips of periodically modulated width. The feasibility of such a device is supported by recent progress in engineering Al-InAs two-dimensional heterostructures showing experimental signatures consistent with Majorana physics [90, 155, 188-196]. A schematic representation of the proposed device is shown in Fig. 6.10 (a). A semiconductor quantum well hosting a twodimensional electron gas (2DEG) with large spin-orbit coupling and g-factor is proximity-coupled to a conventional superconductor (e.g., Al) grown on 
top of the semiconductor heterostructure. The structure is capped by a top gate that can deplete the 2DEG outside the region covered by the superconductor, while the area under the superconductor is screened. As the width of the superconductor is periodically modulated, we obtain a quasi-1D channel with periodic position-dependent width. A top view of the periodic quasi1D channel is shown in Fig. 6.10 (b). As shown explicitly below, the periodic modulation of the superconductor width generates a Majorana wire with effective periodic potential basically equivalent to the 1D model investigated in Sec. 6.1.1.

We model the proposed Majorana waveguide by considering a 2DEG with Rashba spin-orbit coupling and position-dependent confining potential described by the Hamiltonian

$$
\begin{aligned}
H_{2 D E G}= & {\left[-\frac{\hbar^{2}}{2 m^{*}}\left(\partial_{x}^{2}+\partial_{y}^{2}\right)-\mu+V(x, y)\right] \sigma_{o} } \\
& +\alpha_{y} k_{x} \sigma_{y}-\alpha_{x} k_{y} \sigma_{x},
\end{aligned}
$$

where $m^{*}$ is the effective mass, $\mu$ is the chemical potential, $\alpha_{x}$ and $\alpha_{y}$ are Rashba coefficients, and $V(x, y)$ is a confining potential periodic in the $x$ direction with period $\ell, V(x+\ell, y)=V(x, y)$. Let us first consider a "hardwall" confining potential,

$$
V(x, y)= \begin{cases}0, & \text { proximitized region in Fig. } 6.10(b), \\ \infty, & \text { depleted region in Fig. } 6.10(b)\end{cases}
$$

The width of the proximitized region is $x$-dependent, alternating between $W_{1}$ and $W_{2}$, with a bottom offset $w$, as shown in Fig. 6.10 (b). First, we study the spectrum of an infinite channel by imposing periodic boundary conditions in the $x$-direction. In the limit $W_{1}=W_{2}, w=0$, and $\alpha_{x}=0$, the spectrum is trivially given by the analytic expression

$$
\begin{aligned}
E_{n, p, \tau}\left(k_{x}\right)= & \frac{\hbar^{2}}{2 m^{*}}\left(\frac{\pi^{2} n^{2}}{W_{1}^{2}}+\left(k_{x}+\frac{2 \pi p}{\ell}\right)^{2}\right) \\
& -\mu+\left(\sigma_{z}\right)_{\tau, \tau} \alpha_{y} k_{x}
\end{aligned}
$$

where $-\pi / \ell<k_{x} \leq \pi / \ell, n \in \mathbb{Z}^{+}, p \in \mathbb{Z}$, and $\tau=1,2$. The quantum number $n$ indicates the transverse mode, while $p$ is the zone number, and $\tau$ refers to the \pm y spin- $\frac{1}{2}$ eigenstates. Note that the spectrum, which consists of folded and shifted parabolas, is gapless. Allowing $W_{1} \neq W_{2}$ and /or $w \neq 0$ leads to the opening of energy gaps near $k=0$ and $k= \pm \pi / \ell$. This property can be viewed as a consequence of plane waves scattering at the interface between the regions with different widths.

The calculated spectrum of a system with $W_{1} \neq W_{2}$ and $w=0$ is shown in Fig. 6.11 (a). Since the eigenenergies scale as $E \propto W_{1}^{-2}$, as long as the ratios between the spatial variables are held fixed [211], it is natural to define the energy unit as $E_{o}=\left(\pi^{2} \hbar^{2} / 2 m^{*} W_{1}^{2}\right)$, i.e., the confinement energy 


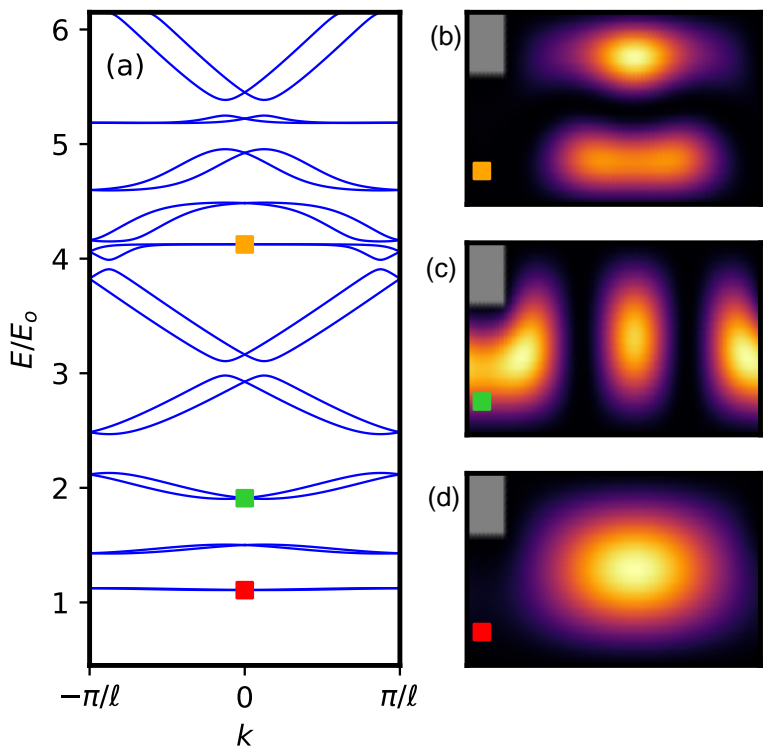

FIGURE 6.11: (a) Spectrum of a quasi-1D system with "hardwall" confining potential. (b-d) Modulus square of the $k=0$ eigenstates marked by colored squares in panel (a). The gray areas outside the narrow regions (of width $W_{2}$ ) are inaccessible due to the "hard-wall" confinement. The system parameters are: $W_{2} / W_{1}=0.68, w=0, L_{1} / W_{1}=2.6, L_{2} / W_{1}=0.4$, $m^{*} \alpha_{y} W_{1} / \hbar^{2}=0.13$, and $\alpha_{x}=0$, and the energy unit is $E_{o}=$ $\left(\pi^{2} \hbar^{2} / 2 m^{*} W_{1}^{2}\right)$.

associated with the first transverse mode in a quasi-1D channel of width $W_{1}$. Notice that the first miniband has its bottom just above $E_{0}$. The (modulus squared of the) wave function corresponding to the lowest energy state [marked by a red square in Fig. 6.11 (a)] is shown in Fig. 6.11 (d). Notice that the wave function has a single transverse lobe localized within the wide region and does not leak significantly into the narrow region. The minimum energy required to have an oscillatory component within the narrow region is $E=E_{o}\left(W_{1} / W_{2}\right)^{2} \approx 2.16 E_{0}$. The wavefunction corresponding to the $k=0$ state of the third minibabd is shown in Fig. 6.11(c). The state is still dominated by the first transverse mode, but it has three maxima within the wide region. States characterized by transverse modes with two (or more) maxima ocur above $E \approx 4 E_{0}$, as expected based on the fact that the confinement energy of the second transverse mode in a uniform channel is $4 E_{o}$. An example of such states is show in Fig. 6.11 (b). Similarly to the first miniband, this miniband is quite flat, as the second transverse mode decays in the narrow regions for energies below $E \approx 8.65 E_{0}$. However, this miniband mixes with other minibands (dominated by the first transverse mode) near the zone edge, where it acquires some dispersion. In general, minibands above $4 E_{o}$ are characterized by strong mixing between different transverse modes. Below this threshold energy, however, the system has a spectrum similar to that of a purely 1D system in the presence of a periodic potential, which can be seen 
by comparing the low energy minibands of Fig. 6.11 (a) and Fig. 6.4 (a). Therefore, we expect the results obtain based on the purely 1D model of Sec. 6.1.1 to hold at least for the first few minibands of the quasi-1D structure.

Next, we investigate numerically the correspondence between the properties of the Majorana waveguide and the predictions of the 1D toy model. For concreteness, we consider a quasi-1D system with effective parameters consistent with an InAs / Al heterostructure implementation of the proposed device: $m^{*}=0.023 m_{0}, \alpha_{x}=\alpha_{y}=100 \mathrm{meV} \cdot \AA, \Delta=0.3 \mathrm{meV}, W_{1}=50 \mathrm{~nm}$, $W_{2}=30 \mathrm{~nm}, L_{1}=130 \mathrm{~nm}, L_{2}=20 \mathrm{~nm}$, and $w=\left(W_{1}-W_{2}\right) / 2=10 \mathrm{~nm}$. The corresponding normal spectrum for $\mu=\Gamma=0$ is shown in Fig. 6.12(a). Note the qualitative similarity with the spectrum in Fig. 6.11(a). The absence of mixing between the flat and the dispersive high-energy minibands is due to a mirror symmetry with respect to the $x$-axis generated by the specific choice of geometric parameters. The corresponding topological phase diagram as a function of Zeeman field, $\Gamma$, and chemical potential, $\mu$, is shown in Fig. 6.12(b). The topologically trivial and non-trivial regions correspond to the gray and colored regions, respectively, with the color scale indicating the size of the topological gap. The phase diagram displays the main qualitative features found in the context of the 1D model (see Fig. 6.4). Again, each miniband edge supports a (low-field) topological phase region, with the flat minibands generating a non-trivial phase only inside thin slices of parameter space. Also, the topological gap generally increases as the chemical potential moves into higher energy minibands, due to a larger effective spin-orbit coupling. Note, however, the effect of higher energy flat minibands (absent in the 1D model), which can create narrow topological regions at higher values of the chemical potential. In practice, these regions are likely irrelevant, due to their small areas (requiring a high degree of fine tuning), and, most importantly, due to their small topological gaps.

Consider now a finite segment of length $L=3.75 \mu \mathrm{m}$ of the quasi-1D system discused above. The dependence of the low-energy spectrum on the applied Zeeman field for $\mu=22.2 \mathrm{meV}$ is shown in Fig. 6.13(a). For comparison, the spectrum of a uniform wire with constant thickness $W_{1}=50 \mathrm{~nm}$ and the chemical potential tuned to the bottom of the first transverse mode (all other parameters being the same) is shown in Fig. 6.13(b). As expected based on our 1D analysis, the periodic system with the chemical potential within a higher energy miniband supports a larger topological gap and has a lower density of states, compared to the uniform system. Also note that the Majorana mode in Fig. 6.13(a) is characterized by larger energy splitting oscillations as compared to its counterpart in the uniform system [see Fig. 6.13(b)], indicating a larger MBS localization length, in agreement with the results of Sec. 6.1.1.

While the "hard-wall" potential is a convenient approximation for initially exploring the physics of Majorana waveguides, in real systems there is a finite length scale, $\chi$, over which the confining potential varies from its value inside the covered region to its value inside the depleted region. In fact, it is important that this "soft-confinement" length scale be large-enough, as 


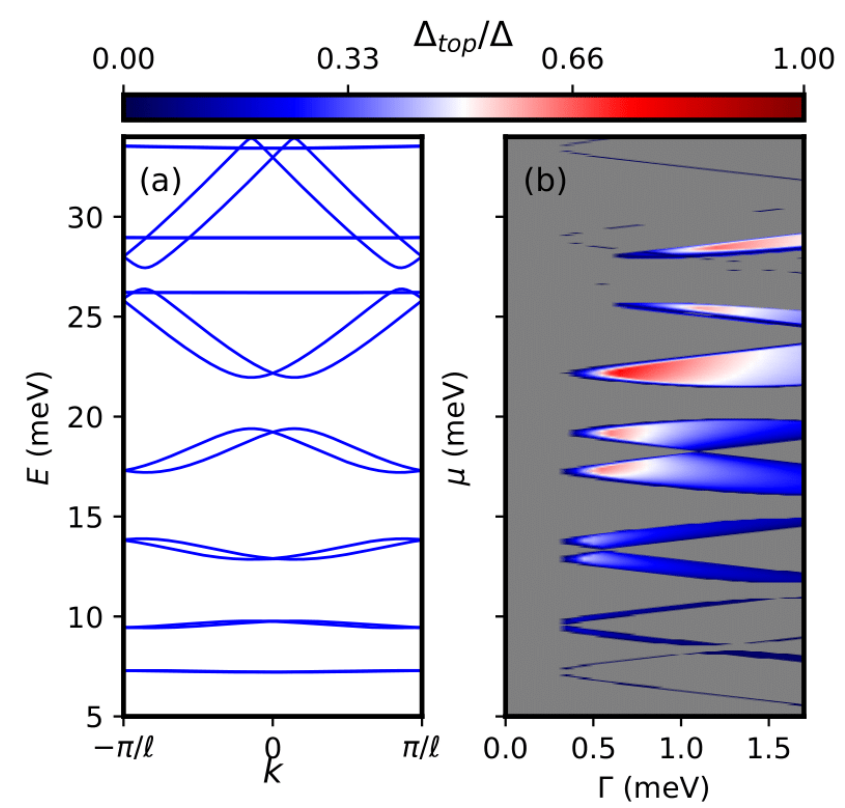

FIGURE 6.12: (a) Spectrum of a quasi-1D periodic system with mirror-symmetric "hard-wall" confining potential. (b) Topological phase diagram of the periodic system as a function of chemical potential, $\mu$, and Zeeman splitting, $\Gamma$. Gray regions are topologically trivial, while colored regions are topologically non-trivial, with the color scale indicating the size of the topological gap. Note that the phase diagram is qualitatively similar to the phase diagram of the 1D model shown in Fig. 6.4(b). The system parameters are: $m^{*}=0.023 m_{0}, \alpha_{x}=\alpha_{y}=100 \mathrm{meV} \cdot \AA$, $\Delta=0.3 \mathrm{meV}, W_{1}=50 \mathrm{~nm}, W_{2}=30 \mathrm{~nm}, L_{1}=130 \mathrm{~nm}$, $L_{2}=20 \mathrm{~nm}$, and $w=\left(W_{1}-W_{2}\right) / 2=10 \mathrm{~nm}$.

this enables the tuning of the chemical potential by the top gate. Quantitatively, the length scale $\chi$, which describes the efficiency of the screening by the superconductor of the potential created by the top gate, depends on the details of the heterostructure and can be estimated by solving a numerically challenging Schrodinger-Poisson problem [116, 126, 127]. Here, we do not address this problem, but instead consider $\chi$ as a phenomenological parameter and investigate the following key question: how are the topological phase diagram and the properties of the Majorana bound states affected by a finite (rather than zero) screening length? To address this question, we model the "smooth" confining potential as

$$
V(x, y)=V_{\max }\left[g(x) V_{1}(y)+(1-g(x)) V_{2}(y)\right]
$$



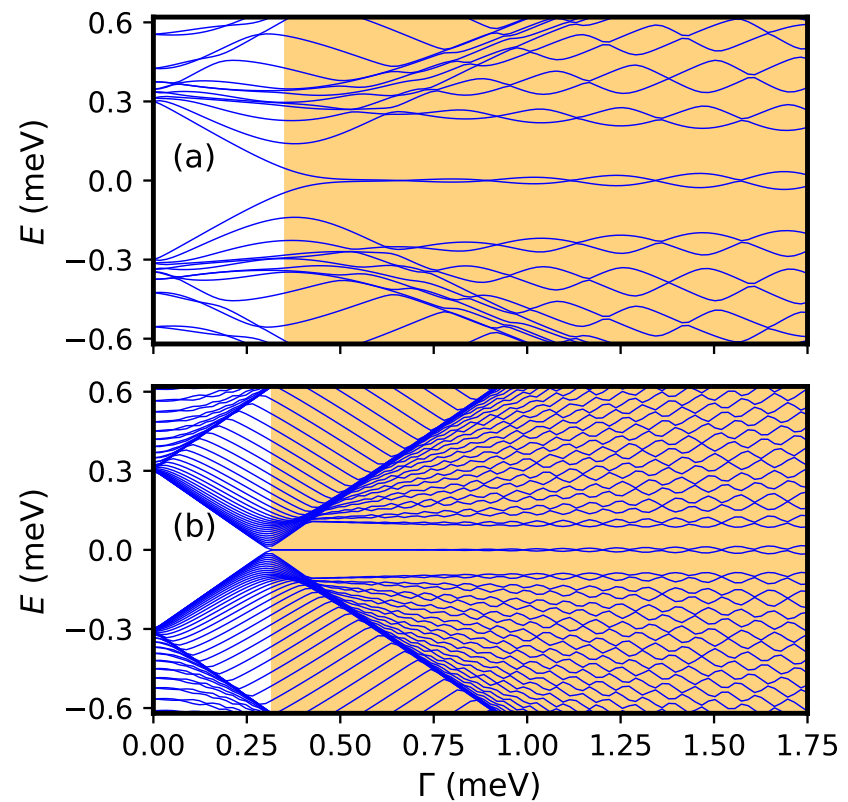

FIGURE 6.13: (a) Low energy spectrum of a finite quasi-1D periodic system as a function of the Zeeman field $\Gamma$. The length of the system is $L=3.75 \mu \mathrm{m}$ and the chemical potential is $\mu=22.2 \mathrm{meV}$. All other parameters are the same as in Fig. 6.12. (b) Spectrum of a uniform system of thickness $W_{1}=50 \mathrm{~nm}$ (same as the wide region of the periodic system) and chemical potential corresponding to the bottom of the first transverse mode. All other parameters are the same as in (a).

where

$$
\begin{aligned}
g(x) & =H(x, 0, x)-H\left(x, L_{1}, x\right), \\
V_{1}(y) & =H(-y, 0, x)+H\left(y, W_{1}, \chi\right), \\
V_{2}(y) & =H(-y, w, \chi)+H\left(y, w+W_{2}, x\right),
\end{aligned}
$$

with $H$ defined in Eq. (6.33). An example of a smooth confining potential landscape is shown in Fig. 6.14. Note that the finite screening length $\chi$ rounds the corners of the wide and narrow regions, resulting in a smooth periodic quasi-1D channel.

The dependence of the phase diagram on the screening length $\chi$ is illustrated in Fig. 6.15. The system is characterized by a soft confinement potential given by Eq. (6.37) with $V_{\max }=75 \mathrm{meV}$ and different values of the screening length. All other parameters are the same parameters as in Fig. 6.13. In the top panel, i.e., Fig. 6.15(a), we have $\chi=0$, which means a sharp transition from the covered region to the depleted region, similar to the hardwall scenario, except for a finite value of the potential in the depleted region. The resulting phase diagram is practically identical to the hard-wall phase diagram in Fig. 6.13(b), except for an overall shift to smaller values of the chemical potential due to a (small) leakage of the wave functions into the depleted 


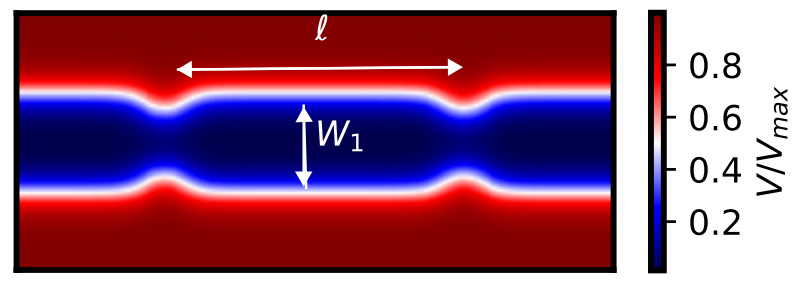

FIGURE 6.14: Confining potential landscape for a smooth potential defined by Eq. (6.37) with parameters: $W_{1}=50 \mathrm{~nm}$, $W_{2}=30 \mathrm{~nm}, L_{1}=130 \mathrm{~nm}, L_{2}=20 \mathrm{~nm}$, and $\chi=10 \mathrm{~nm}$.

region. As the screening length $\chi$ increases, the topological regions move to higher values of $\mu$ as the proximitized (i.e., covered) channel acquires a nonzero potential. Importantly, the phase diagram remains largely unaffected by the finite $\chi$, even for values of the screening length comparable to the width of the channel [e.g., in Fig. 6.15(c) we have $\chi=0.67 W_{2}=0.4 W_{1}$ ]. Of course, the exact shape and location of the phase boundaries change slightly with $\chi$, but the overall topological area, the lowest values of the critical Zeeman field, and the typical size of the topological gap are practically insensitive to changes of the screening length. This insensitivity, combined with the overall shift of the phase diagram, demonstrates the possibility of tuning the chemical potential using the top gate (i.e., changing $V_{\text {max }}$ ) without altering the phase boundaries. For the parameters used in these calculations, a variation

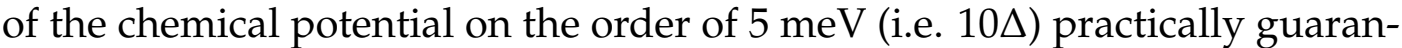
tees access to a miniband edge, which corresponds to a low-field topological phase. In addition, the overall energy scale can be further controlled through the channel geometry, in particular the length scales $L_{1}, L_{2}, W_{1}$, and $W_{2}$ that determine the confining potential landscape. Having demonstrated the basic equivalence between the low-energy physics of the Majorana waveguide and the effective 1D model of Sec. 6.1.1, we conclude that periodic quasi$1 \mathrm{D}$ channels engineered using patterned 2D semiconductor-superconductor structures represent an extremely versatile platform that can provide significant advantages for realizing robust Majorana zero modes.

\subsubsection{Summary and conclusions}

In this work we investigate the emergence of topological superconductivity and Majorana zero modes in periodic structures, focusing on (i) determining the impact of the periodic potential on the robustness of the Majorana modes and (ii) identifying practical solutions for engineering a strongenough periodic potential. First, we consider a simplified one-dimensional model of the periodic structure and derive analytical expressions for the effective parameters that characterize the periodic potential-induced minibands. We find that higher energy minibands are characterized by large values of the effective spin-orbit coupling and low effective masses. In turn, this leads to enhanced values of the topological gap and low densities of bulk 


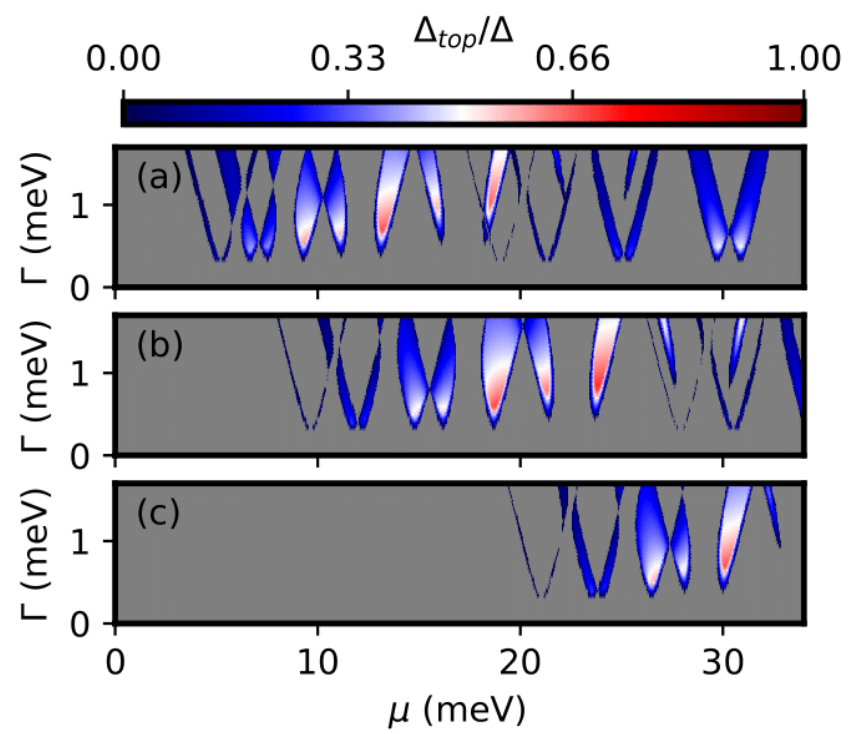

FIGURE 6.15: Topological phase diagram for a system with softconfinement. The confining potential is given by Eq. (6.37), with $V_{\max }=75 \mathrm{meV}$ and different screening lengths: (a) $\chi=0$, (b) $\chi=10 \mathrm{~nm}$, and (c) $\chi=20 \mathrm{~nm}$. Other system parameters are the same as in Fig. 6.13. Increasing the screening length $\chi$ shifts the phase diagram toward larger values of $\mu$, but does not change the main features.

states, creating ideal conditions for the realization of robust topolgical superconductivity. Using numerical simulations, we show explicitly that the presence of a periodic potential reduces the low-field parameter space consistent with topologically trivial low-energy states that mimic the local phenomenology of Majorana zero modes (i.e., ps-ABSs or quasi-Majoranas). Most importantly, the periodic potential enhances the robustness of the Majorana modes against disorder. To demonstrate this point, we introduce an edge-to-edge correlation function that could prove useful in determining the expected output of non-local (three terminal) conductance measurements on Majorana devices. The price for the larger topological gap and the increased robustness against disorder is an enhanced Majorana localization length characterizing the periodic structures. While this implies longer wires for realizing topologically protected Majorana zero modes, in the near term this property (combined with the enhanced robustness against disorder and the reduced susceptibility of generating trivial low-energy states) can prove helpful in demonstrating hybridization-induced Majorana oscillations, a key feature that, so far, has eluded experimental observation, casting doubts regarding the real nature of the observed zero-bias conductance peaks. In addition, the enhancement of the Majorana localization length is rather moderate (a factor of two, or less), provided the periodic potential is strong-enough, a key condition for actualizing the potential benefits of periodic structures.

To address the critical problem of engineering strong-enough periodic potentials, we propose the Majorana waveguide device, a quasi-1D channel 
with periodically modulated width hosted by a semiconductor heterostructure and proximity-coupled to a lithographycally defined superconductor. We show that the periodic modulation of the channel width can generate an effective periodic potential with the required specifications. Moreover, in the regime characterized by a chemical chemical potential smaller than the confinement energy of the second transverse mode, the phase diagram of the Majorana waveguide has all the qualitative features predicted by the 1D effective theory (compare, for example, Figs. 6.4 and 6.13). Finally, we show that the finite length scale associated with the screening by the superconductor has a weak effect on the phase boundaries, basically resulting in an overall shift of the chemical potential. This property suggests the possibility of efficient control of the chemical potential by a top gate. In addition, the effective system parameters can be engineered by controlling the channel geometry, in particular the length scales $L_{1}, L_{2}, W_{1}$, and $W_{2}$ that determine the confining potential landscape. These elements, which support the feasibility of conditions necessary for actualizing the potential benefits of periodic structures identified by our analysis of the effective 1D model, suggest that modulated quasi-1D channels realized in patterned 2D semiconductor-superconductor structures provide a promising platform for realizing robust Majorana zero modes.

Future theoretical efforts that could assist the fabrication and measurement of this type of devices should build on detailed Schrödinger-Poisson simulations of realistic structures. Obtaining quantitative estimates of the screening length will enable the optimization of the channel geometry and the identification of the realistic range for the top gate lever arm. In addition, based on the calculated screening length, one can estimate the strength of the effective disorder generated by patterning imperfections, which addresses the important engineering problem regarding how precise these patterns need to be. Finally, these efforts must be supplemented by calculations of transport properties, including quantitative predictions of edge-to-edge correlations expected in a multi-terminal measurement.

\subsubsection{Acknowledgments}

The work of this section was supported in part by NSF DMR-1414683.

\subsection{Enhanced topological superconductivity in spa- tially modulated planar Josephson junctions}

In this section, we turn our attention to Majorana Josephson junctions (MMJs) within SM-SC planar nanostructures. As mentioned in the introduction of this chapter, a conventional MJJ [197, 198] consists of a 2DEG with strong spin-orbit coupling and large g-factor (InAs/InSb) in proximity to a two large superconductors separated by a narrow uncovered junction region. Compared to the Majorana nanowires and planar SM-SC structures with a single quasi-1D SC strip, the MJJ designs have the additional experimentally 
tunable parameter of the superconducting phase difference $\phi$ between the two SC regions. Interestingly, as $\phi$ is tuned from $\phi=0$ to $\phi=\pi$, the necessary Zeeman splitting $E_{Z}$ needed to drive the system into the topological phase can approach zero [197, 198], $E_{Z} \rightarrow 0$, which is of course an attractive feature of such platforms. Moreover, fine-tuning of the chemical potential $\mu$ in not necessary for $\phi=\pi$ phase bias, in stark contrast to their nanowire counterparts in which $\mu$ must be tuned near the bottom of a subband.

Despite these attractive features, planar SM-SC structures have several potentially serious issues. For the SC strip design, a dramatic suppression of the effective g-factor and spin-orbit coupling may occur if the coupling between the semiconductor and superconductor is strong [145, 168]. In the strong coupling regime, the Zeeman energy necessary to reach the topological phase is no longer $E_{Z, \text { crit }}=\sqrt{\mu^{2}+\Delta_{i n d^{2}}^{2}}$, as found in the minimal 1D Majorana model $[8,9,43,44]$, but rather $E_{Z, c r i t}=\sqrt{\mu^{2}+\widetilde{\gamma}^{2}}$ [145], where $\Delta_{\text {ind }}$ is the induced superconducting gap, $\mu$ is the chemical potential, and $\widetilde{\gamma}$ is the effective SM-SC coupling. Note that $\widetilde{\gamma}$ can be several times larger than $\Delta_{\text {ind }}$, which makes it difficult to reach the topological phase before superconductivity in the parent superconductor is destroyed by the applied magnetic field. Many widely used hybrid structures, which include both nanowires and planar strip-type systems, particularly those characterized by a hard (induced) SC gap, are likely to be in a strong coupling regime since the induced gap is comparable to the parent gap, $\Delta_{\text {ind }} \sim \Delta_{o}$.

The Josephson junction design overcomes the issues associated with strong SM-SC coupling, basically because the "bare" semiconductor parameters are not renormalized by the superconductor within the junction region. Also, this design enables an additional, potentially useful tuning parameter: the superconducting phase difference. However, the Josephson junction system has a major issue that could make these attractive features practically irrelevant: the optimal topological gap is rather small, which makes the topological superconducting phase (and the corresponding MZMs) very fragile. In addition, realizing the conditions consistent with the optimal topological gap is nontrivial, as it involves a highly uniform effective potential throughout the system. More specifically, for realistic device parameters characterizing InAs / Al systems, the topological gap $\Delta_{\text {top }}$ is restricted to rather small fractions of the parent gap $\Delta_{o}$. Combined with the already small parent SC gap of $\mathrm{Al}, \Delta_{o} \approx 0.2-0.3 \mathrm{meV}$, this leads to a very fragile topological phase that can be easily destroyed by a small amount of disorder [74, 76, 77]. Moreover, using superconductors with a larger gap does not automatically fix the issue, since it involves an increase of the Zeeman energy necessary to achieve the optimal topological gap [198]. Finally, the uniformity requirement to be satisfied by the effective potential represents a nontrivial task in the presence of both proximitized and non-proximitized regions [217] due to the strong band bending at the SM-SC interface $[117,126]$, as well as the proximity induced shift of the potential in the presence of finite SM-SC coupling [168, 177].

In this work we propose a planar JJ device with periodically modulated junction width (see Fig. 6.16) as a possible solution to the challenges facing the "standard" JJ design. In essence, the modulation of the junction 
width generates a strong periodic potential, which, in turn, produces minibands with strongly renormalized effective parameters. On the one hand, in the presence of these minibands, the topological phase supported by the hybrid structure with no superconducting phase difference expands significantly within the low-field regime. Most importantly, the topological gap characterizing the low-field topological phase is substantially enhanced as compared to gap associated with the the uniform, non-modulated structure. In turn, this enhances the robustness of the topological phase and the associated MZMs against disorder. This enhancement of the topological gap is, in essence, a direct result of the larger effective spin-orbit coupling that characterizes the minibands induced by the periodic (effective) potential. Note that this type of optimization of the effective parameters by controlling the geometric properties of a nanodevice opens a new and potentially fruitful route to creating materials by design. Finally, we note that optimal regime for operating the proposed modulated structure involves the presence of an attractive potential in the junction region, which can be easily generated using a top gate. This bypasses any potential uniformity requirement that may apply to the non-modulated structure. In addition, the low-field topological phase can be accessed by tuning the top gate potential without the need to apply a nonzero superconducting phase difference. This could significantly simplify the scaling of these devices and the design of multiqubit architectures. Of course, the proposed structure inherits the advantages of the JJ design with respect to the issues arising from strong SM-SC coupling, which can affect nanowire-type devices.

The rest of this section is organized as follows. In Sec. 6.2.1 we introduce the model of the modulated MJJ device, present our numerical techniques, and provide a qualitative discussion of the effects of the junction modulations. Secs. 6.2.2 and 6.2.3 presents and compares numerical results for nonmodulated and modulated systems. We discuss and summarize in section 6.2.4.

\subsubsection{Modeling}

We consider the hybrid system shown schematically in Fig. 6.16, which consists of a 2DEG hosted by a semiconductor quantum well (InAs) that is proximity coupled to a pair of s-wave superconductors (Al) separated by a narrow quasi-1D region, forming a Josephson junction (JJ). The width of the $\mathrm{JJ}$ is periodically modulated, which represents the key new ingredient of our proposed design. Specifically, the width of the junction region varies between two values $W_{1} \geq W_{2}$, where the constricted regions $\left(W_{2}\right)$ have length $\ell$ and the overall length of the unit cell is $L$. In this section we discuss the theoretical model used to describe the system and the method developed to efficiently solve the corresponding quantum mechanics problem. We also provide a qualitative characterization of the effects generated by the periodic modulation of the junction width, which represent key ingredients affecting the low-energy physics of the proposed device. 

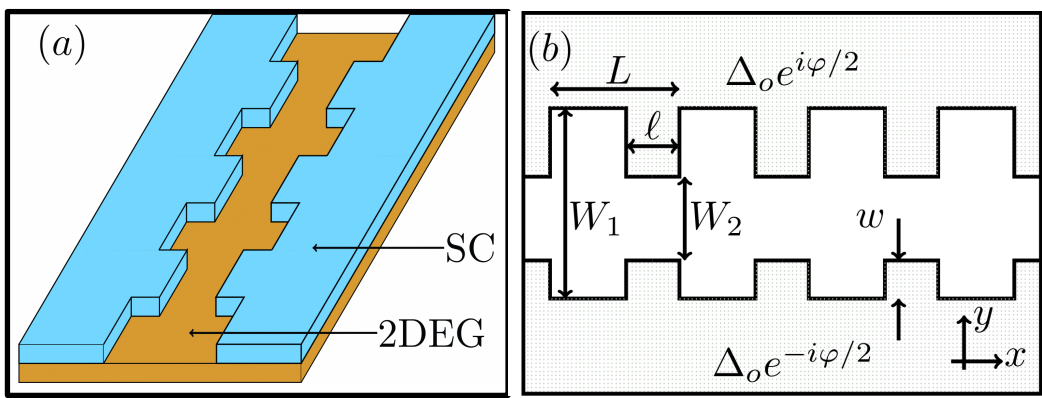

FIGURE 6.16: (a) Schematic representation of the proposed modulated Josephson junction device. A 2DEG hosted by a semiconductor quantum well (orange) is proximity-coupled to a pair of s-wave superconductors (light blue) separated by a quasi-1D, spatially-modulated junction region. (b) Top view of four unit cells of the periodic structure. There is a phase difference $\phi$ between the top and bottom superconductors. Proximitized regions are considered to be semi-infinite in the $y$-direction.

\section{Effective low-energy Hamiltonian}

We assume that the quantum well hosting the 2DEG is narrow, so that its low-energy physics is accurately captured by a single quantized mode along the $z$-direction (i.e., the direction perpendicular to the 2DEG plane). We model the effectively two-dimensional semiconductor-superconductor (SM$\mathrm{SC})$ heterostructure at the mean-field level using the Bogliubov-de Gennes (BdG) Hamiltonian,

$$
\begin{aligned}
H= & {\left[-\frac{h^{2}}{2 m^{*}}\left(\partial_{x}^{2}+\partial_{y}^{2}\right)-\mu+V(x, y)\right] \sigma_{o} \tau_{z} } \\
& -i \alpha_{R}\left(\partial_{x} \sigma_{y} \tau_{z}-\partial_{y} \sigma_{x} \tau_{o}\right)+\widetilde{E}_{Z}(x, y) \sigma_{x} \tau_{z} \\
& +\operatorname{Re}[\Delta(x, y)] \sigma_{y} \tau_{y}+\operatorname{Im}[\Delta(x, y)] \sigma_{y} \tau_{x},
\end{aligned}
$$

where $m^{*}$ is the SM effective mass, $\mu$ is the chemical potential, $V$ is the electrostatic potential, $\alpha_{R}$ is the Rashba spin-orbit coefficient, $\widetilde{E}_{Z}$ is the Zeeman energy, $\Delta$ is the induced SC pairing, and $\sigma_{j}$ and $\tau_{j}$, with $j=0, x, y, z$, are Pauli matrices acting on the spin and particle-hole spaces, respectively. Note that $V, \widetilde{E}_{Z}$, and $\Delta$ are position dependent quantities having a periodic dependence on $x$ (i.e., on the position along the junction) with period $L, V(x+L, y)=V(x, y)$ and similarly for $\widetilde{E}_{Z}$ and $\Delta$. Explicitly, the electrostatic potential is assumed to have the form

$$
V(x, y)= \begin{cases}V_{J}, & (x, y) \in \text { junction region, } \\ 0, & (x, y) \in \text { proximitized regions }\end{cases}
$$


where $V_{J}$ is the junction potential. We note that the potential $V_{J}$ in the junction region can be controlled using a top gate, which will not affect the electrostatic potential of the proximitized regions due to screening by the SCs. The Zeeman energy $\widetilde{E}_{Z}$, which is generated by applying a magnetic field along the $x$-direction, has a spatial dependence given by

$$
\widetilde{E}_{Z}(x, y)= \begin{cases}E_{Z}, & (x, y) \in \text { junction region, } \\ 0, & (x, y) \in \text { proximitized regions }\end{cases}
$$

Here, we assume that the junction region is characterized by a relatively large $g$-factor, while the effective $g$-factor within the proximitixed regions is strongly suppressed as a result of the renormalization generated by a strong SM-SC coupling [145]. For simplicity, we neglect the small Zeeman splitting within the proximitized regions. Finally, the induced SC pairing is assumed to have the form

$$
\Delta(x, y)= \begin{cases}\Delta_{0} e^{i \phi / 2}, & (x, y) \in \text { top SC region, } \\ 0, & (x, y) \in \text { junction region, } \\ \Delta_{0} e^{-i \phi / 2}, & (x, y) \in \text { bottom SC region, }\end{cases}
$$

where $\Delta_{o} \in \mathbb{R}_{0}^{+}$is the magnitude of the induced SC gap in the proximitized regions and $\phi$ is the phase difference between the top and bottom superconductors. A more detailed model would incorporate superconductivity in the proximitized regions through a self-energy [145], but we have assumed an energy independent pairing for simplicity.

We discretize the Hamiltonian in Eq. (6.41) using the finite difference method [218] on a square lattice with lattice constant $a_{x}=a_{y}=5 \mathrm{~nm}$ and solve numerically the corresponding $\mathrm{BdG}$ problem. The model parameters used in the calculation, $m^{*}=0.026 m_{e}, \alpha_{R}=200 \mathrm{meV} \AA$, and $\Delta_{o}=0.3 \mathrm{meV}$, are consistent with InAs/Al structures in the strong SM-SC coupling limit. We note that the effective SM parameters may be renormalized in a strongly confined 2DEG, but incorporating such effects would require a more complicated modeling, e.g., using an 8-band Kane model [219]. The discretized BdG Hamiltonian can be written in second quantized form as

$$
H=\sum_{R, R^{\prime}} \sum_{i, j} \sum_{\mu, v} \psi_{R, i, \mu}^{+} \mathcal{H}_{i \mu, j v}\left(R-R^{\prime}\right) \psi_{R^{\prime}, j, v}
$$

where $\psi_{R, i, \mu}^{\dagger}$ creates a fermion with spin/particle-hole index $\mu$ on site $i$ of the unit cell $R$, while $\mathcal{H}$ represents the corresponding Hamiltonian matrix. Note that the matrix elements are the same within every unit cell, as indicated by the $\left(R-R^{\prime}\right)$ dependence in Eq. (6.45). Due to this periodicity, we can use Bloch's theorem by introducing the representation

$$
\psi_{R, i, \mu}=\frac{1}{\sqrt{N_{x}}} \sum_{q} \widetilde{\psi}_{q, i, \mu} e^{-i q R}
$$


into Eq. (6.45). Note that in Eq. (6.46) $q \in[-\pi, \pi]$ represents the crystal momentum, which is restricted to the first Brillouin zone, and $N_{x}$ is the number of unit cells along the length of the system. The BdG Hamiltonian takes the form

$$
H=\sum_{q} \sum_{i, j} \sum_{\mu, v} \widetilde{\psi}_{q, i, \mu}^{+} \widetilde{\mathcal{H}}_{i \mu, j v}(q) \widetilde{\psi}_{q, j, v}
$$

where $\widetilde{\psi}_{q, i, \mu}^{\dagger}$ creates a fermion with spin/particle-hole index $\mu$ and crystal momentum $q$ on site $i$ of the unit cell. The Bloch Hamiltonian $\widetilde{\mathcal{H}}(q)$ is given by

$$
\widetilde{\mathcal{H}}_{i \mu, j v}(q)=\sum_{r} \mathcal{H}_{i \mu, j v}(r) e^{i q r} .
$$

Note that the Bloch Hamiltonian has (anti-unitary) particle-hole symmetry,

$$
\tau_{x} \widetilde{\mathcal{H}}^{*}(q) \tau_{x}=-\widetilde{\mathcal{H}}(-q),
$$

which is characteristic of all BdG Hamiltonians [16], but time-reversal symmetry is broken due to the applied magnetic field. The system therefore belongs to class $\mathrm{D}$ and is characterized by a $\mathbb{Z}_{2}$ topological invariant,

$$
Q=\operatorname{sign}(\operatorname{Pf}[X(0)] \operatorname{Pf}[X(\pi)])
$$

where Pf indicates the Pfaffian and $i X(q)$ is the imaginary skew-symmetric Hamiltonian matrix in the Majorana basis [16]. The trivial SC phase corresponds to $Q=+1$, while the topological superconducting phase corresponds to $Q=-1$ and, in a finite system, gives rise to Majorana zero modes (MZMs) localized at the ends of the junction. Note that the phase boundaries separating the topological and trivial phases correspond to $\widetilde{\mathcal{H}}(q)$ having zero eigenvalues (i.e., gapless states) at $q=0$ or $q=\pi$. The key questions that we address within this theoretical framework are: 1) Given a set of structural parameters (e.g., $W_{1}, W_{2}, \ell$, and $L$ ), what is the corresponding topological phase diagram as function of the control parameters, $\mu, E_{Z}, V_{J}$, and $\phi$ ? 2) Within the topological region of parameter space, what is the size of the topological gap? Of course, a larger topological gap indicates a more robust topological phase and, implicitly, more robust MZMs. Our main goal is to determine the impact of having a spatially-modulated junction width $\left(W_{1} \neq W_{2}\right)$ on the extent and robustness of the topological phase.

\section{Green's function formalism}

The hybrid system is perfectly well-defined by the Bloch Hamiltonian in Eqs. (6.47) and (6.48), but solving the corresponding quantum mechanics problem using a straightforward, brute force numerical procedure is at least challenging, or even impossible, if we assume that the proximitized regions are semi-infinite in the $y$-direction. We note that numerical calculations involving non-modulated structures (with $W_{1}=W_{2}$ ) do not face a similar challenge because the underlying problem is effectively one-dimensional and a scattering approach can be employed [198]. Alternatively, one can assume 
large, but finite, widths for the proximitized regions [197], which implies solving a one-dimensional lattice problem for a system with $N_{y}$ sites, where $a N_{y}$ is the total width of the system. By contrast, using a similar approach for the modulated system involves a finite lattice with $N_{L} \times N_{y}$ sites, where $L=a N_{L}$ is the length of the unit cell, and the numerical problem becomes significantly more costly. To address this challenge, we use a self-energy approach within the Green's function formalism [220,221] and integrate out the degrees of freedom associated with the large (possibly infinite) proximitized regions. See Appendix D for details regarding lattice Green's functions and the self-energy formalism. Here, we will simply sketch the basis approach. For quadratic Hamiltonians, the retarded Green's function is given by

$$
G(\omega, q)=(\omega-\widetilde{\mathcal{H}}(q)+i \eta)^{-1},
$$

where $\eta$ is an infinitesimally small positive energy that moves the poles of the retarded Green's function to the lower half of the complex plane. The reduced Green's function within the junction region is obtained by integrating out the proximitized regions. Specifically, we have

$$
G_{J}(\omega, q)=\left(\omega-\widetilde{\mathcal{H}}_{J}(q)-\Sigma_{S C}(\omega, q)+i \eta\right)^{-1}
$$

where the subscript $J$ indicates a quantity that is restricted to the junction region and $\Sigma_{S C}$ is the self-energy that captures the contribution of the two proximitized regions. The self-energy can be efficiently calculated numerically using the decimation method of Ref. [149], which takes advantage of the fact that couplings between layers normal to the JJ interface are independent of the layer index. Importantly, the topological index $Q$ can be calculated using the zero frequency Green's function. Furthermore, the topological phase diagram can be efficiently calculated using the energy-independent effective Hamiltonian,

$$
\widetilde{\mathcal{H}}_{J}^{e f f}(q)=\widetilde{\mathcal{H}}_{J}(q)+\Sigma_{S C}(0, q) .
$$

Indeed, the phase boundaries correspond to $G_{J}(0, q)$ having poles at $q=0$ or $q=\pi$, which is equivalent with $\widetilde{\mathcal{H}}_{J}^{\text {eff }}(q)$ having gapless modes at the corresponding values of $q$. Note that the problem is now numerically tractable since $\widetilde{\mathcal{H}}_{J}^{e f f}(q)$ only contains the junction degrees of freedom. Also note that $\Sigma_{S C}(0, q)$ is Hermitian since there exist no states within the SC gap for the isolated SC regions and, therefore, $\widetilde{\mathcal{H}}_{J}^{\text {eff }}(q)$ is also Hermitian. We also calculate the topological gap by finding the poles of Eq. (6.52) using the iterative method discussed in Appendix D.

\section{Qualitative effects of modulating the junction width}

Before presenting the results of our numerical scheme, we briefly discuss the effects of the periodic modulation of the junction width at a qualitative level, to highlight the underlying physics. Consider first the case of $V_{J}=0$, 
when there is no potential difference between the junction and the proximitized regions. In such a situation, the only difference a between modulated and a non-modulated structure (with $W_{2}=W_{1}$ ) arises from the changes in $\Delta(x, y)$ and $\widetilde{E}_{z}(x, y)$ within the region $|y|<W_{1} / 2$ associated with the modulated structure (see Fig. 6.16). The relevant question concerns the existence of geometric parameters (i.e., $\ell, L$, and $W_{2}$ ) consistent with significant deviations from the uniform system. To answer this question, we consider states with energies below the parent gap, $E<\Delta_{0}$, which are classically forbidden within the proximitized region and have a decay length (approximately) given by

$$
\xi=2 \sqrt{\frac{\hbar^{2}}{2 m^{*}} \frac{\mu}{\Delta_{o}^{2}-E^{2}}}=\frac{2 \mu}{k_{F} \sqrt{\Delta_{o}^{2}-E^{2}}},
$$

for $\mu \gg \Delta_{o}$. We expect modulation effects to be important if the weight of the wave function within the "additional" proximitized regions with $|y|<$ $W_{1} / 2$ (see Fig. 6.16) is comparable to the weight of the wave function within the "standard" proximitized regions $\left(|y|>W_{1} / 2\right)$ associated with the uniform system. Considering now, as an example, a system with $\mu=3 \mathrm{meV}$, $E=0$, and the InAs / Al model parameters given above, Eq. (6.54) yields $\xi \approx 440 \mathrm{~nm}$, which is much larger than the values of the geometric parameters considered in this work. This implies that for the uniform system most of the weight associated with the sub-gap state is already within the proximitized regions and that this weight will not change significantly by adding the constrictions. Therefore, we conclude that in the absence of a junction potential $V_{j}$ modulating the junction width has rather small effects, a conclusion that is confirmed by the numerical calculations. Similar considerations hold when the system is characterized by a positive junction potential, $V_{J}>0$. Again, the basic reason is that the relevant wave functions undergo negligible changes in the spatial distribution of their spectral weight upon introducing the "additional" proximitized regions, which translates into the emergence of a very weak effective periodic potential. We dub the regime characterized by $V_{J} \geq 0$ as the "potential barrier" regime. Based on the above considerations, we conclude that modulating the junction width has weak effects on the low-energy physics of a hybrid structure operating in the potential barrier" regime.

Next, we consider the effects of the modulation for a system with $V_{J}<0$, when the junction becomes a quantum well. The normal spectrum of a non-modulated structure in the absence of spin-orbit coupling and Zeeman splitting is shown in Fig. 6.17. Assuming that $\left|V_{J}\right|$ is sufficiently large, discrete bound states form within the junction with energies below the continuum of scattering states. Moreover, in the absence of spin-orbit coupling and modulation the $x$ and $y$ components of the problem can be separated. Consequently, the energies of the eigenstates are simply $E_{n, k}=\varepsilon_{n}+\frac{\hbar^{2} k^{2}}{2 m^{*}}$, where $k$ is the $x$ component of the momentum and $\varepsilon_{n}$ is the eigenenergy of the corresponding transverse mode. In addition, the wavefunction of the states take the simple form $\Psi_{n, k}(\mathbf{r})=\varphi_{n}(y) \exp (i k x)$, where $\varphi_{n}$ is the $k$ independent transverse wave function. If we now add superconductivity to 


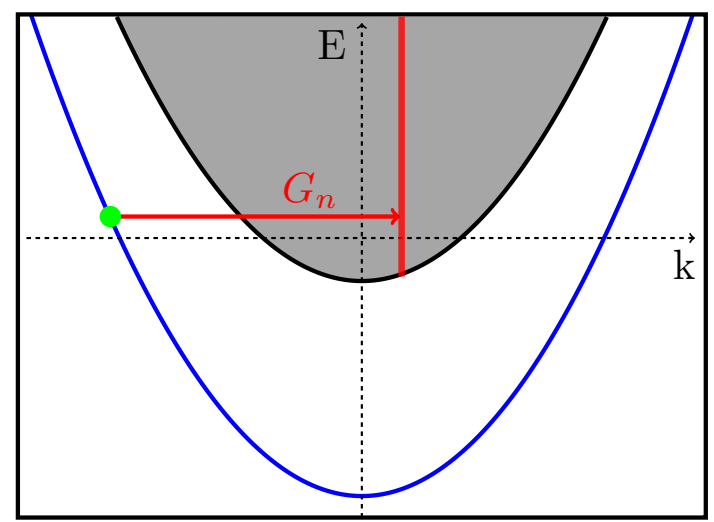

FIGURE 6.17: Spectrum of a non-modulated structure without spin-orbit coupling or Zeeman splitting with a sufficiently deep junction potential $V_{J}<0$ such that a bound state subband (blue line) forms with states heavily localized in the junction region. There also exists a continuum of scatter states (shaded grey region) that extend across the entire device. Upon the introduction of modulations, states with $k$ 's differing by a reciprocal lattice vector $G_{n}=\frac{2 \pi n}{L}$ couple due to the periodic potential. For example, the bound state indicated by the green dot couples to the continuum of scattering states in the red shaded region. This results in a "dressed" bound state with enhanced weight within the proximitized regions.

the non-modulated system, the bound state bands give rise to an extremely small induced gap, since they are heavily localized within the junction region where $\Delta=0$. Any topological phase would be extremely fragile in this regime (characterized by $V_{J}<0$ ), which we dub the "quantum well" regime. Based on these considerations, we conclude that the quantum well regime is basically useless for practical applications involving uniform structures.

However, introducing periodic modulations of the junction width breaks momentum conservation along the $x$-direction, i.e. $k$ is no longer a good quantum number, but, instead, we have a conserved crystal momentum $q$. This allows states of momentum $k_{1}$ and $k_{2}$ to couple through the periodic potential provided $k_{2}=k_{1}+G_{n}$, where $G_{n}$ is a reciprocal lattice vector defined by $G_{n}=\frac{2 \pi n}{L}$ with $n \in \mathbb{Z}$. As illustrated in Fig. 6.17, bound states with energies near the Fermi level can now couple to scattering states with much lower momentum generating "dressed" bound states that have finite weight within the proximitized regions. This strongly alters the previously gapless bound states in the vicinity of the Fermi level, which now acquire a significant induced gap from mixing with (gaped) scattering states. Moreover, the spectrum folds into the first Brillouin zone $(|q| \leq \pi / L)$ and the folded subbands associated with "dressed" bound states become viable for supporting a topological phase for sufficiently large $E_{Z}$. In addition, these folded subbands are characterized by renormalized effective parameters (e.g., effective mass and spin-orbit coupling strength), which can result in a significant increase the (effective) spin-orbit coupling and, consequently, an increase of 


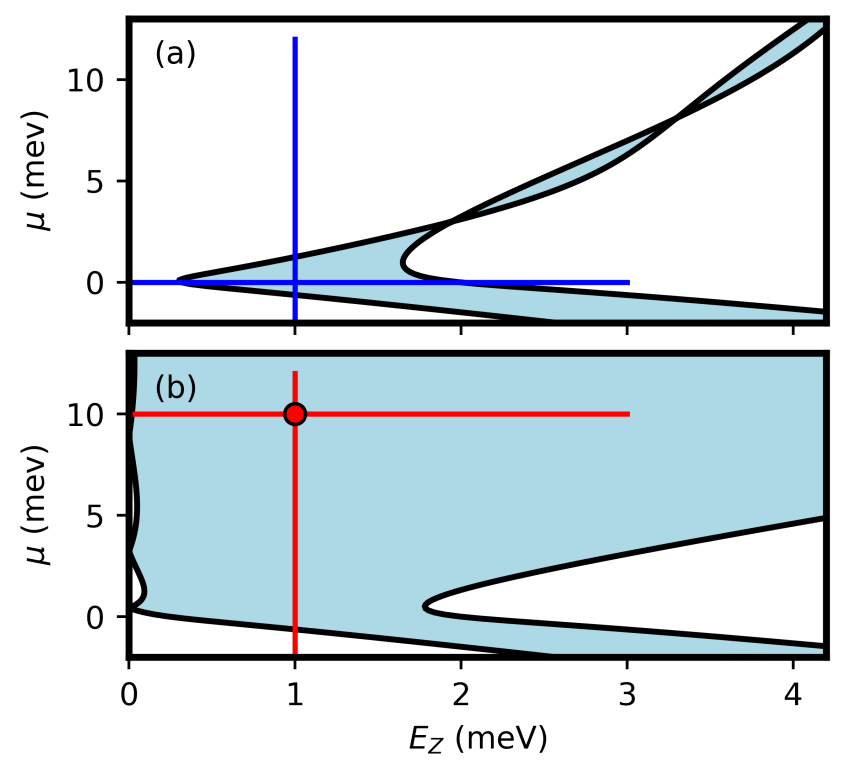

FIGURE 6.18: Phase diagram as a function of Zeeman energy and chemical potential for a uniform, non-modulated system of width $W_{1}=W_{2}=100 \mathrm{~nm}$, with $V_{J}=0$ and a superconducting phase difference (a) $\phi=0$ and (b) $\phi=\pi$. The shaded areas represent the topological superconducting phase. Note the substantial expansion of the topological phase within the low- $E_{Z}$ region in panel (b). The quasiparticle gap values along cuts corresponding to the blue and red lines are shown in Fig. 6.19 .

the topological gap [179]. This qualitative picture, which is confirmed by the numerical calculation presented in the next section, captures the key mechanism responsible for the "topological enhancement" that characterized our proposed modulated devices.

\subsubsection{Uniform Majorana Josephson Junction Results}

We first consider a uniform, non-modulated system, which provides reference results for evaluating the modulated structures. This case also illustrates some of the potential concerns about (uniform) Josephson junction structures, in particular regarding the size of the topological gap that can be realized in this type of system. An example of topological phase diagram as function of the Zeeman splitting $\left(E_{Z}\right)$ and chemical potential $(\mu)$ for a system of junction width $W_{1}=W_{2}=100 \mathrm{~nm}$ and superconducting phase difference (a) $\phi=0$ and (b) $\phi=\pi$ is shown in Fig. 6.18, panels (a) and (b), respectively. Note that the junction potential is set to zero, $V_{J}=0$. The phase diagrams are consistent with previous studies $[197,198]$. We note that the experimentallyrelevant regime corresponds to relatively low values of the Zeeman splitting (e.g., $E_{Z} \lesssim 2 \mathrm{meV}$ ), since applying large magnetic fields is detrimental to superconductivity inside the parent SC (i.e., the Al films that proximitize the 

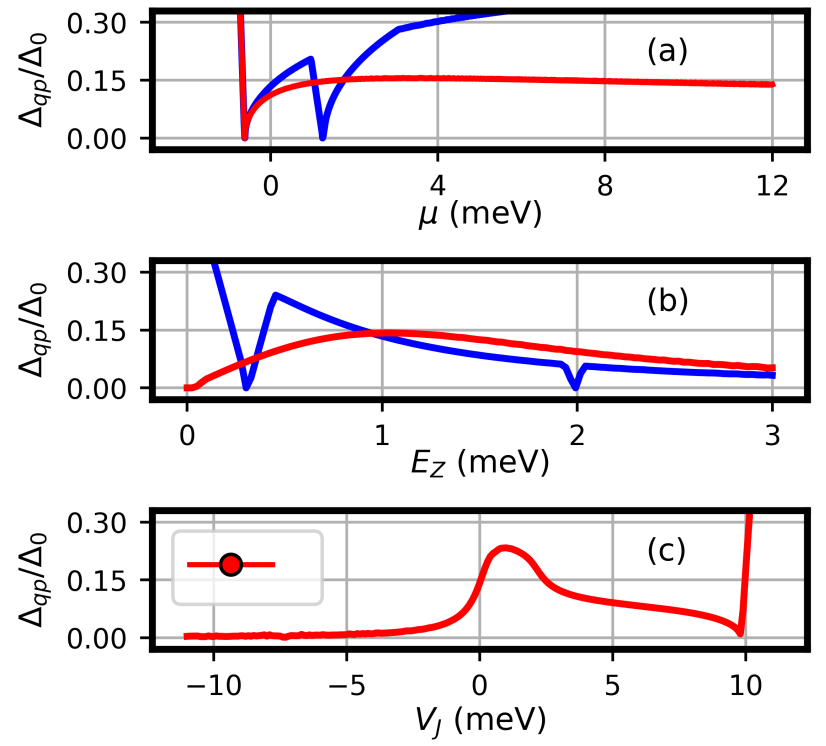

FIGURE 6.19: Quasi-particle gap along representative cuts marked by blue and red lines in Fig. 6.18 as functions of (a) chemical potential, $\mu$, and (b) Zeeman splitting, $E_{z}$. Blue lines correspond to $\phi=0$, while red lines correspond to a system with superconducting phase difference $\phi=\pi$. Panel (c) shows the dependence of the quasiparticle gap on the applied junction potential, $V_{J}$, for a system with parameters corresponding to the red dot in Fig. 6.18 (b). Note that the maximum topological gap for the uniform (non-modulated) system is $\Delta_{\text {top }} \approx 0.23 \Delta_{o}=0.07 \mathrm{meV}$.

2DEG) and, implicitly, to the size of the topological gap. As expected [197, 198], in the presence of a phase difference $\phi=\pi$ practically the entire the low-field region of the phase diagram with $\mu \gtrsim 0$ is covered by the topological superconducting phase. By contrast, the system with $\phi=0$ (i.e., no phase difference) is characterized by a single, relatively narrow topological lobe around $\mu \approx 0$. Additional small topological regions occur at higher $\mu$ and $E_{Z}$ values, but, because they are (mostly) outside the low-field regime, these regions are expected to have rather limited experimental significance. This behavior is due to the fact that the relevant low-energy states leak further into the proximitized regions as $\mu$ increases and, therefore, require larger values of $E_{Z}$ (which is nonzero only inside the junction region) to acquire the effective Zeeman splitting consistent with the emergence of topological superconductivity.

The quasi-particle gap $\Delta_{q p}$ (defined as the lowest positive eigenenergy of the bulk spectrum) corresponding to the cuts marked by blue lines in Fig. 6.18 (a) are shown as blue lines in Fig. 6.19 (a) and (b). Note that $\Delta_{q p}$ is the topological gap, $\Delta_{\text {top }}=\Delta_{q p}$, when the system is in the topological phase. The maximum topological gap along these representative cuts for the system 


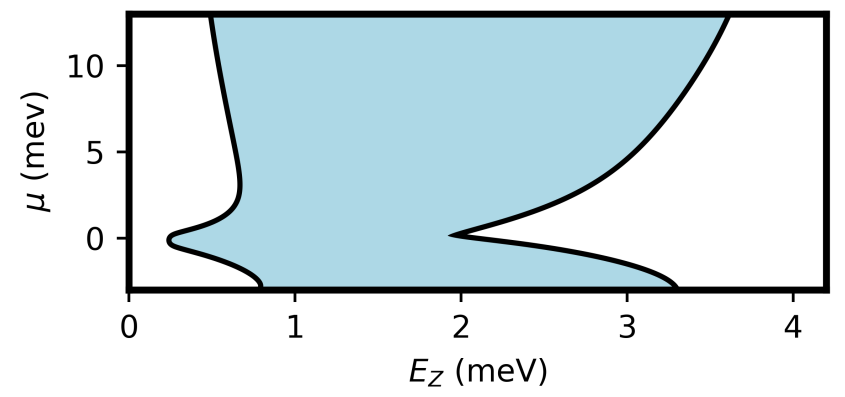

FIGURE 6.20: Topological phase diagram for a uniform system of width $W_{1}=W_{2}=100 \mathrm{~nm}$ with no superconducting phase difference $(\phi=0)$ and optimal junction potential, $V_{J}=V_{J}^{*}(\mu)$ (see main text). Note that the lowest critical Zeeman field in a system with finite chemical potential is always larger than the minimal value $E_{Z}=\Delta_{o}=0.3 \mathrm{meV}$, which corresponds to $\mu=0$.

with no phase difference $(\phi=0)$ is $\Delta_{\text {top }} \approx 0.23 \Delta_{o}=0.07 \mathrm{meV}$. The quasiparticle gap corresponding to the red lines in Fig. 6.18 (b), i.e., for a system with phase difference $\phi=\pi$, are shown as red lines in Fig. 6.19 (a) and (b). Note that, as a function of the applied Zeeman field, the topological gap has a maximum $\Delta_{\text {top }} \approx 0.15 \Delta_{o}=0.045 \mathrm{meV}$ at $E_{Z} \approx 1 \mathrm{meV}$ [see Fig. 6.19 (b)]. The dependence of this maximum value on $\mu$ is rather weak, as shown in Fig. 6.19 (a). The effect of a nonzero junction potential $V_{J}$ on the topological gap is shown in Fig. 6.19 (c) for parameters corresponding to the red dot in Fig. 6.18 (b). Note that for $V_{J}<0$ the topological gap quickly decreases toward zero. This is due to the formation of bound states localized almost entirely within the junction region, which are characterized by a small induced gap, as discussed in Sec. 6.2.1. The maximum of the topological gap is obtained for $V_{J} \approx 1 \mathrm{meV}$ and has a value $\Delta_{\text {top }} \approx 0.23 \Delta_{o}=0.07 \mathrm{meV}$ comparable to the maximum gap of the system with no phase difference $(\phi=0)$. Upon further increasing $V_{J}$ the system becomes non-topological near $V_{J} \approx 10 \mathrm{meV}$, when the junction region becomes depleted (i.e. $V_{J}>\mu$ ).

An important question is whether the low-field topological lobe characterizing the system with $\phi=0$ can be accessed by tuning $V_{J}$ when the system has a finite (possibly large) chemical potential. To address this question, we determine the value $V_{J}^{*}(\mu)$ of the junction potential that minimizes the critical Zeeman field for a given value of the chemical potential. We find that $V_{J}^{*}(\mu) \lesssim \mu$, i.e., the optimal $V_{J}$ is slightly smaller than the value of the chemical potential. The topological phase diagram as a function of $E_{Z}$ and $\mu$ for a system with optimal junction potential $V_{J}=V_{J}^{*}(\mu)$ is shown in Fig. 6.20. Note that the phase boundary shifts from a minimum $E_{Z}=\Delta_{0}=0.3 \mathrm{meV}$ at $\mu=0$ to larger values of the critical Zeeman splitting when $\mu \neq 0$, which can make accessing the topological phase more difficult. We emphasize that the experimentally-relevant situation corresponds to $\mu>0$, which implies that the minimal critical field (obtained by tuning $V_{J}$ ) in a system with no phase difference (i.e., $\phi=0$ ) is always larger than (but comparable to) $\Delta_{o}$. This 


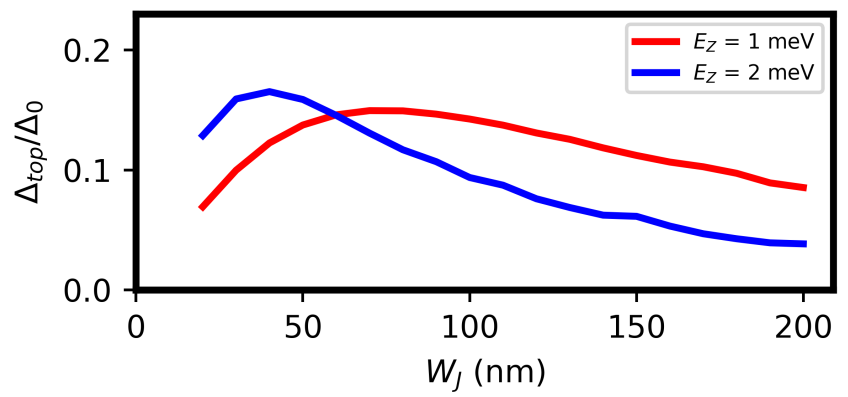

FIGURE 6.21: Dependence of the topological gap on the junction width $W$ for a uniform system with chemical potential $\mu=10 \mathrm{meV}$, phase difference $\phi=\pi$, and two values of the Zeeman field: $E_{Z}=1 \mathrm{meV}$ (red) and $E_{Z}=2 \mathrm{meV}$ (blue). Note that the dependence is non-monotonic. Reducing the width of the junction allows larger values of the maximum topological gap, but this requires larger Zeeman fields that may be outside the (experimentally-relevant) low-field regime.

analysis shows that, as far as the accessibility of the topological phase is concerned, there is no fundamental advantage of being able to apply a nonzero phase difference if the regime $E_{Z} \sim 1 \mathrm{meV}$ is experimentally accessible. In other words, there is no major difference between accessing the topological quantum states in Fig. 6.19(b) by tuning the phase difference and accessing the topological quantum states in Fig. 6.20 by tuning the junction potential. If, on the other hand, $E_{Z} \sim 1 \mathrm{meV}$ is outside of the low-field regime, having $\phi=\pi$ may be a significant advantage. However, this comes with the heavy price of a small topological gap (see Fig. 6.19).

While applying a phase difference $\phi=\pi$ can solve potential problems regarding the accessibility of the topological phase due to finite critical values of $E_{Z}$, there remains the key issue of the relatively small topological gap. Why is the (maximum) topological gap only a small fraction of $\Delta_{o}$ in nonmodulated systems? As pointed out in Ref. [198], the topological gap is expected to be large if $W \lesssim \sqrt{\hbar^{2} /\left(m^{*} \Delta_{0}\right)}$; otherwise, it is inversely proportional to the square of the junction width, $\Delta_{\text {top }} \propto W^{-2}$. For the values of the effective mass and induced gap used in this work, the large gap condition yield $W \lesssim 100 \mathrm{~nm}$, which suggests that a larger topological gap could be obtained by using narrower junctions. This motivates us to calculate the dependence of the topological gap on the junction width. Fig. 6.21 shows $\Delta_{\text {top }}$ as a function of the junction width for a uniform system with $\mu=10 \mathrm{meV}$, $\phi=\pi$ and two different values of $E_{Z}$. Note that the topological gap decreases with increasing the junction width for large-enough $W$ values, as expected based on the asymptotic $\Delta_{\text {top }} \propto W^{-2}$ behavior. Perhaps more surprising is the suppression of the gap in the narrow junction limit, $W \rightarrow 0$. This occurs because the effective Zeeman energy associated with the relevant lowenergy states is proportional to the spectral weight of the states within the junction, which is reduced as $W$ decreases. For small $W$ values, the topological gap will reach its maximum at a higher $E_{Z}$, which may be outside of the 
(experimentally-relevant) low-field regime. The maximum gap corresponding to a given junction width $W$ occurs at a value of the Zeeman splitting that increases with decreasing $W$. Consequently, reducing $W$ cannot be a practical solution to the problem of small topological gaps in uniform systems. Finally, we note that an enhancement of the topological gap could be obtained by significantly increasing the spin-orbit coupling strength [198]. Indeed, according to Ref. [198], the maximum topological gap in the narrow junction regime is $\Delta_{\text {top, } \max } \approx \Delta_{o} \sqrt{\alpha /\left(\hbar v_{F}\right)}$, where $\alpha$ is the spin-orbit coupling and $v_{F}=\sqrt{2 \mu / m^{*}}$ is the Fermi velocity. Unfortunately, $\hbar v_{F} \gg \alpha$ for realistic parameter values. We therefore find that reaching values of the maximum topological gap above $\Delta_{\text {top }} \approx 0.3 \Delta_{o}$ would be extremely difficult within any realistic parameter regime. In the next section we show that a practical solution to "artificially" enhancing the effective spin-orbit coupling involves a periodic potential created by modulating the width of the Josephson junction.

To summarize this subsection, we point out that the most appealing feature of the Josephson junction proposal for realizing topological superconductivity and MZMs - the extensive low-field topological phase emerging in the presence of a phase difference $\phi=\pi$ [see Fig. $6.18(\mathrm{~b})$ ] - is offset by serious limitations regarding the size of the topological gap. For the realistic parameters used in our calculation, the maximum topological gap is $\Delta_{\text {top }} \approx 0.23 \Delta_{o}=0.07 \mathrm{meV}$. Moreover, generating this gap requires not only a finite Zeeman field, $E_{Z} \approx 1 \mathrm{meV}$, but also tuning the Junction potential $V_{J}$ (see Fig. 6.19). However, if the regime $E_{Z} \approx 1 \mathrm{meV}$ is accessible and $V_{J}$ can be tuned, one can realize similar values of the topological gap in a system with no phase difference, by simply tuning the junction potential (see Figs. 6.19 and 6.20). In both cases the major problem is the relatively small topological gap and, consequently, the fragility of the topological phase and of the emerging MZMs. In the next subsection we explore the possibility of enhancing the robustness of the topological superconducting phase by engineering periodically-modulated structures.

\subsubsection{Modulated Majorana Josephson Junction Results}

In this subsection we present the numerical results for the proposed modulated Josephson junction structure and show that, in the absence of a superconducting phase difference (i.e., for $\phi=0$ ) the system operated in the quantum well regime $\left(V_{J}<0\right)(i)$ supports a low-field topological phase that covers a significant area of the phase diagram and (ii) is characterized by an enhanced topological gap that represents a substantial fraction of the induced gap $\Delta_{0}$. For completeness, we also consider the case $\phi=\pi$ and show that having a superconducting phase difference $\phi=\pi$ provides no practical advantage in a system with modulated junction width. We emphasize that the calculations presented in this section should be considered as "proof-ofconcept" examples. Note that we do not explicitly address the issue of optimizing the geometric parameters; this optimization task should be addressed 
in synergy with the materials growth and structure engineering efforts and should target specific materials and hybrid structures.

\section{Modulated Josephson junctions with no phase difference $(\phi=0)$}

Let us consider a two-dimensional semiconductor-superconductor hybrid system with no superconducting phase difference, $\phi=0$, in the presence of a spatial modulation characterized by the geometric parameters $W_{1}=$ $100 \mathrm{~nm}, W_{2}=20 \mathrm{~nm}, L=60 \mathrm{~nm}$, and $\ell=20 \mathrm{~nm}$. We assume that the system has reflection symmetry about the $y$-axis. A negative junction potential $V_{J}=-40 \mathrm{meV}$ puts the system into the quantum well regime. As discussed in Sec. 6.2.1 and as shown numerically in Fig. 6.19 (c), this negative junction potential would essentially produce a gapless spectrum in a uniform structure. However, modulating the junction width significantly enhances the induced gap. The corresponding phase diagram, as a function of $E_{Z}$ and $\mu$, is shown in Fig. 6.22. First, note that the phase diagram is quite complicated, especially at larger values of $E_{Z}$ (i.e., $E_{Z}>2 \mathrm{meV}$ ). This behavior is due to the presence of many folded subbands, which often cross giving rise to a rather atypical phase diagram. Nonetheless, the crucial point is that the low-field topological region of the phase diagram is dramatically enlarged as compared to the corresponding phase diagram of the non-modulated structure [see Fig. 6.18 (a)]. We emphasize that, unlike the uniform structure, in this modulated system the topological phase exists at low Zeeman energies, $E_{Z} \sim \Delta_{0}$, even for relatively large $\mu$ values. This substantial enhancement of the low-field topological region represents the first significant advantage of the modulated structures.

To asses the robustness of the topological phase, we calculate the topological gap along representative cuts through the parameter space, wich are shown as blue lines in Fig. 6.22. The results for the vertical cut (i.e., the dependence on the chemical potential $\mu$ for a fixed value of the Zeeman splitting $E_{Z}=1 \mathrm{meV}$ ) are shown in Fig. 6.23. For convenience, the low-field portion of the phase diagram shown in Fig. 6.22 is reproduced in panel (a), while panel (b) shows the topological gap along the vertical cut marked by the blue line. For simplicity, we do not plot the quasiparticle gap corresponding to the topologically-trivial superconducting phase. Note that the lowfield phase diagram, especially for $\mu>4 \mathrm{meV}$, is qualitatively similar to a "conventional" Majorana phase diagram for a multi-subband hybrid system [37]. In our case, the subbands responsible for the emergence of the topological lobes are actually "minibands" resulting from the folding of bound state bands into the first Brillouin zone associated with the periodic structure. The topological gap for a fixed value of the Zeeman field, $E_{Z}=1 \mathrm{meV}$, is shown in Fig. 6.23 (b). Note that the topological gap corresponding to the top two lobes is quite large, having peak values $\Delta_{\text {top }} \approx 0.47 \Delta_{o}$. This is a significant increase (by a factor of two) as compared to the uniform system, which has a maximum topological gap of $\Delta_{\text {top }} \approx 0.23 \Delta_{o}$. We note that this enhancement was not optimized with respect to the geometric parameters characterizing the modulated junction, or with respect to the applied junction potential $V_{J}$, 


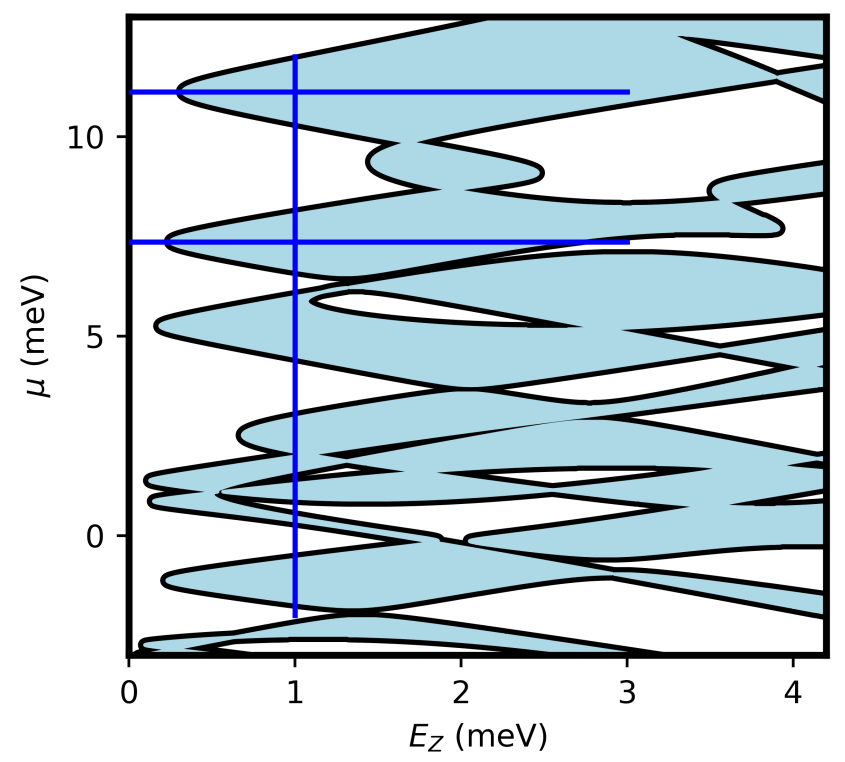

FIGURE 6.22: Phase diagram of a modulated structure with no superconducting phase difference $(\phi=0)$ as a function of Zeeman energy, $E_{Z}$, and chemical potential, $\mu$. The geometric parameters are $W_{1}=100 \mathrm{~nm}, W_{2}=20 \mathrm{~nm}, L=60 \mathrm{~nm}$, $\ell=20 \mathrm{~nm}$, and $w=40 \mathrm{~nm}$ (see Fig. 6.16). The junction potential is negative, $V_{J}=-40 \mathrm{meV}$, which generates several bound states within the junction region. As compared to the phase diagram for the uniform system [see in Fig. 6.18 (a)], the lowfield topological region is dramatically expanded; there exist several topological lobes at low $E_{Z}$ values, each associated with a different folded subband. The quasi-particle gap corresponding the parameter cuts indicated by the blue lines are shown in Figs. 6.23 and 6.24.

and is obtained at a value of the applied Zeeman field comparable to the (optimal) low-field values associated with the uniform system.

The enhanced topological gap exists over a larger range of Zeeman field, as shown in Fig. 6.24. Furthermore, the maximum value corresponding to the parameter range used in this calculation slightly exceeds $0.5 \Delta_{o}$ and is obtained at a relatively low field, $E_{Z} \approx 2.2 \Delta_{o}=0.66 \mathrm{meV}$. These results clearly show that modulating the junction width can lead to a substantial enhancement of the topological gap. The key physical mechanism responsible for the enhancement of the topological gap is associated with the increase of the effective spin-orbit coupling in the presence of a periodic potential [179]. More specifically, the high-order minibands (i.e., the minibands formed from subbands that have folded several times) are characterized by a substantially enhanced effective spin-orbit coupling [179], which, in turn, produces a large topological gap. Note that the lower-order minibands, which are responsible for the topological regions at smaller $\mu$ values, are characterized by a weaker enhancement of the effective spin-orbit coupling and, consequently, a weaker enhancement of the topological gap. On the other hand, further increasing 


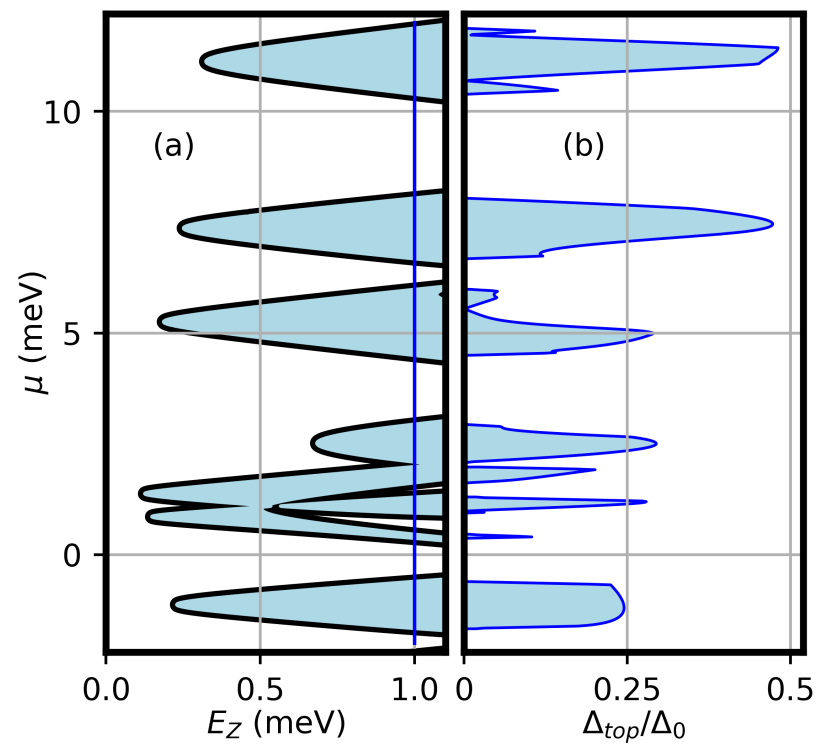

FIGURE 6.23: (a) Low-field phase diagram corresponding to the region $E_{Z}<1.1 \mathrm{meV}$ in Fig. 6.22. Note the presence of several topological lobes (shaded) with $E_{Z, \text { crit }} \sim \Delta_{0}$. (b) Topological gap as a function of the chemical potential $\mu$ for fixed $E_{Z}=$ $1 \mathrm{meV}$ [blue line in (a)]. The quasiparticle gap corresponding to the topologically-trivial phase is not shown. Note that the maximum topological gap approaches $0.5 \Delta_{0}$, which represents a significant increase as compared to the non-modulated structure (see blue lines in Fig. 6.19).

the chemical potential $(\mu>12 \mathrm{meV}$ ) leads to the emergence of topological lobes generated by even higher-order minibands, with extremely large effective spin-orbit coupling, which can push the maximum topological gap closer to $\Delta_{0}$.

The results presented in Figs. 6.22, 6.23, and 6.24 illustrate the main benefits of engineering hybrid structures with periodically modulated junction width: the emergence of multiple low-field topological lobes in the absence of a superconducting phase difference, $\phi=0$, and the enhancement of the topological gap. However, for a given structure the chemical potential $\mu$ is not an experimentally tunable parameter. In fact, $\mu$ is essentially determined by details of the 2DEG-SC heterostructure, such as materials properties, band-bending effects at the SM-SC interface [117, 126], and the strength of the effective SM-SC coupling [145, 168]. On the other hand, the junction potential $V_{J}$ is readily tunable using a top gate and, therefore, represents a relevant control parameter, along with the Zeeman field $E_{Z}$. In other words, the low-field topological lobes generated by the (high-order) minibands and characterized by large values of the topological gap should be accessed by tuning $V_{J}$, rather than the chemical potential. In other words, the experimentally-relevant phase diagram should be given in terms of the control parameters $E_{Z}$ and $V_{J}$. 


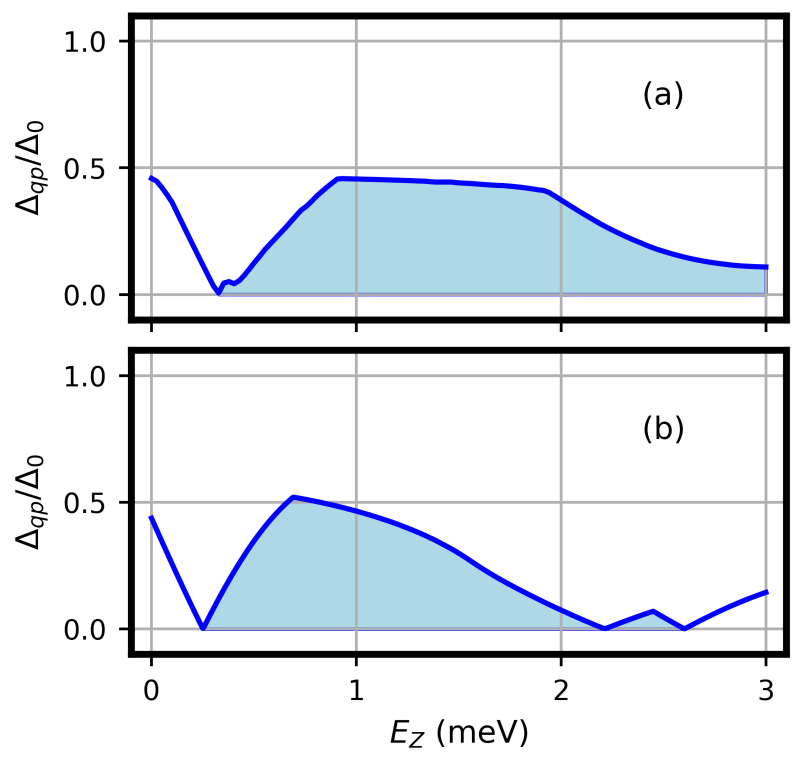

FIGURE 6.24: Quasi-particle gap as a function of Zeeman energy corresponding to the horizontal lines in Fig. 6.22 with: (a) $\mu=11.1 \mathrm{meV}$ and (b) $\mu=7.4 \mathrm{meV}$. Shading indicates the presence of a topological superconducting phase, i.e. $\Delta_{q p}=\Delta_{\text {top }}$. Note that the topological gap remains near its maximum value, $\Delta_{\text {top }} \approx 0.5 \Delta_{0}$, over a significant range of $E_{Z}$.

An example of a phase diagram as function of the Zeeman field, $E_{Z}$, and the applied junction potential, $V_{J}$, for a system with the same structure parameters as in 6.22 and a (fixed) chemical potential $\mu=10 \mathrm{meV}$ is shown in Fig. 6.25 (a). The phase diagram is characterized by several topological regions with low critical Zeeman field, $E_{Z} \sim \Delta_{0}$. As $V_{J}$ is tuned toward more negative values in the presence of a Zeeman field of order $E_{Z} \approx 1 \mathrm{meV}$, we sweep through several topological lobes characterized by large values of the topological gap [see Fig. 6.25(b)]. This corresponds to a series of topological quantum phase transitions that should be experimentallyobservable, provided disorder is low-enough. We also note the presence of a topological region for positive $V_{J}$ values (i.e., in the potential barrier regime) near $V_{J} \approx 5 \mathrm{meV}$. However, the correponding critical Zeeman energy is $E_{Z} \approx 1 \mathrm{meV}$, significantly larger than the lowest critical Zeeman energies in the quantum well regime $\left(V_{J}<0\right)$. This behavior is due a stronger localization of the low-energy states within the junction region in the quantum well regime as compared to the potential barrier case. In turn, since the Zeeman splitting is assumed to be small in the proximitized regions, this results in a larger effective Zeeman energy associated with a given value of $E_{Z}$ for the system in the quantum well regime $\left(V_{J}<0\right)$. Finally, we calculate the topological gap as a function of $V_{J}$ along the cut marked by a blue line in Fig. 6.25(a), which corresponds to $E_{Z}=1 \mathrm{meV}$. The results are shown in Fig. 6.25 (b). Note that several topological lobes are characterized by large maximum 


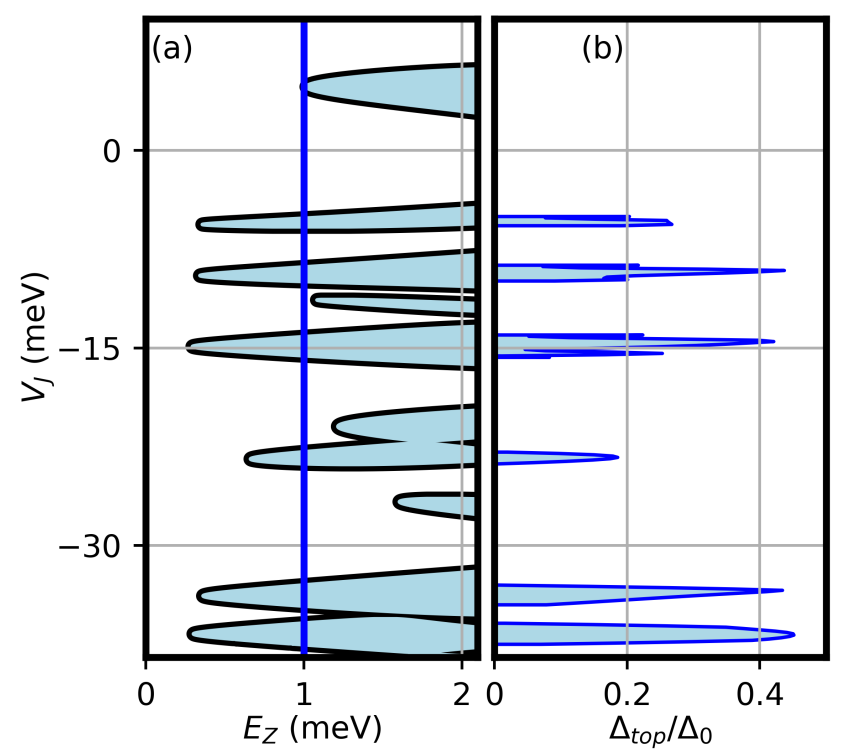

FIGURE 6.25: (a) Phase diagram of a modulated structure with the same parameters as in Fig. 6.22 and $\mu=10 \mathrm{meV}$ as function of the Zeeman field, $E_{Z}$, and the applied junction potential, $V_{J}$. (b) Topological gap along the cut marked by a blue line in (a), wich corresponds to a Zeeman field $E_{Z}=1 \mathrm{meV}$. The trivial quasiparticle gap is not shown.

values of the topological gap, $\Delta_{\text {top }} \approx 0.4-0.45 \Delta_{o}$, which represent a significant enhancement of topological gap as compared to the non-modulated system [see red lines in Fig. 6.19]. Most importantly, the results shown in Fig. 6.25 demonstrate that the enhanced topological regions generated by minibands with strong effective spin-orbit coupling emerging in the presence of a modulation-induced periodic potential can be conveniently accessed by tuning the junction potential $V_{J}$. We emphasize that tuning $V_{J}$ using a potential gate can be done much more efficiently than tuning the effective potential of a hybrid nanowire structure, because of minimal screening by the superconductor. Indeed, the "active region" in a nanowire is relatively close to the superconductor, which drastically limits the effectiveness of a potential gate. By contrast, the junction region is free from this limitation, which enables tuning $V_{J}$ within a large potential window and, consequently, exploring large regions of the parameter space. This is useful not only for optimizing the topological superconducting phase (by maximizing the topological gap), but also for investigating topological quantum phase transitions. Of course, the presence of disorder can seriously limit or completely destroy this physics. 


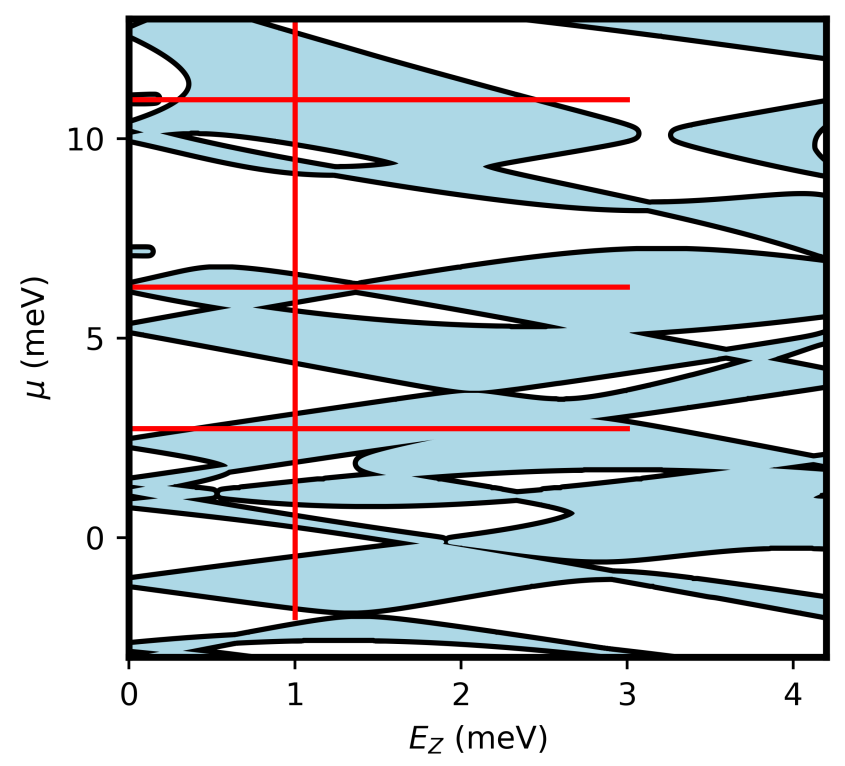

FIGURE 6.26: Phase diagram of a modulated structure with superconducting phase difference $\phi=\pi$ as a function of Zeeman energy, $E_{Z}$, and chemical potential, $\mu$. The geometric parameters are the same as in Fig. 6.22 and the junction potential is negative, $V_{J}=-40 \mathrm{meV}$. The quasiparticle gap calculated along the cuts marked by red lines is shown in Figs. 6.27 (b) and 6.28 .

\section{Modulated Josephson junctions with phase difference $(\phi=\pi)$}

Having demonstrated the enhancement of the topological gap in modulated structures with no superconducting phase difference $(\phi=0)$, the natural question regards the fate of topological superconductivity in the presence of a phase difference $\phi=\pi$. We note that, in general, modulating the junction with of a system operated in the potential barrier regime $\left(V_{J} \geq 0\right)$ generates no advantage with respect to the uniform structure (see discussion in Sec. 6.2.1) and, therefore, we focus on the quantum well regime. Fig. 6.26 shows an example of topological phase diagram as a function of $E_{Z}$ and $\mu$ for a system with the same parameters as in Fig. 6.22 and phase difference $\phi=\pi$. Similarly to the non-modulated system in Fig. 6.18, the phase boundary shifts toward lower values of the Zeeman field, reaching $E_{Z}=0$ at certain values of the chemical potential. The phase diagram exhibits an alternation of topological and trivial phases as the chemical potential is varied in the presence of a finite Zeeman field. Note that the ranges of $\mu$ where the phase boundary approaches $E_{Z}=0$ correspond to the low $E_{Z}$ topological lobes in Fig. 6.22, suggesting that they are associated with the presence of modulation-induced minibands.

Next, we calculate the size of the quasiparticle gap along representative cuts corresponding to the red lines in Fig. 6.26. It is important to emphasize that the system is deep inside the quantum well regime, $V_{J}=-40 \mathrm{meV}$, and that the corresponding uniform system would be essentially gapless, as 




FIGURE 6.27: (a) Low-field phase diagram corresponding to the region $E_{Z}<1.1 \mathrm{meV}$ in Fig. 6.26. (b) Topological gap along the cut marked by the red line in (a), which corresponds to a Zeeman field $E_{Z}=1 \mathrm{meV}$. The trivial quasiparticle gap is not shown. Note that the topological gap is characterized by a huge enhancement as compared to the gap of a uniform system in the quantum well regime $\left[V_{J}=-40 \mathrm{meV}\right.$; see Fig. 6.19 (c)]. However, the gap values are strongly suppressed in comparison to those of a the modulated system with no phase difference [see Fig. $6.23(b)]$.

clearly shown in Fig 6.19 (c). By contrast, the topological gap characterizing the modulated system with $\phi=\pi$ is finite, although significantly smaller that the corresponding gap in the absence of a phase difference (see Figs. 6.23 (b) and 6.24). The results corresponding to the vertical cut are shown in Fig. 6.27, with panel (a) reproducing the low-field region of the phase diagram and panel (b) showing the dependence of the topological gap on the chemical potential for $E_{Z}=1 \mathrm{meV}$. Note that the maximum value of the topological gap is $\Delta_{\text {top }} \approx 0.1 \Delta_{o}$, with typical values of the order $\Delta_{\text {top }} \approx 0.01-$ $0.03 \Delta_{o}$. In addition, by contrast with the system with no phase difference [see Figs. 6.23 (b) and 6.24], the typical gap tends to decrease with the chemical potential. This trend is confirmed by the results in Fig. 6.28, which shows the dependence of the quasiparticle gap on the Zeeman field along the horizontal cuts marked by red lines in Fig. 6.26.

\subsubsection{Summary and conclusions}

In summary, we have explored an alteration of the planar Majorana Josephson junctions in which the junction width is periodically modulated. We have found that the modulated structures offers several advantages over 


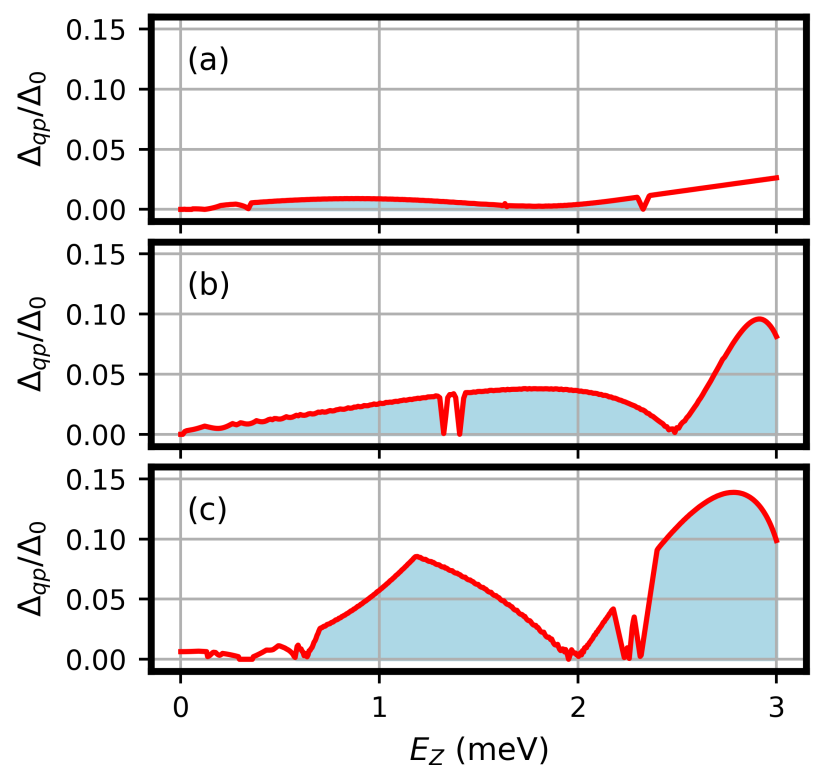

FIGURE 6.28: Quasi-particle gap as a function of $E_{Z}$ along the horizontal cuts marked by red lines in in Fig. 6.26. The chemical potential values are: (a) $\mu=11.0 \mathrm{meV}$, (b) $\mu=6.3 \mathrm{meV}$, and (c) $\mu=2.7 \mathrm{meV}$. Shading indicates the presence of a topological superconducting phase. The topological gap is massively enhanced in comparison to the corresponding gap of a uniform system (in the quantum well regime) [see Fig. 6.19(c)], but is significantly smaller that the gap of the modulated system with no phase difference (Fig. 6.24).

non-modulated structures; 1) The topological region in parameter space is dramatically expanded. This solves the issue of needing small chemical potentials in systems without SC phase bias $\phi=0$ as well as removes the need for a nearly uniform potential in both biased and unbiased systems as one goes from the proximity regions to the junction region. Indeed, we have found that the modulated structure should be operated in the quantum well regime where the junction region has a lower potential than the proximitized regions, forming bound states in the junction. This should be easily attained with a top gate since the junction region of the 2DEG is not electrostatically screened from above. 2) The topological gap is enhanced in modulated structures in comparison to their non-modulated counterparts. This occurs due to an enhanced effective spin-orbit coupling of mini bands deriving from the bound state subbands of the junction region that have folded several times in momentum space. A more detailed explanation of the spinorbit enhancement in periodic Majorana structures can be found in our previous work [179]. Note that the absence of phase bias across the junction, $\phi=0$, is required in the modulated structure to achieve larger topological gaps. Indeed, we found that the topological gap is enhanced for $\phi=0$ but remains relatively unchanged when $\phi=\pi$. Also note that we have not optimized the structure for the topological gap, but rather wished to illustrate the 
basic principle of topological gap enhancement with the addition of junction width modulations. It seems likely that the topological gap can be further enhanced than what was illustrated here by reaching higher-order minibands by making $V_{J}$ even more negative or increasing $\mu$. Future work will address such issues. We note that it's unlikely that the topological gap can be significantly increased in the non-modulated structure with phase bias due to the intrinsically large fermi velocity of InAs at even moderate chemical potentials. Moreover, note that the modulated structure achieves topological gaps of higher fraction of the parent gap than the altered planar structures with phase bias [203]. 3) The device design overcomes the issues of the strong SM-SC coupling regime. This is an important property, as most InAs/Al experimental devices, both nanowires and planar structures, appear to be in this regime, which suppresses the effective g-factor and spin-orbit coupling if not dealt with. Moreover, it removes the engineering requirement of tuning the SM-SC coupling. This should simplify the growth process since growers can focus solely on creating a clean SM-SC interface without having to worry about such things as barrier layers to reduced the SM-SC coupling. This also may expand the materials combinations that can be explored for these structures. For example, $\mathrm{Pb}$ was recently grown epitaxially on InAs nanowires [88]. Importantly $\mathrm{Pb}$ is able to withstand a very large magnetic field without SC being destroyed. In the nanowire experiment, however, the SM-SC coupling is clearly in the strong-regime since the bulk gap of the device never closes, even with fields up to $8 \mathrm{~T}$ ! The topological phase will then not be achieved in InAs/ $\mathrm{Pb}$ nanowires unless the SM-SC coupling is significantly reduced. Provided $\mathrm{Pb}$ can be grown cleanly on InAs, our device design overcomes this strong coupling issue. One then may be able to achieve much larger topological gaps than current InAs/Al devices. This of course increases the robustness of the topological phase against disorder. Note that SCs with larger gaps do not automatically provide significant enhancement of the topological gap of non-modulated structure with phase bias. In fact, they place stricter requirements on the junction width, as well as increase the needed Zeeman energy to reach a significant topological gap.

The most significant potential issue facing the realization of modulated Josephson junction devices concerns the lithography requirements to etch the modulations of the junction geometry. While this may be a challenging engineering and materials growth problem, we estimate that, in the context of a growing interest for nanotechnologies, it is likely that precision lithography will make significant progress in the coming years, making modulated structures feasible and more attractive. Future theoretical work should address the problem of optimizing the geometric features of the device within the limitations imposed by lithography and investigate the effect of geometric imprecision associated with lithography, which may represent a significant source of disorder. Finally, we note that a number of previous studies have investigated the effects of various types of periodic alterations of the "basic" Majorana structures, for both nanowires [62, 128, 208] and planar systems $[179,203,207]$. While significantly different from our proposal, these works have also found certain benefits of adding periodic alterations to the uniform 
structure, which suggests that this type of design deserves further attention.

Lastly, we wish to comment on the bare spin-orbit coupling coefficient. For simplicity we assumed a constant Rashba coefficient $\alpha_{R}$ independent of other parameters. In reality, the spin-orbit coupling strength depends on the electrostatic details, which could be incorporated in a more detailed model of the device [46]. The quantum well regime in which we propose to operate the modulated Josephson junctions likely has a larger bare spin-orbit parameter $\alpha_{R}$ compared to the ideal case of $V_{J}=0$ for the non-modulated structures due to a stronger electric field in the junction region. Therefore we expect that there will be an additional enhancement of the topological gap from the increased bare spin-orbit coefficient which is independent from the topological gap enhancement illustrated in this paper coming from subband folding.

\subsubsection{Acknowledgments}

This work of this section was supported by NSF Grant No. 2014156. The author acknowledges the contributions from Trey Cole and Purna Paudel for the contents of this section. 


\section{Summary and Outlook}

In this thesis, I have presented several theoretical studies on Majorana nanostructures with a focus on understanding and manipulating the electrostatic environment of realistic devices. These studies include the development of self-consistent solvers for the Schrödinger-Poisson equations, which were subsequently applied in chapters 4 and 5 to various problems currently of importance to the community attempting to achieve MZMs in semiconductorsuperconductor (SM-SC) nanostructures. I have also introduced in chapter 6 two original device designs in planar SM-SC nanostructures with periodic modulations of the SC components leading to an increased robustness of the topological superconducting phase and resulting MZMs.

The first example application of our self-consistent Schrödinger-Poisson formalism was studying the effects of the electrostatics on subband occupation in SM-SC hybrid nanowires. For moderate values of the band-bending at the SM-SC interface, we found that it is unlikely that the few-occupied subband regime can be reached before the onset of holes from the valence band. In the many-occupied subband regime, the typical energy separation between subbands is reduced and cause the system to be more susceptible to disorder effects. This problem was shown to be further exacerbated with the addition of positive surface charge known to be present for InAs nanowires, stressing the importance of making material improvements that prevent the charge accumulation on the exposed surfaces of the InAs nanowires. Furthermore, the presence of surface charge was shown to decrease the spinorbit coupling of subbands near the Fermi level due to a decreased electric field across the profile of the nanowire.

In the second application of our formalism, we studied the effects of the electrostatic environment on the magnetic proximity effect in SM-SC-magnetic insulator (MI) heterostructures. The scenario in which the magnetic proximity effect occurred solely through the coupling of SC to the MI was shown to be unlikely, if not impossible. We then found that the strength of the magnetic proximity effect coming from the coupling between the SM and MI is heavily dependent on the geometric layout of the MI and SC components. Essentially, a structure with overlapping MI and SC components has an increased magnetic proximity effect compared to the non-overlapping structure due to the attractive electric field caused by the presence of the SC on top of the MI. Indeed, we found the setup with overlapping MI and SC components may give rise to topological superconductivity for reasonable Zeeman splittings in the MI, while the non-overlapping structure cannot. Note that this is 
consistent with the recent experiment in Ref. [119].

In chapter 5, I turned toward the issue of disorder arsing from charge impurities in the SM nanowire. We first calculated the self-consistent potential arising from a single charge impurity in the $\mathrm{SM}$, including the screening effects of the SC, backgate, and redistribution of free charge around the impurity. The typical amplitude and length scale characterizing the effective perturbation potential arising from the impurity was found to be $\approx 1-5 \mathrm{meV}$ and $\approx 10-20 \mathrm{~nm}$, respectively. Note the energy scale of MZM physics is set by the superconducting gap, which is typically $\Delta \approx 0.2-0.3 \mathrm{meV}$ when aluminum serves as the SC. Therefore, a perturbation to the effective potential coming from a charge impurity is non-negligible in a Majorana device. Indeed, we then studied systems of finite length and found that to consistently achieve well-separated MZMs occurring at the two edges of the system the charge impurity concentration needs to be $n \lesssim 10^{15} \mathrm{~cm}^{-3}$. Based on transport measurements, the current samples likely have charge impurity concentrations several orders of magnitude larger than this threshold. Importantly, however, impurity levels in SMs can in principle made much lower and have actually been achieved in GaAs samples.

Potential non-uniformities in multi-gate nanostructures were also studied in chapter 5 . We showed that inter-subband coupling due to this type of non-uniformity can give rise to trivial ABSs that become pinned near zeroenergy, mimicking much of the local phenomenology of MZMs. This intersubband coupling acts essentially as disorder in the system. Additionally, the effects become more pronounced when many subbands are occupied with small energy-separation between them, which connects to our work on subband occupation previously discussed. Finally, the inter-subband coupling mechanism can occur due to non-uniformities on a much smaller length scale $(\approx 100-200 \mathrm{~nm})$ than other mechanisms, such as soft confinement, that give rise to topologically trivial ABSs.

Finally, in chapter 6 I presented results involving planar SM-SC nanostructures, which serve as an alternative to the "conventional", well-studied hybrid nanowire systems. More specifically, I introduced two original designs of planar SM-SC nanostructures in which the SC in periodically modulated. The first design involved a two-dimensional electron gas (2DEG) proximitized to a quasi-1D SC strip with periodically modulated width. A top gate depletes the 2DEG outside the proximitized region, leaving a quasi-1D channel acting in the same role as the quasi-1D nanowire setups. The periodic modulations of the SC width give rise to an effective periodic potential. In turn, this produces mini-bands with renormalized parameters, including dramatically increased spin-orbit coupling and diminished effective mass, which significantly enhance the topological gap and robustness against disorder. The geometry of the second design is essentially the inverse of the first; Instead of a quasi-1D SC strip with an adjacent 2DEG, the second design had two large proximitized region with a narrow unproximitized junction region in between, forming a Josephson junction (JJ) with periodically modulated width along its length. In contrast to the first design, however, the unproximitized 2DEG is not depleted but serves as a potential well in which bound 
states form. The periodic modulations again give rise to mini-bands with enhanced spin-orbit coupling. Additionally, the periodic modulations are also responsible for increasing the induced superconducting gap of the bound states localized in the junction from practically zero in the absence of modulations to a significant fraction of the gap of the parent superconductor. In addition to increasing the topological gap, this second setup overcomes the issue of strong SM-SC coupling that, if present, dramatically suppress the gfactor and spin-orbit coupling of our first design and "conventional" SM-SC nanowires.

The results of this thesis provide motivation for many future avenues of research in SM-SC Majorana nanostructures. For example, the SchrödingerPoisson equations were self-consistently solved for all of the results of this thesis under the assumption that the SM-SC coupling is small, such that the SC does not enter explicitly into the device Hamiltonian. Note that the SC still plays a crucial role in the electrostatics of our models due to the bandbending near the SM-SC interface. Nonetheless, our formalism can be extended to include the SC explicitly in the Hamiltonian. This can be done in a brute-force fashion by simply extending the effective mass Hamiltonian into the SC. This has been done by other researchers, but increases the necessary computational resources by a large factor and makes the physics somewhat opaque. Rather, I envision a more illuminating method in which the SC is taken into account with a self-energy in a Green's function approach. The quantity of interest is the retarded Green's function restricted to the SM,

$$
G_{S M}(\omega)=\left(\omega-H_{S M}-\Sigma_{S C}(\omega)\right)^{-1},
$$

where $H_{S M}$ is the Hamiltonian of the isolated SM and $\Sigma_{S C}$ is the self-energy due to the SC. At first sight, this does not seem to be an efficient approach since the Green's function $G_{S M}$ is needed for a very large number of $\omega$ values to accurately calculate the density of states and charge density in the SM. Indeed, the self-energy $\Sigma_{S C}$ is strongly dependent on $\omega$ near the Fermi level due to superconductivity. Note, however, that this strong $\omega$ dependence only occurs over an energy scale of the superconducting gap $\Delta_{o}$ of the parent superconductor. This is a much smaller energy scale than the energy scales important to the Schrödinger-Poisson, which is typically tens of meV's. From the perspective of the electrostatic potential, the SC can be therefore be well approximated as a normal metal. Moreover, the large chemical potential of the SC implies that the self-energy $\Sigma_{S C}$ is weakly dependent on $\omega$ over the energy range important for the calculation of the charge density in the SM. ${ }^{1}$ Treating the self-energy $\Sigma_{S C}$ in a static approximation, $\Sigma_{S C}(\omega) \rightarrow \Sigma_{S C}^{\text {static }}$, then yields an energy-independent, effective Hamiltonian for the SM. ${ }^{2}$ This effective Hamiltonian will non-Hermitean, which will cause the density of states peaks to broaden, but the formalism of chapter 4 can be straightforwardly adapted to such a change.

\footnotetext{
${ }^{1}$ When treating the superconductor as a metal

${ }^{2}$ The energy-independent, effective Hamiltonian takes the form $H_{\text {eff }}=H_{S M}+\Sigma_{S C}^{\text {static }}$.
} 
Another avenue of research that the results of this thesis motivate is the study of forms of disorder in SM-SC nanostructures and their effects on Majorana physics other than charge impurity disorder, which was presented in chapter 5 . It should be once again stressed that disorder and other device non-uniformities is likely the key issue inhibiting the consistent achievement of MZMs in experimental devices. It is therefore important to understand the various sources of disorder and their expected magnitudes and length scales as much as possible. For example, in addition to causing high subband occupation, the surface charge occurring on the exposed facets of InAs wires also likely causes fluctuations in the potential along the length of the wire due to a non-uniform distribution of surface charge. What level of potential fluctuations should be expected from such surface charge fluctuations? Apriori, it is not obvious, but a Schrödinger-Poisson calculation along the lines of the charge impurity calculations presented in chapter 5 should provide an estimate. ${ }^{3}$ Another source of disorder is oxidation at the surface of the SC. Previous work [177] indicates that weak disorder in the SC is non-detrimental to Majorana physics, but what level of disorder can be tolerated? It is known that disorder in the SC explains the large induced gap experimentally observed [116], but does the disorder strength necessary for a hard induced gap cause significant fluctuations to the effective potential in the SM? Again, a Schrödinger-Poisson calculation using our formalism (with the extension to explicitly include the SC in the Hamiltonian) should be able to answer this question. Finally, disorder in the nanowire may also come from stacking faults or dislocations during the growth process. While it is not yet clear how to best incorporate such lattice imperfects into the low-energy effective Hamiltonian, the resulting perturbations should be able to be efficiently incorporated into our effective 1D multi-orbital model described in Sec. 5.2 of chapter 5.

Finally, the work presented in chapter 6 on planar SM-SC Majorana nanostructures with periodic modulations should motivate further research within the community. These works clearly show the possible benefits of periodic modulations, which includes increasing spin-orbit coupling, the topological gap, and the robustness against disorder, soft-confinement, and other non-uniformities. While the designs presented in this thesis show promise, further improvements are likely possible. For example, both designs required modulations of the superconductor with precision on the order of nm's, which may be challenging with current lithography techniques. Can this be circumvented through the use of designs in planar SM-SC Majorana nanostructures with periodic arrays of top gates instead of modulating the SC? Alternatively, maybe a clever choice of geometry of the periodic modulations of the superconductor can allow for modulations to occur on a larger length scale, reducing the required precision of the device lithography.

It is unclear when and if quantum computation will become important to the human story. To say the least, humanity is still a long ways away from

\footnotetext{
${ }^{3}$ We are currently performing this type of calculation to provide an estimation of the potential fluctuations from surface charge fluctuations.
} 
large-scale quantum computation. Additionally, it is unknown what platform will win out in the competition to be the most practical and powerful for large-scale quantum computation. Nonetheless, the possibilities brought about through quantum computation make its pursuit exciting and clearly worthwhile. Furthermore, the benefits of topological quantum computation make Majorana zero modes a promising avenue for continued research. The community continues to make significant advances towards consistently and reliably achieving MZMs in SM-SC nanostructures, maintaining the hope that topological qubits composed of MZMs will one day become a reality. 


\section{Appendix A}

\section{Integration over elements in finite element method}

In the finite element method, we confront the repeated task of calculating integrals over the elements of the mesh. In this appendix, we show how to perform these integrals in a systematic way such that automation because easy to implement. We do this for both 1D and 2D elements.

\section{A.1 Integrals over 1-dimensional elements}

In 1 dimension, the elements of the mesh are simply line segments. Each endpoint or vertex of the line segment is associated with a linear basis function, which we label as $g_{1}(x)$ and $g_{2}(x)$ for the basis function associated with the left and right vertices, respectively. Within the line segment of this element, each of these linear basis functions can be written in the form

$$
g_{j}(x)=a_{j}+b_{j} x,
$$

where $a_{j}, b_{j} \in \mathbb{R}$ are constant coefficients. Applying the condition $g_{j}\left(x_{l}\right)=$ $\delta_{j, l}$, where $x_{l}$ is the $\mathrm{x}$-coordinate of the $l^{\text {th }}$ vertex of the element, the coefficients are found to be

$$
\begin{aligned}
& a_{1}=\frac{x_{2}}{x_{2}-x_{1}}, \\
& b_{1}=\frac{-1}{x_{2}-x_{1}}, \\
& a_{2}=\frac{-x_{1}}{x_{2}-x_{1}}, \\
& b_{2}=\frac{1}{x_{2}-x_{1}} .
\end{aligned}
$$

We are then free to use the generic form of $g_{j}$ found in Eq. (A.1) when performing integrals. We often come across integrals with integrands proportional to $x^{n}$, where $n \in \mathbb{Z}$. It is therefore useful to define

$$
\left\langle x^{n}\right\rangle \equiv \int_{x_{1}}^{x_{2}} x^{n} d x= \begin{cases}\frac{1}{n+1}\left(x_{2}^{n+1}-x_{1}^{n+1}\right), & n \neq-1 \\ \ln \left(\frac{x_{2}}{x_{1}}\right), & n=-1\end{cases}
$$


The matrix elements of $x^{n}$ are then found to be

$$
\begin{aligned}
\left\langle j\left|x^{n}\right| l\right\rangle & \equiv \int_{x_{1}}^{x_{2}} g_{j}(x) x^{n} g_{l}(x) d x \\
& =a_{j} a_{l}\left\langle x^{n}\right\rangle+\left(a_{j} b_{l}+a_{l} b_{j}\right)\left\langle x^{n+1}\right\rangle+b_{j} b_{l}\left\langle x^{n+2}\right\rangle,
\end{aligned}
$$

which can be seen to be symmetric, $\left\langle j\left|x^{n}\right| l\right\rangle=\left\langle l\left|x^{n}\right| j\right\rangle$. Note that the overlap matrix elements are simply the $n=0$ version of the above integral. What about integrals involving derivatives? The $\hat{k}_{x}$ momentum operator has matrix elements

$$
\begin{aligned}
\left\langle j\left|\hat{k}_{x}\right| l\right\rangle & \equiv-i \int_{x_{1}}^{x_{2}} g_{j}(x) \partial_{x} g_{l}(x) d x \\
& =\frac{-i}{2} \int_{x_{1}}^{x_{2}} g_{j}(x) \frac{\partial g_{l}(x)}{\partial x} d x+\frac{i}{2} \int_{x_{1}}^{x_{2}} \frac{\partial g_{j}(x)}{\partial x} g_{l}(x) d x \\
& =\frac{-i}{2}\left(a_{j} b_{l}-a_{l} b_{j}\right)\langle 1\rangle,
\end{aligned}
$$

where $\langle 1\rangle=\left\langle x^{o}\right\rangle=x_{2}-x_{1}$. Note that we used integration by parts in the second line of Eq. (A.8) such that the matrix representation of $\hat{k}_{x}$ is obviously Hermitean, i.e. $\left\langle j\left|\hat{k}_{x}\right| l\right\rangle=\left\langle l\left|\hat{k}_{x}\right| j\right\rangle^{*}$. The $\hat{k}_{x}^{2}$ operator has matrix elements

$$
\begin{aligned}
\left\langle j\left|\hat{k}_{x}^{2}\right| l\right\rangle & \equiv-\int_{x_{1}}^{x_{2}} g_{j}(x) \partial_{x}^{2} g_{l}(x) d x \\
& =\int_{x_{1}}^{x_{2}} \frac{\partial g_{j}(x)}{\partial x} \frac{\partial g_{l}(x)}{\partial x} d x \\
& =b_{j} b_{l}\langle 1\rangle,
\end{aligned}
$$

which is obviously Hermitean.

\section{A.2 Integrals over 2-dimensional elements}

The integrals over 2-dimensional elements are significantly more complicated to perform than the integrals we encountered in 1D. An element in a 2-dimensional mesh is a triangle. The $j^{\text {th }}$ vertex has coordinates $\left(x_{j}, y_{j}\right)$ and an associated linear function $g_{j}(x, y)$. Within the triangular element, each of the three linear basis functions can be written in the form

$$
g_{j}(x, y)=a_{j}+b_{j} x+c_{j} y,
$$

where $a_{j}, b_{j}, c_{j} \in \mathbb{R}$ are constant coefficients. Similarly to the 1-dimensional basis functions, we determine the coefficients by the constraint $g_{j}\left(x_{l}, y_{l}\right)=$ 
$\delta_{j, l}$. This results in

$$
\begin{aligned}
b_{1} & =\frac{y_{23}}{x_{12} y_{13}-x_{13} y_{12}}, \\
c_{1} & =\frac{x_{32}}{x_{12} y_{13}-x_{13} y_{12}}, \\
b_{2} & =\frac{y_{13}}{x_{21} y_{23}-x_{23} y_{21}}, \\
c_{2} & =\frac{x_{31}}{x_{21} y_{23}-x_{23} y_{21}}, \\
b_{3} & =\frac{y_{21}}{x_{32} y_{31}-x_{31} y_{32}}, \\
c_{3} & =\frac{x_{12}}{x_{32} y_{31}-x_{31} y_{32}}, \\
\forall j: a_{j} & =1-b_{j} x_{j}-c_{j} y_{j},
\end{aligned}
$$

where $x_{j l}=x_{j}-x_{l}$ and $y_{j l}=y_{j}-y_{l}$. Directly integrating a function over a generic triangular element is awkward. For example, suppose we integrate over the y variable first. We have to split the integral into two $x$-intervals with differing y-integral boundary expressions. It is therefore advisable to perform a coordinate transformation such that the integral is over a right triangle. The coordinate transform is given by

$$
\begin{aligned}
& x=x_{1}+\left(x_{2}-x_{1}\right) u+\left(x_{3}-x_{1}\right) v, \\
& y=y_{1}+\left(y_{2}-y_{1}\right) u+\left(y_{3}-y_{1}\right) v,
\end{aligned}
$$

where $u$ and $v$ are the new coordinate variables. In the $u-v$ plane, the element is a right triangle with vertex coordinates $(0,0),(1,0)$, and $(0,1)$. Integrals then transform as

$$
\begin{aligned}
\int_{\Omega} f(x, y) d x d y & =\int_{0}^{1} \int_{0}^{1-u} f(u, v) \operatorname{det}(J) d v d u \\
& =2 A \int_{0}^{1} \int_{0}^{1-u} f(u, v) d v d u
\end{aligned}
$$

where $J$ is the Jacobian and $A$ is the area of the original triangle. ${ }^{1}$ Integrals of multivariate polynomial will repeatedly occur when evaluating matrix elements. We are therefore interested in evaluating integrals of the form

$$
\left\langle x^{m} y^{n}\right\rangle_{\Omega} \equiv \int_{\Omega} x^{m} y^{n} d x d y
$$

Performing the coordinate transformation and substituting Eqs. (A.18, A.19) for $x$ and $y$, respectively, yields

$$
\begin{aligned}
& \left\langle x^{m} y^{n}\right\rangle_{\Omega}=2 A \int_{0}^{1} \int_{0}^{1-u}(\alpha+\beta u+\gamma v)^{m}(\delta+\epsilon u+\eta v)^{n} d v d u, \\
& { }^{1} A=\frac{1}{2}\left[x_{1}\left(y_{2}-y_{3}\right)+x_{2}\left(y_{3}-y_{1}\right)+x_{3}\left(y_{1}-y_{2}\right)\right] .
\end{aligned}
$$


where $\alpha=x_{1}, \beta=x_{2}-x_{1}, \gamma=x_{3}-x_{1}, \delta=y_{1}, \epsilon=y_{2}-y_{1}$, and $\eta=$ $y_{3}-y_{1}$. For integration over the unit right triangle, we have the integral identity [115],

$$
\int_{0}^{1} \int_{0}^{1-u} u^{m} v^{n} d v d u=\frac{m ! n !}{(2+m+n) !},
$$

where $m, n \in \mathbb{Z}^{*}$. The integral in Eq. (A.22) is evaluated by expanding the integrand into a multivariate polynomial and applying the above integral identity.

With the various multivariate polynomial integrals calculated, we can turn to calculating the matrix elements for various operators. The matrix of an operator $\mathcal{O}$ is defined as

$$
\langle j|\hat{\mathcal{O}}| l\rangle_{\Omega}=\int_{\Omega} g_{j}(x, y) \hat{\mathcal{O}} g_{l}(x, y) d x d y,
$$

where the matrix element is restricted the single element with region $\Omega$. When we obtain the full matrix element between basis functions, we will have to sum over all elements. Below we list matrix representations of operators that we use in this thesis:

$$
\begin{aligned}
\left\langle j\left|x^{m} y^{n}\right| l\right\rangle_{\Omega} & =a_{j} a_{l}\left\langle x^{m} y^{n}\right\rangle_{\Omega} \\
& +\left(a_{j} b_{l}+a_{l} b_{j}\right)\left\langle x^{m+1} y^{n}\right\rangle_{\Omega}+\left(a_{j} c_{l}+a_{l} c_{j}\right)\left\langle x^{m} y^{n+1}\right\rangle_{\Omega} \\
& +b_{j} b_{l}\left\langle x^{m+2} y^{n}\right\rangle_{\Omega}+\left(b_{j} c_{l}+b_{l} c_{j}\right)\left\langle x^{m+1} y^{n+1}\right\rangle_{\Omega}+c_{j} c_{l}\left\langle x^{m} y^{n+2}\right\rangle_{\Omega} \\
\left\langle j\left|\hat{k}_{x}\right| l\right\rangle_{\Omega} & =\frac{-i}{2}\left(a_{j} b_{l}-a_{l} b_{j}\right) A-\frac{i}{2}\left(b_{l} c_{j}-b_{j} c_{l}\right)\left\langle y^{2}\right\rangle_{\Omega} \\
\left\langle j\left|\hat{k}_{y}\right| l\right\rangle_{\Omega} & =\frac{-i}{2}\left(a_{j} c_{l}-a_{l} c_{j}\right) A-\frac{i}{2}\left(b_{j} c_{l}-b_{l} c_{j}\right)\left\langle x^{2}\right\rangle_{\Omega} \\
\left\langle j\left|\hat{k}_{x}^{2}\right| l\right\rangle_{\Omega} & =b_{j} b_{l} A \\
\left\langle j\left|\hat{k}_{x} \hat{k}_{y}\right| l\right\rangle_{\Omega} & =\frac{1}{2}\left(b_{j} c_{l}+b_{l} c_{j}\right) A \\
\left\langle j\left|\hat{k}_{y}^{2}\right| l\right\rangle_{\Omega} & =c_{j} c_{l} A \\
\left\langle j\left|y \hat{k}_{x}\right| l\right\rangle_{\Omega} & =\frac{-i}{2}\left(a_{j} b_{l}-a_{l} b_{j}\right)\langle y\rangle_{\Omega}-\frac{i}{2}\left(b_{l} c_{j}-b_{j} c_{l}\right)\left\langle y^{3}\right\rangle_{\Omega} \\
\left\langle j\left|x \hat{k}_{y}\right| l\right\rangle_{\Omega} & =\frac{-i}{2}\left(a_{j} c_{l}-a_{l} c_{j}\right)\langle x\rangle_{\Omega}-\frac{i}{2}\left(b_{j} c_{l}-b_{l} c_{j}\right)\left\langle x^{3}\right\rangle_{\Omega} \\
\left\langle j\left|x \hat{k}_{x}-\frac{i}{2}\right| l\right\rangle_{\Omega} & =\frac{-i}{2}\left(a_{j} b_{l}-a_{l} b_{j}\right)\langle x\rangle_{\Omega}-\frac{i}{2}\left(b_{l} c_{j}-b_{j} c_{l}\right)\langle x y\rangle_{\Omega} \\
\left\langle j\left|y \hat{k}_{y}-\frac{i}{2}\right| l\right\rangle_{\Omega} & =\frac{-i}{2}\left(a_{j} c_{l}-a_{l} c_{j}\right)\langle y\rangle_{\Omega}-\frac{i}{2}\left(b_{j} c_{l}-b_{l} c_{j}\right)\langle x y\rangle_{\Omega}
\end{aligned}
$$

The operators in Eqs. (A.32 - A.34) occur when including the magnetic orbital effect. 


\section{Appendix B}

\section{Effective mass Hamiltonian with position dependent spin-orbit coupling}

\section{B.0.1 Statement of the problem}

Consider a connected space $\Omega^{\prime} \subseteq \Omega \subseteq \mathbb{R}^{2}$, where $\Omega$ is the domain of the Poisson problem in the previous section. Find Bloch eigenstates $\varphi_{\alpha}$ that satisfy the Bloch-Schrödinger equation

$$
\mathcal{H}_{N}\left(k_{z}\right) \varphi_{\alpha}\left(\mathbf{r}, k_{z}\right)=E_{\alpha}\left(k_{z}\right) \varphi_{\alpha}\left(\mathbf{r}, k_{z}\right),
$$

where $\mathcal{H}_{N}$ is the Bloch Hamiltonian, $E_{n}$ is the eigenenergy, and $\varphi_{\alpha}$ is a twocomponent spinor,

$$
\varphi_{\alpha}\left(\mathbf{r}, k_{z}\right)=\left(\begin{array}{c}
\varphi_{\alpha \uparrow}\left(\mathbf{r}, k_{z}\right) \\
\varphi_{\alpha \downarrow}\left(\mathbf{r}, k_{z}\right)
\end{array}\right) .
$$

The Bloch Hamiltonian is given by

$$
\begin{aligned}
\mathcal{H}_{N}\left(k_{z}\right) & =\mathcal{H}_{o}\left(k_{z}\right)+\mathcal{H}_{\Gamma}+\mathcal{H}_{S O}\left(k_{z}\right), \\
\mathcal{H}_{o}\left(k_{z}\right) & =\frac{\hbar^{2}}{2 m^{*}}\left(-\nabla^{2}+k_{z}^{2}\right)-e \phi(\mathbf{r}), \\
\mathcal{H}_{\Gamma} & =\Gamma \sigma_{z} \\
\mathcal{H}_{S O}\left(k_{z}\right) & =\left(\alpha_{x}(\mathbf{r}) \sigma_{y}-\alpha_{y}(\mathbf{r}) \sigma_{x}\right) k_{z},
\end{aligned}
$$

where $\nabla^{2}=\partial_{x}^{2}+\partial_{y}^{2}, \sigma_{j}$ with $j=\{x, y, z\}$ are the Pauli matrices acting in spin-space, and $\alpha_{x}$ and $\alpha_{y}$ are spin-orbit fields given by

$$
\begin{aligned}
& \alpha_{x}(\mathbf{r})=C \frac{\partial \phi(\mathbf{r})}{\partial x} \\
& \alpha_{y}(\mathbf{r})=C \frac{\partial \phi(\mathbf{r})}{\partial y}
\end{aligned}
$$

where $C \in \mathbb{R}^{+}$is a constant. In other words, the spin-orbit fields are proportional to the local electric field within the nanowire. These spin-orbit fields then produce a position and momentum dependent magnetic field in Eq. (B.6). Note that $k_{z} \in \mathbb{R}$ is the $z$-component of the momentum and is a good 
quantum number. The boundary conditions are given by

$$
\varphi_{\alpha}\left(\mathbf{r}, k_{z}\right)=0, \quad \mathbf{r} \in \partial \Omega^{\prime},
$$

i.e. we assume that the wavefunction vanishes everywhere on the boundary of $\Omega^{\prime}$.

\section{B.0.2 Weak form, basis functions, and matrix assembly}

Just as we did in the Poisson problem, we multiply Eq. (B.1) by a test function $f$ and integrate over all space. The only difference is that our test function needs to have two components, $f_{\uparrow}$ and $f_{\downarrow}$, since $\varphi_{\alpha}$ also has two components. This leads to the integral equation,

$$
\begin{aligned}
\sum_{\sigma \sigma^{\prime}} \int_{\Omega^{\prime}} f_{\sigma} & {\left[\left(-\frac{\hbar^{2}}{2 m^{*}} \nabla^{2}-e \phi+\frac{\hbar^{2} k_{z}^{2}}{2 m^{*}}-E_{\alpha}\right) \delta_{\sigma \sigma^{\prime}}+\Gamma\left(\sigma_{z}\right)_{\sigma \sigma^{\prime}}\right.} \\
& \left.+\left(\alpha_{x}\left(\sigma_{y}\right)_{\sigma \sigma^{\prime}}-\alpha_{y}\left(\sigma_{x}\right)_{\sigma \sigma^{\prime}}\right) k_{z}\right] \varphi_{\alpha \sigma^{\prime}} d \Omega^{\prime}=0
\end{aligned}
$$

where I have notationally suppressed all functional dependence on $\mathbf{r}$ and the sums over $\sigma$ and $\sigma^{\prime}$ run over the two species. The integral equation is brought into weak form using integration by parts. The second-order derivatives are handled just as in the Poisson problem in Sec. 4.2.2. The weak form is then

$$
\begin{aligned}
\sum_{\sigma \sigma^{\prime}} \int_{\Omega^{\prime}} & {\left[\nabla f_{\sigma} \cdot\left(\frac{\hbar^{2}}{2 m^{*}} \delta_{\sigma \sigma^{\prime}}\right) \nabla+f_{\sigma}\left(-e \phi+\frac{\hbar^{2} k_{z}^{2}}{2 m^{*}}\right) \delta_{\sigma \sigma^{\prime}}\right.} \\
& \left.+f_{\sigma}\left(\alpha_{x}\left(\sigma_{y}\right)_{\sigma \sigma^{\prime}}-\alpha_{y}\left(\sigma_{x}\right)_{\sigma \sigma^{\prime}}\right) k_{z}\right] \varphi_{\alpha, \sigma^{\prime}}=E_{\alpha} \sum_{\sigma} \int_{\Omega^{\prime}} f_{\sigma} \varphi_{\alpha, \sigma} d \Omega^{\prime}
\end{aligned}
$$

Note that the first term in the upper line of Eq. (B.11) have had the a partial derivative transferred to the test function component $f_{\sigma}$. Additionally, there are no boundary terms in the weak form equations due to the vanishing Dirichlet boundary condition (B.9) enforced on the wavefunction.

Having transformed the problem into weak form, the eigenstate $\varphi_{\alpha}$ is expanded using a finite basis set $\mathcal{B}$. To do so we

$$
\varphi_{\alpha}\left(\mathbf{r}, k_{z}\right)=\sum_{i=1}^{2} \sum_{m} g_{i m}(\mathbf{r}) D_{i m \alpha}\left(k_{z}\right),
$$

where $D_{\text {im } \alpha} \in \mathbb{C}$ and $g_{\text {im }} \in \mathcal{B}$ is a spinor basis function given by

$$
g_{i m}(\mathbf{r})=g_{m}(\mathbf{r})\left(\begin{array}{c}
\delta_{i 1} \\
\delta_{i 2}
\end{array}\right) .
$$


As in Sec. 4.2.4, the function $g_{m}$ is defined by first introducing a finite element mesh that covers $\Omega$. Next, the function $g_{m}$ is defined as the unique linear piecewise function that satisfies

$$
g_{m}\left(\mathbf{r}_{n}\right)=\left\{\begin{array}{ll}
1, & n=m \\
0, & n \neq m
\end{array},\right.
$$

where $\mathbf{r}_{n}$ is the $n^{\text {th }}$ node of the finite element mesh. Plugging in the basis functions $g_{i, n}$ and $g_{j, m}$ into Eq. (B.11) for the test function $f$ and the wavefunction spinor $\varphi_{\alpha}$, respectively, leads to the generalized eigenvalue equation,

$$
\sum_{j=1}^{2} \sum_{m} h_{n m}^{i j}\left(k_{z}\right) D_{j m \alpha}\left(k_{z}\right)=E_{\alpha}\left(k_{z}\right) \sum_{m} S_{n m} D_{i m \alpha}\left(k_{z}\right),
$$

where $h_{n m}^{i j}$ is the result of evaluating the left hand side of Eq. (B.11) for $f=$ $g_{i n}$ and $\varphi_{\alpha}=g_{j m}$, and $S_{n m}$ is the overlap matrix element given by

$$
S_{n m}=\int_{\Omega^{\prime}} g_{n} g_{m} d \Omega^{\prime}
$$

The eigenstates of Eq. (B.15) are then found using sparse matrix eigenvalue algorithms [169]. 


\section{Appendix C}

\section{Localization length of Majorana zero modes}

In this appendix we derive analytic expressions for the localization length of Majorana zero modes. We also confirm the analytic expressions numerically.

We start by consider a 1D Rashba nanowire in an applied magnetic field and with s-wave superconductivity, which represents the 1D minimal model of a Majorana nanowire [43]. The Hamiltonian is given by

$$
H=\left(\frac{\hbar^{2} k^{2}}{2 m^{*}}-\mu+\alpha k \sigma_{y}+\Gamma \sigma_{z}\right) \tau_{z}-\Delta \sigma_{y} \tau_{y}
$$

where $\mu$ is the chemical potential, $\Gamma$ is the Zeeman energy, $\Delta$ is the induced superconductivity parameter, and $\sigma_{i}$ and $\tau_{i}$ are the Pauli matrices acting in spin and particle-hole space, respectively. This Hamiltonian is known to undergo a topological phase transition at a Zeeman field given by

$$
\Gamma_{c}=\sqrt{\Delta^{2}+\mu^{2}}
$$

with Majorana bound states emerging at the edges of the wire once this critical magnetic field is reached. While the Majorana states will have zero energy for a semi-infinite system, the overlap between the edge Majorana causes oscillations about zero energy as $\Gamma$ is changed. This overlap can be quantified if we know the length scale on which the Majorana bound states are localized at the edge of the wire. Since the Majorana bound state's energy lies inside the bulk energy bands, the states are necessarily composed of evanescent waves that have a complex wave number. The imaginary component of the complex wavenumber causes decay of the edge mode as it enters into the bulk of the wire. We can study the length scale of this decay by studying the complex band structure of Eq. (C.1), where we let $k$ become a complex number. 
To begin we find the eigenenergies of Eq. (C.1), which are found to be

$$
\begin{aligned}
E^{2}(k) & =\left(\frac{\hbar^{2} k^{2}}{2 m^{*}}-\mu\right)^{2}+\Gamma^{2}+\Delta^{2}+\alpha^{2} k^{2} \\
& \pm 2 \sqrt{\left(\frac{\hbar^{2} k^{2}}{2 m^{*}}-\mu\right)^{2}\left(\Gamma^{2}+\alpha^{2} k^{2}\right)+\Gamma^{2} \Delta^{2}} .
\end{aligned}
$$

Note that for any eigen energy $E$, there as also exists and eigen energy $(-E)$ due to the particle-hole symmetry of the Eq. (C.1). Since Majorana bound states are (nearly) zero energy modes, we desire to find $k$ satifying Eq. (C.3) for $E=0$. In principle, this can be done exactly since finding $E=0$ solutions to Eq. (C.3) involves solving a quartic equation for the variable $k^{2}$. The quartic equation solution is too complicated to be of practical use, however, so we instead approach the problem using asymptotic methods [222]. Note that in the limit of $\Delta=0$, the Hamiltonian (C.1) separates into particle and hole components. The spectrum of the isolated particle sector is simply

$$
E(k)=\frac{\hbar^{2} k^{2}}{2 m^{*}}-\mu \pm \sqrt{\Gamma^{2}+\alpha^{2} k^{2}},
$$

and we can easily find the $E=0$ solutions, which are

$$
\begin{aligned}
k_{o}^{2} & =\frac{2 m^{*}}{\hbar^{2}}\left(\mu+2 E_{S O}\right) \\
& \pm \frac{2 m^{*}}{\hbar^{2}} \sqrt{\Gamma^{2}+4 \mu E_{S O}+4 E_{S O}^{2}}
\end{aligned}
$$

where $E_{S O}=m^{*} \alpha^{2} /\left(2 \hbar^{2}\right)$ is the spin-orbit energy. Note that $k_{0}$ represents an asymptotic approximation for $k$ in Eq. (C.3) as $\Delta \rightarrow 0$. To find the leading order correction, we let $k^{2}=k_{o}^{2}+\left(2 m^{*} / \hbar^{2}\right) \Delta p^{2}$ and substitute this expression into Eq. (C.3). Here $p^{2}$ is a dimensionless parameter that we wish to find. A question arises as to what sign to take in Eq. (C.5). To answer this question, notice that for large enough $\Gamma$, we obtain a set of purely real and a set of purely imaginary values for $k_{0}$. The purely real and imaginary sets of eigen values correspond to the low and high energy spin split bands, respectively, where the high energy spin band has imaginary wavenumbers because no propagating bulk states exist at $E=0$ for this band. In the presence of $\Delta \neq 0$, the real eigenvalues are rotated slightly into the complex plane, while the imaginary eigenvalues remain completely imaginary. The localization length of the Majorana modes will be determined by the eigenvalues with the smallest imaginary component. Therefore, the eigenvalues stemming from the real eigenvalues of Eq. (C.5) are the most important, so we take the $(+)$ sign. Upon substituion, we find the asymptotic relation, 


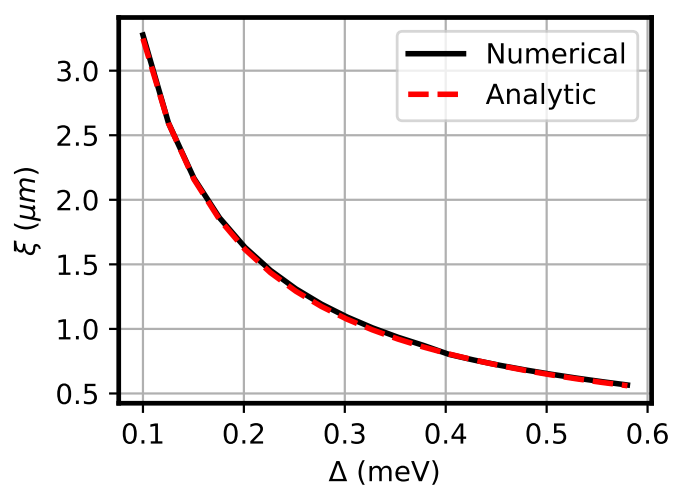

FIGURE C.1: Localization length, $\xi$, vs superconducting gap, $\Delta$, for a long nanowire, $\xi \ll L$, with parameters $m^{*}=0.026 m_{0}$, $\mu=1 \mathrm{meV}, \alpha=50 \mathrm{meV} \cdot \mathrm{nm}$, and $\Gamma=3 \mathrm{meV}$. Numerical localization length is extracted by fitting the Majorana wavefunction to the exponential envelope, $\psi \propto \exp (-x / \xi)$.

$$
p^{2} \sim \pm i \sqrt{\frac{4 E_{S O} k_{o}^{2}}{\Gamma^{2}+4 \mu E_{S O}+4 E_{S O}^{2}}},
$$

as $\Delta \rightarrow 0$. Our total wave number is then given by

$$
\begin{aligned}
& k= \pm \kappa \pm i q, \\
& \kappa \sim k_{o} \\
& q \sim \sqrt{\left(\frac{2 m^{*}}{\hbar^{2}}\right) \frac{E_{S O}}{\Gamma^{2}+4 \mu E_{S O}+4 E_{S O}^{2}} \Delta,}
\end{aligned}
$$

where the two $( \pm)$ in Eq. (C.7) are independent. The localization length is then just the reciprocal of the imaginary part of the wave number, $\xi=q^{-1}$. We find

$$
\xi \sim \ell_{S O}\left(\frac{\Gamma}{\Delta}\right) \sqrt{1+\frac{4 \mu E_{S O}}{\Gamma^{2}}+\frac{4 E_{S O}^{2}}{\Gamma^{2}}},
$$

where $\ell_{S O}=\hbar^{2} /\left(m^{*} \alpha\right)$ is the spin-orbit length. This shows the expected behavior of increased delocalization for large $\Gamma$ and small $\alpha$.

Interestingly, in the limit of $E_{S O} \gg \Gamma$, we actually find increasing localization length for increasing $\alpha$. While this regime is difficult to achieve for the conventional proximitized Rashba nanowire setups, this situation is possible in the higher-energy minibands within a periodic potential setup. A comparison between the numerical and derived analytical localization length of Eq. (C.10) is shown in Fig. C.1 as a function of $\Delta$, showing excellent agreement. We note that starting from the assumption of a small spin-orbit coefficient $\alpha$ in Eq. (C.3), we find a similar and consistent asymptotic expression for the localization length, given by

$$
\xi \sim \ell_{S O} \frac{\sqrt{\Gamma^{2}-\Delta^{2}}}{\Delta},
$$


as $\alpha \rightarrow 0$ in the case of $\mu=0$. 


\section{Appendix D}

\section{Lattice Green's functions and self-energy formalism}

\section{D.1 Green's function and the density of states}

Lattice Green's functions methods are used ubiquitously throughout all of theoretical physics, especially in many-body physics [221] and quantum transport [223], to determine the expectation values and correlations functions without having to have explicit knowledge about the partition function of the system. Indeed, they find use into connecting the observations of experiments through linear response theory, where the system is being probed by an external perturbation, to the underlying physics. They also are the starting point and work horse of many-body diagram techniques [221]

Rather than start from the typical definition for the Green's function and deriving its equation of motion, I will instead take the equation of motion for a non-interacting fermion system as a given and derive the important properties and quantities that concern us in this thesis. Suppose we have a lattice $\mathcal{L}$ with $N$ sites and $M$ degrees of freedom on each site for a total of $\bar{N}=N M$ degrees of freedom. The retarded Green's function $G(\omega)$ is defined by the matrix equation

$$
(\omega-H+i \eta) G(\omega)=I,
$$

where $\omega \in\{x+i y \mid y \geq 0 ; x, y \in \mathbb{R}\}$ is an energy on or above the real axis of the complex plane, $H$ is the matrix representation of the Hamiltonian, $I$ is the identity matrix with dimensions equal to that of $H$, and $\eta$ is an infinitesimal positive real number. Note that the $\omega$ and $\eta$ terms are assumed to be multiplies of $I$ and that $G$ is a matrix of the same dimension as $H$. Also note that it is assumed that the basis states used to create the matrix representation of $H$ are orthonormal, $\langle\alpha \mid \beta\rangle=\delta_{\alpha, \beta}$. In Sec. D.3, the Green's function equation will be generalize to non-orthogonal basis sets. To see what information is contained in the Greens function $G$, let us perform a few manipulations. Suppose

$$
U^{\dagger} H U=\Lambda=\operatorname{diag}\left(\lambda_{1}, \lambda_{2}, \ldots, \lambda_{\bar{N}}\right),
$$

where $\lambda_{n}$ is the the $n^{\text {th }}$ eigenvalue of $H$, and $U$ is a unitary matrix. Multiply Eq. (D.1) from the left and right by $U^{\dagger}$ and $U$, respectively, along with an 
insertion of $I=U U^{\dagger}$ directly before $G$ yields

$$
(\omega-\Lambda+i \eta) U^{\dagger} G U=I .
$$

Being a diagonal matrix, $(\omega-\Lambda)$ has a trivial matrix inverse, and can be carried over to the right hand side of Eq. (D.3). Multiplying by $U$ and $U^{\dagger}$ then yields

$$
G(\omega)=U(\omega-\Lambda+i \eta)^{-1} U^{\dagger},
$$

which has matrix elements

$$
G_{i j}(\omega)=\sum_{n=1}^{P} \frac{U_{i n} U_{j n}^{*}}{\omega-\lambda_{n}+i \eta} .
$$

The form of Eq. (D.4) is known as the spectral representation of the lattice Green's function [220], because of the fact that it obvious in this form that the Green's function has a pole located at $\omega=\lambda_{n}-i \eta$, i.e. at every eigenenergy along the real axis. ${ }^{1}$ Let us now assume $\omega \in \mathbb{R}$ and separate the real and imaginary parts of diagonal matrix elements of the Green's function in Eq. (D.5). This yields

$$
G_{i i}(\omega)=\sum_{n=1}^{P}\left|U_{i n}\right|^{2} \frac{\omega-\lambda_{n}}{\left(\omega-\lambda_{n}\right)^{2}+\eta^{2}}-i \sum_{n=1}^{P}\left|U_{i n}\right|^{2} \frac{\eta}{\left(\omega-\lambda_{n}\right)^{2}+\eta^{2}},
$$

where the first and second sums represent the real and imaginary parts of $G_{i i}$, respectively. Using the identity,

$$
\delta(x)=\pi \lim _{\eta \rightarrow 0^{+}} \frac{\eta}{x^{2}+\eta^{2}}
$$

the imaginary part of the Green's function can be written as

$$
\operatorname{Im}\left[G_{i i}(\omega)\right]=-\frac{1}{\pi} \sum_{n=1}^{P}\left|U_{i n}\right|^{2} \delta\left(\omega-\lambda_{n}\right),
$$

which we recognize as being proportional to the density of states due to the $i^{\text {th }}$ degree of freedom,

$$
\operatorname{LDOS}_{i}(\omega)=-\frac{1}{\pi} \operatorname{Im}\left[G_{i i}(\omega)\right]
$$

The full density of states is found by summing over $i$, which yields

$$
\operatorname{DOS}(\omega)=-\frac{1}{\pi} \operatorname{Im}[\operatorname{Tr}(G(\omega))] .
$$

\footnotetext{
${ }^{1}$ The infinitesimal $\eta>0$ pushes the poles ever so slightly into the lower complex plane. This ensures that the retarded Green's function is analytic in the entire upper half of the complex plane
} 


\section{D.2 Self-energy formalism}

Suppose the Hamiltonian of a system is given by

$$
H=H_{0}+V,
$$

where $H_{o}$ is the unperturbed Hamiltonian and $V$ is a perturbation. How does the Green's function of the total Hamiltonian related to the Green's function of the unperturbed system? Starting from the Green's function equation,

$$
\begin{aligned}
G & =\left(\omega-H_{o}-V+i \eta\right)^{-1} \\
& =\left(g^{-1}-V\right)^{-1}
\end{aligned}
$$

where $g=\left(\omega-H_{o}+i \eta\right)^{-1}$ is the unperturbed Green's function, the Dyson equation can easily be derived,

$$
G=g+g V G
$$

Now suppose a system is composed of two subsystems. The Hamiltonian can then be written in the block form,

$$
H=\left(\begin{array}{cc}
H_{1} & T^{\dagger} \\
T & H_{2}
\end{array}\right),
$$

where $H_{1}$ and $H_{2}$ are the Hamiltonians of the first and second isolated subsystems, respectively, and $T$ represents the coupling between the two systems. Let us identify $T$ as the perturbation. The dressed and unperturbed Green's function then take the block forms,

$$
\begin{aligned}
& G=\left(\begin{array}{ll}
G_{11} & G_{12} \\
G_{21} & G_{22}
\end{array}\right), \\
& g=\left(\begin{array}{cc}
g_{11} & 0 \\
0 & g_{22}
\end{array}\right),
\end{aligned}
$$

where $G_{i j}$ and $g_{i i}$ are block matrices. Note that the off-diagonal blocks of the unperturbed Green's function $g$ vanish because the coupling between the two subsystems does not exist from the perspective of the unperturbed Green's function. Dyson's equation (D.14) then yields

$$
\begin{aligned}
& G_{11}=g_{11}+g_{11} T^{\dagger} G_{21}, \\
& G_{21}=g_{22} T G_{11},
\end{aligned}
$$

where I have used the fact that $g_{21}=0$. Plugging Eq. (D.19) into Eq. (D.18) yields

$$
G_{11}=g_{11}+g_{11} \Sigma_{11} G_{11}
$$

where

$$
\Sigma_{11}=T^{\dagger} g_{22} T
$$


Notice that Eq. (D.20) has the same form as Eq. (D.14), except that it is restricted to subsystem 1. Reversing the derivation of Dyson's equation finally yields

$$
G_{11}(\omega)=\left(\omega-H_{1}-\Sigma_{11}(\omega)+i \eta\right)^{-1}
$$

for the subsystem 1 block of the dressed Greens function. The effects of subsystem 2 on subsystem 1 are all contained in $\Sigma_{11}$, and is therefore called the self-energy on subsystem 1 due to subsystem 2 . Importantly, the dimension of the matrices in Eq. (D.22) are smaller than those in Eq. (D.13) since the degrees of freedom of subsystem 2 have been integrated out. Moreover, no information about the dressed Green's function involving subsystem 2 is necessary. The price to be paid for these advantages is that the self-energy $\Sigma_{11}$ is dependent on the energy $\omega$. In the event that the self-energy is only weakly dependent on $\omega$, a static approximation can be employed in which this $\omega$ dependence is ignored, i.e. $\Sigma_{11}(\omega) \rightarrow \Sigma_{11}^{\text {static }}$. Subsystem 1 can then be viewed as existing in isolation from subsystem 2, but with the modified effective Hamiltonian,

$$
H_{1}^{\text {eff }}=H_{1}+\Sigma_{11}^{\text {static }},
$$

with no dependence on the energy $\omega$. In the case that subsystem 2 has nonzero density of states at the energy used for the static energy, the effective Hamiltonian $H_{1}^{\text {eff }}$ will generically be non-Hermitean, possibly leading to interesting physics [224].

\section{D.3 Green's function representation with a non- orthonormal basis set}

In Sec. D.1, it was assumed that the basis states used to form the matrix representations were orthonormal. This, however, is not the case for the basis functions used in finite element methods as described in Chapter 4. Here we generalize the Green's function to non-orthonormal basis sets.

Let $S$ with elements $S^{i j}=\langle i \mid j\rangle$ be the overlap matrix of basis states. The Green's function obeys the operator equation,

$$
((\omega+i \eta) \hat{I}-\hat{H}) \hat{G}(\omega)=\hat{I},
$$

where the hat denotes an operator as opposed to a matrix, and $\eta$ is again a small infinitesimal positive energy. The Green's function operator can be expanded as

$$
\hat{G}(\omega)=\sum_{i j}|i\rangle G^{i j}(\omega)\langle j|
$$


We sandwich both sides of Eq. (D.24) with $\langle k|$ and $|l\rangle$ from the left and right, respectively, which leads to

$$
\begin{gathered}
\sum_{i j}\left((\omega+i \eta) S^{k i}-H^{k i}\right) G^{i j} S^{j l}=S^{k l} \\
\Longrightarrow((\omega+i \eta) S-H) G(\omega)=I,
\end{gathered}
$$

which is the generalized version of Eq. (D.1) to non-orthonormal basis states. Note that in the case of an orthonormal basis set, $S^{i j}=\delta_{i j}$, Eq. (D.27) reduces to Eq. (D.1).

The self-energy (D.21) also needs to be generalized. The overlap matrix $S$ can be written in block form,

$$
S=\left(\begin{array}{ll}
S_{11} & S_{12} \\
S_{21} & S_{22}
\end{array}\right)
$$

where $S_{11}$ and $S_{22}$ contain the overlap of basis states both within the first and second subsystems, respectively, while $S_{21}$ contains the overlap of basis functions from the first subsystem with the second subsystem. The dressed Green's function block of the first subsystem $G_{11}$ is found to be [150]

$$
G_{11}(\omega)=\left((\omega+i \eta) S_{11}-H_{1}-\Sigma_{11}(\omega)\right)^{-1},
$$

where the self-energy is given by

$$
\Sigma_{11}(\omega)=\left(\omega S_{12}-T^{\dagger}\right)\left(\omega S_{22}-H_{2}\right)^{-1}\left(\omega S_{21}-T\right),
$$

which is the generalization of Eq. (D.21) to non-orthogonal basis sets. Again, note that in the case of an orthonormal basis set, $S^{i j}=\delta_{i j}$, Eq. (D.30) reduces to Eq. (D.21). 


\section{Bibliography}

${ }^{1}$ E. Altman, K. R. Brown, G. Carleo, L. D. Carr, E. Demler, C. Chin, B. DeMarco, S. E. Economou, M. A. Eriksson, K.-M. C. Fu, M. Greiner, K. R. Hazzard, R. G. Hulet, A. J. Kollár, B. L. Lev, M. D. Lukin, R. Ma, X. Mi, S. Misra, C. Monroe, K. Murch, Z. Nazario, K.-K. Ni, A. C. Potter, P. Roushan, M. Saffman, M. Schleier-Smith, I. Siddiqi, R. Simmonds, M. Singh, I. Spielman, K. Temme, D. S. Weiss, J. Vučković, V. Vuletić, J. Ye, and M. Zwierlein, "Quantum simulators: architectures and opportunities", PRX Quantum 2, 017003 (2021).

${ }^{2} \mathrm{M}$. Schlosshauer, "Decoherence, the measurement problem, and interpretations of quantum mechanics", Rev. Mod. Phys. 76, 1267-1305 (2005).

${ }^{3}$ C. Nayak, S. H. Simon, A. Stern, M. Freedman, and S. Das Sarma, “Nonabelian anyons and topological quantum computation", Rev. Mod. Phys. 80, 1083 (2008).

${ }^{4}$ N. Read and D. Green, "Paired states of fermions in two dimensions with breaking of parity and time-reversal symmetries and the fractional quantum hall effect", Phys. Rev. B 61, 10267 (2000).

${ }^{5}$ S. B. Bravyi and A. Y. Kitaev, "Fermionic quantum computation", Annals of Physics 298, 210 -226 (2002).

${ }^{6}$ B. A. Bernevig, Topological Insulators and Topological Superconductors (Princeton University Press, New Jersey, 2013).

${ }^{7}$ T. D. Stanescu, Introduction to topological quantum matter and quantum computation (CRC Press, Taylor \& Francis Group, 2017).

${ }^{8}$ R. M. Lutchyn, J. D. Sau, and S. Das Sarma, "Majorana fermions and a topological phase transition in semiconductor-superconductor heterostructures", Phys. Rev. Lett. 105, 077001 (2010).

${ }^{9}$ Y. Oreg, G. Refael, and F. von Oppen, "Helical liquids and majorana bound states in quantum wires", Phys. Rev. Lett. 105, 177002 (2010).

${ }^{10}$ L. D. Landau and E. M. Lifshitz, Statistical physics (Pergamon Press, Oxford), 1980).

${ }^{11}$ K. v. Klitzing, G. Dorda, and M. Pepper, "New method for high-accuracy determination of the fine-structure constant based on quantized hall resistance", Phys. Rev. Lett. 45, 494-497 (1980).

${ }^{12}$ D. J. Thouless, M. Kohmoto, M. P. Nightingale, and M. den Nijs, “Quantized hall conductance in a two-dimensional periodic potential", Phys. Rev. Lett. 49, 405-408 (1982). 
${ }^{13}$ R. M. Kaufmann, D. Li, and B. Wehefritz-Kaufmann, "Notes on topological insulators", Reviews in Mathematical Physics 28, 1630003 (2016).

${ }^{14} \mathrm{M}$. Nakahara, Geometry, topology and physics, Second Edition (Taylor Francis, 2003).

${ }^{15}$ X.-L. Qi and S.-C. Zhang, "Topological insulators and superconductors", Rev. Mod. Phys. 83, 1057 (2011).

${ }^{16}$ C.-K. Chiu, J. C. Y. Teo, A. P. Schnyder, and S. Ryu, "Classification of topological quantum matter with symmetries", Rev. Mod. Phys. 88, 035005 (2016).

${ }^{17}$ D. C. Tsui, H. L. Stormer, and A. C. Gossard, “Two-dimensional magnetotransport in the extreme quantum limit", Phys. Rev. Lett. 48, 1559-1562 (1982).

${ }^{18}$ E. P. Wigner, Group theory and its application to the quantum mechanics of atomic spectra (Academic Press, 1959).

${ }^{19} \mathrm{M}$. Tinkham, Group theory and quantum mechanics (Dover Publications, 2003).

${ }^{20}$ F. Schindler, A. M. Cook, M. G. Vergniory, Z. Wang, S. S. P. Parkin, B. A. Bernevig, and T. Neupert, "Higher-order topological insulators", Science Advances 4, 10.1126/sciadv . aat0346 (2018).

${ }^{21} \mathrm{~A}$. M. Essin and V. Gurarie, "Bulk-boundary correspondence of topological insulators from their respective green's functions", Phys. Rev. B 84, 125132 (2011).

${ }^{22}$ M. König, S. Wiedmann, C. Brüne, A. Roth, H. Buhmann, L. W. Molenkamp, X.-L. Qi, and S.-C. Zhang, "Quantum spin hall insulator state in hgte quantum wells", Science 318, 766-770 (2007).

${ }^{23}$ D. Hsieh, D. Qian, L. Wray, Y. Xia, Y. S. Hor, R. J. Cava, and M. Z. Hasan, "A topological dirac insulator in a quantum spin hall phase", Nature 452, 970-974 (2008).

${ }^{24} \mathrm{~J}$. Preskill, Lecture notes for ph219/cs219: quantum information, chapter 5, (2015) http://theory.caltech.edu/ preskill/ph219/chap5_15.pdf.

${ }^{25}$ L. K. Grover, "A fast quantum mechanical algorithm for database search", Proceedings of the twenty-eighth annual ACM symposium on Theory of computing - STOC 96, 10.1145/237814.237866 (1996).

${ }^{26} \mathrm{D}$. Coppersmith, "Ran approximate fourier transform useful in quantum factoring", arXiv:quant-ph/0201067 (1994).

${ }^{27} \mathrm{P}$. Shor, "Algorithms for quantum computation: discrete logarithms and factoring", Proceedings 35th Annual Symposium on Foundations of Computer Science, 10.1109/sfcs . 1994.365700.

${ }^{28}$ R. P. Feynman, "Simulating physics with computers", International Journal of Theoretical Physics 21, 467-488 (1982).

${ }^{29}$ J. Preskill, Lecture notes for ph219/cs219: quantum information, chapter 7, (1999)

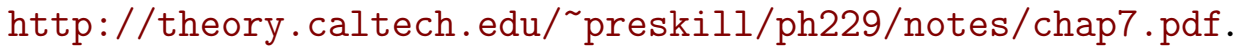


${ }^{30} \mathrm{~A}$. Kitaev, "Fault-tolerant quantum computation by anyons", Annals of Physics 303, 2-30 (2003).

${ }^{31}$ V. Lahtinen and J. K. Pachos, "A Short Introduction to Topological Quantum Computation", SciPost Phys. 3, 021 (2017).

${ }^{32}$ R. B. Laughlin, "Anomalous quantum hall effect: an incompressible quantum fluid with fractionally charged excitations", Phys. Rev. Lett. 50, 13951398 (1983).

${ }^{33}$ S. Das Sarma, M. Freedman, and C. Nayak, "Majorana zero modes and topological quantum computation", Npj Quantum Information 1, 15001 (2015).

${ }^{34}$ J. Alicea, Y. Oreg, G. Refael, F. von Oppen, and M. P. A. Fisher, “Nonabelian statistics and topological quantum information processing in $1 \mathrm{~d}$ wire networks", Nature Physics 7, 412 (2011).

${ }^{35} \mathrm{~J}$. Alicea, "New directions in the pursuit of Majorana fermions in solid state systems", Reports on Progress in Physics 75, 076501 (2012).

${ }^{36}$ C. Beenakker, "Search for Majorana Fermions in Superconductors", Annual Review of Condensed Matter Physics 4, 113 (2013).

${ }^{37}$ T. D. Stanescu and S. Tewari, "Majorana fermions in semiconductor nanowires: fundamentals, modeling, and experiment", J. Phys.: Condens. Matter 25, 233201 (2013).

${ }^{38}$ R. M. Lutchyn, E. P. A. M. Bakkers, L. P. Kouwenhoven, P. Krogstrup, C. M. Marcus, and Y. Oreg, "Majorana zero modes in superconductor-semiconductor heterostructures", Nature Reviews Materials 3, 52 (2018).

${ }^{39}$ K. Flensberg, F. von Oppen, and A. Stern, "Engineered platforms for topological superconductivity and majorana zero modes", arXiv:2103.05548 (2021).

${ }^{40}$ A. Y. Kitaev, "Unpaired Majorana fermions in quantum wires”, PhysicsUspekhi 44, 131 (2001).

${ }^{41} \mathrm{~L} . \mathrm{Fu}$ and C. L. Kane, "Superconducting proximity effect and majorana fermions at the surface of a topological insulator", Phys. Rev. Lett. 100, 096407 (2008).

${ }^{42} \mathrm{~L} . \mathrm{Fu}$ and C. L. Kane, “Josephson current and noise at a superconductor/quantumspin-hall-insulator/superconductor junction", Phys. Rev. B 79, 161408 (2009).

${ }^{43}$ J. D. Sau, R. M. Lutchyn, S. Tewari, and S. Das Sarma, "Generic new platform for topological quantum computation using semiconductor heterostructures", Phys. Rev. Lett. 104, 040502 (2010).

${ }^{44}$ J. D. Sau, S. Tewari, R. M. Lutchyn, T. D. Stanescu, and S. Das Sarma, "Nonabelian quantum order in spin-orbit-coupled semiconductors: search for topological majorana particles in solid-state systems", Phys. Rev. B 82, 214509 (2010).

${ }^{45} \mathrm{~J}$. Alicea, "Majorana fermions in a tunable semiconductor device", Phys. Rev. B 81, 125318 (2010). 
${ }^{46} \mathrm{R}$. Winkler, Spin-orbit coupling effects in two-dimensional electron and hole systems (Springer, 2003).

${ }^{47}$ V. Mourik, K. Zuo, S. M. Frolov, S. R. Plissard, E. P. A. M. Bakkers, and L. P. Kouwenhoven, "Signatures of Majorana Fermions in Hybrid SuperconductorSemiconductor Nanowire Devices", Science 336, 1003 (2012).

${ }^{48}$ M. T. Deng, C. L. Yu, G. Y. Huang, M. Larsson, P. Caroff, and H. Q. Xu, "Anomalous Zero-Bias Conductance Peak in a Nb-InSb Nanowire-Nb Hybrid Device", Nano Letters 12, 6414 (2012).

${ }^{49}$ A. Das, Y. Ronen, Y. Most, Y. Oreg, M. Heiblum, and H. Shtrikman, "Zerobias peaks and splitting in an Al-InAs nanowire topological superconductor as a signature of Majorana fermions", Nature Physics 8, 887 (2012).

${ }^{50}$ A. D. K. Finck, D. J. Van Harlingen, P. K. Mohseni, K. Jung, and X. Li, "Anomalous modulation of a zero-bias peak in a hybrid nanowire-superconductor device", Phys. Rev. Lett. 110, 126406 (2013).

${ }^{51}$ L. P. Rokhinson, X. Liu, and J. K. Furdyna, “The fractional a.c. josephson effect in a semiconductor-superconductor nanowire as a signature of majorana particles", Nature Physics 8, 795 (2012).

${ }^{52} \mathrm{~K}$. Flensberg, "Tunneling characteristics of a chain of majorana bound states", Phys. Rev. B 82, 180516 (2010).

${ }^{53}$ M Wimmer, A. R. Akhmerov, J. P. Dahlhaus, and C. W. J. Beenakker, “Quantum point contact as a probe of a topological superconductor", New Journal of Physics 13, 053016 (2011).

${ }^{54}$ G. Kells, D. Meidan, and P. W. Brouwer, "Near-zero-energy end states in topologically trivial spin-orbit coupled superconducting nanowires with a smooth confinement", Phys. Rev. B 86, 100503 (2012).

${ }^{55} \mathrm{D}$. Bagrets and A. Altland, "Class $d$ spectral peak in majorana quantum wires", Phys. Rev. Lett. 109, 227005 (2012).

${ }^{56}$ J. Liu, A. C. Potter, K. T. Law, and P. A. Lee, "Zero-bias peaks in the tunneling conductance of spin-orbit-coupled superconducting wires with and without majorana end-states", Phys. Rev. Lett. 109, 267002 (2012).

${ }^{57}$ E. Prada, P. San-Jose, and R. Aguado, “Transport spectroscopy of ns nanowire junctions with majorana fermions", Phys. Rev. B 86, 180503 (2012).

${ }^{58}$ D. Roy, N. Bondyopadhaya, and S. Tewari, “Topologically trivial zero-bias conductance peak in semiconductor majorana wires from boundary effects", Phys. Rev. B 88, 020502 (2013).

${ }^{59}$ D. Rainis, L. Trifunovic, J. Klinovaja, and D. Loss, "Towards a realistic transport modeling in a superconducting nanowire with majorana fermions", Phys. Rev. B 87, 024515 (2013).

${ }^{60}$ J. D. Sau and S. Das Sarma, "Density of states of disordered topological superconductor-semiconductor hybrid nanowires", Phys. Rev. B 88, 064506 (2013). 
${ }^{61}$ W. DeGottardi, D. Sen, and S. Vishveshwara, “Majorana fermions in superconducting $1 \mathrm{~d}$ systems having periodic, quasiperiodic, and disordered potentials", Phys. Rev. Lett. 110, 146404 (2013).

${ }^{62}$ I. Adagideli, M. Wimmer, and A. Teker, "Effects of electron scattering on the topological properties of nanowires: majorana fermions from disorder and superlattices", Phys. Rev. B 89, 144506 (2014).

${ }^{63}$ J. Cayao, E. Prada, P. San-Jose, and R. Aguado, "Sns junctions in nanowires with spin-orbit coupling: role of confinement and helicity on the subgap spectrum", Phys. Rev. B 91, 024514 (2015).

${ }^{64}$ W. S. Cole, J. D. Sau, and S. Das Sarma, "Proximity effect and majorana bound states in clean semiconductor nanowires coupled to disordered superconductors", Phys. Rev. B 94, 140505 (2016).

${ }^{65}$ C.-X. Liu, J. D. Sau, T. D. Stanescu, and S. Das Sarma, "Andreev bound states versus majorana bound states in quantum dot-nanowire-superconductor hybrid structures: trivial versus topological zero-bias conductance peaks", Phys. Rev. B 96, 075161 (2017).

${ }^{66}$ C. Reeg, O. Dmytruk, D. Chevallier, D. Loss, and J. Klinovaja, "Zero-energy andreev bound states from quantum dots in proximitized rashba nanowires", Phys. Rev. B 98, 245407 (2018).

${ }^{67}$ C. Moore, T. D. Stanescu, and S. Tewari, "Two-terminal charge tunneling: disentangling majorana zero modes from partially separated andreev bound states in semiconductor-superconductor heterostructures", Phys. Rev. B 97, 165302 (2018).

${ }^{68}$ C. Moore, C. Zeng, T. D. Stanescu, and S. Tewari, “Quantized zero-bias conductance plateau in semiconductor-superconductor heterostructures without topological majorana zero modes", Phys. Rev. B 98, 155314 (2018).

${ }^{69}$ C. Fleckenstein, F. Domínguez, N. Traverso Ziani, and B. Trauzettel, "Decaying spectral oscillations in a majorana wire with finite coherence length", Phys. Rev. B 97, 155425 (2018).

${ }^{70}$ T. D. Stanescu and S. Das Sarma, "Building topological quantum circuits: majorana nanowire junctions", Phys. Rev. B 97, 045410 (2018).

${ }^{71}$ Y. Huang, J. D. Sau, T. D. Stanescu, and S. Das Sarma, “Quasiparticle gaps in multiprobe majorana nanowires", Phys. Rev. B 98, 224512 (2018).

${ }^{72}$ B. D. Woods, J. Chen, S. M. Frolov, and T. D. Stanescu, “Zero-energy pinning of topologically trivial bound states in multiband semiconductorsuperconductor nanowires", Phys. Rev. B 100, 125407 (2019).

${ }^{73}$ Y.-H. Lai, J. D. Sau, and S. Das Sarma, "Presence versus absence of endto-end nonlocal conductance correlations in majorana nanowires: majorana bound states versus andreev bound states", Phys. Rev. B 100, 045302 (2019).

${ }^{74}$ H. Pan and S. Das Sarma, "Physical mechanisms for zero-bias conductance peaks in majorana nanowires", Phys. Rev. Research 2, 013377 (2020). 
${ }^{75}$ E. Prada, P. San-Jose, M. W. A. de Moor, A. Geresdi, E. J. H. Lee, J. Klinovaja, D. Loss, J. Nygård, R. Aguado, and L. P. Kouwenhoven, "From andreev to majorana bound states in hybrid superconductor-semiconductor nanowires", Nature Reviews Physics, 10 . 1038 / s42254-020-0228-y (2020).

${ }^{76}$ B. D. Woods, S. D. Sarma, and T. D. Stanescu, "Charge impurity effects in hybrid majorana nanowires", arXiv:2103.06880 (2021).

${ }^{77}$ H. Pan and S. D. Sarma, "Crossover between trivial zero modes in majorana nanowires", arXiv:2102.07296 (2021).

${ }^{78}$ S. Das Sarma and H. Pan, “Disorder-induced zero-bias peaks in majorana nanowires", Phys. Rev. B 103, 195158 (2021).

${ }^{79}$ J. Cayao and P. Burset, "Confinement-induced zero-bias peaks in conventional superconductor hybrids", arXiv:2105.11560 (2021).

${ }^{80}$ S. Takei, B. M. Fregoso, H.-Y. Hui, A. M. Lobos, and S. Das Sarma, "Soft superconducting gap in semiconductor majorana nanowires", Phys. Rev. Lett. 110, 186803 (2013).

${ }^{81}$ P. Krogstrup, N. L. B. Ziino, W. Chang, S. M. Albrecht, M. H. Madsen, E. Johnson, J. Nygård, C. M. Marcus, and T. S. Jespersen, "Epitaxy of semiconductor-superconductor nanowires", Nature Materials 14, 400 (2015).

${ }^{82}$ W Chang, S. M. Albrecht, T. S. Jespersen, F. Kuemmeth, P. Krogstrup, J. Nygård, and C. M. Marcus, "Hard gap in epitaxial semiconductor-superconductor nanowires", Nat Nano 10, 232 (2015).

${ }^{83}$ S. Gazibegovic, D. Car, H. Zhang, S. C. Balk, J. A. Logan, M. W. A. de Moor, M. C. Cassidy, R. Schmits, D. Xu, G. Wang, P. Krogstrup, R. L. M. O. het Veld, K. Zuo, Y. Vos, J. Shen, D. Bouman, B. Shojaei, D. Pennachio, J. S. Lee, P. J. van Veldhoven, S. Koelling, M. A. Verheijen, L. P. Kouwenhoven, C. J. Palmstrøm, and E. P. A. M. Bakkers, "Epitaxy of advanced nanowire quantum devices", Nature 548, 434 (2013).

${ }^{84}$ J.-H. Kang, A. Grivnin, E. Bor, J. Reiner, N. Avraham, Y. Ronen, Y. Cohen, P. Kacman, H. Shtrikman, and H. Beidenkopf, "Robust epitaxial al coating of reclined inas nanowires", Nano Lett. 17, 7520 (2017).

${ }^{85} \mathrm{H}$ Zhang, O. Gul, S. Conesa-Boj, M. P. Nowak, M. Wimmer, K. Zuo, V. Mourik, F. K. de Vries, J. van Veen, M. W. de Moor, J. D. Bommer, D. J. van Woerkom, D. Car, S. R. Plissard, E. P. Bakkers, M. Quintero-Perez, M. C. Cassidy, S. Koelling, S. Goswami, K. Watanabe, T. Taniguchi, and L. P. Kouwenhoven, "Ballistic superconductivity in semiconductor nanowires", Nat. Commun. 8, 16025 (2017).

${ }^{86}$ J. E. Sestoft, T. Kanne, A. N. Gejl, M. von Soosten, J. S. Yodh, D. Sherman, B. Tarasinski, M. Wimmer, E. Johnson, M. Deng, J. Nygård, T. S. Jespersen, C. M. Marcus, and P. Krogstrup, "Engineering hybrid epitaxial inassb/al nanowires for stronger topological protection", Phys. Rev. Materials 2, 044202 (2018). 
${ }^{87}$ S. Vaitiekenas, A. M. Whiticar, M.-T. Deng, F. Krizek, J. E. Sestoft, C. J. Palmstrøm, S. Marti-Sanchez, J. Arbiol, P. Krogstrup, L. Casparis, and C. M. Marcus, "Selective-area-grown semiconductor-superconductor hybrids: a basis for topological networks", Phys. Rev. Lett. 121, 147701 (2018).

${ }^{88}$ T. Kanne, M. Marnauza, D. Olsteins, D. J. Carrad, J. E. Sestoft, J. de Bruijckere, L. Zeng, E. Johnson, E. Olsson, K. Grove-Rasmussen, and J. Nygård, "Epitaxial pb on inas nanowires", (2020).

${ }^{89}$ J. Chen, P. Yu, J. Stenger, M. Hocevar, D. Car, S. R. Plissard, E. P. A. M. Bakkers, T. D. Stanescu, and S. M. Frolov, "Experimental phase diagram of zero-bias conductance peaks in superconductor/semiconductor nanowire devices", Science Advances 3, e1701476 (2017).

${ }^{90}$ F. Nichele, A. C. C. Drachmann, A. M. Whiticar, E. C. T. O'Farrell, H. J. Suominen, A. Fornieri, T. Wang, G. C. Gardner, C. Thomas, A. T. Hatke, P. Krogstrup, M. J. Manfra, K. Flensberg, and C. M. Marcus, "Scaling of majorana zero-bias conductance peaks", Phys. Rev. Lett. 119, 136803 (2017).

${ }^{91}$ S. Vaitiekenas, M.-T. Deng, J. Nygård, P. Krogstrup, and C. M. Marcus, "Effective $g$ factor of subgap states in hybrid nanowires", Phys. Rev. Lett. 121, 037703 (2018).

${ }^{92}$ H. Zhang, C.-X. Liu, S. Gazibegovic, D. Xu, J. A. Logan, G. Wang, N. van Loo, J. D. Bommer, M. W. de Moor, D. Car, R. L. M. O. het Veld, P. J. van Veldhoven, S. Koelling, M. A. Verheijen, M. Pendharkar, D. J. Pennachio, B. Shojaei, J. S. Lee, C. J. Palmstrom, E. P. Bakkers, S. D. Sarma, and L. P. Kouwenhoven, "Retracted article: quantized majorana conductance", Nature 556, 74 (2018).

${ }^{93}$ H. Zhang, M. W. A. de Moor, J. D. S. Bommer, D. Xu, G. Wang, N. van Loo, C.-X. Liu, S. Gazibegovic, J. A. Logan, D. Car, R. L. M. O. het Veld, P. J. van Veldhoven, S. Koelling, M. A. Verheijen, M. Pendharkar, D. J. Pennachio, B. Shojaei, J. S. Lee, C. J. Palmstrøm, E. P. A. M. Bakkers, S. D. Sarma, and L. P. Kouwenhoven, Large zero-bias peaks in insb-al hybrid semiconductorsuperconductor nanowire devices, 2021.

${ }^{94}$ A. Vuik, B. Nijholt, A. R. Akhmerov, and M. Wimmer, "Reproducing topological properties with quasi-Majorana states", SciPost Phys. 7, 61 (2019).

${ }^{95}$ H. Pan, W. S. Cole, J. D. Sau, and S. Das Sarma, “Generic quantized zerobias conductance peaks in superconductor-semiconductor hybrid structures", Phys. Rev. B 101, 024506 (2020).

${ }^{96} \mathrm{C}$. Zeng, G. Sharma, S. Tewari, and T. Stanescu, “Partially-separated majorana modes in a disordered medium", arXiv:2105.06469 (2021).

${ }^{97}$ P. Yu, J. Chen, M. Gomanko, G. Badawy, E. P. A. M. Bakkers, K. Zuo, V. Mourik, and S. M. Frolov, "Non-majorana states yield nearly quantized conductance in proximatized nanowires", Nature Physics, 10.1038/ s41567-020-01107-w (2021). 
${ }^{98}$ S. Das Sarma, J. D. Sau, and T. D. Stanescu, "Splitting of the zero-bias conductance peak as smoking gun evidence for the existence of the majorana mode in a superconductor-semiconductor nanowire", Phys. Rev. B 86, 220506 (2012).

${ }^{99}$ T. D. Stanescu and S. Tewari, "Nonlocality of zero-bias anomalies in the topologically trivial phase of majorana wires", Phys. Rev. B 89, 220507 (2014).

${ }^{100}$ L. N. Cooper, "Bound electron pairs in a degenerate fermi gas", Phys. Rev. 104, 1189-1190 (1956).

${ }^{101}$ D. LEHMANN, Mathematical methods of many-body quantum field theory (CRC PRESS, 2019).

${ }^{102}$ A. Haim and Y. Oreg, "Time-reversal-invariant topological superconductivity", arXiv 1809.06863 (2018).

${ }^{103}$ A. J. Mildred S. Dresselhaus Gene Dresselhaus, Group theory: application to the physics of condensed matter (Springer, 2008).

${ }^{104}$ D. A. Ivanov, “Non-abelian statistics of half-quantum vortices in $p$-wave superconductors", Phys. Rev. Lett. 86, 268-271 (2001).

${ }^{105}$ A. Manchon, H. C. Koo, J. Nitta, S. M. Frolov, and R. A. Duine, "New perspectives for rashba spin-orbit coupling", Nature Materials 14, 871-882 (2015).

${ }^{106}$ B. Nijholt and A. R. Akhmerov, "Orbital effect of magnetic field on the majorana phase diagram", Phys. Rev. B 93, 235434 (2016).

${ }^{107}$ G. W. Winkler, D. Varjas, R. Skolasinski, A. A. Soluyanov, M. Troyer, and M. Wimmer, "Orbital contributions to the electron $g$ factor in semiconductor nanowires", Phys. Rev. Lett. 119, 037701 (2017).

${ }^{108}$ T. D. Stanescu and S. Tewari, “Robust low-energy andreev bound states in semiconductor-superconductor structures: importance of partial separation of component majorana bound states", Phys. Rev. B 100, 155429 (2019).

${ }^{109}$ B. D. Woods, S. Das Sarma, and T. D. Stanescu, “Electronic structure of fullshell inas/al hybrid semiconductor-superconductor nanowires: spin-orbit coupling and topological phase space", Phys. Rev. B 99, 161118 (2019).

${ }^{110}$ A. Vuik, D Eeltink, A. R. Akhmerov, and M Wimmer, "Effects of the electrostatic environment on the majorana nanowire devices", New Journal of Physics 18, 033013 (2016).

${ }^{111}$ S. Schuwalow, N. B. M. Schroeter, J. Gukelberger, C. Thomas, V. Strocov, J. Gamble, A. Chikina, M. Caputo, J. Krieger, G. C. Gardner, M. Troyer, G. Aeppli, M. J. Manfra, and P. Krogstrup, "Band bending profile and band offset extraction at semiconductor-metal interfaces", arXiv:1910.02735 (2019).

${ }^{112}$ L. Y. V. L. C. and M. Willatzen, The kp method: electronic properties of semiconductors (Springer, 2009).

${ }^{113} \mathrm{P}$. Löwdin, "A note on the quantum-mechanical perturbation theory", The Journal of Chemical Physics 19, 1396-1401 (1951). 
${ }^{114}$ P. Wójcik, A. Bertoni, and G. Goldoni, “Tuning rashba spin-orbit coupling in homogeneous semiconductor nanowires", Phys. Rev. B 97, 165401 (2018).

${ }^{115}$ R. M. L. Ramdas, Finite element and boundary element applications in quantum mechanics (Oxford University Press, 2007).

${ }^{116}$ A. E. Antipov, A. Bargerbos, G. W. Winkler, B. Bauer, E. Rossi, and R. M. Lutchyn, "Effects of gate-induced electric fields on semiconductor majorana nanowires", Phys. Rev. X 8, 031041 (2018).

${ }^{117}$ G. W. Winkler, A. E. Antipov, B. van Heck, A. A. Soluyanov, L. I. Glazman, M. Wimmer, and R. M. Lutchyn, "Unified numerical approach to topological semiconductor-superconductor heterostructures", Phys. Rev. B 99, 245408 (2019).

${ }^{118}$ C.-X. Liu, S. Schuwalow, Y. Liu, K. Vilkelis, A. L. R. Manesco, P. Krogstrup, and M. Wimmer, "Electronic properties of inas/eus/al hybrid nanowires", arXiv:2011.06567 (2020).

${ }^{119}$ S. Vaitiekènas, Y. Liu, P. Krogstrup, and C. M. Marcus, "Zero-bias peaks at zero magnetic field in ferromagnetic hybrid nanowires", Nature Physics 17, 43-47 (2020).

${ }^{120} \mathrm{C}$. Reeg, D. Loss, and J. Klinovaja, "Proximity effect in a two-dimensional electron gas coupled to a thin superconducting layer", Beilstein J. Nanotechnol. 9, 1263-1271 (2018).

${ }^{121}$ B. D. Woods, S. Das Sarma, and T. D. Stanescu, "Subband occupation in semiconductor-superconductor nanowires", Phys. Rev. B 101, 045405 (2020).

${ }^{122}$ J. Chen, B. D. Woods, P. Yu, M. Hocevar, D. Car, S. R. Plissard, E. P. A. M. Bakkers, T. D. Stanescu, and S. M. Frolov, “Ubiquitous non-majorana zerobias conductance peaks in nanowire devices", Phys. Rev. Lett. 123, 107703 (2019).

${ }^{123}$ L. O. Olsson, C. B. M. Andersson, M. C. Håkansson, J. Kanski, L. Ilver, and U. O. Karlsson, "Charge accumulation at inas surfaces", Phys. Rev. Lett. 76, 3626-3629 (1996).

${ }^{124} \mathrm{~J}$. R. Weber, A. Janotti, and C. G. Van de Walle, "Intrinsic and extrinsic causes of electron accumulation layers on inas surfaces", Applied Physics Letters 97, 192106 (2010).

${ }^{125}$ C. W. M. Castleton, A. Höglund, M. Göthelid, M. C. Qian, and S. Mirbt, "Hydrogen on iii-v (110) surfaces: charge accumulation and stm signatures", Phys. Rev. B 88, 045319 (2013).

${ }^{126}$ B. D. Woods, T. D. Stanescu, and S. Das Sarma, "Effective theory approach to the schrodinger-poisson problem in semiconductor majorana devices", Phys. Rev. B 98, 035428 (2018).

${ }^{127}$ A. E. G. Mikkelsen, P. Kotetes, P. Krogstrup, and K. Flensberg, “Hybridization at superconductor-semiconductor interfaces", Phys. Rev. X 8, 031040 (2018). 
${ }^{128}$ S. D. Escribano, A. Levy Yeyati, Y. Oreg, and E. Prada, "Effects of the electrostatic environment on superlattice majorana nanowires", Phys. Rev. B 100, 045301 (2019).

${ }^{129} \mathrm{~J}$. M. Luttinger, "Quantum theory of cyclotron resonance in semiconductors: general theory", Phys. Rev. 102, 1030-1041 (1956).

${ }^{130} \mathrm{C}$. Geuzaine and J. F. Remacle, “Gmsh: a three-dimensional finite element mesh generator with built-in pre- and post-processing facilities", International Journal for Numerical Methods in Engineering 79, 1309 (2009).

${ }^{131}$ D. I. Pikulin, J. P. Dahlhaus, M Wimmer, H Schomerus, and C. W. J. Beenakker, "A zero-voltage conductance peak from weak antilocalization in a majorana nanowire", New Journal of Physics 14, 125011 (2012).

${ }^{132}$ M. W. A. de Moor, J. D. S. Bommer, D. Xu, G. W. Winkler, A. E. Antipov, A. Bargerbos, G. Wang, N. van Loo, R. L. M. O. het Veld, S. Gazibegovic, D. Car, J. A. Logan, M. Pendharkar, J. S. Lee, E. P. A. M. Bakkers, C. J. Palmstrøm, R. M. Lutchyn, L. P. Kouwenhoven, and H. Zhang, "Electric field tunable superconductor-semiconductor coupling in majorana nanowires", New Journal of Physics 20, 103049 (2018).

${ }^{133}$ C. W. J. Beenakker, "Random-matrix theory of majorana fermions and topological superconductors", Rev. Mod. Phys. 87, 1037-1066 (2015).

${ }^{134}$ S. Das Sarma and X. C. Xie, "Calculated transport properties of ultrasubmicrometer quasi-one-dimensional inversion lines", Phys. Rev. B 35, 98759878 (1987).

${ }^{135} \mathrm{G}$. Binnig and H. Rohrer, "Scanning tunneling microscopy-from birth to adolescence", Rev. Mod. Phys. 59, 615-625 (1987).

${ }^{136}$ B. D. Woods, and T. D. Stanescu, “Electrostatic effects and topological superconductivity in semiconductor-superconductor-magnetic insulator hybrid wires", arXiv:2011.01933 (2020).

${ }^{137}$ T. Karzig, C. Knapp, R. M. Lutchyn, P. Bonderson, M. B. Hastings, C. Nayak, J. Alicea, K. Flensberg, S. Plugge, Y. Oreg, C. M. Marcus, and M. H. Freedman, "Scalable designs for quasiparticle-poisoning-protected topological quantum computation with majorana zero modes", Phys. Rev. B 95, 235305 (2017).

${ }^{138}$ Y. Liu, A. Luchini, S. Martí-Sánchez, C. Koch, S. Schuwalow, S. A. Khan, T. Stankevič, S. Francoual, J. R. L. Mardegan, J. A. Krieger, V. N. Strocov, J. Stahn, C. A. F. Vaz, M. Ramakrishnan, U. Staub, K. Lefmann, G. Aeppli, J. Arbiol, and P. Krogstrup, "Coherent epitaxial semiconductor-ferromagnetic insulator inas/eus interfaces: band alignment and magnetic structure", ACS Applied Materials \& Interfaces 12, PMID: 31877013, 8780-8787 (2020).

${ }^{139}$ Y. Liu, S. Vaitiekènas, S. Martí-Sánchez, C. Koch, S. Hart, Z. Cui, T. Kanne, S. A. Khan, R. Tanta, S. Upadhyay, M. E. Cachaza, C. M. Marcus, J. Arbiol, K. A. Moler, and P. Krogstrup, "Semiconductor-ferromagnetic insulator-superconductor nanowires: stray field and exchange field", Nano Letters 20, PMID: 31769993, 456-462 (2020). 
${ }^{140} \mathrm{P} . \mathrm{M}$. Tedrow, J. E. Tkaczyk, and A. Kumar, "Spin-polarized electron tunneling study of an artificially layered superconductor with internal magnetic field: euo-al", Phys. Rev. Lett. 56, 1746-1749 (1986).

${ }^{141} \mathrm{~T}$. Tokuyasu, J. A. Sauls, and D. Rainer, "Proximity effect of a ferromagnetic insulator in contact with a superconductor", Phys. Rev. B 38, 88238833 (1988).

${ }^{142}$ F. S. Bergeret, A. F. Volkov, and K. B. Efetov, “Induced ferromagnetism due to superconductivity in superconductor-ferromagnet structures", Phys. Rev. B 69, 174504 (2004).

${ }^{143}$ E. Strambini, V. N. Golovach, G. De Simoni, J. S. Moodera, F. S. Bergeret, and F. Giazotto, "Revealing the magnetic proximity effect in eus/al bilayers through superconducting tunneling spectroscopy", Phys. Rev. Materials 1, 054402 (2017).

${ }^{144}$ T. T. Heikkilä, M. Silaev, P. Virtanen, and F. S. Bergeret, “Thermal, electric and spin transport in superconductor/ferromagnetic-insulator structures", Progress in Surface Science 94, 100540 (2019).

${ }^{145}$ T. D. Stanescu and S. Das Sarma, "Proximity-induced low-energy renormalization in hybrid semiconductor-superconductor majorana structures", Phys. Rev. B 96, 014510 (2017).

${ }^{146}$ B. S. Chandrasekhar, "A note on the maximum critical field of high-field superconductors", Applied Physics Letters 1, 7-8 (1962).

${ }^{147}$ A. M. Clogston, "Upper limit for the critical field in hard superconductors", Phys. Rev. Lett. 9, 266-267 (1962).

${ }^{148} \mathrm{~W}$. Chen, X. Zhang, and Y. Huang, "Luminescence enhancement of eus nanoclusters in zeolite", Applied Physics Letters 76, 2328-2330 (2000).

${ }^{149}$ M. P. L. Sancho, J. M. L. Sancho, J. M. L. Sancho, and J Rubio, “Highly convergent schemes for the calculation of bulk and surface green functions", Journal of Physics F: Metal Physics 15, 851-858 (1985).

${ }^{150} \mathrm{~J}$. Klimeš, "Inelastic transmission of current through molecular bridge", MA thesis (2007).

${ }^{151}$ N. A. Court, A. J. Ferguson, and R. G. Clark, “Energy gap measurement of nanostructured aluminium thin films for single cooper-pair devices", Superconductor Science and Technology 21, 015013 (2007).

${ }^{152}$ W. Müller and W. Nolting, "Temperature-dependent quasiparticle band structure of the ferromagnetic semiconductor eus", Phys. Rev. B 66, 085205 (2002).

${ }^{153}$ H. O. H. Churchill, V. Fatemi, K. Grove-Rasmussen, M. T. Deng, P. Caroff, H. Q. Xu, and C. M. Marcus, "Superconductor-nanowire devices from tunneling to the multichannel regime: zero-bias oscillations and magnetoconductance crossover", Phys. Rev. B 87, 241401 (2013).

${ }^{154}$ S. M. Albrecht, A. P. Higginbotham, M. Madsen, F. Kuemmeth, T. S. Jespersen, J. Nygård, P. Krogstrup, and C. M. Marcus, "Exponential protection of zero modes in majorana islands", Nature 531, 206 (2016). 
${ }^{155}$ H. J. Suominen, M. Kjaergaard, A. R. Hamilton, J. Shabani, C. J. Palmstrøm, C. M. Marcus, and F. Nichele, "Zero-energy modes from coalescing andreev states in a two-dimensional semiconductor-superconductor hybrid platform", Phys. Rev. Lett. 119, 176805 (2017).

${ }^{156}$ M.-T. Deng, S. Vaitiekenass, E. Prada, P. San-Jose, J. Nygård, P. Krogstrup, R. Aguado, and C. M. Marcus, "Nonlocality of majorana modes in hybrid nanowires", Phys. Rev. B 98, 085125 (2018).

${ }^{157}$ A. Grivnin, E. Bor, M. Heiblum, Y. Oreg, and H. Shtrikman, “Concomitant opening of a bulk-gap with an emerging possible majorana zero mode", Nature Communications 10, 10.1038/s41467-019-09771-0 (2019).

${ }^{158}$ S. Vaitiekenas, G. W. Winkler, B. van Heck, T. Karzig, M.-T. Deng, K. Flensberg, L. I. Glazman, C. Nayak, P. Krogstrup, R. M. Lutchyn, and C. M. Marcus, "Flux-induced topological superconductivity in full-shell nanowires", Science 367, 10.1126/science . aav3392 (2020).

${ }^{159} \mathrm{~J}$. Klinovaja and D. Loss, "Fermionic and majorana bound states in hybrid nanowires with non-uniform spin-orbit interaction", The European Physical Journal B 88, 62 (2015).

${ }^{160}$ D. Chevallier, D. Sticlet, P. Simon, and C. Bena, “Mutation of andreev into majorana bound states in long superconductor-normal and superconductornormal-superconductor junctions", Phys. Rev. B 85, 235307 (2012).

${ }^{161}$ O. A. Awoga, J. Cayao, and A. M. Black-Schaffer, "Supercurrent detection of topologically trivial zero-energy states in nanowire junctions", Phys. Rev. Lett. 123, 117001 (2019).

${ }^{162}$ C. Zeng, C. Moore, A. M. Rao, T. D. Stanescu, and S. Tewari, “Analytical solution of the finite-length kitaev chain coupled to a quantum dot", Phys. Rev. B 99, 094523 (2019).

${ }^{163}$ S. T. Pantelides, "The electronic structure of impurities and other point defects in semiconductors", Rev. Mod. Phys. 50, 797-858 (1978).

${ }^{164}$ M. W. A. de Moor, J. D. S. Bommer, D. Xu, G. W. Winkler, A. E. Antipov, A. Bargerbos, G. Wang, N. van Loo, R. L. M. O. het Veld, S. Gazibegovic, D. Car, J. A. Logan, M. Pendharkar, J. S. Lee, E. P. A. M. Bakkers, C. J. Palmstrøm, R. M. Lutchyn, L. P. Kouwenhoven, and H. Zhang, "Electric field tunable superconductor-semiconductor coupling in majorana nanowires", New Journal of Physics 20, 103049 (2018).

${ }^{165}$ J. S. Lee, S. Choi, M. Pendharkar, D. J. Pennachio, B. Markman, M. Seas, S. Koelling, M. A. Verheijen, L. Casparis, K. D. Petersson, I. Petkovic, V. Schaller, M. J. W. Rodwell, C. M. Marcus, P. Krogstrup, L. P. Kouwenhoven, E. P. A. M. Bakkers, and C. J. Palmstrøm, "Selective-area chemical beam epitaxy of in-plane inas one-dimensional channels grown on inp(001), inp(111)b, and inp(011) surfaces", Phys. Rev. Materials 3, 084606 (2019). 
${ }^{166}$ J. D. S. Bommer, H. Zhang, O. Gül, B. Nijholt, M. Wimmer, F. N. Rybakov, J. Garaud, D. Rodic, E. Babaev, M. Troyer, D. Car, S. R. Plissard, E. P. A. M. Bakkers, K. Watanabe, T. Taniguchi, and L. P. Kouwenhoven, "Spin-orbit protection of induced superconductivity in majorana nanowires", Phys. Rev. Lett. 122, 187702 (2019).

${ }^{167}$ J. Shen, G. W. Winkler, F. Borsoi, S. Heedt, V. Levajac, J. Y. Wang, D. van Driel, D. Bouman, S. Gazibegovic, R. L. M. O. H. Veld, D. Car, J. A. Logan, M. Pendharkar, C. J. Palmstrom, E. P. A. M. Bakkers, L. P. Kouwenhoven, and B. van Heck, A full parity phase diagram of a majorana island, 2020.

${ }^{168}$ C. Reeg, D. Loss, and J. Klinovaja, "Metallization of a rashba wire by a superconducting layer in the strong-proximity regime", Phys. Rev. B 97, 165425 (2018).

${ }^{169}$ R. B. Lehoucq, D. C. Sorensen, and C. Yang, Arpack users' guide (Society for Industrial and Applied Mathematics, 1998).

${ }^{170}$ G. E. Blonder, M. Tinkham, and T. M. Klapwijk, "Transition from metallic to tunneling regimes in superconducting microconstrictions: excess current, charge imbalance, and supercurrent conversion", Phys. Rev. B 25, 4515 (1982).

${ }^{171}$ J. Stenger and T. D. Stanescu, "Tunneling conductance in semiconductorsuperconductor hybrid structures", Phys. Rev. B 96, 214516 (2017).

${ }^{172}$ C.-X. Liu, J. D. Sau, and S. Das Sarma, "Role of dissipation in realistic majorana nanowires", Phys. Rev. B 95, 054502 (2017).

${ }^{173} \mathrm{M}$. Wimmer and K. Richter, "Optimal block-tridiagonalization of matrices for coherent charge transport", Journal of Computational Physics 228, 8548-8565 (2009).

${ }^{174}$ D. S. Fisher and P. A. Lee, "Relation between conductivity and transmission matrix", Phys. Rev. B 23, 6851-6854 (1981).

${ }^{175}$ J. D. Sau and E. Demler, "Bound states at impurities as a probe of topological superconductivity in nanowires", Phys. Rev. B 88, 205402 (2013).

${ }^{176}$ T. Karzig, W. S. Cole, and D. I. Pikulin, "Quasiparticle poisoning of majorana qubits", Phys. Rev. Lett. 126, 057702 (2021).

${ }^{177}$ T. D. Stanescu, R. M. Lutchyn, and S. Das Sarma, “Majorana fermions in semiconductor nanowires", Phys. Rev. B 84, 144522 (2011).

${ }^{178}$ S. Das Sarma, A. Nag, and J. D. Sau, "How to infer non-abelian statistics and topological visibility from tunneling conductance properties of realistic majorana nanowires", Phys. Rev. B 94, 035143 (2016).

${ }^{179}$ B. D. Woods and T. D. Stanescu, “Enhanced topological protection in planar quasi-one-dimensional channels with periodically modulated width", Phys. Rev. B 101, 195435 (2020).

${ }^{180}$ L. Pfeiffer, K. W. West, H. L. Stormer, and K. W. Baldwin, “Electron mobilities exceeding $10^{7} \mathrm{~cm}^{2} / \mathrm{V}$ s in modulation-doped GaAs", Applied Physics Letters 55, 1888-1890 (1989). 
${ }^{181}$ W. Bauriedl, P. Ziemann, and W. Buckel, "Electron-tunneling observation of impurity bands in superconducting manganese-implanted lead", Phys. Rev. Lett. 47, 1163-1165 (1981).

${ }^{182}$ A. Yazdani, B. A. Jones, C. P. Lutz, M. F. Crommie, and D. M. Eigler, "Probing the local effects of magnetic impurities on superconductivity", Science 275, 1767-1770 (1997).

${ }^{183}$ I. Martin and D. Mozyrsky, "Nonequilibrium theory of tunneling into a localized state in a superconductor", Phys. Rev. B 90, 100508 (2014).

${ }^{184}$ C. W. Groth, M. Wimmer, A. R. Akhmerov, and X. Waintal, “Kwant: a software package for quantum transport", New Journal of Physics 16, 063065 (2014).

${ }^{185}$ F. J. C. Dominguez, P. San-Jose, R. Aguado, A. L. Yeyati, and E. Prada, "Zero-energy pinning from interactions in majorana nanowires", npj Quantum Materials 2, 13 (2016).

${ }^{186}$ S. D. Escribano, A. L. Yeyati, and E. Prada, "Interaction-induced zeroenergy pinning and quantum dot formation in Majorana nanowires", Beilstein J. Nanotechnol 9, 2171-2180 (2018).

${ }^{187}$ O. Gül, H. Zhang, F. K. de Vries, J. van Veen, K. Zuo, V. Mourik, S. ConesaBoj, M. P. Nowak, D. J. van Woerkom, M. Quintero-Pérez, M. C. Cassidy, A. Geresdi, S. Koelling, D. Car, S. R. Plissard, E. P. A. M. Bakkers, and L. P. Kouwenhoven, "Hard superconducting gap in insb nanowires", Nano Letters 17, 2690 (2017).

${ }^{188}$ J. Shabani, M. Kjaergaard, H. J. Suominen, Y. Kim, F. Nichele, K. Pakrouski, T. Stankevic, R. M. Lutchyn, P. Krogstrup, R. Feidenhans'1, S. Kraemer, C. Nayak, M. Troyer, C. M. Marcus, and C. J. Palmstrøm, "Two-dimensional epitaxial superconductor-semiconductor heterostructures: a platform for topological superconducting networks", Phys. Rev. B 93, 155402 (2016).

${ }^{189}$ M. Kjaergaard, F. Nichele, H. J. Suominen, M. P. Nowak, M. Wimmer, A. R. Akhmerov, J. A. Folk, K. Flensberg, J. Shabani, C. J. Palmstrøm, and C. M. Marcus, "Quantized conductance doubling and hard gap in a two-dimensional semiconductor-superconductor heterostructure", Nature Communications 7, 12841 (2016).

${ }^{190}$ E. C. T. O'Farrell, A. C. C. Drachmann, M. Hell, A. Fornieri, A. M. Whiticar, E. B. Hansen, S. Gronin, G. C. Gardner, C. Thomas, M. J. Manfra, K. Flensberg, C. M. Marcus, and F. Nichele, "Hybridization of subgap states in one-dimensional superconductor-semiconductor coulomb islands", Phys. Rev. Lett. 121, 256803 (2018).

${ }^{191}$ A. Fornieri, A. M. Whiticar, F. Setiawan, E. Portolés, A. C. C. Drachmann, A. Keselman, S. Gronin, C. Thomas, T. Wang, R. Kallaher, and et al., "Evidence of topological superconductivity in planar josephson junctions", Nature 569, 89-92 (2019). 
${ }^{192}$ J. S. Lee, B. Shojaei, M. Pendharkar, A. P. Mcfadden, Y. Kim, H. J. Suominen, M. Kjaergaard, F. Nichele, H. Zhang, C. M. Marcus, and et al., "Transport studies of epi-al/inas two-dimensional electron gas systems for required building-blocks in topological superconductor networks", Nano Letters 19, 3083-3090 (2019).

${ }^{193}$ H. Ren, F. Pientka, S. Hart, A. T. Pierce, M. Kosowsky, L. Lunczer, R. Schlereth, B. Scharf, E. M. Hankiewicz, L. W. Molenkamp, and et al., "Topological superconductivity in a phase-controlled josephson junction", Nature 569, 93-98 (2019).

${ }^{194}$ N. Pankratova, H. Lee, R. Kuzmin, K. Wickramasinghe, W. Mayer, J. Yuan, M. G. Vavilov, J. Shabani, and V. E. Manucharyan, “Multiterminal josephson effect", Phys. Rev. X 10, 031051 (2020).

${ }^{195}$ W. Mayer, W. F. Schiela, J. Yuan, M. Hatefipour, W. L. Sarney, S. P. Svensson, A. C. Leff, T. Campos, K. S. Wickramasinghe, M. C. Dartiailh, and et al., "Superconducting proximity effect in inassb surface quantum wells with in situ al contacts", ACS Applied Electronic Materials 2, 2351-2356 (2020).

${ }^{196}$ M. C. Dartiailh, W. Mayer, J. Yuan, K. S. Wickramasinghe, A. Matos-Abiague, and J. Shabani, "Phase signature of topological transition in josephson junctions", Phys. Rev. Lett. 126, 036802 (2021).

${ }^{197}$ M. Hell, M. Leijnse, and K. Flensberg, “Two-dimensional platform for networks of majorana bound states", Phys. Rev. Lett. 118, 107701 (2017).

${ }^{198}$ F. Pientka, A. Keselman, E. Berg, A. Yacoby, A. Stern, and B. I. Halperin, "Topological superconductivity in a planar josephson junction", Phys. Rev. X 7, 021032 (2017).

${ }^{199}$ M. Hell, K. Flensberg, and M. Leijnse, “Coupling and braiding majorana bound states in networks defined in two-dimensional electron gases with proximity-induced superconductivity", Phys. Rev. B 96, 035444 (2017).

${ }^{200}$ F. Setiawan, C.-T. Wu, and K. Levin, "Full proximity treatment of topological superconductors in josephson-junction architectures", Phys. Rev. B 99, 174511 (2019).

${ }^{201}$ F. Setiawan, A. Stern, and E. Berg, "Topological superconductivity in planar josephson junctions: narrowing down to the nanowire limit", Phys. Rev. B 99, 220506 (2019).

${ }^{202}$ B. Scharf, F. Pientka, H. Ren, A. Yacoby, and E. M. Hankiewicz, “Tuning topological superconductivity in phase-controlled josephson junctions with rashba and dresselhaus spin-orbit coupling", Phys. Rev. B 99, 214503 (2019).

${ }^{203}$ T. Laeven, B. Nijholt, M. Wimmer, and A. R. Akhmerov, “Enhanced proximity effect in zigzag-shaped majorana josephson junctions", Phys. Rev. Lett. 125, 086802 (2020).

${ }^{204}$ Y. Zhang, K. Guo, and J. Liu, “Transport characterization of topological superconductivity in a planar josephson junction", Phys. Rev. B 102, 245403 (2020). 
${ }^{205}$ T. Zhou, M. C. Dartiailh, W. Mayer, J. E. Han, A. Matos-Abiague, J. Shabani, "Phase control of majorana bound states in a topological X junction", Phys. Rev. Lett. 124, 137001 (2020).

${ }^{206}$ J. D. Pakizer, B. Scharf, and A. Matos-Abiague, “Crystalline anisotropic topological superconductivity in planar josephson junctions", Phys. Rev. Research 3, 013198 (2021).

${ }^{207}$ O. Lesser, A. Saydjari, M. Wesson, A. Yacoby, and Y. Oreg, "Phase-induced topological superconductivity in a planar heterostructure", Phys. Rev. Lett. 124, 137001 (2021).

${ }^{208}$ Y. Levine, A. Haim, and Y. Oreg, "Realizing topological superconductivity with superlattices", Phys. Rev. B 96, 165147 (2017).

${ }^{209}$ Y. Lu, W.-Y. He, D.-H. Xu, N. Lin, and K. T. Law, "Platform for engineering topological superconductors: superlattices on rashba superconductors", Phys. Rev. B 94, 024507 (2016).

${ }^{210}$ F. Krizek, J. E. Sestoft, P. Aseev, S. Marti-Sanchez, S. Vaitiekenas, L. Casparis, S. A. Khan, Y. Liu, A. M. Whiticar, A. Fursina, F. Boekhout, R. Koops, E. Uccelli, L. P. Kouwenhoven, C. M. Marcus, J. Arbiol, and P. Krogstrup, "Field effect enhancement in buffered quantum nanowire networks", Phys. Rev. Materials 2, 093401 (2018).

${ }^{211}$ A. Weisshaar, J. Lary, S. M. Goodnick, and V. K. Tripathi, "Analysis and modeling of quantum waveguide structures and devices", Journal of Applied Physics 70, 355 (1991).

${ }^{212}$ J. D. Sau, S. Tewari, and S. Das Sarma, "Experimental and materials considerations for the topological superconducting state in electron- and holedoped semiconductors: searching for non-abelian majorana modes in $1 \mathrm{~d}$ nanowires and 2d heterostructures", Phys. Rev. B 85, 064512 (2012).

${ }^{213}$ H.-Y. Hui, J. D. Sau, and S. Das Sarma, "Bulk disorder in the superconductor affects proximity-induced topological superconductivity", Phys. Rev. B 92, 174512 (2015).

${ }^{214}$ P. W. Brouwer, M. Duckheim, A. Romito, and F. von Oppen, “Topological superconducting phases in disordered quantum wires with strong spinorbit coupling", Phys. Rev. B 84, 144526 (2011).

${ }^{215} \mathrm{M}$. Deserno, "How to generate exponentially correlated gaussian random numbers", Phys. Rev. Materials 2, 093401 (2002).

${ }^{216}$ O. Motrunich, K. Damle, and D. A. Huse, “Griffiths effects and quantum critical points in dirty superconductors without spin-rotation invariance: one-dimensional examples", Phys. Rev. B 63, 224204 (2001).

${ }^{217}$ J. P. T. Stenger, B. D. Woods, S. M. Frolov, and T. D. Stanescu, “Control and detection of majorana bound states in quantum dot arrays", Phys. Rev. B 98, 085407 (2018).

${ }^{218}$ G. D. Smith, Numerical solution of partial differential equations: finite difference methods, Vol. 2 (Clarendon Press, 2008), p. 093401. 
${ }^{219}$ E. O. Kane, "Band structure of indium antimonide", Journal of Physics and Chemistry of Solids 1, 249 -261 (1957).

${ }^{220}$ M. M. Odashima, B. G. Prado, and E. Vernek, "Pedagogical introduction to equilibrium greens functions: condensed-matter examples with numerical implementations", Revista Brasileira de Ensino de Física 39, 093401 (2016).

${ }^{221}$ W. Nolting and W. D. Brewer, Fundamentals of many-body physics, Vol. 2 (Springer, 2018), p. 093401.

${ }^{222}$ C. M. Bender, Advanced mathematical methods for scientists and engineers: asymptotic methods and perturbation theory, Vol. 2 (Springer, 1999), p. 093401.

${ }^{223}$ S. Datta, Electronic transport in mesoscopic systems, Vol. 2 (Cambridge University Press, 1999), p. 093401.

${ }^{224}$ Y. Ashida, Z. Gong, and M. Ueda, "Non-hermitian physics", Advances in Physics 69, 249-435 (2020). 